Florida International University FIU Digital Commons

5-31-2011

\title{
Hydroxyapatite-Nanotube Composites and Coatings for Orthopedic Applications
}

Debrupa Lahiri

Florida International University, dlahi001@fiu.edu

DOI: $10.25148 /$ etd.FI1 1080203

Follow this and additional works at: https://digitalcommons.fiu.edu/etd

\section{Recommended Citation}

Lahiri, Debrupa, "Hydroxyapatite-Nanotube Composites and Coatings for Orthopedic Applications" (2011). FIU Electronic Theses and Dissertations. 444.

https://digitalcommons.fiu.edu/etd/444 


\title{
FLORIDA INTERNATIONAL UNIVERSITY
}

\author{
Miami, Florida
}

\section{HYDROXYAPATITE-NANOTUBE COMPOSITES AND COATINGS FOR ORTHOPEDIC APPLICATIONS}

A dissertation submitted in partial fulfillment of the

requirements for the degree of

DOCTOR OF PHILOSOPHY

in

MATERIALS SCIENCE AND ENGINEERING

by

Debrupa Lahiri

2011 


\section{To: Dean Amir Mirmiran}

College of Engineering and Computing

This dissertation, written by Debrupa Lahiri, and entitled Hydroxyapatite-Nanotube Composites and Coatings for Orthopedic Applications, having been approved in respect to style and intellectual content, is referred to you for judgment.

We have read this dissertation and recommend that it be approved.

$\begin{array}{r}\text { Lidia Kos } \\ \hline \text { W. Kinzy Jones } \\ \hline \text { Norman D.H. Munroe } \\ \hline \text { Arvind Agarwal, Major Professor }\end{array}$

Arvind Agarwal, Major Professor

Date of Defense: May 31, 2011

The dissertation of Debrupa Lahiri is approved.

Dean Amir Mirmiran College of Engineering and Computing

Interim Dean Kevin O'Shea University Graduate School

Florida International University, 2011 


\section{DEDICATION}

I dedicate this dissertation to my parents, son and husband - without their energetic support and love, it would have been impossible to complete this research. 


\section{ACKNOWLEDGMENTS}

I sincerely thank my advisor, Dr. Arvind Agarwal, for his extensive support throughout the span of my $\mathrm{PhD}$ research - without his continued, enthusiastic and critical guidance, it would not have been possible for me to write this page today. Starting from his vibrant classroom teaching to energetic discussions about research planning or results, he was successful to induce a high level of liveliness to his students. Working closely with him for almost four years, I learnt a lot about multi-tasking ability and time management. He has devoted long hours of discussions during the course of this research, which helped me immensely to understand the issues much thoroughly. Apart from mentoring to sharpen my technical abilities, he was equally careful for my personality development, too. His constant support has helped me to gain important confidence, which, I think, will be helpful for my future career also. In short, his dedication towards his students' career is unlimited and it is impossible for me to narrate all those great efforts in this short paragraph. He has set an example how an advisor should be and I cannot think one could be better than that. I wish I could follow his footsteps to be an advisor like him, which is extremely difficult.

I would like to offer my heartfelt thanks to Dr. Lidia Kos and her PhD student Ms. Ana Paula Benaduce for their continued and extensive support regarding the biological experiments of this research. Dr. Kos has spent lot of time in advising, planning and discussing minute steps in necessary bio-studies, while Ana Paula was extremely sincere to devote hours together to make those plans a reality. She was always available for clarifying my queries - be it early morning or late evening. I learnt a lot from both of them regarding biology. I could not have imagined of performing the bio-related studies 
in my $\mathrm{PhD}$ research without them. I am also thankful to Dr. Rouzaud - he was extremely helpful in my initial stages of research while I was being introduced to the bio-part of it. I learnt a lot from him.

I sincerely thank Dr. W. Kinzy Jones and Dr. Norman D.H. Munroe for serving on my dissertation committee and for their timely advice and support throughout my $\mathrm{PhD}$ research. Dr. Jones is always very supportive with his advices and provided me immense help with the research facilities. Dr. Munroe was always so helpful towards my research and available whenever I needed his support.

My special thanks to all my group mates - especially, Dr. Kantesh Balani and Dr. Anup Kumar Keshri. I found them always beside me, whenever I need any help, be it technical or personal. In my initial days, I learnt a lot from Kantesh and even today when I need any technical help, I do not hesitate to contact him. I tried my best to learn from him about the passion to feel for everyone around. Anup has been a very good friend of mine and I could share all my failures and successes with him. In today's world, it is difficult to find friends like them. I can never forget those moments I spent with Kantesh or Anup - those days in Miami are my treasures. 'Thanks' is a small word to express my feelings for them - I found true friends in them.

My sincere thanks to Dr. Yao Chen and Dr. Srinivasa Rao Bakshi - I had many useful technical discussion sessions with them and I gained much from those sessions. I also wish thank my other group mates - Dr. Ruben, Riken, Jorge, Di, Sam and Sunil. Cheng deserves a special mention - due to his energetic presence in the lab, during last phase of my $\mathrm{PhD}$. Jonathan Solomon and Sanat Ghosh are two very intelligent undergraduate interns - I enjoyed working closely with them and I wish them the best in 
their careers. I am also thankful to all the undergraduate and high-school interns, who have worked in our group during last four years - they have made my stay in Miami a lovely experience.

I am also thankful to Dr. Sudipta Seal (UCF, Orlando, FL, USA) and his PhD student Virendra for the excellent HRTEM studies. Virendra was always very coordinating and his excellent HRTEM expertise has taken the analysis of our results to a different level. He was always available and spent hours together for related discussion, which was very helpful towards my research.

I sincerely acknowledge Dr. Nadia Jessel (University of Strasbourg, France) and her PhD student, Sybille for the in vivo studies. It was a very meaningful collaboration with them that has helped a lot in fulfillment of the aim of my dissertation. They were very nice hosts during my visit to University of Strasbourg, Francs in February, 2010. I learnt a lot about biology and animal studies during that visit from Dr. Jessel, Sybille and other group members, for which I am grateful to them.

My sincere thanks to Dr. Surendra Saxena (FIU) for allowing me to use Raman facilities in his lab and to Dr. Jiuhua Chen for his generous support during my studies.

My heartfelt thanks to Dr. Liu and Neal Ricks - they were supportive to my experiments throughout my research period. Without their extensive help, it could not have been possible for me to finish my studies in a timely manner. I have learnt a lot from both of them about different characterization techniques and have always found them ready to help going beyond their limits. I am also thankful to our department staffs - Maite and Martha - they always offered a warm welcome and shared their experience in reducing my involvement in many administrative and purchase related duties. 
I wish to thank my good friend, Vennila - she is another true friend of mine. She was one of the persons, who has made my initial days in Miami as comfortable as my home. I also wish to thank Dr. Anuradha Godavarty. Though I was never technically related with her, but she was very caring and encouraging and gave me lot of confidence in finishing my $\mathrm{PhD}$ research timely.

I also thank Puneet, Ali (Karbasi), Lyci, Sarah, Shadab, Shabnam and Rupak they have extended their hands whenever I needed any help from them during my research.

I am thankful to University Graduate School, FIU for supporting me through Dissertation Evidence Acquisition (DEA) and Dissertation Year Fellowship (DYF) awards. I also acknowledge financial support from NSF grant (NSF-DMI-0547178).

I am grateful to my parents - they were the source of energy for me to join $\mathrm{PhD}$ and finish it in a timely manner. Apart from his inspiration and support, my father, Dr. Sisir Mondal, has also helped me technically during this research. Being a professor in Biology, my father taught me many biological issues in simple terms - it could have taken long time for me otherwise, if I had to learn them by self-study. His discussion and tutelage was very helpful in understanding many critical issues related to the bio-aspect of my research, which was not my expertise. My mother, Mrs. Ila Mondal, was always extra supportive for my $\mathrm{PhD}$. She discontinued her own $\mathrm{PhD}$ research to take care of me, after my birth; so she wanted to make sure that I could finish my PhD without any hassle. They have taken the responsibility of growing up my son, so that I can concentrate on my studies. And, I don't know how to express my feelings for my sweet, little son Dibyanshu. He probably did not understand what he missed, as my parents have not left 
any effort to give him parents' love and comfort. I missed him throughout these 4 years it was really painful to stay away from him. I hope to be with him very soon and give him extra love and care that was due from my side in his childhood days. I do not find any word when I talk about these three most influencing person in life - this dissertation is a small gift to them.

I also thank my brother, Rudra. Sweet memories of growing up together, especially when we both were kids, were always inspiring for me.

Lastly, this section will be incomplete without mentioning about another influential person in my life - Indranil. He is my husband, best friend and the person who is always with me, through ups and downs of life in last 14 years and I learnt a lot from him in different aspects of life. I would not have dared to start PhD after a discontinuation of 7 years from academic environment, without his support. Apart from all these, having similar academic background, his discussions have supported me a lot in this research. He was always available for his inputs in all the critical moments of this research. His round-the-clock encouragement has energized me to finish this research. This dissertation is a small gift to him, too. 


\title{
ABSTRACT OF THE DISSERTATION \\ HYDROXYAPATITE-NANOTUBE COMPOSITES AND COATINGS \\ FOR ORTHOPEDIC APPLICATIONS
}

\author{
by
}

Debrupa Lahiri

Florida International University, 2011

Miami, Florida

\section{Professor Arvind Agarwal, Major Professor}

Hydroxyapatite (HA) has received wide attention in orthopedics, due to its biocompatibility and osseointegration ability. Despite these advantages, the brittle nature and low fracture toughness of HA often results in rapid wear and premature fracture of implant. Hence, there is a need to improve the fracture toughness and wear resistance of HA without compromising its biocompatibility.

The aim of the current research is to explore the potential of nanotubes as reinforcement to HA for orthopedic implants. HA- 4 wt.\% carbon nanotube (CNT) composites and coatings are synthesized by spark plasma sintering and plasma spraying respectively, and investigated for their mechanical, tribological and biological behavior. CNT reinforcement improves the fracture toughness $(>90 \%)$ and wear resistance $(>66 \%)$ of HA for coating and free standing composites. CNTs have demonstrated a positive influence on the proliferation, differentiation and matrix mineralization activities of osteoblasts, during in-vitro biocompatibility studies. In-vivo exposure of HA-CNT coated titanium implant in animal model (rat) shows excellent histocompatibility and neobone integration on the implant surface. The improved osseointegration due to presence of 
CNTs in HA is quantified by the adhesion strength measurement of single osteoblast using nano-scratch technique.

Considering the ongoing debate about cytotoxicity of CNTs in the literature, the present study also suggests boron nitride nanotube (BNNT) as an alternative reinforcement. BNNT with the similar elastic modulus and strength as CNT, were added to HA. The resulting composite having 4 wt.\% BNNTs improved the fracture toughness ( $\sim 5 \%)$ and wear resistance $(\sim 75 \%)$ of HA in the similar range as HA-CNT composites. BNNTs were found to be non-cytotoxic for osteoblasts and macrophages. In-vitro evaluation shows positive role of BNNT in osteoblast proliferation and viability. Apatite formability of BNNT surface in $\sim 4$ days establishes its osseointegration ability. 


\section{TABLE OF CONTENTS}

CHAPTER

PAGE

1.0 INTRODUCTION 1

1.1 Hydroxyapatite for Orthopedic Applications- Advantages and

Limitations and solution

1

1.2 Carbon Nanotubes: Potential Reinforcement to Hydroxyapatite 4

1.2.1 Scope of Improvement in Mechanical And Tribological

Properties

1.2.2 Issues Related to Biocompatibility

4

4

1.3 Boron Nitride Nanotubes: Alternative Reinforcement to Hydroxyapatite

5

$\begin{array}{lll}1.4 & \text { Objectives of the Current Research } & 6\end{array}$

$\begin{array}{lll}1.5 & \text { References } & 10\end{array}$

2.0 LITERATURE REVIEW 15

2.1 Hydroxyapatite-Carbon Nanotube Composite Systems for $\begin{array}{ll}\text { Orthopedic Application } & 15\end{array}$

2.1.1 Synthesis of Composite 16

2.1.1.1 Composite Powder Preparation 17

2.1.1.2 Consolidation of Composite Structure 27

2.1.1.3 Dispersion of Carbon Nanotubes in Composite 44

2.1.1.4 Phase Stability and Crystallinity of HA in
Composite

2.1.2 Mechanical Properties of HA-CNT Composites 53

2.1.2.1 Fracture Toughness $\quad 54$

2.1.2.2 Elastic Modulus $\quad 59$

2.1.2.3 Hardness $\quad 63$

2.1.2.4 Strength $\quad 66$

2.1.3 Tribological Properties of HA-CNT Composites 68

2.1.4 Biocompatibility of HA-CNT Composites $\quad 70$

2.1.4.1 In-vitro Assessment of Biocompatibility 77

2.1.4.2 In-vivo Assessment of Biocompatibility 81

2.2 Boron Nitride Nanotube: Alternative to Carbon Nanotube 83

2.2.1 What Is Boron Nitride Nanotube? 83

2.2.2 Boron Nitride Nanotube Reinforced Composites 87

2.2.3 Biocompatibility of Boron Nitride Nanotube 90

2.2.4 Predicted Advantages of Boron Nitride Nanotube over
Carbon Nanotube in HA for Orthopedics

2.3 Scope of Improvement of Nanotube Reinforced

$\begin{array}{ll}2.4 \text { References } & 96\end{array}$ 
3.1 Synthesis of Hydroxyapatite-Carbon Nanotube Composite 111

3.1.1 HA-CNT Composite Powder Preparation: Spray Drying 111

3.1.2 HA-CNT Composite Coating Synthesis: Plasma Spraying 113

3.1.3 Free Standing HA-CNT Composite Synthesis: Spark Plasma Sintering

3.2 Synthesis of Hydroxyapatite-Boron Nitride Nanotube

Composite

114

3.2.1 HA-BNNT Composite Powder Preparation: Wet Chemistry/Ultrasonication

3.2.2 Free Standing HA-BNNT Composite Synthesis: Spark Plasma Sintering

115

3.3 Microstructural Characterization

116

3.3.1 Scanning Electron Microscopy: Powder Morphology and Microstructure of Composites and Coatings

3.3.2 High Resolution Transmission Electron Microscopy: Interface of Hydroxyapatite and Nanotubes

3.3.3 Steoreological Analysis using Image Processing Software 117

3.3.4 X-Ray Diffraction: Phases and Crystallinity Determination

3.3.5 Raman Spectroscopy 118

3.3.6 Density and Surface Roughness Measurement 119

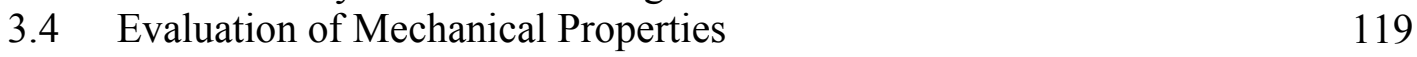

3.4.1 Nano-Indentation: Elastic Modulus and Hardness 119

3.4.2 Micro-Indentation: Fracture Toughness and Hardness 121

$\begin{array}{ll}3.5 & \text { Evaluation of Tribological Behavior } \\ & 122\end{array}$

3.5.1 Tribology: Macro-scale Wear 122

3.5.2 Nano-Scratch: Micro/Nano-scale Wear 123

$\begin{array}{lll}3.6 & \text { Evaluation of Biocompatibility } & 124\end{array}$

3.6.1 In-Vitro Evaluation 125

3.6.1.1 Osteoblast Cell Culture 125

3.6.1.2 Proliferation and Viability Study 125

3.6.1.3 BrDU Expression for Proliferation Kinetics 126

3.6.1.4 Gene Expression Related to Differentiation 127

3.6.1.5 Alkaline Phosphatase Expression Related to Mineralization 129

3.6.1.6 Actin Expression Related to Adhesion 129

3.6.1.7 Cytotoxicity of Wear Debris and BNNTs with
Osteoblasts and Macrophages

3.6.2 In-Vivo Evaluation of Plasma sprayed HA-CNT Coating 131

3.6.2.1 Implantation using Rat Model 132

3.6.2.2 Histology of Retrieved Bone with Implant 133

3.6.2.3 TEM Study of Retrieved Bone with Implant 133

3.7 Evaluation of Osseointegration 134 
3.7.1 Quantifying Adhesion of Osteoblasts on Composite

Surface: Nano-Scratch Technique

134

3.7.2 Determining Mechanical Compatibility of Implant-Bone Interface: Modulus Mapping Across Interface using Nano-DMA Technique

3.7.3 Apatite Formability of Boron Nitride Nanotube by Simulated Body Fluid Immersion

138

3.8 References

139

4.0 RESULTS AND DISCUSSION

4.1 Microstructural Evolution, Mechanical and Tribological

Performance of Composites

141

4.1.1 Hydroxyapatite-Carbon Nanotube Composite

4.1.1.1 Composite Coating by Plasma Spraying

141

142

4.1.1.1.1 Distribution and Structural Integrity of

Carbon Nanotube in Plasma Sprayed

Coating

142

4.1.1.1.2 Phase Stability and Crystallinity of HA in Plasma Sprayed Coating

4.1.1.1.3 Elastic Modulus and Fracture Toughness of Plasma Sprayed HA-CNT Coating

147

4.1.1.1.4 Wear Behavior of Plasma Sprayed HA-CNT Coating

150

4.1.1.1.5 Wear in Simulate Body Fluid (SBF) Environment

4.1.1.2 Free Standing Composite by Spark Plasma Sintering (SPS)

160

4.1.1.2.1 Distribution and Structural Integrity of Carbon Nanotube in SPS Structure

4.1.1.2.2 Phase Stability and Crystallinity of HA in SPS Structure

164

4.1.1.2.3 Role of Carbon Nanotube in Microstructural Evolution through Spark Plasma Sintering

4.1.1.2.4 Hydroxyapatite-Carbon Nanotube Interface in SPS Structure

173

4.1.1.2.5 Elastic Modulus and Fracture Toughness of SPS HA-CNT

177

4.1.1.2.6 Wear Behavior SPS HA-CNT Across Multiple Length Scale

4.1.2 Hydroxyapatite-Boron Nitride Nanotube Composite by Spark Plasma Sintering

193

4.1.2.1 Role of Boron Nitride Nanotube in Microstructural Evolution of SPS HA-BNNT composite

4.1.2.2 Distribution and Structural Integrity of Boron

Nitride Nanotube in SPS Structure 
4.1.2.3 Phase Stability and Crystallinity of HA in SPS HABNNT Structure

4.1.2.4 Hydroxyapatite-Boron Nitride Nanotube Interface in SPS Structure

4.1.2.5 Elastic Modulus and Fracture Toughness of SPS HA-BNNT

4.1.2.6 Wear Behavior SPS HA-BNNT 206

4.2 Biocompatibility of HA-Nanotube Composites and Coatings

4.2.1 Biocompatibility of Hydroxyapatite-Carbon Nanotube Composite

4.2.1.1 In-Vitro Assessment of HA-CNT

4.2.1.1.1 Osteoblast Viability

4.2.1.1.2 Proliferation Kinetics of Osteoblast

4.2.1.1.3 Evaluation of Osteoblast Differentiation through Gene Expression

4.2.1.1.4 Assessment of Mineralization through Alkaline Phosphatase Expression

4.2.1.1.5 Cytotoxicity Evaluation for Wear Debris with Osteoblasts and Macrophages

4.2.1.2 In-Vivo Evaluation of HA-CNT Coating

4.2.1.2.1 Implantation using Rat Model

4.2.1.2.2 Histology of Retrieved Bone with Implant

4.2.1.2.3 TEM Study of Retrieved Bone with Implant

4.2.2 In-vitro Biocompatibility of Hydroxyapatite-Boron

Nitride Nanotube Composite

4.2.2.1 Cytotoxicity of BNNTs to Osteoblasts and Macrophages

4.2.2.2 Osteoblast Proliferation and viability of SPS HA and HA-BNNT

4.3 Ossoeintegration Ability of Implant

4.3.1.1 Adhesion Quantification Techniques for a Single Cell

4.3.1.2 Quantification of Adhesion Force and Energy of Osteoblast on Composite Surface by Nano-scratch Technique

4.3.1.3 Osteoblast Adhesion Mechanism through Actin Expression

4.3.1.4 Role of CNT in Adhesion of Osteoblasts

4.3.2 Mechanical Compatibility of Implant-Bone Interface

4.3.2.1 Gradient of Elastic Modulus Across Bone-Implant Interface

4.3.2.2 Analyzing Role of CNT in Mechanical Health of Implanted Bone 
4.3.3 Apatite Formation on Boron Nitride Nanotube in Simulated Body Fluid

4.3.3.1 Morphology of Apatite Precipitate on BNNT

4.3.3.2 Compositional Analysis and Comparative Volume Determination of Apatite Precipitate

4.3.3.3 Nucleation and Growth Mechanism of Apatite Precipitate

4.4 Comparison of CNT vs. BNNT: As Reinforcement to HA 


\section{LIST OF TABLES}

TABLE

PAGE

Table 2.1: Summary of biocompatibility studies on HA-CNT composite systems

Table 2.2: Mechanical and physical properties of BNNT in comparison to CNT

Table 2.3: $\quad$ Summary of BNNT reinforced composite systems

Table 3.1: Plasma spraying parameters for HA/HA-CNT coating deposition

Table 3.2: $\quad$ Chemical composition of Simulated Body Fluid (SBF)

Table 4.1: Comparative quantification of microstructural features in spark plasma sintered HA and HA-CNT pellets

Table 4.2: Mechanical properties measured by nanoindentation and Vickers indentation methods and calculated through micromechanics models

Table 4.3: $\quad$ Percentage LDH absorbance values from live cells obtained through cytotoxicity tests indicating cytotoxicity level in osteoblasts \& macrophages cultured with and without wear debris ( $p$ value $<0.05$ )

Table 4.4: Adhesion force and energy of osteoblast on permanox plastic, HA and HA-CNT for 1, 3 and 5 days of incubation periods

Table 4.5: Comparison of spark plasma sintered HA-CNT and HABNNT composites 


\section{LIST OF FIGURES}

FIGURE

PAGE

Figure 1.1: Schematic of an orthopedic hip implant in human body and picture of a metallic hip implant

Figure 1.2: Flow chart of the research plan

Figure 2.1: Year-wise publication chart for HA-CNT system (source: scopus.com)

Figure 2.2: Classification of techniques adopted for better dispersion of CNT in composite powder/precursor (for Coatings)

Figure 2.3: TEM images of CNTs-HA (a) before and (b) after hydrothermal treatment. Inset shows the EDS spectrum for HA layer in (a)

Figure 2.4: TEM image of Fe-catalyst particles encapsulated in CVD grown CNT on HA powder

Figure 2.5: $\quad$ SEM images of ultrasonically mixed HA-CNTs powder at (a) low and (b) high magnifications

Figure 2.6: Classification of consolidation methods for HA-CNT composites

Figure 2.7: $\quad$ SEM micrographs of HA-CNT composites sintered in (a) argon and (b) vacuum

Figure 2.8: $\quad$ SEM micrographs of fracture surface for SPS processed HA-CNT composites sintered at (a) $900^{\circ} \mathrm{C}$; (b) $1000^{\circ} \mathrm{C}$; (c) $1100^{\circ} \mathrm{C}$; (d) $1200^{\circ} \mathrm{C}$

Figure 2.9: $\quad$ SEM micrographs of coating cross sections synthesized by plasma spraying of (a) HA and (b) HA-CNT

Figure 2.10: SEM micrographs of the HA-CNT coatings electrophoretically deposited for 8 minutes with DC voltages: (a) $10 \mathrm{~V}$; (b) $20 \mathrm{~V}$; and (c) $40 \mathrm{~V}$

Figure 2.11: SEM micrographs of electrophoretically deposited HACNTs coating on $\mathrm{Ti}$ alloy wire as a function of deposition time, (a) $1 \mathrm{~min}$, (b) $2 \mathrm{~min}$ and (c) $3 \mathrm{~min}$ 
Figure 2.12: SEM micrograph of electrophoretically deposited HACNT coating suggesting good dispersion of CNT in HA

Figure 2.13: SEM micrograph of electrophoretically deposited HACNT coating from chemically precipitated composite powder

Figure 2.14: $\quad$ SEM micrographs of uniformly distributed CNT in (a) CVD processed composite powder and (b) sintered composite fracture surface

Figure 2.15: Fracture surface of sintered HA-Carbon nano-fiber composite showing the uniform distribution of reinforcement phase

Figure 2.16: X-ray diffraction pattern of SPS sintered HA, HAAl2O3 (HA-A) and HA-A12O3-CNT (HA-A16C) composites

Figure 2.17: X-ray diffraction pattern of plasma sprayed HA coatings showing increased crystallinity in presence of CNT

Figure 2.18: Protruded CNT on fracture surface of HA-CNT composite synthesized by (a) hot pressing; (b) aerosol deposition

Figure 2.19: Percentage improvement in KIC as a function of CNT content in HA-CNT systems synthesized through different process routes

Figure 2.20: Change in the fracture toughness as a function of CNT content in a hot pressed HA-CNT system

Figure 2.21: Percentage improvement in elastic modulus (E) as a function of CNT content in HA-CNT systems synthesized through different process routes

Figure 2.22: Percentage improvement in hardness $(\mathrm{H})$ as a function of CNT content in HA-CNT systems synthesized through different process routes

Figure 2.23: Change in the bending strength as a function of CNT content in a hot pressed HA-CNT system 
Figure 2.24: Variation in coefficient of friction for plasma sprayed $\mathrm{HA}, \mathrm{HA}-\mathrm{Al}_{2} \mathrm{O}_{3}$ and $\mathrm{HA}-\mathrm{Al}_{2} \mathrm{O}_{3}-\mathrm{CNT}$ coatings (the undulation in the profile is due to surface roughness and structural heterogeneity)

Figure 2.25: SEM images showing the adhesion of fibroblast on vertically-aligned MWCNT after 7 days

Figure 2.26: SEM micrographs of pre-osteoblast MC3T3-E1 cells attached to (a) HA and (b) HA-CNT coatings for $5 \mathrm{hr}$

Figure 2.27: Pathological micrographs of striated mouse muscle after HA-CNT composite was embedded for (a) 1; (b) 3; (c) 5; (d) 7 and (e) 14 Days

Figure 2.28: Schematic of rolled h-BN sheet forming single walled boron nitride nanotube

Figure 2.29: Year-wise publication chart for BNNT reinforced composites (source: scopus.com)

Figure 3.1: Schematic of formation of spray dried HA-CNT composite powder from HA-nanorods and CNTs and SEM micrographs of (a) HA-nanorods, (b) multi-walled CNTs, (c) spray dried HA-CNT agglomerate, (d) distribution of CNT in spray-dried HA-CNT and (e) some CNT clusters on HA-CNT agglomerate surface

Figure 3.2: (a) SEM and (b) TEM images of the as-received BNNTs showing the presence of both tubular and bamboo type structures

Figure 3.3: (a) Schematic of the nano-scratch technique for measuring adhesion of individual osteoblast cell on substrate. (b) Representative lateral (shear) force vs. displacement curves for nano-scratches on HA-CNT substrate with osteoblast grown for 3 days. The curves show the variation in lateral force required to make a scratch in presence of a cell and on bare substrate. Different regions of the curve are correlated with the stages of scratch in schematic (a) with roman numbers (I, II and III). The shaded area denotes the adhesion energy and the height of the hump shows adhesion force of an osteoblast 
Figure 3.4: (a) Semi-thin (1-2 mm) section cut from the rat's bone with implant embedded inside. (b) Optical crosssectional micrograph of the retrieved implant showing Ti substrate, HA/HA-CNT coating and bone with marked regions for modulus mapping studies. Position 1 denotes the region in titanium part of the implant near coating. Positions $2-6$ are equally placed locations through the thickness of HA/HA-CNT coating starting from titanium side and going towards the bone. Position 7 is in the newly grown bone along the implant surface and position 8 is in a distant region that represents the normal bone

Figure 4.1: (a) High magnification SEM image of spray dried HACNT agglomerate showing homogeneous distribution of CNTs in HA nano-rods, (b) cross-section of plasma sprayed HA-CNT coating showing the splat structure, cracks and porosity, (c) fracture surface showing homogeneous distribution of CNT in HA matrix and (d) precipitation of HA crystals on CNTs

Figure 4.2: $\quad$ Raman spectra of HA-CNT powder and plasma sprayed HA-CNT coating showing D and G peaks of CNT

Figure 4.3: X-ray diffraction patterns for HA and HA-CNT powders and plasma sprayed coatings

Figure 4.4: (a) Load vs. displacement curves for HA and HA-CNT coatings obtained by nanoindentation, (b) statistical distribution of $\mathrm{E}$ value in $\mathrm{HA}$ and $\mathrm{HA}-\mathrm{CNT}$, (c) protruding $\mathrm{CNT}$ from HA-CNT fracture surface showing absence of crack or gap at matrix/CNT interface

Figure 4.5: SEM images of radial cracks emerging from 148 microindents on (a) HA and (b) HA-CNT coatings

Figure 4.6: CNT bridges offering crack propagation resistance in HA-CNT coating

Figure 4.7: Coefficient of friction for HA and HA-CNT coatings plotted against sliding distance

Figure 4.8: Three dimensional optical profiles of the wear tracks and a two dimensional profile across the track on (a) 
HA and (b) HA-CNT - at $5 \mathrm{~N}$ load and $100 \mathrm{~m}$ of sliding distance

Figure 4.9: Wear debris size distribution for HA and HA-CNT coatings

Figure 4.10: Wear tracks of (a) HA and (b) HA-CNT coatings showing abrasion and fracture mode of mass removal

Figure 4.11: Presence of CNTs in the wear debris generated from HA-CNT coating

Figure 4.12: Coefficient of friction for HA and HA-CNT coatings in SBF immersion plotted against sliding distance

Figure 4.13: Three dimensional optical profile of a section of the wear tracks on (a) HA and (b) HA-CNT; (c) two dimensional profiles across the tracks for HA and HACNT coatings - at $5 \mathrm{~N}$ load and $100 \mathrm{~m}$ of sliding distance while immersed in SBF

Figure 4.14: (a) Raman spectrum for HA-CNT powder, SPS pellet and wear track on SPS pellet showing D and G peak of CNT and phosphate peak of HA, (b) HRTEM of asreceived defect-free CNT, (c-d) HRTEM images of CNT in SPS HA-CNT pellet showing defects induced in CNT

Figure 4.15: X-ray diffraction patterns of spray dried HA and HACNT powders and SPS pellets

Figure 4.16: SEM micrographs of fracture surface of SPS pellets showing (a) monolithic and fine grain region in HA, (b) monolithic and fine grain region in HA-CNT, (c) partially sintered HA structure with fine grain region and porosity, (d) fully densified fine grain region of HA-CNT with homogeneously distributed CNTs, (e) monolithic region of HA-CNT with CNTs embedded in HA matrix and (f) CNT clusters at fine grain region of HA-CNT

Figure 4.17: (a) SEM micrograph of fracture surface of SPS-HA pellet showing porosity and (b) histogram showing bimodal porosity distribution in SPS-HA pellet 
Figure 4.18: Schematic showing effect of SPS and CNT on the consolidation mechanism and microstructural evolution in HA and HA-CNT pellets

Figure 4.19: HRTEM image of CNT and HA interface. FFT analysis reveals the CNT wall spacing and HA lattice spacing at the interface

Figure 4.20: (a) Schematic of atomic arrangement at the interface of basal plane of HA and graphene sheets on CNT surface and (b) Schematic of CNT open end showing alignment of CNT walls with (211) planes of HA

Figure 4.21: (a) Load vs. displacement plot for HA and HA-CNT composite obtained by nanoindentation, (b) statistical distribution of $\mathrm{E}$ value in HA and HA-CNT composites measured for more than 100 nano-indents in each sample

Figure 4.22: High magnification SEM micrographs of HA-CNT fracture surface showing (a) protruded CNTs and (b) strong and defect free interface of CNT with HA matrix

Figure 4.23: SEM micrographs of polished cross sections in HA and HA-CNT showing (a) Vickers indents on HA and (b) radial crack from the indent propagating in fine grain region of HA, (c) Vickers indent on HA-CNT, (d) deflection and arrest of radial crack at fine grain region in HA-CNT, (e) crack bridging by CNTs and (f) a single CNT bonded with HA matrix at both ends and forming bridge on crack.

Figure 4.24: (a) Wear volume loss and (b) coefficient of friction for HA and HA-CNT plotted against sliding distance during ball-on-disk wear, (c-d) high magnification SEM micrographs of broken and damaged CNTs observed in the HA-CNT wear track

Figure 4.25: Nano-scale (a) wear volume loss, (b) coefficient of friction and (c) percentage elastic depth recovery during nano-scratch of HA and HA-CNT as a function of normal load

Figure 4.26: Schematic diagram showing effective surface area (SAeff) for nano-scratch study 
Figure 4.27: Schematic diagram showing effective surface area ( $\left.\mathrm{SA}_{\mathrm{eff}}\right)$ for macro-wear study

Figure 4.28: Fracture surface of (a) HA and (b) HA-BNNT sintered pellets revealing the porosity content

Figure 4.29: (a-b) HRTEM images of BNNT in the sintered HABNNT pellet retaining their defect free structure; (c) Raman spectrum of HA and HA-BNNT powders and sintered pellet

Figure 4.30: X-ray diffraction pattern of HA and HA-BNNT sintered pellet showing HA and BN peaks

Figure 4.31: (a) HRTEM image of BNNT and HA interface. FFT analysis reveals the BNNT wall spacing and HA lattice (211) spacing at the interface; (b) schematic of atomic arrangement at the interface with basal plane of HA and coinciding h-BN sheet on BNNT outer wall; (c) schematic of BNNT open end showing alignment of h$\mathrm{BN}$ walls with (211) planes of HA

Figure 4.32: (a) Load vs. displacement plot for HA and HA-BNNT composite obtained by nanoindentation; (b) statistical distribution of $\mathrm{E}$ value in HA and HA-BNNT composites measured for more than 100 nano-indents in each sample

Figure 4.33: Vickers indent impressions showing radial crack generation in (a) HA and (b) HA-BNNT sintered pellets

Figure 4.34: BNNTs bridging the radial crack generated from microindent

Figure 4.35: (a) Coefficient of friction and (b) wear volume loss for HA and HA-BNNT plotted against sliding distance during ball-on-disk wear

Figure 4.36: Ball-on disc wear tracks on (a, c) HA and (b, d) HABNNT revealing different morphology

Figure 4.37: 'Sword in sheathe' type structure shown by BNNT upon application of stress

Figure 4.38: BNNT bridging of mass on wear track of HA-BNNT 
Figure 4.39: Fluorescent images of osteoblast cells grown for 3, 5 and 10 days on (a-c) HA and (d-f) HA-CNT coatings. The live cells are stained in green color with FDA and dead cells in red with PI

Figure 4.40: Osteoblast cell viability on HA and HA-CNT coatings for $1,3,5$ and 10 days of culture $(\mathrm{p}<0.05)$

Figure 4.41: Fluorescent images of osteoblasts on HA and HA-CNT coatings, exposed with BrDU after 1 day of incubation. The cells stained in red indicate total number of osteoblasts on substrate, whereas the cells proliferated during $1 \mathrm{hr}$ of BrDU exposure are stained in green

Figure 4.42: Fluorescent images of osteoblasts on HA and HA-CNT coatings, exposed with BrDU after 3 days of incubation

Figure 4.43: Fluorescent images of osteoblasts on HA and HA-CNT coatings, exposed with BrDU after 5 days of incubation

Figure 4.44: Proliferation rate of osteoblasts on HA and HA-CNT coatings after 1,3 and 5 days of exposure

Figure 4.45: Over expression of osteopontin, osteocalcin and RunX2 for osteoblasts cultured on HA-CNT for 5 days as compared to the cells on HA surface

Figure 4.46: Alkaline phosphatase expression for osteoblasts cultured on HA and HA-CNT surfaces for 5 days

Figure 4.47: (a) Ti-6Al-4V Alloy rod - coated with HA-CNT by plasma spraying; (b) top surface of plasma sprayed HACNT coating revealing embedded CNTs in HA matrix

Figure 4.48: (a) Retrieved femoral bone of rat with HA-CNT coated Ti-alloy rod implanted; (b) X-ray images of rat femoral bones after one month implantation. The distal part of femur contains (1) uncoated Ti-alloy implant, (2) HA coated Ti-alloy implant, (3) HA-CNT composite coated Ti-alloy and (4) no implant (normal)

Figure 4.49: Ex-vivo semi-thin (1-2 mm) sections cut from the rat's bone with implant embedded inside (magnification 5X)

Figure 4.50: Histological results (40X) for rat bones presented through Mallory coloration images for uncoated, HAcoated and HA-CNT coated implants. These images 
show normal, thick trabecular and hematopoietic marrow bones, without any inflammatory reactions and tissues. A layer is observed attached on the bone closed to the cavity $(\mathrm{C})$ caused by retrieved implant

Figure 4.51: Histological image (5X) of HA-CNT coated implant in rat, with toluidine blue coloration, shows HA-CNT coating strongly attached to newly grown bone tissues

Figure 4.52: TEM images of bone on HA-CNT coated implant at 2200X magnification

Figure 4.53: TEM images of (a) bone on uncoated Ti implant; (b) bone on HA coated implant; (c) normal bone at $2200 \mathrm{X}$ magnification

Figure 4.54: Cytotoxicity result of bare BNNTs with (a) osteoblasts and (b) macrophages obtained through LDH assay

Figure 4.55: Fluorescent images of osteoblasts grown on HA and HA-BNNT surface for (a-b) 1 day and (c-d) 3 days. The live cells are stained in green color with FDA and dead cells in red with PI

Figure 4.56: Osteoblast viability on HA and HA-BNNT surfaces for 1,3 and 5 days of culture $(\mathrm{p}<0.05)$

Figure 4.57: Optical Images of osteoblast on permanox surface grown for 3 days (A1) before and (B1) after nanoscratch. A2 and B2 show the magnified part of images $\mathrm{A} 1$ and $\mathrm{B} 1$, respectively, to clearly visualize the displacement of the cell by nano-scratching

Figure 4.58: Lateral force vs. displacement curves obtained from nano-scratch tests of (a) osteoblasts grown on both HA and HA-CNT substrate for 5 days and the bare substrates in same condition; (b) osteoblasts grown on HA-CNT surface for 1, 3 and 5 days and (c) osteoblasts grown on HA surface for 1, 3 and 5 days

Figure 4.59: Adhesion energy of osteoblast on permanox plastic, HA and HA-CNT substrates for 1,3 and 5 days of incubation periods

Figure 4.60: Fluorescence images of stained cytoskeleton showing actin network of osteoblasts grown for 1, 3 and 5 days 
on HA and HA-CNT surfaces. All the images were taken at 40X magnification

Figure 4.61: (a) Image analysis-based actin expression for substrates incubated with osteoblast for 1 day - showing number of stained pixels/cell for HA and HA-CNT substrates; (b) fraction of area covered by actin network (in \% of total area) at different substrates for different incubation periods - determined by evaluating fraction of stained pixels out of $\sim 8 \times 10^{6}$ pixels in each substrates (images of similar magnification were used for the measurements)

Figure 4.62: Schematic for proposed model of focal adhesion mechanism for osteoblast showing (a) osteoblast grown on HA-CNT coating; (b) SEM micrograph of HA-CNT coating top surface revealing exposed surfaces of embedded CNTs; (c) osteoblast focal adhesion assembly showing integrin mediated attachment of actin to CNT surface

Figure 4.63: Optical cross-sectional micrograph of the rat femur with HA-CNT coated implant, showing the locations of modulus mapping measurements by Arabic numbers. Position 1 denotes the region in titanium part of the implant near coating. Positions $2-6$ are equally placed locations through the thickness of HA/HA-CNT coating starting from titanium side and going towards the bone. Position 7 is in the newly grown bone along the implant surface and position 8 is in a distant region that represents the normal bone

Figure 4.64: Modulus map from different regions in implant/coating/bone interfaces

Figure 4.65: Gradient of elastic modulus across bone/implant interface for (a) uncoated Ti implant; (b) HA and HACNT coated Ti implants. Elastic modulus values were averaged from the modulus maps presented in figure 4.64

Figure 4.66: SEM images of (a) as-received BNNTs and apatite precipitation on BNNTs after (b) 7; (c) 14; (d) 28 days of soaking in SBF 
Figure 4.67: EDS spectra of SBF soaked BNNTs for (a) 7; (b) 14 and (c) 28 days. (d) Increasing Ca wt.\% in precipitate as a function of days of soaking

Figure 4.68: Raman spectra of as-received BNNTs and BNNTs soaked in SBF showing h-BN peaks of BNNT and phosphate peaks of HA

Figure 4.69: TEM image of BNNTs in (a) as-received condition; soaked in SBF for (b) 7; (c) 14 days and (d) 28 days. (e) Selected Area Diffraction (SAD) pattern of 28 days sample in figure ' $d$ ' showing the presence of crystalline HA

Figure 4.70: Distribution of HA needle length in 7 and 14 days samples

Figure 4.71: TEM images of precipitates showing HA needles and amorphous HA for (a) 7; (b) 14 and (c) 28 days

Figure 4.72: HRTEM image of HA precipitate on BNNT surface after 28 days of soaking in SBF, revealing both amorphous and crystalline nature of the precipitate

Figure 4.73: HA needles nucleated and grown on BNNT parallel to surface as well as making different angles 


\subsection{INTRODUCTION}

The aim of this study is to develop a composite material by reinforcing hydroxyapatite (HA) with nanotubes for orthopedic application. The idea is to develop a material that overcomes the existing limitations and disadvantages of hydroxyapatite in terms of poor fracture toughness and wear resistance. An in-depth analysis of mechanical and tribological properties of the newly developed material is conducted to establish its benefits over HA. Considering the application of HA-nanotube composite in orthopedics, different aspects of biocompatibility of the composite is evaluated using in-vitro and invivo studies.

\subsection{Hydroxyapatite for Orthopedic Applications - Advantages, Limitations and Solution}

The field of biomaterials is a rapidly emerging one, due to its direct relation with the healthcare and impact on human health related issues. The biomaterials device market was $\$ 115.4$ billion in the year 2008 and is expected to be $\$ 252.7$ billion in 2014 [1]. The largest market size amongst all biomaterial products belongs to orthopedic biomaterials. As a result, development and improvement of orthopedic related biomaterials is a very active and growing research field.

Orthopedic biomaterials are mainly of two types. The first ones are implants and fixing accessories, which are generally made of metals, ceramics, hard polymers or their composites. Second category consists of scaffolds for tissue regeneration, which are based on polymers - preferably the biodegradable ones and their composites. Hydroxyapatite (HA) is very attractive for orthopedic implants as its chemical 
composition is similar to the mineral crystallites present in human bone. Hydroxyapatite is basically a calcium phosphate based bioceramic with the chemical formula as: $\mathrm{Ca}_{10}\left(\mathrm{PO}_{4}\right)_{6}(\mathrm{OH})_{2}$. The bioactivity and osteoconductivity of HA offers a suitable surface for new bone growth and integration [2-6]. Thus, HA is being vastly researched and clinically used as free-standing implant, coating on metallic implants and also as reinforcement to polymer scaffold material for tissue regeneration [2, 5, 7-13]. Figure 1.1 presents a schematic of a hip implant in human body and a picture of a metallic hip implant with different parts [14-15]. As shown in the schematic, the stem part of the implant goes inside the cavity of femoral bone. HA coating is applied on the stem part, in order to have good bonding between the femoral bone and implant surface. The upper surface of the shell is also coated with HA in some cases to have good bonding with the pelvis.

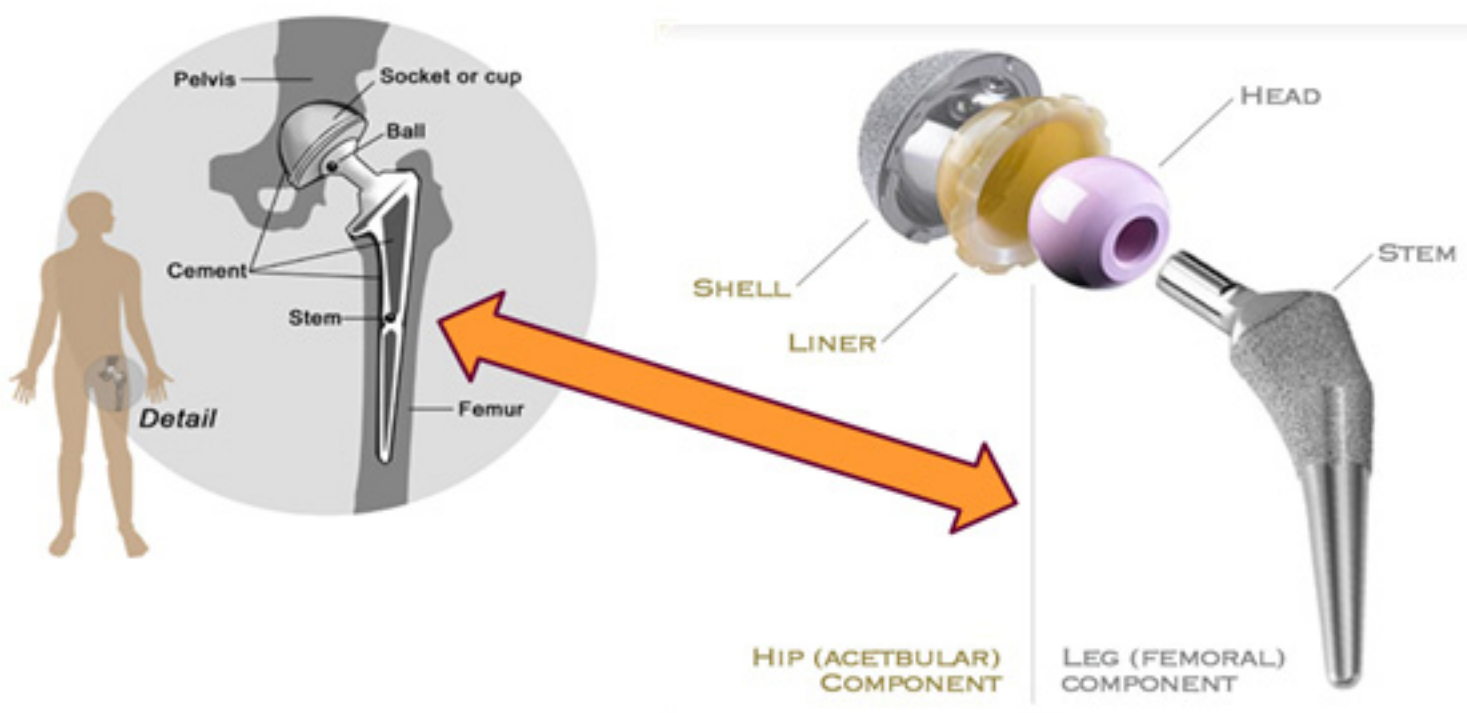

Figure 1.1: Schematic of an orthopedic hip implant in human body and picture of a metallic hip implant [14-15]. 
But the main shortcomings of freestanding HA implant or HA coatings are their poor fracture toughness and wear resistance [3-5, 16-17]. Fracture toughness of dense HA $\left(1 \mathrm{MPa} \cdot \mathrm{m}^{0.5}\right)$ is significantly lower than the minimum reported value for the cortical bone $\left(2 \mathrm{MPa} \cdot \mathrm{m}^{0.5}\right)$ [3]. Bones are the load bearing parts of a living body. They need to possess good fracture toughness $\left(\mathrm{K}_{\mathrm{IC}}\right)$ to prevent cracking and fracture on the application of high and cyclic loading during limb movement and actions. Thus, to replace bone as an implant or coating, fracture toughness of HA needs to be improved. Poor fracture toughness also results in low wear resistance of HA as wear volume loss in ceramics is directly related to its fracture toughness [18]. Poor wear resistance causes greater volume loss for HA due to continuous shearing force applied on implant surface during movement of limbs. Apart from abrasion of the coating, generation of more wear debris is also a problem inside the living body.

One of the possible solutions is reinforcement of HA by a second phase material that can help in improving the fracture toughness and wear resistance of the HA matrix. Researchers have also explored the possibility of using other hard ceramics [19-24], polymers [25-26] and bio glasses [27]. Keeping in concern the biocompatibility of the composite structure, the ideal reinforcement material is the one that can increase the fracture toughness and wear resistance significantly with a low content of reinforced phase. The lower content of reinforcement phase ensures introduction of minimum foreign element inside living body. HA gets integrated with bone as it has similar chemical composition to the mineral part of bone. Thus, it is important that the reinforcement phase possesses excellent elastic modulus and strength, so that its 
minimum content can increase the fracture toughness and wear resistance of HA significantly.

\subsection{Carbon Nanotubes: Potential Reinforcement to Hydroxyapatite}

\subsubsection{Scope of Improvement in Mechanical and Tribological Properties}

Carbon nanotube (CNT), with its excellent stiffness and strength, has excellent potential as reinforcement to $\mathrm{HA}$ for overcoming its limitation related to fracture toughness and wear resistance. CNT possesses Young's modulus in the range of 200$1000 \mathrm{GPa}$ [28] and tensile strength of 11-63 GPa [29]. Several studies on CNT reinforced metal/ceramic/polymer matrix composites have successfully demonstrated its capability of improving the structural properties, e.g., the strength, elastic modulus, fracture toughness, wear resistance etc. [30-32]. Apart from improvement in elastic modulus, CNT can improve the fracture toughness of any ceramic based composite system by absorbing energy through crack deflection and crack bridging. The possible roles played by CNT in improving the wear resistance are (i) increasing fracture toughness and (ii) providing lubrication on wear track through peeling of graphene layers from CNT surface.

\subsubsection{Issues Related to Biocompatibility}

The intended orthopedic application of HA-CNT composite demands a thorough understanding of its biocompatibility. HA is already a clinically proven biomaterial and in use as coating for metallic implants [33]. But, the biocompatibility of CNT is still 
under debate [34]. Though, comprehensive reviews on this issue have agreed that the reported cytotoxic response of CNT is mostly not due to CNT itself, but is associated with the impurities and catalyst particles, degree of agglomeration, surface defects and also on the effect of cell culture medium, secondary chemicals, and $\mathrm{pH}$ values [34-37]. Further, the biocompatibility of CNTs in orthopedic application is also established by studies showing accelerated bone growth (in-vivo) [38] and increased proliferation and differentiation of osteoblast (in-vitro) [39-41] in the presence of CNTs. The recent report on biodegradation of CNT by human neutrophil and macrophage [42] strengthens the drive for bio-related applications of CNTs. All these findings fortify the candidature of HA-CNT composite in orthopedic application.

\subsection{Boron Nitride Nanotubes: Alternative Reinforcement to Hydroxyapatite}

The ongoing debate on the biocompatibility of CNTs also demands search for an alternative reinforcement to HA. The alternative reinforcement phase should have the strength and modulus similar or better than CNTs in addition to being biocompatibile. Boron nitride nanotube (BNNT) has the capability to serve as an alternative. BNNT is a structural analogue of $\mathrm{CNT}$ - formed with tubular shaped hexagonal boron nitride (hBN) sheet. BNNT possesses elastic modulus (750-1200 GPa [43-44] and tensile strength ( $>24$ GPa [45]) similar to CNT, which makes it a potential reinforcement for HA. BNNTs are more flexible and elastic and can withstand heavy deformation without having damage in the structure [46]. BNNT withstands higher fracture strain than CNT, which can lead to higher fracture toughness for HA-BNNT as compared to HA-CNT [47]. These properties 
are attractive when BNNTs are used as reinforcement for strengthening of ceramic/polymer matrix. Moreover BNNTs show higher chemical stability than CNTs in oxidative atmosphere with the oxidation starting temperature of $1223 \mathrm{~K}$, as compared to $773 \mathrm{~K}$ for CNT [46]. This chemical inertness of BNNT remains an added advantage for high temperature processing of BNNT reinforced ceramic composite at oxidative atmosphere.

Another important consideration for using BNNT for orthopedic applications is its biocompatibility. As BNNT is very new to the field of biomedical, only few studies are available on the cytotoxicity of BNNTs but none has reported negative effect on different cell types [49-50]. BNNTs are found to be non-cytotoxic to human embryonic kidney cells (HEK-293) [50] and human neuroblastoma cell line (SH-SY5Y) [48].

Thus, theoretically, boron nitride nanotube has the capabilities to serve as an alternative to $\mathrm{CNT}$ as reinforcement to HA in orthopedic application.

\subsection{Objectives of the Current Research}

The overall objective of this research is to reveal and establish the potential of nanotubes (CNT or BNNT) reinforced HA based composite in orthopedic application - in terms of mechanical properties; tribological behavior and biocompatibility (in-vivo and in-vitro). This overall objective can be achieved through the following specific objectives:

- $\quad$ Analyzing the role of CNT reinforcement in fracture toughness and tribological behavior of HA based composites and coatings. 
- Understanding the role of CNT dispersion and HA-CNT interfacial bonding on the performance of the composite.

- $\quad$ Exploring the potential of BNNT as an alternative reinforcement to HA in terms of fracture toughness and tribological property improvement.

- In-vitro evaluation of biocompatibility of HA-nanotube composites by proliferation, viability, differentiation and cytotoxicity assays using bone cell.

- In-vivo evaluation of biocompatibility of HA-nanotube composites through animal study.

- Analyzing osseointegration ability and mechanical compatibility of HA-nanotube composite surfaces through evaluation of

- apatite formability of the surface

- adhesion of bone cells on the surface

- mechanical property gradient through implant-bone interface

Figure 1.2 presents a summary of the research carried out during this study.

The dissertation has been arranged in different chapters, sections and subsections to present a clear picture about the background and the state of the art; the methods adopted in this study; the analysis of the outcomes with scientific interpretation and the future scope of research and improvement. Chapter 2 provides a comprehensive literature review on this topic. This chapter also highlights those research areas that have not been paid much attention yet. Third chapter presents a detailed account of the methodology adopted in this research. Explanation of the results and scientific analysis of the outcomes in context with the objective of this research is discussed in chapter 4 . The key points of 
the research with an overview of the main outcomes are described as conclusion in chapter 5. Chapter 6 presents further scope of research and recommendations for research on HA-nanotube composite that would take this orthopedic implant material to clinical application stage. 


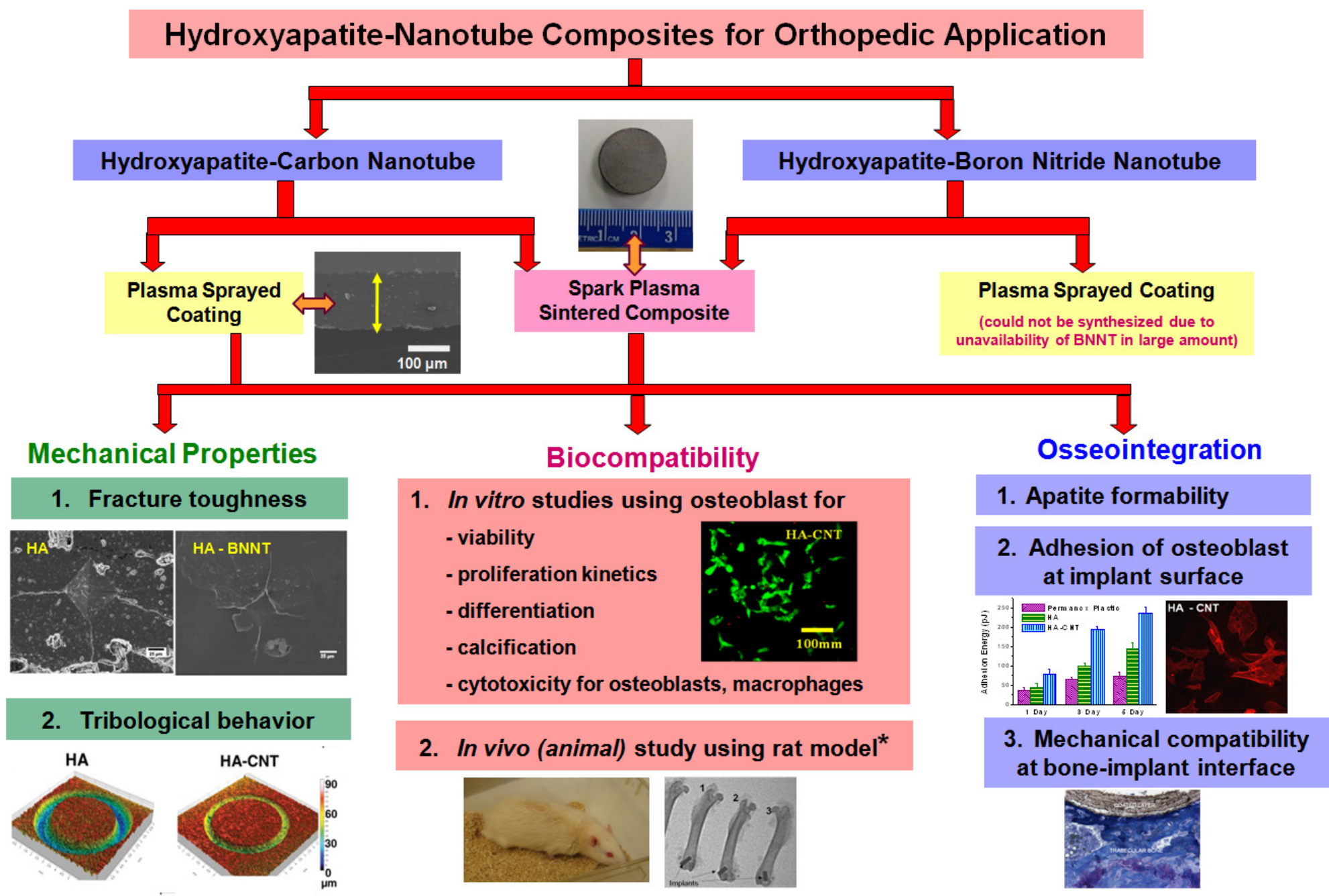

Figure 1.2: Flow chart of the research plan.

* Animal study on HA-nanotube composite is carried out at University of Strasbourg, France by collaborating research group 


\subsection{References}

1. F. Moussy. Biomaterials for the developing world. 2010, J. Biomed. Mater. Res. A, Vol. 94A, pp. 1001-1003.

2. Y.W. Gu, N.H. Loha, K.A. Khor, S.B. Tor, P. Cheang. Spark plasma sintering of hydroxyapatite powders. 2002, Biomaterials, Vol. 23, pp. 37-43.

3. A.A. White, S.M. best, I.A. Kinloch. Hydroxyapatite-carbon nanotube composite for biomedical applications: A review. 2007, Int. J. Appl. Ceram. Technol., Vol. 4, pp. 1-13.

4. L.G. Yu, K.A. Khor, H. Li, P. Cheang. Effect of spark plasma sintering on microstructure and in vitro behavior of plasma sprayed HA coatings. 2003, Biomaterials, Vol. 24, pp. 2695-2705.

5. Y.W. Gu, K.A. Khor, P. Cheang. Bone-like apatite layer formation on hydroxyapatite prepared by spark plasma sintering (SPS). 2004, Biomaterials, Vol. 25 , pp. 4127-4134.

6. S. Oh, N. Oh, M. Appleford, J.L. Ong. Bioceramics for Tissue Engineering Applications - A Review. 2006, Am. J. Biochem. Biotechnol., Vol. 2, pp. 49-56.

7. H. Li, K.A. Khor, V. Chow, P. Cheang. Nanostructural characteristics, mechanical properties, and osteoblast response of spark plasma sintered hydroxyapatite. 2007 , J. Biomed Mater. Res. A, Vol. 82, pp. 296-303.

8. X. Guo, J.E. Gough, P. Xiao, J. Liu, Z. Shen. Fabrication of nanostructured hydroxyapatite and analysis of human osteoblast cellular response. 2007, J. Biomed. Mater Res. A, Vol. 82, pp. 1022-1032.

9. W. Wang, J. Chen, D.K. Agarwal, A.P. Mashle, H. Liu. Improved mechanical properties of nanocrystalline hydroxyapatite coating for dental and orthopedic implants. 2009, Mater. Res. Soc. Sump. Proc., Vol. 1140, DOI: 10.1557/PROC1140-HH03-03.

10. L. Sun, C.C. Berdnt, K.A. Gross, A. Kucuk. Material Fundamentals and Clinical Performance of Plasma-Sprayed Hydroxyapatite Coatings: A Review. 2001, J. Biomed. Mater. Res. A, Vol. 58, pp. 570-592.

11. R. Petit. The use of hydroxyapatite in orthopedic surgery: A ten-year review. 1999, Eur. J. Orthp. Surg. Traumatol., Vol. 9, pp. 71-74.

12. K. Pielichowska, S. Blazewicz. Bioactive Polymer/Hydroxyapatite (Nano)composites for Bone Tissue Regeneration. 2010, Adv. Polym. Sci., Vol. 232, pp. 97-207. 
13. L.M. Mathieu, P.E. Bourban, J.A.E. Manson. Processing of homogeneous ceramic/polymer blends for bioresorbable composites. 2006, Compos. Sci. Tech., Vol. 66, pp. 1606-1614.

14. http://www.ocw.cn/OcwWeb/Biological-Engineering/20-441Fall2003/CourseHome/index.htm.

15. evertsmith.com/img/content/480-hip-components.jpg.

16. K. Balani, Y. Chen, S.P. Harimkar, N.B. Dahotre, A. Agarwal. Tribological behavior of plasma-sprayed carbon nanotube-reinforced hydroxyapatite coating in physiological solution. 2007, Acta Biomater., Vol. 3, pp. 944-951.

17. Y. Chen, T.H. Zhang, C.H. Gan, G. Yu. Wear studies of hydroxyapatite composite coating reinforced by carbon nanotubes. 2007, Carbon, Vol. 45, pp. 998-1004.

18. A.G. Evans, B. Marshall. Wear mechanisms in ceramics. In: Editor - D.A. Rigney. Fundamentals of Friction and Wear of Materials, 1981, Materials Park, Ohio: American Society of Metals, pp. 439-452.

19. J. Li, H. Liao, L. Hermansson. Sintering of partially-stabilized zirconia and partially-stabilized zirconia-hydroxyapatite composites by hot isostatic pressing and pressureless sintering. 1996, Biomaterials, Vol. 17, pp. 1787-1790.

20. J. Li, B. Fartash, L. Hermansson. Hydroxyapatite - alumina composites and bonebonding. 1995, Biomaterials, Vol. 16, pp. 417-422.

21. S. Gautier, E. Champion, D. Bernache-Assollant. Processing, microstructure and toughness of Al2O3 platelet-reinforced hydroxyapatite. 1997, J. Eu. Ceram. Soc., Vol. 17, pp. 1361-1369.

22. Z. Avis, R.H. Doremus. Coatings of hydroxyapatite - nanosize alpha alumina composites on Ti-6Al-4V. 2005, Mater. Lett., Vol. 59, pp. 3824-3827.

23. K.H. Im, S.B. Lee, K.M. Kim, Y.K. Lee. Improvement of bonding strength to titanium surface by sol-gel derived hybrid coating of hydroxyapatite and titania by sol-gel process. 2007, Surf. Coat. Technol., Vol. 202, pp. 1135-1138.

24. M. Inuzuka, S. Nakamura, S. Kishi, K. Toshida, K. Hashimoto, Y. Toda, K. Yamashita. Hydroxyapatite-doped zirconia for preparation of biomedical composites ceramics. 2004, Solid State Ionics, Vol. 172, pp. 509-513.

25. W. Bonfield, M.D. Grynpas, A.E. Tully, J. Bowman, J. Abram. Hydroxyapatite reinforced polyethylene - a mechanically compatible implant material for bone replacement. 1981, Biomaterials, Vol. 2, pp. 185-186. 
26. R.E. Neuendorf, E. Saiz, A.P. Tomsia, R.O. Ritchie. Adhesion between biodegradable polymers and hydroxyapatite: Relevance to synthetic bone-like materials and tissue engineering scaffolds. 2008, Acta Biomater., Vol. 4, pp. 12881296.

27. G. Goller, H/. Demirkiran, F.N. Oktar, E. Demirkessen. Processing and characterization of bioglass reinforced hydroxyapatite composites. 2003, Ceram. Int., Vol. 29, pp. 721-724.

28. S. Singh, Y. Pei, R. Miller, P.R. Sundarrajan. Long range, entangled carbon nanotube networks in polycarbonate. 2003, Adv. Func. Mater., Vol. 13, pp. 868872 .

29. M.F. Yu, O. Lourie, M.J. Dyer, K. Moloni, T.F. Kelly, R.S. Rouff. Strength and breaking mechanism of multiwalled carbon nanotube under tensile load. 2000, Science, Vol. 287, pp. 637-640.

30. A. Agarwal, S.R. Bakshi, D. Lahiri. Carbon Nanotubes: Reinforced metal Matrix Composites. 2010, Boca Raton: CRC Press, ISBN: 9781439811498.

31. S.C. Tjong. Carbon Nanotube Reinforced Composites: Metal and Ceramic Matrices. 2009, Weinheim: Wiley-VCH, ISBN: 9783527408924.

32. V. Mittal. Polymer Nanotube Nanocomposites: Synthesis, Properties, and Applications. 2010, New Jersey: Wiley Publishers, ISBN: 9780470625927.

33. S. Pramanik, A.K. Agarwal, K.N. Rai. Chronology of Total Hip Joint Replacement and Materials Development. 2005, Trends Biomater. Artif. Organs, Vol. 19, pp. 1526.

34. S. Fioritto. Carbon Nanotube: Angels or Demons? 2008, Singapore: Pan Stanford Publications, ISBN: $9814241016,9789814241014$.

35. H.X. Ren, X. Chen, J.H. Liu, N. Gu, X.J. Huang. Toxicity of single-walled carbon nanotube: How we were wrong? 2010, Mater. Today, Vol. 13, pp. 6-8.

36. M.A. Hussain, M.A. Kabir, A.K. Sood. On the cytotoxicity of carbon nanotubes. 2009, Current Sci., Vol. 96, pp. 664-673.

37. J. muller, F. Huaux, A. Fonseca, J.B. Nagy, N. Moreau, M. Delos, E. RaymundoPinero, F. Beguin, M. Kirsch-Volders, I. Fenoglio, B. Fubini, D. Lison. Structural Defects Play a Major Role in the Acute Lung Toxicity of Multiwall Carbon Nanotubes: Toxicological Aspects. 2008, Chem. Res. Toxicol., Vol. 21, pp. 16981705.

38. Y. Usui, K. A., N. Narita, N. Murakami, I. Nakamura, K. Nakamura, N. Ishigaki, H. Yamazaki, H. Horiuchi, H. Kato, S. Taruta, Y. A. Kim, M. Endo, N. Saito. Carbon 
Nanotubes with High Bone-Tissue Compatibility and Bone-Formation Acceleration Effects. 2008, Small, Vol. 4 (2), pp. 240-246.

39. M. Kalbacova, M. Kalbac, L. Dunsch, U. Hempel. Influence of single-walled carbon nanotube films on metabolic activity and adherence of human osteoblasts. 2007, Carbon, Vol. 45, pp. 2266-2272.

40. M. Matsuoka, T. Akasaka, Y. Totsuka, F. Watari. Strong adhesion of Saos-2 cells to multi-walled carbon nanotubes. 2010, Mater. Sci. Eng. B, Vol. 173, pp. 182-186.

41. T. Akasaka, A. Yokoyama, M. Matsuoka, T. Hashimoto, F. Watari. Thin films of single-walled carbon nanotubes promote human osteoblastic cells (Saos-2) proliferation in low serum concentrations. 2010, Mater. Sci. Eng. C, Vol. 30, pp. 391-399.

42. V.E Kagan, N.V. Konduru, W. Feng, B.L. Allen, J. Conroy, Y. Volkov, I.I. Vlasova, N.A. Belikova, N. Yanamala, A. Kapralov, Y.Y. Tyurina, J. Shi, E.R. Kisin, A.R. Murray, J. Franks, D. Stolz, P. Gou, J.K. Seetharaman, B. Fadeel, A. Star, A.A. Shvedova. Carbon nanotubes degraded by neutrophil myeloperoxidase induce less pulmonary inflammation. 2010, Nat. Nanotech., Vol. 5, pp. 354-359.

43. N.G. Chopra, A. Zetll. Measurement of the elastic modulus of a multi walled boron nitride nanotubes. 1998, Solid State Commun., Vol. 105, pp. 297-300.

44. A.P. Suryavanshi, M.F. Tu, J. Wen, C. Tang, Y. Bando. Elastic modulus and resonance behavior of boron nitride nanotubes. 2004, Appl. Phys. Lett., Vol. 84, pp. 2527-2529.

45. H. Shen. Thermal-conductivity and tensile-properties of BN, SiC and Ge nanotubes. 2009, Comp. Mater. Sci., Vol. 47, pp. 220-224.

46. D. Golberg, Y. Bando, C. Tang, C.Zhi. Boron Nitride Nanotubes. 2007, Adv. Mater., Vol. 19, pp. 2413-2432.

47. H.M. Ghassemi, C.H. Lee, Y.K. Yap, R.S. Yassar. In situ observation of reversible rippling in multi-walled boron nitride nanotubes. 2011, Nanotechnology, Vol. 22, p. 115702.

48. G. Ciofani, V. Raffa, A. Menciassi, A. Cuschieri. Cytocompatibility, interactions, and uptake of polyethyleneimine-coated boron nitride nanotubes by living cells: confirmation of their potential for biomedical application. 2008, Biotech. Bioeng., Vol. 101, pp. 850-858.

49. G. Ciofani, V. Raffa, A. Menciassia, A. Cuschieria. Boron nitride nanotubes: An innovative tool for nanomedicine. 2009, Nanotoday, Vol. 4, pp. 8-10. 
50. X. Chen, P. Wu, M. Rousseas, D. Okawa, Z. Gartner, Z.A. Zetll, C.R. Bertozzi. Boron nitride nanotubes are noncytotoxic and can be functionalized for interaction with proteins and cells. 2009, J. Am. Chem Soc., Vol. 131, pp. 890-891. 


\subsection{LITERATURE REVIEW}

This chapter presents a comprehensive summary of investigations by other researchers on mechanical properties and biocompatibility of HA-nanotube composites. A review and analysis of the past and on-going research in the field helped in finding the areas that need attention and led to the planning of this study.

\subsection{Hydroxyapatite-Carbon Nanotube Composite Systems for Orthopedic Application}

CNT is emerging as a suitable reinforcement of HA to solve its fracture toughness and wear resistance related problems. Research on HA-CNT composites has started very recently in 2004 [1-2]. The chronological trend of publications on HA-CNT system, as shown in figure 2.1, presents a clear picture of the growing interest in this field. A significant increase in the number of publication in 2010 indicates the importance of this topic in the contemporary research.

The research on HA-CNT composite is mainly around its synthesis, evaluation of mechanical and tribological properties and assessment of its biocompatibility. The success of CNT reinforcement is governed significantly by composite synthesis techniques. The mechanical and tribological performance of HA-CNT is dependent on the dispersion of CNT, its interfacial bonding with HA, densification of composite and phase stability of HA in the composite, which are manipulated greatly through synthesis techniques. Biocompatibility of HA-CNT composite is of immense importance considering the final application in orthopedics. This chapter provides a comprehensive 
literature review on synthesis, mechanical properties and biocompatibility of HA-CNT composites.

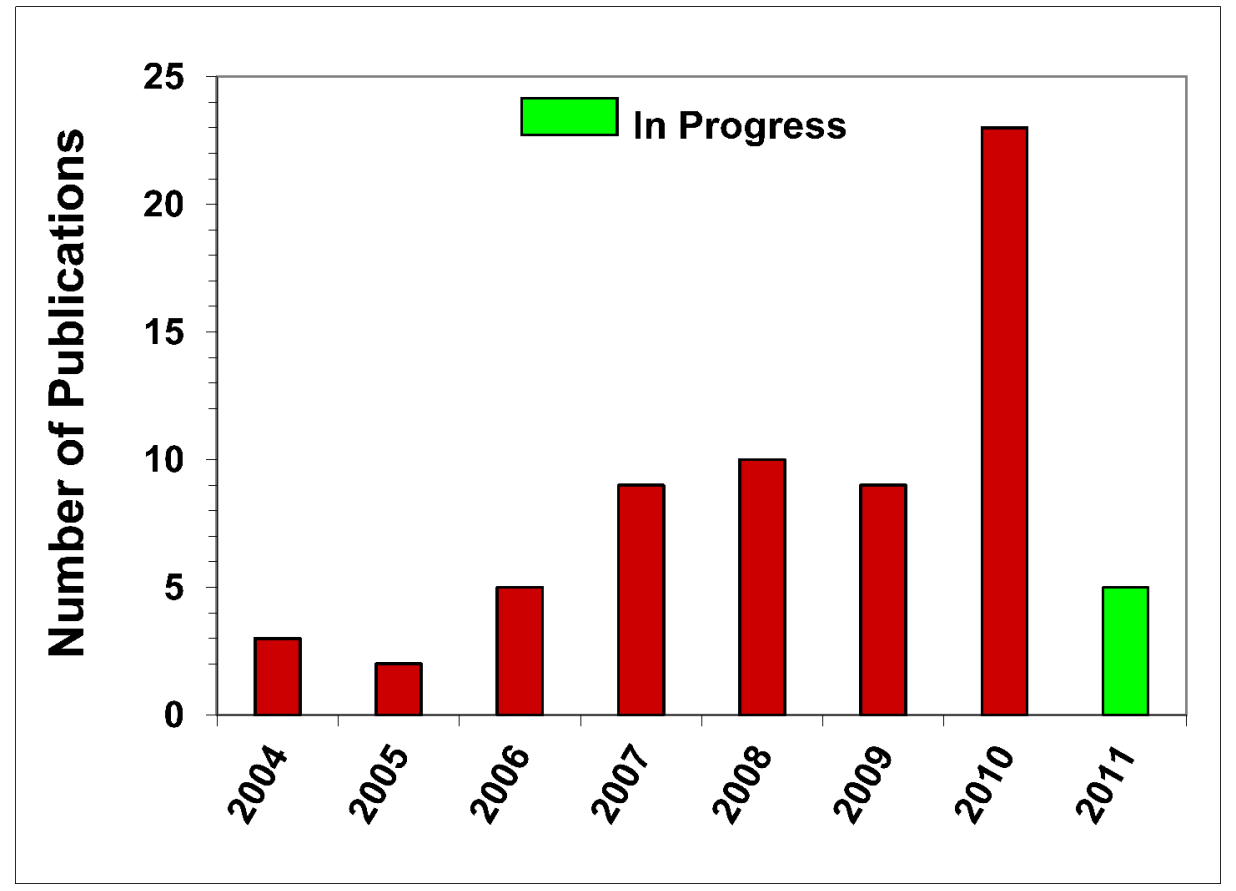

Figure 2.1: Year-wise publication chart for HA-CNT system (source: scopus.com).

\subsubsection{Synthesis of Composite}

The synthesis of HA-CNT CNT is divided into four major subsections. The first two are related to the processing of the composite, viz. (i) composite powder preparation and (ii) composite synthesis/consolidation of the composite powder. These two sections would discuss about different processing techniques and their advantages/disadvantages for HA-CNT composite preparation. The last two subsections are (i) dispersion of CNT in HA, which is a result of powder preparation techniques and (ii) phase stability and 
crystallinity of HA in composite, which is controlled by the consolidation method involved.

\subsubsection{Composite Powder Preparation}

Homogeneous distribution of the second phase reinforcement plays a key role on the performance of HA-CNT, like all other composite systems. The issue of dispersion becomes even more critical owing to the natural tendency of CNTs to form agglomerates due to its high aspect ratio and higher surface energy. Several modifications in the composite processing techniques have been adopted by researchers to ensure homogeneous dispersion of CNT in HA at powder/precursor stage itself.

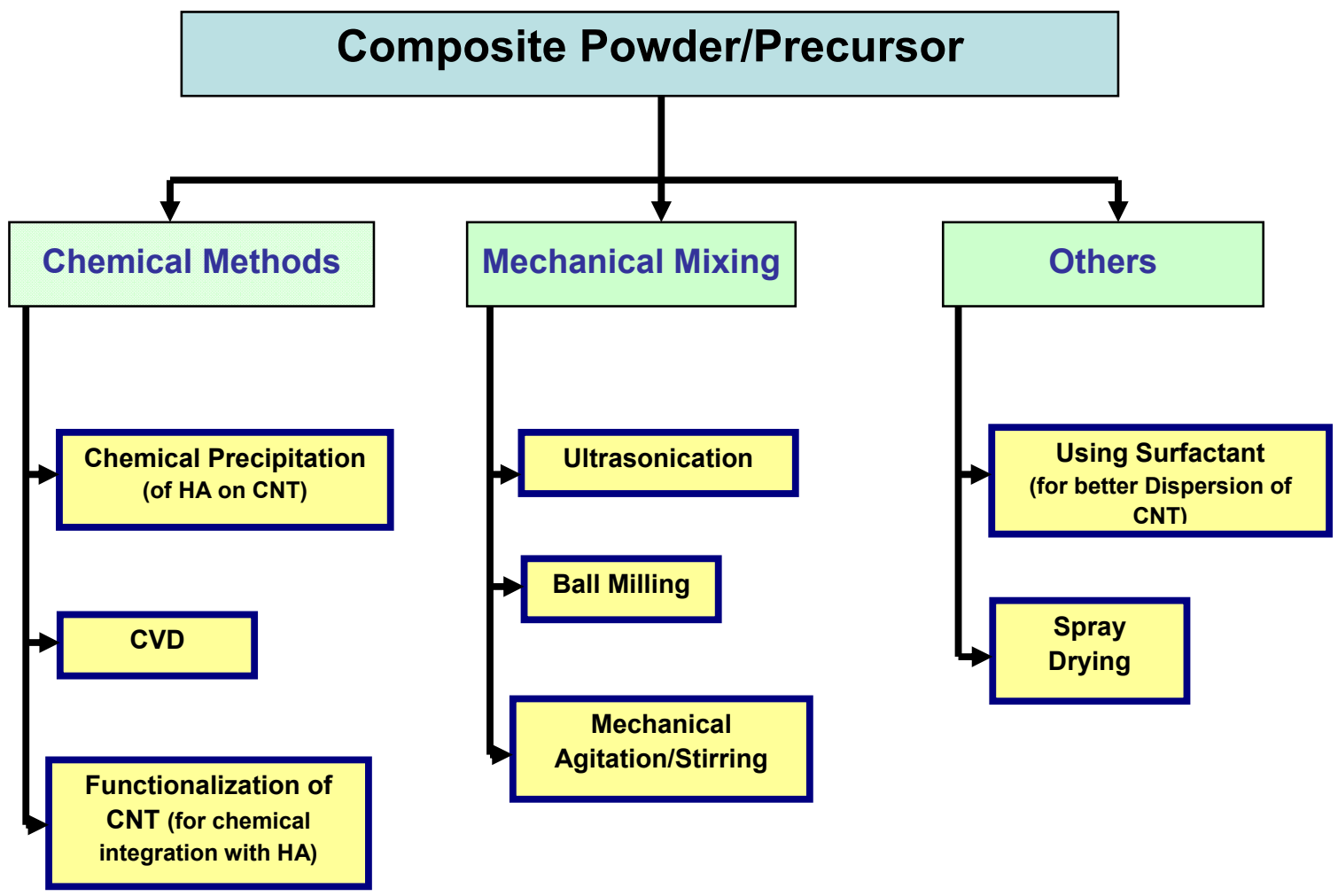

Figure 2.2: Classification of techniques adopted for better dispersion of CNT in composite powder/precursor (for Coatings). 
Figure 2.2 presents the classification of different powder/precursor processing techniques reported for HA-CNT system. The main aims of these methods are (i) to ensure better dispersion and avoid agglomeration of CNT in HA matrix; (ii) better interaction of CNT with HA at the interface to ensure good bonding in the composite. The following sub-sections briefly discuss each of the techniques with a summary of their outcomes.

\section{Chemical Mixing Methods}

Chemical methods include chemical synthesis of HA on CNT surface and vice versa or chemical modification of CNT surfaces for homogeneous distribution of the two phases in powder/precursor stage.

\section{Chemical Precipitation of HA on CNT}

Chemical precipitation of HA on CNT surface is generally performed by dispersing CNTs in the chemical bath from which HA is precipitated. CNT, thus coated with HA, ensures uniform distribution of HA and CNT, as well as, has a potential to improve the adhesion between nanotube and matrix. Most of the studies have suspended CNT in calcium nitrate bath followed by stirring. Afterwards, diamonium hydrogen phosphate is added to the bath with vigorous agitation to form HA precipitate on the surface of suspended CNT [1, 3-10]. Some of the studies have replaced calcium nitrate with calcium chloride [11-12]. Optimization of the precipitation parameters and specially $\mathrm{pH}(>10)$ ensures precipitation of $\mathrm{HA}$ phase and avoids formation of $\mathrm{CaHPO}_{4} \cdot 2 \mathrm{H}_{2} \mathrm{O}$ or $\mathrm{Ca}_{3}\left(\mathrm{PO}_{4}\right)_{2}$ phases $[4,9-10]$. Further study by Lu et al. shows the precipitated HA crystal 
size is a function of the temperature [9]. It is also mentioned that HA crystals prefer growing along c-axis and bigger crystals are not good for the coating due to their small contact area [9].

Most of the studies report uniform distribution of CNTs in the HA precipitate in the powder form using chemical precipitation method. The only exception is reported by Keally et al. on formation of CNT agglomeration in the resulting precipitate, though, no major change in the precipitation parameters are noted [8]. The HA precipitate formed on the CNT surface by chemical precipitation route is reported to have an amorphous structure that requires hydrothermal treatment to transform into crystalline structure $[1$, 12]. Figure 2.3 shows uniformly coated CNT with amorphous and crystalline HA precipitates, before and after hydrothermal treatment respectively, as observed by Zhao and Gao [1].
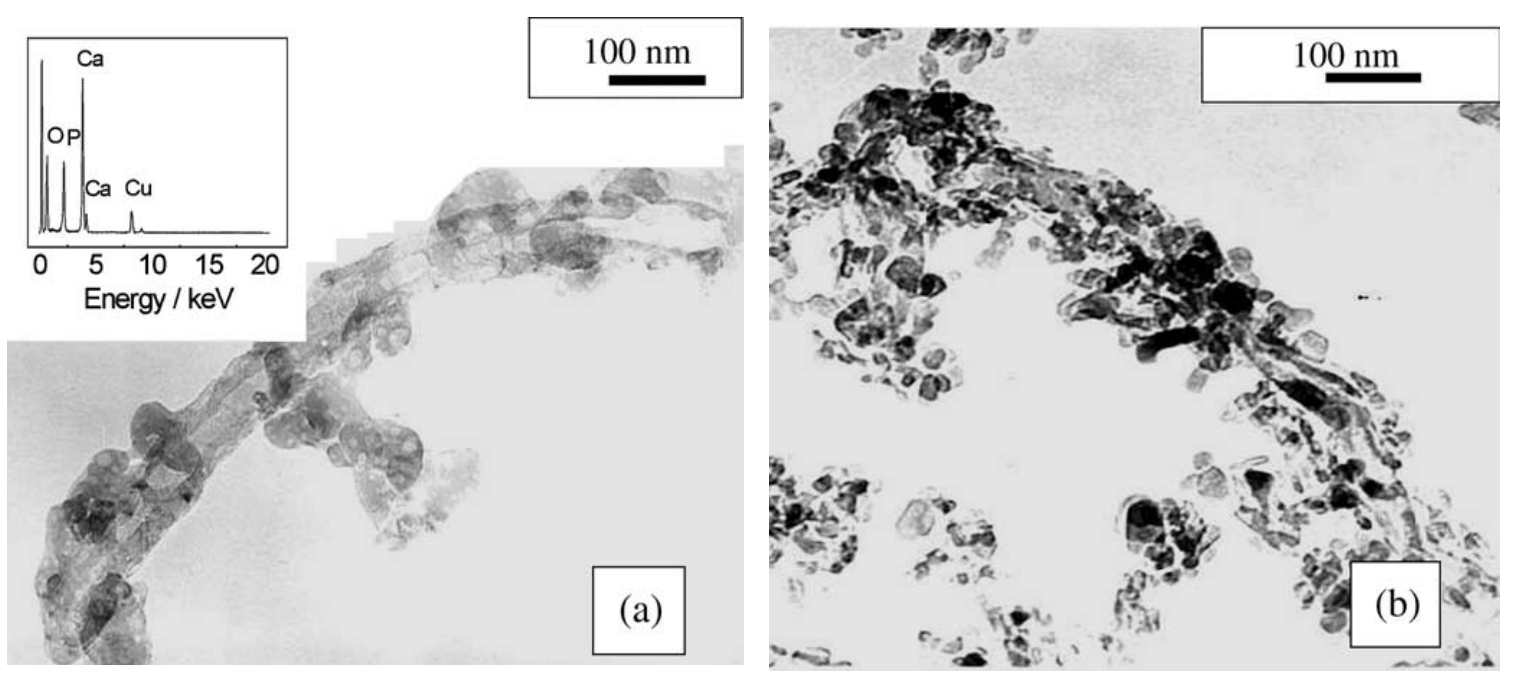

Figure 2.3: TEM images of CNTS-HA (a) before and (b) after hydrothermal treatment. Inset shows the EDS spectrum for HA layer in (a) [1]. 


\section{Functionalization of CNT}

Functionalization of CNT involves acid etching of surface and attaching functional groups like $-\mathrm{COOH},-\mathrm{OH}$ and $-\mathrm{C}=\mathrm{O}$, resulting in a negatively charged nanotube surface. These anionic groups attract positively charged ions and particles to get attached on the CNT surface. The purpose of functionalization is twofold: (i) forming good bonding between HA-and CNT at powder/precursor stage and (ii) obtaining homogeneous dispersion of CNT by forming coating of HA on its surface. Functionalization of CNT is carried out by refluxing with $\mathrm{HNO}_{3}-\mathrm{H}_{2} \mathrm{SO}_{4}$ mixture $[7,13-$ 18] or $\mathrm{HNO}_{3}$ only $[9,11-12,19]$.

Functionalization of CNTs is found as an essential step before chemical precipitation of HA on CNT by several research groups [7, 9, 11-12]. The negatively charged functional groups on CNT surface first gets attached with the $\mathrm{Ca}^{2+}$ ions through electrostatic attraction. After that, when $\mathrm{PO}_{4}{ }^{3-}$ ions are introduced in the bath, they react with attached $\mathrm{Ca}^{2+}$ and forms HA precipitates. Functionalization of CNT is also found effective in preparing the precursor for deposition of coatings using electrophoretic [13$14,17]$ and aerosol deposition [16]. The aqueous suspension of $\mathrm{HA}$ is adjusted $(\mathrm{pH} \sim 4$ ) to get the HA particles positively charged for the uniform dispersion in the precursor. Functionalized CNTs, being introduced in the suspension, attract positively charged HA particles through electrostatic force to form coating on them [17]. TEM and SEM observations of the composite powder from such precursor have found CNTs mostly covered by fine HA particles, revealing effectiveness of functionalization [14, 16-17]. 


\section{Chemical Vapor Deposition of CNT on HA}

Chemical vapor deposition (CVD) process is used to grow CNT on HA powder to ensure better dispersion and bonding of CNT with HA. Another advantage of using CVD grown CNT in composite is claimed to be retaining the undamaged pristine CNT structure while powder processing [20-22]. Researchers have used CVD technique successfully for different metal and ceramic matrix composites to get homogeneous dispersion of CNT in the matrix material [23-24]. CVD route for preparing composite HA-CNT powders is proposed by two different groups [20-22, 25]. Li et al. used Fe as catalyst $(0.4-10$ wt.\%) on HA powder to grow CNT and reported increasing CNT content in composite powder with increasing Fe catalyst content [20]. But, Lu et al. have directly used HA powders as catalyst to grow CNT, thus avoiding addition of metallic catalyst particles [21]. Though, they have observed decomposition of HA to some extent in process of in-situ CNT growth. Catalyst particles (Fe and HA) were found encapsulated in the as-grown CNT in both the studies. Figure 2.4 shows Fe catalyst particles encapsulated in CVD grown multiwall CNT on HA, as observed by Li et al. [20]. In another study, Li et al. have performed a comparative evaluation of the effect of $\mathrm{Fe}, \mathrm{Ni}$ and Co catalyst particles on the quality of CVD grown CNTs on HA powder [22]. Their study revealed that highest growth rate and crystallinity of CNTs occurred using Fe, whereas lowest growth rate and crystallinity were observed for Co. HA-CNT composite powder, prepared though CVD route are claimed to possess homogeneous dispersion of CNT and attribute towards strong bonding at reinforcement-matrix interface in the final composite structure [20]. 


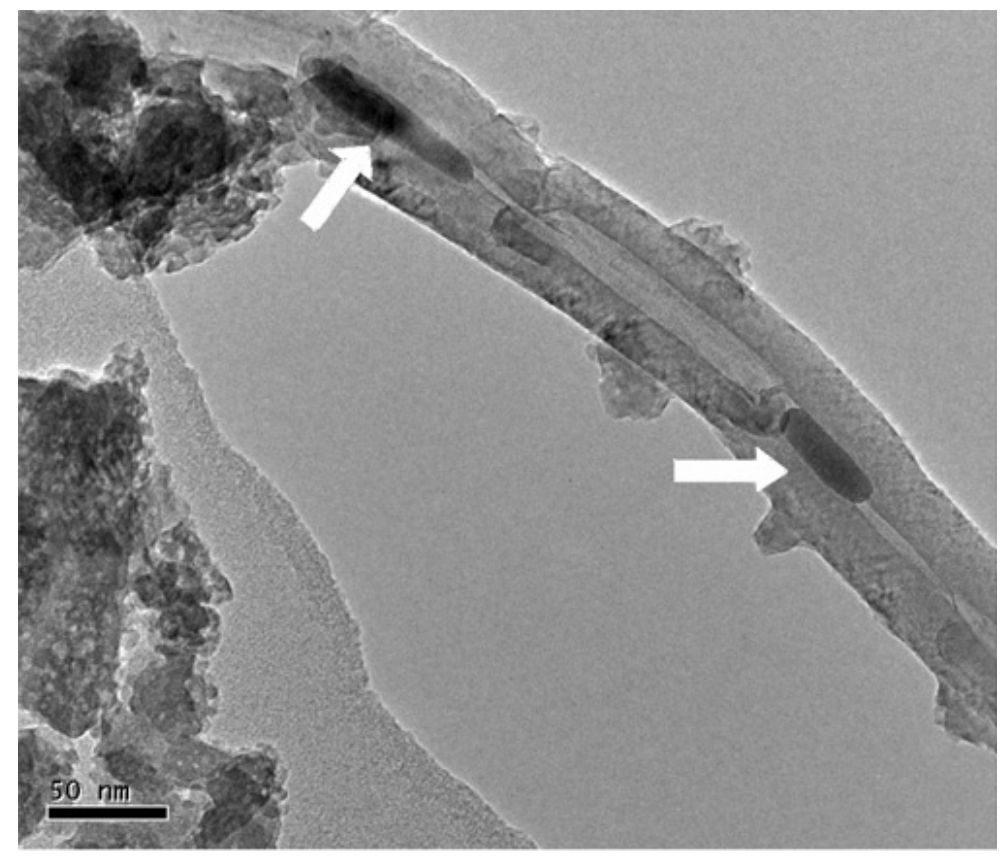

Figure 2.4: TEM image of Fe-catalyst particles encapsulated in CVD grown CNT on HA powder [20].

\section{Mechanical Mixing Methods}

Mechanical methods of composite powder processing involve physical mixing of HA and CNT using some mechanical forces. These are sometimes associated with chemical mixing routes also to enhance the CNT dispersion.

\section{Ball Milling}

Ball milling is used to disperse CNT in HA to prepare composite powders for fabricating both coatings and sintered parts. Chen et al. have used ball-milling to mix up to 20 wt.\% CNTs in HA for laser surface alloyed coatings [26-28]. Other research groups have used ball-milled HA-CNT composite powders for preparing composites using sintering [29], hot pressing [18, 30] and spark plasma sintering routes [31]. Sarkar et al. 
have reported considerable dispersion of CNTs in HA powder with presence of some agglomerates in the ball-milled composite powder [31]. But, these studies have not specifically mentioned about the effectiveness of ball-milling for dispersing CNT in HA $[18,26-31]$.

\section{$\underline{\text { Ultrasonication }}$}

Ultrasonication has been used both independently and in conjunction with chemical mixing methods to prepare HA-CNT composite powder/precursor. The very first study on HA-CNT mixing by ultrasonication has reported this method to be more effective in dispersion than ball milling [29]. Ultrasonication is mostly used in preparing HA-CNT composite precursor for ambient temperature coatings techniques, e.g. electrophoretic [12-14, 17, 32-35] and aero-sol depositions [16]. These studies have used ultrasonication bath to mix functionalized [12-14, 16-17] or pristine (non-functionalized) CNTs [33-35] with HA. The studies have reported uniform dispersion of CNTs in the precursor and coating stages. Kaya et al. have observed coating of HA particles on CNT surface after ultrasonication of functionalized CNTs with HA [17]. Figure 2.5 presents uniformly distributed non-functionalized CNTs in HA powder mixed using ultrasonication [34]. Some of the studies have used surfactant in the ultrasonication bath to improve the dispersion of CNTs in HA $[32,36]$. Composite powder processed by ultrasonication has been used in fabricating free standing composite parts through sintering route $[29,36]$. Wei et al. have used ultrasonication as an aid for the improved dispersion of CNTs in chemically precipitated composite powder [3]. 

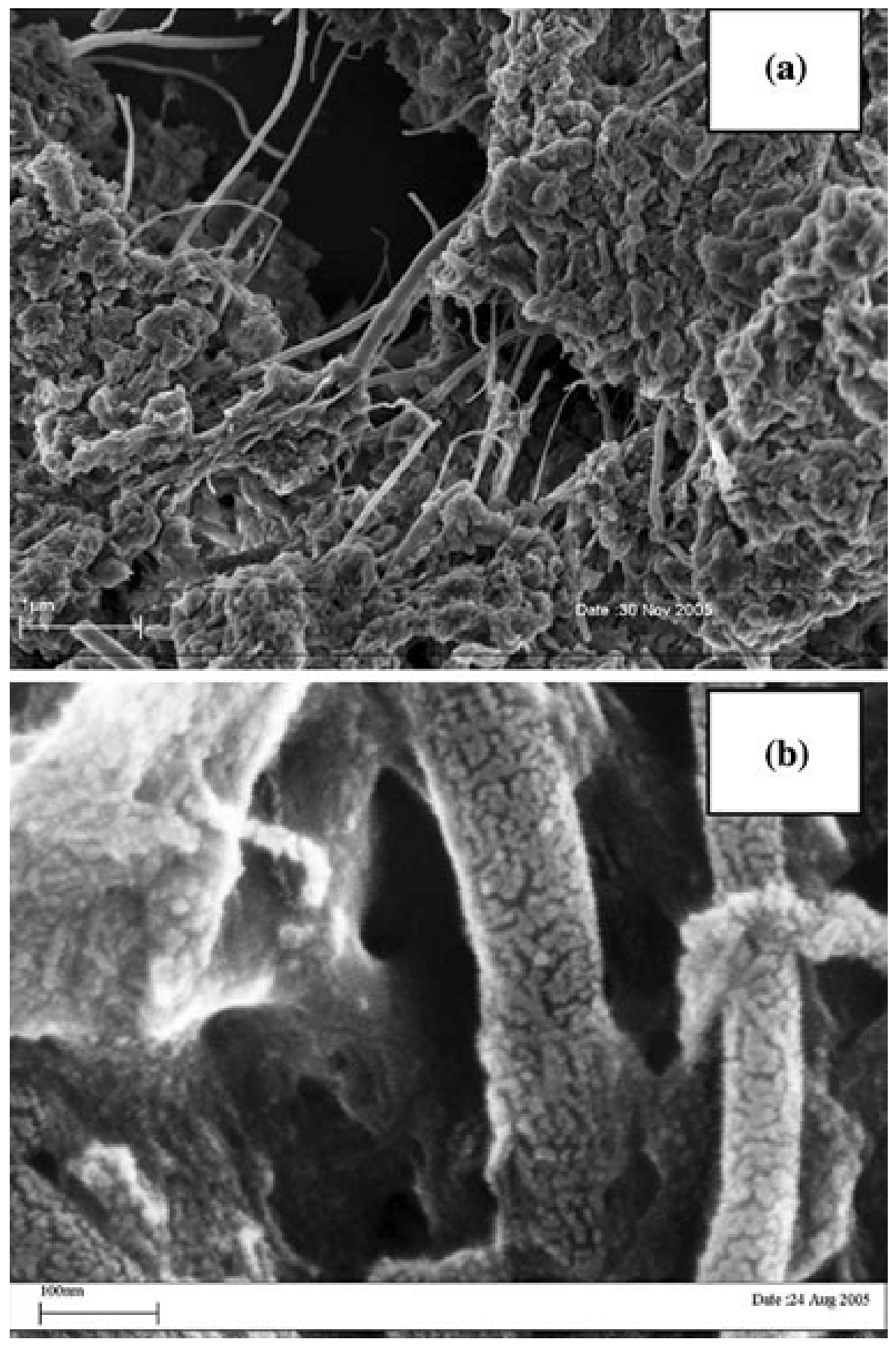

Figure 2.5: SEM images of ultrasonically mixed HA-CNTs powder at (a) low and (b) high magnifications [34]. 


\section{Mechanical Agitation/Stirring}

Different research groups have used various mechanical agitation methods other than ball-milling and ultrasonication to prepare HA-CNT composite powder. HA-CNT composite powder, prepared by blending HA powders with CNT in jar mill has been used for plasma sprayed coatings [37-40] and spark plasma sintered composite parts [41]. CNT gets well dispersed in HA powder through this process. Mechanical stirring in ethanol up to 5 days have been also used for preparing composite HA-CNT powder as feedstock for spark plasma sintering [42-44]. Functionalization of CNTs and dispersion using surfactant in conjunction with mechanical mixing is also used for preparing

composite powder $[7,15,45]$. A comparative study by White et al. have shown that functionalization of CNTs prior to mechanical mixing with HA improves the degree of dispersion [7]. The above mentioned mechanical mixing methods have reported good dispersion of CNTs in the HA matrix. But, use of agate mortar to make a slurry and then defoaming mixing by Tanaka et al. have resulted in inefficient dispersion and formation of CNT agglomerate in the composite structure [46]. Similar observation is made by Keally et al. also, while using gyroscopic tumbler of HA-CNT mixing [8].

\section{Other Mixing Methods}

Some of the methods used for aiding the dispersion of CNT in HA at powder stage cannot be classified under purely chemical or mechanical mixing methods. Methods that use surfactant for dispersion of CNT and spray drying for preparing HA-CNT composite are described briefly in the following subsections. 


\section{Using Surfactant for Dispersion of CNT}

Surfactants are mostly used in conjugation with other powder preparation techniques to prepare the composite powder. A thorough study by Meng et al. [45] on different surfactants for dispersing CNT shows anionic surfactants, e.g., sodium dodecyl sulphate (SDS) or sodium dodecyl benzene sulphonate (SDBS) makes better dispersion of CNTs in de-ionized water. Because the hydrophilic end of anionic surfactants are anionic and thus the cationic ends attach with negatively charged surface of the functionalized CNTs. Meng et al. have also observed that HA powders dispersed using cationic surfactants, e.g., cetyl trimethyl ammonium bromide (CTAB), gets mixed more uniformly when dispersed in CNT bath with anionic surfactant. HA powder without any surfactant does not show as good dispersion with CNT. The reason is natural attraction between the hydrophilic ends of cationic and anionic surfactants, helping in attachment of HA and CNT in the dispersion [45]. Few other studies have also used surfactant to disperse CNT and then mechanically mixing HA to prepare powder for composite parts $[18,31,36]$ or precursor for coating [32].

\section{Spray Drying}

Spray drying is a process of preparing micron sized agglomerates of nano-sized powder by atomization. Balani et al. have used spray dried HA powder for plasma sprayed coating, but CNTs were separately blended to spray dried HA in those studies [37-38]. Few studies have used spray dried HA powder and spray dried composite alumina-CNT powder to mix together mechanically [39-41]. The homogeneous dispersion of CNT in alumina is reported to result in good dispersion of CNT in the final 
HA-alumina-CNT composite powder. Thus, spray drying could be a promising technique for HA-CNT composite powder preparation, which was to be explored before this research.

A review of HA-CNT composite powder/precursor processing techniques suggests two methods to be most suitable for uniform dispersion and bonding of CNT with HA. These methods are: (i) chemical precipitation of HA on functionalized CNT and (ii) CVD assisted growth of CNT on HA powder. Apart from the uniform HA precipitation on CNT, chemical precipitation method also ensures good bonding of HA on functionalized CNT surface. Electrostatic attraction of '+ve' charged Ca of HA and 've' charged $-\mathrm{COOH}$ functionalized group on CNT surface is responsible for this good bonding. In CVD process, the CNTs are individually grown on HA surface, forming a good attachment and dispersion. But, the major problem inherited with both these processes is related to the large scale synthesis of composite powder. Mechanical mixing methods are suitable for bulk production, but are not as good in dispersion as chemical precipitation or CVD. Functionalization of CNTs and use of surfactant helps in improving the quality of dispersion in mechanical mixing methods. Spray drying method appears to be a good compromise between large scale synthesis and CNT dispersion but suffers with loss of powder during synthesis. The suitability of all these methods in terms of degree of CNT dispersion is critically discussed in section 2.1.1.3.

\subsubsection{Consolidation of Composite Structure}

Several consolidation methods have been explored for fabricating HA-CNT composite using powders mixed through various routes as discussed in the previous 
section. Most of the techniques uses high temperature for consolidation, owing to high melting point $\left(1614{ }^{\circ} \mathrm{C}\right)$ of $\mathrm{HA}[47]$. The consolidated HA-CNT composites can be classified into two groups based on the targeted application. They are: (i) free standing composite bodies used as prosthesis or its part and (ii) composite coatings on metallic implants.

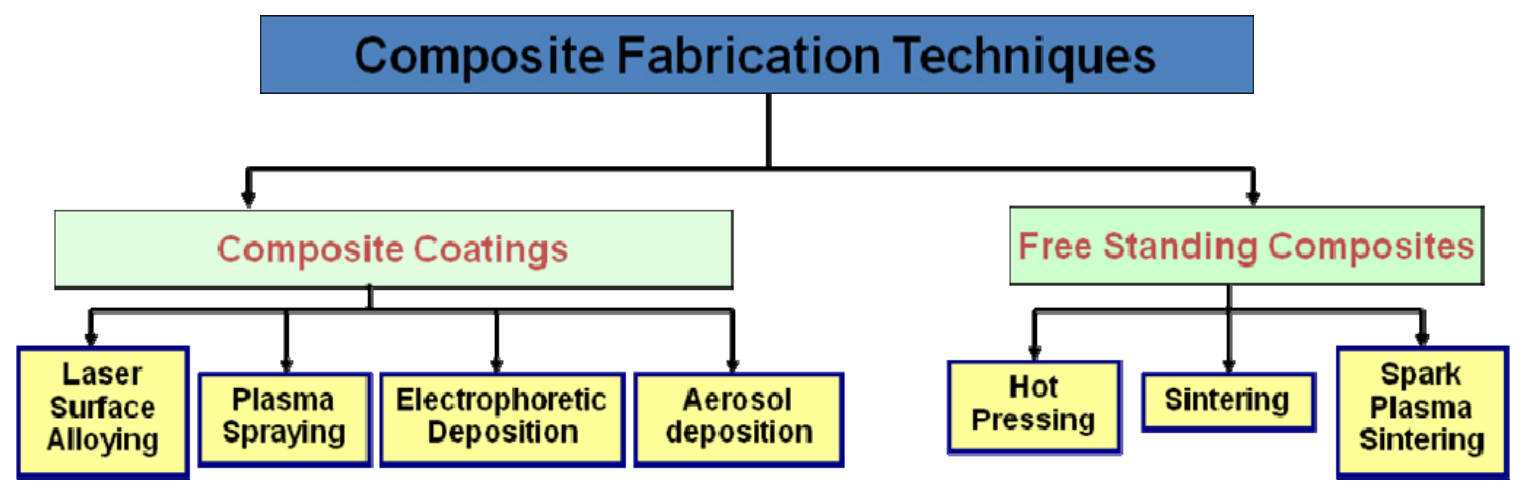

Figure 2.6: Classification of consolidation methods for HA-CNT composites.

Researchers have studied different techniques in each of the groups to successfully fabricate HA-CNT composite. Figure 2.6 presents a classification of fabrication techniques used to synthesize different HA-CNT composites. The main considerations during consolidation are to control the porosity, maintain good dispersion of CNT and at the same time, minimize the chemical dissociation of HA, when exposed to high temperature. Controlling the dissociation of HA to other phases such as tri calcium phosphate (TCP), is required to maintain the mechanical strength of the composite. Controlling the porosity and distribution of CNT is mainly to achieve the good mechanical properties of the composite structure. Though, lowest porosity gives the best mechanical property, but it is not desired for orthopedic application. Porosity assists 
in tissue in-growth and bone integration on implant/prosthesis surface. Hence, the intention of adding CNTs to HA is to improve the mechanical property of the composite, while maintaining some degree of porosity. Following sub-sections deal with the effectiveness of each technique in consolidation of HA-CNT composite.

\section{Free Standing HA-CNT Composite Parts/Structures}

Conventional powder metallurgy routes of powder processing and high temperature consolidation has mainly been applied for fabricating free standing HA-CNT composite structures, with certain modifications to minimize high temperature exposure for long time.

\section{Sintering}

Consolidation of HA-CNT composite by sintering is always performed after green compaction of the powder $[7,20,25,29,36,48]$. Both uniaxial $[7,48]$ and isostatic pressing [36] have been tried for the green compaction. Few studies performed an additional post sintering pressing for better compaction[20, 25]. The major concern with conventional sintering of $\mathrm{HA}$ is associated with the exposure to high temperature for long time, which causes dissociation of $\mathrm{HA}$ to TCP or $\mathrm{CaO}$. A wide range of sintering temperatures starting from $650^{\circ} \mathrm{C}$ up to $1200^{\circ} \mathrm{C}$ with a dwell time of $2-5 \mathrm{hrs}$ has been adopted for HA-CNT consolidation. But, temperatures below $1000^{\circ} \mathrm{C}$ were not found suitable for achieving sufficient densification. White et al. have reported a density of 63 $\% \mathrm{TD}$ (theoretical density) for $\mathrm{HA}$ and $51 \% \mathrm{TD}$ for $\mathrm{HA}-\mathrm{CNT}$ when sintered at $800^{\circ} \mathrm{C}$ for 
$2 \mathrm{hrs}$ [7]. Higher temperature and longer time of heat treatment increases the density and crystallinity of HA [7]. But, sintering at higher temperature increases the chance of dissociation of HA [36, 49]. White et al. have also found the density of HA-CNT composite structure is lower than HA, when sintered at same conditions [7]. They have inferred that CNTs hold the grains of the matrix apart and thus prevent closure of the porosity. These findings suggest conventional sintering as an unsuitable process for HACNT consolidation. Sintering of HA-CNT composites are mostly carried out in vacuum $20,25,29,36]$ or inert atmosphere, e.g., nitrogen [36] and argon [7, 29, 36]. An interesting study about the effect of sintering atmosphere on the degree of consolidation for HA-CNT composite has been carried out by Li et al. [36]. Their findings suggest vacuum to be the best sintering atmosphere. Both HA and CNT can absorb gas intensively, which makes the HA-CNT interface bonding weak and increases the porosity content of the sintered structure, when sintered in presence of any gas. Figures 2.7(a) and 2.7(b) shows the porous HA-CNT structure sintered in argon compared to its consolidated counterpart sintered in vacuum, respectively. As a result, the composite with same CNT content ( 3 wt. $\%$ ) sintered in vacuum offers $\sim 200 \%$ more fracture toughness than the one sintered in argon atmosphere [36]. But, a recent study by White et al. have shown a sintering atmosphere, consisted of $\mathrm{CO}$ and $\mathrm{H}_{2}$, to be the best considering the retention of HA and CNT in the final sintered structure [48]. Sintering atmosphere with a positive pressure of $\mathrm{CO}$ and $\mathrm{H}_{2}$ can effectively suppress the dissociation of water molecule from HA and its subsequent reaction with CNT. 
(a)

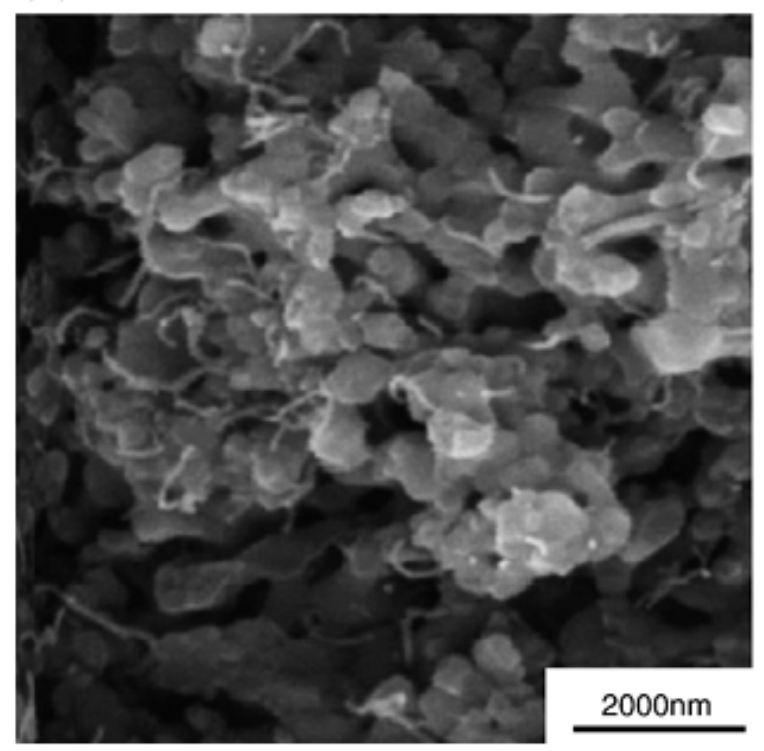

(b)

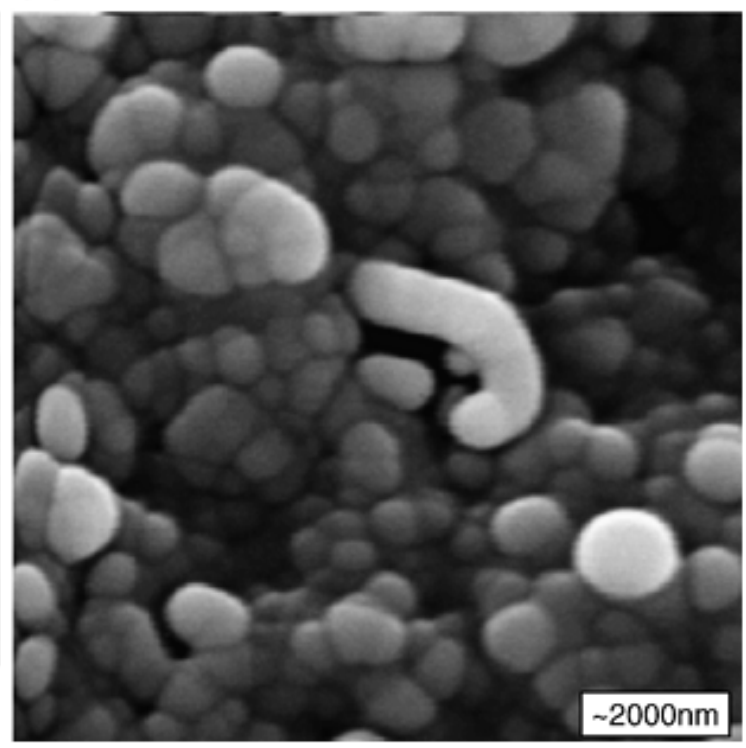

Figure 2.7: SEM micrographs of HA-CNT composites sintered in (a) argon and (b) vacuum [36].

\section{Pressure Assisted Sintering}

In order to overcome the problem of HA dissociation and obtain better densification, researchers have attempted application of pressure during sintering to synthesize HA-CNT composites $[1,4-6,8,15,18,30,45]$. With application of pressure, densification as high as $96 \% \mathrm{TD}$ is achieved at temperature as low as $900^{\circ} \mathrm{C}$ [8]. Lower sintering temperature reduces the chance of dissociation of HA. Researchers have also reported sintering at high temperature $\left(1200^{\circ} \mathrm{C}\right)$ with application of pressure, without resulting in dissociation of HA $[15,18]$. Meng et al. have suggested that application of pressure could inhibit the dehydration of $\mathrm{HA}$ even at higher temperature, thus suppressing its dissociation $[15,18]$. Both uniaxial $[6,18]$ and isostatic hot pressing $[4-5$, 8] with a widely varying pressure range of $28-100 \mathrm{MPa}$ and argon $[4-6,8,30]$ or nitrogen $[15,18,45]$ atmosphere is used to consolidate HA-CNT. Unlike conventional 
sintering, sintering under high pressure in presence of inert gas does not create a problem in consolidation and interfacial bonding between HA and CNT. Thus, the mechanical property of the composite is reported to improve over their HA counterpart, whether it is fracture toughness $[8,15,45]$, flexural strength $[15,45]$, hardness [8] or compressive strength [1]. The application of pressure might help in driving out the entrapped or absorbed gas on HA and CNT surface and results in lesser porosity in the consolidated product, in contrast to conventional sintering. The sintering pressure influences the density of HA-CNT composite, but shows no effect on HA without CNTs. Kobayashi et al. have reported a $10 \%$ increase in the relative density of HA-CNT composite when the pressure increases from 7.81 $\mathrm{MPa}$ to $15.6 \mathrm{MPa}$ at $1200^{\circ} \mathrm{C}$ [30]. They have concluded lower sinterability of the composite in the presence of a second phase.

\section{$\underline{\text { Spark Plasma Sintering }}$}

Few research groups have used spark plasma sintering (SPS) technique to consolidate the HA-CNT composites [2, 19, 31, 41-44, 46]. The advantages of using SPS for HA-based composite are two-fold :(i) retention of fine grain structure and (ii) reduction in HA dissociation. SPS is a very promising technique for processing nanostructured materials, as it retains the fine grain size after sintering [50-54]. It is more attractive for ceramics, as grain size refinement could simultaneously increase hardness and fracture toughness of ceramic structure due to the deflection of propagating crack and the change of cracking mode from transgranular to intergranular [55]. Requirement of high temperature and longer hold time in conventional sintering causes severe grain 
growth. Whereas, due to simultaneous application of pressure, electrical current and rapid heating rate, SPS results into better densification of nanoceramic particles in much lower temperature [50-52]. Sintering time for SPS is generally few minutes, compared to few hours in conventional sintering. Due to shorter time of sintering, SPS retains the nanostructure and improves the mechanical property of the sintered body [19, 50-54]. In addition, minimal dwell at high temperature causes lesser dissociation of HA into TCP [56-61].SPS of HA-CNT has employed temperatures in the range of $900-1250{ }^{\circ} \mathrm{C}[42$, 46], pressure of 7.5- $120 \mathrm{MPa}[42,46]$ in vacuum [41] or argon [31]. The systematic studies on the determination of ideal SPS temperature for HA-CNT system found $1100^{\circ} \mathrm{C}$ as the optimum sintering temperature [31, 42]. Lower temperature causes poor consolidation with lots of residual pores, whereas higher temperature results in an excessive grain growth [31, 42]. Figures 2.8 (a-d) presents HA-CNT SPS structures sintered at different temperatures, showing porosities at $900^{\circ} \mathrm{C}$ and $1000^{\circ} \mathrm{C}$ and excessive grain growth at $1200^{\circ} \mathrm{C}$, whereas densified fine grained structure at $1100^{\circ} \mathrm{C}$ [42]. However, a thorough understanding about role of CNT in microstructural evolution of SPS processed HA-CNT is not available in the published literature.

HA-CNT composites processed through SPS route shows increase in mechanical properties, e.g. fracture toughness [31, 41, 46], elastic modulus [42], hardness[41-42] and bending strength [46]. Thus, SPS is found to be a suitable consolidation process for synthesizing free standing HA-CNT composite parts. The only drawback of SPS with respect to HA-CNT processing is its application of high pressure, which can cause damage to CNT structure and thus have negative effect on the strengthening of the 
composite. CNTs are reported to collapse or forming, kinks, caps, onion structure and corrugated walls due to application of pressure during SPS processing [62-63].

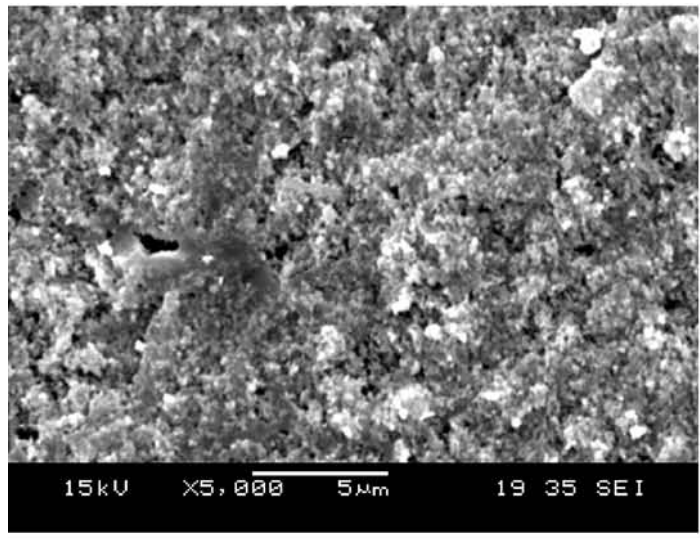

(a)

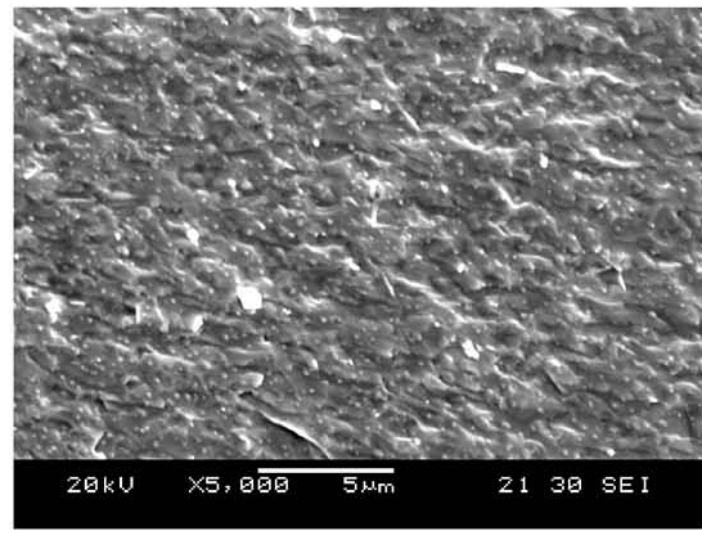

(c)

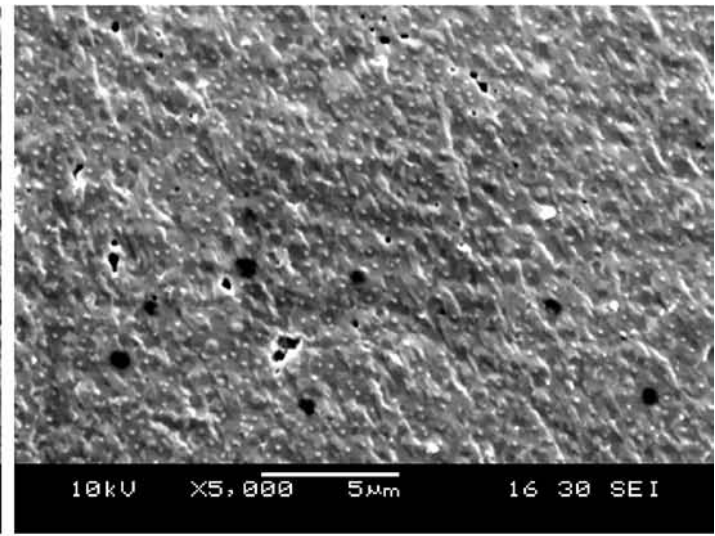

(b)

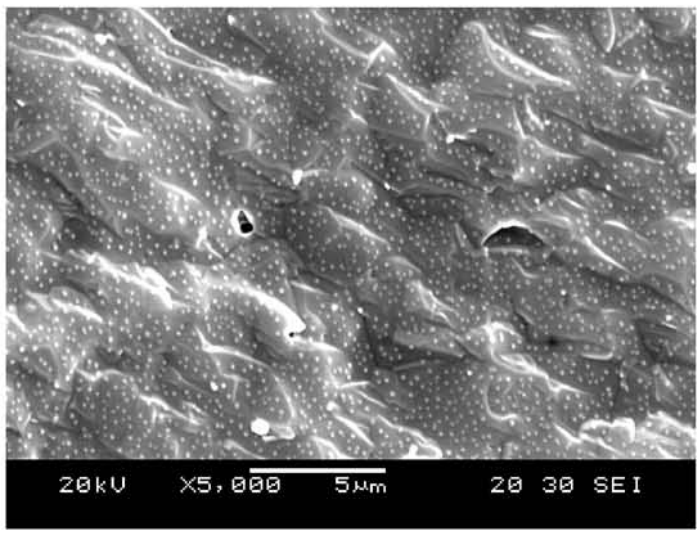

(d)

Figure 2.8: SEM micrographs of fracture surface for SPS processed HA-CNT composites sintered at (a) $900{ }^{\circ} \mathrm{C}$; (b) $1000^{\circ} \mathrm{C}$; (c) $1100^{\circ} \mathrm{C}$; (d) $1200{ }^{\circ} \mathrm{C}$ [42].

\section{CNT Reinforced HA Coatings}

Since late 1990s, HA is in clinical use as a coating on metallic orthopedic implants [64]. Development of CNT reinforced HA based composite coatings on the metallic substrate are of utmost importance for its projected scope of real life application. The research on synthesis of HA-CNT coatings was started with laser surface alloying 
and followed up with other techniques, e.g., plasma spraying, electrophoretic deposition and aerosol deposition. Following subsections summarize the significant outcomes of the studies on HA-CNT coating synthesis techniques.

\section{$\underline{\text { Laser Surface Alloying }}$}

The first HA-CNT composite coating on metallic surface was synthesized by Chen et al. using laser surface alloying (LSA) technique [26]. The high power density applied in LSA acts as a heat sink, causing localized melting of the substrate and forming good adhesion with coating [65]. Thus, it is effective in forming good bonding of ceramic coating on metallic surface. Chen et al. have used Ti-6Al-4V alloy as the substrate for HA-CNT coating [26-28]. Ti-6Al-4V is a well-established alloy for orthopedic application and also in use with HA coating. The substrates were preheated to reduce the residual thermal stress that can cause cracking at interface and delamination of coating. The coating was synthesized using a Nd:YAG laser operating at $400 \mathrm{~W}$ power. High temperature exposure in LSA causes dissociation of $\mathrm{HA}$ to $\mathrm{TCP}$ and $\mathrm{CaO}$, which is not favorable for its intended orthopedic application [26, 28, 45]. Moreover, CNTs reacted with $\mathrm{Ti}$ to form $\mathrm{TiC}$. In the LSA process, the Ti alloy substrate gets melted and becomes more prone to react with $\mathrm{CNT}$ to form $\mathrm{TiC}$. This phenomenon has two major shortcomings for HA-CNT coating synthesis. Firstly, presence of $\mathrm{TiC}$ in the coating might affect the biocompatibility of the coating, especially when exposed to body fluid and blood stream during wear or dissolution of the HA coating from the implant surface. Secondly, reaction of CNT with Ti will destroy the CNTs and will require increased CNT 
content to offer required strengthening to HA. But, increase in CNT content results in increased in TiC content also [27]. Though, a portion of CNT reacts with Ti-alloy substrate, remaining CNTs were found in the HA coating, maintaining their cylindrical structure [26]. LSA synthesized HA-CNT coating displayed improvement in the elastic modulus, hardness [26-27] and wear resistance [28].

\section{Plasma Spraying}

In plasma spraying, the micron sized powders are fed through plasma gun and sprayed on preheated substrate to deposit a coating. While passing through the plasma plume, powder particles gets exposed to very high temperature $\left(>10,000{ }^{\circ} \mathrm{C}\right)$ for milliseconds, which causes melting. These molten particles are propelled on the substrate with high velocity to get deformed/flattened through impact and rapidly solidify. Layer by layer deposition of these molten powder particles causes the typical lamellar structure of the plasma sprayed coatings. High temperature exposure in plasma spraying is suitable for preparing ceramic coatings on substrates with good adhesion strength. It is a suitable technique for synthesizing uniform and controlled thickness coating on substrates with complex geometry. Plasma spraying is US Food and Drug Administration (FDA) approved technique for coating implants with biomaterials [66] and is routinely used for depositing HA coating on implants [67-68]. Thus, plasma spraying is a viable option to synthesize HA-CNT composite coating. In plasma spraying, only powders are exposed to very high temperature and not the substrate. Hence, the chance of $\mathrm{TiC}$ formation due to melting of substrate (as in laser surface alloying) does not exist. All available studies on plasma spray formed HA-CNT coatings till date have been carried out by our research 
group [37-40]. Figure 2.9 shows HA and HA-CNT coatings on Ti-6Al-4V substrate deposited using plasma spraying [37]. The cross sectional microstructures of the coatings reveal typical lamellar structure of plasma sprayed coating with uniform thickness. No sign of delamination and cracking at the interface denotes good bonding between Ti-alloy substrate and HA/HA-CNT coating.
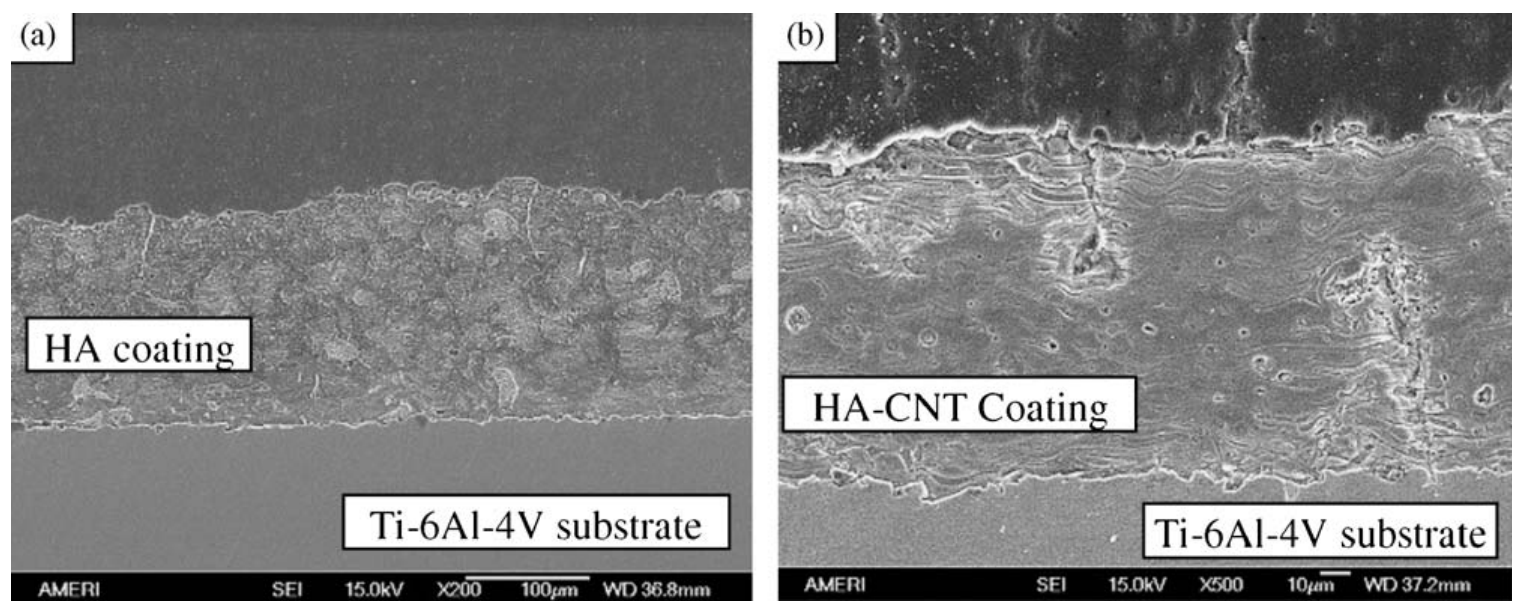

Figure 2.9: SEM micrographs of coating cross sections synthesized by plasma spraying of (a) HA and (b) HA-CNT [37].

Both SEM micrographs of fracture surface and Raman spectrum proves the survival of CNT in the composite coating after being exposed to plasma [37]. CNTs survive the high temperature due to the shorter exposure time in plasma (in milliseconds) which is not sufficient for their oxidation and destruction. Moreover, ceramic melts during plasma spraying form a protective layer on CNTs [69-70]. Powder carrier gas (argon) also creates inert shroud over the particles that shields against oxidation of CNTs. X-ray diffraction study on the HA-CNT composite coatings reveals much lower intensity $\beta$-TCP peaks compared to peaks from HA phase $[37,40]$. A negative effect of very fast cooling rate in plasma spraying is decreased crystallinity of HA in the coating. But, 
presence of CNT increases the crystallinity of the composite coating, due to its higher heat capacity. A more detailed discussion on this has been presented in section2.1.1.4, while discussing phase stability and Crystallinity of HA in composite. Similar relative density of both HA and HA-CNT coatings indicate that CNT does not create any problem in densification. Plasma sprayed HA-CNT composite coatings show improvement in fracture toughness [37, 39-40], hardness [39] and wear resistance [38-39] compared to the HA coatings. Thus, plasma spraying is an effective technique for synthesizing HA coatings reinforced with CNT.

\section{Electrophoretic Deposition}

Electrophoretic deposition (EPD) is a colloidal process in ceramic production that is used for preparing cost-effective coatings on substrates with complex geometry. In EPD, charged powder particles, suspended in a liquid medium, are attracted and deposited onto a conductive substrate of opposite charge when DC electric field is applied. It is a widely used coating technique for ceramics [71-72] and is being vastly researched for biomaterials [73-74] and CNT reinforced composite ceramic coatings [75]. Few studies have been conducted on synthesizing HA-CNT coating for bioimplant surfaces using EPD [12-14, 17, 33-35]. For the effective deposition, the $\mathrm{pH}$ of the suspension medium is maintained in the range of $3.5-5$ [13-14, 17, 33-34]. Applied DC voltage has significant effect on the final coating morphology. A systematic study by Bai et al. shows $20 \mathrm{~V}$ to be the optimum voltage to form uniform and continuous coating with no cracks [12]. Increase in the voltage to $40 \mathrm{~V}$ causes lot of cracks and porosity in the 
coating due to significant hydrogen evolution at cathode. Figure 2.10 shows the effect of applied DC voltage on EPD coating morphology [12].
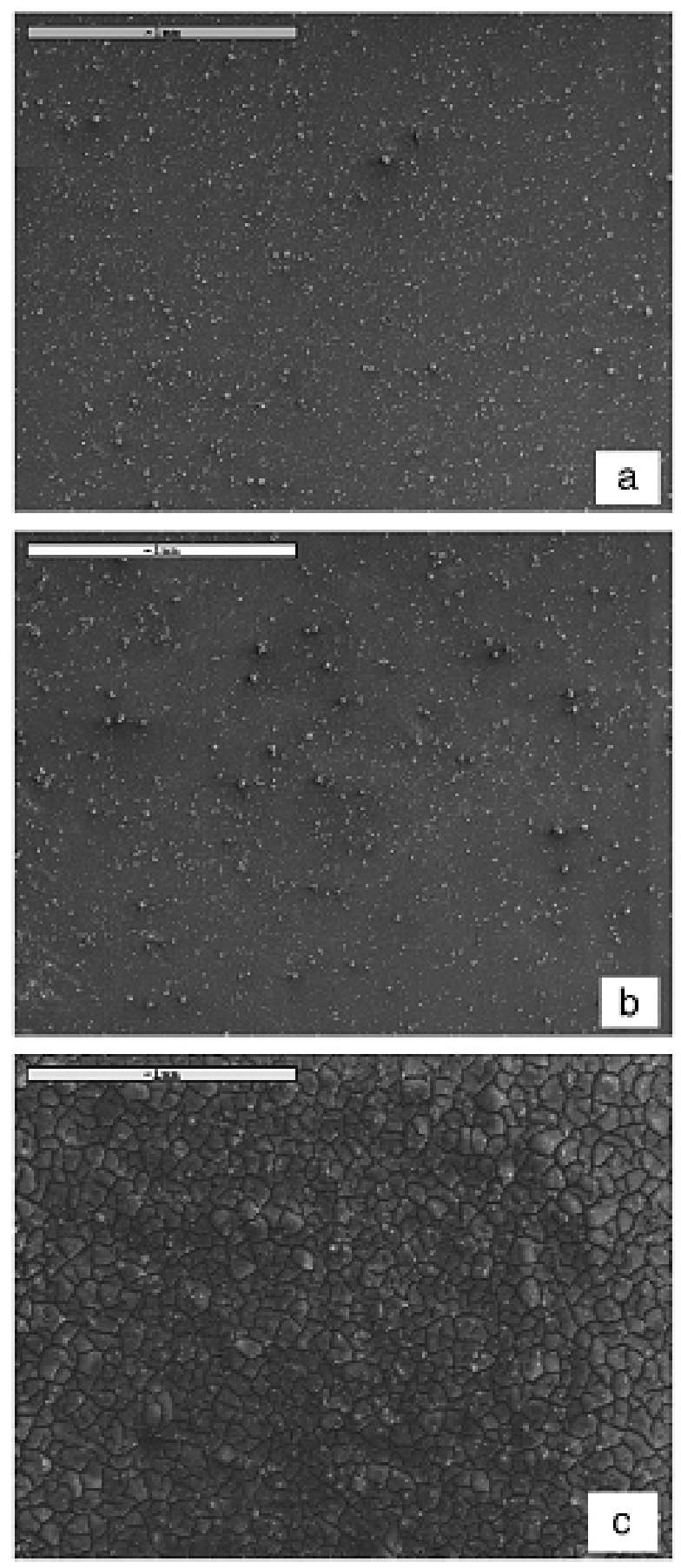

Figure 2.10: SEM micrographs of the HA-CNT coatings electrophoretically deposited for 8 minutes with DC voltages: (a) $10 \mathrm{~V}$; (b) $20 \mathrm{~V}$; and (c) $40 \mathrm{~V}$ [12]. 
HA particles and CNTs are separately added to the suspension with opposite surface charges on them. Thus, they attach to each other to form composite particle, which ensures uniform dispersion of CNT in the composite coating [75]. A low temperature $\left(600-700{ }^{\circ} \mathrm{C}\right)$ post-coating sintering cycle in inert atmosphere is always assisted with EPD to have better densification of the coatings [13-14, 17, 33]. But, differential shrinkage of coating and substrate during sintering also causes formation of cracks in the coating [17]. EPD synthesized HA-CNT coatings shows absence of delamination and good adhesion with the Ti-alloy substrates [13, 17, 33]. Increasing CNT content increases the adhesion strength of coating with substrate $[13,33]$. Incorporation of CNT in HA by EPD is found effective in enhancing the elastic modulus [14], hardness [14] and inter-laminar shear strength [14] of the coating. The main advantage of EPD for synthesizing HA based coating is its room temperature operation followed by a low temperature sintering cycle, which helps in maintaining the crystallinity of HA in coating $[13,33]$. Moreover, absence of high temperature exposure does not create any problem regarding dissociation of $\mathrm{HA}$, which is a problem for most of the other processing techniques $[13,33]$. But, the major problem with EPD technique is severe cracking of the coating [76], especially with increasing coating thickness. None of the studies on HACNT till date have reported synthesizing a coating with $>25 \mu \mathrm{m}$ thickness with insignificant amount of cracks $[12,14,17,34]$. Presence of cracks deteriorates the mechanical property and strength of the coating and makes it incompatible for the intended orthopedic applications. Figure 2.11 presents electrophoretically deposited HACNT coatings on Ti alloy, revealing the intensity of cracking with increasing time of deposition [34]. The thickness of the coating in figure 2.11a with 1 min deposition time is 
reported to be $12 \mu \mathrm{m}$. Considerable amount of effort is needed to overcome the problem of cracking in order to have EPD as an efficient technique for synthesizing HA-CNT composite coatings.
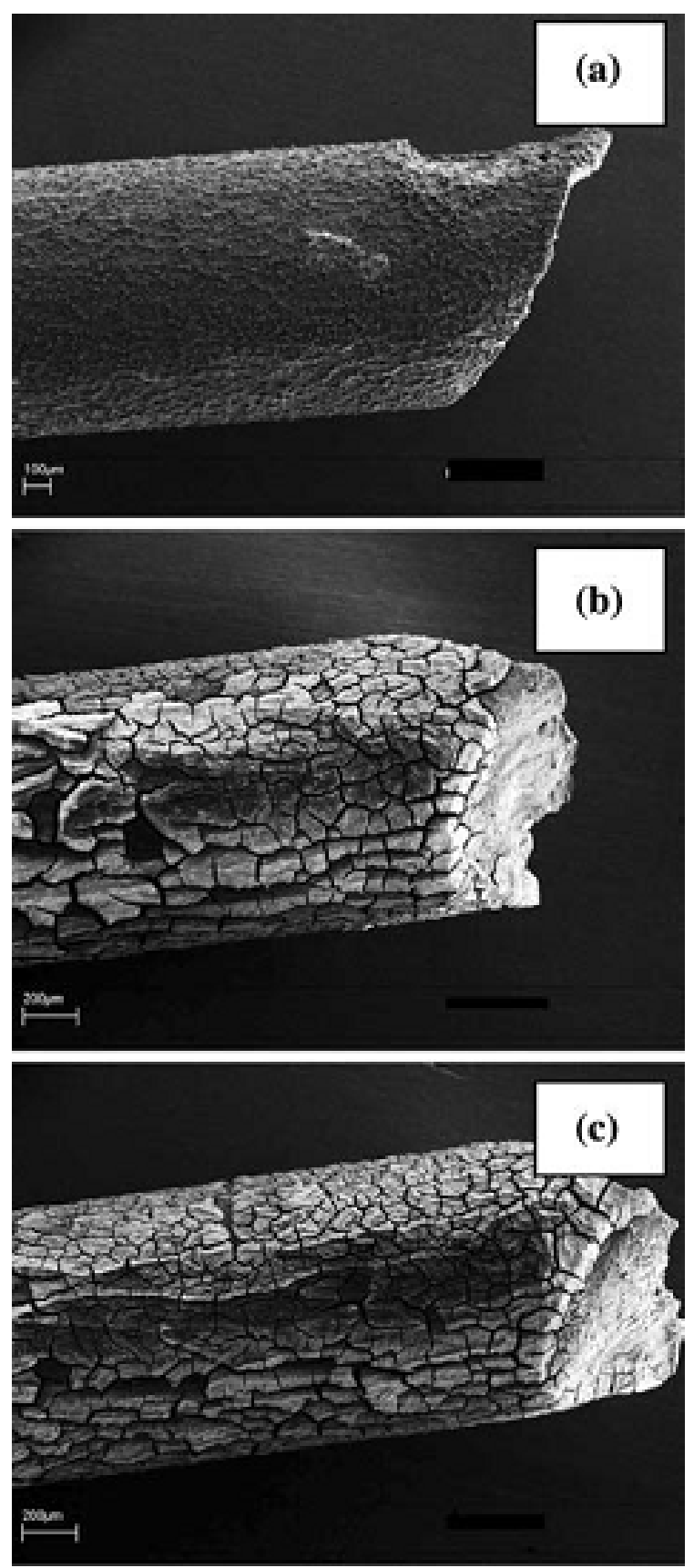

Figure 2.11: SEM micrographs of electrophoretically deposited HA-CNTs coating on Ti alloy wire as a function of deposition time, (a) $1 \mathrm{~min}$, (b) $2 \mathrm{~min}$ and (c) $3 \mathrm{~min}$ [34]. 


\section{$\underline{\text { Aerosol Deposition }}$}

Aerosol deposition (AD) uses shock-consolidation principle to form dense and hard coating of fine ceramic powders on metallic, ceramic and plastic surfaces. In AD process, submicron size ceramic particles are accelerated through air/gas flow with a velocity of several hundreds of $\mathrm{m} / \mathrm{s}$ to collide on the substrate and form a coating. This technique is successfully employed to form ceramic coatings for different applications [77-79]. The only study on synthesizing HA and HA-CNT coating using AD process is conducted by Hahn et al. [16]. A pore and micro-crack free HA-CNT composite coating with an uniform thickness of $5 \mu \mathrm{m}$ is deposited on Ti plate using AD process. CNT addition does not influence the deposition behavior of the coating. HA crystallite size in the coating $(5-20 \mathrm{~nm})$ reduces to one tenth of the size in powder stage. Finer crystallite size in coating is due to the fracture of particles during high energy collision with surface, which bonds together in the coating. Low processing temperature of AD process has specific advantage for HA based coating, in maintaining the crystallinity and preventing the HA phase dissociation. Broadening of X-ray diffraction peaks of HA in the coating from the powder stage is due to the fragmentation of crystals in nano-size. But, very low intensity of HA peaks, with respect to that of Ti peaks is not justified, considering the coating thickness of $5 \mu \mathrm{m}$. CNT reinforcement in coating is successful in increasing the hardness, but elastic modulus is not improved significantly. The adhesion strength of HA and HA-CNT coatings with Ti substrate remains similar and is claimed to be in the same range of plasma sprayed coatings [16]. Adhesion of ceramic coating with substrate in AD is only due to physical bonding. Considering this fact, an in-depth explanation on the bonding mechanism is needed to justify the reported high adhesion strength, which is 
absent in the publication [16]. At present, with only one study being done, AD technique is in its infancy to be suggested for HA-CNT coating application. Further studies, addressing the issues and concerns raised, could project aerosol deposition to be a viable and effective option to synthesize HA-CNT coatings on metallic implant surfaces.

Amongst different consolidation techniques used for HA-CNT composite, conventional sintering is not a suitable one due to its high temperature exposure for long time, which poses the risk of aggravated grain growth of ceramic and dissociation of HA into unwanted phases. Sintering in vacuum, in the presence of pressure or in specialized atmosphere of $\mathrm{CO}$ and $\mathrm{H} 2$ are better options for overcoming these risks. SPS is the most suitable method for synthesizing free standing HA-CNT composite body, due to its short exposure at high temperature, which restricts HA-dissociation and grain growth. Moreover, presence of CNT is an added advantage for better consolidating through SPS. Higher thermal and electrical conductivity of CNT helps uniform distribution of thermal energy significantly in very short sintering time of SPS. LSA is an effective method for synthesizing a strongly adherent and well densified HA-CNT composite coating, though results into dissociation of HA and formation of reaction products like TiC, which are not suitable for orthopedic application. Plasma spraying is a clinically proven method for HA coating and found suitable for HA-CNT. Plasma spraying of HA might cause dissociation of HA and loss of crystallinity. But addition of CNT in HA does not aggravate its limitations, rather causes improvement in crystallinity. This issue has been addressed in details in section 5. Application of low temperature in electrophoretic deposition and aerosol depositions makes them very potential option for HA-CNT coating synthesis. But the major limitation of these processes is the thickness of coating along with the severe 
cracking. Electrophoretic deposition methods have not been extensively studied for HA or HA-CNT coating synthesis. Effect of composite consolidation techniques on phase transformation and crystallinity of HA-CNT system is addressed in details in section 2.1.1.4.

\subsubsection{Dispersion of Carbon Nanotubes in Composite}

Uniform distribution of CNTs in the HA matrix is very critical to ensure uniform improvement in mechanical and tribological performance of the composite structure. Agglomeration of CNTs will cause heterogeneous structure leading to poor densification, weak interfacial bonding and stress concentration points in the structure that will aggravate its failure. The natural tendency of agglomeration in CNTs, due to their high aspect-ratio, nano-sized diameter and high surface energy, makes the job of effective dispersion even more challenging. A closer look on the HA-CNT literature reveals that the distribution of CNT in the HA matrix at the powder stage is carried forward in the consolidated structures for both free standing parts and coatings. Singh et al. have also found the good distribution of CNTs in HA powder, achieved by ultrasonication (figure 2.5) results into uniform dispersion of CNT in electrophoretically deposited composite coating (figure 2.12) [34]. Chemical precipitation of HA on functionalized CNT helps in uniform mixing of HA and CNT. Bai et al. have reported achieving uniform CNT distribution on electrophoretic coating synthesized using chemically precipitated composite powder in precursor (figure 2.13)[12]. Figure 2.14 is the evidence of uniform distribution of CVD grown CNTs on HA in powder and the sintered structure [20]. Kobayashi et al. have reported effective dispersion of carbon nano-fiber in HA by ball 
milling, which is maintained in the hot pressed composite structure (figure 2.15) [30]. Hence, dispersion of CNTs at powder stage is more critical to attain its final homogeneous distribution in the composite.

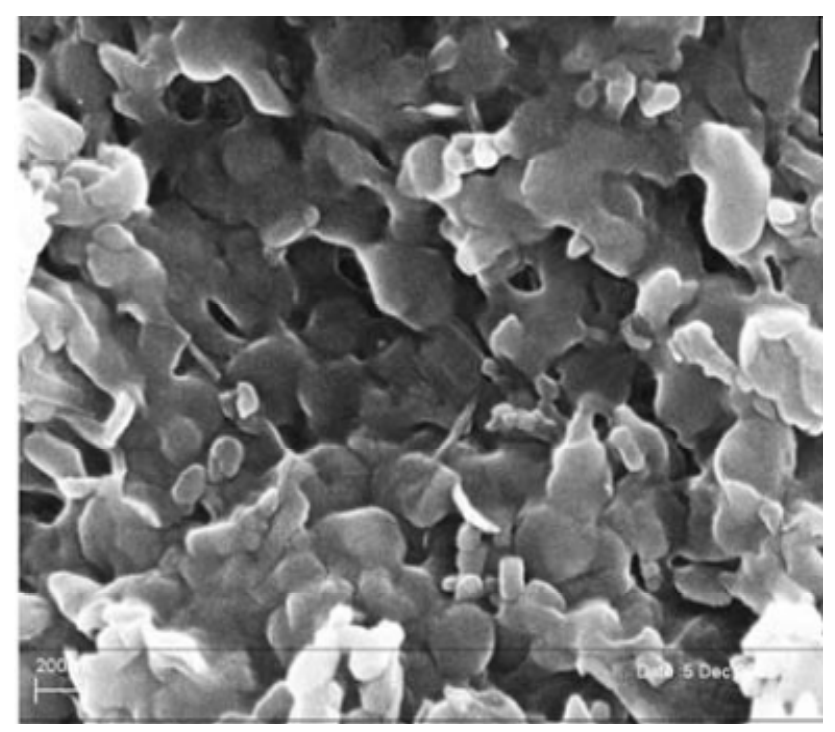

Figure 2.12: SEM micrograph of electrophoretically deposited HA-CNT coating suggesting good dispersion of CNT in HA [34].

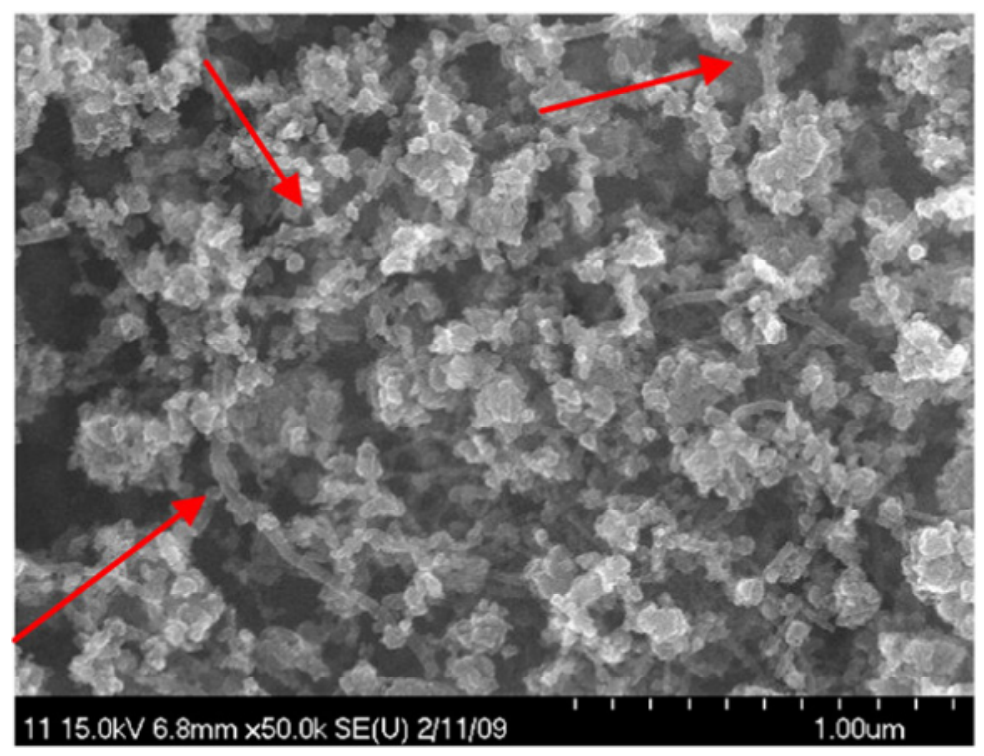

Figure 2.13: SEM micrograph of electrophoretically deposited HA-CNT coating from chemically precipitated composite powder [12]. 

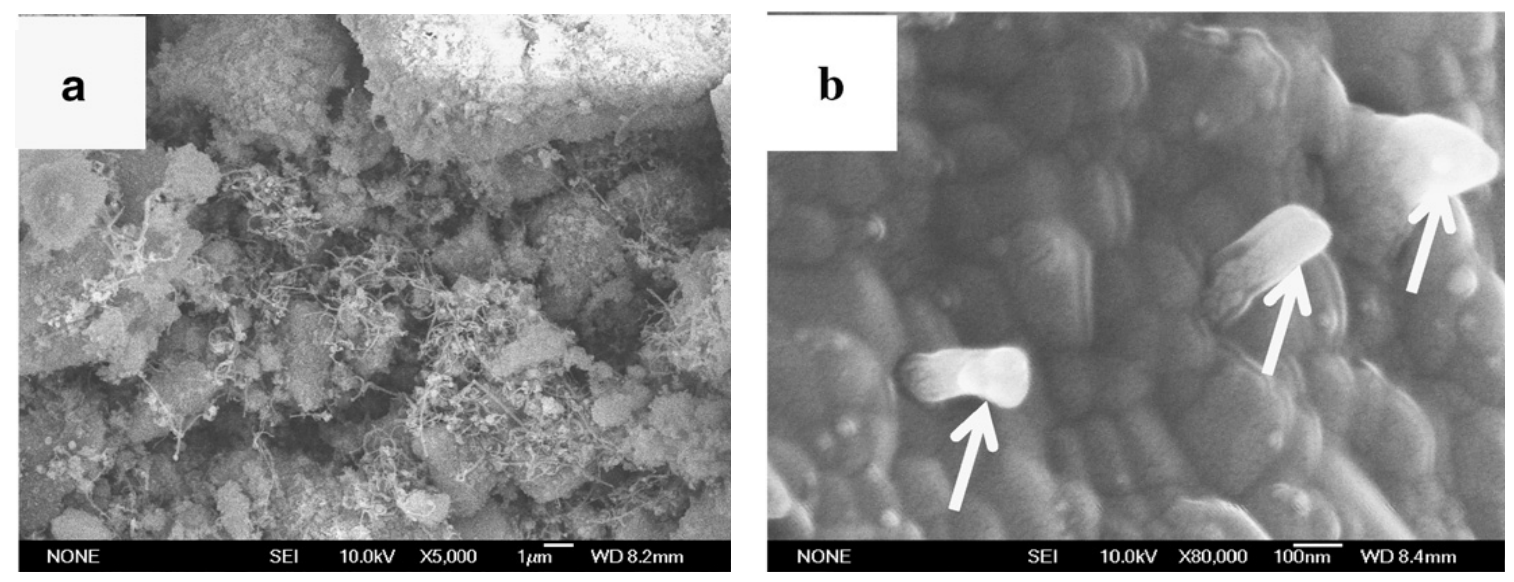

Figure 2.14: SEM micrographs of uniformly distributed CNT in (a) CVD processed composite powder and (b) sintered composite fracture surface [20].

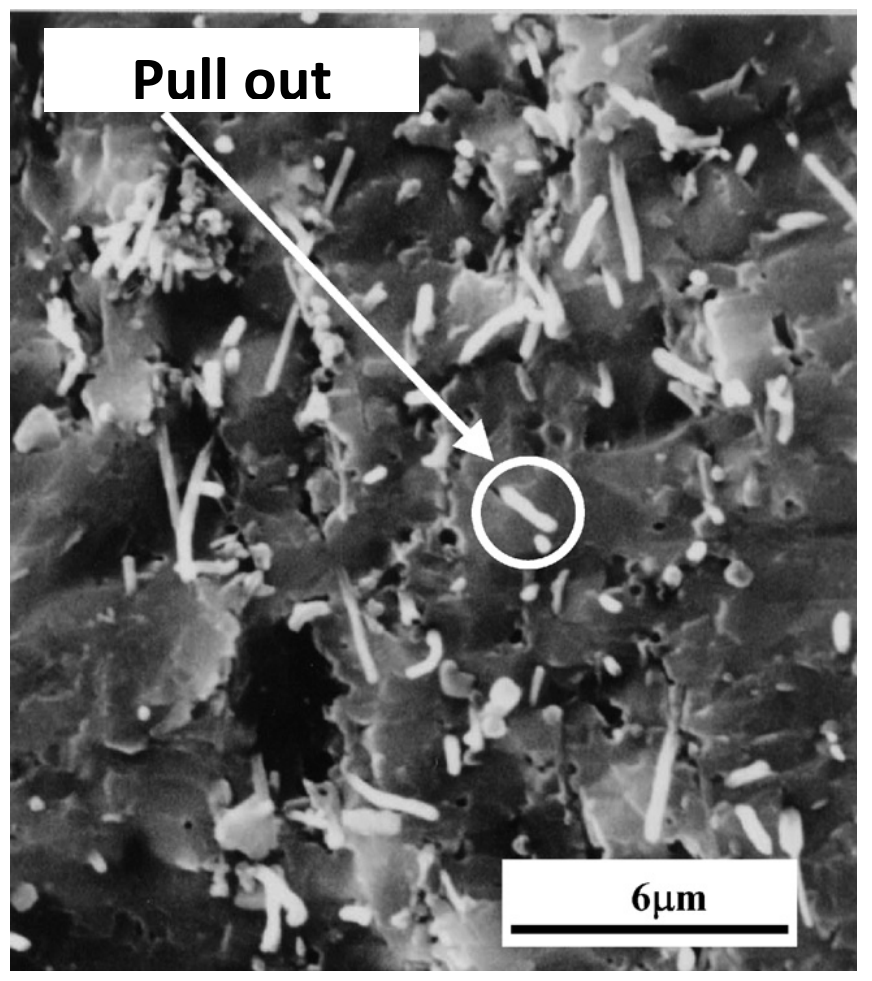

Figure 2.15: Fracture surface of sintered HA-Carbon nano-fiber composite showing the uniform distribution of reinforcement phase [30]. 
Dispersion of CNTs in HA at the powder stage has been tried using several techniques, as discussed in section 2. Most of the studies have used more than one technique simultaneously to achieve the best dispersion. Comparative studies have shown that the degree of CNT dispersion in HA remains similar for both chemical precipitation and mechanical mixing methods [5,7]. Functionalization of CNTs is effective in enhancing the dispersion of CNT in HA followed by both chemical precipitation and mechanical mixing routes [7]. Forming HA-CNT nano-composite particles in electrophoretic deposition bath using opposite surface charge between HA and CNT is also effective in homogeneous distribution of CNTs in composite coating [75]. But, functionalization using acid treatment poses high chance of damage to CNT's outer wall(s) which could lead to lowering in the elastic modulus and strength of the composite $[1,80]$, thus defeating the aim of adding CNTs to HA. Similarly, ball milling also generates defects in CNTs and shortens them by breakage, lowering the effectiveness of the reinforcement [80]. Ultrasonication and other mechanical mixing methods could also pose problem if used excessively [80]. Thus, it is very important to assess the damage on CNTs as an effect of powder mixing procedure. CVD is a very useful method for uniform dispersion of CNTs by growing them directly on the surface of ceramic particles such as HA. But, using metallic catalyst particles for CVD growth may create problem at a later stage, due to cytotoxicity related issues. The process proposed by $\mathrm{Lu}$ et al. for CVD growth of CNT directly on HA is a suitable solution, though the decomposition of HA in this process needs to be taken care of [21]. Hence, an optimum powder processing method is required for the effective CNT dispersion at powder-mixing and consolidation stages. 


\subsubsection{Phase Stability and Crystallinity of $\mathrm{HA}$ in Composite}

One of the main concerns regarding high temperature processing of $\mathrm{HA}$ is the loss of hydroxyl group to form oxyapatite and oxyhydroxyapatite, which starts at $900^{\circ} \mathrm{C}$ in air and $850^{\circ} \mathrm{C}$ in water free atmosphere [81-83]. Further dissociation of $\mathrm{HA}$ at higher temperatures $\left(1200-1450{ }^{\circ} \mathrm{C}\right)$ are very much sensitive to the $\mathrm{Ca} / \mathrm{P}$ ratio, which is 1.67 . In case of $\mathrm{Ca} / \mathrm{P}<1.67$, HA dissociate into $\beta$-TCP ( $\alpha$-TCP at higher temperature) and tetra calcium phosphate (TCP). HA dissociate to $\mathrm{CaO}$ when $\mathrm{Ca} / \mathrm{P}>1.67$ [83-84]. The dissociation of HA into TCP or other phases has two major effects on the consolidated structure. Firstly, the dissociated phases causes problem in densification significantly lower the strength of the structure $[83,85]$. Secondly, the phases formed by dissociation of HA leads to considerable increase of the dissolution rate in physiological $\mathrm{pH}$, as confirmed by the in-vitro tests [86]. Dissociation of HA inside living body is unsuitable for orthopedic applications.

Similar problems also exist for HA-CNT composite system. Moreover, $\mathrm{H}_{2} \mathrm{O}$ formed due to dehydroxylation of HA at higher temperature can also react with CNT to cause damage to nanotubes. The problem of HA dissociation does not exist for synthesis routes that employ lower temperature, e.g. electrophoretic and aerosol deposition of coatings $[13,16,33]$. Electrophoretic deposition generally requires a post-sintering treatment. But, the sintering temperature $\left(600-700{ }^{\circ} \mathrm{C}\right)$ is lower than the HA dissociation start temperature $\left(850-900{ }^{\circ} \mathrm{C}\right)$. The problem regarding phase transformation of HA is largely associated with the high temperature synthesis routes. Conventional sintering of HA-CNT composite leads to significant HA dissociation $[6,36]$. Phase dissociation is 
mainly detected by analysis of x-ray diffraction (XRD) pattern of the composite structure. JCPDS PDF No. 9-432 is used for the detection of characteristics diffraction peaks from HA. Presence of dissociated phases is also detected by referring to JCPDS data-base, e.g., PDF No. 9-169 for $\beta$-TCP, PDF no. 29-359 for $\alpha$-TCP etc. The reference XRD data for CNT is available in JCPDS PDF No. 23-0064 for graphite. CVD growth of CNTs on HA powder also introduces transformation phases in the composite powder [21]. The study by Li et al. reveals the effect of CNT in increasing dissociation of HA during sintering at $1200{ }^{\circ} \mathrm{C}$ [36]. Sintering of pure HA at $1200^{\circ} \mathrm{C}$ produces $\beta$-TCP. But, the presence of CNT causes further decomposition of HA into $\alpha$-TCP and $\gamma-\mathrm{Ca}_{2} \mathrm{P}_{2} \mathrm{O}_{7}$, which requires higher heat input. High specific heat of CNTs [87] can enable them to work as heat sink and assist in further dissociation of HA in the neighboring region. A recent study by White et al. suggests that combination of $\mathrm{CO}$ and $\mathrm{H}_{2}$ is ideal sintering atmosphere for HA-CNT (48). Presence of carbon monoxide and hydrogen in atmosphere shifts the equilibrium of reaction between $\mathrm{H}_{2} \mathrm{O}$ and $\mathrm{C}$, and thus retains $\mathrm{CNT}$ and prevents dehydroxylation of HA. Application of pressure during sintering is found effective in partially suppressing dissociation of HA in most HA-CNT systems $[4,15,18]$. Meng et al. have suggested that application of pressure could reduce the dehydration and decomposition of HA [18].

In this regard, rapid processing routes such as SPS or plasma spraying become attractive. The faster synthesis time can reduce the dissociation of HA to some extent by defeating the kinetics of the phase transformation reactions. SPS processed HA-CNT systems shows partial decomposition of HA into a very small amount of $\beta$-TCP in the 
sintered structure [31, 42]. Kalmodia et al. have reported presence of $\beta$-TCP in their SPS sintered HA- 18.4 wt. $\% \mathrm{Al}_{2} \mathrm{O}_{3}-1.6$ wt.\% CNT composite structure even at a higher heating rate [41]. But, closer observation of the XRD pattern (figure 2.16) presented in the same study reveals that $100 \%$ intensity peak of $\beta$-TCP $\left(2 \theta=30.9^{\circ}\right)$ is not present in the pure $\mathrm{HA}$ sample, whereas it is present in other two samples containing $\mathrm{Al}_{2} \mathrm{O}_{3}$ and $\mathrm{Al}_{2} \mathrm{O}_{3}+\mathrm{CNT}$ respectively. As all three samples were sintered using same SPS parameters, the dissociation of HA in the composite cannot be attributed solely to sintering. Though not reported by the authors of the same study [41], but the dissociation of $\mathrm{HA}$ could be due to $\mathrm{Al}_{2} \mathrm{O}_{3}$. Previous studies have shown effect of $\mathrm{Al}_{2} \mathrm{O}_{3}$ on increasing dissociation of HA at high temperature exposure [88-89].

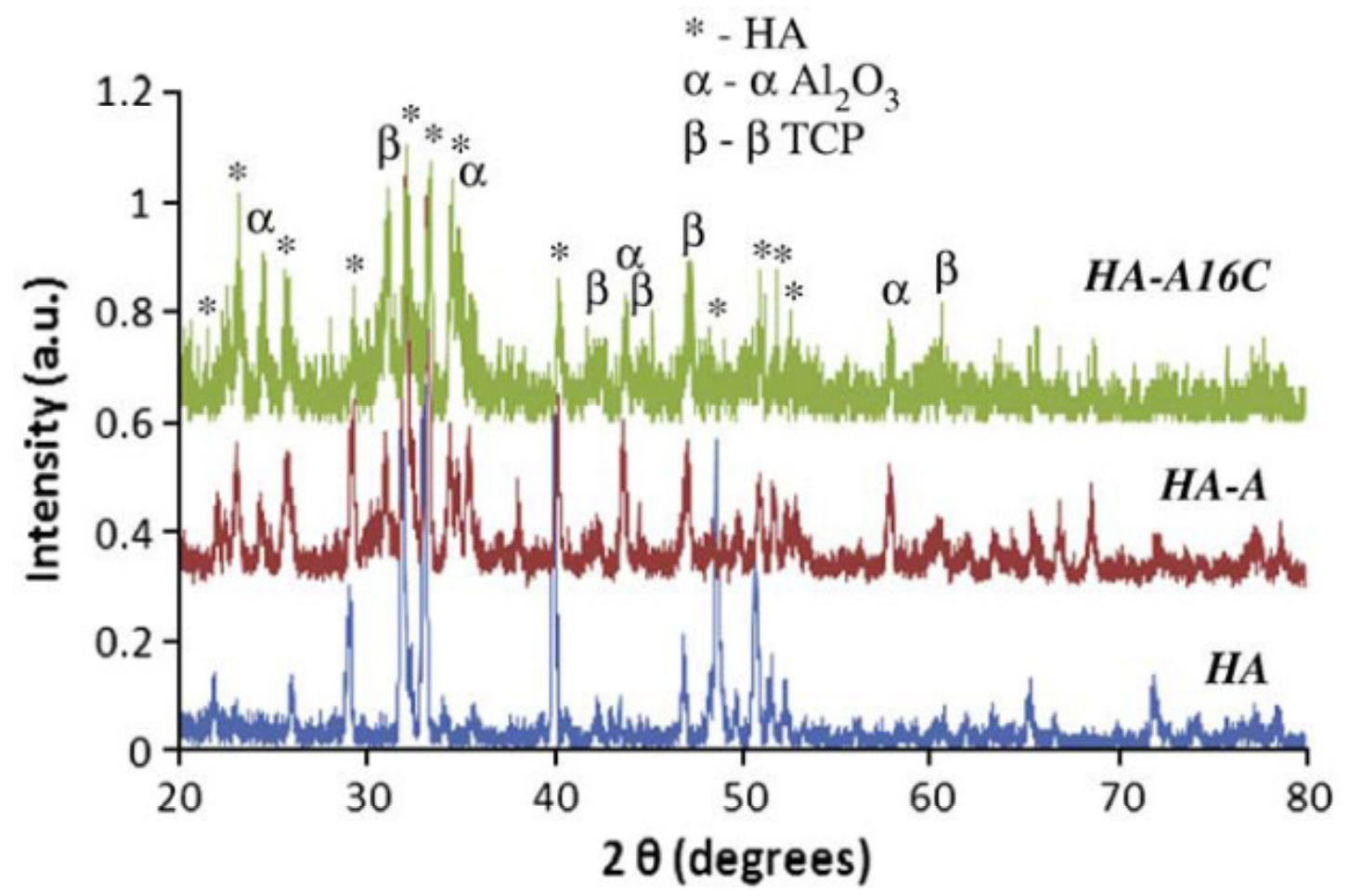

Figure 2.16: X-ray diffraction pattern of SPS sintered $\mathrm{HA}, \mathrm{HA}-\mathrm{Al}_{2} \mathrm{O}_{3}$ (HA-A) and $\mathrm{HA}$ $\mathrm{Al}_{2} \mathrm{O}_{3}$-CNT (HA-A16C) composites [41]. 
Plasma spray forming of HA- $4 \mathrm{wt} \%$ CNT composite coating also reveals minimal dissociation of HA to form a small amount of $\beta$-TCP, which is evident from the very low intensity of the TCP peaks compared to much higher HA peaks [37]. However, laser surface alloyed HA- (5/10/20 wt.\%) CNT composite coatings show very significant amount of HA dissociation [26]. Melting of the substrate along with the coating material during laser surface alloying exposes HA to high temperature for longer duration as compared to plasma spraying, and hence results in its dissociation. None of these rapid processing routes report increase in HA dissociation in the presence of CNT. Higher specific heat of CNTs might not induce significant effect due to faster consolidation time for SPS and plasma spraying, as it does in case of conventional sintering. One important issue, while discussing the phase dissociation of HA in HA-CNT system, is the solubility or diffusion of carbon atoms in HA lattice. But, no report is available on the diffusion of C in HA lattice and corresponding change in HA cell volume. On the contrary, Rietveld analysis of the neutron diffraction data by Keally et al. indicates no change in the structural parameter (cell constant) of HA in HA-2 wt.\% CNT system sintered up to $1000^{\circ} \mathrm{C}[4]$.

Crystallinity of HA is another important variable as it influences the mechanical properties. The presence of amorphous phases increases the dissolution/resorption rate of HA, which is not suitable for its intended orthopedic applications [90-91]. Amorphization of HA is not a concern for most of the synthesis processes used in consolidation of HACNT composites, except plasma spraying, which involves extremely high cooling rates, in the range of $10^{5}-10^{8} \mathrm{Ks}^{-1}$. A study by Keally et al. on hot pressing of HA- 2 wt.\% CNT composite has shown fully crystalline HA consolidated structure with no significant 
effect of CNT addition on the unit cell parameters [4]. Another study by the same group have reported an increase in the crystallite size of HA with $5 \mathrm{wt} . \%$ CNT addition [6]. But the crystallite size was found to be decreasing for higher CNT content (12 and 25 wt. \%). The initial increase in HA crystallite size could be due to higher specific heat and thermal conductivity of CNT, which provides more heat to neighboring regions aiding in grain growth. But, with increasing CNT content, pinning action offered by CNTs at grain boundaries becomes dominating to reduce the crystallite size. Inhomogeneous distribution of CNTs in the reported structure is also responsible for such behavior [8]. White et al. have also reported increasing crystallinity in HA- 5 wt.\% CNT composite system with increasing sintering temperature [7].

As mentioned earlier, formation of amorphous phases is a concern in HA coatings synthesized using plasma spraying. But, addition of CNTs is found to act as a solution of this problem. Balani et al. reported that plasma sprayed HA-CNT coating shows significantly increased crystallinity $(80.4 \%)$ compared to HA coating $(53.7 \%)$ synthesized using the same parameters (figure 2.17) [37]. Increased crystallinity in HA- 4 wt.\% CNT system is attributed to higher thermal conductivity of CNTs compared to HA, which helps in nucleation and precipitation of HA. Higher heat capacity of CNT can absorb more heat when exposed to the plasma and then higher thermal conductivity of CNT can create a thermal pool around while cooling. Both of these phenomenon provides an enhanced cooling time to the HA matrix in the neighboring region of CNTs, which helps in its recrystallization. Another study by Tercero et al. on plasma sprayed HA-18.4 wt. $\% \mathrm{Al}_{2} \mathrm{O}_{3}-1.6$ wt.\% CNT coating, provides further evidence on effect of CNT in increased crystallinity of $\mathrm{HA}$ based composite coating [40]. Both the $\mathrm{HA}$ and $\mathrm{HA}-\mathrm{Al}_{2} \mathrm{O}_{3}$ - 
coatings show same type of amorphous hump in the XRD pattern, whereas, sharp crystalline HA peaks with reduced amorphous hump is observed with the CNT addition. It can be inferred from these observations that $\mathrm{Al}_{2} \mathrm{O}_{3}$ does not contribute towards the crystallinity of HA composite coating due to its poor thermal conductivity. Thus, presence of CNTs tends to improve the crystallinity of HA in composite systems.

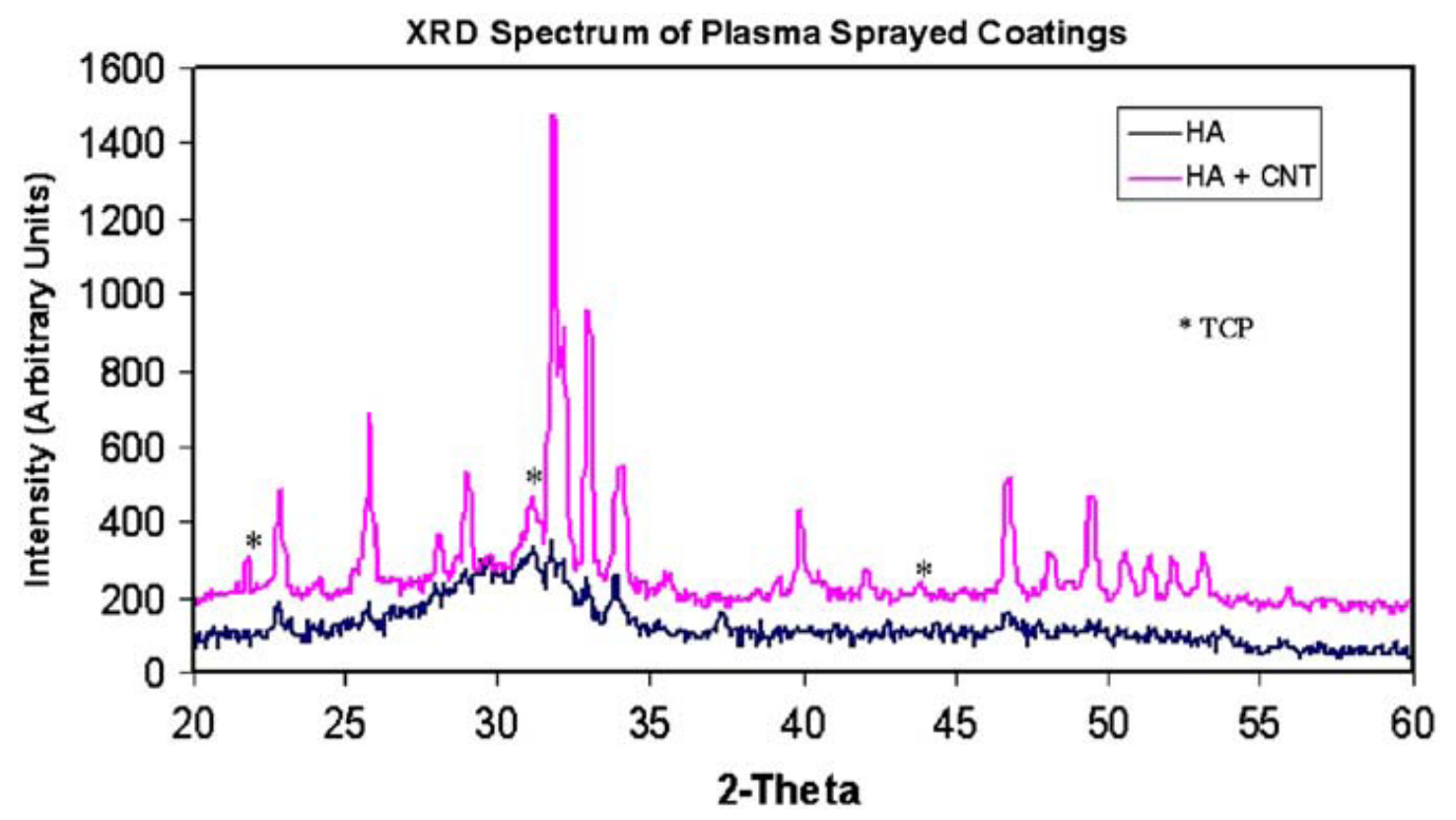

Figure 2.17: X-ray diffraction pattern of plasma sprayed HA coatings showing increased crystallinity in presence of CNT [37].

\subsubsection{Mechanical Properties of HA-CNT Composites}

The main aim of CNT addition to HA is to improve the overall mechanical properties of the composite. CNT is found effective in increasing the fracture toughness, elastic modulus, hardness and bending strength of HA-CNT free standing parts and coatings. Another important consideration for composite coating is its adhesion strength with the substrate. Researchers have studied the effect of CNT addition on adhesion 
strength of HA based coating with underlying titanium substrate $[13-14,16,33]$. The following subsections present a comprehensive summary of the effect of CNT addition on the fracture toughness, elastic modulus, hardness, and strength of the composite.

\subsubsection{Fracture Toughness}

Bones are the load bearing parts of a living body. Thus, they need to possess good fracture toughness $\left(\mathrm{K}_{\mathrm{IC}}\right)$ to prevent cracking and fracture on the application of high and cyclic loading during limb movement and actions. Fracture toughness of dense HA (1 MPa. $\mathrm{m}^{0.5}$ ) is significantly lower than the minimum reported value for cortical bone ( 2 MPa.m ${ }^{0.5}$ [ [84]. Thus, to replace bone as an implant or coating, fracture toughness of HA needs to be improved. CNTs have been explored as a second phase reinforcement for improving the fracture toughness of HA based composite parts and coatings. The role of CNT in improving the fracture toughness of the composite system can be explained using three main toughening mechanisms - (i) crack deflection; (2) CNT pull-out and (3) crack bridging.

(1) Crack Deflection: When a crack propagates through the matrix and reaches a CNT across its path, the crack gets deflected and absorbs some energy resulting in toughening of the matrix. Chen et al. have developed an analytical model to predict the deflection of crack and energy release rate due to the deflection for ceramic-CNT composite system [92]. Studies on HA-CNT system mentions about crack deflection as one of the toughening mechanism contributing towards higher $\mathrm{K}_{\mathrm{IC}}$ of composite than only HA $[15,18,45]$. 
(2) CNT Pull-out: Pulling out of CNTs from the ceramic matrix can dissipate energy due to binding and friction and subsequently, leads to toughening. Figures $2.14 \mathrm{~b}$ and 2.18 show SEM images of CNT pull-out from the fracture surfaces of HACNT composite, reported in different studies 15-16, 20, 30-31, 39, 40, 79].

(3) Crack Bridging: CNTs can act as bridges and restrict the widening of the cracks. CNT bridges require more energy for opening up of the cracks and cause toughening. Different studies have also shown evidence of CNT bridging as an effective mechanism for the toughening of HA-CNT composite $[15,18,37,41]$.
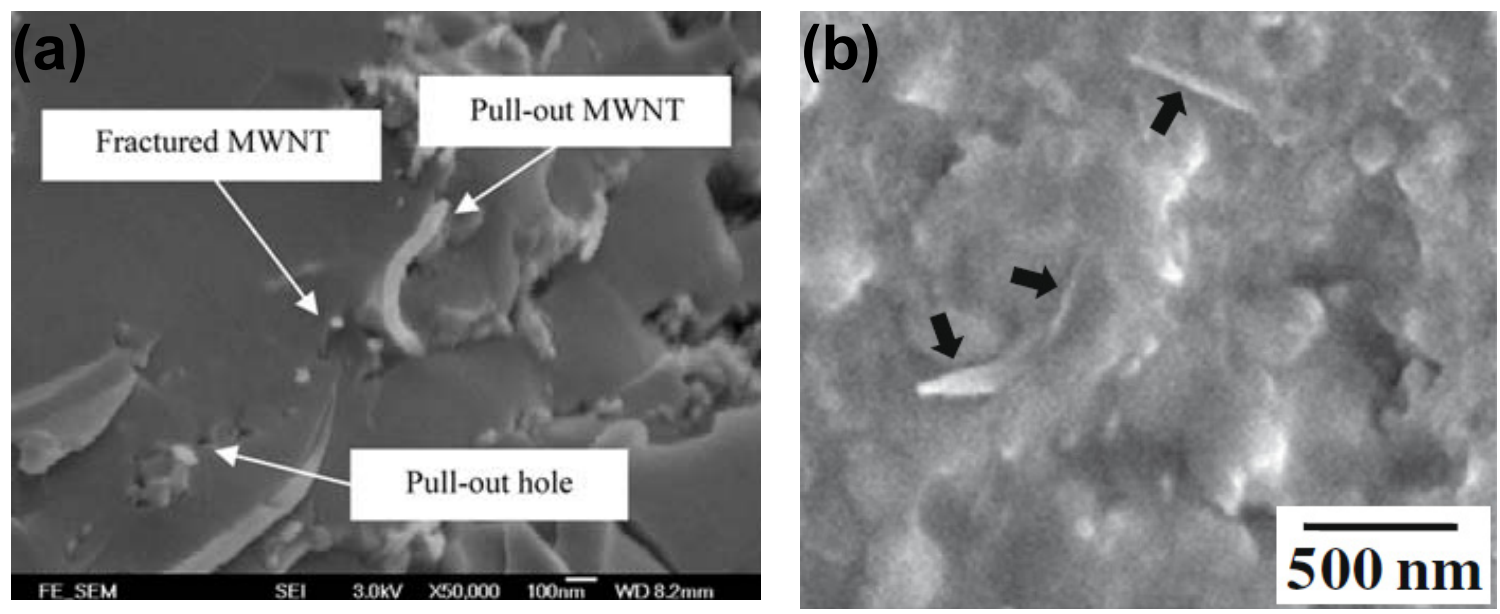

Figure 2.18: Protruded CNT on fracture surface of HA-CNT composite synthesized by (a) hot pressing [15]; (b) aerosol deposition [16].

Most of the studies have used indentation based technique for the measurement of fracture toughness. Figure 2.19 shows a comparison of the published data on percentage improvement in the fracture toughness as a function of CNT content in the composite structure. The value of $\mathrm{K}_{\mathrm{IC}}$ in each study depends on several factors including processing route, powder morphology, structure type - i.e. free standing or coating. Thus, the 
percentage improvement of $\mathrm{K}_{\mathrm{IC}}$ in HA-CNT system is calculated with reference to $\mathrm{K}_{\mathrm{IC}}$ of the HA structure (without CNT) reported in the same study. A few studies have adopted $\mathrm{Al}_{2} \mathrm{O}_{3}$ or $\mathrm{ZrO}_{2}$ in addition to $\mathrm{CNT}$, which also contribute towards toughening of the HA matrix [18, 39-41]. In those cases, the comparison has been made between the two compositions having $\mathrm{HA}-\mathrm{Al}_{2} \mathrm{O}_{3} / \mathrm{ZrO}_{2}$ and $\mathrm{HA}-\mathrm{Al}_{2} \mathrm{O}_{3} / \mathrm{ZrO}_{2}-\mathrm{CNT}$. The CNT content in figure 2.19 is presented in weight percentage. In cases where CNT content is reported as volume percentage, it is converted to weight percentage using the density of HA and $\mathrm{CNT}$ as $3.2 \mathrm{~g} / \mathrm{cc}$ and $2.1 \mathrm{~g} / \mathrm{cc}$ respectively.

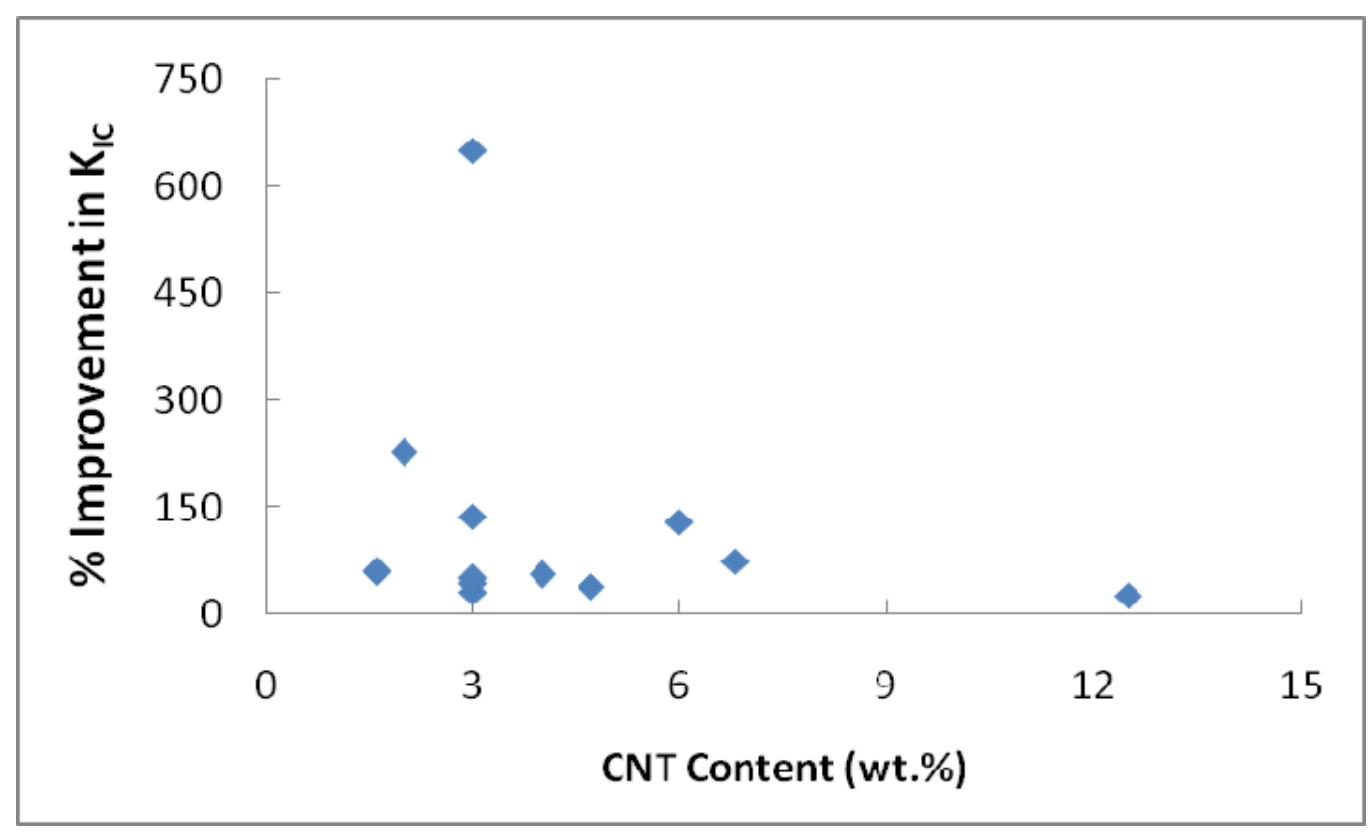

Figure 2.19: Percentage improvement in $K_{I C}$ as a function of CNT content in HA-CNT systems synthesized through different process routes.

The highest improvement in $\mathrm{K}_{\mathrm{IC}}$ of $\sim 650 \%$ is obtained by $\mathrm{Li}$ et al. with 3 wt.\% CNT addition [36]. HA was sintered in air, whereas, the HA- 3 wt.\% CNT system that shows maximum improvement in $\mathrm{K}_{\mathrm{IC}}$ was sintered in vacuum. This study has also 
mentioned that sintering in presence of any gas is detrimental for HA based system. HA surface can absorb gas that deteriorates its densification and sintering in vacuum produces best densification. Thus, $650 \%$ improvement in $\mathrm{K}_{\mathrm{IC}}$ in this study is a cumulative effect of CNT reinforcement and better densification in absence of gas. On closer observation of other studies that has shown more than $100 \%$ improvement in $\mathrm{K}_{\mathrm{IC}}$, the effect of uniform dispersion of CNT becomes more evident. CVD grown CNTs on HA powder by Li et al. ensures good dispersion at powder and consolidated stage. A225\% improvement in fracture toughness was observed with only 2 wt.\% CNTs grown via CVD [20, 25]. The surface adsorption force at CVD grown CNT and HA particles interface plays an important role in the high performance of the composite.

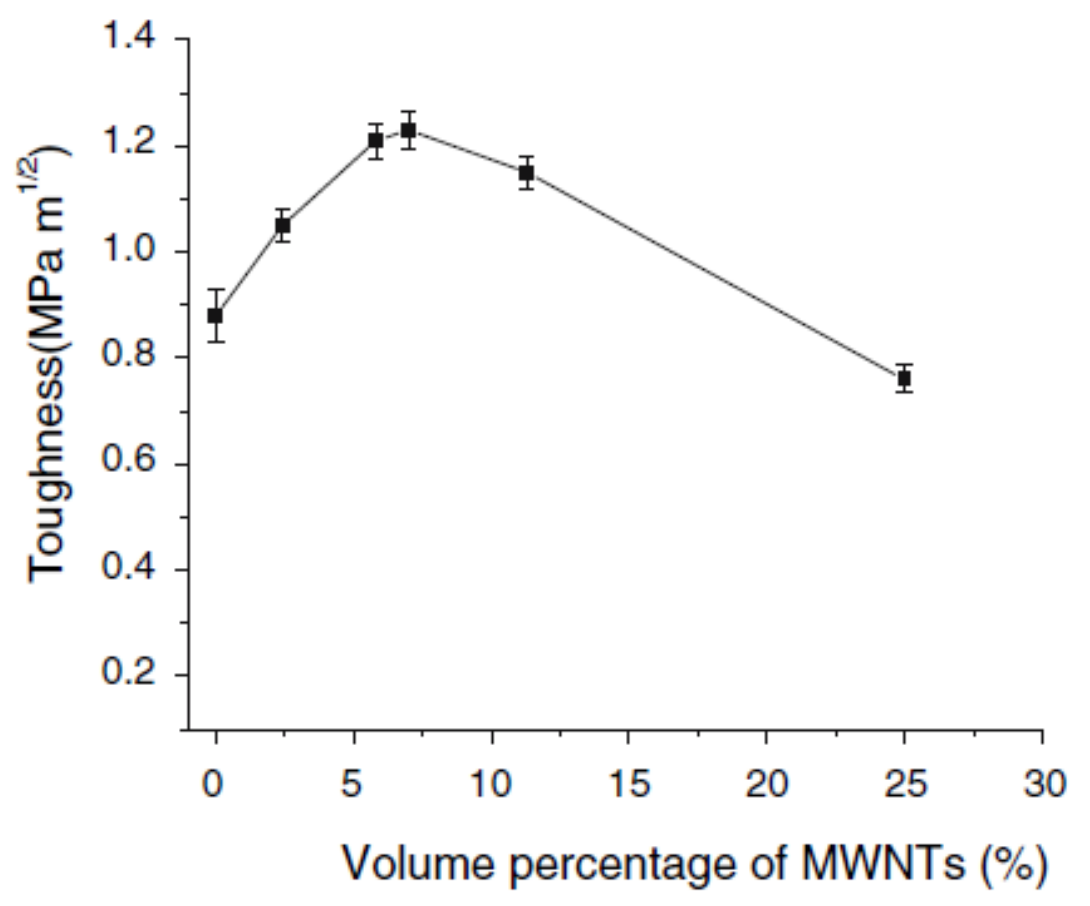

Figure 2.20: Change in the fracture toughness as a function of CNT content in a hot pressed HA-CNT system [15]. 
Lower increase in $\mathrm{K}_{\mathrm{IC}}$ can be associated with the existence of agglomerated CNTs in the sintered structures, due to poor CNT dispersion at the powder stage $[8,15,31]$. Meng et al. have provided a direct evidence of effect of CNT dispersion on $\mathrm{K}_{\mathrm{IC}}$ of hot pressed HA-CNT composite [45]. Better dispersion of CNT with similar content (3 wt.\%) is reported to increase $\mathrm{K}_{\mathrm{IC}}$ by $5 \%$. Another study by Meng et al. on optimization of CNT content in hot pressed composite has shown 7 vol.\% ( 4.7 wt.\%) CNT addition causes the maximum improvement in $\mathrm{K}_{\mathrm{IC}}$, as presented in figure 2.20 [15]. Further increase in CNT content causes agglomeration and deterioration of fracture toughness. The studies, which have used an additional reinforcement phase $\left(\mathrm{Al}_{2} \mathrm{O}_{3}\right.$ or $\left.\mathrm{ZrO}_{2}\right)$ than $\mathrm{CNT}$, show relatively lower degree of $\mathrm{K}_{\mathrm{IC}}$ improvement due to CNTs [18, 40-41]. $\mathrm{K}_{\mathrm{IC}}$ of HA in those cases is already improved significantly with the $\mathrm{Al}_{2} \mathrm{O}_{3}$ or $\mathrm{ZrO}_{2}$, leaving less room for improvement by CNT addition. The only study that shows agglomeration of CNT and still higher improvement in $\mathrm{K}_{\mathrm{IC}}(\sim 130 \%)$ is by Tanaka et al. on SPS processed HA- 6 wt.\% CNT system [46]. But, the study does not provide a suitable justification for such behavior, contradictory to all other studies on HA-CNT systems. Tanaka et al. have very briefly mentioned about anisotropic nature of CNT clumps that can prevent grain boundary cracking from extension. But, CNT clumps are supposed to create a very weak interface with HA matrix, which could have severe detrimental effect on the toughening of the composite. Sintering temperature in SPS processing is also found to have direct effect on the fracture toughness of the HA-CNT composite. Sarkar et al. have reported fracture toughness of HA-2.5 vol.\% (1.65 wt. \%) CNT synthesized by spark plasma sintering at 1000,1100 and $1200{ }^{\circ} \mathrm{C}[31]$. Lower temperature $\left(1000{ }^{\circ} \mathrm{C}\right)$ leaves significant porosity, whereas higher temperature $\left(1200^{\circ} \mathrm{C}\right)$ results in excessive grain growth. As a 
result, transgranular type of fracture with easily propagating cracks dominates in both the cases; having deteriorating effect on the fracture toughness. HA-CNT composite sintered at $1100^{\circ} \mathrm{C}$ shows fine grained consolidated structure. Intergranular fracture with $\mathrm{CNT}$ pull-outs is prevalent in this structure, which absorb energy while cracks propagate and increase the fracture toughness [31, 42]. Moreover, the fine grained structure, generated due to grain boundary pinning action of $\mathrm{CNT}$, is also effective in increasing the fracture toughness of the composite structure [55].

The dispersion of CNT in HA matrix and densification are two most critical factors for improvement of fracture toughness in HA-CNT system. A comprehensive outcome of all the studies indicate $\leq 5 \mathrm{wt} . \% \mathrm{CNT}$ is effective for improvement in fracture toughness, irrespective of the processing technique. Agglomeration of CNTs at higher content is responsible for deterioration in $\mathrm{K}_{\mathrm{IC}}$. CNT improves the $\mathrm{K}_{\mathrm{IC}}$ of composite by absorbing the dissociation energy, crack deflection, and crack bridging in HA matrix. CNT induced toughening is highly effective even in porous and heterogeneous structures such as plasma sprayed HA coatings, indicating dominance of toughening effect over porosity.

\subsubsection{Elastic Modulus}

Elastic modulus (E) of human cortical bone is reported to be in the range of 15-25 $\mathrm{GPa}$ [93-94], whereas it is much higher $(\sim 100 \mathrm{GPa})$ for consolidated monolithic HA [95]. Mismatch of $\mathrm{E}$ at bone-implant interface might pose a risk of fracture or delamination of implant. But, osseointegration ability of HA creates a strong bonding at HA- bone 
interface, which reduces the chance of delamination and fracture. Thus, unlike fracture toughness, elastic modulus of HA need not be improved to match with bone. But, increase in E directly influences improvement in the fracture toughness in ceramic based composite systems. Fracture toughness of brittle ceramic system is mainly estimated from indentation cracking using Anstis' equation [97], median crack equation by Lawn [98] or Evans' relationship [99] as following:

Anstis' Equation: $K_{I C}=0.016\left(\frac{E}{H}\right)^{0.5} \frac{P}{C^{1.5}} \ldots \ldots \ldots .(1)$

Median Crack Equation: $\quad K_{I C}=0.018\left(\frac{E}{H}\right)^{0.5}\left(\frac{a}{C^{1.5}}\right) \ldots \ldots \ldots . .(2)$

Evans and Charles Equation: $K_{I C}=0.16 H a^{0.5}\left(\frac{C}{a}\right)^{-1.5}$, Where $c / a \geq 3$

Expression (3) was later modified with a non-dimensional factor $(\mathrm{E} / \mathrm{H})^{\mathrm{n}}[100]$, where, $\mathrm{K}_{\mathrm{IC}}$ - is the fracture toughness, $\mathrm{E}$ - is the elastic modulus, $\mathrm{H}$ - is the hardness, $\mathrm{a}-$ is the half diagonal length of indent and c- is the radial crack length.

The studies on HA-CNT systems have used above-listed three equations for determining the fracture toughness $[15,20,30-31,37,39-40]$. It is clear that an increase in $\mathrm{E}$ is beneficial for the increased fracture toughness, which is essential for application of HA-CNT for orthopedic applications.

Improvement of elastic modulus in HA-CNT system is due to the high elastic modulus of CNT (200-1000 GPa [101] and good bonding at HA-CNT interface. Upon application of a stress on HA-CNT, the matrix starts deforming first due to its lower elastic modulus. If the HA matrix has a strong interfacial bonding with much stiffer 
CNTs, then effective transfer of stress can take place from matrix to the reinforcement. CNTs can absorb much more stress than HA to produce similar amount of the elastic strain. Thus, the resultant elastic strain generated in the composite structure is reduced compared to HA for the same applied stress, resulting in increased effective elastic modulus for HA-CNT. A cross-linking model for estimating the Young's modulus of HA-functionalized CNT composite proposed by Saffar et al., shows that the improvement in modulus of the composite depends on the CNT content, its aspect ratio, number of cross links and characteristics of interfacial reaction [102]. Cross links are the points where HA attaches to functionalized sites on CNT surfaces, and are considered as active load transfer points through HA-CNT interface during modeling.

Figure 2.21 presents percentage improvement of $\mathrm{E}$ in HA-CNT system as a function of CNT content summarized from all the publications. Comparative analysis of the HA-CNT literature data in figure 26 reveals no improvement in E for CNT content beyond 4-5 wt.\%. All the studies referred in Figure 26 have used nanoindentation technique to measure the elastic modulus for HA-CNT, from the slope of the unloading curve using Oliver-Pharr method [103]. Most of the studies have reported the increase in E up to $70 \%$ with CNT content varying in a range of 1.3-12.5 wt.\%. Homogeneous dispersion of CNTs and good HA-CNT interfacial bonding plays key role in increasing E of the composite $[14,26,42]$. On the contrary, agglomerated CNTs with weak interfacial bonding reduce the elastic modulus of the composite as compared with pure HA [8, 41]. Keally et al. has reported $2 \%$ decrease in E with increasing CNT content, which is attributed towards agglomeration of CNT in the structure [8]. Whereas, Kalmodia et al. 
have found weak interface, yielding easier sliding in nano-scale length, to be responsible for a $22 \%$ decrease of $\mathrm{E}$ after $1.6 \mathrm{wt} . \% \mathrm{CNT}$ reinforcement [41].

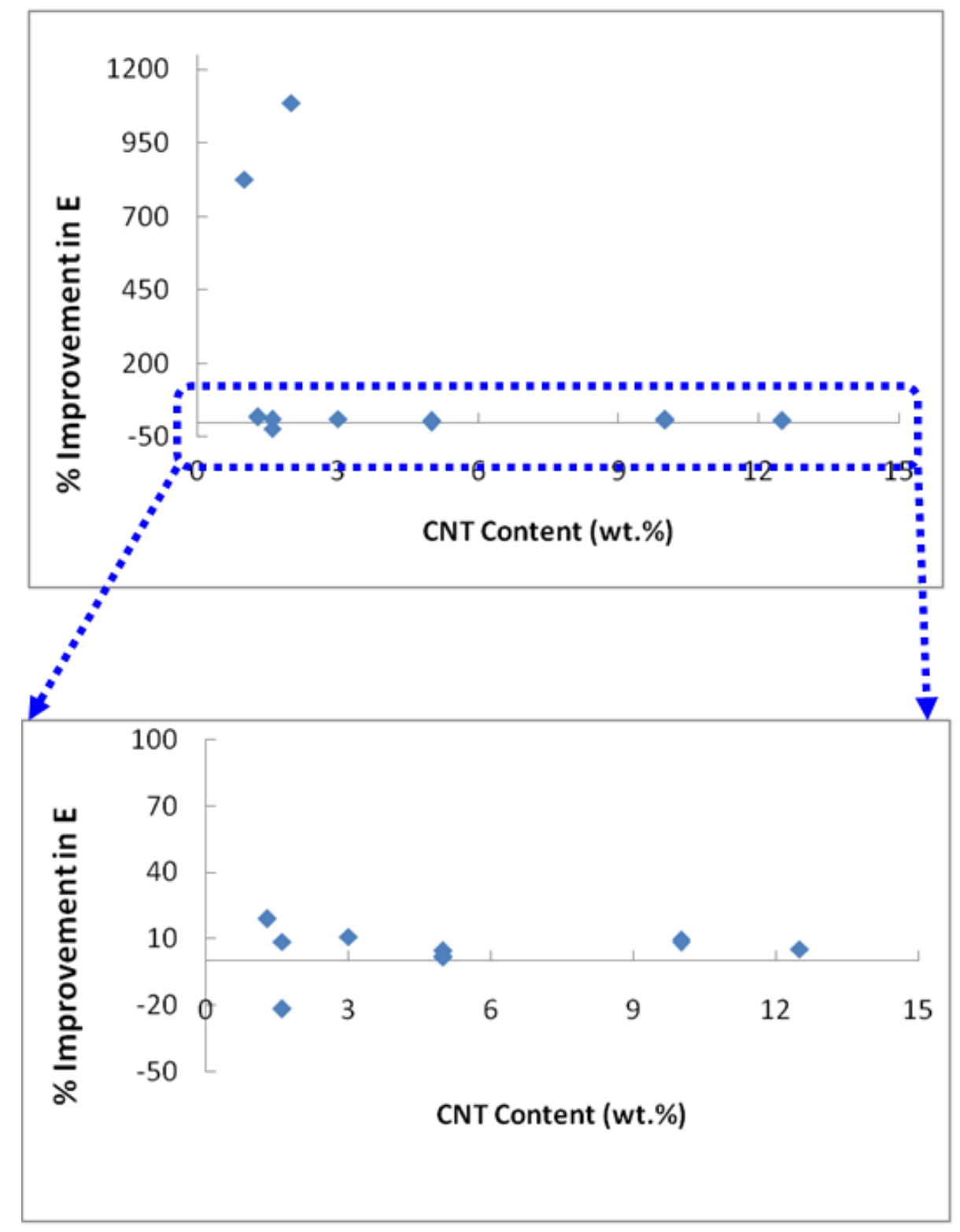

Figure 2.21: Percentage improvement in elastic modulus (E) as a function of CNT content in HA-CNT systems synthesized through different process routes.

Similar to $\mathrm{K}_{\mathrm{IC}}$, optimum sintering temperature is found to have a direct relationship with E of the SPS processed HA- 2 vol.\% (1.3 wt. \%) CNT structure [42]. 
The $\mathrm{E}$ of composite sintered at $1100^{\circ} \mathrm{C}$ shows the best improvement. Both lower and higher temperature of sintering causes reduction in $\mathrm{E}$ due to poor consolidation and excessive grain growth respectively. Lower E at higher consolidation temperature could also be due to possible damage in CNT structure [42].

The only study that has reported a very impressive increase in E with CNT addition is by Kaya for electrophoretically deposited HA coatings [14]. Addition of 1 and 2 wt. $\%$ CNT increases $\mathrm{E}$ of the composite coating by $827 \%$ and $1087 \%$ respectively, when compared with HA coating. No detailed explanation has been provided for such a huge increase in E. But, the E value reported for the HA coating is significantly low (15 $\mathrm{GPa}$ ), as compared to other studies, which report $\mathrm{E}$ of pure $\mathrm{HA}$ in 50-150 GPa [27] range. The study on electrophoretically deposited HA-CNT coatings did not provide details of the indentation experiments or load vs. displacement curves to better understand such an impressive improvement.

CNTs are found having significant effect on the improvement of elastic modulus (E) for HA-CNT composite, which has a direct positive influence on $\mathrm{K}_{\mathrm{IC}}$ of the structure. The degree of dispersion of CNTs and bonding at HA-CNT interface are two most critical factors that govern E of the composite system. Similar to fracture toughness, the elastic modulus improvement of HA-CNT system is most effective at $\leq 5$ wt.\% CNT content (Figure 2.21).

\subsubsection{Hardness}

Addition of CNT also influences the hardness for HA based composite. The higher stiffness of CNT provides a strengthening effect in HA matrix, which causes 
hindrance to plastic deformation. Instrumented indentation has always recorded lower penetration depth with similar load application in HA-CNT compared to HA [26-27, 39]. Significant decrease in pile up at the edge of nano-indents with increasing CNT content is a direct evidence of higher resistance to plastic deformation [27]. Grain boundary pinning and refinement of structure by CNT also helps in simultaneous increase in hardness and fracture toughness $[31,42,55]$.

Researchers have used Vicker's hardness measurement [31, 41] and instrumented nanoindentation technique $[8,14,16,19,26-27,39,42]$ to quantify hardness of HA-CNT composite. Figure 2.22 presents percentage improvement of $\mathrm{H}$ in HA-CNT system as a function of CNT content.

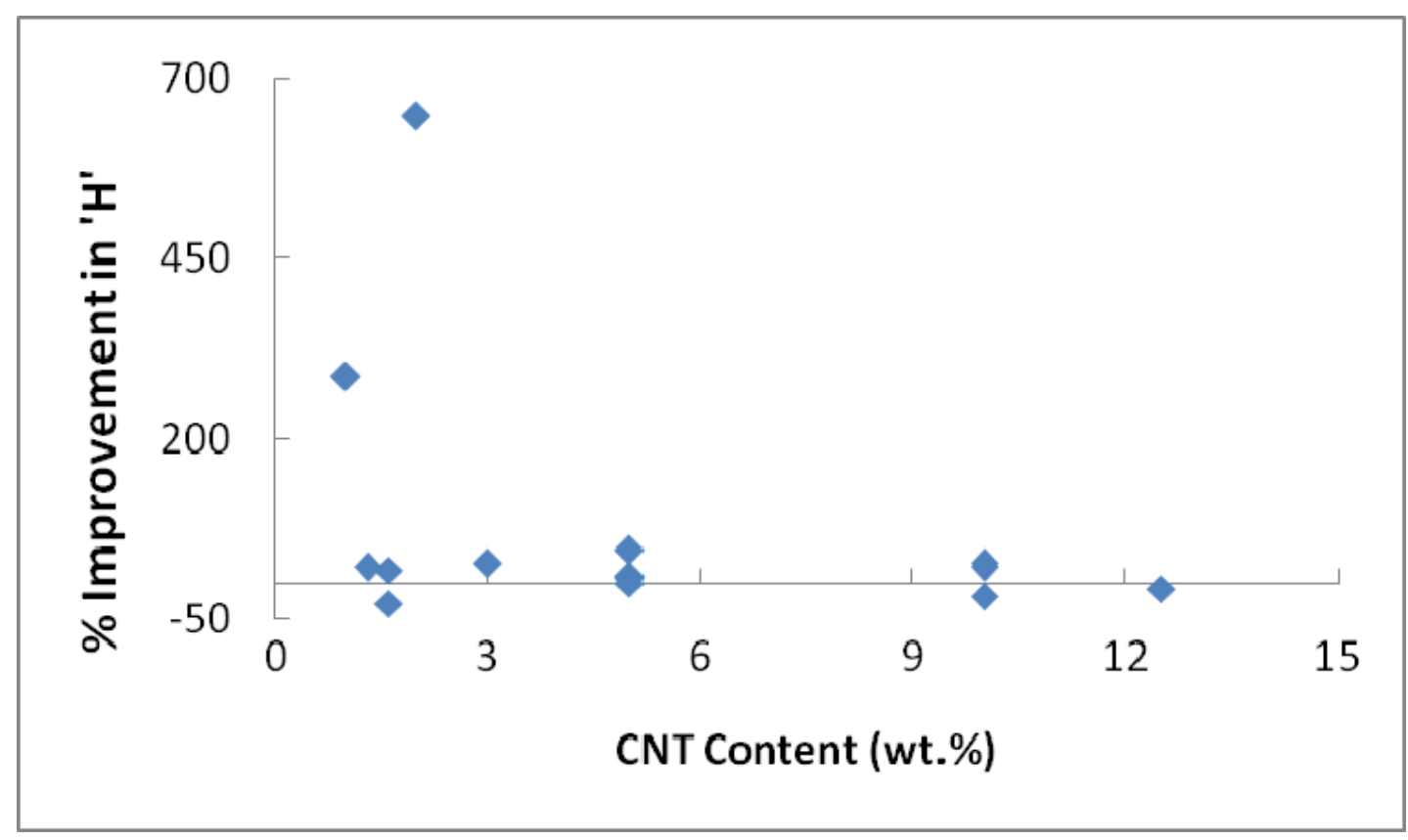

Figure 2.22: Percentage improvement in hardness $(H)$ as a function of CNT content in HA-CNT systems synthesized through different process routes. 
Based on this comparative data, addition of CNT beyond 4-5 wt.\% is found ineffective in terms of hardness improvement of the composite structure. Comparatively higher increase in hardness is noted for composite coatings processed by laser surface alloying [26-27] and electrophoretic deposition [14]. In case of laser surface alloyed HACNT coatings on Ti-6Al-4V substrate, formation of TiC ceramic adds to the overall hardness [27]. Electrophoretically deposited HA-CNT coating shows very high improvement in hardness of $287 \%$ and $647 \%$ with 1 and 2 wt.\% CNT addition respectively [14]. An $\mathrm{H}$ value of $36.44 \mathrm{GPa}$ with 2 wt.\% CNT addition has been reported, which is very high. The same study has also reported high E value (178 GPa) measured through nanoindentation study. No adequate explanation has been provided for such extraordinary improvement in the mechanical properties. Similar to elastic modulus, agglomeration and weak interface causes lower increase and/or decrease in hardness with CNT addition. Researchers have observed decrease (18-8 \%) in $\mathrm{H}$ with 10-12.5 wt.\% CNT addition, which is due to the agglomeration of CNT into SPS and hot pressed composites $[8,19]$. A $28 \%$ decrease in $\mathrm{H}$ with $1.6 \mathrm{wt} . \% \mathrm{CNT}$ addition to $\mathrm{HA}-\mathrm{Al}_{2} \mathrm{O}_{3}(18.4$ wt.\%) system is recorded by Kalmodia et.al, which has been attributed to the incomplete densification of the structure in presence of CNT [41]. Higher consolidation temperature $\left(1200^{\circ} \mathrm{C}\right)$ can also have adverse effect on hardness, due to possible damage and destruction of CNT structure, as reported by Xu et al. [42]. But the study by Sarkar et al. [31] has contradicted the findings by Xu et al.[42] in terms of hardness of SPS processed HA-CNT composite. The former one reports gradual increase in hardness with sintering temperature up to $1200^{\circ} \mathrm{C}$, in spite of grain coarsening at that temperature [31]. Though, no further explanation has been provided. 
Addition of CNT improves the hardness of HA impressively due to (i) strengthening of matrix and (ii) grain size refinement - both of which hinders plastic deformation. Agglomeration of CNT in HA matrix is detrimental to hardness improvement.

\subsubsection{Strength}

Strength of HA-CNT system is measured mostly in tensile mode using flexural or bending strength $[15,18,20,25,30,36,45-46]$ with only one study on compressive strength [1]. Bending strength has been measured for sintered $[20,25,36]$, hot pressed [1, $15,18,30,45]$ and SPS processed [46] free standing composite using three point $[18,46]$ or four point [30] bending tests. Strengthening of HA-CNT composite structure is governed by two major factors; (1) dispersion of CNTs; (2) bonding at HA-CNT interface. Homogeneous dispersion of CNTs helps in the uniform distribution of stress in the structure and hence, uniform strengthening. The agglomeration of CNTs causes stress concentration points and non-uniform distribution of stress. Moreover, interface of HA and agglomerated CNTs being weak act as source of failure under stress. Li and coworkers have shown broken and pulled out CNTs on the fracture surface of sintered composite, indicating that CNTs can share significant portion of load transmitted from the HA matrix $[20,25]$. They have reported $49 \%$ increase in bending strength with 2 wt.\% CNT addition and attributed to uniform dispersion of CVD grown CNTs on HA and good interfacial bonding. Other studies have also shown similar improvement in bending strength with 3-4 wt.\% CNT addition in HA [30, 45]. Meng et al. have presented the bending strength of hot pressed HA-CNT composite as a function of CNT content 
(Figure 2.23) [15]. This systematic study on optimization of CNT content shows a maximum bending strength achieved with 7 vol.\% (5 wt.\%) CNT. The bending strength of the composite decreases with larger CNT content, as CNT tends to agglomerate. The highest improvement in bending strength of HA-CNT (164\%) is reported by Li et al. with 3 wt. \% CNT addition [36]. But, the sintering condition for HA and HA-CNT being different, the properties cannot be compared directly.

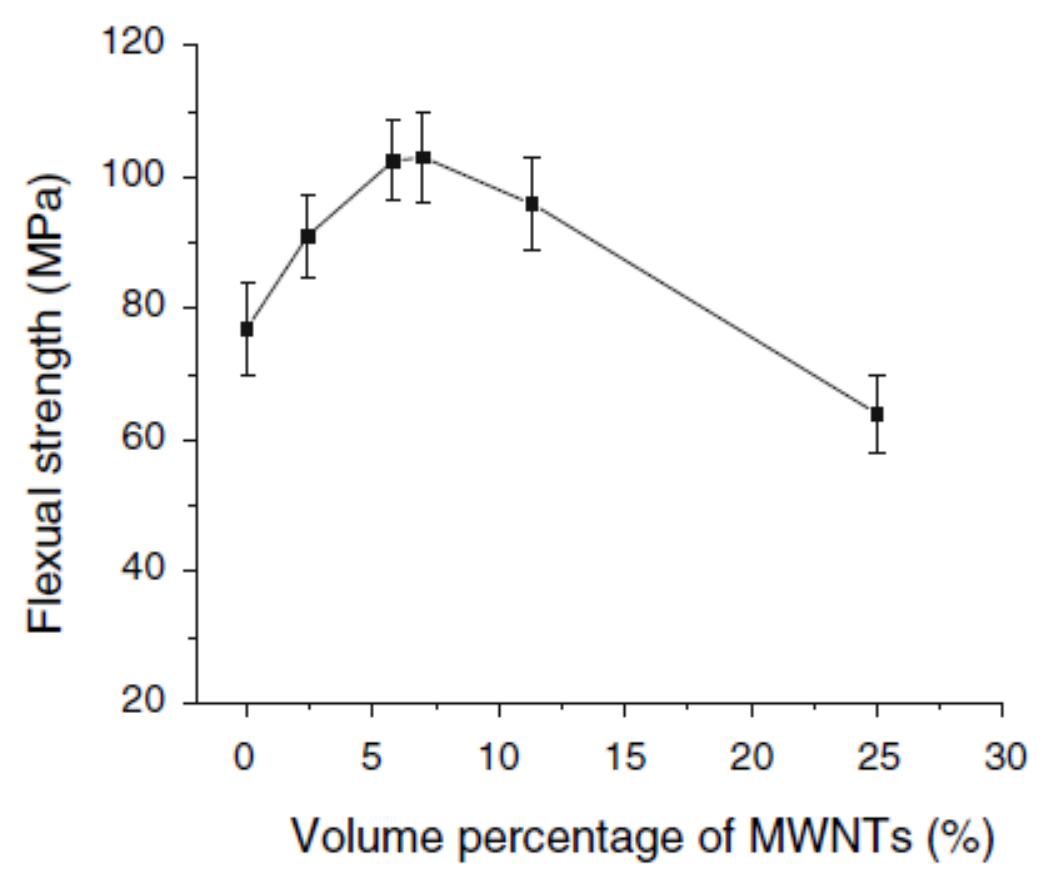

Figure 2.23: Change in the bending strength as a function of CNT content in a hot pressed HA-CNT system [15].

The only study that reports compressive strength of HA-CNT composite is by Zhao and Gao on hot pressed structure [1]. HA-2 wt.\% CNT possesses a compressive strength of $102 \mathrm{MPa}$, which is $61 \%$ improvement over HA. It was also concluded that chemically modified CNTs result in more effective bonding with HA and results in 23\% more compressive strength as compared to unmodified CNTs in HA-CNT composite. 
Reinforcement of CNTs in HA improves the strength of matrix, whether it is tensile (flexural) or compressive. Similar to other mechanical properties, the improvement in strength is also dependant on dispersion of CNT and bonding at HA-CNT interface.

\subsubsection{Tribological Properties of HA-CNT Composites}

Poor wear resistance of HA is one of the shortcomings for its application as orthopedic implant $[28,38,59,61,84]$. Poor fracture toughness causes low wear resistance of HA. Wear volume loss is a combined function of the elastic modulus, hardness and fracture toughness of ceramics. A well established model by Evans and Marshall for estimation of the wear volume in brittle ceramic shows dependence of the wear resistance on the mechanical properties as following [104]:

$$
V=P^{1.125} K_{I C}{ }^{-0.5} H^{-0.625}\left(\frac{E}{H}\right)^{0.8} S \ldots \ldots \ldots \text { (4) }
$$

where, $\mathrm{V}$ is the wear volume, $\mathrm{P}$-is the normal load, and $\mathrm{S}$ is the total travelling distance on wear track.

One of the main objectives for adding CNT in HA is to improve its wear resistance. Only three studies have reported the effect on CNT addition on the wear resistance of HA-CNT composite and coatings [28, 38-39]. These studies have investigated wear at macro-scale in physiological solution using pin-on-disk method [38] and at nano-scale length, in dry condition, by nano-scratches using nanoindenter [28, 39]. The outcomes of all the studies have merged in to a common agreement that CNT reinforcement improves the wear resistance of HA- in any environment (wet or dry) at macro through nano-scale lengths. 
Balani et al. have performed nano-scale wear study on plasma sprayed HA based coating reinforced with both 18.4 wt.\% aluminum oxide and 1.6 wt.\% CNTs [39]. The comparison of properties have been carried out for the two compositions having $H A+A l$ ${ }_{2} \mathrm{O}_{3}$ and $\mathrm{HA}+\mathrm{Al}_{2} \mathrm{O}_{3}+\mathrm{CNT}$, in order to separate out the reinforcing effect from CNT only. CNT reinforcement has not posed any significant effect on CoF of the coatings, as observed in figure 2.24 .

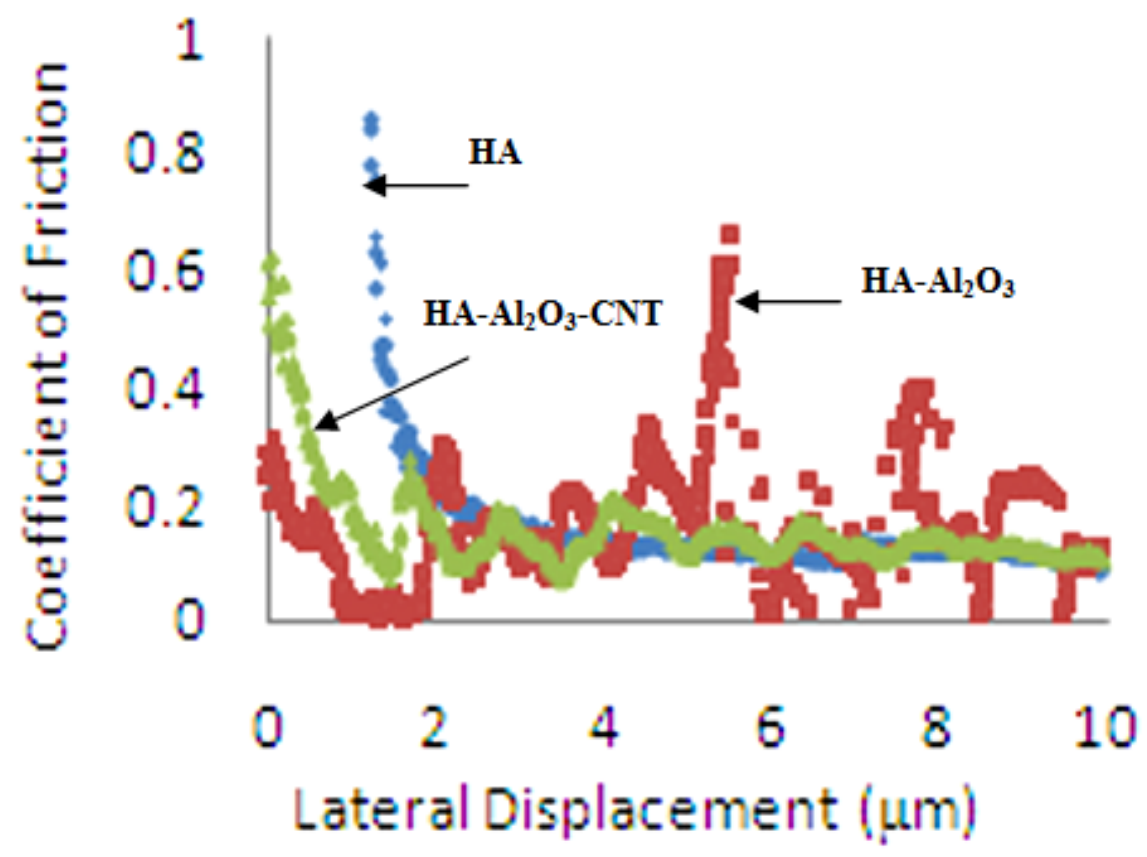

Figure 2.24: Variation in coefficient of friction for plasma sprayed $\mathrm{HA}, \mathrm{HA}-\mathrm{Al}_{2} \mathrm{O}_{3}$ and $\mathrm{HA}-\mathrm{Al}_{2} \mathrm{O}_{3}$-CNT coatings (the undulation in the profile is due to surface roughness and structural heterogeneity) [39].

Balani et al. have also studied the wear resistance of HA- 4 wt.\% CNT coating immersed in simulated body fluid (SBF) and concluded that CNT reinforcement improves the wear resistance [38]. Nano-scale wear study on laser surface alloyed HA(5-20 wt.\%) CNT coatings by Chen et al. reports lower depth of scratch, indicating 
increased wear resistance [28]. The increasing wear resistance in this case is attributed to the enhanced $\mathrm{K}_{\mathrm{IC}}$ as well as the increasing TiC phase content with CNT addition. The density and length of cracks on wear track decreases with increasing CNT content. The CoF also decreases with CNT content, probably due to graphene peeling and thus increased lubrication in HA-CNT wear track [28].

One important issue related to the wear of HA-CNT is the generation of CNT containing debris, which may have significance on its biocompatibility. The role of CNT containing wear debris on the biocompatibility is not yet addressed in the open literature.

\subsubsection{Biocompatibility of HA-CNT Composites}

HA-CNT composite systems are intended for orthopedic application in a living body. Hence, it is extremely important to assess their biocompatibility, by means of invitro and in-vivo studies, before suggesting their clinical use. HA is already a clinically proven biomaterial and in use as coating for metallic implants. But, the biocompatibility of CNT is still under debate [105]. Several studies have been carried out to study the cytotoxicity of carbon nanotubes since 2001 [105-114]. , The topic remains controversial till date with contradictory reports by different research groups [106, 108-110, 113]. For example, Cherukuri et al. have reported CNTs can be ingested by macrophages without producing any toxic effect [106]. But, Cheng et al. have reported frustrated/incomplete phagocytosis of CNT in macrophages, causing oxidative stress and cell death [109]. A recent study by Kagan et al. reveals ingestion and biodegradation of carbon nanotube by human macrophages and neutrophils without any inflammatory or toxic response [110]. Comprehensive reviews on this issue have agreed that the reported cytotoxic response of 
CNT is mostly not due to CNT itself, but is associated with the impurities and catalyst particles, degree of agglomeration, surface defects and also on the effect of cell culture medium, secondary chemicals, and $\mathrm{pH}$ values $[105,107,113,115]$. Thus, Ren et al. have suggested standardization of the assessment method to comment on the cytotoxic effect of carbon nanotube on the cells, organ, or whole organism [113]. While considering the application of HA-CNT composite in orthopedic implants, there is at least three major points in favor. Firstly, CNTs have cytotoxic effect mainly when they are suspended in fluid medium, while they appear nontoxic if immobilized to a matrix or to a culture dish [115]. Secondly, there are several reports of CNTs having positive response to bones and bone cells. Usui et al. have found that CNTs show good bone-tissue compatibility without any toxic effect [111]. CNTs, when implanted with collagen in a mouse skull, aids in accelerated bone growth [111]. Bone forming cells (osteoblasts and fibroblasts) show normal growth and proliferation on CNTs [116-119]. Figure 2.25 shows fibroblast cells grown on vertically aligned carbon nanotubes [116]. Recent studies have reported increased proliferation and adhesion of osteoblast cells on CNT surface, due to the selective absorption of proteins on the CNT surface from cell culture medium [120-121]. Osteoblast cells are reported to attach better on CNT surface than collagen, which is the main component of natural bone and widely used in orthopedic scaffolds [122]. Thirdly, if the CNTs are released in the blood stream along with the wear debris generated from the composite surface, they are either biodegraded completely by neutrophils and macrophages [110] or safely cleared out from the body by renal excretion route very quickly, without causing toxic effect in any organ $[108,123]$. Hence the use of CNT in HA based composite should not have negative impact on the biocompatibility. 

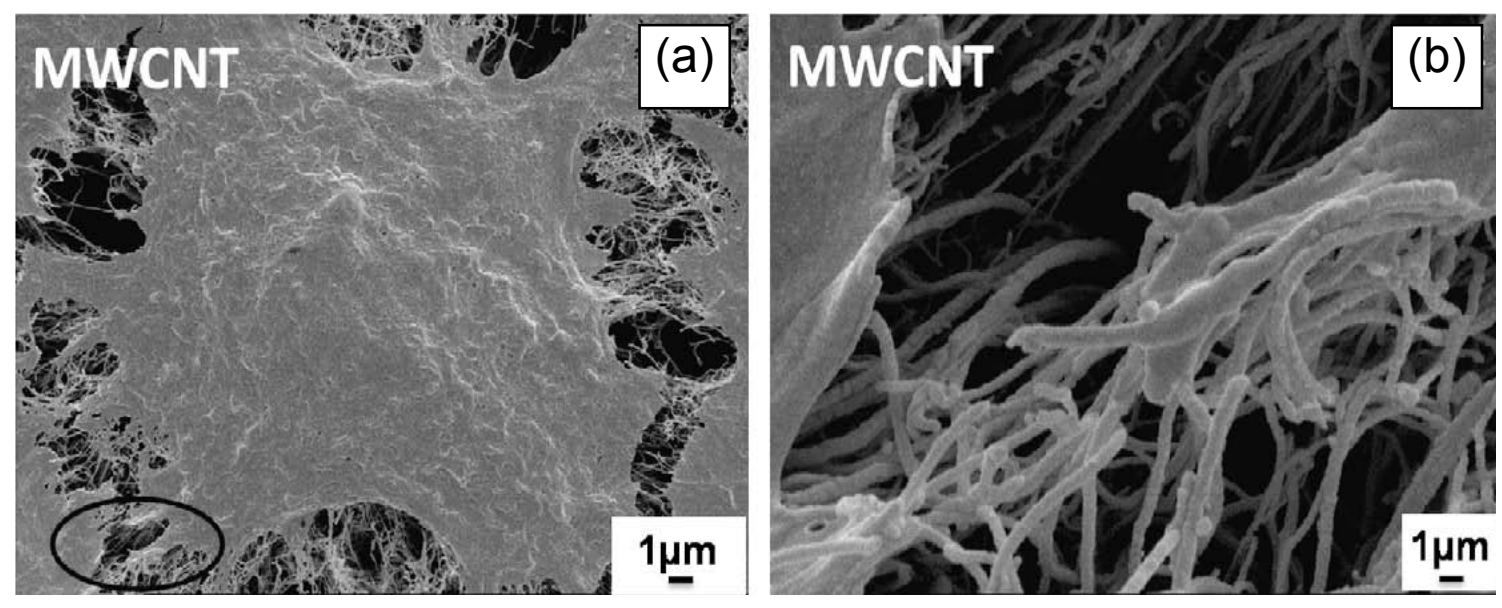

Figure 2.25: SEM images showing the adhesion of fibroblast on vertically-aligned MWCNT after 7 days [116].

The precipitation of HA on the CNT surface adds to the biocompatibility by offering better integration with the newly formed bone [37]. Several reports are available on apatite formation ability of CNT-surface, when immersed in simulated body fluid [124-129]. Still, a thorough investigation of the biocompatibility of HA-CNT composites, prepared by different techniques, is mandatory for the clinical application. Several research groups have carried out different types of in-vitro biocompatibility assessments for HA-CNT composites, whereas, only one report is available on in-vivo study [36]. Table 2.1 summarizes all the biocompatibility studies with the brief description of methodology, cells-lines, assessment techniques used and the major outcomes. The following subsections briefly presents the important outcomes of the biocompatibility studies with possible explanations of results and their significance in orthopedic biocompatibility of HA-CNT system. 
Table 2.1: Summary of biocompatibility studies on HA-CNT composite systems

\begin{tabular}{|c|c|c|c|c|}
\hline $\begin{array}{c}\text { HA-CNT } \\
\text { Composition/Processing }\end{array}$ & $\begin{array}{l}\text { Type of Bio- } \\
\text { study }\end{array}$ & Details of the Study & Major Findings & Reference \\
\hline $\begin{array}{l}3 \text { wt. \% CNT - Hot } \\
\text { Pressing }\end{array}$ & $\begin{array}{l}\text { Immersion in } \\
\mathrm{SBF}\end{array}$ & $\begin{array}{l}\text { - Immersed in SBF for 3, } 10 \\
\text { and } 17 \text { Days }\end{array}$ & $\begin{array}{l}\text { - Apatite growth rate is lower in } \\
\text { first few days - after wards } \\
\text { becomes higher - composite is } \\
\text { claimed to have high bioactivity }\end{array}$ & {$[45]$} \\
\hline $\begin{array}{l}4 \text { wt. } \% \text { CNT - Plasma } \\
\text { Spraying }\end{array}$ & $\begin{array}{l}\text { Cultured } \\
\text { osteoblast cells on } \\
\text { coatings -SEM }\end{array}$ & $\begin{array}{l}\text { - Human osteoblasts were } \\
\text { cultured on coatings for } 3 \\
\text { days } \\
\text { - Cells were fixed and } \\
\text { observed under SEM }\end{array}$ & $\begin{array}{l}\text { - Human osteoblasts grow on HA- } \\
\text { CNT surface - alongside CNTs } \\
\text { - HA can precipitate on CNT } \\
\text { surface }\end{array}$ & [37] \\
\hline $\begin{array}{l}3 \text { wt.\% CNT }- \text { Cold } \\
\text { Compaction and } \\
\text { Sintering }\end{array}$ & $\begin{array}{l}\text { In-vivo- } \\
\text { embedded in } \\
\text { striated mouse } \\
\text { muscle }\end{array}$ & $\begin{array}{l}\text { - The cylindrical composite } \\
\text { samples were embedded } \\
\text { into stripped mouse muscle } \\
\text { - The mice were sacrificed at } \\
\text { 1, 3, 5, } 7 \text { and } 14 \text { Days } \\
\text { - The tissues around were } \\
\text { observed under microscope } \\
\text { - Molecular biology } \\
\text { experiments on the tissues }\end{array}$ & $\begin{array}{l}\text { - Initially some immune activity is } \\
\text { observed - inflammatory cell } \\
\text { infiltration, blood vessel dilation, } \\
\text { lymphocyte infiltration and } \\
\text { monocyte production is observed } \\
\text { - At } 14 \text { days the inflammatory } \\
\text { reactions are reduced } \\
\text { - No necrosis of tissue occurs } \\
\text { - The nearby tissue remains normal } \\
\text { - Composite is found non-toxic }\end{array}$ & {$[36]$} \\
\hline $\begin{array}{l}20 \text { wt. \% CNT, } \\
\text { Electrophoretic } \\
\text { Deposition }\end{array}$ & $\begin{array}{l}\text { Osteoblast like } \\
\text { MG63 cell culture } \\
\text { on coating }\end{array}$ & $\begin{array}{l}\text { - Cells cultures on composite } \\
\text { coatings for } 3 \text { Days } \\
\text { - Fixed and observed in SEM }\end{array}$ & $\begin{array}{l}\text { - Cells grow and attach well on } \\
\text { HA-CNT surface }\end{array}$ & {$[33]$} \\
\hline
\end{tabular}




\begin{tabular}{|c|c|c|c|c|}
\hline $\begin{array}{l}2 \text { vol.\% CNT - Spark } \\
\text { Plasma Sintering }\end{array}$ & $\begin{array}{l}\text { Protein profile of } \\
\text { osteoblast } \\
\text { cultured on } \\
\text { composite surface } \\
\text { using iTRQ- } \\
\text { coupled 2D LC } \\
\text { MS/MS Analysis }\end{array}$ & $\begin{array}{l}\text { - Cells were cultured on till } \\
\text { they reached } 90 \% \\
\text { confluence } \\
\text { - Cells were lysed and } \\
\text { extracted protein was } \\
\text { quantified } \\
\text { - Antibodies were used to } \\
\text { detect different proteins } \\
\text { - Cells were also fixed for } \\
\text { SEM study }\end{array}$ & $\begin{array}{l}\text { - Most of the protein shows similar } \\
\text { expression on substrates. } \\
\text { - Upregulation of Feutin A is } \\
\text { observed in case of both HA and } \\
\text { HA-CNT - which is associated } \\
\text { with suppressed calcification. } \\
\text { - Relatively high EF2 level was } \\
\text { detected in HA-CNT than in HA } \\
\text { - which participate in the } \\
\text { elongation phase during protein } \\
\text { biosynthesis on the ribosome } \\
\text { - CNT slightly promote the protein } \\
\text { expression and cytoskeleton } \\
\text { protein distribution }\end{array}$ & {$[43]$} \\
\hline $\begin{array}{l}2 \text { vol.\% CNT - Spark } \\
\text { Plasma Sintering }\end{array}$ & $\begin{array}{l}\text { Protein profile of } \\
\text { osteoblast } \\
\text { cultured on } \\
\text { composite surface } \\
\text { using iTRQ- } \\
\text { coupled 2D LC } \\
\text { MS/MS Analysis }\end{array}$ & $\begin{array}{l}\text { - Cells were cultured on till } \\
\text { they reached } 90 \% \\
\text { confluence in } 4 \text { Days } \\
\text { - Cells were lysed and } \\
\text { extracted protein was } \\
\text { quantified } \\
\text { - Antibodies were used to } \\
\text { detect different proteins } \\
\text { - Cells were also fixed for } \\
\text { SEM study }\end{array}$ & $\begin{array}{l}\text { - Major cytoskeletal proteins } \\
\text { slightly decreased level on HA- } \\
\text { CNT than HA - denotes less } \\
\text { adhesion on surface } \\
\text { - For metabolic enzymes - related } \\
\text { to osteoblast proliferation - some } \\
\text { shows increased and some of the } \\
\text { decreased level on HA-CNT } \\
\text { compared to HA } \\
\text {-EF2 shows higher level on HA- } \\
\text { CNT - responsible for } \\
\text { accelerated } \mathrm{Ca}^{2+} \text { dissolution from } \\
\text { composite surface }\end{array}$ & {$[44]$} \\
\hline $\begin{array}{l}\text { HA- } 1 \text { wt. } \% \text { and } 3 \text { wt. } \% \\
\text { composite coating on Ti }\end{array}$ & $\begin{array}{l}\text { Cytotoxity, } \\
\text { Differentiation by } \\
\text { ALP activity and }\end{array}$ & $\begin{array}{l}\text { - SEM on cells fixed after } 5 \\
\text { hrs of incubation } \\
\text { - Cytotoxicity assessed }\end{array}$ & $\begin{array}{l}\text { - Cells on HA-CNT surface } \\
\text { spreads better and has longer } \\
\text { filopods than on HA and bare Ti }\end{array}$ & {$[16]$} \\
\hline
\end{tabular}




\begin{tabular}{|c|c|c|c|c|}
\hline - Aerosol Deposition & $\begin{array}{l}\text { SEM of pre- } \\
\text { osteoblast cells } \\
(\mathrm{MC} 3 \mathrm{~T} 3-\mathrm{E} 1)\end{array}$ & $\begin{array}{l}\text { evaluated using LDH Assay } \\
\text { after } 24 \text { hrs of incubation. } \\
\text { - Cell Differentiation } \\
\text { evaluated by measuring } \\
\text { ALP activity on cells } \\
\text { cultured on coatings for } 10 \\
\text { Days }\end{array}$ & $\begin{array}{l}\text { surface } \\
\text { - HA-CNT surface shows lower } \\
\text { cytotoxicity than HA and Bare Ti } \\
\text { - HA-CNT surface shows better } \\
\text { proliferation and significantly } \\
\text { higher ALP Activity than HA } \\
\text { and Ti }\end{array}$ & \\
\hline $\begin{array}{l}\mathrm{HA}-\mathrm{Al}_{2} \mathrm{O}_{3}-1.6 \mathrm{wt} . \% \\
\text { CNT composite coating } \\
\text { on Ti-Alloy - Plasma } \\
\text { Spraying }\end{array}$ & $\begin{array}{l}\text { Osteoblast } \\
\text { quantification and } \\
\text { SEM observation }\end{array}$ & $\begin{array}{l}\text { - Cells were fixed and } \\
\text { observed in SEM after } 3 \\
\text { Days of culture } \\
\text { - Cells were counted using } \\
\text { hemocytometer after 1, } 3 \\
\text { and } 7 \text { Days of culture }\end{array}$ & $\begin{array}{l}\text { - Morphology of fixed cells reveal } \\
\text { normal attachment on all the } \\
\text { coatings } \\
\text { - CNT reinforced composite shows } \\
\text { initially delayed but prolonged } \\
\text { and accelerated proliferation } \\
\text { afterwards. }\end{array}$ & {$[40]$} \\
\hline $\begin{array}{l}\text { HA-2 vol.\% CNT - } \\
\text { Spark Plasma Sintering }\end{array}$ & $\begin{array}{l}\text { Osteoblast } \\
\text { quantification and } \\
\text { SEM observation }\end{array}$ & $\begin{array}{l}\text { - Cells were fixed and } \\
\text { observed in SEM after } 1 \\
\text { Days of culture } \\
\text { - Cells were counted using } \\
\text { hemocytometer after } 2 \text { and } \\
4 \text { Days of culture } \\
\end{array}$ & $\begin{array}{l}\text { - Osteoblast cells flattened and } \\
\text { attached well on HA-CNT } \\
\text { Surface } \\
\text {-Number of cells was higher on } \\
\text { HA-CNT than on HA. }\end{array}$ & [42] \\
\hline $\begin{array}{l}\text { HA-CNT composite - } \\
\text { prepared by chemical } \\
\text { precipitation method - dip } \\
\text { coated from solution on } \\
\text { glassy carbon electrode - } \\
\text { hemoglobin deposited by } \\
\text { immersing in its } \\
\text { phosphate buffer solution }\end{array}$ & $\begin{array}{l}\text { Application as } \\
\text { biosensor and } \\
\text { bioelectrocatalyst }\end{array}$ & $\begin{array}{l}\text { - } \text { Biosensor capability } \\
\text { studied by amperometric } \\
\text { response to } \mathrm{H}_{2} \mathrm{O}_{2} \\
\text { - Electrocatalytic activity is } \\
\text { evaluated by } \\
\text { electrochemical reduction } \\
\text { of Trichloroacetic Acid }\end{array}$ & $\begin{array}{l}\text { - HA-CNT }-\mathrm{Hb} \text { shows fast } \\
\text { amperometric response } \\
\text { - Shows a good bioelectrocatalytic } \\
\text { activity }\end{array}$ & {$[11]$} \\
\hline
\end{tabular}




\begin{tabular}{|c|c|c|c|c|}
\hline $\begin{array}{l}\mathrm{HA}-\mathrm{ZrO}_{2}-3 \text { wt. } \% \text { CNT } \\
\text { - Hot Pressing }\end{array}$ & SBF Immersion & $\begin{array}{l}\text { - Immersion of composite in } \\
\text { SBF for } 10 \text { Days } \\
\text { - Apatite formed from SBF } \\
\text { observed using SEM and } \\
\text { EDS. }\end{array}$ & $\begin{array}{l}\text { - Needle like apatite crystal } \\
\text { precipitates on composite surface } \\
\text { - Thickness of apatite layer } \\
\text { increases with time } \\
\text { - The apatite growing process in } \\
\text { similar for HA and composite }\end{array}$ & {$[18]$} \\
\hline $\begin{array}{l}\text { HA-CNT - } \\
\text { Electrophoretic } \\
\text { Deposition }\end{array}$ & $\begin{array}{l}\text { MTT assay and } \\
\text { SEM observation } \\
\text { on mouse } \\
\text { Osteoblasts } \\
\text { (MC3T3-E1) }\end{array}$ & $\begin{array}{l}\text { - Cells were fixed and } \\
\text { observed in SEM after } 2 \\
\text { Days of culture } \\
\text { - MTT Assay was carried } \\
\text { out for proliferation } \\
\text { evaluation after } 2 \text { and } 4 \\
\text { Days of culture. }\end{array}$ & $\begin{array}{l}\text { - Osteoblast cells get flattened and } \\
\text { attached to substrate. } \\
\text { - Optical density of cells increases } \\
\text { with number of Days and is more } \\
\text { on HA-CNT surface than HA. }\end{array}$ & [12] \\
\hline $\begin{array}{l}\mathrm{HA}-\mathrm{Al}_{2} \mathrm{O}_{3}-1.6 \text { wt. } \% \\
\text { CNT - Spark Plasma } \\
\text { Sintering }\end{array}$ & $\begin{array}{l}\text { MTT assay and } \\
\text { SEM observation } \\
\text { on mouse } \\
\text { Fibroblasts } \\
\text { (L929) }\end{array}$ & $\begin{array}{l}\text { - Cells were fixed and } \\
\text { observed in SEM after } 3 \\
\text { Days of culture } \\
\text { - MTT Assay was carried } \\
\text { out for proliferation } \\
\text { evaluation after } 3 \text { and } 5 \\
\text { Days of culture. }\end{array}$ & $\begin{array}{l}\text { - Fibroblast viability on } \mathrm{HA}-\mathrm{Al}_{2} \mathrm{O}_{3} \\
\text { - } \mathrm{CNT} \text { surface remains similar to } \\
\mathrm{HA} \text { in } 3 \text { days, but decreases after } \\
5 \text { days. } \\
\text { - Fibroblast viability on } \mathrm{HA}-\mathrm{Al}_{2} \mathrm{O}_{3} \\
\text { is minimum for both } 3 \text { days and } 5 \\
\text { days }\end{array}$ & [41] \\
\hline $\begin{array}{l}\text { HA-2 wt. } \% \text { CNT (CVD } \\
\text { grown on HA) - Sintering }\end{array}$ & $\begin{array}{l}\text { Viability Study } \\
\text { by MTT Assay on } \\
\text { L929 mouse } \\
\text { fibroblasts }\end{array}$ & $\begin{array}{l}- \text { MTT assay was performed } \\
\text { after culturing the cells on } \\
\text { HA-CNT for } 2,24,48,72 \text {, } \\
96 \mathrm{hrs}\end{array}$ & $\begin{array}{l}\text { - The viability is higher the } 75 \% \\
\text { at } 2 \text { hrs and increases to } 100 \% \\
\text { later } \\
\text { - HA-CNT composite from CVD } \\
\text { grown CNT powder is non- } \\
\text { cytotoxic }\end{array}$ & {$[25]$} \\
\hline
\end{tabular}




\subsubsection{In-vitro Assessment of Biocompatibility}

In-vitro biocompatibility studies are generally carried out by growing bone cells (osteoblasts, fibroblasts) on HA-CNT composite surface or by SBF-immersion of the composite to study the apatite formation.

\section{Osteoblast/Fibroblast Proliferation and Viability}

The very first study on growing of osteoblast cells on plasma sprayed HA- 4 wt.\% CNT coating surface was carried out by Balani et al. [37]. SEM image of fixed osteoblast cells reveals good spreading on CNT reinforced HA surface.. Few other studies have also reported proliferation and attachment of osteoblast cells on HA- 20 wt. $\%$ CNT composite surface [33]. Presence of CNTs in HA is reported to improve bone cell proliferation [12, 16, 42, 44]. Viability assessment also shows increasing percentage of live cells in presence of CNT $[12,25,41]$. Liang et al. have reported increasing viability of fibroblasts with incubation time on HA-CNT surface up to $100 \%$ in 4 days [25]. Osteoblasts attach better on HA surface in the presence of CNT, as seen in figure 2.26 [16]. The cell on HA3 wt.\% CNT surface has formed several filipods to get attached after 5 hrs of seeding, whereas the cell on HA surface still maintains the globular shape and is yet to spread out (figure 2.26). Lee et al. have also reported active spreading of osteoblasts on HA-CNT surface with several filopodia protrusions [130]. Higher ALP (alkaline phosphatase) activity for osteoblasts on HA-CNT surface denotes increasing differentiation [16]. Several factors are claimed to cause better proliferation and viability of osteoblast on HA-CNT composite surface, viz. (1) attachment of proteins on the CNT surface from the cell culture medium [120-121]; (2) the special porous structure and high surface area of 
CNT [33]; (3) higher porosity content in HA-CNT composite [41] and (4) bioactive nature of $\mathrm{CNT}$ [41-42]. HA-18.4 wt.\% $\mathrm{Al}_{2} \mathrm{O}_{3}-1.6$ wt.\% $\mathrm{CNT}$ composites prepared by plasma spraying and SPS also shows similar trend of osteoblast proliferation and viability [40-41]. Minimum osteoblast proliferation and viability is noted for $\mathrm{HA}-20$ wt. $\% \mathrm{Al}_{2} \mathrm{O}_{3}$ surface and it increases with CNT addition. But both these composites show lesser proliferation and viability than $\mathrm{HA}$. It seems that bio-inertness of $\mathrm{Al}_{2} \mathrm{O}_{3}$ poses a negative effect on the osteoblast behavior. At the same time, addition of CNTs plays a positive role in reviving the proliferation of osteoblast on the composite surface. Increased roughness in the composite coating structure is also reported as a cause of delayed proliferation of osteoblast [40].
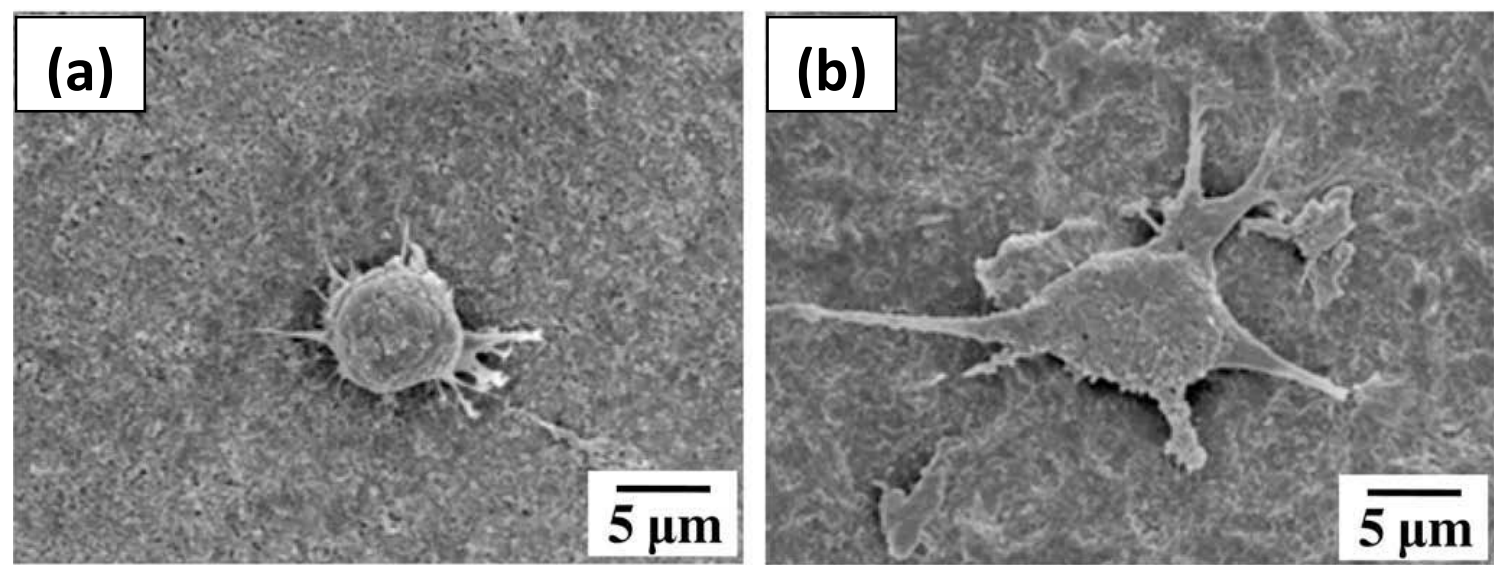

Figure 2.26: SEM micrographs of pre-osteoblast MC3T3-E1 cells attached to (a) $H A$ and (b) HA-CNT coatings for 5 hr [16].

\section{$\underline{\text { Protein Profile Study of Osteoblasts }}$}

$\mathrm{Ju}$ et al. have studied the profile of cytoskeletal proteins, metabolic enzymes, signaling proteins and cell growth proteins that regulate osteoblast cell growth and 
proliferation on HA and HA- 2 vol.\% (1.3 wt.\%) CNT surfaces [43-44]. Cytoskeletal proteins show higher expression on HA than HA-CNT surface, which means better osteoblast cell adhesion on HA. This observation contradicts the SEM image of fixed cells by Hahn et al. (figure 2.26) that shows better osteoblast attachment in HA- 3 wt.\% CNT surface [16]. Further studies are needed to resolve the contradiction between the direct visualization of cell growth and protein profile results. Metabolic enzymes involved in the cell proliferation show higher expression on HA-CNT surface, suggesting enhanced osteoblast proliferation in the presence of CNTs. This observation is supported by an increased cell density count on HA-CNT than HA [44]. Analysis of signaling and cell growth proteins show higher expression of EF-2 (elongation factor-2) on HA-CNT surface, which indicates accelerated dissolution of $\mathrm{Ca}^{2+}$ from surface. On the contrary, Narita et al. have shown CNTs inhibit osteoclast proliferation and reduce the apatite resorption [131]. Thus HA-CNT surface has the potential to make a balance for dissolution of calcium ion from the implant surface.

$\underline{\text { Immersion in Simulated Body Fluid }}$

Apatite precipitation ability of any surface during SBF immersion is a preliminary indicator of its capability to get integrated with new bone in-vivo. The suitability of SBF immersion test as an indicator of biocompatibility could be best justified through a statement made by Kokubo (the inventor of SBF) and Takadama, - "examination of apatite formation on a material in SBF is useful for predicting the in vivo bone bioactivity of a material, and the number of animals used in and the duration of animal experiments can be reduced remarkably by using this method" [132]. It is also well 
understood that the new bone integration on an orthopedic surface is closely related and governed by bone cell (osteoblast) attachment, proliferation and differentiation, which predicts the mineralization of apatite on it [133]. Thus, the behavior of osteoblast is also very important to determine biocompatibility of orthopedic surface along with the apatite formability.

Studies by different research groups have found CNT surfaces suitable for apatite precipitation [124-126]. Akasaka et al. have reported a 14 days incubation period for apatite precipitation on CNT surface when immersed in standard SBF [126]. Incubation time for apatite precipitation on CNT is found accelerated with carboxyl group functionalized CNT surfaces [124] and also with increasing ion concentration in a supersaturated SBF [126]. These studies indicate that presence of CNT on HA surface should not have a negative effect on its apatite formability. Reports are also available on apatite precipitation-ability of HA-CNT composite surfaces [18, 45, 129]. Meng et al. have shown gradual formation of thick and dense apatite layer on hot pressed HA-3 wt.\% CNT surfaces immersed in SBF up to 17 days $[18,45]$. Their observation reveals a slower initial precipitation rate in the presence of CNTs, which increases afterwards. Though the authors have not provided any explanation of such behavior, but the delay could be due to initial incubation period of 14 days for apatite precipitation on CNT surface, as reported by Akasaka et al. [126]. This delay in incubation is justified considering the dissimilar chemical and crystalline structure of the two species involved.

Several in-vitro biocompatibility studies for HA-CNT composite have revealed compatible and positive role of CNT towards proliferation, viability, attachment, differentiation and apatite formation in most of the cases. In-vitro studies carried out till 
date on HA-CNT systems can claim the presence of CNT in HA to be biocompatible; in fact advantageous in some aspects. Though, the only report on protein profile analysis of osteoblasts, cultures on HA-CNT surface, contradicts all other in-vitro studies in issues related to osteoblast attachment and calcium dissolution. This demands further followups on this issue to confirm the results. Moreover, the issue of wear debris related cytotoxicity is not addressed so far.

\subsubsection{In-vivo Assessment of Biocompatibility}

There is a single in-vivo study on HA- 3 wt.\% CNT system. [36]. Sintered HACNT composite was embedded in a striated rat muscle. The big white rats were sacrificed in $1,3,5,7$ and 14 days to assess the histocompatibility of HA-CNT composite. Figure 2.27 presents the pathological micrographs of the striated rat muscle after HA-CNT composite is embedded for different periods. The striated muscle after 1 day shows little inflammatory cells and lymphocyte infiltration. The inflammatory cell infiltration increases in day 3 with sign of blood vessel dilation. Both of these phenomena increase gradually on 5 and 7 days. But, after 14 days, the inflammatory cell infiltration decreases significantly and mild dilation is observed. The muscle tissues after 14 days look similar to natural undamaged tissue, which indicates recovery. This study presents the first indication of in-vivo non-toxicity for HA-CNT composite. However, this study was done on the muscle tissue. Further studies are needed by implanting HA-CNT composites in bones to establish their in-vivo biocompatibility. The analysis of outcomes from such invivo studies would be able to predict the suitability of HA-CNT for clinical use in orthopedic implants. 

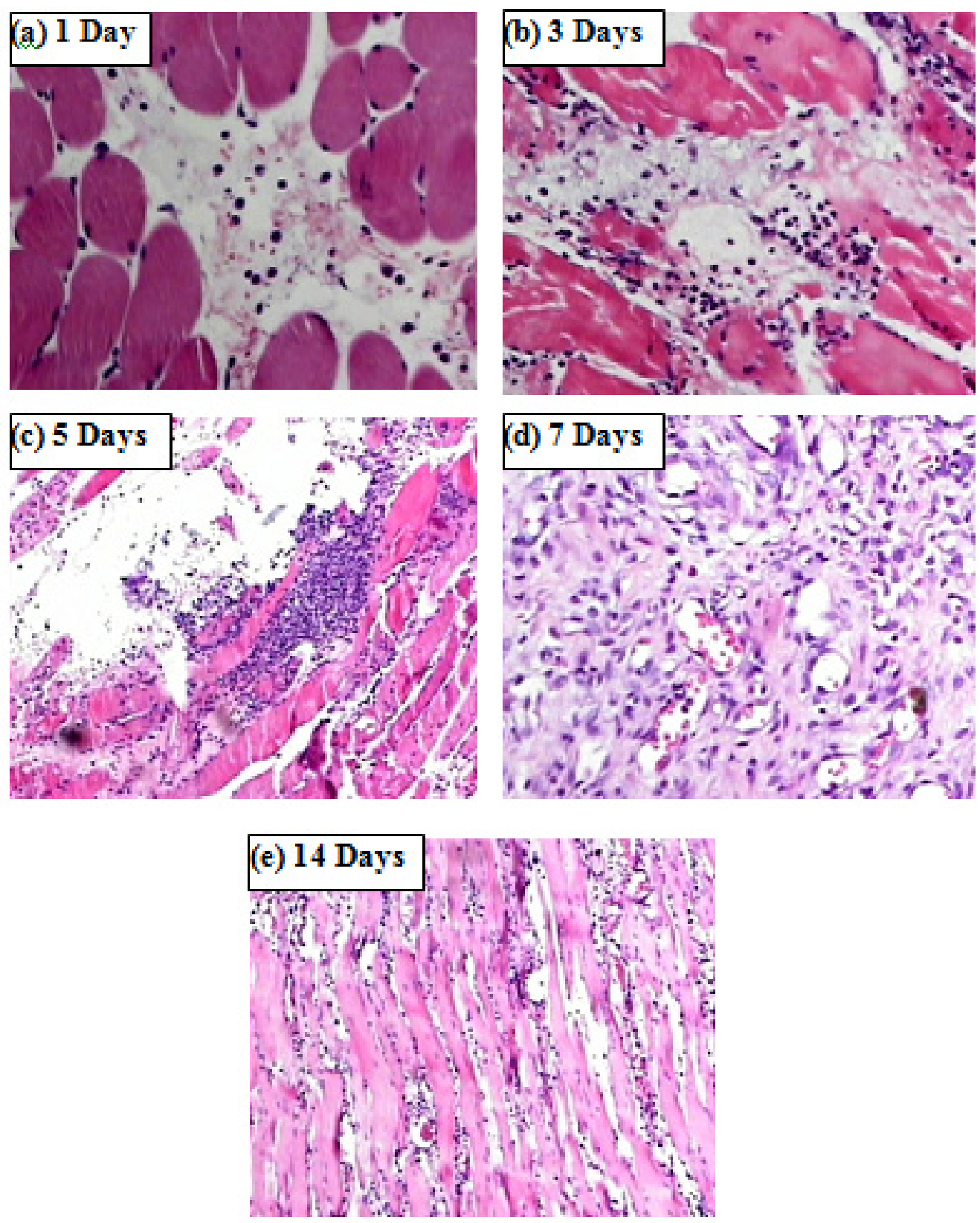

Figure 2.27: Pathological micrographs of striated mouse muscle after HA-CNT composite was embedded for (a) 1; (b) 3; (c) 5; (d) 7 and (e) 14 Days [36].

The review of literature indicates an increasing amount of the research interest in exploring HA-CNT system for orthopedic application. The effect of CNT reinforcement 
in strengthening of HA based composite system has been much in focus; though biocompatibility still remains a topic of debate. In this scenario, one of the two main objectives of this research was to explore the untouched areas related to orthopedic application of HA-CNT, both from mechanical and biocompatibility point of view. In addition, another objective of this study was to find a suitable alternative of CNT for this application, which is presented in details in the following section 2.2.

\subsection{Boron Nitride Nanotube: Alternative to Carbon Nanotube}

In this study, boron nitride nanotubes (BNNT) are chosen as an alternative to CNTs as reinforcement in HA for orthopedic applications. The main reasons for which BNNT was chosen are: (i) excellent elastic modulus (750-1200 GPa [134-135]), (ii) excellent tensile strength ( $>24 \mathrm{GPa}[136])$, similar range to CNT, and (iii) positive reports on its biocompatibility [137-141]. It is emphasized that no study existed on HA-BNNT composite before the work reported in this dissertation. The following subsections would elaborate on this novel material BNNT, its role as reinforcement to different composites, biocompatibility and the potential as reinforcement to HA in orthopedics as compared to CNT.

\subsubsection{What is Boron Nitride Nanotube?}

Boron nitride nanotubes (BNNT) are structural analogue of carbon nanotubes (CNT), formed by tubular shaped hexagonal boron nitride (h-BN) sheets (figure 2.30 [142]). BNNTs generally exist as multiwall structures. Single wall BNNT is rarely synthesized. It has been established that $\mathrm{B}$ and $\mathrm{N}$ atomic planes are slightly shifted in a 
direction perpendicular to the BNNT tube axis [143]. Owing to this and also due to the partially ionic character of $\mathrm{B}-\mathrm{N}$ bonding in a $\mathrm{BN}$ layer, BNNT becomes stabilized in double or multi-layered nanotube morphologies owing to strong "lip-lip" interactions between adjacent layers [144].BN tubular shells are separated by an average inter-shell distance of $0.33-0.34 \mathrm{~nm}$ (similar to MWNTs), which is a characteristic of $\mathrm{d}_{0002}$ spacing in a hexagonal $\mathrm{BN}$. Individual tubes within the bundles interact through weak van der Waals forces and are packed in a honeycomb-like array in cross-section. B and $\mathrm{N}$ atomic planes in h-BN sheet are slightly shifted in a direction perpendicular to the tube axis. Partially ionic character of B-N bonding in a BN layered material may stabilize doubleor multi-layered morphologies owing to strong "lip-lip" interactions between adjacent layers. These interactions favor the placement of B atoms strictly above or below $\mathrm{N}$ atom [144-145]. Table 2.2 presents some of the unique mechanical, thermal and electrical properties of BNNT.
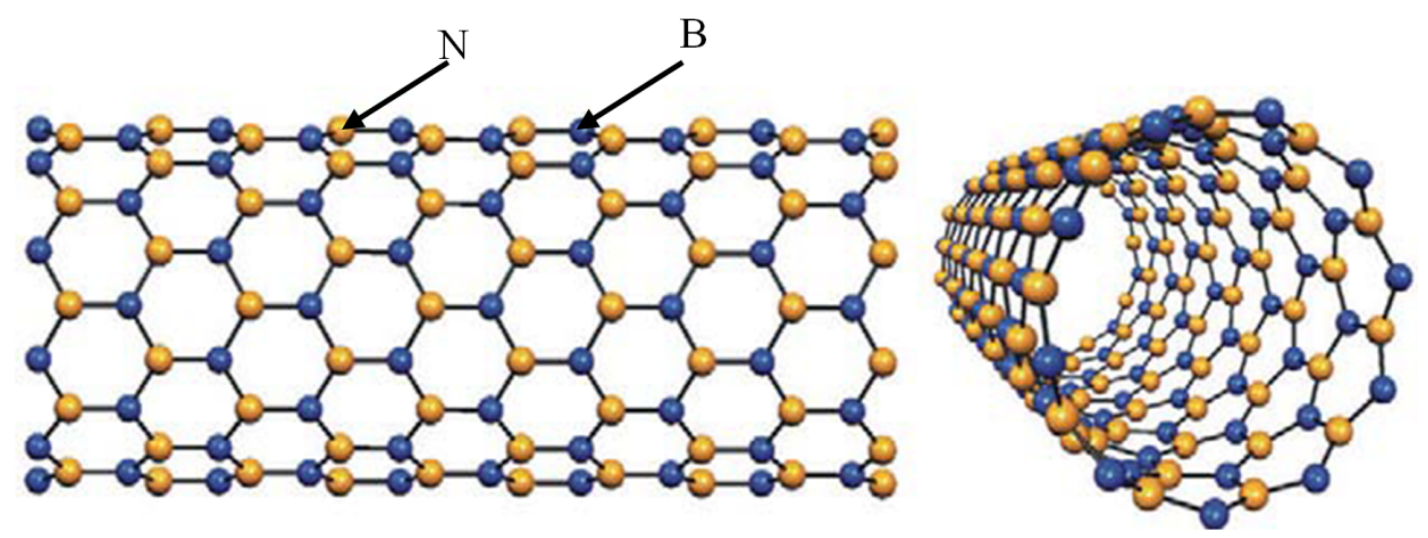

Figure 2.28: Schematic of rolled h-BN sheet forming single walled boron nitride nanotube [142].

Table 2.2: Mechanical and physical properties of BNNT in comparison to CNT 


\begin{tabular}{|l|l|l|}
\hline \multicolumn{1}{|c|}{ Property } & \multicolumn{1}{c|}{ BNNT } & \multicolumn{1}{c|}{ CNT } \\
\hline Elastic Modulus (GPa) & $750-1200[146-147]$ & $270-950 \mathrm{GPa}[148]$ \\
\hline Tensile Strength (GPa) & $>24[149]$ & $11-63 \mathrm{GPa}[148]$ \\
\hline $\begin{array}{l}\text { Specific Heat Capacity } \\
\text { (at 300K), J kg }{ }^{-1} \mathrm{~K}^{-}\end{array}$ & $\sim 1000[150]$ & $\sim 480[87]$ \\
\hline $\begin{array}{l}\text { Thermal Conductivity } \\
\text { at RT, W m } \mathrm{K}^{-1}\end{array}$ & $200-300[151-152]$ & $3000[153]$ \\
\hline $\begin{array}{l}\text { Electrical Conductivity, } \\
\text { S.cm }{ }^{-1}\end{array}$ & $3.3 \times 10^{-3}[154]$ & $1850[155]$ \\
\hline Band Gap (eV) & $5-6[144]$ & $0.2-2 \mathrm{eV} \mathrm{[156]}$ \\
\hline $\begin{array}{l}\text { Oxidation start } \\
\text { temperature (K) }\end{array}$ & $1223[144]$ & $773[144]$ \\
\hline
\end{tabular}

A

pplications of BNNTs have not been explored as vastly as CNTs. With increasing interest in BNNT and its composites, several potential applications are being proposed. The insulating nature of BNNT could be taken advantage for electrically insulated nanocables with embedded metallic or semiconducting nanowires. Such cables may be utilized in downsized electrical devices and complex multi-cable circuits, where each cable should perform independently, without current leakage between them [144]. Carbon doped BNNTs are suitable for field emitters with better environmental stability [144]. BNNTs possess piezoelectric characteristics which could be used in precision piezoelectric devices to measure or apply force at high resolution [157]. BNNT also has bright prospect for non-linear optical and optoelectronic applications. BNNTs may be ideal 
candidates for optical devices working in the UV regime [158]. Gas adsorption ability of BNNT may also be used for hydrogen storage and thus offering solution to current environmental pollution [159]. Apart from the above listed applications, BNNT is gaining popularity as reinforcement in polymer and ceramic matrix composites due to its excellent mechanical and thermal properties.

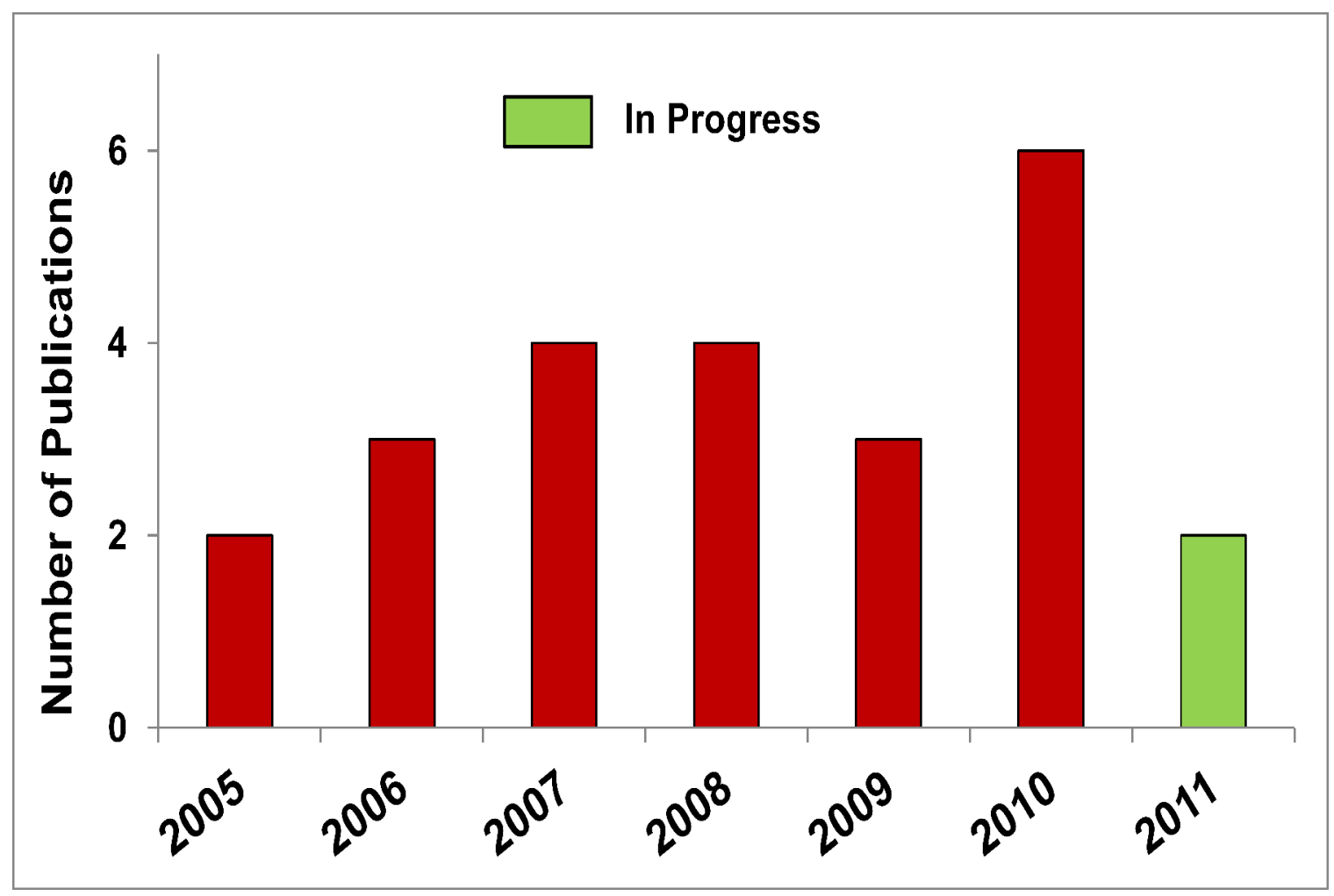

Figure 2.29: Year-wise publication plot for BNNT reinforced composites (source: scopus.com).

Figure 2.29 presents year wise publication plot for BNNT reinforced composites, which reveals its early stage of development. But, the plot shows a trend of growing 
research interest in the field, which would establish BNNT as a potential reinforcement to various material systems (polymer/metal/ceramic) in near future.

\subsubsection{Boron Nitride Nanotube Reinforced Composites}

Randomly and aligned BNNTs reinforced in polymer matrix improves the thermal, mechanical and optical properties of polymers [151-152, 160-162]. Only one study is available on ceramic $\left(\mathrm{Al}_{2} \mathrm{O}_{3}\right.$ and $\left.\mathrm{Si}_{3} \mathrm{~N}_{4}\right)$ based BNNT composites [163]. BNNT introduces high temperature superplasticity in the ceramics by controlled dynamic grain growth and energy absorption mechanism. The hardness of the ceramic-BNNT composite is reported to increase, but not the elastic modulus [163]. The use of BNNT as reinforcement to biodegradable polymer scaffold in orthopedic application has also been established by few recent studies [139]. In the case of biodegradable scaffold, BNNT reinforcement has improved the mechanical strength (109\%) and elastic modulus (1370\%) of the polymer and simultaneously enhanced the osteoblast proliferation and differentiation [139]. Table 2.3 presents a comprehensive summary of the studies on BNNT reinforced composites, which shows the role of BNNT in the property enhancement. However, the role of BNNT as a mechanical reinforcement in orthopedics has not been explored except by our research group [139]. 
Table 2.3: Summary of BNNT reinforced composite systems

\begin{tabular}{|c|c|c|c|c|}
\hline Matrix & $\begin{array}{c}\text { BNNT } \\
\text { Content }\end{array}$ & Composite Properties & $\begin{array}{c}\text { Other Observation/ Potential } \\
\text { Application }\end{array}$ & References \\
\hline $\mathrm{Al}_{2} \mathrm{O}_{3}$ and $\mathrm{Si}_{3} \mathrm{~N}_{4}$ & 0.5 wt. $\%$ & $\begin{array}{l}\underline{\mathrm{Al}}_{2} \underline{\mathrm{O}}_{3}-\mathrm{BNNT} \\
\text { - Hardness increases by } 18 \% \\
\text { - Elastic modulus decreases by } 5 \% \\
\underline{\mathrm{Si}}_{3} \underline{\mathrm{N}}_{4}-\text { BNNT } \\
\text { - Hardness decreases by } 12 \% \\
\text { - Elastic modulus decreases by } 17 \%\end{array}$ & $\begin{array}{l}\text { Composites show high } \\
\text { temperature superplastic } \\
\text { deformation capability }\end{array}$ & [163] \\
\hline AlN & $\begin{array}{l}1-10 \\
\text { vol. } \%\end{array}$ & $\begin{array}{l}\text { - Thermal conductivity increases } \\
\text { with sintering temperature }\end{array}$ & $\begin{array}{c}\text { Chance of damage to nanotubes at } \\
\text { sintering temperature as high as } \\
1700^{\circ} \mathrm{C}\end{array}$ & {$[164]$} \\
\hline Polyaniline & $\begin{array}{l}\text { Not } \\
\text { mentioned }\end{array}$ & $\begin{array}{l}\text { - Optical properties may be } \\
\text { interesting }\end{array}$ & $\begin{array}{c}\text { Strong interaction between } \\
\text { polymer and BNNT, polymer } \\
\text { becomes more ordered }\end{array}$ & {$[160]$} \\
\hline Polystyrene & 1 wt. $\%$ & $\begin{array}{l}\text { - Elastic modulus increases by } 21 \% \\
\text { - Increasing resistance to oxidation } \\
\text { - Lower glass transition temperature }\end{array}$ & $\begin{array}{l}\text { Composite film shows good } \\
\text { transparency, }\end{array}$ & {$[161]$} \\
\hline $\begin{array}{l}\text { Polymethyl } \\
\text { methacrylate }\end{array}$ & 1 wt. $\%$ & $\begin{array}{l}\text { - Elastic modulus increases by } 19 \% \\
\text { - Thermal conductivity increases by } \\
3 \text { times } \\
\text { - Composite shows high electrical } \\
\text { breakover voltage }\end{array}$ & $\begin{array}{c}\text { Promising nanofiller for } \\
\text { improvement in mechanical and } \\
\text { thermal conductivity of polymer, } \\
\text { while maintaining electrical } \\
\text { insulation }\end{array}$ & {$[165]$} \\
\hline $\begin{array}{l}\text { Co-polymer of } \\
\text { Vinylidene chloride } \\
\text { and acrylonitrile }\end{array}$ & $\begin{array}{l}0.5-1.5 \\
\text { wt. } \%\end{array}$ & $\begin{array}{l}\text { - Shows excellence transparency } \\
\text { - Better barrier properties } \\
\text { - Better thermal stability }\end{array}$ & $\begin{array}{l}\text { Application in packaging of } \\
\text { organic photovoltaic devices }\end{array}$ & [162] \\
\hline $\begin{array}{l}\text { Polymethyl } \\
\text { methacrylate, } \\
\text { polystyrene, }\end{array}$ & $\begin{array}{c}18-37 \\
\text { wt. } \%\end{array}$ & $\begin{array}{l}\text { - Increase in thermal conductivity } \\
\text { by } 20 \text { times }\end{array}$ & $\begin{array}{c}\text { Promising nanofiller for } \\
\text { improvement in thermal } \\
\text { conductivity of polymer, lowering }\end{array}$ & {$[151]$} \\
\hline
\end{tabular}




\begin{tabular}{|c|c|c|c|c|}
\hline $\begin{array}{l}\text { polyvinyl butyral, } \\
\text { polyethylene vinyl } \\
\text { alcohol } \\
\end{array}$ & & $\begin{array}{l}\text { - Lowering in Coeff. of thermal } \\
\text { expansion by } 11 \text { times } \\
\text { - Good hardness }\end{array}$ & $\begin{array}{l}\text { coeff. of thermal expansion, while } \\
\text { maintaining electrical insulation }\end{array}$ & \\
\hline $\begin{array}{l}\text { Polyvinyl formal, } \\
\text { Polyvinyl alcohol }\end{array}$ & 3 wt. $\%$ & $\begin{array}{l}\text { - } 270 \% \text { increase in thermal } \\
\text { conductivity }\end{array}$ & $\begin{array}{l}\text { Catechin modified BNNT surface } \\
\text { leads to stronger interfacial } \\
\text { reaction with polymer }\end{array}$ & {$[166]$} \\
\hline Polyvinyl Alcohol & $<5$ vol. $\%$ & $\begin{array}{l}\text { - Up to } 137 \% \text { increase in thermal } \\
\text { conductivity depending on } \\
\text { alignment of BNNT in polymer } \\
\text { matrix }\end{array}$ & $\begin{array}{l}\text { Alignment of BNNT in matrix has } \\
\text { significant effect on properties of } \\
\text { the composite }\end{array}$ & [152] \\
\hline $\begin{array}{l}\text { Low molecular } \\
\text { weight gelator }\end{array}$ & $\begin{array}{l}0-2.5 \\
\text { wt. } \%\end{array}$ & $\begin{array}{l}\text { - Bulk modulus, yield stress and } \\
\text { thermal conductivity of the } \\
\text { composite increases }\end{array}$ & $\begin{array}{l}\text { BNNT causes densely packed } \\
\text { fibrous network of gelator } \\
\text { molecules, increases sol-gel } \\
\text { transition temperature }\end{array}$ & [167] \\
\hline $\begin{array}{l}\text { Polylactide- } \\
\text { polycaprolactone } \\
\text { copolymer }\end{array}$ & $0-5$ wt. $\%$ & $\begin{array}{l}\text { - } 1370 \% \text { improvement in elastic } \\
\text { modulus } \\
\text { - } 109 \% \text { increase in tensile strength }\end{array}$ & $\begin{array}{l}\text { Osteoblast proliferation, viability } \\
\text { and differentiation shows positive } \\
\text { effect on presence of BNNT - } \\
\text { suitable composite for orthopedic } \\
\text { scaffold }\end{array}$ & [139] \\
\hline Epoxy & $5 \mathrm{wt} . \%$ & $\begin{array}{l}\text { - } 69 \% \text { improvement in thermal } \\
\text { conductivity }\end{array}$ & $\begin{array}{l}\text { Promising nanofiller for } \\
\text { improvement in thermal } \\
\text { conductivity of polymer, while } \\
\text { maintaining electrical insulation }\end{array}$ & [168] \\
\hline $\begin{array}{l}\text { Barium calcium } \\
\text { aluminosilicate glass }\end{array}$ & 4 wt.\% & $\begin{array}{l}\text { - Strength increases by } 90 \% \\
\text { - Fracture Toughness increases by } \\
35 \%\end{array}$ & $\begin{array}{l}\text { To be used in Solid Oxide Fuel } \\
\text { Cell (SOFC) sealing }\end{array}$ & [169-170] \\
\hline
\end{tabular}




\subsubsection{Biocompatibility of Boron Nitride Nanotube}

BNNT is new to the field of biomaterials and biomedicine. It has been studied for drug delivery system for last couple of years [140,171]. The cytotoxicity studies on BNNT have not reported any negative effect on different cell types [137-141]. Our research group has found encouraging results on osteoblast proliferation, viability and differentiation in the presence of BNNTs [139]. Gene expression studies have indicated that BNNT influence the accelerated differentiation of osteoblast cells [139].BNNTs are also found highly internalized by mouse myoblast (muscle) cells (C2C12), with neither adversely affecting its viability nor significantly interfering with myotube formation 138]. Chen et. al. [141] have shown BNNTs to be non-cytotoxic to human embryonic kidney cells [HEK-293] and reported that BNNTs do not inhibit cell proliferation even after 4-days. Ciofani et al. [137] demonstrated good cytocompatibility and cellular uptake of polyethyleneimine (PEI)-coated BNNTs in a human neuroblastoma cell line (SHSY5Y). In addition, BNNTs also favor attachment of protein on their surface and thus, are potentially suitable for nanobiological applications [172]. All these studies indicate safe and a bright future of BNNTs in bio-application.

\subsubsection{Predicted Advantages of Boron Nitride Nanotube over Carbon Nanotube in HA for Orthopedics}

BNNT is chosen as an alternative to CNT considering its mechanical properties, viz., excellent elastic modulus (750-1200 GPa [134-135]), strength (>24 GPa [136]), which are comparable to CNT. In addition, BNNTs are more flexible and elastic and can 
withstand heavy deformation without having damage in the structure [144]. A recent study has shown BNNT possess higher fracture strain than CNT [173]. These properties are attractive when BNNTs are used as reinforcement for strengthening of ceramic/polymer matrix. BNNTs also show higher chemical stability than CNTs in oxidative atmosphere with the oxidation starting temperature of $1223 \mathrm{~K}$ as compared to $773 \mathrm{~K}$ of CNT [144]. This chemical inertness of BNNT remains an added advantage for high temperature processing of BNNT reinforced ceramic composites at oxidative atmosphere. There has been no adverse report on biocompatibility of BNNT whereas biocompatibility of CNT is yet being debated. Thus BNNT offer a potential alternative to CNT as reinforcement to HA.

\subsection{Scope of Improvement of Nanotube Reinforced Hydroxyapatite Composites for Orthopedics}

The review of literature indicates an increasing amount of the research in exploring HA-CNT system for orthopedic application. The effect of CNT reinforcement in strengthening of HA based composite system has been much in focus; though biocompatibility still remains a topic of debate. Based on the critical analysis of the state of the art, following specific research areas have been selected in the present research to push the boundaries of the existing knowledge.

- Uniform dispersion of CNTs in HA is very important for improved mechanical and tribological performances. Spray drying is a promising method for preparing composite powders, especially for plasma spraying. This research has adopted 
spray drying route for the preparation of HA-CNT composite powder for effective dispersion of CNTs in HA at the powder stage.

- Plasma spraying is the FDA approved process for synthesizing coating on orthopedic implants. Current research would explore plasma spraying for synthesizing HA-CNT composite coatings on implant alloy (Ti-6Al-4V) surface. The aim is to improve the fracture toughness, elastic modulus and tribological behavior of the coating.

- $\quad$ Spark plasma sintering is an excellent method to consolidate free standing HACNT composites. But, the reports available till date on SPS of HA-CNT do not present an in-depth analysis on the microstructural development, which governs its performance. Another important aspect is the dissociation of HA during sintering, which is detrimental to its orthopedic application. The present study utilizes a modified sintering cycle to suppress the dissociation of HA. A detailed analysis on the evolution of microstructure in SPS processed HA-CNT is also presented. This analysis would help in understanding the role of CNT in the microstructural development of the composite during fast heating under pressure.

- Knowledge about HA-CNT interface is vital in determining the mechanical and tribological performance of the composite. No information is available about HACNT interface. An initiative is taken to understand the nature of bonding at HACNT interface with an estimation of the interfacial fracture strength. 
- Biological fate of the CNT containing HA wear debris is of great concern, considering in-vivo implantation of the composite. No report is available which addresses this concern. Understanding the wear behavior of HA-CNT composite and the fate of wear generated debris under biological conditions is an interesting research area which is yet to be explored. The present study evaluates the cytotoxicity of CNT containing wear debris with related cell lines (osteoblasts and macrophages).

- In-vitro studies, performed by culturing bone cells on HA-CNT surface are required to establish its biocompatibility in orthopedics. In-vitro assessment of osteoblast viability, proliferation kinetics, differentiation and mineralization (calcification), reveals a comprehensive understanding on suitability of HA-CNT surface for orthopedics.

- $\quad$ The only available in-vivo study of HA-CNT composite is for implantation in muscle tissue and for only 2 weeks [36]. Implantation in bone tissue and for longer period is required for thorough assessment of in-vivo biocompatibility to establish the application of HA-CNT in orthopedic implants. This dissertation proposes and includes results from implantation of HA-CNT coated bio-implant in rat model (the implantation related surgery and characterization is carried out by our collaborator at University of Strasbourg, France).

- Osseointegration is very crucial for orthopedic surfaces. The adhesion of neobone on HA-CNT surface is an important factor for implantation, which will 
determine the life-time of the implants. Poor adhesion of neo-bone on implant surface can cause delamination. This issue is mainly addressed till date by evaluating apatite formability of HA-CNT surfaces when immersed in SBF. But, the adhesion of bone cells on implant surface plays an important role in osseointegration. Because, the biological cascade of proliferation, differentiation and mineralization of anchorage dependent bone cells are governed by initial adhesion on implant surface. The smooth operation of this biological cascade ultimately leads to neo-bone integration on the implant surface. Thus, quantifying adhesion of bone cells on differently processed HA-CNT surfaces is vital to understand their in-vivo performance. The current research developed a novel technique for the quantification of cellular adhesion on substrate up to a single cell level. The role of CNT on the adhesion of bone cells on HA based surface is also analyzed.

- Large difference in the elastic modulus at implant-bone interface can cause differential strain during loading, leading to fracture. Thus, evaluation of elastic modulus and stress distribution/gradient at bone-implant interface is very important to evaluate its in-vivo mechanical health. The presence of CNTs in HA coating on implant surface can modify the elastic modulus profile at implant-bone interface. Hence, studies are necessary to analyze such role of nanotubes. No such information is available in the literature for HA and HA-CNT coated implants. The present study determines the gradient of elastic modulus through the boneimplant interface using the retrieved implants from in-vivo studies. The aim of 
this study is to understand the difference in elastic modulus gradient across the interface for HA-coated, HA-CNT coated and uncoated implants.

As mentioned earlier, the cytotoxicity of CNTs is still under debate. Thus, the present research intends to evaluate BNNT (reinforcement to HA) as an alternative to CNT in orthopedic application. The evaluation should include synthesis of HA-BNNT composite, characterization of its mechanical properties (fracture toughness, elastic modulus and hardness), tribological behavior and biocompatibility. Following research tasks were carried out to investigate HABNNT composite.

- Wet chemistry method is proposed to synthesize HA-BNNT composite powder.

- Free-standing HA-BNNT composites were processing by SPS technique. Synthesis of HA-BNNT coating by plasma spraying was not possible due to unavailability of BNNTs in large amount. It must be noted that plasma spraying requires a large amount of powder feedstock due to volumetric nature of the industrial scale powder feeder.

- The mechanical properties (fracture toughness, elastic modulus and hardness)of HA-BNNT composite are investigated. The nature and strength of interfacial bonding between HA and BNNT is also elucidated.

- Role of BNNT in tribological behavior of HA based composite is studied. Since BNNT is being proposed for the first time for orthopedic application, its 
cytotoxicity needs to be evaluated. . Cytotoxicity of bare BNNTs is evaluated for the two main cell lineages related to the bone: (i) the bone forming cells (osteoblasts) and (ii) the phagocytic cells (macrophages).

- Biocompatibility of HA-BNNT composite surface is also studied using invitro methods through osteoblast viability and proliferation.

- Osseointegration in the presence of BNNTs is evaluated by the apatite formability of BNNTs when immersed in simulated body fluid.

Successful completion of the above listed tasks and understanding of the outcomes would ensure a step forward towards clinical application of nanotube reinforced HA composites in orthopedics.

\section{$2.4 \quad$ References}

1. L. Zhao, L. Gao. Novel in situ synthesis of MWNTs-hydroxyapatite composites.2004, Carbon, Vol. 42, pp. 423-460.

2. M. Omori, A. Okubo, M. Otsubo, T. Hashida, K. Tohji. Consolidation of multiwalled carbon nanotube and hydroxyapatite coating by spark plasma system (SPS).2004, Key Eng. Mater., Vols. 254-256, pp. 395-398.

3. Q. Wei, X.P. yang, G.Q. Chen, J.T. Tang, X.L. Deng. Ultrasonic assisted synthesis of nanohydroxyapatite and MWNT/hydroxyapatite composites. 2005, New Carbon Mater., Vol. 20, pp. 164-170.

4. C. Keally, M. Elcombe, A.V. Riessen, B. Ben-Nissan. Development of carbon nanotube-reinforced hydroxyapatite bioceramics. 2006, Physica B, Vols. 385-386, pp. 496-498.

5. C. Keally, B. Ben-Nissan, A.V. Riessen, M. Elcombe. Development of carbon nanotube reinforced hydroxyapatite bioceramics. 2006, Key Eng. Mater., Vols. 309-311, pp. 597-602. 
6. C. Keally, M. Elcombe, A.V. Riessen. Microstrain in hydroxyapatite carbon nanotube composites. 2008, J. Synchrotron Radiation, Vol. 15, pp. 86-90.

7. A.A. White, A.H. Windle, I.A. Kinloch, S.M. Best. Preparation and properties of carbon nanotube reinforced hydroxyapatite. 2008, Key Eng. Mater., Vol. 361-363, pp. 419-422.

8. C.S. Keally, B.A. Latella, A.V. Reissen, M.M. Elcombe, B. Ben-Nissan. Micro and nano-indentation of a hydroxyapatite-carbon nanotube composite.2008, J. Nanosci. Nanotech., Vol. 8, pp. 3936-3941.

9. Z. Lu, K. Sun, S. ren, X. Li. Synthesis and characterization of multi-walled carbon nanotube/hydroxyapatite composite.2007, J. Chinese Ceram.Soc., Vol. 35, pp. 687689.

10. Z.H. Lu, K.N. Sun, D.M. Zhao, A.M. Li, X.N. Sun. Preparation and characterization of hydroxyapatite/carbon nanotubes composites. 2007, J. Synthetic Crystals, Vol. 36, pp. 728-731.

11. H.Y. Zhao, X.X. Xu, J.X. Zhang, W. Zheng, Y.F. Zheng. Carbon nanotubehydroxyapatite-hemoglobin nanocomposites with high bioelectrocaytalytic activity. 2010, Bioelectrochemistry, Vol. 78, pp. 124-129.

12. Y. Bai, M.P. Neupane, I.S. Park, M.H. Lee, T.S. Bae, F. Watari, M. Uo. Electrophoretic deposition of carbon nanotubes-hydroxyapatite nanocomposites on titanium substrate.2010, Mater. Sci. Eng. C, Vol. 30, pp. 1043-1049.

13. C. Kaya, I. Singh, A.R. Boccaccini. Multi-walled carbon nanotube-reinforced hydroxyapatite layers on Ti6Al4V medical implants by electrophoretic deposition (EPD). 2008, Adv. Mater. Eng., Vol. 10, pp. 131-138.

14. C.Kaya. Electrophoretic deposition of carbon nanotube-reinforced hydroxyapatite bioactive layers on Ti-6Al-4V alloys for biomedical applications. 2008, Ceram. Int., Vol. 34, pp. 1834-1847.

15. Y.H. Meng, C.Y. Tang, C.P. Tsui, D.Z. Chen. Fabrication and characterization of needle-like nano-HA and HA/MWNT composites.2008, J. Mater. Sci.: Mater. Med., Vol. 19, pp. 75-81.

16. B.D. Hahn, J.M. Lee, D.S. Park, J.J. Choi, J. Ryu, W.H. Yoon, B.K. lee, D.S. Shin, H.E. Kim. Mechanical and in vitro biological performances of hydroxyapatitecarbon nanotube composite coatings deposited on Ti by aerosol deposition. 2009, Acta Biomater., Vol. 5, pp. 3205-3214.

17. C. Kaya, F. Kaya, J. Cho, J.A. Roether, A.R. Boccaccini. Carbon nanotubereinforced hydroxyapatite coatings on metallic implants using electrophoretic deposition. 2009, Key Eng. Mater., Vol. 412, pp. 93-97. 
18. Y.H. Meng, C.Y. Tang, C.P. Tsui, P.S. Uskokovic. Fabrication and Characterization of HA-ZrO2-MWCNT ceramic composites.2010, J. Compos. Mater., Vol. 44, pp. 871-882.

19. J. Zhu, S.C. Tjong, X.Q. Li. Spark plasma sintered hydroxyapatite/multiwalled carbon nanotube composites with preferred crystal orientation. 2010, Adv. Eng. Mater., Vol. 12 (11), pp. 1161-1165.

20. H. Li, N. Zhao, Y. Liu, C. Liang, C. Shi, X. Du, J. Li. Fabrication and properties of carbon nanotubes reinforced Fe/hydroxyapatite composites by in situ chemical vapor deposition. 2008, Composites: Part A, Vol. 39, pp. 1128-1132.

21. X. Lu, H. Wang, S. Xia, J.X. Wang, J. Weng. In situ growth of carbon nanotubes in hydroxyapatite matrix by chemical vapor deposition.2009, Adv. Mater. Res., Vols. 79-82, pp. 1671-1674.

22. H. Li, L. Wang, C. Liiang, Z. Wang, W. Zhao. Dispersion of carbon nanotubes in hydroxyapatite powder by in situ chemical vapor deposition.2010, Mater. Sci. Eng. B, Vol. 166, pp. 19-23.

23. C. He, N. Zhao, C. Shi, X. Du, J. Li, H. Li, Q. Cui. An Approach to Obtaining Homogeneously Dispersed Carbon Nanotubes in Al Powders for Preparing Reinforced Al-Matrix Composites. 2007, Adv. Mater., Vol. 19, pp. 1128-1132.

24. A.K. Keshri, J. Huang, V. Singh, W.B. Choi, S. Seal, A. Agarwal. Synthesis of aluminum oxide coating with carbon nanotube reinforcement produced by chemical vapor deposition for improved fracture and wear resistance. 2010, Carbon, Vol. 48, pp. 431-442.

25. C. Liang, H. Li, L. Wang, X. Chen, W. Zhao. Investigation of the cytotoxicity of carbon nanotubes using hydroxyapatite as a nano-matrix towards mouse fibroblast cells.2010, Mater. Lett., Vol. 124, pp. 21-24.

26. Y. Chen, C. Gan, T. Zhang, G. Yu, P. Bai, A. Kaplan. Laser-surface-alloyed carbon nanotubes reinforced hydroxyapatite composite coatings. 2005, Appl. Phys. Lett., Vol. 86, p. 251905.

27. Y. Chen, Y.Q. Zhang, T.H. Zhang, C.H. Gan, C.Y. Zheng, G. Yu. Carbon nanotube reinforced hydroxyapatite composite coatings produced through laser surface alloying. 2006, Carbon, Vol. 44, pp. 37-45.

28. Y. Chen, T.H. Zhang, C.H. Gan, G. Yu. Wear studies of hydroxyapatite composite coating reinforced by carbon nanotubes. 2007, Carbon, Vol. 45, pp. 998-1004.

29. K.N. Sun, A.M. Li, Y.S. Yin, Z.Y. Yu. Study on the fabrication of hydroxyapatite and carbon nanotube composites. 2004, Chinese J. Biomed. Eng., Vol. 23, pp. 573578. 
30. S. Koyayashi, W. Kawai. Development of carbon nanofiber reinforced hydroxyapatite with enhanced mechanical properties.2007, Composites: Part A, Vol. 38, pp. 114-123.

31. S.K. Sarkar, M.H. Youn, I.H. Oh, B.T. Lee. Fabrication of CNT reinforced HAp composites by spark plasma sintering.2007, Mater. Sci. Forum, Vols. 534-536, pp. 893-896.

32. T. Shokuhfar, A. Makradi, E. Titus, G. Cabral, S. Ahzi, A.C.M. Sousa, S. Belouettar, J. Gracio. Prediction of the mechanical properties of hydroxyapatite/polymethyl methacrylate/carbon nanotube nanocomposite.2008, J. Nanosci. Nanotech., Vol. 8, pp. 4279-4284.

33. C. Lin, H. Han, F. Zhang, A. Li. 2008, Electrophoretic deposition of HA/MWNTs composite coating for biomedical applications. J. Mater Sci.: Mater. Med., Vol. 19, pp. 2569-2574.

34. I. Singh, C. Kaya, M.S.P. Shaffer, B.C. Thomas, A.R. Boccaccini. Bioactive ceramic coatings containing carbon nanotubes on metallic substrates by electrophoretic deposition.2006, J. Mater. Sci., Vol. 41, pp. 8144-8151.

35. B. Zhang, C.T. Kwok, F.T. Cheng, H.C. Man. Fabrication of nano-structured HA/CNT coatings on Ti6Al4V by electrophoretic deposition for biomedical applications.2010, Vols. Nanoelectronics Conference (INEC), 2010 3rd International, Hongkong, IEEE, ISBN-978-1-4244-3543-2, pp. 813-814, p. DOI: 10.1109/INEC.2010.5425191 .

36. A. Li, K. Sun, W. Dong, D. Zhao. Mechanical properties, microstructure and histocompatibility of MWCNTs/HAp biocomposites.2007, Mater. Lett., Vol. 61, pp. 1839-1844.

37. K.Balani, R. Anderson, T. Laha, M. Andara, J. Tercero, E. Crumpler, A. Agarwal. Plasma-sprayed carbon nanotube reinforced hydroxyapatite coatings and their interaction with human osteoblasts in vitro.2007, Biomaterials, Vol. 28, pp. 618624.

38. K. Balani, Y. Chen, S.P. Harimkar, N.B. Dahotre, A. Agarwal. Tribological behavior of plasma-sprayed carbon nanotube-reinforced hydroxyapatite coating in physiological solution.2007, Acta Biomater., Vol. 3, pp. 944-951.

39. K. Balani, D. Lahiri, A.K. Keshri, S.R. Bakshi, J.E. Tercero, A. Agarwal. The nanoscratch behavior of biocompatible hydroxyapatite reinforced with aluminum oxide and carbon nantube.2009, J. Mineral Met. Mater. Soc., Vol. 61, pp. 63-66.

40. J.E. Tercero, S. Namin, D. Lahiri, K. Balani, N. Tsoukias, A. Agarwal. Effect of carbon nanotube and aluminum oxide addition on plasma-sprayed hydroxyapatite 
coating's mechanical properties and biocompatibility.2009, Mater. Sci. Eng. C, Vol. 29, pp. 2195-2202.

41. S. Kalmodia, S. Goenka, T. Laha, D. Lahiri, B. Basu, K. Balani. Microstructure, mechanical properties, and in vitro biocompatibility of spark plasma sintered hydroxyapatite-aluminum oxide-carbon nanotube composite. 2010, Vol. 30, pp. 1162-1169.

42. J.L. Xu, K.A. Khor, J.J. Sui, W.N. Chen. Preparation and characterization of a novel hydroxyapatite/carbon nanotubes composite and its interaction with osteoblast-like cells. 2009, Mater. Sci. Eng. C, Vol. 29, pp. 44-49.

43. J.L. Xu, K.A. Khor, J. J. Sui, W.N. Chen. Investigation of multiwalled carbon nanotube modified hydroxyapatite on human osteoblast cell line using iTRAQ petromics technology. 2008, Key Eng. Mater., Vols. 361-363, pp. 1047-1050.

44. J. Xu, K.A. Khor, J. Sui, J. Zhang, T.L. Tan, W.N. Chen. Comparative petromics profile of osteoblasts cultured on dissimilar hydroxyapatite biomaterials: An iTRAQ-coupled 2-D LC-MS/MS analysis.2008, Petromics, Vol. 8, pp. 4249-4258.

45. Y. H. Meng, C.Y. tang, C.P. Tsui. Fabrication and characterization of $H A / C N T$ bioceramics.2006, Mater. Sci. Forum, Vols. 532-533, pp. 201-204.

46. M. Tanaka, T. Onoki, M. Omori, A. Okobu, T. Hashida. Mechanical Properties of Carbon Nanotubes / Hydroxyapatite Composites Prepared by Spark Plasma Sintering.2006, The second International Conference on Flow Dynamics, Sendai, Japan, 16-18 November 2005, AIP Conference Proceedings, Vol. 832, pp. 430-432.

47. K. Teshima, S.H. Lee, M. Sakuri, Y. Kameno, K. Yubuta, T. Suzuki, T. Shishido, M. Endo, S. Oishi. Well-Formed One-Dimensional Hydroxyapatite Crystals Grown by an Environmentally Friendly Flux Method.2009, Crystal Growth and Design, Vol. 9, pp. 2937-2940.

48. A.A. White, I.A. Kinloch, A.H. Windle, S.M. Best. Optimization of the sintering atmosphere for high-density hydroxyapatite -carbon nanotube composites.2010, J. Royal Soc. Interface, Vol. 7, pp. S529-S539.

49. A. Li, K. Sun, Y. Yin, Z. Yu, Z. Lu, J. Bi. Mechanical properties and microstructure of hydroxyapatite/carbon nano-tube composite.2004, Acta Mater. Compos. Sinica, Vol. 21, pp. 98-102.

50. K. Lu. Sintering of nanoceramics.2008, International Materials Review, Vol. 53, pp. 21-37.

51. R. Chaim, M. levin, A. Shlayer, C. Estournes. Sintering and densification of nanocrystalline ceramic oxide powders: A review.2008, Adv. Appl. Ceram., Vol. 107, pp. 159-169. 
52. A.V. Ragulya. Consolidation of ceramic nanopowders. 2008, Adv. Appl. Ceram., Vol. 107, pp. 118-134.

53. E.A. Olevsky, S. Kandukuri, L. Froyen. Consolidation enhancement in spark plasma sintering: Impact of high heating rates.2007, J. Appl. Phys., Vol. 102, p. 114913.

54. Z.A. Munir, U.A. Tamburini, M. Ohyanagi. The effect of electric field and pressure on the synthesis and consolidation of materials: A review of the spark plasma sintering method. 2006, J. Mater. Sci., Vol. 41, pp. 763-777.

55. J. wang, L.L. Shaw. Nanocrystalline hydroxyapatite with simultaneous enhancements in hardness and toughness.2009, Biomaterials, Vol. 30, pp. 65656572.

56. J.L. Xu, K.A. Khor, R. Kumar. Physicochemical differences after densifying radio frequency plasma sprayed hydroxyapatite powders using spark plasma and conventional sintering techniques. 2007, Mater. Sci. Eng. A, Vol. 457, pp. 24-32.

57. S. Li, H. Izui, M. Okano. Densification, microstructure and behavior of hydroxyapatite ceramics sintered by using spark plasma sintering.2008, J. Eng. Mater. Tech., Vol. 130, p. 031012.

58. Y.W. Gu, N.H. Loha, K.A. Khor, S.B. Tor, P. Cheang. Spark plasma sintering of hydroxyapatite powders.23, 2002, Biomaterials, pp. 37-43.

59. Y.W. Gu, K.A. Khor, P. Cheang. Bone-like apatite layer formation on hydroxyapatite prepared by spark plasma sintering (SPS).2004, Biomaterials, Vol. 25, pp. 4127-4134.

60. H. Li, K.A. Khor, V. Chow, P. Cheang. Nanostructural characteristics, mechanical properties, and osteoblast response of spark plasma sintered hydroxyapatite. 2007 , J. Biomed. Mater. Res. A, Vol. 82, pp. 296-303.

61. L.G. Yu, K.A. Khor, H. Li, P. Cheang. Effect of spark plasma sintering on microstructure and in vitro behavior of plasma sprayed HA coatings.2003, Biomaterials, Vol. 24, pp. 2695-2705.

62. K. Yang, J. He, J.B. Reppert, M.J. Skove, T.M. Tritt, A.M. Rao. Inter-tube bonding, graphene formation and anisotropic transport properties in spark plasma sintered multi-wall carbon nanotube arrays.2010, Carbon, Vol. 48, pp. 756-762.

63. S.R. Bakshi, V. Singh, D.G. McCartney, S. Seal, A. Agarwal. Deformation and damage mechanisms of multiwalled carbon nanotubes under high-velocity impact.2008, Scripta Mater., Vol. 59, pp. 499-502. 
64. S. Pramanik, A.K. Agarwal, K.N. Rai. Chronology of Total Hip Joint Replacement and Materials Development.2005, Trends Biomater. Artif. Organs, Vol. 19, pp. 1526.

65. C.W. Drapers, C.A. Ewing. Laser surface alloying: A bibliography.1984, J. Mater. Sci., Vol. 19, pp. 3815-3825.

66. A.A. Campbell. Bioceramics for implant coatings. 2003, Mater. Today, Vol. 6, pp. 26-30.

67. Application of plasma coatings in artificial joints: An overview. H. Liang, B. Shi, A. Fairchild, T. Cale. 2004, Vacuum, Vol. 73, pp. 317-26.

68. L. Sun, C.C. Berndt, K.A. Gross, A. Kucuk. Material fundamentals and clinical performance of plasma-sprayed hydroxyapatite coatings: A review.2001, J. Biomed. Mater. Res., Vol. 58, pp. 570-592.

69. K. Balani, A. Agarwal. Wetting of carbon nanotubes by aluminum oxide.2008, Nanotechnology, Vol. 19, p. 165701.

70. K. Balani, S.R. Bakshi, Y. Chen, T. Laha, A. Agarwal. Role of powder treatment and carbon nanotube dispersion in the fracture toughening of plasma sprayed aluminum oxide-carbon nanotube nano-composite. 2007, J. NanoSci. Nanotech., Vol. 7, pp. 1-10.

71. P. Sarkar, P.S. Nicholson. Electrophoretic deposition: mechanisms, kinetics and application to ceramics.1996, J. Am. Ceram. soc., Vol. 79, pp. 1987-2002.

72. L. Besra, M. Liu. A review on fundamentals and applications of electrophoretic deposition (EPD).2007, Prog. Mater. sci., Vol. 52, pp. 1-61.

73. A.R. Boccacini, S. Keim, R. Ma, Y. li, I. Zhitomirsky. Electrophoretic deposition of biomaterials.2010, J. Royal Soc. Interface, Vol. 7, pp. S581-S613.

74. A.R. Boccaccini, S. Keim, R. Ma, Y.Li, I. Zhitomirsky. Electrophoretic deposition of biomaterials.2010, J. Royal Soc. Interface, Vol. 7, pp. S581-S613.

75. A.R. Boccaccini, J. Cho, T. Subhani, C. Kaya, F. Kaya. Electrophoretic deposition of carbon-nanotube ceramic nanocomposites.2010, J. Eu. Ceram. Soc. ,Vol. 30,pp. 1115-1129.

76. S.V. Dorozhkin. Calcium Orthophosphates in Nature, Biology and Medicine.2009, Materials, Vol. 2, pp. 399-498.

77. J. Akedo. Aerosol Deposition Method for Fabrication of Nano Crystal Ceramic Layer. 2004, Mater. Sci. Forum, Vols. 449-452, pp. 43-48. 
78. K. Sahner, M. Kaspar, R. Moos. Assessment of the novel aerosol deposition method for room temperature preparation of metal oxide gas sensor films.2009, Sens. Actuators B: Chem., Vol. 139, pp. 394-399.

79. B.D. Hahn, D.S. Park, J.J. Choi, W.H. Yoon, J. Ryu, D.Y. Kim. Effects of Zr/Ti ratio and post-annealing temperature on the electrical properties of PZT thick films fabricated by aerosol deposition.2008, J. Mater. Res., Vol. 23, pp. 226-235.

80. P.C. Ma, N.A. Siddiqui, G. Maron, J.K. Kim. Dispersion and functionalization of carbon nanotubes for polymer-based nanocomposites: A review.2010, Composites: A, Vol. 41, pp. 1345-1367.

81. P.E. Wang, T. K. Chaki. Sintering behavior and mechanical properties of hydroxyapatite and di-calcium phosphate.1993, J. Mater. Sci., Vol. 4, pp. 150-158.

82. J. Zhou, X. zhang, J. Chen, S. Zeng, K. De Groot. High temperature characteristics of synthetic hydroxyapatite.1993, J. Mater. Sci.: Mater. Med., Vol. 4, pp. 83-85.

83. A.J. Ruys, M. Wei, C.C. Sorrell, M.R. Dickson, A. Brandwood, B.K. Milthorpe. Sintering effects on the strength of hydroxyapatite.1995, Biomaterials, Vol. 16, pp. 409-415.

84. A.A. White, S.M. best, I.A. Kinloch. Hydroxyapatite-carbon nanotube composite for biomedical applications: A review.2007, Int. J. Appl. Ceram. Technol., Vol. 4, pp. 1-13.

85. M. Jarcho, C.H. Bolen, M.B. Thomas, J. Bobick, J.F. Kay, R.H. Doremus. Hydroxyapatite synthesis and characterization in dense polycrystalline form.1976, J. Mater. Sci., Vol. 11, pp. 2027-2035.

86. S.R. Radin, P. Ducheyne. Plasma spraying induced changes of calcium phosphate ceramic characteristics and the effect on in vitro stability. 1992, J. Mater. sci.: Mater. Med., Vol. 3, pp. 33-42.

87. W. Yi, L. Lu, D.L. Zhang, Z.W. Pan, S.S. Xie. Linear specific heat of carbon nanotubes. 1999, Phys. Rev. B, Vol. 59, pp. 9015-9018.

88. H.W. Kim, Y.H. Koh, S.B. Seo, H.E. Kim. Properties of fluoridated hydroxyapatite-alumina biological composites densified with addition of CaF2. 2003, Mater. Sci. Eng. C, Vol. 23, pp. 515-521.

89. S.J. Kim, H.G. Bang, J.H. Song, S.Y. Park. Effect of fluoride additive on the mechanical properties of hydroxyapatite/alumina composites.2009, Ceram. Int., Vol. 35, pp. 1647-1650. 
90. Q. Zhang, J. Chen, J. Feng, Y. Cao, C. Deng, X. Zhang. Dissolution and mineralization behaviors of $H A$ coatings.2003, Biomaterials, Vol. 24, pp. 47414748.

91. P. Cheang, K.A. Khor. Addressing processing problems associated with plasma spraying of hydroxyapatite coatings. 1996, Biomaterials, Vol. 17, pp. 537-544.

92. Y. Chen, K. Balani, A. Agarwal. Analytical model to evaluate interface characteristics of carbon nanotube reinforced aluminum oxide nanocomposites. 2008, Appl. Phys. Lett., Vol. 92, p. 011916.

93. M. Cuppone, B.B. Seedhom, E. Berry, A.E. Ostell. The Longitudinal Young's Modulus of Cortical Bone in the Midshaft of Human Femur and its Correlation with CT Scanning Data.2004, Calcif. Tissue Int., Vol. 74, pp. 302-309.

94. J.Y. Rho, R.B. Ashman, C.H. Turner. Young's modulus of trabecular and cortical bone material: Ultrasonic and microtensile measurements.1993, J. Biomech., Vol. 26, pp. 111-119.

95. A. Ravaglioli, A. Krajewski. Bioceramics: Materials, properties, Applications. 1992, London: Chapman and Hall, pp. 44.

96. Y.C. Yang, E. Chang, B.H. Hwang, S.Y. Lee. Biaxial residual stress states of plasma-sprayed hydroxyapatite coatings on titanium alloy substrate.2000, Biomaterials, Vol. 21, pp. 1327-1337.

97. G.R. Anstis, P. Chantikul, B.R. Lawn, D.B. Marshall. A critical evaluation of indentation technique for measuring fracture toughness: I. Direct crack measurements. 1981, J. Am. Ceram. Soc., Vol. 64 (9), pp. 533-538.

98. B.R. Lawn, A.G. Evans, D.B. Marshall. Elastic/Plastic Indentation Damage in Ceramics: The Median/Radial Crack System.1980, J. Am. Ceram. Soc., Vol. 63, pp. 574-581.

99. A.G. Evans, E.A. Charles. Fracture toughness determination by indentations. 1976, J. Am. Ceram. Soc., Vol. 59, pp. 371-372.

100. R. Spiegler, S. Schmauder, L.S. Sigl. Fracture Toughness Evaluation of WC-Co Alloys by indentation testing. 1990, J. Hard Mater., Vol. 1 (3), pp. 147-158.

101. S. Singh, Y. Pei, R. Miller, P.R. Sundarrajan. Long range, entangled carbon nanotube networks in polycarbonate.2003, Adv. Func. Mater., Vol. 13, pp. 868872.

102. K.P. A. Saffar, A.R. Arshi, N.J. Pour, A.R. Najafi, G. Rouhi, L. Sudak. A crosslinking model for estimating Young's modulus of artificial bone tissue grown on carbon nanotube scaffold.2010, J. Biomed. Mater. Res. A, Vol. 94A, pp. 594-602. 
103. W.C. Oliver, G.M. Pharr. An improved technique for determining hardness and elastic modulus using load and displacement sensing indentation experiments. 1992, J. Mater. Res., Vol. 7, pp. 1564-1583.

104. A.G. Evans, B. Marshall. Wear mechanisms in ceramics. Editor: D.A. Rigney. Fundamentals of Friction and Wear of Materials, 1981, Materials Park, Ohio, pp. 439-452.

105. S. Fioritto. Carbon Nanotube: Angels or Demons? 2008, Singapore: Pan Stanford Publications, ISBN: $9814241016,9789814241014$.

106. P. Cherukuri, S.M. Bachilo, S.H. Litovsky, R.B. Weisman. Near-Infrared Fluorescence Microscopy of Single-Walled Carbon Nanotubes in phagocytic cells.2004, J. Am, Chem. Soc., Vol. 126, pp. 15638-15639.

107. J. Muller, F. Huaux, A. Fonseca, J.B. Nagy, N. Moreau, M. Delos, E. RaymundoPinero, F. Beguin, M. Kirsch-Volders, I. Fenoglio, B. Fubini, D. Lison. Structural Defects Play a Major Role in the Acute Lung Toxicity of Multiwall Carbon Nanotubes: Toxicological Aspects.2008, Chem. Res. Toxicol., Vol. 21, pp. 16981705 .

108. R. Singh, D. Pantarotto, L. Lacerda, G. Pastorin, C. Klumpp, M. Prato, A. Bianco, K. Kostarelos. Tissue biodistribution and blood clearance rates of intravenously administered carbon nanotube radiotracers. 2006, Proc. Natl. Acad. Sci. Am., Vol. 103 (9), pp. 3357-3362.

109. C. Cheng, K.H. Muller, K.K.K. Koziol, J.N. Skepper, P.A. Midgley, M.E. Welland, A.E. Porter. Toxicity and imaging of multi-walled carbon nanotubes in human macrophage cells.2009, Biomaterials, Vol. 30, pp. 4152-4160.

110. V.E Kagan, N.V. Konduru, W. Feng, B.L. Allen, J. Conroy, Y. Volkov, I.I. Vlasova, N.A. Belikova, N. Yanamala, A. Kapralov, Y.Y. Tyurina, J. Shi, E.R. kisin, A.R. Murray, J. Franks, D. Stolz, P. Gou, J.K. Seetharaman, B. Fadeel, A. Star, A.A. Shvedova. Carbon nanotubes degraded by neutrophil myeloperoxidase induce less pulmonary inflammation. 2010, Nat. Nanotech., Vol. 5, pp. 354-359.

111. Y. Usui, K. A., N. Narita, N. Murakami, I. Nakamura, K. Nakamura, N. Ishigaki, H. Yamazaki, H. Horiuchi, H. Kato, S. Taruta, Y. A. Kim, M. Endo, N. Saito. Carbon Nanotubes with High Bone-Tissue Compatibility and Bone-Formation Acceleration Effects.2008, Small, Vol. 4 (2), pp. 240-246.

112. X. Deng, F. Wu, Z. Liu, M. Luo, L. Li, Q. Ni, Z. Jiao, M. Wu, Y. Liu. The splenic toxicity of water soluble multi-walled carbon nanotubes in mice.2009, Carbon, Vol. 47, pp. 1421-1428. 
113. H.X. Ren, X. Chen, J.H. Liu, N. Gu, X.J. Huang. Toxicity of single-walled carbon nanotube: How we were wrong?2010, Mater. Today, Vol. 13, pp. 6-8.

114. J. Kolosnjaj-Tabi, K.B. Hartman, S. Boudjemma, J.S. Ananta, G. Morgrant, H. Szwart, L.J. Wilson, F. Moussa. In Vivo Behavior of Large Doses of Ultrashort and Full-Length Single-Walled Carbon Nanotubes after Oral and Intraperitoneal Administration to Swiss Mice.2010, ACS Nano, Vol. 4 (3), pp. 1481-1492.

115. M.A. Hussain, M.A. Kabir, A.K. Sood. On the cytotoxicity of carbon nanotubes.2009, Current Sci., Vol. 96, pp. 664-673.

116. A.O. Lobo, M.A.F. Corat, E.F. Antunes, M.B.S. Palma, C. Pacheco-Soares, E.E. Garcia, E.J. Corat. An evaluation of cell proliferation and adhesion on verticallyaligned multi-walled carbon nanotube films.2010, Carbon, Vol. 48, pp. 245-254.

117. S. Ginnova, I. Firkowska, J. Rojas-Chapana, M. Giersig. Vertically aligned carbon nanotubes as cytocompatible material for enhanced adhesion and proliferation of osteoblast-like cells.2007, J. Nanosci. Nanotechnol., Vol. 7, pp. 1679-1683.

118. Y.H. Yun, Z. Dong, Z. Tan, M.J. Schulz, V. Shanov. Fibroblast cell behavior on chemically functionalized carbon nanomaterials.2009, Mater. Sci. Eng. C, Vol. 29, pp. 719-725.

119. M. Kalbacova, M. K., L. Dunsch, U. Hempel. Influence of single-walled carbon nanotube films on metabolic activity and adherence of human osteoblasts.2007, Carbon, Vol. 45, pp. 2266-2272.

120. M. Matsuoka, T. Akasaka, Y. Totsuka, F. Watari. Strong adhesion of Saos-2 cells to multi-walled carbon nanotubes. 2010, Mater. Sci. Eng. B, Vol. 173, pp. 182-186.

121. T. Akasaka, A. Yokoyama, M. Matsuoka, T. Hashimoto, F. Watari. Thin films of single-walled carbon nanotubes promote human osteoblastic cells (Saos-2) proliferation in low serum concentrations.2010, Mater. Sci. Eng. C, Vol. 30, pp. 391-399.

122. X. Li, Y. Fan, F. Watari. Current investigations into carbon nanotubes for biomedical application.2010, Biomed. Mater., Vol. 5, p. 022001 (12 pp).

123. K. Kostarelos. Carbon nanotubes fibrillar pharmacology. 2010, Nature Mater., Vol. 9, pp. 793-795.

124. J. Beuvelot, C. Bergeret, R. Mallet, V. Fernandez, J. Cousseau, M.F. Basle, D. Chappard. In vitro calcification of chemically functionalized carbon nanotubes.2010, Acta Biomater., Vol. 6, pp. 4111-4117. 
125. S. Aryal, S.R. Bhattarai, K.C. R. Bahadur, M.S. Khil, D.R. Lee, H.Y. Kim. Carbon nanotubes assisted biomimetic synthesis of hydroxyapatite from simulated body fluid.2006, Mater. Sci. Eng. A, Vols. 426 (1-2), pp. 202-207.

126. T. Akasaka, F. Watari, Y. Sato, K. Tohji. Apatite formation on carbon nanotubes.2006, Mater. Sci. Eng. C, Vol. 26, pp. 675-678.

127. F. Zhang, A. Weidman, J.B. Nebe, E. Burkel. Osteoblast cell response to surfacemodified carbon nanotubes. 2010, Mater. Sci. Eng. C, DOI:10.1016/j.msec.2010.07.007.

128. F. Watari, T. Akasaka, K. Ishikawa, M. Matsuoka, E. Hirata, N. Terada, A. Yokoyama, M. Uo, S. Itoh, Y. Yakawa, M. Suzuki, N. Takashi,Y. Totsuka, Y. Kitagawa, S. Abe, I.D. Roska, Y. Kuboki, Y. Bando. Various nanotube scaffolds for cell proliferation. 2010, Mater. Sci. Forum, Vols. 631-632, pp. 181-186.

129. L. Niu, H. Kua, D.H.C. Chua. Bonelike Apatite Formation Utilizing Carbon Nanotubes as Template.2010, Langmuir, Vol. 26, pp. 4069-4073.

130. H-H. lee, U.S. Shin, J.E. won, H.W. Kim. Preparation of hydroxyapatite-carbon nanotube composite nanopowders.2011, Mater. Lett., Vol. 65, pp. 208-211.

131. N. Narita, Y. Kobayashi,H. Nakamura, K. Maeda, A. Ishihara, T. Mizoguchi, Y. Usui, K. Aoki, M. Simizu, H. Kato, H. Ozawa, N. Udagawa, M. Endo, N. Takahashi, N. Saito. Multiwalled Carbon Nanotubes Specifically inhibit osteoclast Differentiation and Function.2009, Nano Lett., Vol. 9, pp. 1406-1413.

132. T. Kokubo, H. Takadama. How useful is SBF in predicting in vivo bone bioactivity?2006, Biomaterials, Vol. 27, pp. 2907-2915.

133. H. Pan, X. Zhao, B.W. Darvell, W.W. Lu. Apatite-formation ability - predictor of "bioactivity"?2010, Acta Biomater., Vol. 6, pp. 4181-4188.

134. N.G. Chopra, A. Zetll. Measurement of the elastic modulus of a multi walled boron nitride nanotubes. 1998, Solid State Commun., Vol. 105, pp. 297-300.

135. A.P. Suryavanshi, M.F. Yu, J. Wen, C. Tang, Y. Bando. Elastic modulus and resonance behavior of boron nitride nanotubes.2004, Appl. Phys. Lett., Vol. 84, pp. 2527-2529.

136. H. Shen. Thermal-conductivity and tensile-properties of BN, SiC and Ge nanotubes.2009, Comput. Mater. Sci., Vol. 47, pp. 220-224.

137. G. Ciofani, V. Raffa, A. Menciassi, A. Cuschieri. Cytocompatibility, interactions, and uptake of polyethyleneimine-coated boron nitride nanotubes by living cells: confirmation of their potential for biomedical application. 2008, Biotech.Bioeng., Vol. 101, pp. 850-858. 
138. G. Ciofani, L. Ricotti, S. Danti, S. Moscato, C. Nesti, D. D'Alessandro, D. Dinucci, F. Chiellini, A. Pietrabissa, M. Petrini, A. Menciassi. Investigation of interactions between poly-l-lysine-coated boron nitride nanotubes and C2C12 cells: up-take, cytocompatibility , and differentiation.2010, Int. J. Nanomed., Vol. 5, pp. 285-298.

139. D. Lahiri, F. Rouzaud, T. Richard, A.K. Keshri, S.R. Bakshi, L. Kos, A. Agarwal. Boron nitride nanotube reinforced polylactide-polycaprolactone copolymer composite: Mechanical properties and cytocompatibility with osteoblasts and macrophages in vitro. 2010, Acta Biomater., Vol. 6, pp. 3524-3533.

140. G. Ciofanni, V. Raffa, A. Mencissia, A. Cuschieria. Boron nitride nanotubes: An innovative tool for nanomedicine.2009, Nanotoday, Vol. 4, pp. 8-10.

141. X. Chen, P. Wu, M. Rousseas, D. Okawa, Z. Gartner, Z.A. Zetll, C.R. Bertozzi. Boron nitride nanotubes are noncytotoxic and can be functionalized for interaction with proteins and cells. 2009, J. Am. Chem. Soc. Commun., Vol. 131, pp. 890-891.

142. M. Terrones, J.M. Romoherrera, E. Cruz-Silva, F. Lopez-Urias, E. MunozSandoval, J.J. Velaquez-Salazar, H. Terrones, Y. Bando, D. Golberg. Pure and doped boron nitride nanotubes.2007, Mater. Today, Vol. 10 (5), pp. 30-38.

143. X. Blase, A. De Vita, J.C. Charlier, R. Car. Frustration Effects and Microscopic Growth Mechanisms for BN Nanotubes. 1998, Phys. Rev. Lett., Vol. 80, pp. 16661669.

144. D. Golberg, Y. Bando, C. Tang, C.Zhi. Boron Nitride Nanotubes.2007, Adv. Mater., Vol. 19, pp. 2413-2432.

145. D. Golberg, Y. Bando, Y. Huang, T. Terao, M. Mitome, C. Tang, C. Zhi. 2010, Boron nitride nanotubes and nanosheets. ACS Nano, Vol. 4, pp. 2979-2993.

146. N.G. Chopra, A. Zetll. Measurement of the elastic modulus of a multi walled boron nitride nanotubes. 1998, Solid State Commun., Vol. 105, pp. 297-300.

147. A.P. Suryavanshi, M.F. Tu, J. Wen, C. Tang, Y. Bando. Elastic modulus and resonance behavior of boron nitride nanotubes.2004, Appl. Phys. Lett., Vol. 84, pp. 2527-2529.

148. M.F. Yu, O. Laurie, M.J. Dyer, K. Moloni, T.F. Keally, R.S. Rouff. Strength and breaking mechanism of multiwalled carbon nanotubes.2000, Science, Vol. 287, pp. 637-640.

149. H.Shen. Thermal-conductivity and tensile-properties of BN, SiC and Ge nanotubes. 2009, Comp. Mater. Sci., Vol. 47, pp. 220-224. 
150. Y. Xiao, X.H. Yan, J. Xiang, Y. L. Mao, Y. Zhang, J.X. Cao, J.W. Ding. Specific heat of single-walled boron nitride nanotubes.2004, Appl. Phys. Lett., Vol. 84, pp. 4626-4628.

151. C. Zhi, Y. Bando, T. Terao, C. Tang, H. Kuwahara, D. Golberg. Towards thermoconductive, electrically insulating polymeric composites with boron nitride nanotubes as fillers.2009, Adv. Func. Mater., Vol. 19, pp. 1857-1862.

152. T. Terao, C. Zhi, Y. Bando, M. Mitome, C. Tang, D. Golberg. Alignment of boron nitride nanotubes in polymeric composite films for thermal conductivity improvement.2010, J. Phys. Chem., Vol. 114, pp. 4340-4344.

153. P. Kim. L. Shi, A. Majumdar, P.L. McEuen. Thermal transport measurements of individual multiwalled nanotubes.2001, Phys. Rev. Lett., Vol. 87, p. 215502.

154. C. Tang, Y. Bando, Y. Huang, S. Yue, C. Gu, F. Xu, D. Golberg. Fluorination and Electrical Conductivity of BN Nanotubes.2006, J. Am. Chem. Soc., Vol. 127, pp. 6552-6553.

155. Y. ando, X. Zhao, H. Shimoyama, G. Sakai, K. Kaneto. Physical properties of multiwalled carbon nanotubes. 1999, Int. J. Inorg. Mater., Vol. 1, pp. 77-82.

156. J.X. Zhao, B.Q. Dai. DFT studies of electro-conductivity of carbon-doped boron nitride nanotubes. 2004, Mater. Chem. Phys. Vol. 88, pp. 244-249.

157. X. Bai, D. Golberg, Y. Bando, C. Zhi, C. Tang, M. Mitome, K. Kurashima. Deformation-Driven Electrical Transport of Individual Boron Nitride Nanotubes.2007, Nano Lett., Vol. 7, pp. 632-637.

158. J. Wu, W.Q. Han, W. Walukiewicz, J.W. Ager III, W. Shan, E.E. Haller, A. Zetll. Raman spectroscopy and time-resolved photoluminiscence of $B N$ and $B x C y N z$ Nanotubes.2004, Nano Lett., Vol. 4, pp. 647-650.

159. R. Ma, Y. Bando, H. Zhu, T. Saito, C. Xu, D. Wu. 2002, Hydrogen Uptake in Boron Nitride Nanotubes at Room Temperature. J. Am. Chem. Soc., Vol. 124, pp. 7672-7673.

160. C. Zhi, Y. Bando, C. Tang, S. Honda, K. Sato, H. Kuwahara, D. Golberg. Characteristics of boron nitride nanotube-polyaniline composites.2005, Angew. Chem., Vol. 44, pp. 7929-7932.

161. C. Zhi, Y. Bando, C. Tang. Boron nitride nanotube/polystyrene composites.2006, J. Mater. Res., Vol. 21, pp. 2794-2800.

162. J. Ravichandran, A.G. Manoj, J. Liu, I. Manna, D.L. Carroll. A novel polymer nanotube composite for photovoltaic packaging applications.2008, Nanotechnology, Vol. 19, p. 085712. 
163. Q. Huang, Y. Bando, X. Xu, T. Nishimura, C. Zhang, C. Tang, F. Xu, L. Gao, D. Golberg. Enhancing superplasticity of engineering ceramics by introducing $B N$ nanotubes.2007, Nanotechnology, Vol. 18, p. 485706.

164. Y. Li, H. Liang, J. Zhang. 2010, Fabrication and properties of AlN/carbon-doped boron nitride nanotubes composites. J. Chinnese Ceram. Soc., Vol. 38, pp. 14401444.

165. C.Y. Zhi, Y. Bando, W.L. Wang, C.C. Tang, H. Kuwahara, D. Golberg. Mechanical and Thermal Properties of Polymethyl Methacrylate-BN Nanotube Composites.2008, J. Nanomater., Vol. 2008, Article ID: 642036.

166. T. Terao, Y. Bando, M. Mitome, C. Zhi, C. Tang, D. Golberg. Thermal conductivity improvement of polymer films by catechin-modified boron nitride nanotubes.2009, J. Phys. Chem. C, Vol. 113, pp. 13605-13609.

167. S.K. Samanta, A. Gomati, S. Bhattacharya, C.N.R. Rao. Novel nanocomposites made of boron nitride nanotubes and a physical gel. 2010, Langmuir, Vol. 26, pp. 12230-12236.

168. C.Y. Zhi, Y. Bando, T. Terao, C. Tang, D. Golberg. Dielectric and thermal properties of epoxy/boron nitride nanotube composites.2010, Pure Appl. Chem., Vol. 82, pp. 2175-2183.

169. N.P. Bansal, J.B. Hurst, S.R. Choi. Boron Nitride Nanotubes-Reinforced Glass Composites. 2006, J. Am. Ceram. Soc., Vol. 89, pp. 388-390.

170. S.R. Choi, N.P. Bansal, A. Garg. Mechanical and microstructural characterization of boron nitride nanotubes-reinforced SOFC seal glass composite.2007, Mater. Sci. Eng. A, Vols. 460-461, pp. 509-515.

171. G.Ciofani. Potential Applications of boron nitride nanotube as drug delivery systems.2010, Expt Opin. Drug Deliv., Vol. 7, pp. 889-893.

172. C. Zhi, Y. Bando, C. Tang, D. Golberg. Immobilization of protein on boron nitride nanotubes.2005, J. Am. Chem. Soc., Vol. 127, pp. 17144-17145.

173. H.M. Ghassemi, C.H. Lee, Y.K. Yap, R.S. Yassar. In situ observation of reversible rippling in multi-walled boron nitride nanotubes.2011, Nanotechnology, Vol. 22, p. 115702 . 


\subsection{MATERIALS AND METHODS}

This chapter provides a detailed account of the synthesis methods of HAnanotube composite and its characterization for mechanical properties, tribological behavior and biocompatibility.

\subsection{Synthesis of Hydroxyapatite-Carbon Nanotube Composite}

Synthesis comprises of two stages - (i) composite powder preparation and (ii) consolidation of the composite powder to obtain free standing composite or coating. Spray drying technique has been used for preparing hydroxyapatite-carbon nanotube composite powder, to ensure good dispersion and attachment of CNT on HA particles. HA-CNT composite powder is consolidated as coating by plasma spraying whereas spark plasma sintering is adopted for free standing parts.

\subsubsection{HA-CNT Composite Powder Preparation: Spray Drying}

Spray drying is a technique to process agglomerated powder particles of micrometer size starting from nano-sized powders. The nano-sized powders are first dispersed in water soluble organic binder to form slurry. The slurry is then atomized in a chamber and dried subsequently to obtain micron size porous spherical agglomerates. Our research group has established spray drying as an effective tool for the dispersion of CNTs in ceramic $\left(\mathrm{Al}_{2} \mathrm{O}_{3}\right)$ powder [1]. 


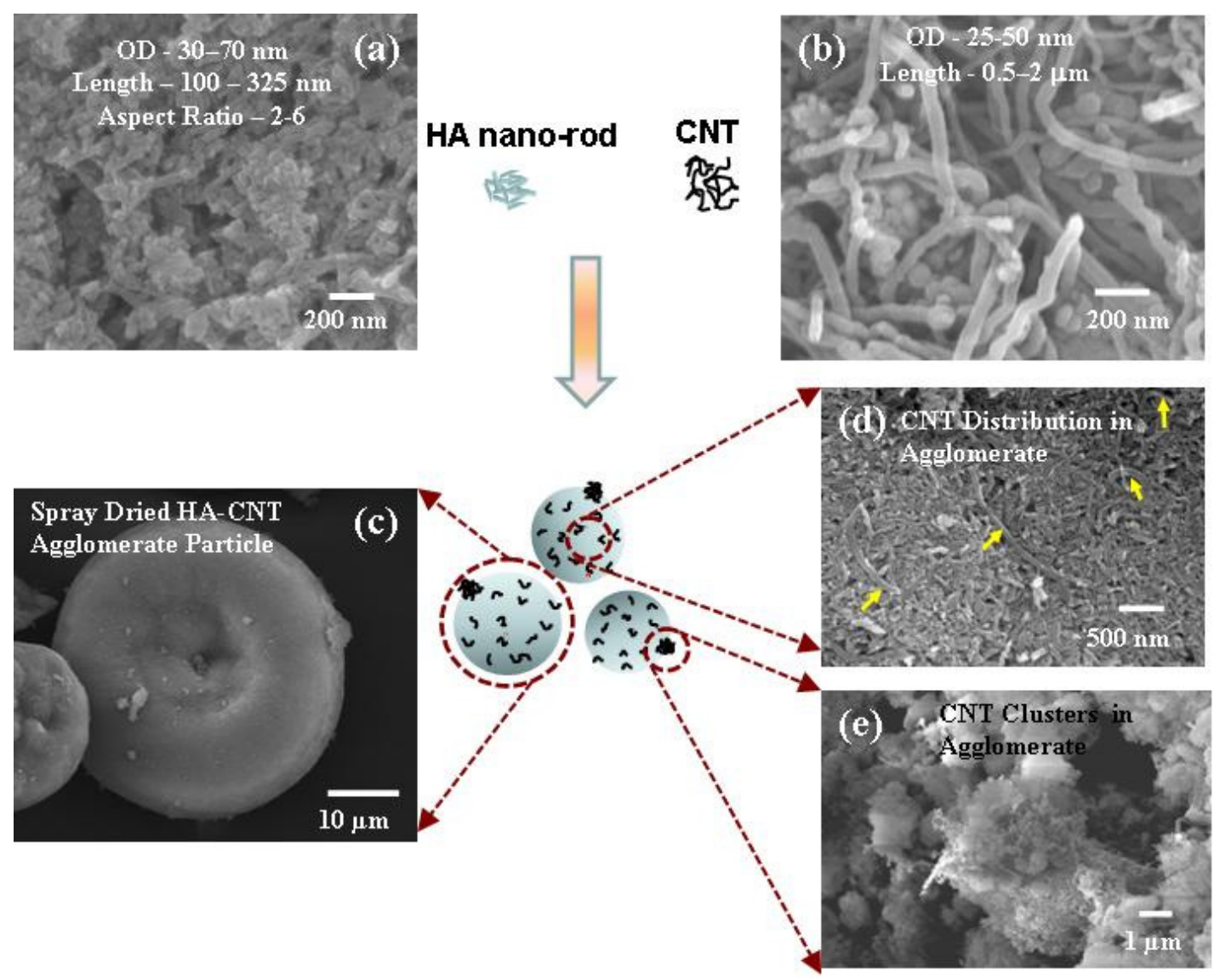

Figure 3.1: Schematic of formation of spray dried HA-CNT composite powder from HAnanorods and CNTs and SEM micrographs of (a) HA-nanorods, (b) multi-walled CNTS, (c) spray dried HA-CNT agglomerate, (d) distribution of CNTs in spray-dried HA-CNT and (e) some CNT clusters on HA-CNT agglomerate surface.

HA nano-rods (length: $100-325 \mathrm{~nm}$, diameter: 25-50 nm, density: 3.2 g.cm ${ }^{-3}$ ) and multi-walled carbon nanotubes (95\% purity, 25-50 nm OD, 0.5-2 $\mu \mathrm{m}$ in length: density: $2.1 \mathrm{~g} . \mathrm{cm}^{-3}$ ), procured from Inframat Corporation, (Willington, CT, USA), are used as precursor powder as shown in figures 3.1a and 3.1b, respectively. Figure 3.1 shows a schematic of spray dried HA-CNT composite powder preparation with SEM images of powder at different stages. HA nano-rods and CNTs are spray-dried together to synthesize the composite agglomerates with a size of $15-55 \mu \mathrm{m}$ (figure 3.1c). Spray dried 
powder enables a higher degree of homogeneity of CNT distribution in the HA matrix as observed in high magnification images of powder agglomerates in figure 1d. HA-CNT powder used in this study contains 4 wt.\% CNT. HA powder is also spray dried and used as the control sample.

\subsubsection{HA-CNT Composite Coating Synthesis: Plasma Spraying}

Spray dried HA and HA- 4 wt.\% CNT powders were plasma sprayed using SG 100 gun (Praxair Surface Technology, Danbury, CT) on $2.5 \mathrm{~mm}$ thick Ti-6Al-4V medical grade alloy substrate. Plasma spraying process is used to synthesize the coating because it is FDA approved process for coating deposition on orthopedic implants [2]. Table 3.1 presents the plasma spraying parameters used for HA and HA-4 wt.\% CNT coating deposition. The deposited coating had a uniform thickness of $\sim 150 \mu \mathrm{m}$.

Table 3.1: Plasma spraying parameters for HA/HA-CNT coating deposition

\begin{tabular}{|l|c|}
\hline Plasma Power & $23 \mathrm{~kW}(575 \mathrm{~A}, 40 \mathrm{~V})$ \\
\hline Primary Gas (Argon) Flow Rate & $30 \mathrm{slpm} *$ \\
\hline Auxiliary Gas (Helium) Flow Rate & $28 \mathrm{slpm} *$ \\
\hline Powder Carrier Gas (Argon) Flow Rate & $25.5 \mathrm{slpm} *$ \\
\hline Powder Feed Rate & $4.5{\mathrm{~g} . \mathrm{min}^{-1}}^{-1}$ \\
\hline Stand-off Distance & $0.1 \mathrm{~m}$ \\
\hline
\end{tabular}

*slpm - standard liter per minute

\subsubsection{Free Standing HA-CNT Composite Synthesis: Spark Plasma Sintering}

Consolidation of spray dried HA and HA-4 wt.\% CNT powders is performed using the spark plasma sintering facility at Thermal Technology LLC, Sana Rosa, 
California, USA. The SPS is carried out at $60 \mathrm{MPa}$ pressure and $1373 \mathrm{~K}$ in vacuum using a graphite die. The temperature and pressure of sintering is chosen based on the outcomes in published literature [3-4]. A rapid heating rate of $360 \mathrm{~K} / \mathrm{min}$ with a hold time of 5 minute at $1373 \mathrm{~K}$ is used. Pellets of $19.5 \mathrm{~mm}$ diameter and $\sim 5 \mathrm{~mm}$ thickness are consolidated. HA-4 wt.\% compositions (coating and sintered) would be referred to as HA-CNT hereafter.

\subsection{Synthesis of Hydroxyapatite-Boron Nitride Nanotube Composite}

HA-BNNT composite powder is prepared by wet chemistry method. The consolidation of the powder is performed using spark plasma sintering. Plasma spraying of HA-BNNT is not carried out due to unavailability of BNNT in large amount. Plasma spraying requires a large amount (e.g. 50-100 g) of powder feedstock due to volumetric nature of the industrial scale powder feeder. Synthesis of BNNT is still in the developmental stage and its bulk production has not yet been commercialized.

\subsubsection{HA-BNNT Composite Powder Preparation: Wet Chemistry/}

\section{Ultrasonication}

Boron nitride nanotubes, obtained from Nanoamor, Houston, USA, comprised of nodular (bamboo type), cylindrical nanotubes, and few nano-rods. Figure 3.2 presents the TEM image of as-received BNNTs showing nodular and cylindrical nanotubes. BNNTsused in this study are of length: $0.43-5.8 \mu \mathrm{m}$; outer diameter: $10-145 \mathrm{~nm}$ and 
density 2.25 g.cm ${ }^{-3}$. HA nano-rods used in this composite are same as the ones used for HA-CNT composite preparation.
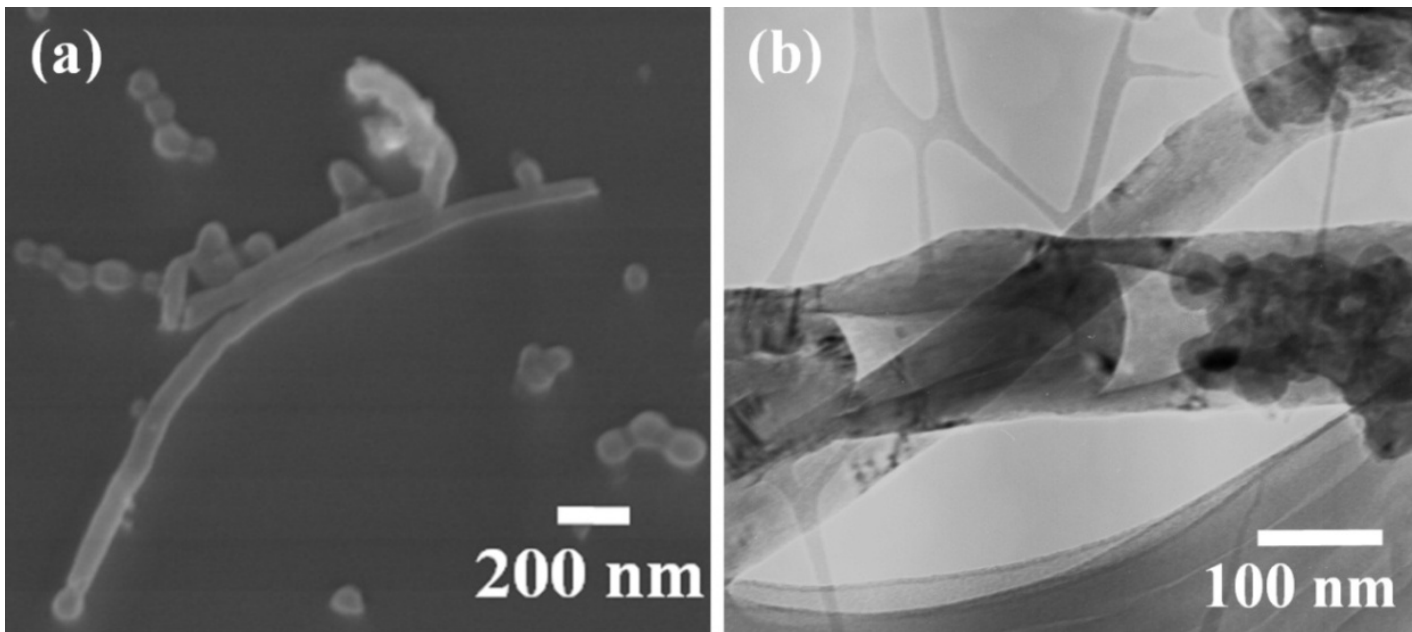

Figure 3.2: (a) SEM and (b) TEM images of the as-received BNNTs showing the presence of both tubular and bamboo type structures.

The powder feedstock for sintering is prepared with two composition, viz. $100 \%$ HA and HA-4 wt. \% BNNT. The composite powder is prepared in batches of $0.5 \mathrm{~g}$ using ultrasonication for uniform dispersion of BNNTs in HA. In each batch, $0.02 \mathrm{~g}$ of BNNT is mixed in $20 \mathrm{ml}$ of acetone and ultrasonicated for $3 \mathrm{~h}$. Subsequently, $0.48 \mathrm{~g}$ of HA is mixed in the dispersion and ultrasonicated for $1 \mathrm{~h}$. Finally the dispersion is dried in an oven at $348 \mathrm{~K}$ for $3 \mathrm{~h}$.

\subsubsection{Free Standing HA-BNNT Composite Synthesis: Spark Plasma Sintering}

Spark plasma sintering of HA and HA- 4 wt.\% BNNT composite powders is carried out in vacuum at $1373 \mathrm{~K}$ and $70 \mathrm{MPa}$ pressure using the spark plasma sintering facility at Thermal Technology LLC, Sana Rosa, CA, USA. A fast heating rate of 360 $\mathrm{K} / \mathrm{min}$ is employed with a soaking time of $5 \mathrm{~min}$ at maximum temperature. HA and HA- 
4 wt.\% BNNT pellets of $20 \mathrm{~mm}$ diameter and $\sim 5 \mathrm{~mm}$ thickness are consolidated. HA4wt.\% BNNT composite would be referred as HA-BNNT hereafter in this document.

\subsection{Microstructural Characterization}

Characterization techniques for evaluating physical properties and microstructure of HA-CNT and HA-BNNT composites and coating are described below.

\subsubsection{Scanning Electron Microscopy: Powder Morphology and Microstructure of Composites and Coatings}

JEOL JSM-633OF field emission scanning electron microscope (FE-SEM), operating at $15 \mathrm{kV}$, was used for the characterization of powders and consolidated composites and coatings. Samples were sputter coated with gold before observing in SEM. Microscopic characterization of powders was performed by dispersing them on a glass slide or silicon wafer. The consolidated samples were sectioned with a diamond blade, mounted and metallographically polished for the microstructural observation through cross section. Fracture surfaces of the sintered pellets or peeled off coatings were also observed under SEM to analyze the quality of nanotube dispersion and its bonding with HA matrix.

\subsubsection{High Resolution Transmission Electron Microscopy: Interface of Hydroxyapatite and Nanotubes}

Transmission electron microscopy images of as-received nanotubes were captured using Philips PW 6061 TEM system (model CM 200, Eindhoven, Netherlands) to 
analyze the dimension and structure of nanotubes. Philips/FEI Tecnai F30 high resolution transmission electron microscope (HRTEM) was used to study HA-nanotube composite structure. Lattice level resolution of HRTEM was used to understand the nature and bonding at HA-CNT interface as a result of consolidation. Forward and inverse Fourier transform (FFT \& Inverse-FFT) analysis, using Gatan, Inc. Digital Micrograph software, was utilized for an accurate calculation of the lattice spacing and determination of crystallographic relationship at interface. Nucleation and growth of apatite precipitation on BNNT surface was also studied using HRTEM images.

\subsubsection{Stereological Analysis using Image Processing Software}

Stereological analysis was carried out by ImageJ software [5]. Quantitative analysis of the particle size in HA-CNT wear debris was performed using multiple SEM images. HA precipitate needle length on BNNT was quantified using HRTEM images. Osteoblasts viability was quantified using multiple fluorescent micrographs of stained cells. The image based quantification of actin expression for osteoblasts was performed using color based analysis mode in ImageJ to obtain the fraction of pixels with similar color in an image.

\subsubsection{X-Ray Diffraction: Phases and Crystallinity Determination}

X-ray diffraction (XRD) studies were carried out to determine the HA phases present in powder and consolidated stages. Dissociation of HA during consolidation is very crucial for its orthopedic application. XRD was carried out using $\mathrm{Cu} \mathrm{K}_{\alpha}(\lambda=1.542$

$\AA$ ) radiation in a Siemens D-500 X-ray diffractometer operating at $40 \mathrm{kV}$ and $40 \mathrm{~mA}$. A 
scan rate of $0.2^{\circ} / \mathrm{min}$ and a step size of $0.01^{\circ}$ have been used. The phases present in powder and sintered structure were determined using the JCPDS standard database. The XRD data files that were used are: hydroxyapatite (JCPDS PDF No. 9-432), $\alpha$-TCP (JCPDS PDF No. 29-359), $\beta$-TCP phase (JCPDS PDF No. 9-169), graphite for CNT (JCPDS PDF No. 23-0064) and hexagonal BN for BNNT (JCPDS PDF No. 34-0421).

\subsubsection{Raman Spectroscopy}

Raman spectroscopy was carried out to confirm the retention of nanotube structure in the consolidated structure after exposure to high temperature and pressure. Raman spectrum shows characteristics plot for all specific type of bonds present in the sample, which could be correlated with the presence of a chemical entity or compound. Shift in wave number (peak position) in Raman spectra signifies change in the bond length and thus the stress present in material. Also, the relative intensity of the signature peaks of CNT (D and G) denotes the defect in CNT structure. All these characteristics are taken advantage of for analyzing the composite microstructure in the current research. The variation in Raman peak intensity is also used to qualitatively assess the volume of apatite precipitate on nanotube surface. Raman spectra of powders, consolidated structures and precipitates are obtained by using argon ion $\left(\mathrm{Ar}^{+}\right)$laser system (Spectra Physics, model 177G02) of wavelength $514.5 \mathrm{~nm}$. Backscattered spectra are collected by high throughput holographic imaging spectrograph (Kaiser Optical Systems, model HoloSpec $f / 1.8 \mathrm{i}$ ) with volume transmission grating, holographic notch filter, and charge coupled device detector (Andor Technology). Raman system has a spectral resolution of $4 \mathrm{~cm}^{-1}$ and the spectra were collected at an exposure of $300 \mathrm{~s}$. 


\subsubsection{Density and Surface Roughness Measurement}

Density of free standing composite structures and peeled off coatings were measured through Archimedes principle using water as an immersion medium. Surface roughness of the composite surfaces was measured by contact type surface roughness tester (TR200 from Micro Photonics Inc, Irvine, USA) using a contact-type stylus with a $5 \mu \mathrm{m}$ radius diamond tip (TS-120).

\subsection{Evaluation of Mechanical Properties}

Elastic modulus, hardness and fracture toughness of HA-nanotube composites and coatings have been studied. Nanoindentation and microindentation techniques were used to measure mechanical properties at multiple length scales.

\subsubsection{Nano-Indentation: Elastic Modulus and Hardness}

Hysitron Triboindenter TI-900 (Hysitron Inc., Minneapolis, MN, USA) with 100 $\mathrm{nm}$ Berkovich pyramidal tip, was used in quasi-static indentation mode to measure the elastic modulus and hardness of the sintered pellets and coatings. Tip-area calibration was done using a standard fused quartz substrate of known modulus (69.6 GPa). Indentation was performed with a constant loading/unloading rate for $10 \mathrm{~s}$ and $3 \mathrm{~s}$ hold at the peak load of $2500 \mu \mathrm{N}$. Elastic modulus (E) was calculated from the unloading segment of the load-displacement curves using Oliver-Pharr method [6]. Following procedure has been adopted for $\mathrm{E}$ and $\mathrm{H}$ calculation: 
(i) The selected portion of the unloading curve (upper $95 \%$ to lower $20 \%$ ) is fit to the power law relation:

$$
P=\alpha h^{m} \ldots \ldots . .(3.1)
$$

where, $P$ is the applied load, $h$ is the depth of penetration and $\alpha$ and $m$ are constants.

(ii) The derivative of the power law relation with respect to $h$ is evaluated at the maximum load to calculate the contact stiffness, $S$.

(iii) The contact depth, $h_{c}$, is calculated using $S$ as:

$$
h_{c}=h_{\max }-0.75 \cdot \frac{P_{\max }}{S}
$$

(iv) The hardness is calculated as:

$$
H=\frac{P_{\max }}{A\left(h_{c}\right)}
$$

where, $A\left(h_{c}\right)$ is the area as a function of contact depth, obtained from the tip area calibration function.

(v) The reduced modulus is calculated as:

$$
E_{r}=\frac{\sqrt{\pi}}{2 \sqrt{A\left(h_{c}\right)}} . S \ldots \ldots \ldots(3.4)
$$

(vi) The elastic modulus of sample $\mathrm{E}$ is calculated as:

$$
\frac{1}{E_{r}}=\left(\frac{1-v^{2}}{E}\right)_{\text {sample }}+\left(\frac{1-v^{2}}{E}\right)_{\text {indenter }} \ldots(3.5)
$$

where, $v$ is Poisson's ratio. The indenter used is diamond with $\mathrm{E}=1140 \mathrm{GPa}$ and $v=$ 0.07 .

Nanoindentation provides localized mechanical properties. In order to get an impression of the bulk mechanical properties of the composites, more than 100 indents were made at randomly chosen regions throughout the polished cross-section of each of the composites. In each region, the indents were made at a distance of $9 \mu \mathrm{m}$ from each 
other. Total area covered by the indents was $>5000 \mu \mathrm{m}^{2}$ in each sample. The statistical distribution of elastic modulus, measured from individual indents, thus provides the mechanical properties of the composite at macro-scale length.

\subsubsection{Micro-Indentation: Fracture Toughness and Hardness}

Microhardness was measured using a microhardness tester (Shanghai Taiming Optical Instrument Co. Ltd., model HXD-1000 TMC, Shanghai) with Vickers probe and application of $1 \mathrm{~kg}$ load for 15 seconds of dwell time. Microindents in the consolidated composites were performed to determine the fracture toughness by initiating the cracks. Fracture toughness was evaluated using Anstis' equation [7] expressed as:

$$
K_{I C}=0.016\left(\frac{E}{H}\right)^{1 / 2} \frac{P}{c^{3 / 2}}
$$

where, $\mathrm{P}$ is the applied load, $\mathrm{E}$ is the elastic modulus, $\mathrm{H}$ is the Vickers hardness and $\mathrm{c}$ is the radial crack length (measured from the center of the indent). For an accurate measurement of radial crack length, the indents were also observed under SEM. Elastic modulus values for the composites were estimated from nanoindentation. The microindents on the polished cross-section and the radial cracks generated were observed through high resolution SEM imaging to understand the role of nanotubes in toughening of the composite. 


\subsection{Evaluation of Tribological Behavior}

Tribological behavior of the composite is analyzed at multiple length scales by estimating the wear volume and the coefficient of friction. Wear volume is indicative and inversely related to the wear resistance. Wear at macro scale was performed using ballon-disk method. Nano-scale tribological studies were performed using nano-scratch.

\subsubsection{Tribology: Macro-scale Wear}

Ball-on disk tribometer (Nanovea, Micro Photonics Inc., CA) was used to evaluate the macro-scale wear resistance and coefficient of friction $(\mathrm{CoF})$ of sintered pellets and coatings. Samples were polished to a roughness $\left(R_{a}\right)$ of $0.5 \mu \mathrm{m}$ or less. Macro-wear studies are performed at 50 RPM speed with a circular track of $2 \mathrm{~mm}$ radius and a total travel distance of $100 \mathrm{~m}$. The linear speed of wear probe on wear track was $10.5 \mathrm{mms}^{-1}$. An alumina ball of $3 \mathrm{~mm}$ diameter is used as the counter surface (probe). The lateral force between the alumina ball and the composite surface and depth of wear track is measured by the linear variable differential transformer (LVDT) sensor. The coefficient of friction data is acquired at a frequency of $16.67 \mathrm{~Hz}$. In case of HA-CNT and HA-BNNT sintered pellets, the wear volume is measured by considering the depth of wear track from LVDT and the geometry of the wear probe. In case of plasma sprayed HA/HA-CNT coatings, the wear track profiles across the tracks are obtained using Nanovea ST400 Optical Profiler. Wear volume is computed using the depth profile from the wear tracks. The depth from optical profile is in good agreement with the ones measured by LVDT in this case, proving the accuracy of the LVDT data used in other cases. Macro-wear tracks are observed closely along with the wear debris to understand 
the wear mechanism in HA-nanotube composite. Elemental analysis of the samples, as required is some cases, is performed using energy dispersive spectroscope (EDS) attached with a JEOL JSM 5910LV scanning electron microscope.

Wear study in physiological condition, is performed by immersing the sample in simulated body fluid (SBF), while carrying out the ball-on-disk wear using the same testing condition and wear probe as dry wear. The SBF is prepared using Kokubo's recipe [8] with the chemical composition as presented in table 3.2.

Table 3.2: Chemical composition of Simulated Body Fluid (SBF)

\begin{tabular}{|c|c|}
\hline Ingredient & Amount (g/l) \\
\hline $\mathrm{NaCl}$ & 7.996 \\
\hline $\mathrm{NaHCO}_{3}$ & 0.350 \\
\hline $\mathrm{KCl}$ & 0.224 \\
\hline $\mathrm{K}_{2} \mathrm{HPO}_{4} \cdot 3 \mathrm{H}_{2} \mathrm{O}$ & 0.228 \\
\hline $\mathrm{MgCl}_{2} \cdot 6 \mathrm{H}_{2} \mathrm{O}$ & 0.305 \\
\hline $\mathrm{CaCl}_{2}$ & 0.278 \\
\hline $\mathrm{Na}_{2} \mathrm{SO}_{4}$ & 0.071 \\
\hline$\left(\mathrm{CH}_{2} \mathrm{OH}\right)_{3} \mathrm{CNH}$ & 6.057 \\
\hline $1 \mathrm{kmol} / \mathrm{m}^{3} \mathrm{HCl}$ & To adjust the pH - 7.25 \\
\hline $\mathrm{Ultrapure}$ water & To make volume upto 1 litre \\
\hline
\end{tabular}

\subsubsection{Nano-Scratch: Micro/Nano-scale Wear}

Hysitron Triboindenter TI-900 (Hysitron Inc., Minneapolis, MN, USA) with 100 $\mathrm{nm}$ Berkovich pyramidal tip, is used in 2D scratch mode for nano-wear studies. The scratches of $10 \mu \mathrm{m}$ length are made with constant normal loads of $3500 \mu \mathrm{N}$ and $4500 \mu \mathrm{N}$ 
on the metallographically polished SPS HA-CNT composite cross sections. Lateral force and scratch depth along the scratch length is recorded by two different piezoelectric sensors working in directions perpendicular to each other. Scratch profiles are obtained by scanning probe microscopy (SPM) with the same tip at a set point load of $2 \mu \mathrm{N}$. The topography image processing is performed using Scanning Probe Image Processor (SPIP) version 4.5.1 (Image Metrology, Denmark) [9]. Scratch volume calculation has been performed from the geometry of the scratches obtained through 2D profiles of scratch along the length and width, using the following expression:

$$
V=\int_{0}^{l} h^{2} \tan \theta \cdot d l
$$

where, $V$ is the volume of the scratched groove, $h$ is the height of the groove (obtained from 2D SPM profile of scratch along the length), $\theta$ is the average angle of the groove measured at five points along the scratch length using 2D SPM profile and $l$ is the length of the scratch. The detailed procedure of this volume calculation is available in our earlier publication [10].

\subsection{Evaluation of Biocompatibility}

Biocompatibility of HA-nanotube composites is evaluated for assessing the suitability for orthopedic applications based on ISO and ASTM guidelines [11-15]. The standard guidelines for biocompatibility tests are available in 'Appendix-1'. Following subsections present a detailed description of in-vivo and in-vitro biocompatibility studies carried out in this research. 


\subsubsection{In-Vitro Evaluation}

In-vitro biocompatibility evaluation for HA-nanotube composites is carried out by culturing osteoblast and/or macrophage cells on the coatings or sintered pellet surfaces. Osteoblasts and macrophage cells are also used for studying cytotoxicity of the bare nanotubes and wear debris.

\subsubsection{Osteoblast Cell Culture}

The substrates (coatings and free standing pellets) with $5 \mathrm{~mm}$ x $5 \mathrm{~mm}$ surface area were washed with 95\% ethyl alcohol, washed 3 times with fresh medium and left for 3 hours in a hood under UV light for sterilization. They were then placed into 6-well polystyrene petri dishes (Corning, New York). Human osteoblasts ATCC CRL-11372 (ATCC, Manassas, VA) were seeded at a density of 1000 cells per well (with one substrate in each well) in 6-well polystyrene petri dishes (Corning, New York) at $310 \mathrm{~K}$ $\left(37{ }^{\circ} \mathrm{C}\right), 5 \% \mathrm{CO}_{2}$ in a 1:1 mixture of Ham's F12 Medium Dulbecco's Modified Eagle's Medium, with $2.5 \mathrm{mM}$ L-glutamine. The phenol red-free base media was supplemented with 10\% Fetal Bovine Serum (Atlanta Biologicals, Lawrenceville, GA), $100 \mathrm{UI} / \mathrm{ml}$ of penicillin and $100 \mu \mathrm{g} / \mathrm{ml}$ of streptomycin (MP Biomedicals, Irvine, CA).

\subsubsection{Proliferation and Viability}

For cell viability studies, osteoblasts were seeded at a density of 5000 cells per well in $2.5 \mathrm{ml}$ of medium and grown in an incubator at $310 \mathrm{~K}\left(37^{\circ} \mathrm{C}\right), 5 \% \mathrm{CO}_{2}$ following the procedure given above. After fixed culture period, cells grown on the substrate were stained for 2 minutes with a Phosphate Buffer Saline $1 \mathrm{X}$ solution containing $15 \mu \mathrm{g} / \mathrm{ml}$ of 
Fluorescein Di-Acetate (FDA) (MP Biomedicals, Irvine, CA) and $4.5 \mu \mathrm{g} / \mathrm{ml}$ of Propidium Iodide (PI) (Fisher Scientific, Waltham, MA) ${ }^{23}$ before visualization on a Leica Leitz DM RB fluorescent microscope (Leica, Bannockburn, IL). Digital pictures were captured with a Leica DM 500 camera. Live (green) versus dead (red) cells counting was manually performed using Image J software. 'Student t' test was performed to find the $95 \%$ confidence interval for the viability data. Number of green cells gives the idea about proliferation and the ratio of green vs. red cells denotes the viability.

\subsubsection{BrDU Expression for Proliferation Kinetics}

Proliferation kinetic assay was done using Bromodeoxyuridine (BrdU) labeling and Detection Kit I (Roche Products, Hertforshire, UK) following the manufacturer's recommendations. Osteoblasts were seeded at a density of 100 cells per well in $2.5 \mathrm{ml}$ of medium following the same procedure described before. After 1, 3 and 5 days, cells grown on the HA and HA-CNT coating surfaces were incubated at $37{ }^{\circ} \mathrm{C}, 5 \% \mathrm{CO}_{2}$ for 1hour with $10 \mu \mathrm{M}$ BrdU labeling reagent diluted in fresh osteoblast medium. BrdUtreated cells were rinsed with washing buffer and fixed at $-20{ }^{\circ} \mathrm{C}$ for $30 \mathrm{~min}$ in an ethanol fixative solution with $\mathrm{pH} \sim 2.0$. Subsequently, cells were washed with washing buffer and incubated with anti-BrdU solution for $30 \mathrm{~min}$ at $37^{\circ} \mathrm{C}$ in incubator. After the incubation period, cells were washed with washing buffer and then incubated with anti-mouse-Igfluorescein solution for $30 \mathrm{~min}$ at $37^{\circ} \mathrm{C}$. Next, the cells were washed with washing buffer and incubated with $4.5 \mu \mathrm{g} / \mathrm{ml}$ propidium iodide (PI) (Fisher Scientific, Waltham, MA) for 2 min. Cells were washed again with washing buffer and mounted using Citifluor 
Mounting media (Ted Pella). Finally, the stained cells were examined with a Leica Leitz DM RB fluorescent microscope (Leica, Bannockburn, IL) and digital images were captured with a Leica DM 500 camera. The cells proliferated during BrDU exposure only appears green in green-filter, whereas all the cells appear red in red filter. The number of proliferated cells over the total number of cells provides the proliferation rate of osteoblasts.

\subsubsection{Gene Expression Related to Differentiation}

Prior to this experiment, HA and HA-CNT coating surfaces $(10 \mathrm{~mm} \times 10 \mathrm{~mm}$ surface area) were sterilized in the same way described above and then placed into 12well polystyrene petri dishes (Corning, New York). For gene expression studies, osteoblasts were seeded at a density of 1000 cells per well in $2.5 \mathrm{~mL}$ of medium and grown in an incubator at $37^{\circ} \mathrm{C}, 5 \% \mathrm{CO}_{2}$. Medium used is the same as described earlier. After 5 days, cells grown on the pellets were trypsinized for $3 \mathrm{~min}$, neutralized with fresh osteoblast medium, centrifuged $\left(1,000 \mathrm{~g}, 5 \mathrm{~min}\right.$ at $\left.4{ }^{\circ} \mathrm{C}\right)$ and lysed with $500 \mu \mathrm{L}$ of TRIzol Reagent (Invitrogen). Samples were centrifuged $\left(12,000 \mathrm{~g}, 10 \mathrm{~min}\right.$ at $\left.4^{\circ} \mathrm{C}\right)$ again, followed by removing the supernatant and incubating at room temperature for $5 \mathrm{~min}$. Subsequently, $100 \mu \mathrm{L}$ of chloroform was mixed to each supernatant and samples were incubated for $3 \mathrm{~min}$ at room temperature. Samples were centrifuged (12,000 g, $10 \mathrm{~min}$ at $4{ }^{\circ} \mathrm{C}$ ) again, aqueous clear phase was collected. $250 \mu \mathrm{L}$ of cold isopropanol was added to the samples and incubated at room temperature for 10 min followed by centrifuging $\left(12,000 \mathrm{~g}, 10 \mathrm{~min}\right.$ at $\left.4{ }^{\circ} \mathrm{C}\right)$. Following this, $500 \mu \mathrm{L}$ of $75 \%$ cold ethanol was added to 
each substrate; vortexed and centrifuged $\left(7500 \mathrm{~g}, 5\right.$ min at $\left.4{ }^{\circ} \mathrm{C}\right)$ again. Substrates were left to dry and later resuspended in $15 \mu \mathrm{L}$ of RNase-free water. The total RNA was reverse transcribed into cDNA using Maxima First Strand cDNA Synthesis Kit for RTqPCR (Fermentas Life Sciences), following the manufacturer's recommendations. Subsequently, cDNA was used for the real time PCR amplification with SYBRGreen in a ABI 7300 Cycler (Applied Biosystems, Foster City, CA, USA). Differentiation of osteoblasts, cultured for 5 days on HA and HA-CNT pellet substrates was assessed by measuring the expression of Runx2, osteopontin and osteocalcin using the following primers:

Runx2 - Fw CCA CCA CTC ACT ACC ACA CCT ACC,

- Rv CAT GGC GGA AGC ATT CTG GAA GG,

Osteopontin - Fw TGA AAG TGA CTG ATT CTG CGA,

- Rv GGA CGA TTG GAG TGA AAG TGT,

Osteocalcin - Fw AAT CCC CTT GGC TTC TGA CT,

- Rv TCT AGC CCT CTG CAG GTC AT.

Normalization was performed in relation to the transcript levels of GAPDH housekeeping gene using the primers: Fw CCA CCC ATG GCA AAT TCC and Rv TGGGAT TTC CAT TGA TGA CAA G. Transcript levels of cells grown on HA surfaces were used as a reference to calculate expression of cells grown on HA-CNT surfaces. The numbers of fold activation were calculated as following:

$\mathrm{nb}$ fold $=2^{\text {(copies target }- \text { copies reference) }}$. 


\subsubsection{Alkaline Phosphatase Expression Related to Mineralization}

Osteoblast cells were cultured on HA and HA-CNT surfaces following the same procedure as in gene expression study. After 5 days, cells grown on the pellets were trypsinized for $3 \mathrm{~min}$, neutralized with fresh osteoblast medium and centrifuged $(1,000 \mathrm{~g}$, 5 min at $4{ }^{\circ} \mathrm{C}$ ). Subsequently, cells were lysed by $0.1 \%$ Triton (Sigma-Aldrich) and sonicated to destroy cell membranes. Samples were centrifuged $(21,000 \mathrm{~g}, 10 \mathrm{~min}$ at 4 ${ }^{\circ} \mathrm{C}$ ) and supernatant was used for DNA quantification and alkaline phosphatase activity. DNA quantification was done using the Fluorescent DNA Quantification Kit (Bio-rad Laboratories, CA) following the manufacturer's recommendations. $10 \mu \mathrm{L}$ of the supernatant of each sample was transferred to a 96 well plate. $200 \mu \mathrm{L}$ of $2 \mu \mathrm{g} / \mathrm{mL}$ Hoechst dye was alsoadded to each well. Fluorescent emission from samples was quantified with Bio-tek Synergy ${ }^{\mathrm{TM}}$ HT Multi-Detection Microplate Reader, excited at $360 \mathrm{~nm}$ and the emission monitored at $460 \mathrm{~nm}$. To determine alkaline phosphatase activity, $20 \mu \mathrm{L}$ of the prepared supernatant was incubated with $100 \mu \mathrm{L}$ of pnitrophenylphosphatase liquid substrate (Sigma-Aldrich) in a well of a 96 well plate. Samples were incubated at $37{ }^{\circ} \mathrm{C}$ for 3 hours and read in a spectrophotometer. An absorbance at $405 \mathrm{~nm}$ was recorded indicating the alkaline phosphatase activity. Alkaline phosphatase activity was normalized by total DNA amount of each sample.

\subsubsection{Actin Expression Related to Adhesion}

For actin imaging studies, osteoblasts were grown on HA and HA-CNT coatings for 1, 3 and 5 days. After incubation, the coatings were washed with phosphate-buffered 
saline (PBS), fixed with 3.7\% paraformaldehyde for 20 minutes, and permeabilized with $0.2 \%$ Triton X-100 for 5 minutes. Subsequently, cells were washed with PBS and incubated with tetramethylrhodamine B isothiocyanate (TRITC)-conjugated phalloidin (Sigma-Aldrich) diluted 1:2000 in 1\% BSA/PBS for 1hour to visualize F-actin. Cells were washed again with PBS and then mounted using Citifluor Mounting media (Ted Pella). Finally, the samples were examined using a Leica Leitz DM RB fluorescent microscope (Leica, Bannockburn, IL). Digital images were captured with a Leica DM 500 camera.

\subsubsection{Cytotoxicity of Wear Debris and BNNTs with Osteoblasts and Macrophages}

Cytotoxicity of HA/HA-CNT wear debris and BNNTs was evaluated separately using the procedure described here. These two separate studies should not be construed as one. Human osteoblasts were seeded at a density of 1000 cells per well in 6-well polystyrene petri dishes. Murine macrophages (J774 Eclone, provided by Dr. M.A. Barbieri, Biological sciences, Florida International University) were seeded in the same manner in Dulbecco's Modified Eagle's Medium, supplemented with 5\% Fetal Bovine Serum and 1\% Sodium Pyruvate (Atlanta Biologicals, Lawrenceville, GA), $100 \mathrm{UI} / \mathrm{ml}$ of penicillin and $100 \mu \mathrm{g} / \mathrm{ml}$ of streptomycin (MP Biomedicals, Irvine, CA) at $310 \mathrm{~K}\left(37^{\circ} \mathrm{C}\right.$ ). Osteoblasts and macrophages were allowed to attach to the plastic surface for 24 hours, after which the medium was replaced by a fresh medium with HA and HA-CNT wear debris at $1 \mu \mathrm{g} / \mathrm{ml}$ concentration (typically $2 \mathrm{ml}$ of medium were added to the 3 experimental wells). A similar study with BNNTs added to medium was used for checking cytotoxicity of BNNTs. Prior to mixing in the culture medium; the wear 
particles (or BNNTs) were washed with 95\% ethyl alcohol, washed 3 times with fresh medium and left for 3 hours in a hood under UV light. Both types of cells were cultured for 3 days with the wear debris (or BNNTs) prior to the cytotoxicity test, which was performed with the CytoTox 96 Non-Radioactive Cytotoxicity Assay kit (Promega, Madison, WI) following the manufacturer's recommendations. Cytotoxicity test, performed in this study, isa colorimetric assay that quantitatively measures lactate dehydrogenase (LDH), a stable cytosolic enzyme released into the culture medium upon lysis of cell. Released LDH in culture supernatant was measured with a coupled enzymatic assay which results in the formation of a red formazan product that can be measured at $490 \mathrm{~nm}$ with a spectrophotometer and is proportional to the number of cells lysed. 'Student $\mathrm{t}$ ' test was performed to find out the $95 \%$ confidence interval. The absorbance value of the culture medium without any cells or debris (or BNNTs)was considered as the background and subtracted from the experimental absorbance values obtained for the cells cultured with and without debris (or BNNTs). LDH absorbance was obtained for cells that died over the 3 day experimental period. Cells that remained alive by the end of the experiment were detached and lyzed for the release of LDH, which was then measured.

\subsubsection{In-Vivo Evaluation of Plasma Sprayed HA-CNT Coating}

In-vivo study of biocompatibility for plasma sprayed HA-nanotube composite coating was performed by implanting HA-CNT coated titanium alloy (Ti-6Al-4V) rods in rat's femur. The experiments related to implantation in rat, retrieving the implants and their characterization for biocompatibility through TEM and histological 
characterization were carried out by our collaborating researchers at Dr. Nadia Jessel's Laboratory in University of Strasbourg, France. The results are included in this dissertation for the sake of completeness in terms of biocompatibility studies intended for orthopedic applications.

\subsubsection{Implantation using Rat Model}

Three types of implants were used for this research, viz., (i) titanium rods without any coating; (ii) with HA coating and (iii) with HA-CNT coating. Titanium rods are 10 $\mathrm{mm}$ long and $2.5 \mathrm{~mm}$ in diameter. Titanium rods were plasma sprayed to deposit a coating of 100-150 $\mu \mathrm{m}$ thickness. A total of 6 male Wistar rats from C. River (520-630 grams, 17 weeks old) were used for this study. Coated/uncoated titanium rods were implanted inside distal part of external condyle of the femoral of rats. For all in vivo studies, animals were acclimatized for a minimum of two weeks prior to the experimentation. All procedures were performed with prior received ethical approval and carried out in accordance with the regulations laid down for the animals. Each animal received only one type of implant, in order to avoid systemic responses.

The rats were anesthetized with an intraperitoneal injection of ketamine (75 $\mathrm{mg} / \mathrm{Kg})$ and xylasine $(10 \mathrm{mg} / \mathrm{kg})$. The surgical procedure involves removal of the hair over the external part of right lower limb via shaving and cleaning. The animals were placed on ventral decubitus, with external rotation and abduction of the lower limb. With strict aseptic conditions, after skin incision, patella and quadriceps tendon were released. A circular bone defect (hole) of $3 \mathrm{~mm}$ diameter was made on external femoral condyle using an electrical drill with a sterile round bur under irrigation of sterile normal saline. 
Through the bony defect, implant rods were completely introduced inside the distal femoral bone. Patella and quadriceps tendon were replaced without sutures and the skin was closed with non-absorbable sutures.

All animals were examined on a weekly basis after implantation, for any sign of infection or discomfort on the lower limb for a period of one month. One month after implantation; rats were sacrificed with an overdose of ketamine and xylasine. For different ex vivo analysis, all parts of the femoral bone, from femoral head to knee joint, were excised and cleaned off from the soft tissue. The bones with the implants were sectioned to a thickness of 1-2 $\mathrm{mm}$, with a low speed diamond saw for further characterization.

\subsubsection{Histology of Retrieved Bone with Implant}

For histological observations, the sections of rat bones were fixed in neutral formalin solution of $20 \%$, then decalcified inside acetic acid solution of $10 \%$ for 4 days and embedded in paraffin. Ultrathin sections of $70 \mu \mathrm{m}$ thickness were cut using ultra microtome. These sections were stained with mallory coloration and histologically analyzed by light microscopy at different magnifications $(5,10,20$ and 40X).

\subsubsection{TEM Study of Retrieved Bone with Implant}

For TEM study, bone sections were fixed in $4 \%$ buffered paraformaldehyde solution, decalcified inside acetic acid solution of $10 \%$ during 4 days, post fixed with $1 \%$ osmium tetroxide in $0.1 \mathrm{M}$ cacodylate buffer for 1 hour at $4{ }^{\circ} \mathrm{C}$, dehydrated through graded alcohol and embedded in Epon 812. Ultrathin sections $(70 \mu \mathrm{m})$ were cut with a 
diamond knife and stained with uranyl acetate and lead nitrate and observed with a Morgagni 268 transmission electron microscope.

\subsection{Evaluation of Osseointegration}

Osseointegration is evaluated for the HA-nanotube composites through three different methods, viz.(i) quantifying the adhesion strength of osteoblasts on plasma sprayed HA and HA-CNT coating surfaces; (ii) measuring the gradient of elastic modulus through implant-bone interface for the retrieved femur bones from animal study and (iii) determining the apatite formability on BNNTs when immersed in simulated body fluid. The detailed account of the experimentations, involved in all three of these studies, is described below.

\subsubsection{Quantifying Adhesion of Osteoblasts on Composite Surface: Nano-Scratch}

\section{Technique}

Plasma sprayed HA and HA-CNT coated surfaces $(10 \mathrm{~mm}$ x $10 \mathrm{~mm}$ area) were washed with $95 \%$ ethanol followed by washing three times with fresh medium and left for $3 \mathrm{~h}$ in a hood under UV light before cell culture. Permanox plastic chamber slides (Fisher Scientific, Pittsburgh, PA) were also used for cell culture for the comparison purpose. HA and HA-CNT coatings were polished with alumina powder suspension (up to $0.3 \mu \mathrm{m}$ size) to have similar surface roughness. The surface roughness of HA and HACNT coatings, measured using TR200 instrument (Micro Photonics Inc., Irvine, CA), is $80( \pm 25)$ and $70( \pm 15) \mathrm{nm}$ respectively. The surface roughness $\left(\mathrm{R}_{\mathrm{a}}\right)$ of permanox is 50 $( \pm 10) \mathrm{nm}$. Human osteoblasts were cultured on HA and HA-CNT coated substrates and 
permanox plastic chamber slides using the same procedure described in section 3.6. Nano-scratch studies for adhesion force measurement of osteoblast were performed using 2D scratch mode of Hysitron Triboindenter TI 900 with a load resolution of $1 \mathrm{nN}$ and lateral displacement resolution of $4 \mathrm{~nm}$. All the tests are performed within 1 hour of collecting the substrates with cells from the incubator. Osteoblasts were kept immersed in the culture medium during the nano-scratch study. A long, conospherical fluid cell tip of $1 \mu \mathrm{m}$ radius was used to minimize the meniscus forces caused by the culture medium. A $15 \mu \mathrm{m}$ long scratch is made, with $15 \mu \mathrm{N}$ normal force and $0.5 \mu \mathrm{m} / \mathrm{s}$ velocity, starting on the substrate $(\sim 5 \mu \mathrm{m}$ away from cell) and going towards the cell to push and detach it from the surface. Scratch tests were performed for more than 8 osteoblasts on each substrate.

The schematic of the technique is presented in figure 3.3a, which includes a cell on the substrate with indenter at different stages of scratching. Corresponding regions are also labeled in a schematic load vs. displacement curve presented in figure 3.3b. The adhesion force was measured for osteoblasts cultured for 1,3 and 5 days on permanox plastic slides, HA and HA-CNT coated surfaces. Scratches were also made on all surfaces without any cell to evaluate the effect of the substrate and culture medium in the same loading condition. The value of the lateral force on the bare substrate (without cell) was subtracted from the lateral force curve obtained by nano-scratch of the cell (figure 3.3b) to calculate the adhesion force of a cell. The area under the lateral force vs. displacement curve for the cell was calculated to estimate the adhesion energy of a cell (figure 3.3b). This technique enables an accurate quantification of the cell adhesion on a substrate avoiding errors introduced due to substrate and culture medium. 
(a)
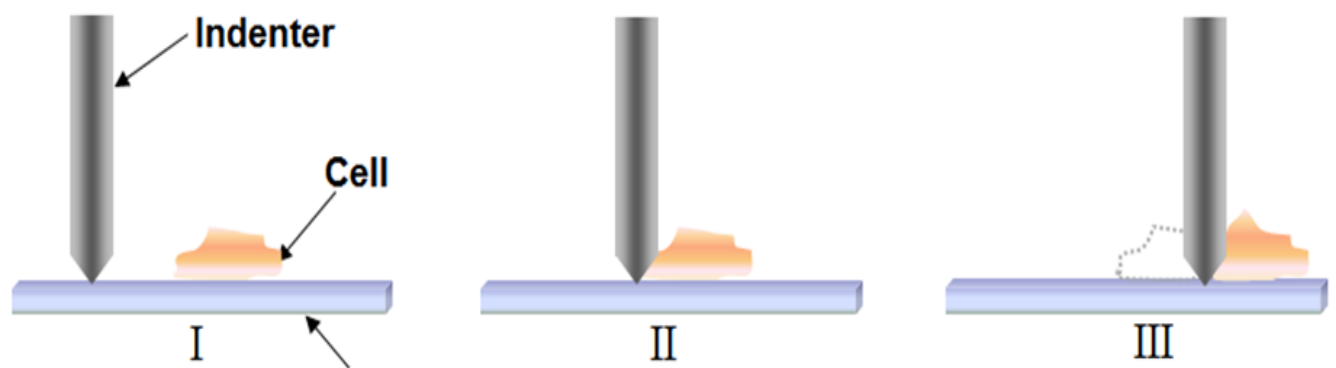

\section{Substrate}

(b)

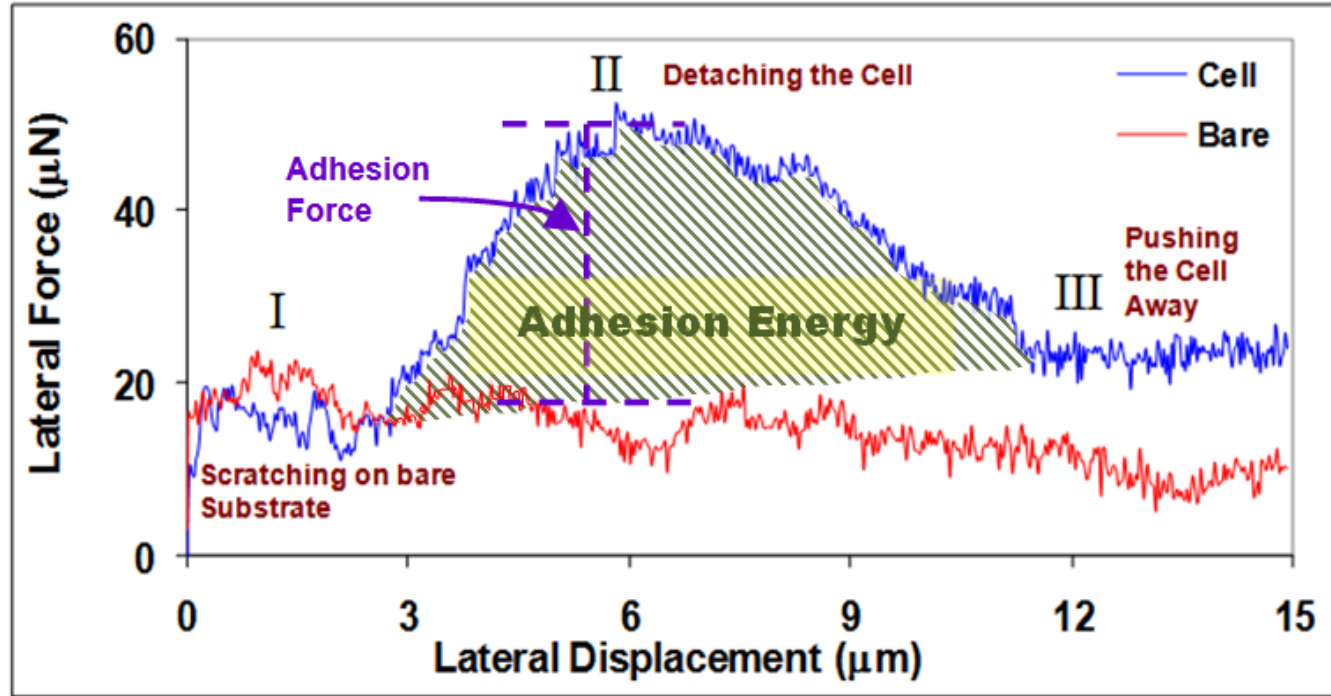

Figure 3.3: (a) Schematic of the nano-scratch technique for measuring adhesion of individual osteoblast cell on substrate. (b) Representative lateral (shear) force vs. displacement curves for nano-scratches on HA-CNT substrate with osteoblast grown for 3 days. The curves show the variation in the lateral force required to make a scratch in presence of a cell and on bare substrate. Different regions of the curve are correlated with the stages of scratch in schematic (a) with roman numbers (I, II and III). The shaded area denotes the adhesion energy and the height of the hump shows adhesion force of an osteoblast.

\subsubsection{Determining Mechanical Compatibility of Implant-Bone Interface: Modulus} Mapping Across Interface using Nano-DMA Technique 
Distribution and gradient of elastic modulus (E) of at bone-implant interface in the retrieved implants was evaluated using dynamic nanoindentation in order to check the mechanical compatibility of the implant with bone. The cross sections of the retrieved implant bones were cleaned by removing the attached tissues. Figure 3.4 (a) shows a cut and cleaned cross section of retrieved femur with embedded implant. The elastic modulus across the implant/coating/bone interfaces was determined using 'modulus mapping' in nanodynamic analysis (nano DMA) mode. The tests were carried out on the samples hydrated in formalin. Hysitron Triboindenter is utilized to produce 2D maps of elastic modulus of the sample surface. Figure 3.4 (b) shows the optical micrograph of cross section of femur with HA-CNT coated Ti implant, indicating the regions where modulus mapping was carried out.

In order to prepare the sample surface for nanoindentation experiments, implant cross-sections were polished using wet cloth without any abrasive particles. Abrasive particles were not used to prevent the impregnation of bone surface with the harder particles, which could influence the mechanical properties. A Berkovich indentation probe of $100 \mathrm{~nm}$ radius was used for the measurements. The static and dynamic loads for the measurements were $3 \mu \mathrm{N}$ and $1.5 \mu \mathrm{N}$ respectively with an applied frequency of 200 Hz. Each 2D modulus map presented in this study covers a $5 \mu \mathrm{m}$ X $5 \mu \mathrm{m}$ area on the sample surface that includes a matrix of equally spaced 256 X 256 points, resulting in 65536 measurements in each scanning. At least three areas of $5 \mu \mathrm{m}$ X $5 \mu \mathrm{m}$ were used for 2D modulus mapping from each region to get the representative value of the elastic modulus. During the measurement of elastic modulus (E), the bone samples were kept hydrated by adding formalin using a dropper to carry out the tests in wet condition. 


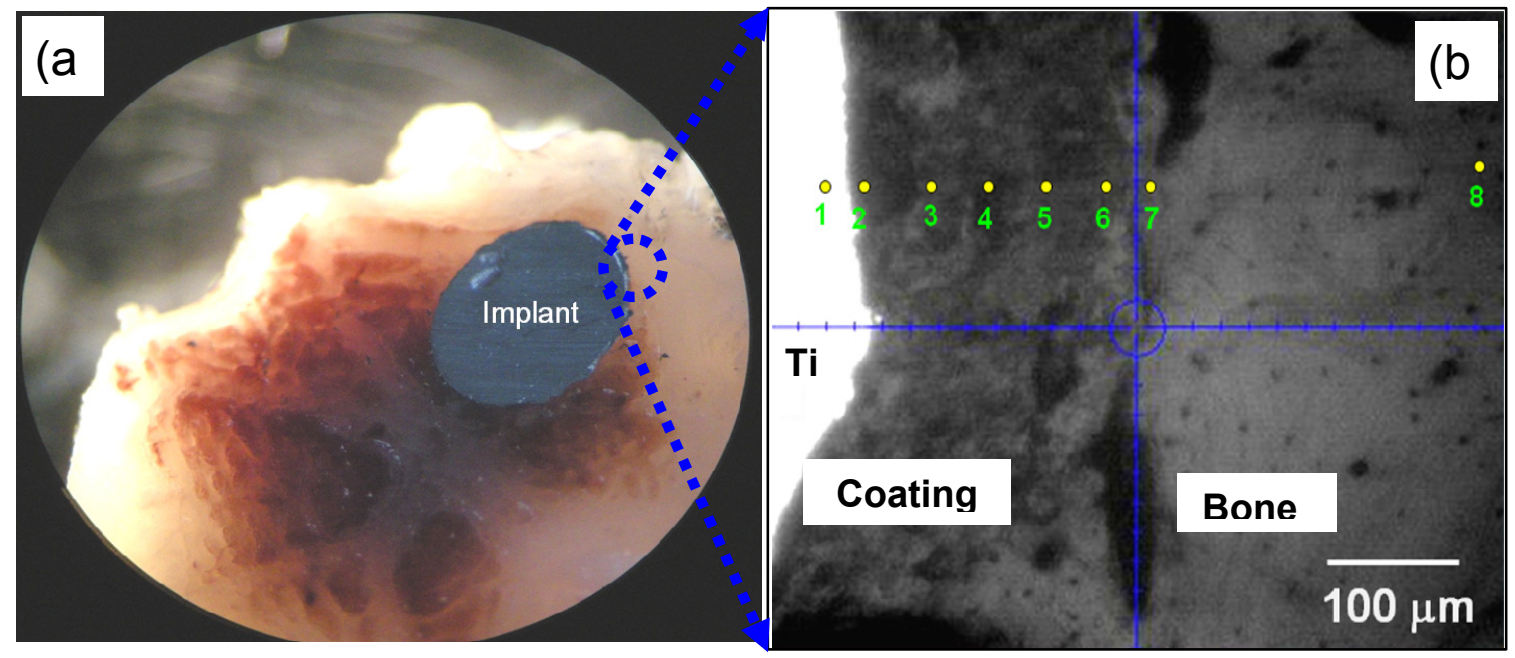

Figure 3.4: (a) Semi-thin $(1-2 \mathrm{~mm})$ section cut from the rat's bone with implant embedded inside. (b) Optical cross-sectional micrograph of the retrieved implant showing Ti substrate, HA/HA-CNT coating and bone with marked regions for modulus mapping studies. Position 1 denotes the region in titanium part of the implant near coating. Positions $2-6$ are equally placed locations through the thickness of HA/HACNT coating starting from titanium side and going towards the bone. Position 7 is in the newly grown bone along the implant surface and position 8 is in a distant region that represents the normal bone.

\subsubsection{Apatite Formability of Boron Nitride Nanotube by Simulated Body Fluid Immersion}

Apatite formability of BNNTs was studied by immersing them in simulated body fluid (SBF) to determine their osseointegration ability. We did not carry out similar study with CNTs as such studies are already available in the literature [16-18]. Simulated body fluid was prepared following Kokubo's recipe given in table 3.2. BNNTs were immersed in SBF in glass vials and kept at physiological temperature of $310 \mathrm{~K}\left(37^{\circ} \mathrm{C}\right)$ and $5 \% \mathrm{CO} 2$ 
inside incubator for 7, 14 and 28 days. Soaking periods were selected based on the fact that most of the new bone formation starts between 2-3 weeks during post-implantation healing. Thus, it is effective to check the apatite precipitation ability of a surface during 1-4 weeks. After the soaking period, the precipitates were separated from SBF by centrifuging and washed with deionized water for 3 times. The precipitates were dried at the ambient temperature. Three different precipitate samples were collected after 7, 14 and 28 days for further characterization. These samples were analyzed using SEM, EDS, Raman Spectroscopy and HRTEM to understand the apatite precipitation mechanism on BNNT. The results of the experiments are presented and analyzed in the following chapter 4 .

\subsection{References}

1. K. Balani, S.R. Bakshi, Y. Chen, T. Laha, A. Agarwal. Role of powder treatment and carbon nanotube dispersion in the fracture toughening of plasma sprayed aluminum oxide-carbon nanotube nano-composite. 2007, J. NanoSci. Nanotech., Vol. 7, pp. 1-10.

2. A.A. Campbell. Bioceramics for implant coatings. 2003, Mater. Today, Vol. 6, pp. 26-30.

3. J.L. Xu, K.A. Khor, J.J. Sui, W.N. Chen. Preparation and characterization of a novel hydroxyapatite/carbon nanotubes composite and its interaction with osteoblast-like cells.2009, Mater. Sci. Eng. C, Vol. 29, pp. 44-49.

4. S.K. Sarkar, M.H. Youn, I.H. Oh, B.T. Lee. Fabrication of CNT reinforced HAp composites by spark plasma sintering. 2007, Mater. Sci. Forum, Vols. 534-536, pp. 893-896.

5. M.D. Abramoff, P.J. Magelhaes PJ, S.J. Ram. Image processing with ImageJ.2004, J. Biophotonics Int., Vol. 11, pp. 36-42.

6. W.C. Oliver, G.M. Pharr. An improved technique for determining hardness and elastic modulus using load and displacement sensing indentation experiments.1992, J. Mater. Res., Vol. 7, pp. 1564-1583. 
7. G.R. Anstis, P. Chantikul, B.R. Lawn, D.B. Marshall. A critical evaluation of indentation technique for measuring fracture toughness: I. Direct crack measurements. 1981, J. Am. Ceram. Soc., Vol. 64(9), pp. 533-538.

8. T. Kokubu, H. Kushitani, S. Sakka, T. Kitsugi, T. Yamamuro. Solutions able to reproduce in vivo surface-structure changes in bioactive glass-ceramic A-W.1990, J. Biomed. Mater. Res., Vol. 24, pp. 721-734.

9. Image Processing Software for Microscopy, version 4.5.1, Image Metrology, DK2970 Hørsholm, Denmark.

10. S.R. Bakshi, D. Lahiri, R. Patel, A. Agarwal. Nanoscratch behavior of carbon nanotube reinforced aluminum coatings. 2010, Thin Solid Films, Vol. 518, pp. 1703-1711.

11. G. Kotzar, M. Freas, P. Abel, A. Fleischman, S. Roy, C. Zorman, J.M. Moran, J. Melzak.Evaluation of MEMS materials of construction for implantable medical devices.2002, Biomaterials, Vol. 23, pp. 2737-2750.

12. http://www.fda.gov/medicaldevices/deviceregulationandguidance/guidancedocu ments.

13. http://www.astm.org/Standards/medical-device-and-implant-standards.html.

14. http://www.iso.org/iso/iso_catalogue/catalogue_tc/catalogue_detail.htm?csnum ber $=364$.

15. http://www.iso.org/iso/iso_catalogue/catalogue_tc/catalogue_detail.htm?csnum ber $=4$.

16. S. Aryal, S.R. Bhattarai, K.C. R. Bahadur, M.S. Khil, D.R. Lee, H.Y. Kim. Carbon nanotubes assisted biomimetic synthesis of hydroxyapatite from simulated body fluid. 2006, Mater. Sci. Eng. A, Vols. 426, pp. 202-207.

17. T. Akasaka, F. Watari, Y. Sato, K. Tohji. Apatite formation on carbon nanotubes. 2006, Mater. Sci. Eng. C, Vol. 26, pp. 675-678.

18. J. Beuvelot, C. Bergeret, R. Mallet, V. Fernandez, J. Cousseau, M.F. Basle, D. Chappard. In vitro calcification of chemically functionalized carbon nanotubes.2010, Acta Biomater., Vol. 6, pp. 4111-4117. 


\subsection{RESULTS AND DISCUSSION}

The objective of this research is to explore the overall potential of HA-nanotube composites for orthopedic applications. This chapter is the nucleus of the dissertation, which presents a detailed description of the results obtained through experimentations and scientific analysis of the outcomes.

\subsection{Microstructural Evolution, Mechanical and Tribological Performance of Composites}

One of the important issues in this research is to tailor the mechanical and tribological properties of HA by reinforcing with nanotubes. Addition of nanotubes in HA modifies its microstructure and thus controls its performance. Following subsections presents a detailed description on the role of CNT and BNNT in modifying the microstructure and performance (mechanical and tribological) of HA based composite structures.

\subsubsection{Hydroxyapatite-Carbon Nanotube Composite}

In this study, HA-CNT composites are synthesized as plasma sprayed coating and spark plasma sintered structures. Due to the huge difference in the consolidation techniques, the two composites show vastly different microstructure. The role played by $\mathrm{CNT}$ in evolution of the microstructure in these two cases is also very different, resulting in different level of contribution towards strengthening and toughening, as discussed below. 


\subsubsection{Composite Coating by Plasma Spraying}

HA-CNT composite coating is deposited by plasma spraying of spray dried powder on Ti alloy substrate. HA coating prepared using same plasma spray parameters is used as the control sample for studying the effect of CNT on HA based coating. The polished cross-section of HA-CNT coating reveals typical signatures of plasma sprayed structure, such as wavy splats, lamellar cracks and porosity (figure 4.1b). Density of HA and HA-CNT coatings, measured using Archimedes principle and water as immersion medium, is 93.7 and $94.0 \%$ TD respectively. The densities are comparable for HA and HA-CNT coatings. This observation indicates no negative role played by CNT in consolidation of HA by plasma spraying, which was a concern during conventional sintering of HA, as CNTs were reported to hold grains apart and hinder closure of porosity [1]. The probable reason could be rapid solidification during plasma spraying as well as good wetting of CNT with molten ceramic [2] due to momentary high temperature during plasma spraying.

\subsection{Distribution and Structural Integrity of Carbon Nanotube in Plasma Sprayed Coating}

Fracture surface of the HA-CNT composite coating (figure 4.1c) shows uniformly dispersed and embedded CNTs protruding out of HA matrix. The homogeneous dispersion of CNT in HA at powder stage in spray dried agglomerates (figure 4.1a) is carried forward to the plasma sprayed coating. Thus, spray drying route for composite powder preparation is an effective method for homogeneous dispersion of CNTs, which is required to obtain uniform mechanical properties. High magnification SEM image of 
the HA-CNT coating reveals attachment of fine HA particles on the individual CNT surface (figure 4.d). Higher thermal conductivity of CNT makes the HA nucleation and precipitation easier on the CNT surface [3].
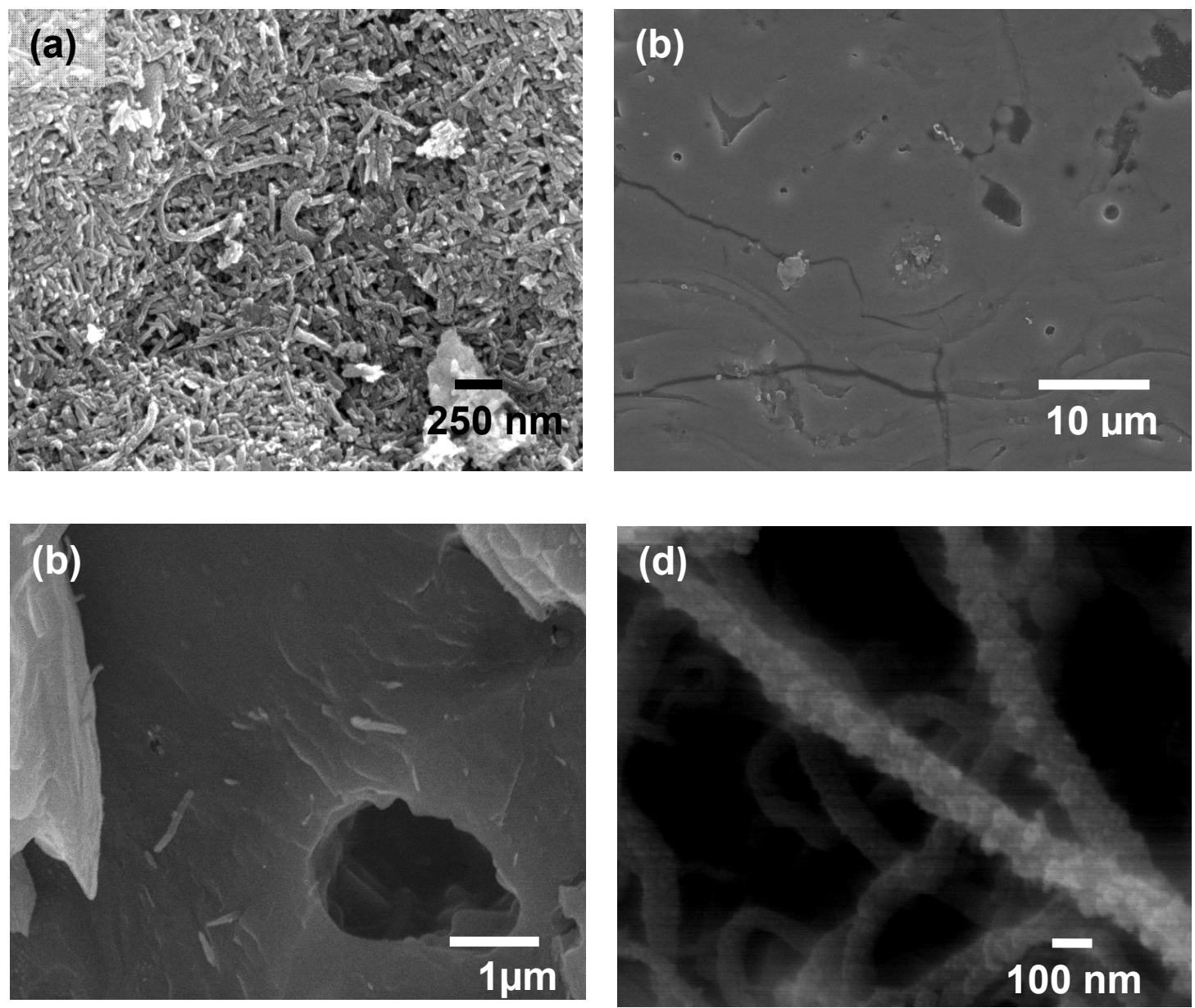

Figure 4.1: (a) High magnification SEM image of spray dried HA-CNT agglomerate showing homogeneous distribution of CNTs in HA nano-rods, (b) cross-section of plasma sprayed HA-CNT coating showing the splat structure, cracks and porosity, (c) fracture surface showing homogeneous distribution of CNT in HA matrix and (d) precipitation of HA crystals on CNTs. 
High temperature exposure during plasma spraying makes it necessary to check the survival of CNT structure in the final coating. Micro Raman spectrum (figure 4.2) of HA-CNT powder and coating shows the presence of D and G peaks coming from the C-C bond CNT. The appearance of G peaks at around $1580 \mathrm{~cm}^{-1}$ is the signature of highly pure graphitic structure whereas D-peak (at around $1350 \mathrm{~cm}^{-1}$ ) originates from a disorderinduced mode in graphite. The signature from the Raman spectrum along with the tubular structure visible in the fracture surface (figure 4.1c) ensures the presence of CNT in the plasma sprayed composite coating. Even after being exposed to several thousand degrees, the survival of CNTs during plasma spraying could be justified for the following reasons. The short exposure (milliseconds) to high temperature is not sufficient for the oxidation and destruction of CNTs. In addition, the ceramic melts during plasma spraying and forms protective coating on the CNT surface $[2,4]$. The inert carrier gas (argon) also creates a shroud over the sprayed particles, which acts as a shield against oxidation. A reduction in the peak intensity ratio $\left(\mathrm{I}_{\mathrm{D}} / \mathrm{I}_{\mathrm{G}}\right)$ in plasma sprayed coating $(0.39)$ from powder (0.60) is observed, which indicates a decrease of defect density in CNT in the coating. Similar observations have been reported by Keshri et al. for plasma sprayed aluminaCNT coatings [5]. The decrease in defects in CNTs has been attributed to the increasing degree of graphitization in CNTs due to high temperature exposure. Decrease in the strain energy during annealing decreases the interlayer spacing between CNT walls. Consequently, the defect density in CNT also decreases during annealing [6-7]. 


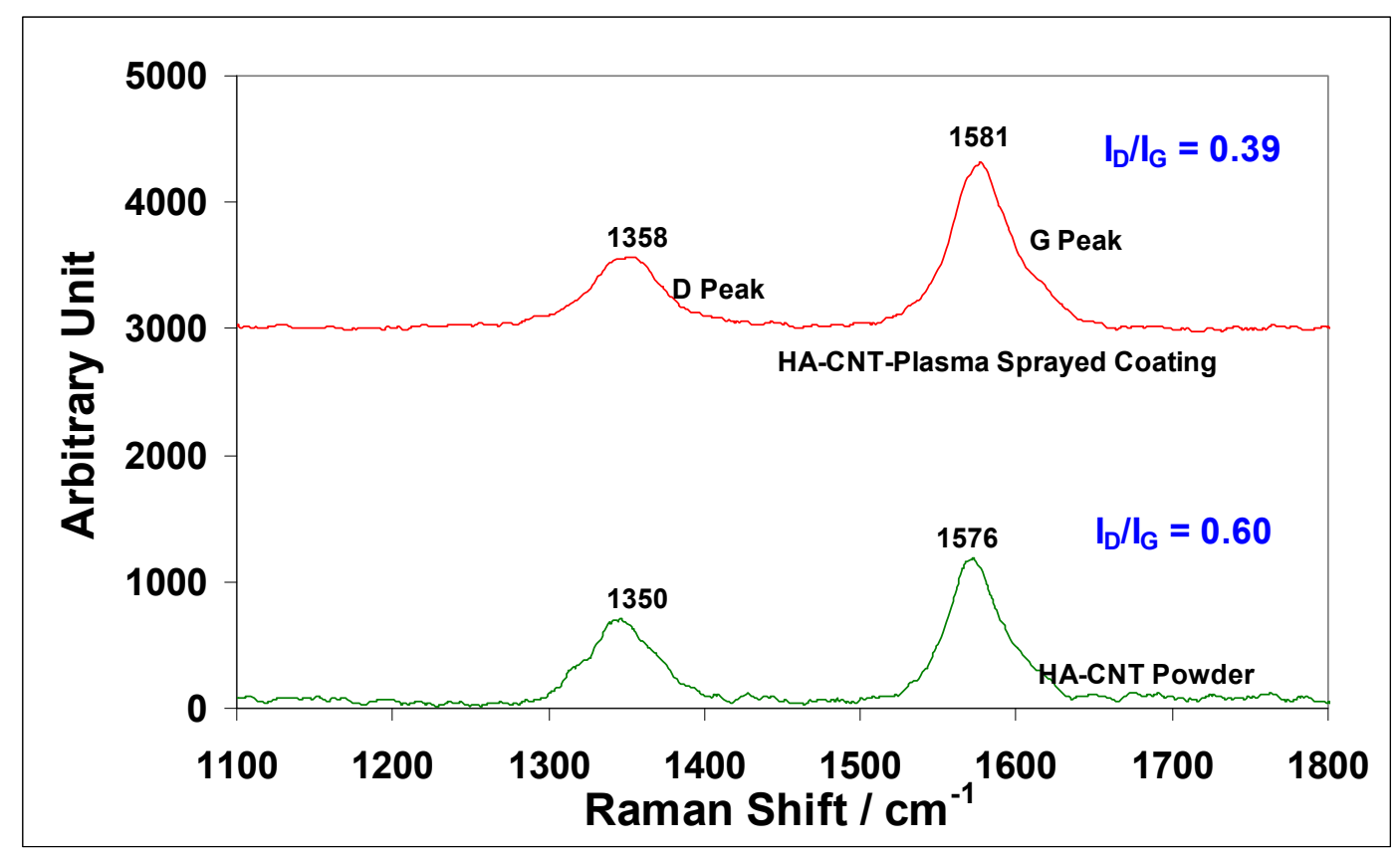

Figure 4.2: Raman spectra of HA-CNT powder and plasma sprayed HA-CNT coating showing D and G peaks of CNT.

\subsection{Phase Stability and Crystallinity of HA in Plasma Sprayed Coating}

The evolution/transformation of phases and crystallinity of HA, as a result of rapid consolidation during plasma spraying, can be evaluated comparing the X-ray diffraction patterns of HA and HA-CNT powder and coatings, as presented in figure 4.3. The patterns show presence of HA phase mainly, with some graphite peaks in HA-CNT compositions, which are overlapped with HA peaks. XRD patterns indicate minimal dissociation of HA to product phases. During plasma spraying, HA is exposed to much higher temperature than its dissociation point $\left(\sim 1200^{\circ} \mathrm{C}\right)$. But the exposure is for milliseconds, which might have restricted dissociation of HA in the plasma sprayed structure. Amorphous humps are present in both HA and HA-CNT coatings, due to some 
degree of amorphisation introduced during rapid cooling. But, the hump is smaller in case of HA-CNT than in HA. Increased crystallinity in HA-CNT system is attributed to higher thermal conductivity of CNTs ( $2980 \mathrm{~W} / \mathrm{mK}$ [8]) compared to HA(1.25 W/mK [9]), which helps in nucleation and precipitation of HA. Higher heat capacity of CNT $(480 \mathrm{~J} / \mathrm{kgK}$ [10]) can absorb more heat when exposed to the plasma and then higher thermal conductivity of CNT can create a thermal pool around while cooling. Both of these phenomenon provides an enhanced cooling time to the HA matrix in the neighboring region of CNTs, which helps in its recrystallization. SEM image presented in figure 4.1d shows the evidence of nucleation and precipitation of fine HA crystals on CNTs during plasma spraying.

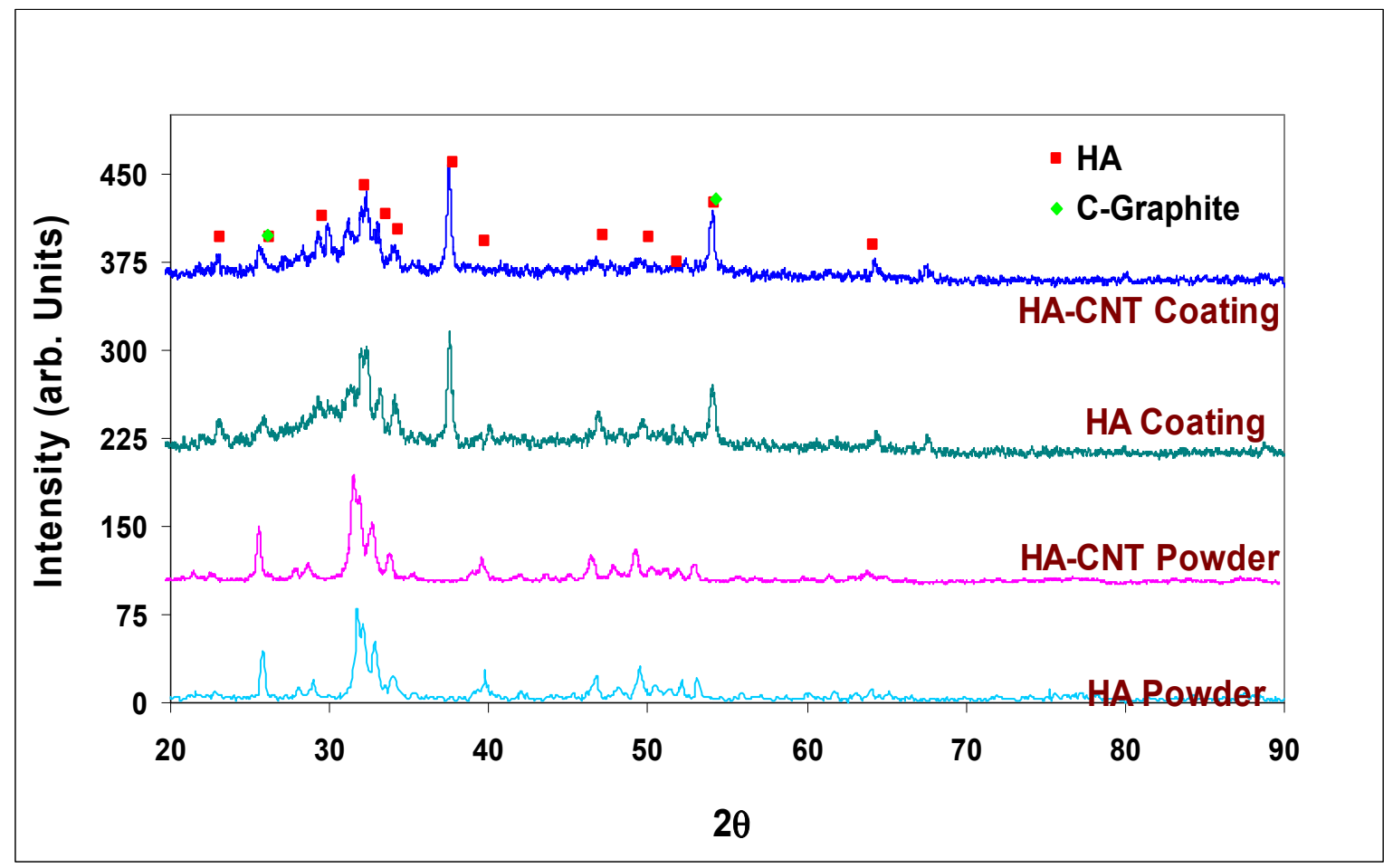

Figure 4.3: X-ray diffraction patterns for $H A$ and $H A-C N T$ powders and plasma sprayed coatings. 
Presence of a very sharp (220) peak at $38.15^{\circ}$ for coating, which is absent in powder and (a very low intensity peak according to JCPDS data of HA), indicates the formation of some kind of texture in the coating, which could be due to exposure at high temperature as well as the stress related to thin [11-13].

\subsection{Elastic Modulus and Fracture Toughness of Plasma Sprayed HA-CNT}

\section{Coating}

One of the main intent for adding CNT to HA is to improve its elastic modulus (E) and fracture toughness $\left(\mathrm{K}_{\mathrm{IC}}\right)$. Elastic modulus $(\mathrm{E})$ of the coatings has been evaluated using nanoindentation, which is a well-established technique for brittle ceramic coatings [14]. Plasma sprayed composite coating is heterogeneous in nature. Single indent provides localized mechanical properties. Hence, more than 100 indents were made at randomly chosen regions throughout the polished cross-section of the coatings to obtain the bulk elastic modulus. Figure 4.4a shows a typical load-displacement curve obtained through nanoindentation of $\mathrm{HA}$ and HA-CNT composite coatings. The statistical distribution of the elastic modulus (figure 4.4b), measured from the individual indent, thus provides the elastic modulus of the composite coating at macro-scale length. The elastic modulus for $\mathrm{HA}$ and $\mathrm{HA}-\mathrm{CNT}$ coating is $51 \pm 4 \mathrm{GPa}$ and $88 \pm 10 \mathrm{GPa}$ respectively. A $72.5 \%$ improvement in elastic modulus is attributed to the high $\mathrm{E}$ value of $\mathrm{CNT}$ and effective reinforcement of $\mathrm{CNT}$ in HA matrix. Effective reinforcement is justified in terms of uniform distribution of CNT in HA matrix (figure 4.1c) and good interfacial bonding of each CNT with the HA matrix. Figure $4.4 \mathrm{c}$ shows a protruded CNT 
from the HA matrix. Strong bonding at HA-CNT interface is inferred from the absence of any crack or gap at the interface.

(a)

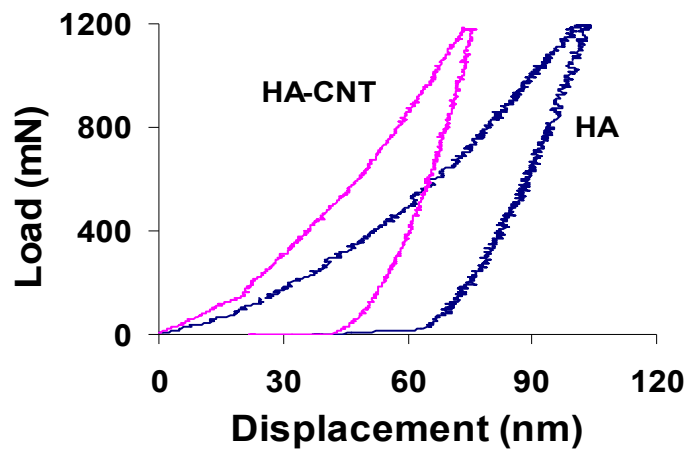

(b)

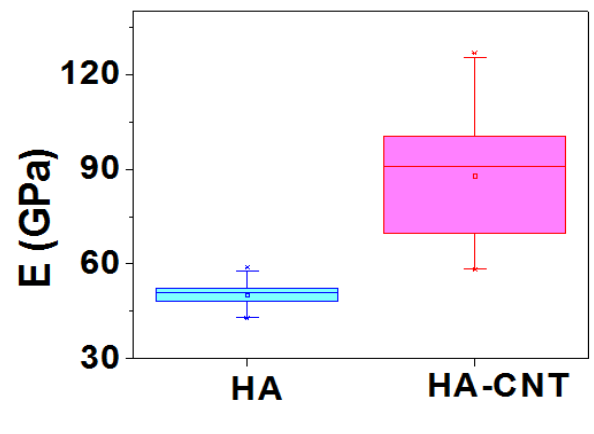

\section{(c)}

$100 \mathrm{~nm}$

Figure 4.4: (a) Load vs. displacement curves for HA and HA-CNT coatings obtained by nanoindentation, (b) statistical distribution of $E$ value in HA and HA-CNT, (c) protruding CNT from HA-CNT fracture surface showing absence of crack or gap at matrix/CNT interface.

Fracture toughness $\left(\mathrm{K}_{\mathrm{IC}}\right)$ of the coatings is evaluated using the length of the radial crack generated in microindentation. The impression of Vickers indent was observed under SEM for an accurate measurement of the radial crack lengths. Figure 4.5 shows SEM images of indents on HA and HA-CNT coatings. The microhardness of HA and 
HA-CNT coatings is $1.30 \pm 0.01 \mathrm{GPa}$ and $2.43 \pm 0.02 \mathrm{GPa}$ respectively. Fracture toughness of the composite structures has been evaluated using Anstis' equation [15] expressed as:

$$
K_{I C}=0.016\left(\frac{E}{H}\right)^{1 / 2} \frac{P}{c^{3 / 2}}
$$

where, $\mathrm{P}$ is the applied load, $\mathrm{E}$ is the elastic modulus, $\mathrm{H}$ is the Vickers hardness and $\mathrm{c}$ is the radial crack length (measured from the center of the indent). E values, measured by nanoindentation, were used to compute $\mathrm{K}_{\mathrm{IC}}$ of the composite. The radial crack length in HA was more than $125 \mu \mathrm{m}$. But, the radial cracks are of much smaller length $(\sim 50 \mu \mathrm{m})$ in HA-CNT coating. The decrease in radial crack length causes a higher fracture toughness of $3 \mathrm{MPa} . \mathrm{m}^{0.5}$ for HA-CNT as compared to $0.64 \mathrm{MPa}^{0.5}$ for HA.
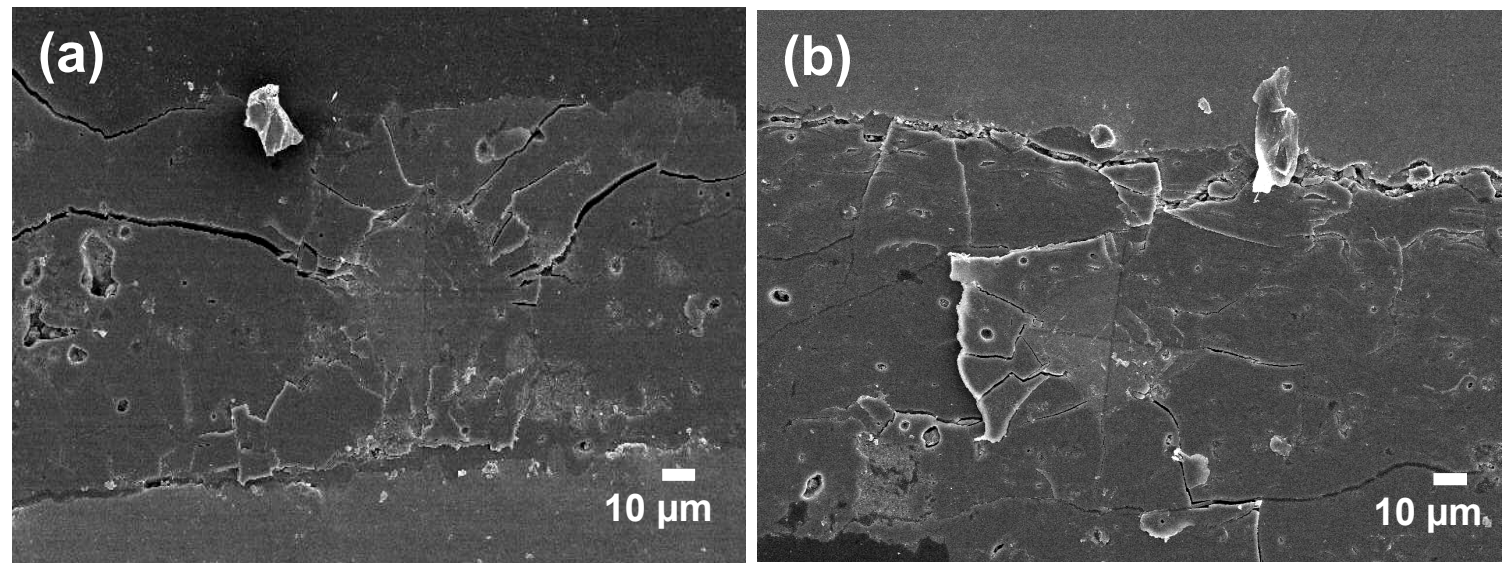

Figure 4.5: SEM images of radial cracks emerging from microindents on (a) HA and (b) HA-CNT coatings.

CNT addition improved the fracture toughness of HA coating by $\sim 350 \%$ which is attributed to (i) higher E value with CNT addition and (ii) crack bridging effect offered by CNT. Figure 4.6 shows high magnification SEM image of CNT bridges within a radial 
crack generated during microindentation on HA-CNT coating. CNTs act as bridges restricting the crack propagation. Hence, crack propagating through HA matrix gets restricted when comes in the vicinity of a CNT reinforcement.

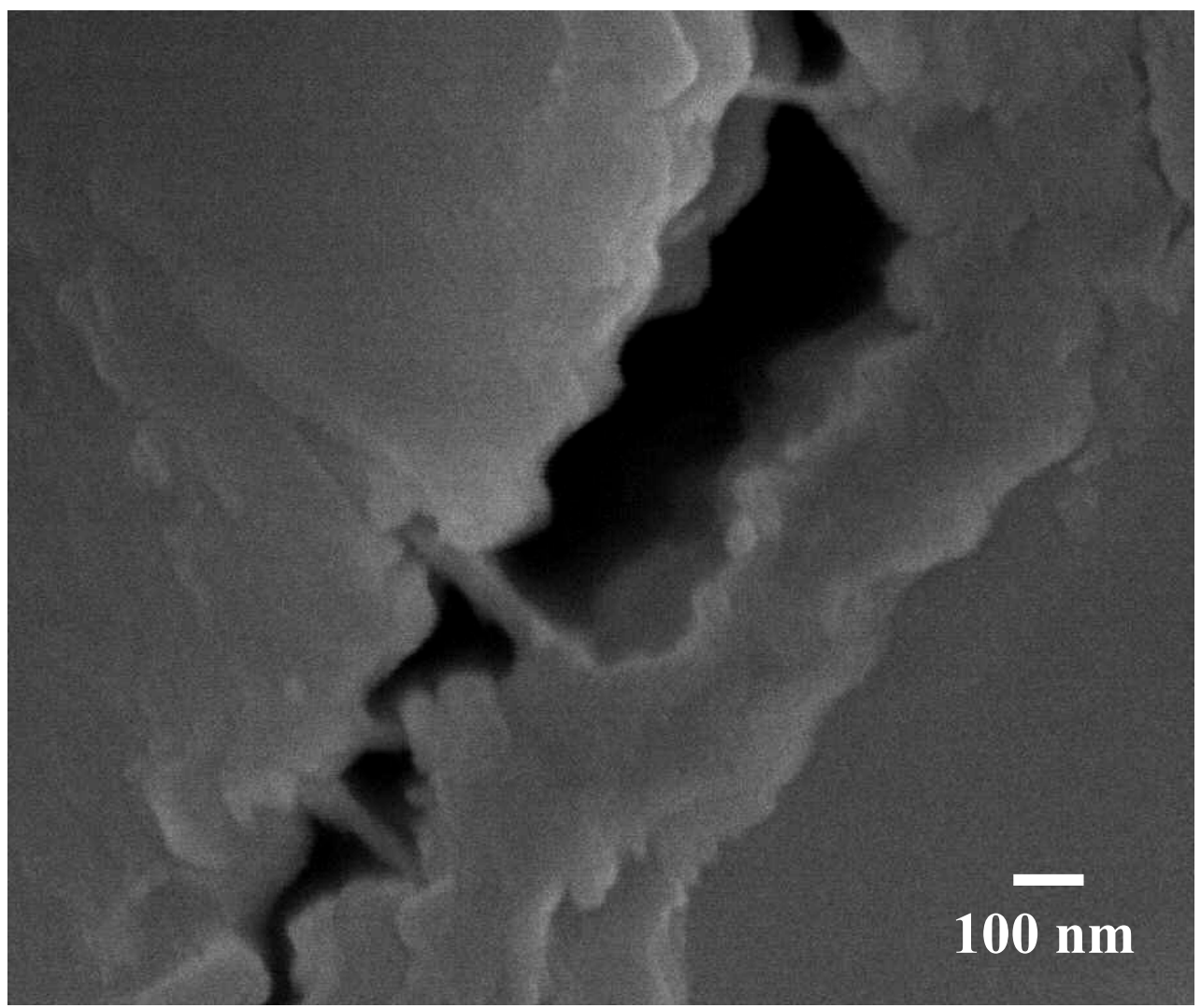

Figure 4.6: CNT bridges offering crack propagation resistance in HA-CNT coating.

\subsection{Wear Behavior of Plasma Sprayed HA-CNT Coating}

Improvement in the wear resistance of HA is of concern due to the biological response of wear debris in-vivo. Generation of more volume of debris results in higher amount of foreign element in contact with the bone cells and in body fluid, which is always undesired. The amount of debris and its local concentration is reported to have adverse effect on osteoblasts viability at the implant surface [16]. Moreover, the wear 
debris can stimulate cellular responses, which in turn may cause excess osteoclastic differentiation, reduced numbers of bone-forming cells or stimulation of osteoblastic cells to release bone resorbing mediators [17-21]. All these phenomena lead to osteolysis and as a result loosening and failure of the implant. Hence, controlling the amount of debris generated is extremely important. The volume of debris generated is inversely related to the wear resistance of the coating. Further, the morphology of the wear debris is directly related to the wear mechanism controlling the tribological behavior of the coatings. The size and morphology of the wear debris is one of the important factors that regulate the cytotoxic response of osteoblast and macrophage cells.

\section{Choice of Wear parameters}

Parameters for the tribological study were selected considering the wear conditions of an orthopedic implant inside the human body. Hip joint is one of the major load-bearing parts that face severe frictional forces during movement. HA is usually coated on the stem of the femoral part and outer surface of the acetabular cup of the hip joint. The frictional forces faced by these parts are much lesser than the mating surface of the femoral head and the inside surface of acetabular cup. The wear parameters for the present study are selected based on the wear conditions faced by the femoral head inside the acetabular cup. The stress in a hip joint during walking is $0.8-2.5 \mathrm{MPa}$ [22]. HA coating is expected to withstand high frictional forces for a minimum of 20 years [23]. Since wear experiments cannot be continued for such a long period, the normal load during the test is kept at a high value of $5 \mathrm{~N}$, to obtain significant wear loss data. Considering the wear probe as a ball of $3 \mathrm{~mm}$ diameter, the stress exerted on the wear 
track (coating) is determined. The calculated stress on the wear track for $5 \mathrm{~N}$ normal load is $\sim 12 \mathrm{GPa}$, which is 3 orders of magnitude higher than the actual loading condition at the hip joint. Wear speed is selected by considering a normal walking speed of a healthy adult as $4 \mathrm{~km} / \mathrm{h}$. The average step size of a $6 \mathrm{ft}$ tall man is $0.91 \mathrm{~m}$. Assuming the swinging action of a $25 \mathrm{~mm}$ diameter acetabular cup as the cause for wear during walking, the calculated speed for wear is $955 \mathrm{~mm} / \mathrm{min}$. Hence, the wear speed of the present study is fixed to $\sim 950 \mathrm{~mm} / \mathrm{min}(50 \mathrm{RPM})$.

\section{Coefficient of Friction}

Coefficient of friction $(\mathrm{CoF})$ is obtained continuously during sliding of the alumina ball on the wear track. Figure 4.7 shows the CoF for plasma sprayed HA and HA-CNT coatings with $5 \mathrm{~N}$ normal load up to $100 \mathrm{~m}$ of distance. CoF decreases from 0.9 to 0.68 with the CNT addition to HA matrix. The decrease in CoF in presence of CNT is due to the lubrication offered by the peeled-off graphite layers from the CNT surface. Removal of a single graphite layer from multiwall CNT requires a tensile force $\geq 11 \mathrm{GPa}$ along its axial direction [24]. Lateral force applied in the wear causes shearing removal of mass on the surface, which causes tensile stress along the surface of wear track. Our previous study has showed that computed tensile stress in the wear track was $\sim 12 \mathrm{GPa}$, which is sufficient to remove graphite layer from the CNT within the wear track. Hence, it is possible to have peeled-off graphite layers in wear track of HA-CNT coating. 


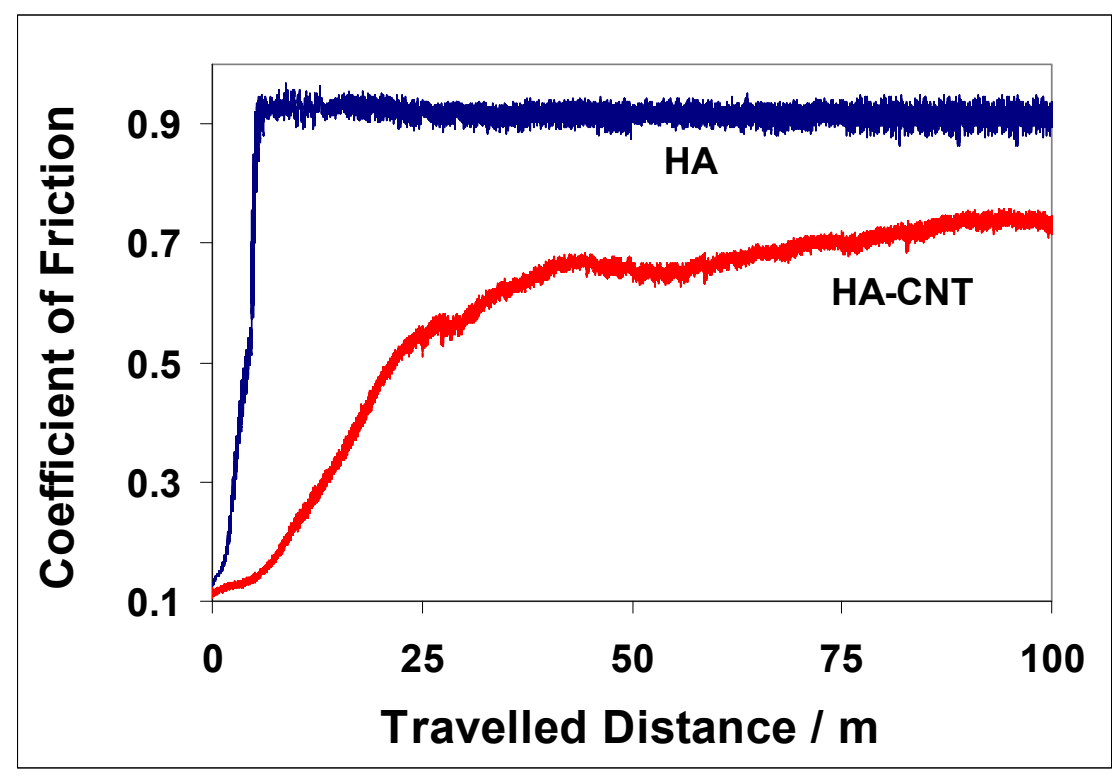

Figure 4.7: Coefficient of friction for $H A$ and $H A-C N T$ coatings plotted against sliding distance.

\section{$\underline{\text { Wear Resistance }}$}

Figure 4.8 shows the $3 \mathrm{D}$ profile of the entire wear track and 2D line profile across the wear track for HA and HA-CNT coatings. The volume of the wear track, calculated from the profiles, is $1.23 \mathrm{~mm}^{3}$ and $0.24 \mathrm{~mm}^{3}$ respectively, for HA and HA-CNT. Wear volume calculated for three tracks in each sample shows a standard deviation of less than $10 \%$. Addition of CNT results in decrease in wear volume of HA coating by $80 \%$. The improvement in wear resistance means generation of less volume of debris, resulting in reduced probability of disturbance in the biological environment around the implant. Two major factors are responsible for the increase in wear resistance of HA-CNT coating. The first factor is the decrease in $\mathrm{CoF}$. With decreasing CoF, the effective lateral force on wear track is small, resulting in low wear volume. The second and dominating factor is the toughening of the HA coating with CNT reinforcement, which makes the 
removal of mass difficult. Improvement in fracture toughness (350\%) and elastic modulus (72\%) of HA with CNT addition indicates a very significant toughening.

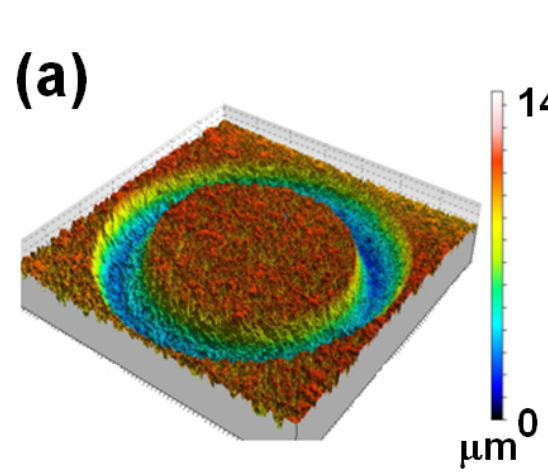

\section{HA}

$140 \mu \mathrm{m}$

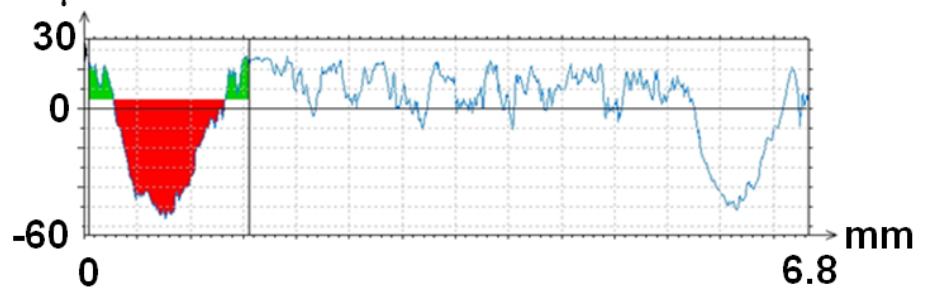

HA-CNT

(b)

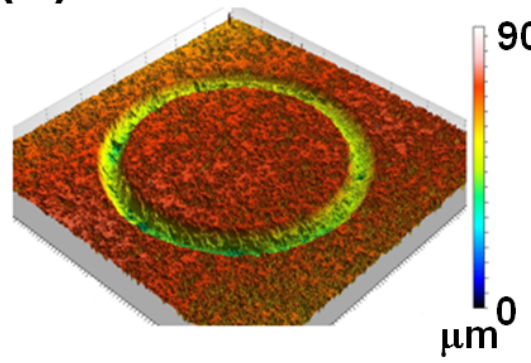

$90 \mu \mathrm{m}$

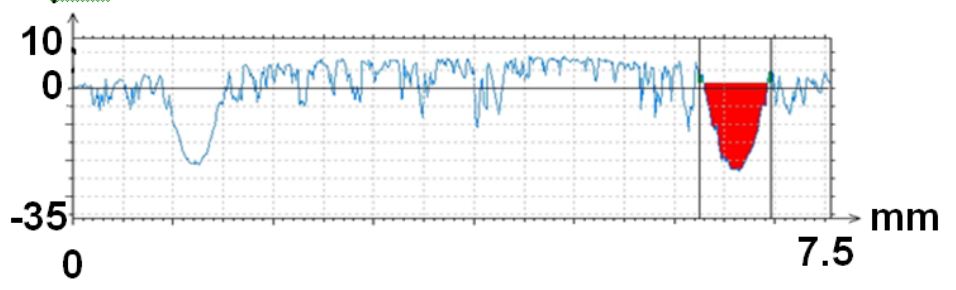

Figure 4.8: Three dimensional optical profiles of the wear tracks and a two dimensional profile across the track on (a) $\mathrm{HA}$ and (b) $\mathrm{HA}-\mathrm{CNT}$ - at $5 \mathrm{~N}$ load and $100 \mathrm{~m}$ of sliding distance.

Wear volume loss is a combined function of mechanical properties of material, viz., $\mathrm{K}_{\mathrm{IC}}, \mathrm{E}$ and $\mathrm{H}$. The model proposed by Evans and Marshall estimates the wear volume loss for brittle ceramics as a function of their mechanical properties [25]. The relationship is as follows:

$$
V=P^{1.125} K_{I C}^{-0.5} H^{-0.625}\left(\frac{E}{H}\right)^{0.8} S
$$


where, $\mathrm{V}$ is the wear volume loss, $\mathrm{P}$ is normal load, $\mathrm{K}_{\mathrm{IC}}$ is the fracture toughness, $\mathrm{H}$ is the hardness, $\mathrm{E}$ is the elastic modulus and $\mathrm{S}$ is the total travelling distance on wear track. The computed volume loss for the present study shows a $70 \%$ reduction in the wear volume with CNT addition. The computed improvement shows a good match with $80 \%$ wear resistance improvement for HA-CNT, obtained experimentally. The slight discrepancy between computed and experimental results can be explained by the lubrication available from the peeling of graphite layer, which is not accounted by the computation model suggested by Evans and Marshall.

\section{Wear Debris Size Distribution and Prediction of Wear Mechanisms}

It is possible to predict the wear mechanism from the size and morphology of wear debris and the wear track. Figure 4.9 shows a statistical 'box-plot' presenting the size distribution of wear debris for HA and HA-CNT coatings. The debris from HA coating shows a wide range of size distribution $(0.3-9.5 \mu \mathrm{m})$ with an average particle size of $\sim 3 \mu \mathrm{m}$. The average debris particle size in HA-CNT is much lower $(0.6 \mu \mathrm{m})$ with a smaller size range $(0.1-3.1 \mu \mathrm{m})$. The wide variation in the HA debris size indicates that wear in HA coating is governed by abrasion as well as fracture and chipping. Finer

particles are generated during abrasive wear, whereas the larger particles are the result of fracture and chipping. In case of HA-CNT coating, the majority of the debris particle is fine indicating predominantly abrasive wear. 


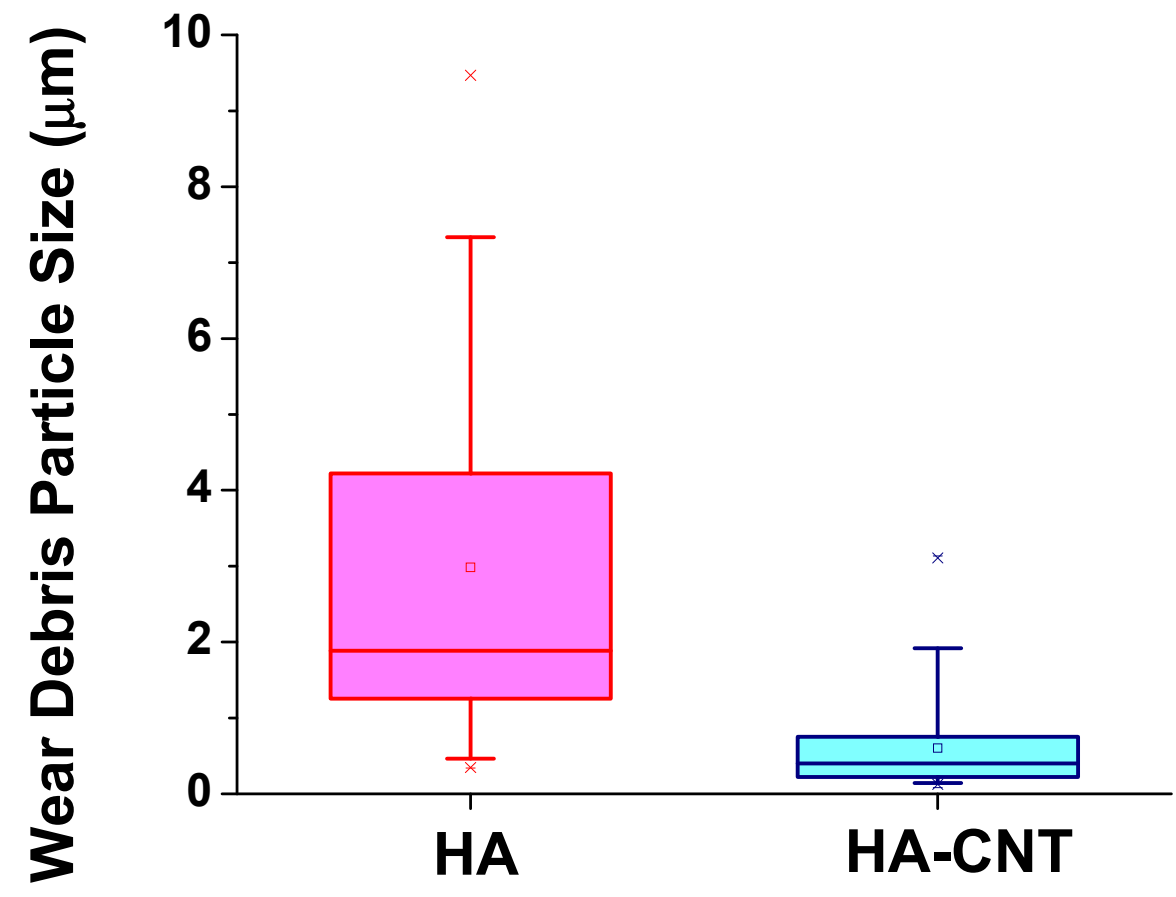

Figure 4.9: Wear debris size distribution for HA and HA-CNT coatings.

The images of wear track (figures 4.10a and 4.10b) reveal smaller size of craters and large area of abraded surface in HA-CNT wear track as compared to HA. The crater is created by fracture and chipping, whereas the rough surface is the result of abrasive wear. The mode of wear and debris size is directly related to the fracture toughness of the coating. Relatively higher fracture and chipping takes place in HA wear track, due to its lower $\mathrm{K}_{\mathrm{IC}}$. On the other hand, smaller size of wear particles in HA-CNT coating is the result of the improved fracture strength compared to HA. Figure 4.11 shows the presence of CNTs in the wear debris, which might influence their cytotoxic response to osteoblast and macrophage cells. 


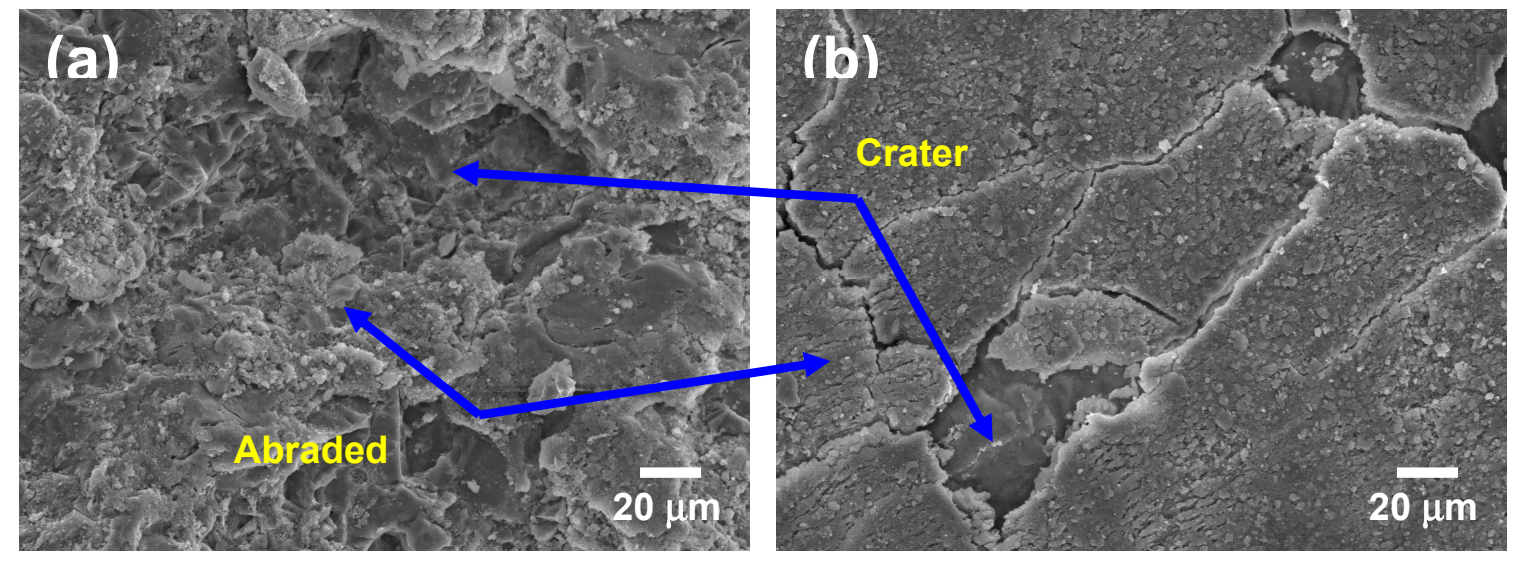

Figure 4.10: Wear tracks of (a) HA and (b) HA-CNT coating showing abrasion and fracture mode of mass removal.

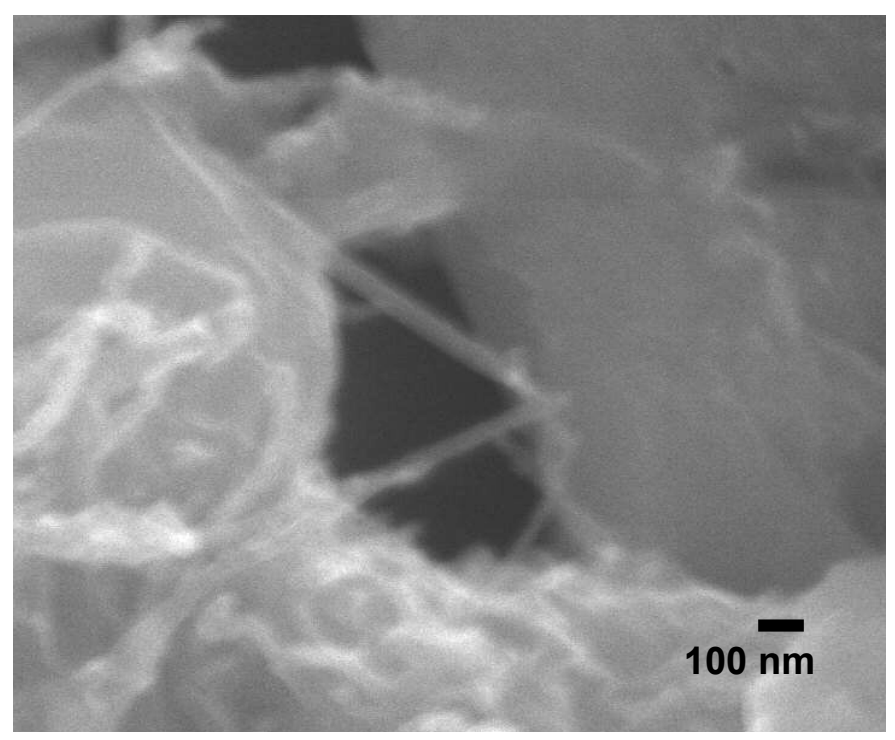

Figure 4.11: Presence of CNTs in the wear debris generated from HA-CNT coating.

\subsection{Wear in Simulated Body Fluid (SBF) Environment}

The wear studies for HA and HA-CNT plasma sprayed coatings were also carried out in simulated body fluid (SBF) to simulate the in-vivo condition. The test parameters and other conditions were kept same as dry wear testing. Figure 4.12 shows the CoF for 
plasma sprayed HA and HA-CNT coatings with $5 \mathrm{~N}$ normal load up to $100 \mathrm{~m}$ of distance carried out under SBF immersed condition. The coefficient of friction is 0.35 for HA and reduces to $\sim 0.2$ for HA-CNT surface in SBF. These CoF values are much lower than 0.9 and 0.68 , recorded for HA and HA-CNT respectively in dry condition. The comparative CoF for the coatings in dry and wet condition clearly indicates the extra lubrication offered in the presence of SBF. Thus, the coatings are expected to behave even better inside living body, as the lubrication offered by body fluid on implant surface would decreases amount of wear debris generated in-vivo. In addition to the lubrication offered by $\mathrm{SBF}, \mathrm{CNT}$ also plays an active role in decreasing the CoF of HA-CNT coating as compared to HA in wet condition. Moreover, the CoF on HA-CNT coating decreases with time in SBF, which is not the case for HA. The probable cause is the good spreading of the peeled off graphene sheet on wear track due to presence of liquid medium, which leads to more effective lubrication with time.

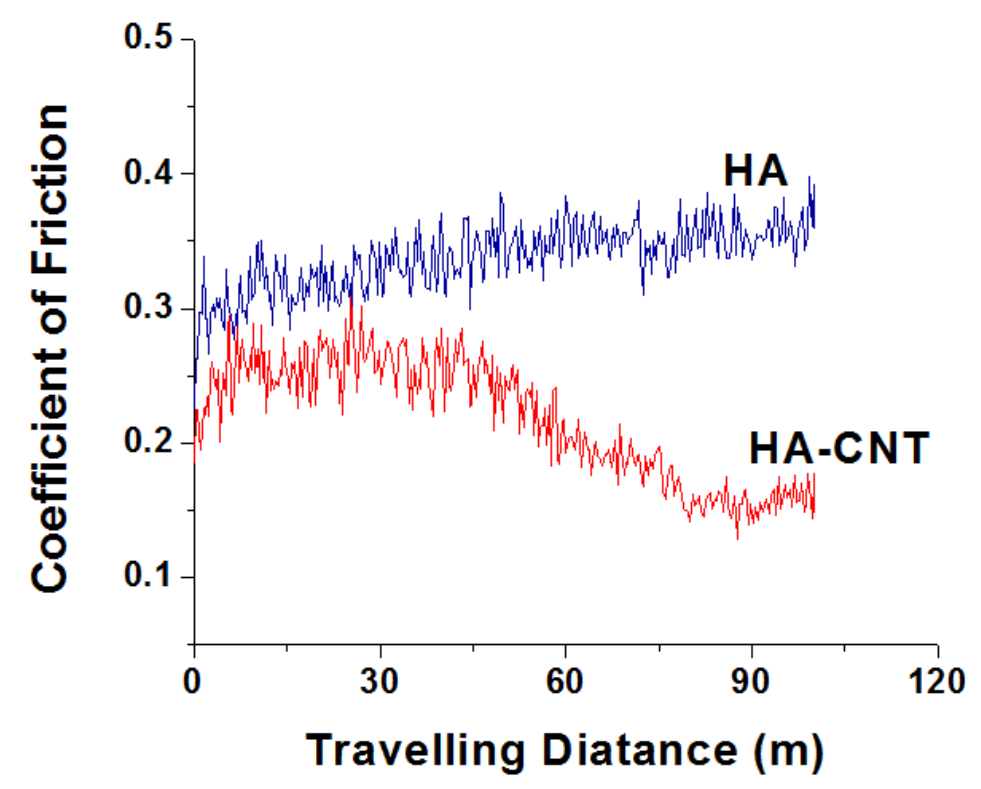

Figure 4.12: Coefficient of friction for $H A$ and HA-CNT coatings in SBF immersion plotted against sliding distance. 

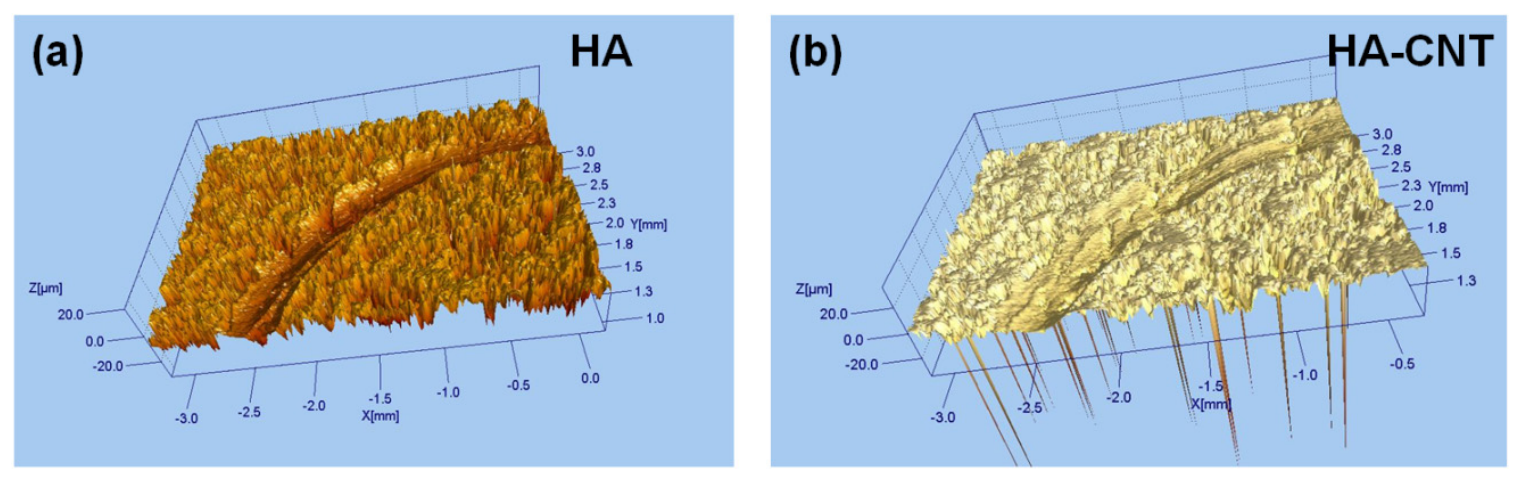

(c)

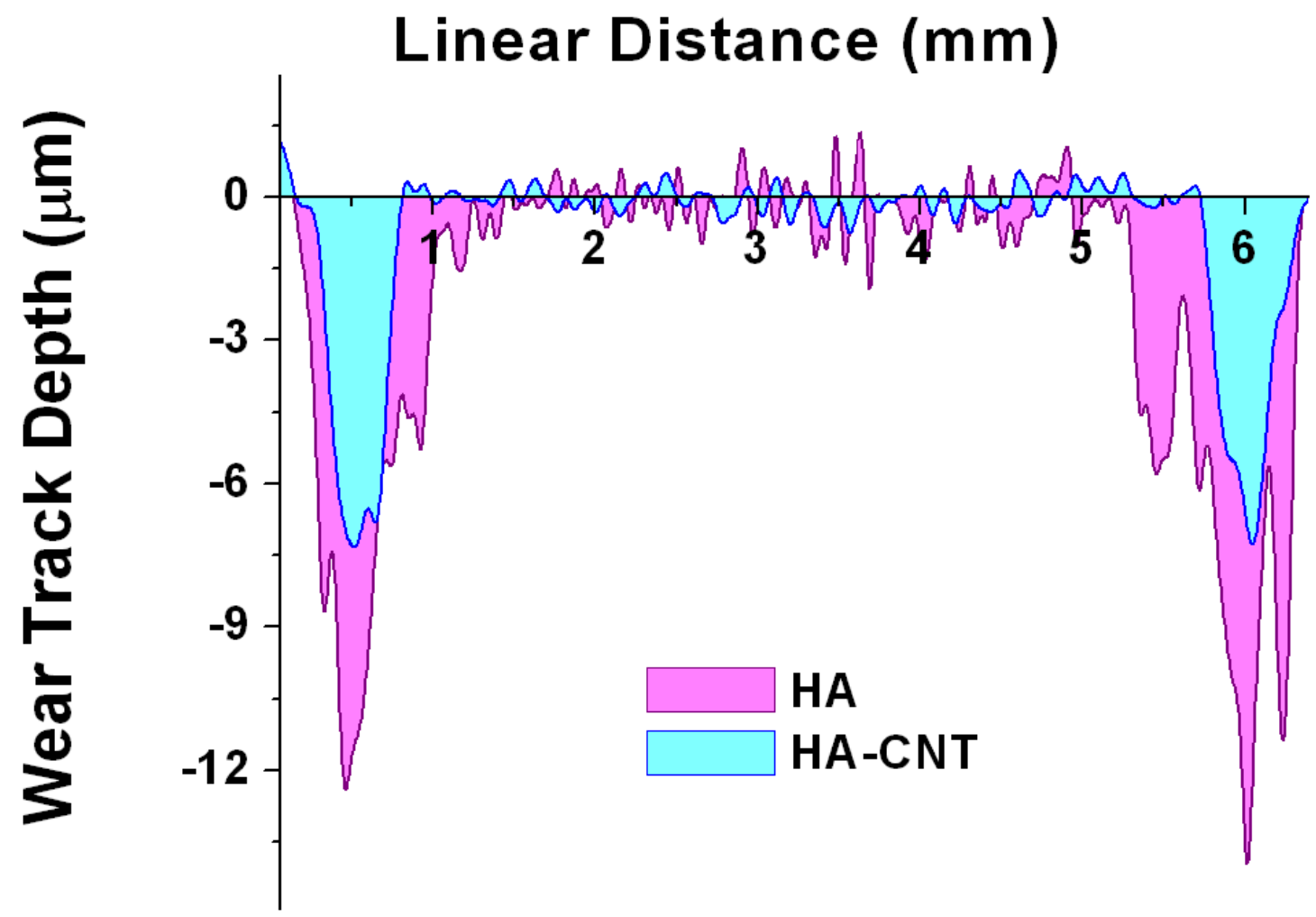

Figure 4.13: Three dimensional optical profile of a section of the wear tracks on (a) $H A$ and (b) HA-CNT; (c) two dimensional profiles across the tracks for HA and HA-CNT coatings - at $5 \mathrm{~N}$ load and $100 \mathrm{~m}$ of sliding distance while immersed in SBF.

The 3D images of the part of wear tracks on HA and HA-CNT coatings, captured using optical profilometer and processed with SPIP software is presented in figure 4.13 (a and b). The images gives a clear visualization of the differential track depths in HA 
coatings with HA and HA-CNT. Figure 4.13c presents the line profiles of the coating surfaces across the wear tracks on HA and HA-CNT. The maximum depth of wear track is $\sim 7 \mu \mathrm{m}$ for HA-CNT and $>12 \mu \mathrm{m}$ for HA coating. The $42 \%$ lower track depth in HA$\mathrm{CNT}$ indicates its higher wear resistance in SBF immersed condition. The wear volume for HA and HA-CNT coatings are estimated from the optical profile of two tracks in each coating. The wear volume of HA $\left(0.118 \pm 0.021 \mathrm{~mm}^{3}\right)$ is $58 \%$ higher than HA-CNT

coating $\left(0.049 \pm 0.001 \mathrm{~mm}^{3}\right)$. Similar to dry condition, the higher wear resistance of HA$\mathrm{CNT}$ is attributed to higher fracture toughness and extra lubrication through peeled off graphene sheets.

The summary of the above finding reveal that addition of $4 \mathrm{wt} . \%$ CNTs to plasma sprayed HA coating improves the wear resistance by $80 \%$ and results in less volume of debris generation. The elastic modulus and fracture toughness of the plasma sprayed coating increases by $72.5 \%$ and $350 \%$ respectively with CNT addition. Release of the graphite layer from CNT provides lubrication and decreases the CoF on HA-CNT surface. Presence of CNTs decrease the size of wear particles (HA: $0.3-9.5 \mu \mathrm{m}$, HACNT: $0.1-3.1 \mu \mathrm{m}$ ).The focus of the next section is to investigate the similar properties of HA-4 wt.\% CNT composite processed through spark plasma sintering (SPS).

\subsubsection{Free Standing Composite by Spark Plasma Sintering (SPS)}

Free standing HA- 4wt.\% CNT composite is studied to understand the role of CNT in microstructural evolution of HA-based composite during SPS process. This is carried out by studying the grain size, and porosity and correlating with the physical properties of CNTs and thermal and electrical conductivity experienced during SPS 
processing. The effect of CNT modified microstructure and HA/CNT interface on elastic modulus and fracture toughness of the composite is also elucidated. Macro and nanoscale tribological properties of SPS HA-CNT composite is studied in terms of wear resistance and coefficient of friction. The differential roles played by CNT in tribological properties, over multiple length scale is also addressed.

HA-CNT composite powder, synthesized through spray drying process, shows bimodal distribution of CNT (figure 3.1). CNTs were found nicely dispersed inside the porous spray dried agglomerate, whereas, loose clusters of CNTs were also present on the surface. Spark plasma sintering leads to the consolidation of both HA and HA-CNT powders into the pellets with a measured density of 94.9 and $96.5 \% \mathrm{TD}$, respectively. The density values reveals slightly better densification in HA-CNT.

\subsection{Distribution and Structural Integrity of Carbon Nanotube in SPS Structure}

Raman spectrum of HA-CNT powder and sintered pellet shows the presence of G and D peaks of CNT (figure 4.14a), which are characteristics of $\mathrm{sp}^{2}$ and $\mathrm{sp}^{3}$ bonds of hexagonal carbon structure [26]. The peak at the lower wave number $\left(950-960 \mathrm{~cm}^{-1}\right)$ is from the $v_{1}$ symmetric stretching vibration of phosphate anions in HA [27]. The appearance of $\mathrm{G}$ peaks at around $1580 \mathrm{~cm}^{-1}$ is the signature of highly pure graphitic structure whereas D-peak (at around $1350 \mathrm{~cm}^{-1}$ ) originates from a disorder-induced mode in graphite. The intensity ratio of $D$ and $G$ peaks $\left(I_{D} / I_{G}\right)$ is the index of defect density present in CNT. Figure 4.14a shows an increase in $I_{D} / I_{G}$ from the powder stage to the SPS HA-CNT composite. This observation indicates increase in the defect density in $\mathrm{CNT}$ as a result of SPS processing. A shift in the G band towards higher wave number 
has also been observed after SPS. This observation concurs with Das et al. on shift of the G peak to higher wave number with an increase in defect density in graphite [28]. Absence of shift in D peak and the phosphate peak (HA) rules out the possibility of presence of compressive stress in the composite.
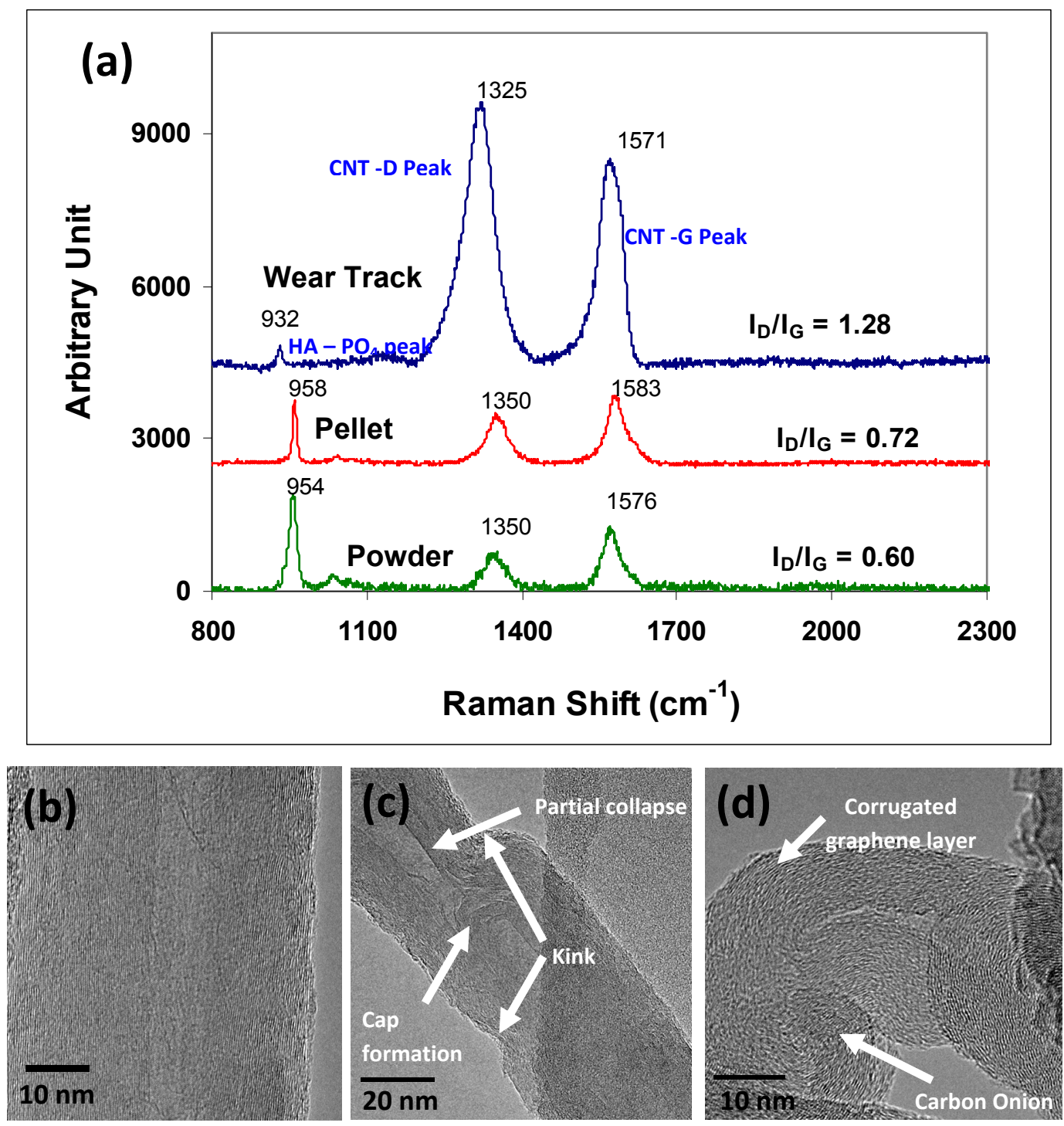

Figure 4.14: (a) Raman spectrum for HA-CNT powder, SPS pellet and wear track on SPS pellet showing D and G peak of CNT and phosphate peak of HA, (b) HRTEM of asreceived defect-free CNT, (c-d) HRTEM images of CNT in SPS HA-CNT pellet showing defects induced in CNT. 
Figure 4.14b shows HRTEM image of as-received CNT which is largely defectfree. The defect induced in CNTs during SPS processing are observed in figure $4.14 \mathrm{c}$ and 4.14d. Disturbed arrangement of concentric tubular walls in CNTs with presence of different types of defects, e.g. carbon onion, cap formation, kink, partial collapse of nanotube walls and corrugated structure of graphene layers are observed in figures $4.14 \mathrm{c}$ and $4.14 \mathrm{~d}$. All these features are results of high temperature, pressure and current density applied in SPS processing. Kink and cap formation and partial or full collapse of CNT walls upon application of high pressure has also been observed in cold spraying and SPS [29-30].

Moreover, such broken graphitic layers are also prone to bending and forming a close structure in order to reduce the surface energy. Thus, they result into a carbononion structure, as observed in figure $4.14 \mathrm{c}$. Wei et al. have shown that longer and thinner CNTs have higher energy per carbon atom than spherical network. At high temperature and pressure condition, when enough energy is provided, the spherical structures are favored over tubular form, resulting in carbon onion formation [31].

Presence of high electrical current density during SPS processing also plays an important role in defect site increment of CNT. The larger current density $\left(\sim 237 \mathrm{~A} / \mathrm{cm}^{2}\right)$ and pulsed DC nature of current causes the knock-on collision of electrons with carbon atoms, resulting into creation of vacancies and interstitials. Kim et al. have observed the breaking of $\mathrm{sp}^{2} \mathrm{C}-\mathrm{C}$ bonds with application of pulsed DC which continues in the direction of current, unzipping the CNT layers [32]. This breaking occurs by the energy absorption of $\mathrm{C}-\mathrm{C}$ bonds with higher energy atoms in tubular CNT structure [31]. In the present 
study, the CNTs are not aligned along the current flow. Hence, localized breaking of $\mathrm{sp}^{2}$ bonding takes place creating corrugated graphene layers on CNT walls (figure 4.14c).

\subsection{Phase Stability and Crystallinity of HA in SPS Structure}

X-ray diffraction patterns of HA and HA-CNT at both stages, viz. powder and after SPS consolidation are presented in figure 4.15. The major peaks in all the four patterns are from hydroxyapatite (JCPDS PDF No. 9-432). Low intensity graphite peaks are observed in HA-CNT powder and SPS pellet. The graphite peaks look weak because of presence of strong HA peaks in the vicinity. The $100 \%$ peak of $\beta$-TCP (at $2 \theta=30.9^{\circ}$ ) and the third highest intensity peak $\left(2 \theta=27.67^{\circ}\right)$ are absent in the XRD plots after SPS. The second highest intensity peak of TCP $\left(2 \theta=34.18^{\circ}\right)$ overlaps with another peak of HA $\left(2 \theta=34.01^{\circ}\right)$. Similarly, the highest peak of $\alpha$-TCP $\left(2 \theta=30.69^{\circ}\right)$ is also absent and the second highest peak $\left(2 \theta=22.88^{\circ}\right)$ overlaps with a HA peak $\left(2 \theta=22.77^{\circ}\right)$. Hence, the presence of $\alpha$-TCP (JCPDS PDF No. 29-359) or $\beta$-TCP phase (JCPDS PDF No. 9-169) cannot be concluded. These observations prove that HA does not dissociate into TCP during SPS processing for both compositions. Previous studies on SPS HA-CNT composites report partial decomposition of HA into TCP [33-34]. But, the heating rate used in those studies $(100 \mathrm{~K} / \mathrm{min})$ is 3.6 times lower than the heating rate in the present study $(360 \mathrm{~K} / \mathrm{min})$. The faster heating rate has reduced the total time of sintering, thus preventing the dissociation of HA into TCP.

The HA peaks were more sharp after SPS processing, which indicates increase in the crystallinity of HA through accelerated grain boundary and lattice diffusion. In the 
HA-CNT composite, the relative height of the first and second highest intensity peaks gets reversed after SPS. The intensity of $(002)$ peak in the powder $\left(2 \theta=25.87^{\circ}\right)$ reduces in the sintered pellet whereas intensity of (210) peak in the powder $\left(2 \theta=28.95^{\circ}\right)$ increases significantly after sintering. A plausible explanation for such behavior is the evolution of preferred crystallographic orientation in HA and HA-CNT due to high temperature exposure [11-13].

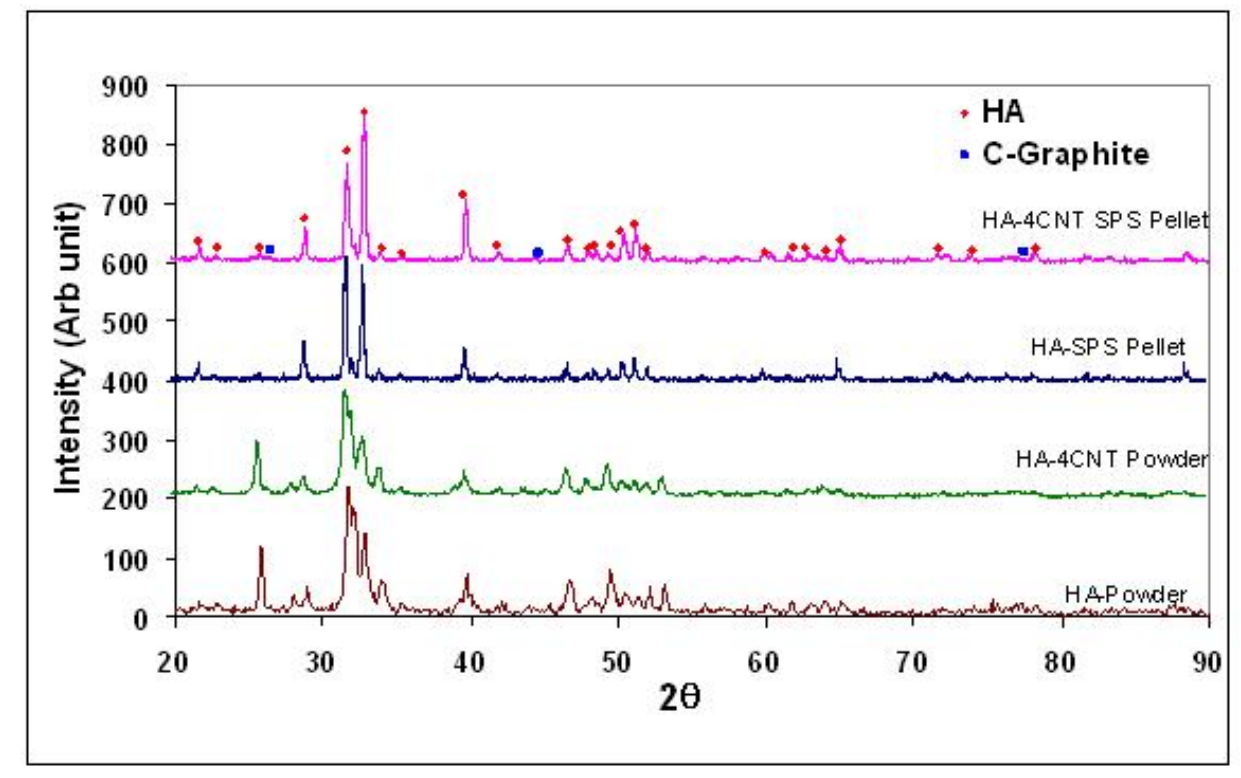

Figure 4.15: X-ray diffraction patterns of spray dried HA and HA-CNT powders and SPS pellets.

\subsection{Role of Carbon Nanotube in Microstructural Evolution through Spark Plasma}

\section{Sintering}

Figure 4.16 presents the fracture surface of sintered HA and HA-CNT pellets at different magnifications. The microstructures reveal two prominent regions present in both HA and HA-CNT. They are (i) larger, fully densified monolithic regions and (ii) small grain regions (figure 4.16a and 4.16b). Apart from these two features, porosity is 

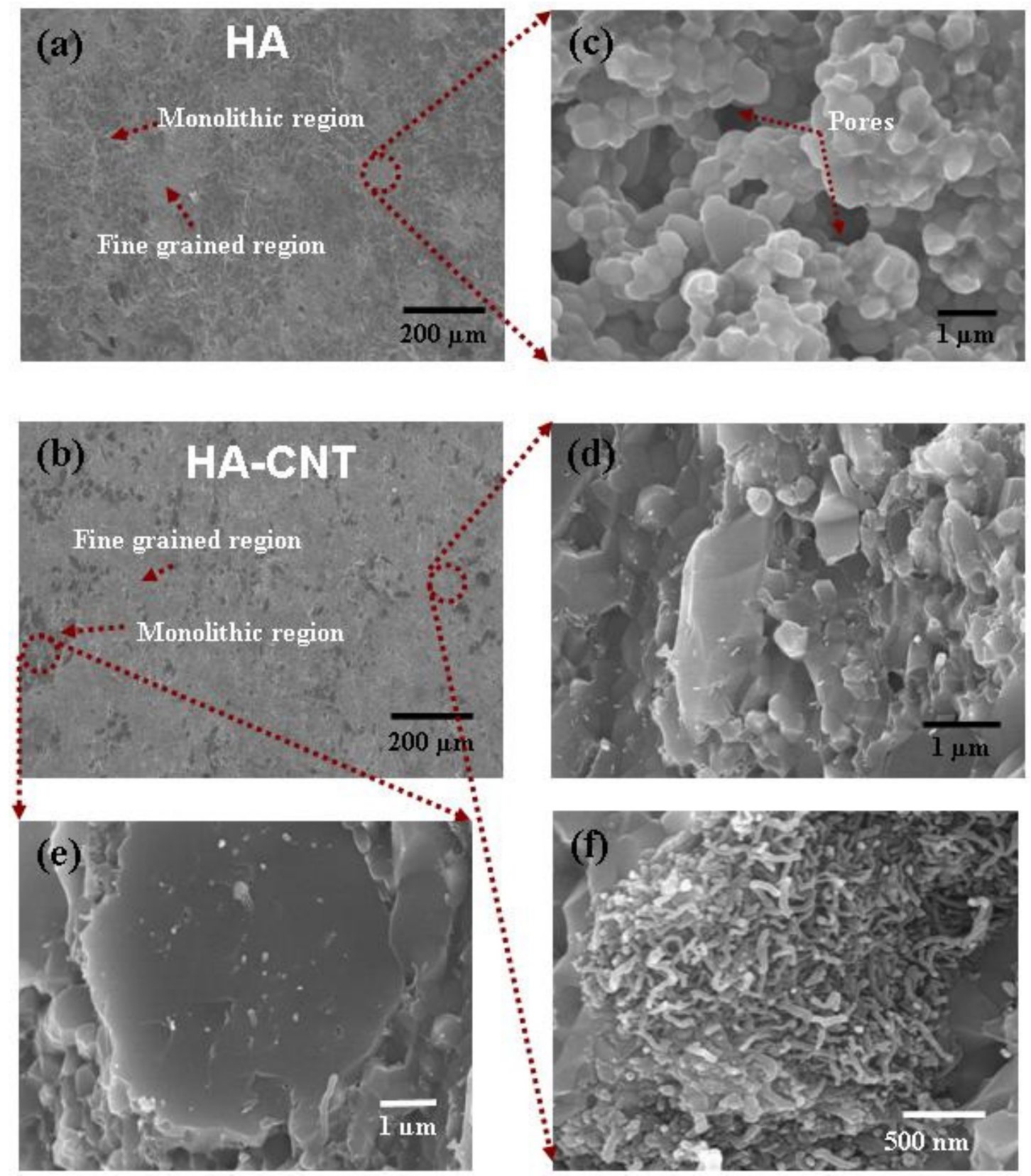

Figure 4.16: SEM micrographs of fracture surface of SPS pellets showing (a) monolithic and fine grain region in $H A$, (b) monolithic and fine grain region in $H A-C N T$, (c) partially sintered HA structure with fine grain region and porosity, (d) fully densified fine grain region of $H A-C N T$ with homogeneously distributed CNTs, (e) monolithic region of HA-CNT with CNTs embedded in HA matrix and (f) CNT clusters at fine grain region of $H A-C N T$. 
also present in HA fracture surface (figures 4.16c and 4.17a). HA-CNT structure does not show porosity. The distribution of CNT in the sintered HA-CNT pellet is directly related to their distribution in the powder stage. Uniformly distributed CNTs are found in both fine grained and monolithic regions (figure 4.16d and 4.16e), whereas CNT clusters are largely found in and around fine grained region (figure 4.16f). It is also observed that distribution and morphology of monolithic region, fine grain regions and pores vary from the periphery to the centre of the pellets for both compositions. Table 4.1 summarizes the microstructural variations in quantitative terms. The values presented in Table 4.1 are based on the area fraction of microstructural features in the micrographs. All these features are attributed to SPS processing in conjunction with the CNTs presence. A detailed explanation of microstructure evolution is discussed below in terms of three main microstructural features, viz.(i) monolithic region, (ii) fine grained region and (iii) porosity in HA or HA/CNT clusters.

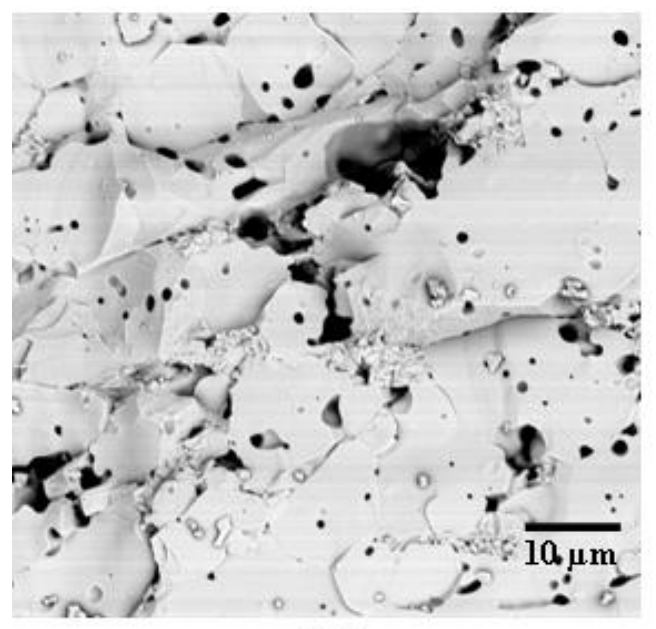

(a)

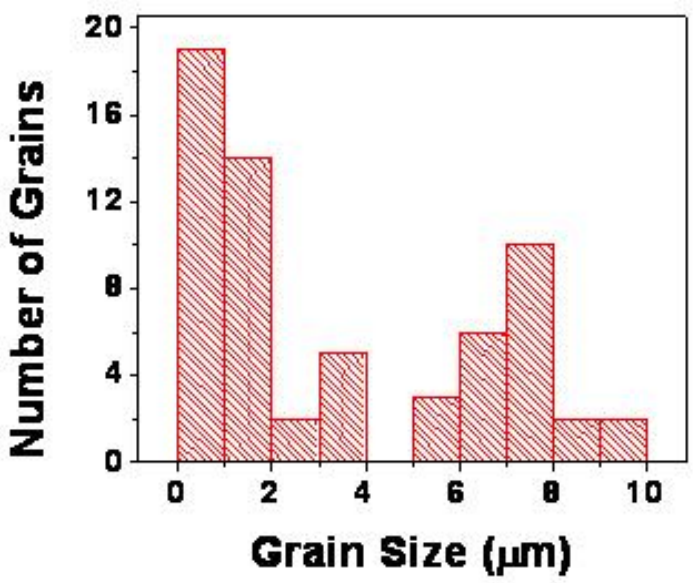

(b)

Figure 4.17: (a) SEM micrograph of fracture surface of SPS-HA pellet showing porosity and (b) histogram showing bimodal porosity distribution in SPS-HA pellet. 
Table 4.1: Comparative quantification of microstructural features in spark plasma sintered HA and HA-CNT pellets

\begin{tabular}{|c|c|c|c|c|}
\hline \multirow{2}{*}{ Microstructural Features } & \multicolumn{2}{|c|}{ HA -Pellet } & \multicolumn{2}{|c|}{ HA-CNT-Pellet } \\
\hline & Periphery & Centre & Periphery & Centre \\
\hline Monolithic region size $(\mu \mathrm{m})$ & $207 \pm 50$ & $179 \pm 41$ & $15 \pm 6$ & $14 \pm 6$ \\
\hline Small Grain Size $(\mu \mathrm{m})$ & $0.6 \pm 0.2$ & $0.5 \pm 0.1$ & $0.4 \pm 0.1$ & $0.6 \pm 0.1$ \\
\hline$\%$ Monolithic region & $48 \pm 3$ & $35 \pm 2$ & $21 \pm 3$ & $18 \pm 3$ \\
\hline$\%$ Porosity & $5 \pm 1$ & $10 \pm 1.5$ & $* \pm 1.8$ & ${ }^{*} 5 \pm 2.5$ \\
\hline$\%$ Small grain region & $47 \pm 2$ & $55 \pm 2$ & $74 \pm 2$ & $77 \pm 3$ \\
\hline
\end{tabular}

* indicates CNT clusters (no visible pores are observed in HA-CNT)

\# Values presented in the table are based on \% area measurement from the micrographs

\section{Monolithic Region}

The monolithic region forms due to rapid grain growth which is also known as dynamic ripening [35]. The heating rate used in this study is $360 \mathrm{~K} / \mathrm{min}$. The rapid heating rate in achieved through application of high current density that causes localized joule heating at particle interfaces. Such localized heating helps in accelerated thermal diffusion, melting within inter-particle contact areas and also creates thermal stresses which enable dislocation creep. Thus, spark plasma sintering by-passes the initial stage of surface diffusion, as in conventional sintering, and achieves accelerated densification by early activation of diffusion mechanisms, like grain boundary and lattice diffusion and power-law dislocation creep [36-38], leading to formation of consolidated monolithic regions. 
But, HA is a ceramic with poor thermal conductivity $((1.25 \mathrm{~W} / \mathrm{mK}[9])$. As the heating source is the graphite die in contact with the periphery of the green pellet, a thermal gradient is created along the pellet thickness with the lowest temperature at the centre. This non-uniform temperature distribution promotes a densification gradient along the pellet thickness $(\sim 5 \mathrm{~mm})$ resulting into microstructure variations. Poor electrical conductivity of HA $\left(7 \times 10^{-7} \mathrm{Scm}^{-1}\right)$ [39], adds to the through thickness density gradient. Joule heating and electrical field assisted mass transport decreases towards the center of the pellet due to existing electrical field gradient across the thickness. As a result, HA pellet goes through more densification at the periphery than in centre. Such differential densification through thickness leads to more monolithic regions at the periphery (48\%) as compared to the centre (35\%) in the sintered structure (table 4.1).

On the contrary, thermal conductivity of CNT (2980 W/mK [8]) is three orders of magnitude higher than HA $(1.25 \mathrm{~W} / \mathrm{mK})$, which results into higher effective thermal conductivity of the HA-CNT composite powder. Similarly, higher electrical conductivity of CNT $\left(2 \times 10^{4} \mathrm{Scm}^{-1}\right)$ [40] than HA $\left(7 \times 10^{-7} \mathrm{Scm}^{-1}\right)$, increases the effective electrical conductivity of HA-CNT composite and reduces the electrical field gradient across the green HA-CNT pellet. The reduction in both, thermal and electrical gradient results in reducing the densification gradient along the thickness of HA-CNT pellet. Thus, in case of HA-CNT pellet, only 3\% difference in monolithic content is observed from the periphery $(21 \%)$ to the center $(18 \%)$ as presented in table 4.1 . The increase in effective thermal and electrical conductivity of HA-CNT also helps in overall better densification than HA, as evident from the measured density of 96.5 and $94.9 \% \mathrm{TD}$, respectively. Application of pressure in SPS also helps in densification by increasing particle surface 
contact through particle rearrangement, breaking of CNT agglomerates and thus promoting easy diffusion at lower temperature, which is absent in conventional sintering. Effect of thermal, electrical and pressure gradient on microstructural evolution during SPS of HA and HA-CNT has been explained through a schematic diagram in figure 4.18.

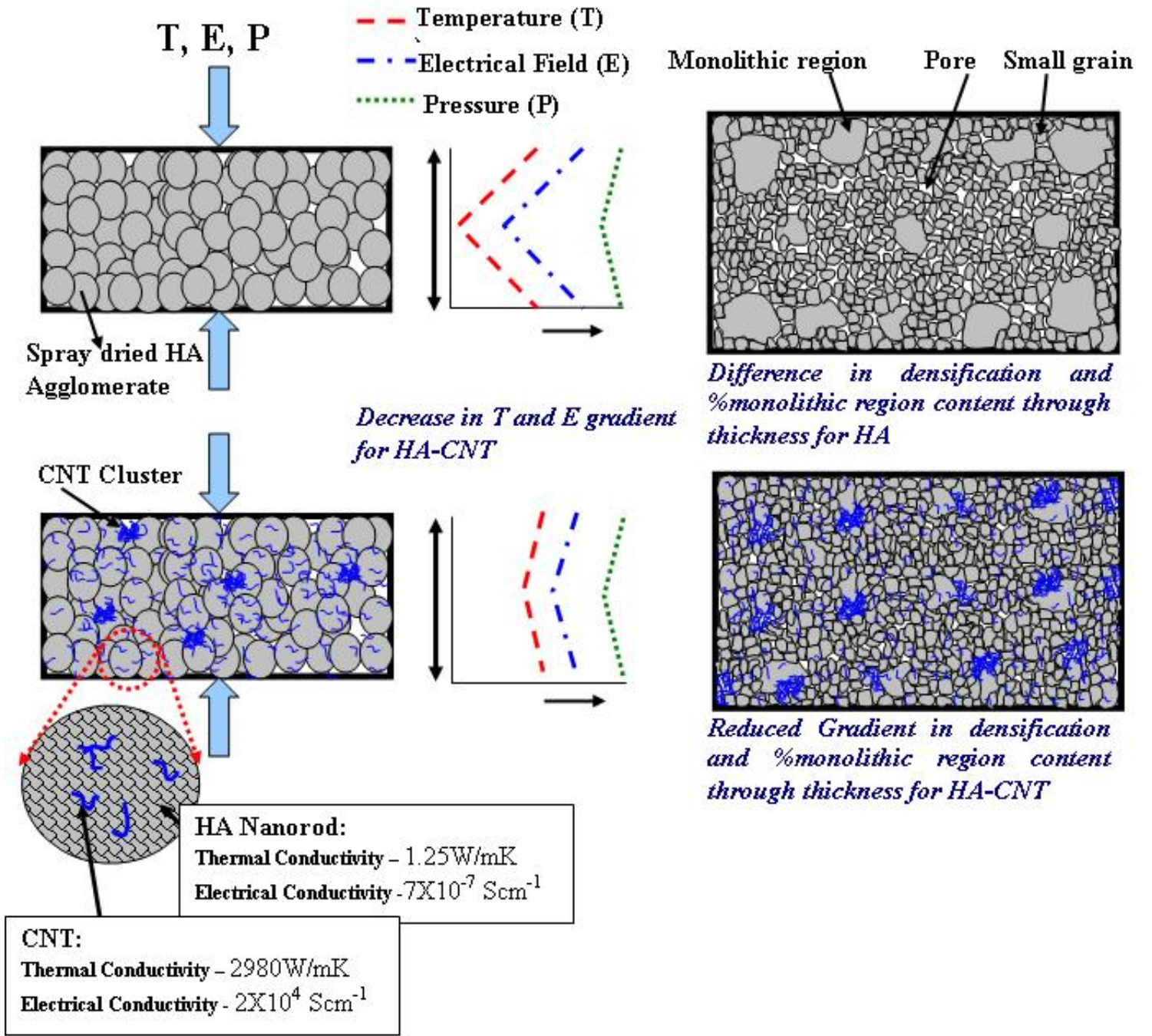

Figure 4.18: Schematic showing effect of SPS and CNT on the consolidation mechanism and microstructural evolution in HA and HA-CNT pellets. 


\section{Fine grained region}

Fine grain regions are observed in both HA and HA-CNT pellets. But, the origin of fine grain region is different in HA and HA-CNT, which is evident from figures 4.16a4.16d. Fine grain regions in SPS HA pellet is generated due to incomplete sintering or less densification. SEM micrograph of fine grain region in HA shows presence of porosity and partially sintered structure (figure 4.16c). Due to less densification at the centre, fraction of fine grain region is also higher at the centre $(55 \%)$ than in periphery (47\%) in HA pellet (table-4.1). HA-CNT pellet contains higher fraction $(\sim 70 \%)$ of fine grained region than HA pellet as shown in figure 4.16b. The fine grain structure in HACNT is the result of grain boundary pinning of HA by CNTs. The grain growth is controlled by the random dispersion of rigid immobile spherical second phase particle through grain boundary pinning and the maximum attainable grain size $R$ can be expressed as,

$$
R=a \frac{r}{f^{b}}
$$

where, $r$ is the radius of the second phase particle, $f$ is the volume fraction of the second phase particle and $a, b$ are constants [41]. The Zener pinning factor $Z$ is defined as,

$$
Z=\frac{f}{r}
$$

Sun et al. have modified expression of $Z$ to get rid of the assumption on second phase particle shape and size [42]. According to their findings,

$$
Z=\frac{S_{V}}{4}
$$


$S_{v}$ is defined as the surface area of particle per unit volume. $S_{v}$ is very high for CNTs due to their large surface to volume ratio. As the $S_{v}$ increases, $R$ decreases, which means CNTs have great potential of grain size refinement. Pinning at grain boundary also retards the dynamic ripening process that results in smaller size of monolithic regions. Thus, HA-CNT structure contains higher fraction of fine grained region as well as smaller size of monolithic regions $(\sim 15 \mu \mathrm{m})$ than HA $(\sim 200 \mu \mathrm{m})$. Presence of CNTs at the HA grain boundary in fine grain region of HA-CNT fracture surface (figure 4.16) provides the visual evidence of grain boundary pinning by CNT. The fraction of fine grain region in HA-CNT remains same in centre $(\sim 77 \%)$ and periphery region $(\sim 74 \%)$, as presented in table-4.1. This uniformity could be attributed to the uniform distribution of CNT in the pellet, which controls the formation of fine grain region in HA-CNT.

\section{Porosity}

Porosity present in HA shows a bimodal distribution (figure 4.17a \&4.17b). The big pores are generated from the inter-particle gap, which are more pronounced at the center of the doughnut shaped spray dried agglomerates (figure-3.1c). The small pores originate from the regions of agglomerates having loosely bound HA particles. Due to poor thermal and electrical conductivity of HA, the inter-particle distances could not be covered fully during diffusion/densification. Presence of a sharp porosity gradient through thickness, with higher porosity in the centre (10\%) than periphery $(5 \%)$ (table4.1 ) is the result of densification gradient. Increase in the porosity content due to presence of thermal gradient in SPS of ceramic has also been observed by other researchers [43]. HA-CNT composite fracture surface shows presence of some CNT clusters and absence 
of porosity (figure 4.16b). The absence of porosity is already justified in terms of better densification achieved due to better thermal and electrical conductivity of CNT. The dense CNT clusters prevent the penetration of HA particles between them due to the short sintering period restricting long distance mass transport. The content of CNT cluster is the same for periphery $(5 \%)$ and centre $(5 \%)$ of the sintered pellet.

\subsection{Hydroxyapatite-Carbon Nanotube Interface in SPS Structure}

The crystallographic arrangement at the interface plays a major role in determining its strength. The strength of the interfacial bond is mainly governed by the work of adhesion. HA-CNT interface has been investigated using HRTEM images (figure 4.19). Fourier transform (FFT and inverse-FFT) analysis of the lattice images at interface reveals the presence of CNT and HA. No reaction product was observed at the interface. It is difficult to predict the nature of bonding at HA-CNT interface, due to complex crystal structure of HA. Though the probability of Van der Waal bond is higher between two chemically non-reacting solids like HA and CNT. CNT shows an inter-wall distance of $0.348 \mathrm{~nm}$, which is slightly higher than defect free CNT inter-wall distance of 0.34 $\mathrm{nm}$. This mismatch is due to the SPS induced defect density in CNT structure as discussed in section 4.1.1.2.1. HA particles are identified by the lattice spacing of 0.282 $\mathrm{nm}$ for the (211) plane, which produces the $100 \%$ intensity peak in XRD pattern of hexagonal HA (figure 4.19). 


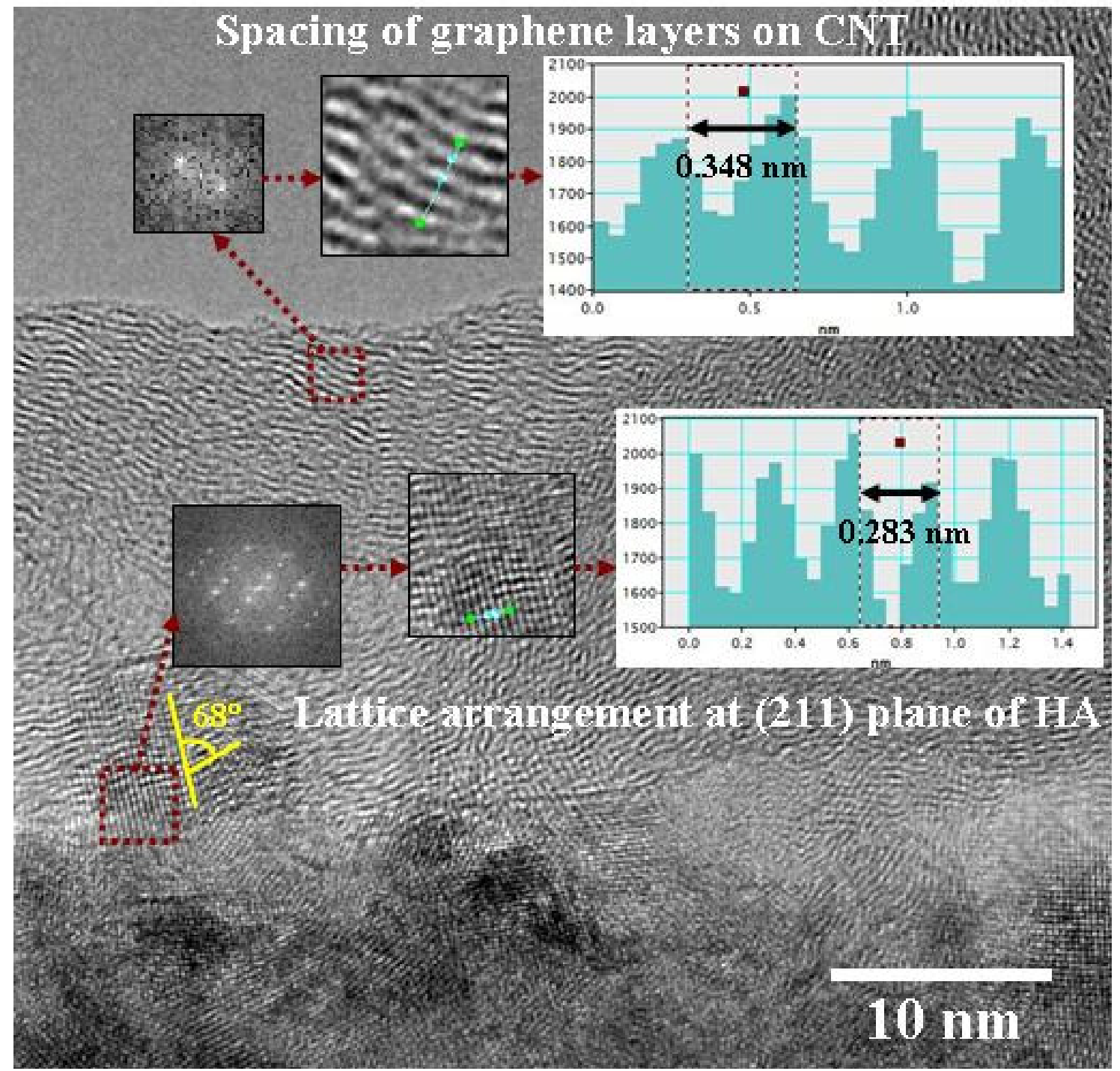

Figure 4.19: HRTEM image of CNT and HA interface. FFT analysis reveals the CNT wall spacing and HA lattice spacing at the interface.

Two different types of interfaces are possible in HA-CNT system. They are: (i) HA crystals attached to CNT wall and (ii) HA crystals along the CNT end (cross section). Good interfacial bonding could be achieved only in case of small lattice mismatch $(\delta)$ giving rise to minimum lattice strain. Absence of lattice strain improves the "work of adhesion' at the interface, which further increases the fracture energy of the interface and 
thus makes it strong [44]. The interface becomes incoherent when $\delta>0.25$ [45]. As observed in figure 4.19 , the (211) planes in HA makes $68^{\circ}$ angle with CNT walls. The angle between the basal plane (001) and (211) of HA is $65^{\circ}$. Hence, there is a greater possibility that basal planes of HA are parallel to the graphene sheet on CNT wall. Both of them are symmetric in nature due to the hexagonal arrangement of both $\mathrm{C}$ (CNT) and $\mathrm{Ca}$ (HA) atoms. The distance between $\mathrm{C}$ atoms in graphene is $0.142 \mathrm{~nm}$. The basal plane of HA has Ca atoms at each corner of the hexagon and the distance between each pair of $\mathrm{Ca}$ atom is $0.94 \mathrm{~nm}$. Schematic diagram presented in figure $4.20 \mathrm{a}$ shows a HA basal plane superimposed on a graphene sheet. HA crystals try to align on CNT surface resulting in minimum atomic distance mismatch. The distance between carbon atoms $(\mathrm{C} 1$ and $\mathrm{C} 2$ in figure $4.20 \mathrm{a})$, nearest to the $\mathrm{Ca}$ atoms (Ca-1 \& $\mathrm{Ca}-2)$ along one side of HA basal plane, is $1.026 \mathrm{~nm}$. As the distance between corresponding Ca atoms of HA basal plane (Ca-1 and Ca-2) is $0.94 \mathrm{~nm}$, the mismatch $(\delta)$ between the inter-atomic distance of these two pairs of $\mathrm{C}$ and $\mathrm{Ca}$ atoms is 0.09 , which is much lower than 0.25 . Hence, basal plane of HA forms a strong and coherent interfacial bond with CNT wall. Thus, basal planes of HA crystals prefer to align on CNT surface. The only disturbance to this strong interfacial bonding is the defects on CNT walls introduced by SPS processing. These defects are probable sites for CNT debonding from the HA matrix causing pull-outs.

At the open ends of CNT, the graphene walls are exposed with an inter-wall spacing of $0.34 \mathrm{~nm}$. The lattice spacing of (211), the highest intensity plane of HA (XRD), is $0.282 \mathrm{~nm}$. The lattice mismatch $(\delta)$ between (211) plane of HA and CNT walls is 0.20 , which is also lower than 0.25 . Thus open ends of CNT forms a semi-coherent 


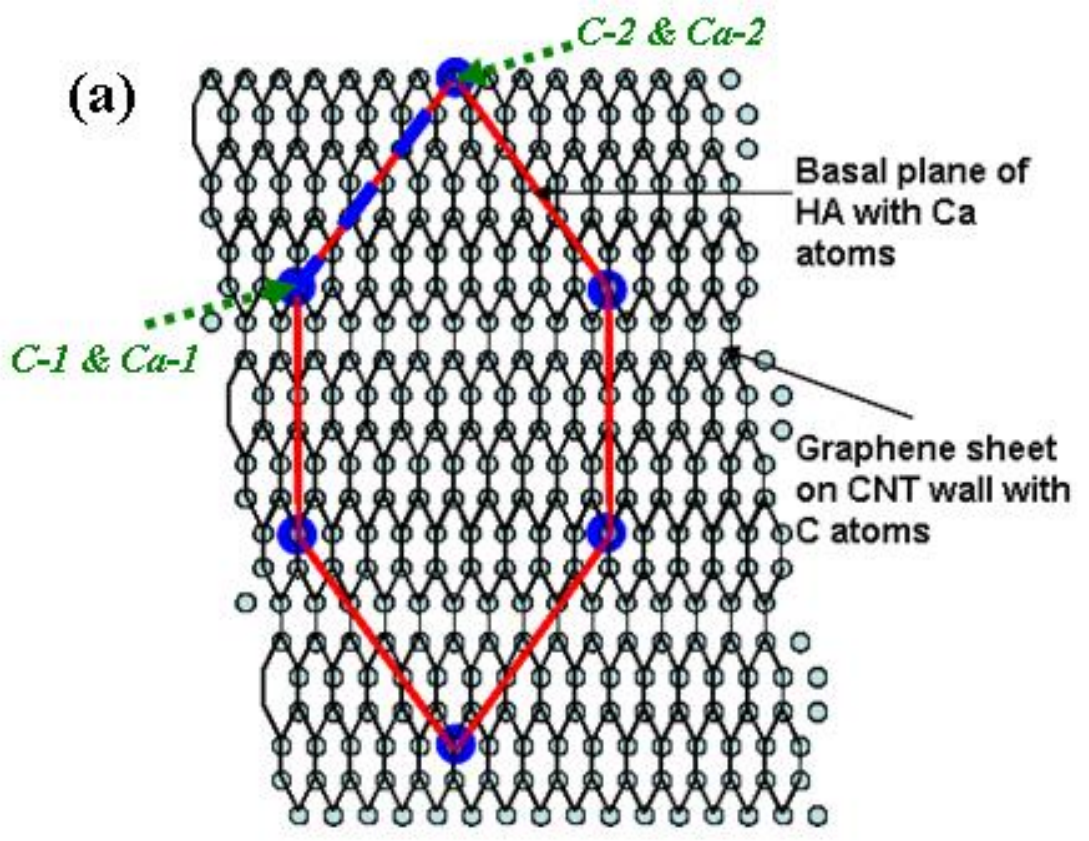

- $1.026 \mathrm{~nm}$ (distance between the two indicated $\mathrm{C}$ atoms in Graphene)

$0.94 \mathrm{~nm}$ (distance between $\mathrm{Ca}$ atoms in $\mathrm{HA}$ )

(b)

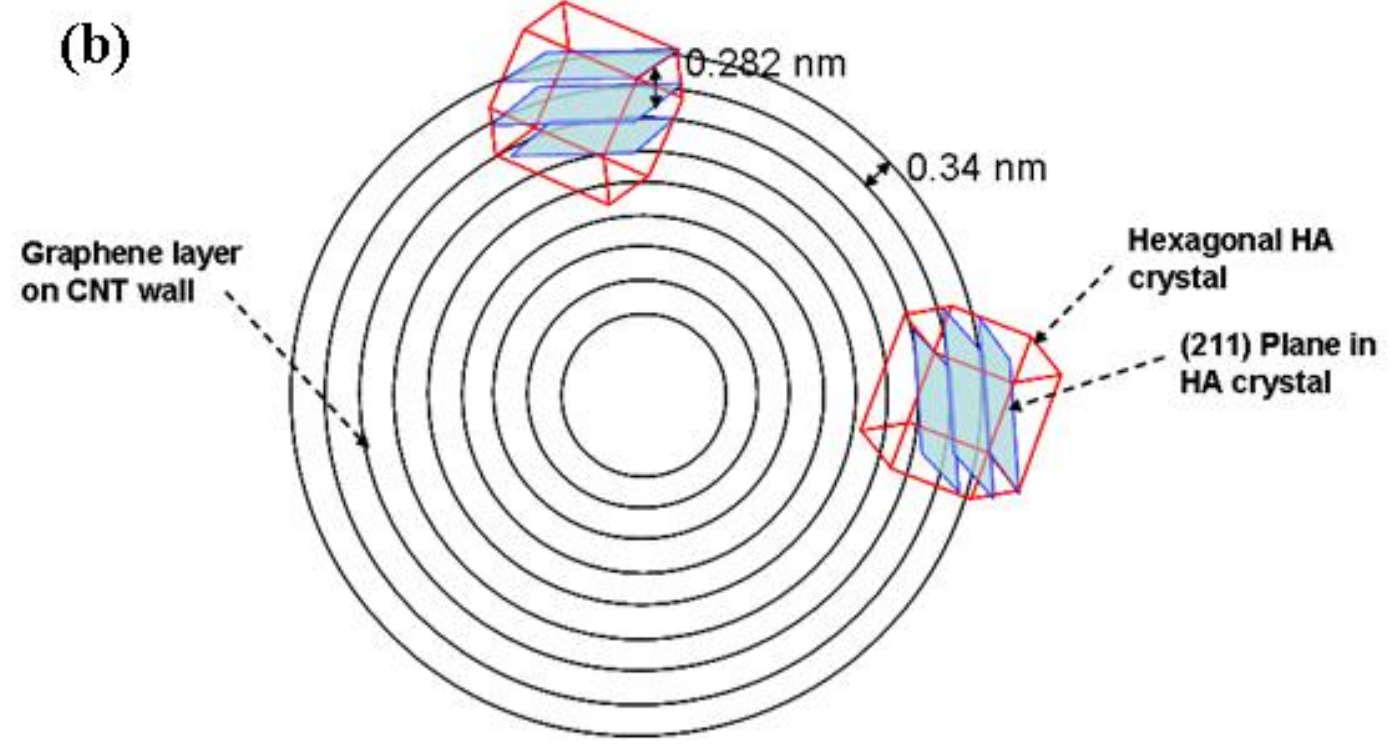

Figure 4.20: (a) Schematic of atomic arrangement at the interface of basal plane of HA and graphene sheets on CNT surface and (b) Schematic of CNT open end showing alignment of CNT walls with (211) planes of HA. 
interface with HA crystals with an orientation such that (211) planes are parallel to graphene walls, as shown in figure $4.20 \mathrm{~b}$. The strong and coherent interface with higher work of adhesion between CNT and HA without other reaction product is the key factor for the improvement of the mechanical properties.

\subsection{Elastic Modulus and Fracture Toughness of SPS HA-CNT}

\section{Elastic Modulus}

Measurement of the elastic modulus $(E)$ of the SPS HA and HA-CNT composites has been performed using nanoindentation technique [14]. More than 100 indents were made at randomly chosen regions throughout the cross-section of the pellets.

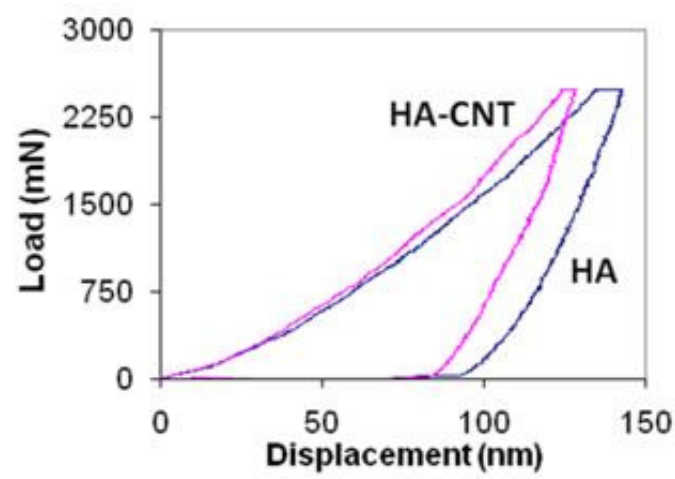

(a)

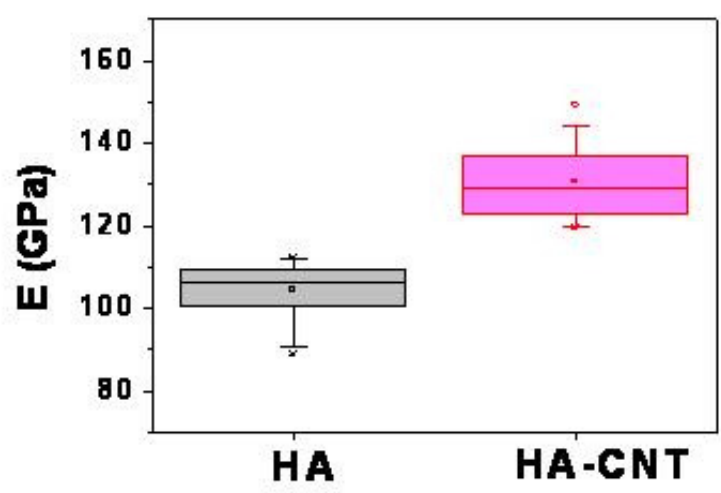

(b)

Figure 4.21: (a) Load vs. displacement plot for HA and HA-CNT composite obtained by nanoindentation, (b) statistical distribution of E value in HA and HA-CNT composites measured for more than 100 nano-indents in each sample.

A representative load vs. displacement curve for both HA and HA-CNT is shown in figure 4.21a. Low indentation depth of HA-CNT sample in figure 4.21a indicates higher hardness. Elastic modulus, calculated from the unloading part of the load- 
displacement curves (table-4.2), shows a 25\% improvement with CNT reinforcement in HA. The measured $E$ value of $130 \mathrm{GPa}$ in the present study agrees well with the reported $E$ for HA-CNT composite processed through SPS route [33]. The spread in $E$, as presented in the statistical distribution plot in figure $4.21 \mathrm{~b}$, is due to the localized nature of the measurement technique. Similar amount of spread in $E$ value for both HA and HACNT indicates homogeneous improvement in effective elastic modulus at macro-scale length.

Fraction of the plastic and elastic work during indentation for the composite structure is given by the following expressions [46],

$$
\begin{aligned}
& W_{t}=W_{e}+W_{p} \\
& \frac{W_{p}}{W_{t}}=1-\left[\frac{1-3\left(\frac{h_{f}}{h_{m}}\right)^{2}+2\left(\frac{h_{f}}{h_{m}}\right)^{3}}{1-\left(\frac{h_{f}}{h_{m}}\right)^{2}}\right]
\end{aligned}
$$

where, $W_{t}, W_{p}$ and $W_{e}$ are the total, plastic and elastic (reversible) work done respectively, $h_{m}$ is the depth of the indent at the peak load and $h_{f}$ is the final depth of indentation after recovery. Table- 4.2 shows the ratio of elastic work in HA-CNT (0.64) increases by $20 \%$ than HA (0.53). This calculation shows that CNT reinforcement makes the HA matrix more prone to recovery upon deformation.

The increase in the elastic modulus of HA-CNT composite is attributed to three major factors: (i) higher $E$ value of CNT reinforcement (200-1000 GPa [47]), (ii) 
homogeneous distribution of CNT in HA matrix and (iii) strong HA/CNT interface. The overall effective elastic modulus of the composites has also been computed using Eshelby [48] and Mori-Tanaka [49] micromechanics models. A lower value of E of CNT 200 GPa was used for these computations as defects have been introduced in the CNT structure during SPS, which will have negative effect on the elastic modulus and tensile strength of a pristine CNT. The computed $E$ values, presented in table 4.2 , are in the similar range as of experimentally measured $\mathrm{E}$ values. The mismatch between the computed and measured $E$ values could be due to several reasons. Firstly, the effect of porosity present in the composite has not been taken care in the computed model. Secondly, presence of few CNT clusters also causes the localized decrease in E values. Moreover, both the models consider the ellipsoid reinforcement particles, whereas CNTs are tubular in shape.

Table 4.2: Mechanical properties measured by nanoindentation and Vickers indentation methods and calculated through micromechanics models.

\begin{tabular}{|c|c|c|c|c|c|c|}
\hline Sample & E (GPa) & E (GPa) & $\begin{array}{c}\text { E (GPa) } \\
\text { (measured) } \\
\text { (Eshelby) } \\
\text { Tanaka) }\end{array}$ & $\mathbf{W}_{\text {elas }} / \mathbf{W}_{\text {tot }}$ & $\begin{array}{c}\text { H (GPa) } \\
\text { (Vickers) }\end{array}$ & K (Anstis' equation) \\
\hline \hline HA & $104 \pm 6$ & - & - & 0.53 & $7 \pm 0.2$ & $1.25 \pm 0.91$ \\
\hline HA-4CNT & $130 \pm 8$ & 159 & 160 & 0.64 & $9 \pm 0.4$ & $2.4 \pm 0.60$ \\
\hline
\end{tabular}

Good bonding at the HA/CNT interface also plays a role in improving the E for the composite structure. When a stress is applied on a composite material, the matrix deforms first due to lower elastic modulus. A strong matrix/reinforcement interface 
would resist the selective elastic deformation of matrix by transferring the stress on the reinforcement. Thus, the effective elastic strain generated in the composite matrix reduces with application of elastic stress resulting in an increase in $\mathrm{E}$ for the composite. Figure 4.22a presents the fracture surface with CNTs protruded from the HA matrix. The protruded CNTs in figure 4.22a shows much shorter length $(100-300 \mathrm{~nm})$ compared to the length of CNTs used in this study $(1-3 \mu \mathrm{m})$, which indicates a strong bonding between matrix and reinforcement. Strong interfacial bond hinders the sliding between reinforcement and matrix and increases the fiber-pull out stress. As a result, only partial debonding of reinforcement from the matrix occurs, resulting into shorter length of pullouts. Figure 4.22b shows high magnification SEM image of a CNT pull-out. CNT/HA interface shows a very good bonding without any sign of loosening or cracks.
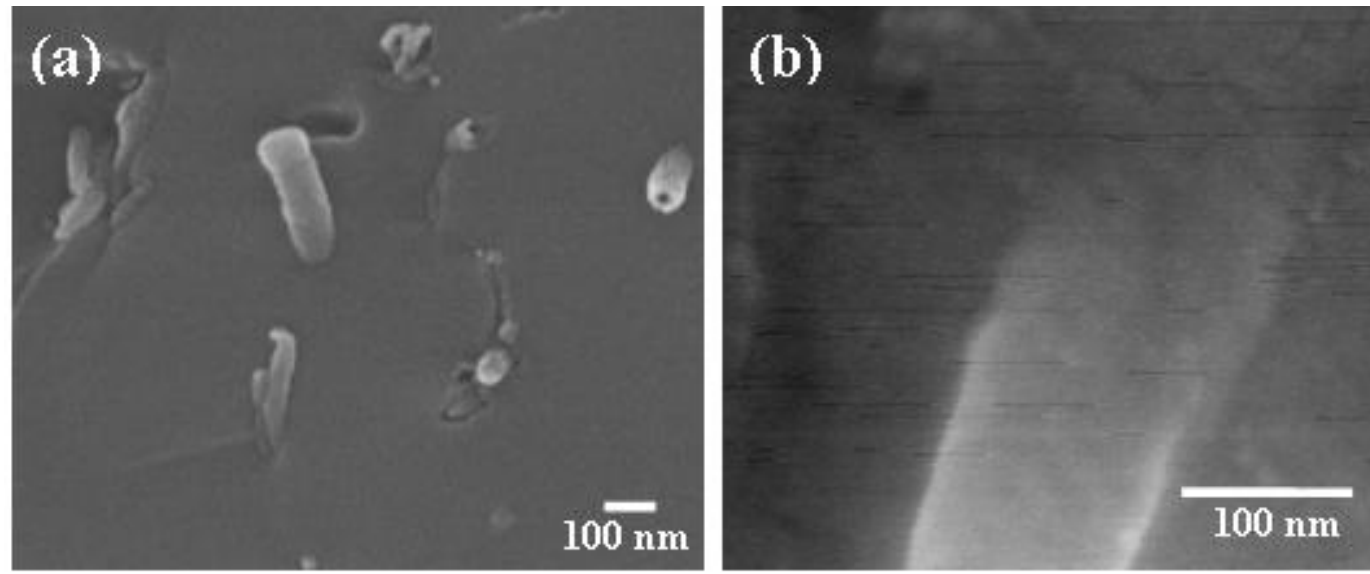

Figure 4.22: High magnification SEM micrographs of HA-CNT fracture surface showing (a) protruded CNTs and (b) strong and defect free interface of CNT with HA matrix.

\section{Fracture Toughness}

Fracture toughness was evaluated using indentation cracking method. Vickers indentation at $1 \mathrm{~kg}$ of normal load was employed to initiate cracking in the composite. Figures 4.23a and 4.23c show Vickers indents on the cross-sections of HA and HA-CNT 
pellets, respectively. Indent on HA-CNT shows a smaller impression with shorter radial cracks as compared to HA. The indents were observed at high magnification under SEM for the accurate measurement of the crack lengths. Fracture toughness of the composite structures has been evaluated using Anstis' equation [equation 4.1]

$K_{I C}$ shows a $92 \%$ improvement with CNT addition in HA, whereas hardness increases by $29 \%$ (table 4.2). $K_{I C}$, calculated for HA-CNT composite in the present study $\left(2.4 \mathrm{MPa} \cdot \mathrm{m}^{0.5}\right)$ is much higher than the only available literature value of $1.27 \mathrm{MPa} . \mathrm{m}^{0.5}$ [34]. The improvement of $K_{I C}$ in the same study is $30 \%$, which is much less than $92 \%$ improvement reported in the present study. One of the probable reasons is the lower content of CNT (2.5 vol. \%) used in the Ref [34], as compared to the present study (4 wt. $\% \approx 6$ vol. $\% \mathrm{CNT}$ ). Further, the use of spray dried HA-CNT composite powder in the present study ensures the better distribution and good bonding of CNT with matrix in the sintered structure. The detrimental effect of few CNT clusters was minimal in comparison to benefits from uniformly dispersed CNTs. Detailed SEM investigations inside the indentation cracks reveal two factors responsible for the improvement in fracture toughness of HA-CNT composite. One of them is the resistance to crack propagation at fine grain region of HA-CNT composite. Figure $4.23 \mathrm{c}$ shows the deflection and arrest of crack at fine grained region in HA-CNT. In contrast, HA fine grain region does not arrest the crack as shown in figure $4.23 \mathrm{~b}$. This is attributed to the difference in the densification level of the fine grain region in HA and HA-CNT. HACNT composite has high fraction of fine grained region (table-4.1), causing the crack propagation more difficult. The second mechanism that resists the crack propagation is 
CNT-bridging. High magnification SEM micrographs inside the cracks (figures $4.23 \mathrm{e}$ \&4.23f) show individual CNTs forming bridges on the crack and resisting its propagation.

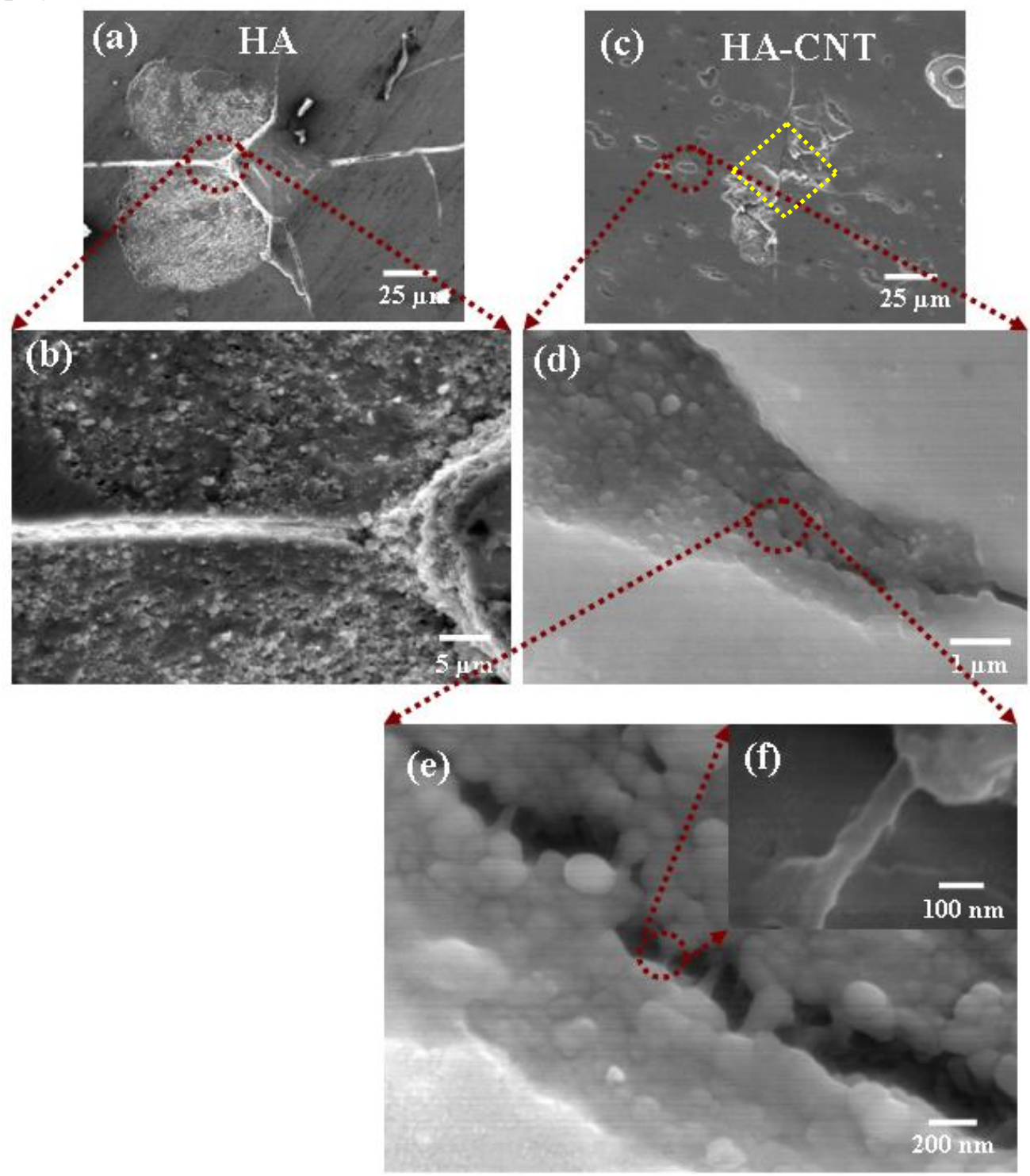

Figure 4.23: SEM micrographs of polished cross sections in HA and HA-CNT showing (a) Vickers indents on HA and (b) radial crack from the indent propagating in fine grain region of $H A$, (c) Vickers indent on $H A-C N T$, (d) deflection and arrest of radial crack at fine grain region in $H A-C N T$, (e) crack bridging by CNTs and (f) a single CNT bonded with HA matrix at both ends and forming bridge on crack. 
Further evaluation of toughening mechanism in HA-CNT composite has been studied using a model developed by Chen et al. that computes interfacial shear strength and reinforcement pull-out energy for CNT-ceramic composites [50]. The effective area of load carrying outer layers $\left(A_{e f f}\right)$ of multiwalled CNTs has been calculated using the following expression:

$A_{\text {eff }}=\pi \sum_{m=1}^{N}\left\{\left[R_{C N T}-(m-1) h-(m-1) h^{\prime}\right]^{2}-\left[R_{C N T}-m h-(m-1) h^{\prime}\right]^{2}\right\}$

where, $R_{C N \mathrm{~T}}$ is the outer radius of CNT (25-50 $\mathrm{nm}$ in this study), $h$ is the effective layer (wall) thickness $(\sim 0.075 \mathrm{~nm}), d$ is the spacing between each graphene layer $(\sim 0.34 \mathrm{~nm})$ and $h^{\prime}=d-h$, and $N$ is the number of outer layers carrying load. The CNTs used in this study has 28 to 51 graphene layers (measured from HRTEM images). But, the CNTs having SPS induced defects, only the outer 5 layers have been considered as active load carrying components in this calculation. Cox model has been used to compute the interfacial shear strength $(\tau)$ between HA and CNT. Cox model assumes the fiber at the centre of a coaxial cylinder of the matrix (of radius $R$ ) to calculate $\tau$, which is expressed as following:

$\tau=\frac{E_{C N T} \times e \times A_{e f f} \times \beta}{2 \pi R_{C N T}} \times \frac{\sinh \beta\left(\frac{L}{2}-x\right)}{\cosh \beta \frac{L}{2}}$

where, $\beta=\sqrt{\left(\frac{G_{H A}^{\prime}}{E_{C N T}}\right)\left(\frac{2 \pi}{A_{e f f} \ln \left(\frac{R}{R_{C N T}}\right)}\right)}$ 
$E_{C N T}$ is the elastic modulus of CNT, used as $200 \mathrm{GPa}$ for this study. The applied strain $e$ has been taken as 0.04 , the fracture strain of HA [51]. $L$ is the length of CNT, which is 1$3 \mu \mathrm{m}$ in this study and $x$ is the distance from end of $\mathrm{CNT}$. $\mathrm{G}_{\mathrm{HA}}^{\prime} \sim 45 \mathrm{GPa}$ [52] is the shear modulus of HA. The radius of matrix coaxial cylinder, $R$, has been calculated using the following relationship,

$\left(\frac{R}{R_{C N T}}\right)^{2}=\frac{\pi}{4 V_{f}}$

$V_{f} \sim 6$, is the volume fraction of CNT in HA matrix. The calculated interfacial shear stress at HA-CNT interface, $\tau$, is $0.3-0.35 \mathrm{GPa}$. Hence, a shear stress greater than this value has to be applied to interface in-order to cause CNT debonding from HA matrix. The computed $\tau$ has been used in calculation of pull-out energy $\left(G_{\text {pullout }}\right)$ for HA-CNT system [50], using equation 12 .

$$
G_{\text {pullout }}=\frac{V_{f} l^{2} \tau}{3 R_{C N T}}
$$

where, $l$ is the pullout length of CNTs $(100-300 \mathrm{~nm})$ measured from SEM images. The computed $G_{\text {pullout }}$ for CNT from HA matrix is $1.5-22 \mathrm{~J} / \mathrm{m}^{2}$. The fracture energy for monolithic HA is $1 \mathrm{~J} / \mathrm{m}^{2}$ [53]. The CNT pull-out energy being higher than fracture energy of HA, cracks propagate through HA, but gets restricted when comes in the vicinity of CNT, as more energy is required for interface debonding. Hence, CNT bridges shown figures $4.23 \mathrm{e}$ and $4.23 \mathrm{f}$, absorb more energy and provides resistance to crack growth. Chen et al. has also observed crack deflection at the alumina-CNT composite due to debonding of CNT from matrix prior to fracture in matrix [50]. 


\subsection{Wear Behavior of SPS HA-CNT Across Multiple Length Scale}

This study reports macro and nano-scale tribological behavior of the SPS processed HA-CNT composite. Macro-scale wear was conducted using ball-on-disk tribometer whereas nano-scale wear was evaluated using nano-scratch studies by nanoindenter.

\section{Macro-Scale Wear}

The wear resistance has been defined in terms of volume loss of the wear track at different traverse distances. The instantaneous depth of wear track is measured using 'linear variable differential transformer' (LVDT). The cumulative volume loss and coefficient of friction (CoF) for HA and HA-CNT composite are shown in figures 4.24a and $4.24 \mathrm{~b}$, respectively. Each point on figure $4.24 \mathrm{~b}$ shows an average value of CoF for 25 m interval. The error bars on figure 4.24 are based on three wear tracks studied for each sample. Wear resistance increases by $66 \%$, whereas CoF decreases by $60 \%$ with CNT addition in HA.

The decrease in CoF for HA-CNT indicates more lubrication in the wear track, which could be due to graphene layer removal from CNT by application of shearing force during macro-wear. This assumption is indirectly supported by the change in relative intensity of D and G peaks of CNT in Raman spectroscopy plots of wear track (figure 4.14a). The $\mathrm{I}_{\mathrm{D}} / \mathrm{I}_{\mathrm{G}}$ ratio increases from 0.72 in SPS HA-CNT pellet to 1.28 in the wear track of the same sample. 

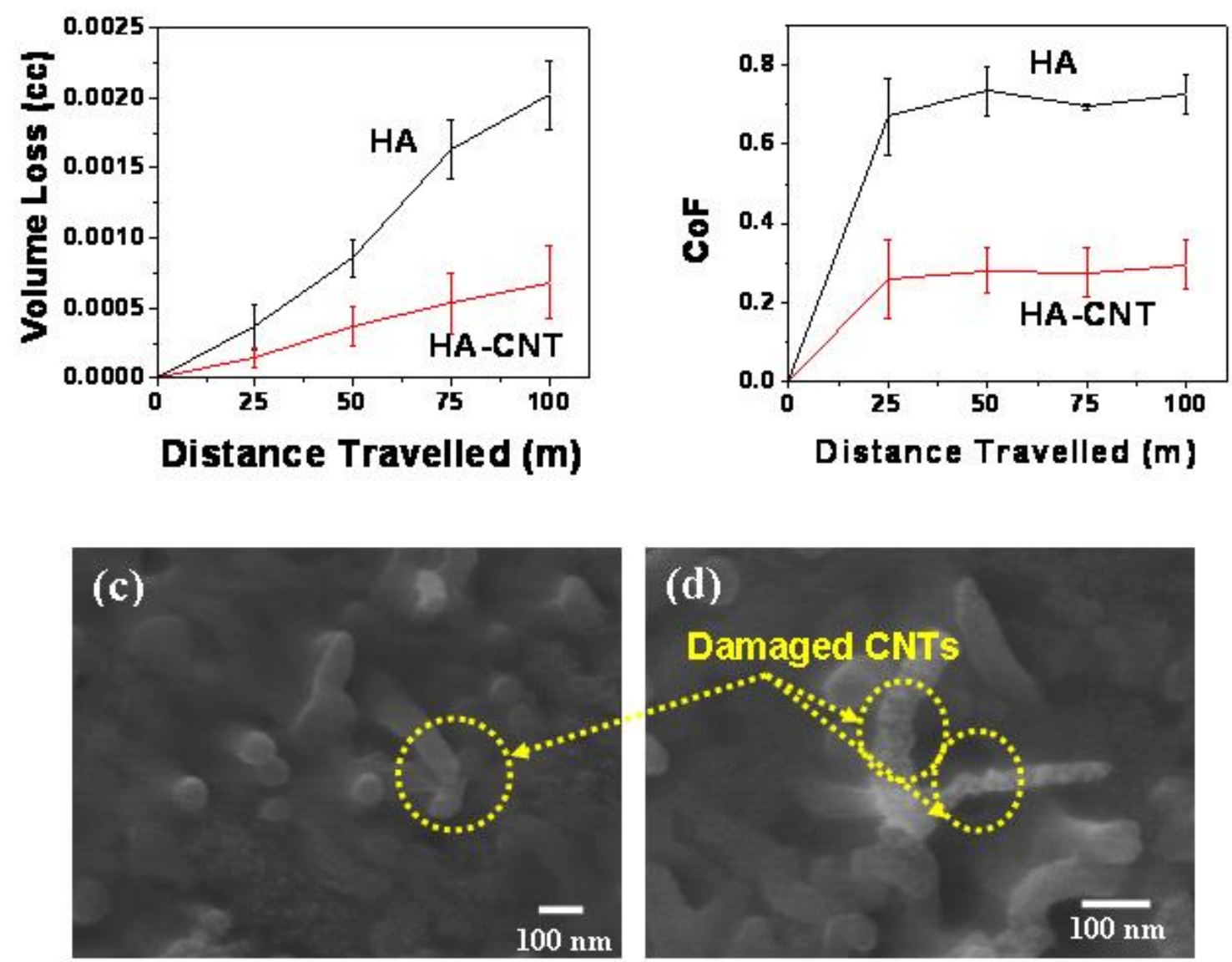

Figure 4.24: (a) Wear volume loss and (b) coefficient of friction for $H A$ and $H A-C N T$ plotted against sliding distance during ball-on-disk wear, (c-d) High magnification SEM micrographs of broken and damaged CNTs observed in the HA-CNT wear track.

Increase in $\mathrm{I}_{\mathrm{D}} / \mathrm{I}_{\mathrm{G}}$ ratio indicates more defects getting introduced in the CNTs during wear, which is resulted from tearing off of graphene layers. Further, a shift of D and $\mathrm{G}$ peak of CNT towards lower wave number is also observed in the wear track, which is attributed to the tensile stress present due to shearing force applied on wear track [54-56]. The shift of the D and G peaks to lower wave numbers is best understood on the basis of an elongation of the carbon-carbon bonds under tension, which makes the bond weaker and therefore lowers the vibrational frequency [54]. Same is true for the 
phosphate peak of HA in wear track also. The extent of shift is more in D and phosphate peaks than in G peak. This is because G peak undergoes a shift to higher wave number due to increase in defect density [28] and simultaneously to lower wave number due to tensile stress induced in it. Hence, the observed shift in G peak is the resultant of these two opposing effects. Increase in the defect density in CNTs during wear is visually observed from the SEM micrographs in figures $4.24 \mathrm{c}$ and $4.24 \mathrm{~d}$, showing presence of damaged and broken CNTs in the wear track. Graphene layer peeling from CNTs by application of shear force is in agreement with a study by Li et al. on SPS consolidated structure [57]. The peeled off graphene layers cover the wear track during wear, thus providing lubrication and decreasing the coefficient of friction. CNT clusters provide more lubrication due to increased probability of graphene layer formation.

Increase in the wear resistance for HA-CNT composite is a combined effect of improvement in the mechanical properties $\left(\mathrm{E}, \mathrm{H}\right.$ and $\left.\mathrm{K}_{\mathrm{IC}}\right)$ caused by $\mathrm{CNT}$ reinforcement and decrease in the coefficient of friction. Improvement in $\mathrm{E}, \mathrm{H}$ and $\mathrm{K}_{\mathrm{IC}}$ increases the resistance to removal of mass with the same amount of applied load. At the same time, decrease in CoF (ratio of lateral and normal force) decreases the effective lateral force in the wear track for same amount of normal force. In the present study, the effective lateral force in HA-CNT is as low as $2.5 \mathrm{~N}$, compared to $\sim 7 \mathrm{~N}$ in $\mathrm{HA}$ sample, due to decrease in CoF. Decrease in the lateral force due to graphite induced lubrication causes lesser wear volume loss in case of HA-CNT.

\section{Nano-scale Wear}


Nano-scale tribological properties of HA and HA-CNT composite have been measured by making nano-scratches with different normal loads. The volume loss has been calculated from the geometry of scratch profile obtained through SPM imaging of the scratch after elastic recovery has taken place, using the following expression:

$$
V=\int_{0}^{l} h^{2} \tan \theta \cdot d l
$$

where, $V$ is the volume of the scratched groove, $h$ is the height of the groove (obtained from 2D SPM profile of scratch along the length), Ais the average angle of the groove measured at five points along the scratch length using 2D SPM profile and $l$ is the length of the scratch. The detailed procedure of the volume measurement in explained in a study by Bakshi et al. [58].

Figures $4.25 \mathrm{a}$ and $4.25 \mathrm{~b}$ show the wear volume loss and CoF, respectively, for HA and HA-CNT at normal loads of $3500 \mu \mathrm{N}$ and $4500 \mu \mathrm{N}$. The error bars in figure 4.25 are based on three scratches made at each load on each sample. Wear volume loss increases with increase in load and is $\sim 45 \%$ higher for HA for both the loads. CoF remains similar at different loads in each of the compositions, but HA-CNT shows $14 \%$ increase in CoF. Decrease in the wear volume loss for HA-CNT indicates its higher wear resistance, which is due to the improvement in $E, H$ and $K_{I C}$ as discussed in case of macro-wear. Increase in $\mathrm{CoF}$ in HA-CNT indicates the absence of any significant lubrication in case of nano-wear. 
Another important observation from the nano-scratch study is the effect of CNT addition on the recovery of HA matrix, which has been calculated using depth of the scratched-groove during scratch and after recovery. HA-CNT shows increase in the recovery at both $3500 \mu \mathrm{N}$ and $4500 \mu \mathrm{N}$ loads suggesting higher elastic deformation in the presence of CNTs (figure 4.25c). This observation directly matches with the higher fraction of elastic work in HA-CNT as calculated from the nanoindentation studies (table 4.2).
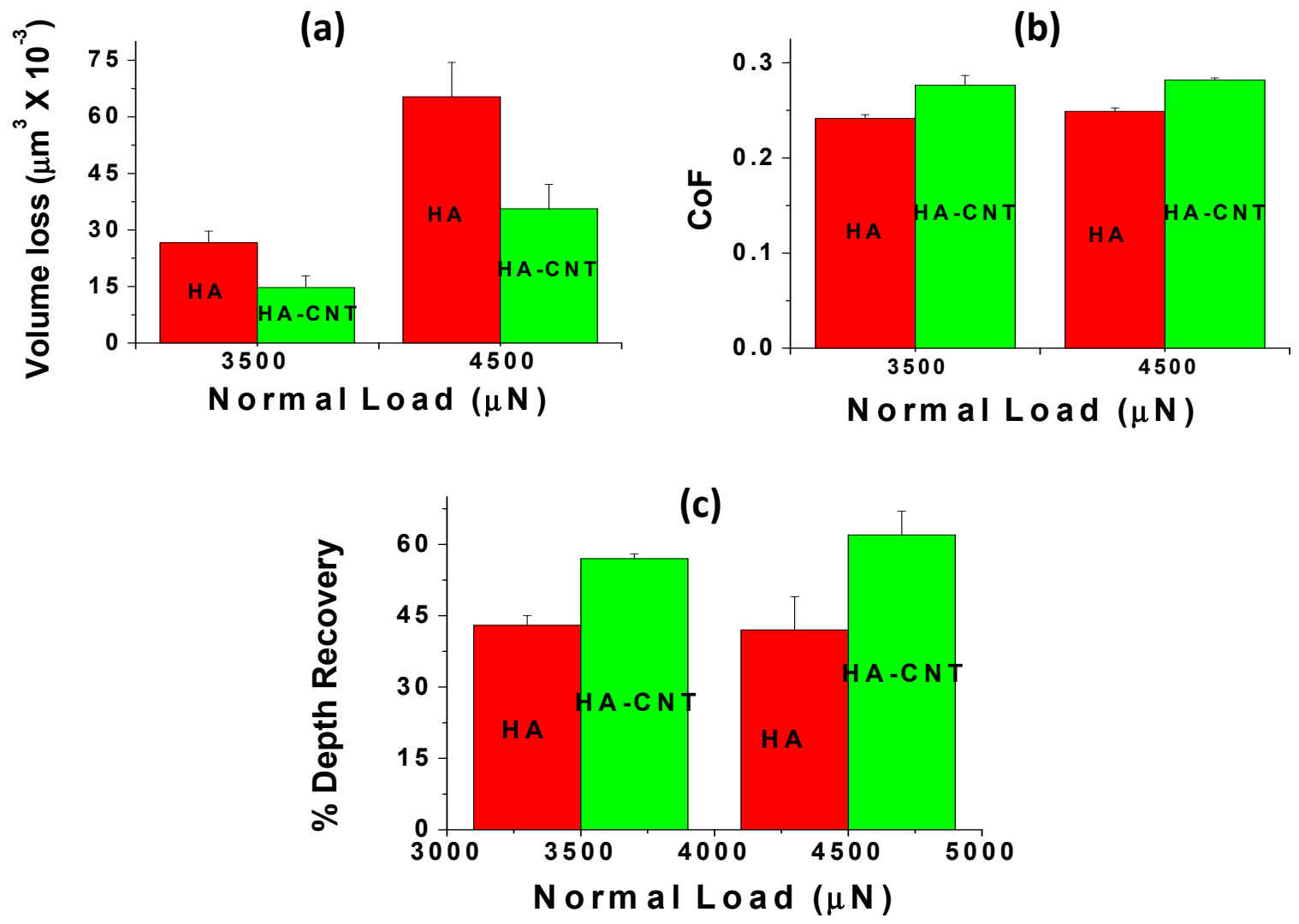

Figure 4.25: Nano-scale (a) wear volume loss, (b) coefficient of friction and (c) percentage elastic depth recovery during nano-scratch of $H A$ and $H A-C N T$ as a function of normal load. 


\section{Differential role of CNT at varying length scales of wear}

Tribological properties of HA and HA-CNT composites show distinct differences at macro and nano length scale. With CNT addition, $\mathrm{CoF}$ decreases by $60 \%$ in case of macro-wear, whereas it increases by $14 \%$ for nano-wear. Further, macro-wear shows 66\% increase in wear volume resistance with CNT reinforcement, whereas nano-wear study reveals increase in wear resistance by $45 \%$.

Increase in $\mathrm{CoF}$ in the case of macro-wear results from the lubrication offered by peeled graphene layers from CNT walls. Lower lubrication in nano-wear is attributed to the absence of damage and breaking of CNT walls. The effective lateral stress exerted on the CNT walls during macro and nano-scale further elucidates this phenomenon.
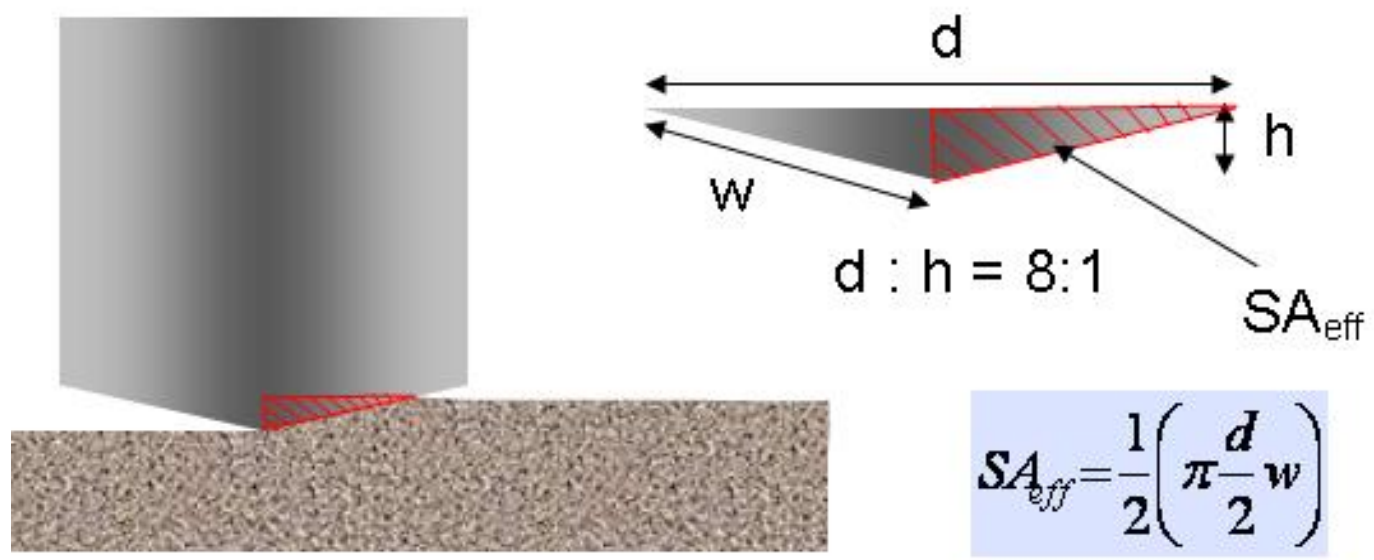

Figure 4.26: Schematic diagram showing effective surface area (SA $\left.A_{\text {eff }}\right)$ for nano-scratch study.

Effective lateral stress on the sample surface is same as the stress on the wear probe in contact with sample during removal of mass. The contact area of probe with the sample surface, is referred as effective surface area $\left(S A_{\text {eff }}\right)$. Figure 4.26 shows a schematic for the calculation of $S A_{\text {eff }}$ for Berkovich indenter tip used for the nano- wear study. The three sided pyramid shape of Berkovich tip has been approximated to a conical shape, 
keeping the aspect ratio of height to base (1:8) same. For the scratches made with 4500 $\mu \mathrm{N}$ normal load, the average depth of scratch is $300 \mathrm{~nm}$ which corresponds to $S A_{\text {eff }}$ as $4.66 \mu \mathrm{m}^{2}$. The CoF for the same case being 0.29 , the lateral force for the scratch is $\sim 1300$ $\mu \mathrm{N}$. These conditions generate a stress of $300 \mathrm{MPa}$ at the Berkovich tip surface in contact with the HA-CNT sample.

In the case of macro-wear, the normal force applied is $10 \mathrm{~N}$. The CoF being 0.25 , the effective lateral force on the wear track is $2.5 \mathrm{~N}$. Maximum wear depth achieved in the track is $0.097 \mathrm{~mm}$. For a total wear distance of $100 \mathrm{~m}$, it takes $8 \times 10^{3}$ revolutions in a 2 $\mathrm{mm}$ radius wear track. Hence, the average wear depth increase per revolution is $1.25 \mathrm{X}$ $10^{-5} \mathrm{~mm}$. A $3 \mathrm{~mm}$ diameter alumina ball has been used as wear probe. Schematic in figure 15 presents the $S A_{\text {eff }}$ for macro-wear. The wear depth per revolution being very small with respect to the radius of wear probe, the surface area of sphere has been approximated to the circular area of the truncated sphere as shown in figure 4.27 . The $S A_{\text {eff, }}$, thus calculated, is $1.18 \times 10^{-4} \mathrm{~mm}^{2}$. Hence, the lateral stress achieved in case of macro-wear is $\sim 21 \mathrm{GPa}$.
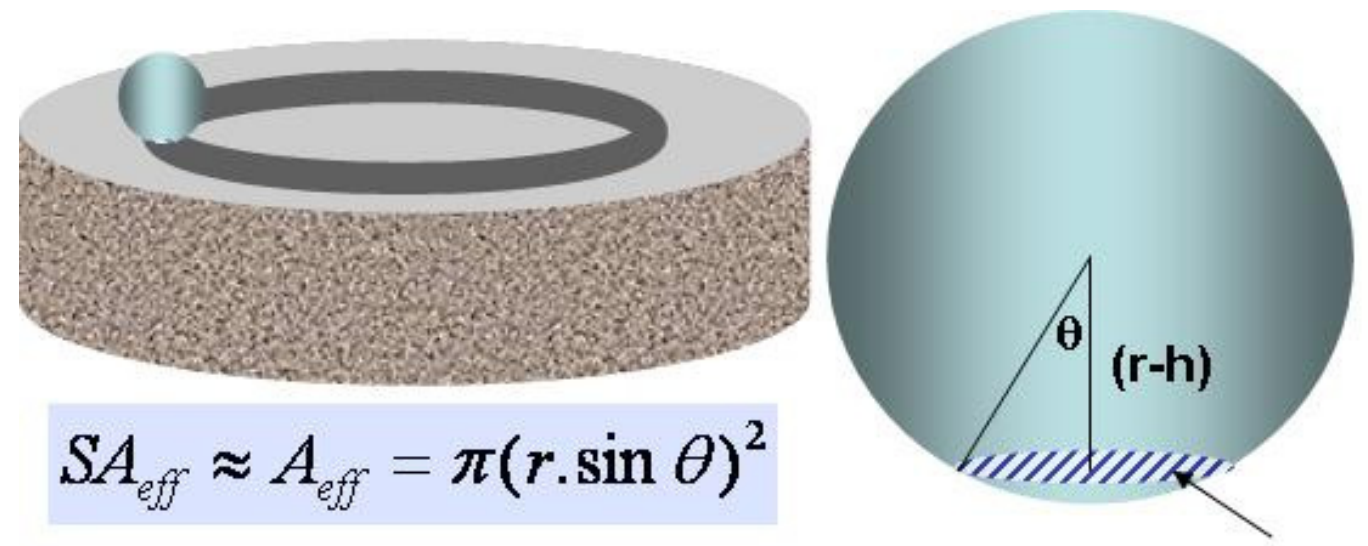

$\mathbf{A}_{\text {eff }}$

Figure 4.27: Schematic diagram showing effective surface area ( $\left.S A_{\text {eff }}\right)$ for macro-wear study. 
These calculations show that macro-wear applies 70 times higher lateral stress on wear track than nano-wear. Yu et al. have reported that removal of a single graphene layer from multi-walled CNT requires a tensile force in range of 11-63 GPa, with varying OD and length of nanotubes [24]. Lateral force during wear causes shearing removal of mass on the surface, which causes tensile stress on the newly exposed surface after mass removal. As the force applied in nano-wear (300 MPa) is much smaller than the minimum stress required for graphene layer peeling from CNT (11 GPa), no lubrication is available in this case. On the contrary, the available lateral stress in case of macro-wear (21 GPa) is enough to remove graphene layer which causes a significant decrease in CoF. Increase in lubrication also contributes towards increasing wear resistance for HA-CNT.

Research on SPS processed HA-CNT composite indicates positive role of CNT reinforcement towards mechanical properties and tribological behavior of the composite. Rapid heating rate for SPS processing prohibits the dissociation of HA into TCP. Good electrical and thermal conductivity of CNT plays important roles in improved densification of microstructures of HA-CNT composite. CNT also pins the grain boundaries and retain HA nanostructure, which helps in improving elastic modulus and fracture toughness. A $92 \%$ increase in the fracture toughness of HA-CNT was obtained due to a combined effect of improved modulus, higher fraction of nanocrystalline HA region and crack bridging by CNTs. Macro-scale dry sliding wear of HA-CNT composite showed a $66 \%$ improvement in the wear resistance and $60 \%$ reduction in $\mathrm{CoF}$. Increase in the wear resistance is due to improvement in mechanical properties $\left(\mathrm{E}, \mathrm{H}\right.$ and $\mathrm{K}_{\mathrm{IC}}$ ) as well as decrease in CoF for HA-CNT. The decrease in CoF is due to peeling of graphene layer from CNTs that provide lubrication. In case of nano-scale wear, a $45 \%$ increase in 
the wear resistance is observed. Nano-scale CoF increase by $14 \%$ in presence of CNT, due to absence of lubrication from graphene layer peeling. The effective shear stress during macro-wear causes graphene layers to peel-off from CNTs. The effective shear stress in nano-wear is significantly lower than the minimum stress required for removing a graphene layer from CNT surface.

This study also proposes BNNT to be a suitable alternative to CNT for similar application. The following section presents and discusses the microstructural, mechanical and tribological behavior of spark plasma sintered HA-BNNT composite.

\subsubsection{Hydroxyapatite-Boron Nitride Nanotube Composite by Spark Plasma} Sintering

HA-BNNT composites are synthesized by spark plasma sintering. The microstructural evolution and interfacial orientation relationship between BNNT and HA is analyzed. Effect of BNNT addition on the elastic modulus and fracture toughness of the composite is investigated. Tribological performance of HA-BNNT composite and the wear mechanism in presence of BNNT is also studied.

\subsubsection{Role of Boron Nitride Nanotube in Microstructural Evolution of SPS HA- BNNT Composite}

SEM image from the fracture surface of HA (figure 4.28) reveals partially sintered grain structure with porosity. On the contrary, HA-BNNT shows a dense and fully sintered fracture surface with negligible porosity (figure-4.28b). The density of HA- 
BNNT $(97.0 \pm 0.3 \%$ TD $)$ was higher than HA $(92.2 \pm 1.1 \%$ TD $)$. The densification in spark plasma sintering is achieved by extremely high heating rate that activates diffusion mechanisms, like grain boundary and lattice diffusion and power-law dislocation creep [36-38]. Thus, conduction of heat throughout the pellet during sintering is important for achieving better densification. The heating source is the graphite die in contact with periphery of the green pellet. A thermal gradient is generated through the thickness of the green pellet due to lower thermal conductivity of HA (1.25 W/mK [9]), which leads to incomplete sintering and porous structure. But, the thermal conductivity of BNNT is much higher than HA, with a reported value of $200-300 \mathrm{~W} / \mathrm{mK}$ in axial and $20-30 \mathrm{~W} / \mathrm{mK}$ in transverse direction [59-60]. Hence expected increase in thermal conductivity of HA with BNNT reinforcement results in accelerated diffusion and better densification of HABNNT. BNNTs have also displayed $160 \%$ increase in the thermal conductivity of PVA-5 vol. \% BNNT composite as compared to PVA [60].
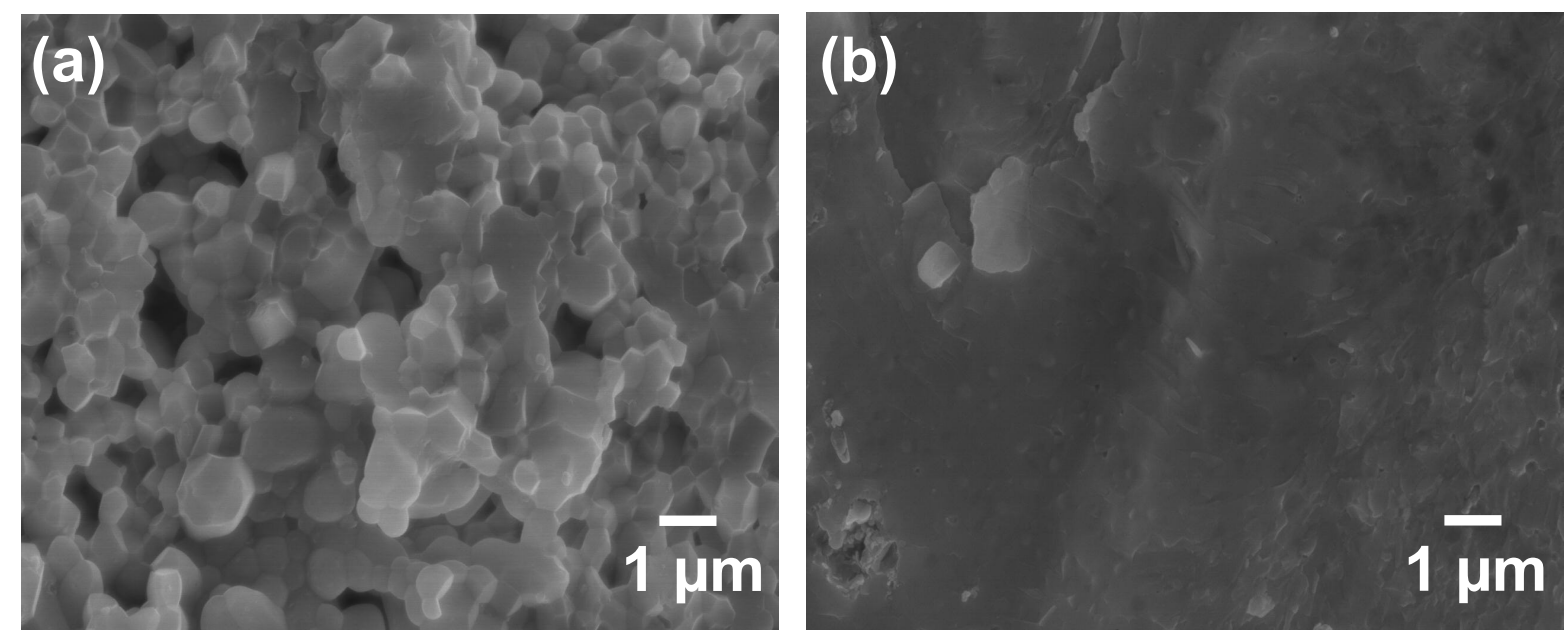

Figure 4.28: Fracture surface of (a) HA and (b) HA-BNNT sintered pellets revealing the porosity content. 
BNNTs play an active role in grain refinement of the sintered structure. HABNNT composite has a grain size of $0.17 \pm 0.1 \mu \mathrm{m}$, which is nearly three times finer than HA having a grain size of $0.61 \pm 0.16 \mu \mathrm{m}$. The presence of BNNTs has also lead to grain refinement in $\mathrm{A} 12 \mathrm{O} 3$ and $\mathrm{Si3} \neg \mathrm{N} 4$ based composites [61]. High surface to volume ratio makes BNNT more active for grain boundary pinning, as found in the case of CNT, the structural analogue of BNNT (section 4.1.1.2.3).

\subsubsection{Distribution and Structural Integrity of Boron Nitride Nanotube in SPS} Structure

The application of high temperature and pressure during spark plasma sintering necessitates investigation on the survival of BNNT structure in the sintered composite. Evidence of the existence of BNNTs in the sintered pellet is provided by the HRTEM images presented in figures $4.29 \mathrm{a}$ and $4.29 \mathrm{~b}$.

The defect free lattice images of BNNT in the sintered pellet rule out damage due to application of high pressure and temperature during SPS, as observed in case of CNTs [29]. High flexibility of BNNT along-with the ability to withstand high deformation without getting damaged [62] is attributed for their defect free structure in SPS pellet.

Figure 4.29c presents the Raman spectrum for HA and HA-BNNT at powder and sintered stages. The peak at $\sim 964 \mathrm{~cm}-1$, present in all spectra, is from the $v 1$ symmetric stretching vibration of phosphate anions in HA [27]. The peak at $\sim 1367 \mathrm{~cm}-1$, present in HA-BNNT powder and sintered pellet, is an E2g mode peak from h-BN [63-65]. Presence of h-BN peak in Raman spectrum of the pellet along with the tubular structures 
in HRTEM images (figures 4.29a and 4.29b) further establishes the survival of BNNT through spark plasma sintering process.
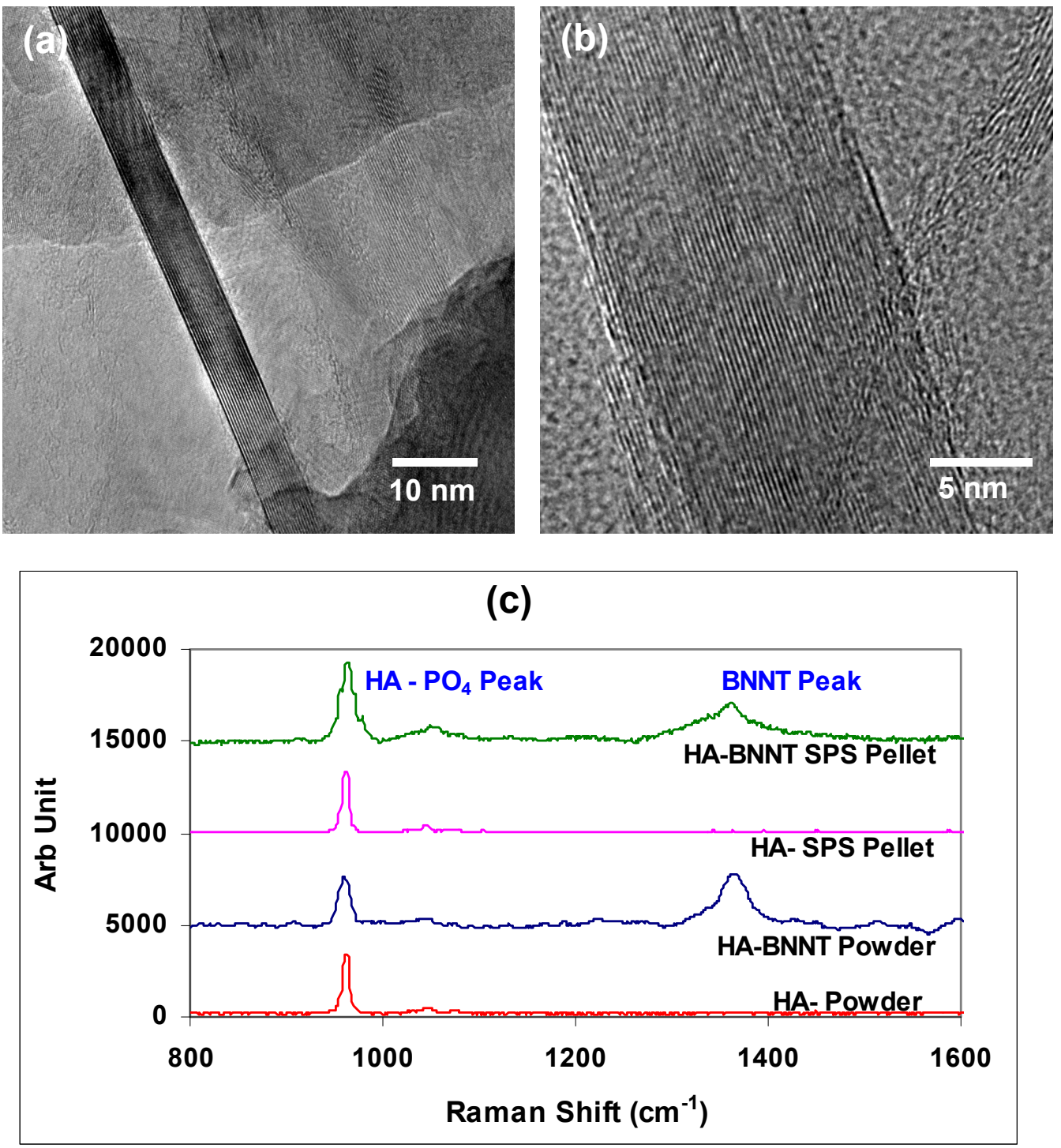

Figure 4.29: (a-b) HRTEM images of BNNT in the sintered HA-BNNT pellet retaining their defect free structure; (c) Raman spectrum of HA and HA-BNNT powders and sintered pellet.

\subsubsection{Phase Stability and Crystallinity of HA in SPS HA-BNNT Structure}

X-ray diffraction (XRD) patterns of the sintered HA and HA-BNNT pellets are shown in figure 4.30. The major peaks in both diffraction patterns are from 
hydroxyapatite (JCPDS PDF No. 9-432). Low intensity boron nitride (BN) peaks in HABNNT are generated from hexagonal (JCPDS PDF No. 34-0421) and tetragonal (JCPDS PDF No.-25-0098) crystal structures. XRD patterns reveal presence of some tetragonalBN impurity in the as-received BNNTs. Some of the BN peaks overlap with HA peaks in the vicinity (figure-4). None of the highest peaks of $\beta$-TCP (JCPDS PDF No. 9-169) and $\alpha$-TCP (JCPDS PDF No. 29-359) is present. These observations prove that HA does not significantly dissociate into TCP during SPS processing for both compositions. The SPS of HA-CNT composite using same processing parameters also reveals similar observation (section 4.1.1.2.2). Faster heating rate in SPS has prevented the long exposure of HA at high temperature and thus the dissociation into TCP.

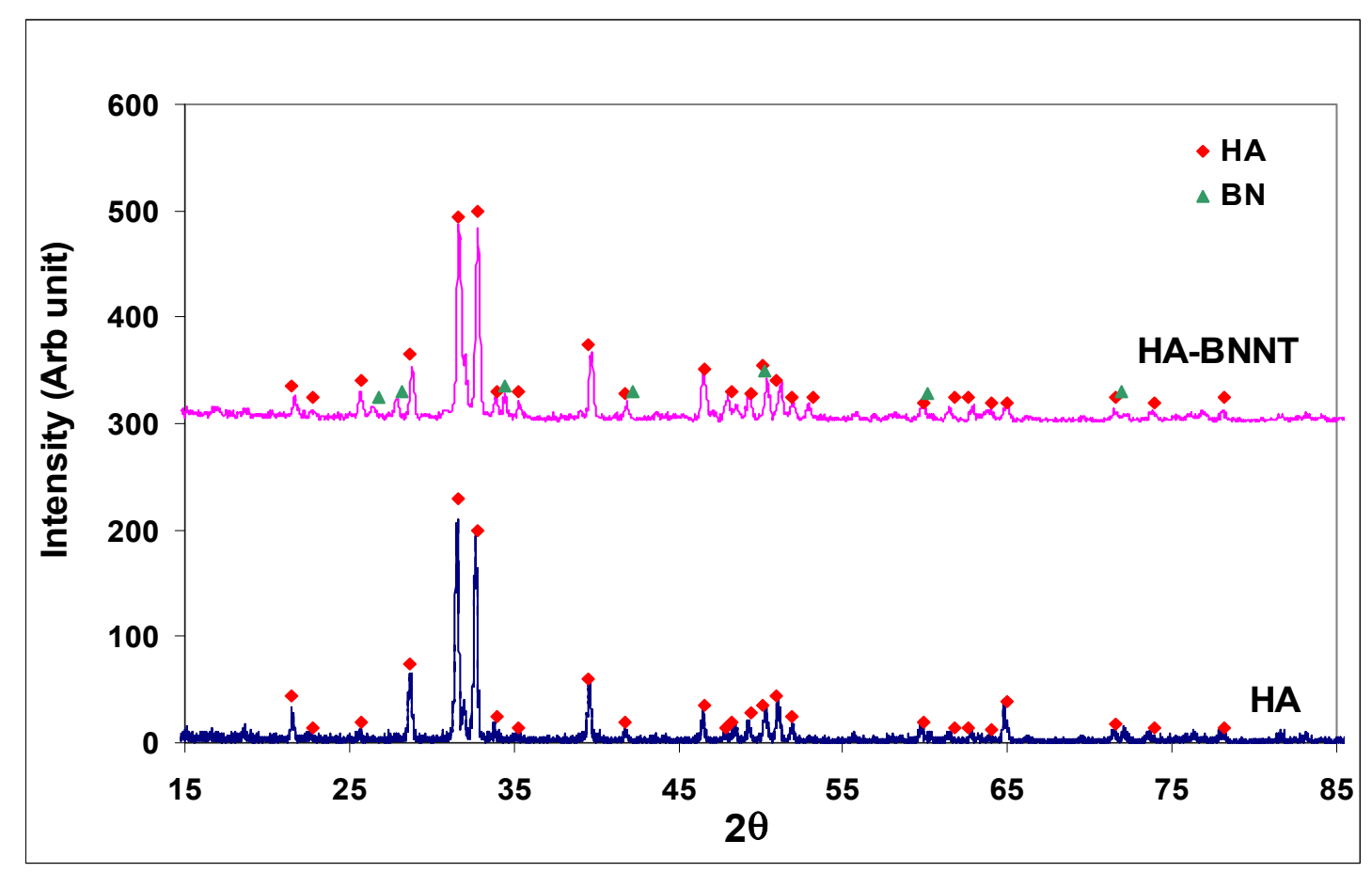

Figure 4.30: X-ray diffraction pattern of $H A$ and $H A-B N N T$ sintered pellet showing $H A$ and $B N$ peaks. 


\subsubsection{Hydroxyapatite-Boron Nitride Nanotube Interface in SPS Structure}

HA and BNNT are chemically non-reacting species. HRTEM image of HABNNT interface reveals absence of any reaction product (figure 4.31a), ruling out presence of ionic or covalent bond. Thus, Van der Waal's bond is the most probable at HA-BNNT interface. Hence the interfacial strength is mainly governed by the work of adhesion, which is dependent on the lattice arrangement at interface. Work of adhesion is higher when the lattice strain due to mismatch is minimal. A higher lattice mismatch, $\delta>$ 0.25 leads to incoherent interface and poor bonding [45]. Fourier transform (FFT and inverse-FFT) analysis of the lattice images from HRTEM micrograph (figure 4.31a) reveals the crystallographic orientation at HA-BNNT interface. BNNT shows h-BN walls with inter-wall spacing of $0.33 \mathrm{~nm}$, specific to boron nitride nanotube structure [66]. HA crystals are recognized from the lattice images of (211) planes with a lattice spacing of $0.282 \mathrm{~nm}$. The (211) planes of HA are arranged at an angular range of $65^{\circ}-68^{\circ}$ to the outer wall of BNNT. The basal plane in hexagonal HA structure also creates $65^{\circ}$ angle with (211) planes. Thus, probability of alignment of HA crystals on BNNT surface with basal planes being parallel to the outer h-BN wall is very strong. The symmetric hexagonal structure for both basal planes of $\mathrm{HA}$ and h-BN makes the alignment more evitable. Figure $4.31 \mathrm{~b}$ represents a schematic of the basal plane of HA superimposed on the h-BN wall. The basal plane of HA has Ca atoms sitting at each corner of the hexagon with a distance of $0.94 \mathrm{~nm}$ [67]. The distance between two neighboring atoms in h-BN is $1.44 \AA$ [68]. As shown in schematic in figure 4.31b, the distance between two B atoms on $\mathrm{h}$-BN, coinciding with the $\mathrm{Ca}$ atoms of superimposed HA basal plane, is $1.04 \mathrm{~nm}$. So, the 


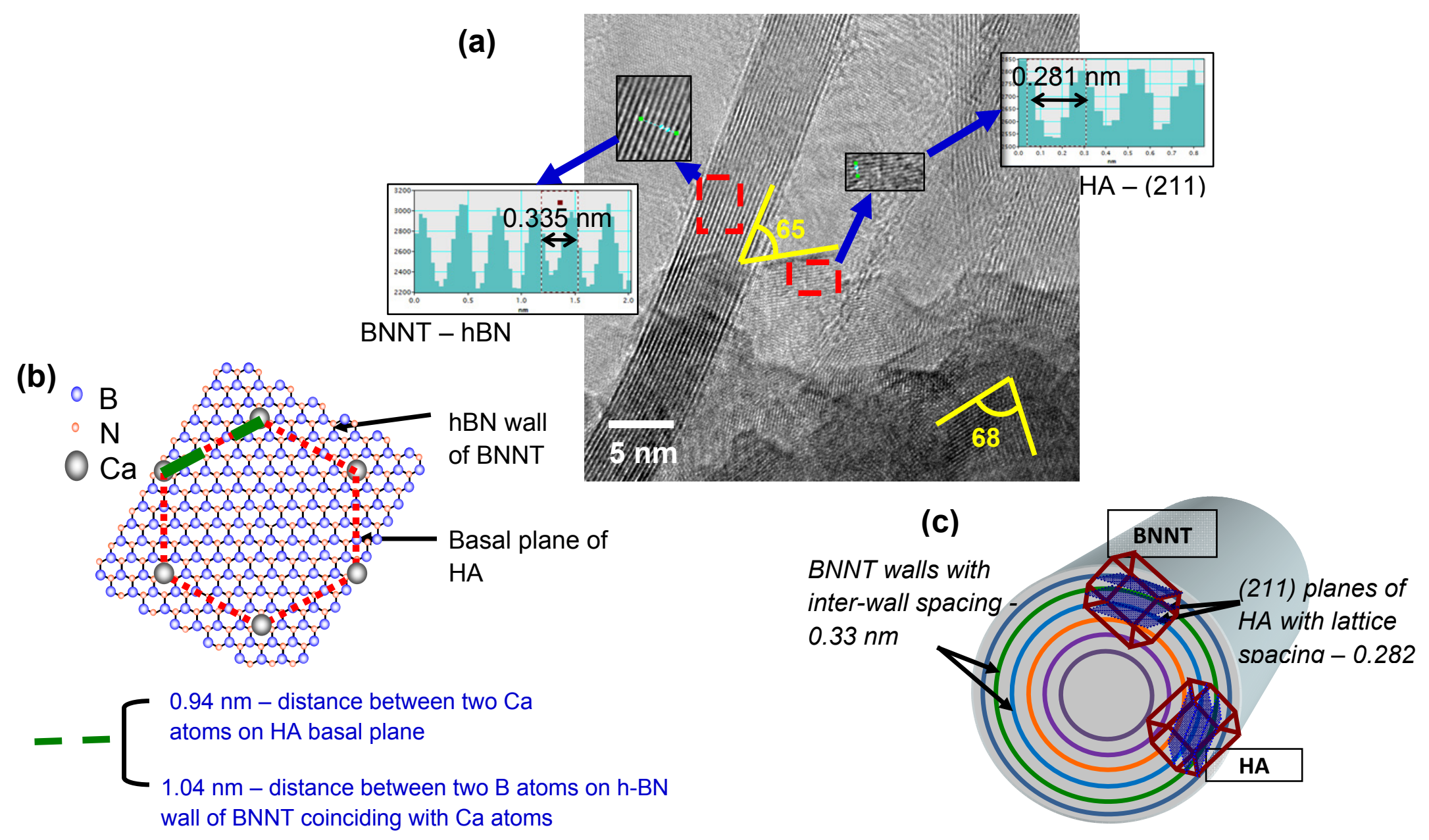

Figure 4.31: (a) HRTEM image of BNNT and HA interface. FFT analysis reveals the BNNT wall spacing and HA lattice (211) spacing at the interface; (b) schematic of atomic arrangement at the interface with basal plane of HA and coinciding $h$-BN sheet on BNNT outer wall; (c) schematic of BNNT open end showing alignment of h-BN walls with (211) planes of HA. 
mismatch between two superimposed pair of $\mathrm{Ca}$ (in $\mathrm{HA}$ ) and B (in h-BN) atoms is $\delta \sim$ 0.11 , which is much lower than the incoherence limit of 0.25 . Thus, the preferential alignment of HA crystals on BNNT surface suggest strong coherent interfacial bond with minimal lattice strain. Similar observation is made for interface of HA and CNT of SPS processed composite structure (section 4.1.1.2.4). HA can also form another interface with BNNT at its open end, as shown in figure 4.31c. BNNT has an interwall spacing of $0.33 \mathrm{~nm}$. The lattice spacing of (211) set of HA plane is $0.282 \mathrm{~nm}$. The lattice mismatch ( $\delta$ ) between (211) plane of HA and BNNT walls is 0.17 , which is also lower than 0.25 . Hence, open ends of BNNT forms a semi-coherent interface with HA crystals having (211) planes parallel to h-BN walls of BNNT.

The strength of the HA-BNNT interface can be estimated based on the model proposed by Chen et al. for $\mathrm{Al}_{2} \mathrm{O}_{3}-\mathrm{CNT}$ system [50] as adopted for SPS HA-CNT structure. BNNT is structural analogue of CNT with similar elastic modulus and tensile strength. The effective area of load carrying outer layers $\left(A_{\text {eff }}\right)$ of a multiwall BNNT is calculated using the following expression:

$$
A_{\text {eff }}=\pi \sum_{m=1}^{N}\left\{\left[R_{C N T}-(m-1) h-(m-1) h^{\prime}\right]^{2}-\left[R_{C N T}-m h-(m-1) h^{\prime}\right]^{2}\right\}
$$

where, $R_{B N N T}$ is the outer radius of BNNT (5-70 nm in this study), $h$ is the effective layer thickness ( $\sim 0.25 \mathrm{~nm}[69]), d$ is the spacing between each h-BN layer $(\sim 0.33 \mathrm{~nm}[66]), h^{\prime}$ $=d-h$, and $N$ is the number of outer layers carrying load. BNNTs have $\sim 10-50$ walls as observed from HRTEM images. For a conservative estimate of the strength, it has been assumed that load is borne by 5 outer walls of BNNT. Cox model is used to compute the interfacial shear strength ( $\tau$ ) between BNNT and HA. According to Cox model, the fiber 
at the centre of a coaxial cylinder of the matrix (of radius $R$ ) is used to calculate $\tau$ using the following expression:

$$
\tau=\frac{E_{B N N T} \times e \times A_{e f f} \times \beta}{2 \pi R_{B N N T}} \times \frac{\sinh \beta\left(\frac{L}{2}-x\right)}{\cosh \beta \frac{L}{2}}
$$

where,

$$
\beta=\sqrt{\left(\frac{G_{H A}^{\prime}}{E_{B N N T}}\right)\left(\frac{2 \pi}{A_{e f f} \ln \left(\frac{R}{R_{B N N T}}\right)}\right)}
$$

$E_{B N N T}$ is the elastic modulus of BNNT, used as $750 \mathrm{GPa}$ [70], which is the lower end of reported range of values. The applied strain $\varepsilon$ has been taken as 0.04 which is the fracture strain of HA [51]. $L$ is the average length of BNNT used in this study $(2 \mu \mathrm{m}), x$ is the distance from end of BNNT. $\mathrm{G}_{\mathrm{HA}}^{\prime} \sim 45 \mathrm{GPa}$ [71-72] is the shear modulus of HA. The radius of matrix coaxial cylinder, $R$, has been calculated using the following relationship,

$\left(\frac{R}{R_{B N N T}}\right)^{2}=\frac{\pi}{4 V_{f}}$

$V_{f} \sim 0.059$, is the volume fraction of BNNT in HA matrix. The calculated interfacial shear stress at HA-BNNT interface, $\tau$, is $0.35-3 \mathrm{GPa}$. Thus, BNNT debonding from HA matrix requires a minimum shear stress $\geq 0.35 \mathrm{GPa}$. The computed $\tau$ has been used in calculation of pull-out energy $\left(G_{\text {pullout }}\right)$ for HA-BNNT system, using equation 5.

$$
G_{\text {pullout }}=\frac{V_{f} l^{2} \tau}{3 R_{B N N T}}
$$


where, $l$ is the pullout length of BNNTs $(100-800 \mathrm{~nm})$ measured from SEM images of fracture surface. The computed $G_{\text {pullout }}$ for BNNT from HA matrix is $2-100 \mathrm{~J} / \mathrm{m}^{2}$, which is greater compared to the fracture energy of monolithic HA of $1 \mathrm{~J} / \mathrm{m}^{2}$ [73]. The higher value of BNNT pullout energy from HA matrix highlights its effectiveness as reinforcement for toughening.

\subsubsection{Elastic Modulus and Fracture Toughness of SPS HA-BNNT}

Measurement of the elastic modulus (E) is performed on the polished cross section of the sintered pellets using nanoindentation technique. More than 100 indents were made at randomly chosen regions in each sample. The representative load vs. displacement curves for HA and HA-BNNT are shown in figure 4.32a.
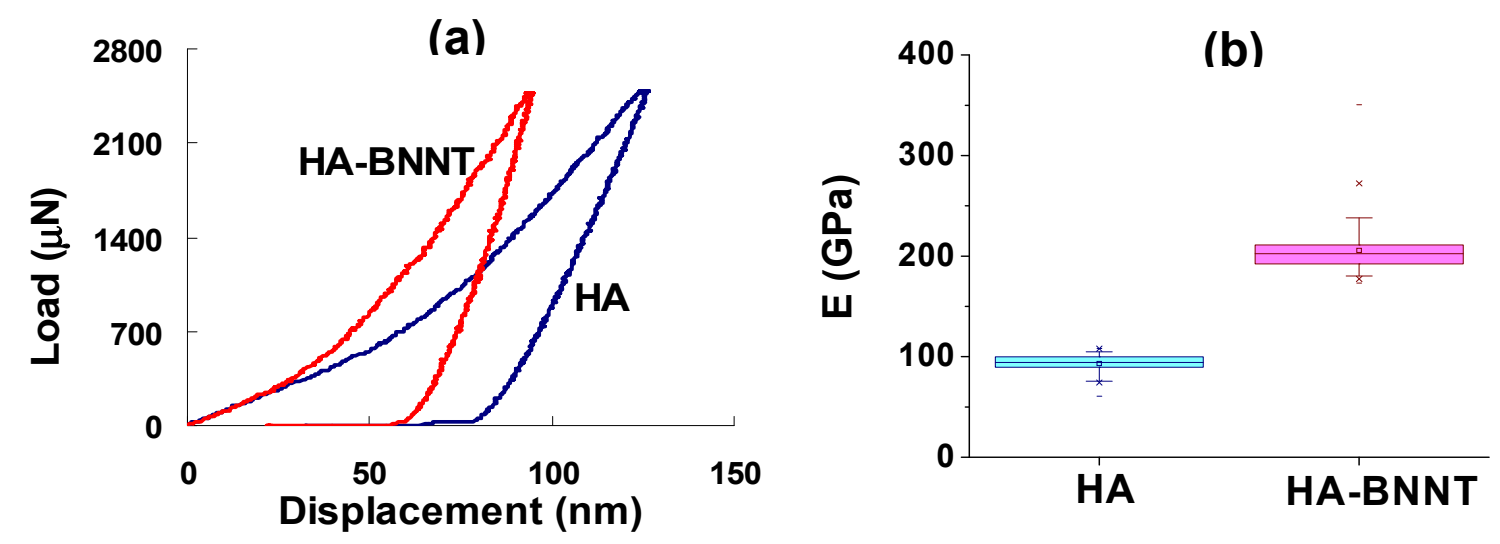

Figure 4.32: (a) Load vs. displacement plot for HA and HA-BNNT composite obtained by nanoindentation; (b) statistical distribution of $E$ value in HA and HA-BNNT composites measured for more than 100 nano-indents in each sample.

Low indentation depth indicates higher hardness for HA-BNNT $(12 \pm 2 \mathrm{GPa})$, which is $100 \%$ increase over HA $(6 \pm 1 \mathrm{GPa})$. Elastic modulus values, calculated from 
the unloading part of the load-displacement curves, shows a higher value of $205 \pm 15$ GPa for HA-BNNT compared to $93 \pm 9$ GPa for HA (figure 4.32b). BNNT reinforcement increases the E of HA matrix by $120 \%$. Statistical distribution of $\mathrm{E}$ values shows similar spread for HA and HA-BNNT (figure 4.32b) indicating uniform improvement in elastic modulus throughout the HA matrix with BNNT reinforcement. The improvement in elastic modulus with BNNT addition is attributed to two major factors: (i) higher elastic modulus of BNNT reinforcement and (ii) strong bonding at HA-BNNT interface. An E value of BNNT (750-1200 GPa [70, 74]) is much higher than that of HA (100 GPa [75]). Further, BNNT retains the defect free structure during SPS, which makes its contribution more effective towards improving the elastic modulus. HA-BNNT exhibits strong coherent interface, as discussed in section 4.1.2.4. During application of stress, strong bonding at the interface helps in transferring the load effectively from HA matrix to BNNT. As a result, with same amount of stress, the resultant strain in the HA-BNNT composite is lower than HA. Reduction in the elastic strain causes increase in E for the composite. Apart from these two, the increased density in HA-BNNT composite also contributes towards the increasing E. Density of the composite structure increased by $5 \%$ with BNNT addition to HA. However, the effect of density on strengthening of composite structure cannot be isolated from the contribution of BNNTs. In fact, higher density of the sintered structure is caused by BNNT content, as processing conditions were same for HA with and without BNNTs. The role of BNNTs in toughening mechanism is discussed below.

Vickers indentation method has been used to determine the fracture toughness of the composite using radial crack measurement method [15]. Figures 4.33a and 4.33b 
show Vickers indents on the polished cross-sections of HA and HA-BNNT pellets, respectively.
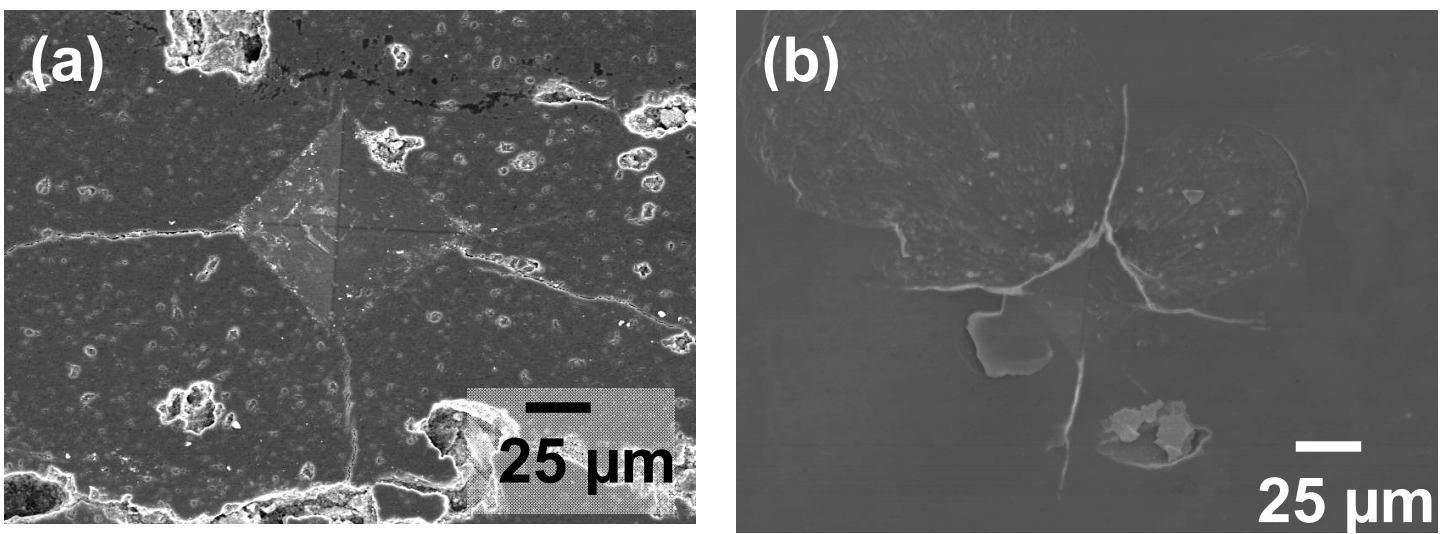

Figure 4.33: Vickers indent impressions showing radial crack generation in (a) HA and (b) HA-BNNT sintered pellets.

Indent on HA-BNNT shows significantly smaller impression with shorter radial cracks as compared to HA. Smaller indentation with same load indicates higher hardness for HA-BNNT $(5.5 \pm 0.12 \mathrm{GPa})$ than $\mathrm{HA}(2.4 \pm 0.05 \mathrm{GPa})$. The absolute values of hardness are different in Vickers and nanoindentation experiments. But the direct comparison is not justified owing to the difference in tip geometry, measurement length scale and the vast difference in applied load. On a relative scale, Vickers' hardness shows a $129 \%$ increase with BNNT addition, which is comparable to $100 \%$ increase in hardness from nanoindentation experiments. Comparable improvement in the mechanical properties at multiple length scales can be attributed to homogeneous distribution of BNNTs in HA matrix and higher density of the composite structure. Significant improvement in the hardness for HA-BNNT could also be due to its finer grain size. Increase in the hardness with decreasing grain size in metals and ceramics, including HA, could be explained through Hall-Petch mechanism $[61,76]$. 
$E$ values measured by nanoindentationare used to compute $K_{I C}$ of the composite. $K_{I C}$ for HA-BNNT is $1.6( \pm 0.3)$ MPa.m ${ }^{0.5}$, which is $86 \%$ higher than HA with $0.85( \pm 0.3)$ MPa.m ${ }^{0.5}$. Due to similar amount of improvement in $\mathrm{E}$ and $\mathrm{H}$, the $\mathrm{E} / \mathrm{H}$ ratio does not have significant contribution in improvement of $\mathrm{K}_{\mathrm{IC}}$ in HA-BNNT. Hence, the radial crack length remains the determining factor. Shorter length of radial crack in HA-BNNT is attributed to two major factors - (i) grain size refinement and (ii) crack bridging by BNNTs. Wang and Shaw have reported simultaneous improvement in hardness and toughness in sintered HA pellet due to refinement in grain size [76]. Deflection of crack and transition of cracking mode from transgranular to intergranular are the reasons for the improvement in fracture toughness of HA with refined grain size. Presence of BNNT causes grain size refinement in HA matrix. Further, due to higher pullout energy of BNNT from HA matrix (section 4.1.2.4) cracks propagate through HA, but gets restricted in the vicinity of BNNT, as more energy is required for interface debonding. Figure 4.34 shows BNNT bridges in a radial crack generated by the indentation. Deflection in the cracking path at each BNNT bridge reveals the absorption of fracture energy at strong HA-BNNT interface and thus increases fracture toughness.

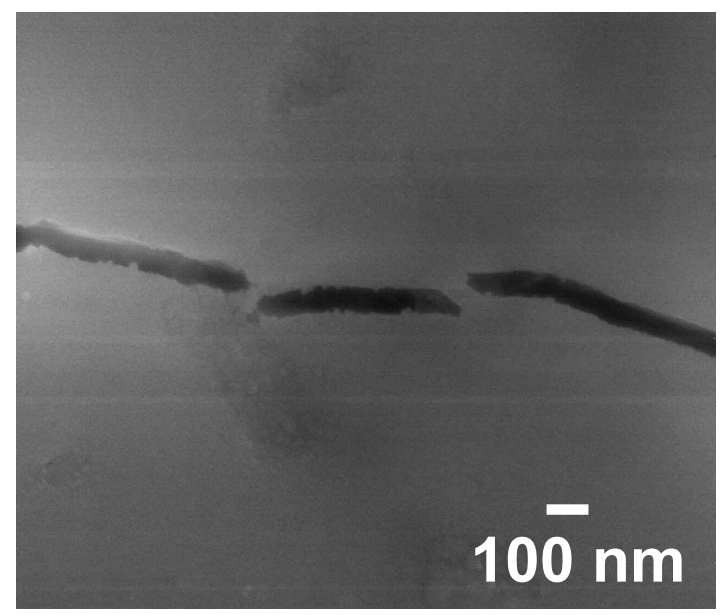

Figure 4.34: BNNTs bridging the radial crack generated from microindent. 


\subsubsection{Wear Behavior of SPS HA-BNNT}

Tribological behavior of HA and HA-BNNT composite is quantified in terms of wear volume loss and coefficient of friction (CoF). Wear volume loss is inversely related to the wear resistance. The CoF and cumulative volume loss for HA and HA-BNNT sintered pellets are shown in figures $4.35 \mathrm{a}$ and $4.35 \mathrm{~b}$, respectively. Each point on figure 4.35a shows an average value of $\mathrm{CoF}$ for $25 \mathrm{~m}$ interval. The error bars on figure 4.35 are based on three wear tracks studied for each composition.

(a)

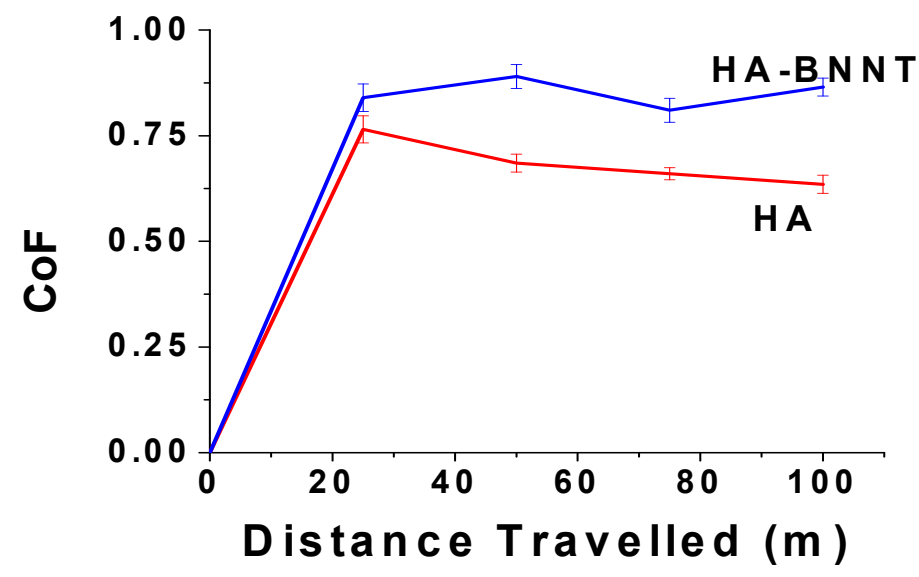

(b)

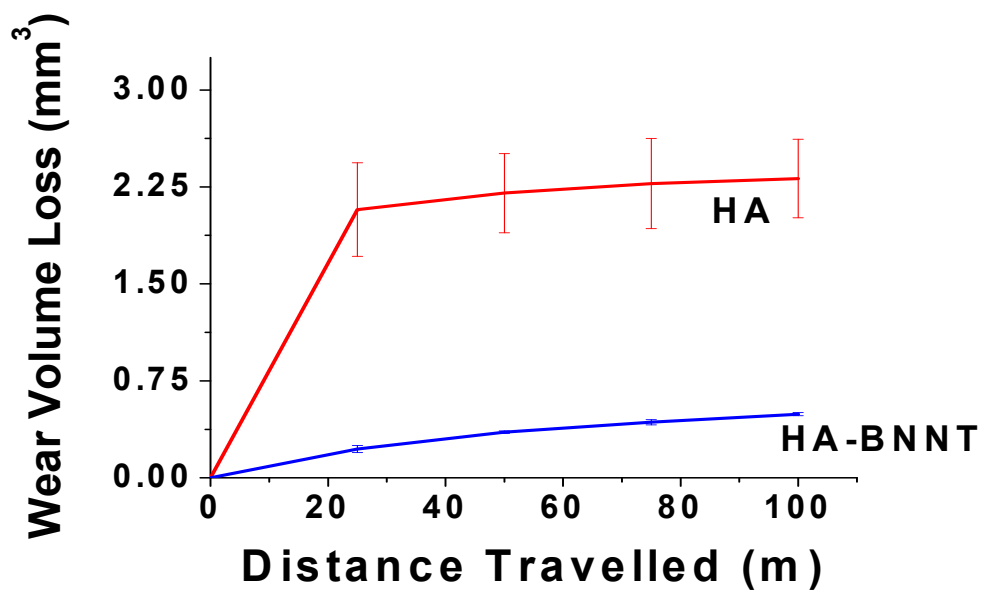

Figure 4.35: (a) Coefficient of friction and (b) wear volume loss for HA and HA-BNNT plotted against sliding distance during ball-on-disk wear. 
The $\mathrm{CoF}$ increases by $\sim 25 \%$ with $\mathrm{BNNT}$ reinforcement in HA (figure $4.35 \mathrm{a}$ ). Higher $\mathrm{K}_{\mathrm{IC}}$ and $\mathrm{E}$ of HA-BNNT composite cause more resistance to mass removal and as a result increase in the lateral (transverse) force. Increase in the lateral force with a constant normal force causes higher CoF for HA-BNNT. Hexagonal boron nitride sheet is known as a good lubricator and its presence is reported to decrease the CoF of the system [77-78]. Hence, increase in CoF in HA-BNNT could be due to absence of peeled off h-BN sheet on wear track. This observation is in contrary to the presence of graphene sheets providing lubrication in wear track of HA-CNT composite. This discrepancy can be explained in terms of the capability of BNNT to withstand high amount of deformation without getting damaged [62]. Shear force applied by wear probe may not be sufficient to peel h-BN layer from the BNNT surface.

Presence of BNNT decreases the wear volume loss of HA matrix by $75 \%$ (figure 4.35b). Similar amount of wear loss for different tracks (indicated as error bars in plots) shows the homogeneous tribological behavior of the composite structure. Higher error bars in wear loss for HA could be due its higher porosity and inhomogeneous microstructure. The increase in the wear resistance of HA-BNNT is the result of its improved mechanical property $\left(\mathrm{E}, \mathrm{H}\right.$ and $\left.\mathrm{K}_{\mathrm{IC}}\right)$. Toughened matrix of BNNT reinforced structure inhibits loss of mass due to fracture and chipping during wear. In order to find out the effect of $\mathrm{E}, \mathrm{H}$ and $\mathrm{K}_{\mathrm{IC}}$ on the wear volume loss, the model for brittle ceramic proposed by Evans and Marshall has been employed [25] using equation 4.2. The computed volume loss for the present study shows a $65 \%$ reduction in the wear volume with BNNT addition. Comparable outcomes of wear loss improvement from experiment 
(75\%) and computation (65\%) supports the hypothesis of wear resistance being governed by the mechanical property improvement in HA-BNNT composite structure.
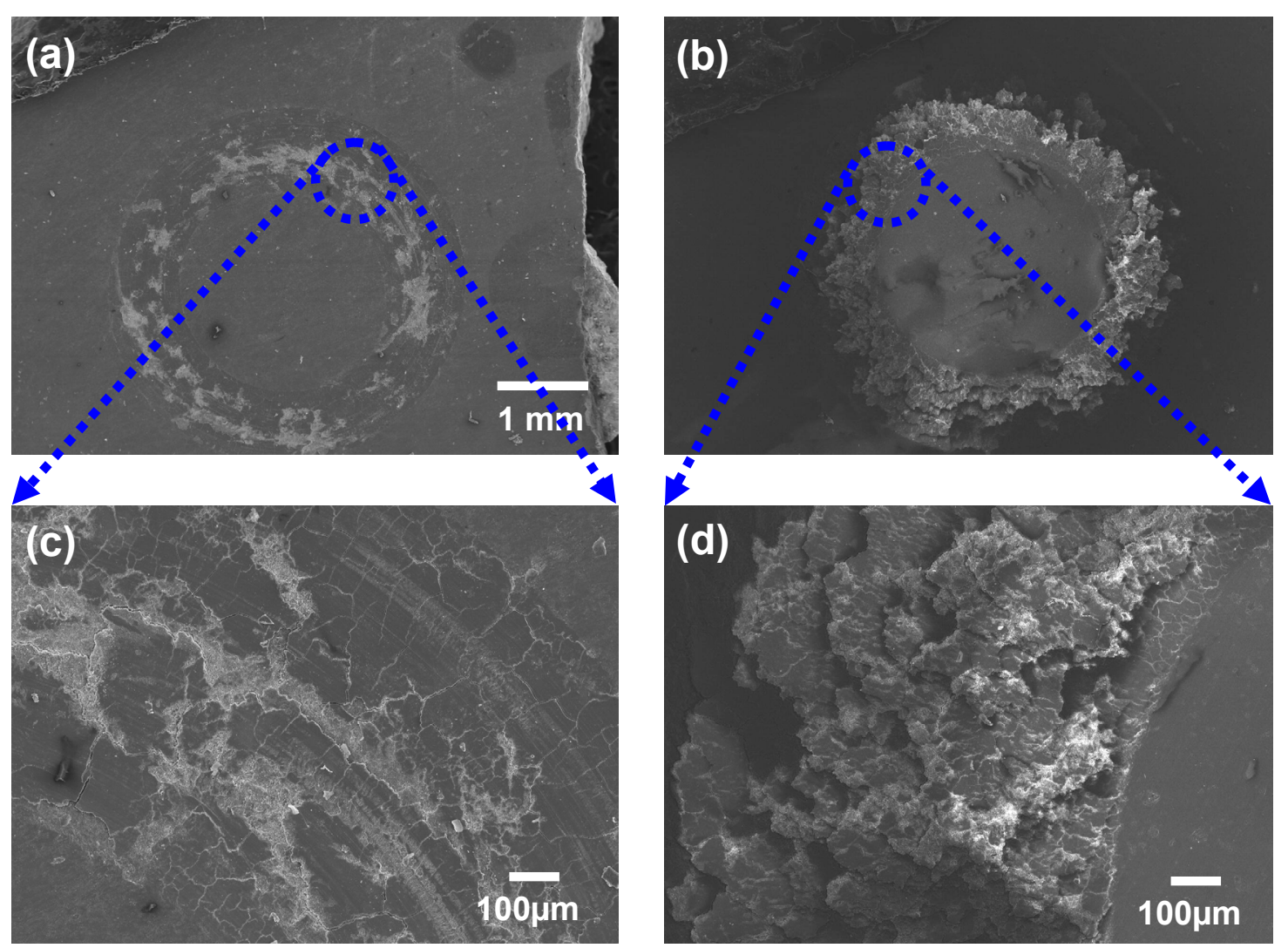

Figure 4.36: Ball-on disc wear tracks on $(a, c) H A$ and $(b, d) H A-B N N T$ revealing different morphology.

Further insight into differential wear behavior of HA and HA-BNNT sintered structure is obtained by investigating the morphology of wear track. Figures $4.36 \mathrm{a}$ and 4.36c show flat morphology in the HA wear track which is an indicator of abrasive wear mechanism with mass being totally removed. The wear track on HA-BNNT shows displacement of mass towards the outer edge of the track, resulting in pile-up (figures $4.36 \mathrm{~b}$ and $4.36 \mathrm{~d})$. Such behavior is specific to plastic deformation, which is not common 
in brittle ceramics like HA. Reinforcement of BNNT in HA matrix is responsible for such behavior. Huang et al. have also reported superplasticity introduced in $\mathrm{Al}_{2} \mathrm{O}_{3}$ and $\mathrm{Si}_{3} \mathrm{~N}_{4}$ ceramics as a result of BNNT reinforcement [61]. Though the study by Huang et al. reported the high temperature property of the composite, but the superplastic behavior is attributed to mainly two factors - (i) obstacle in dynamic grain growth at higher temperature and (ii) the 'sword in sheathe' phenomenon of load transfer in BNNT [61]. Although wear tests in this study were conducted at room temperature and in dry condition, localized high temperature at the point of contact between two surfaces may exist due to high friction. Such localized high temperature may aid the plastic deformation as seen in figure $4.36 \mathrm{~d}$.

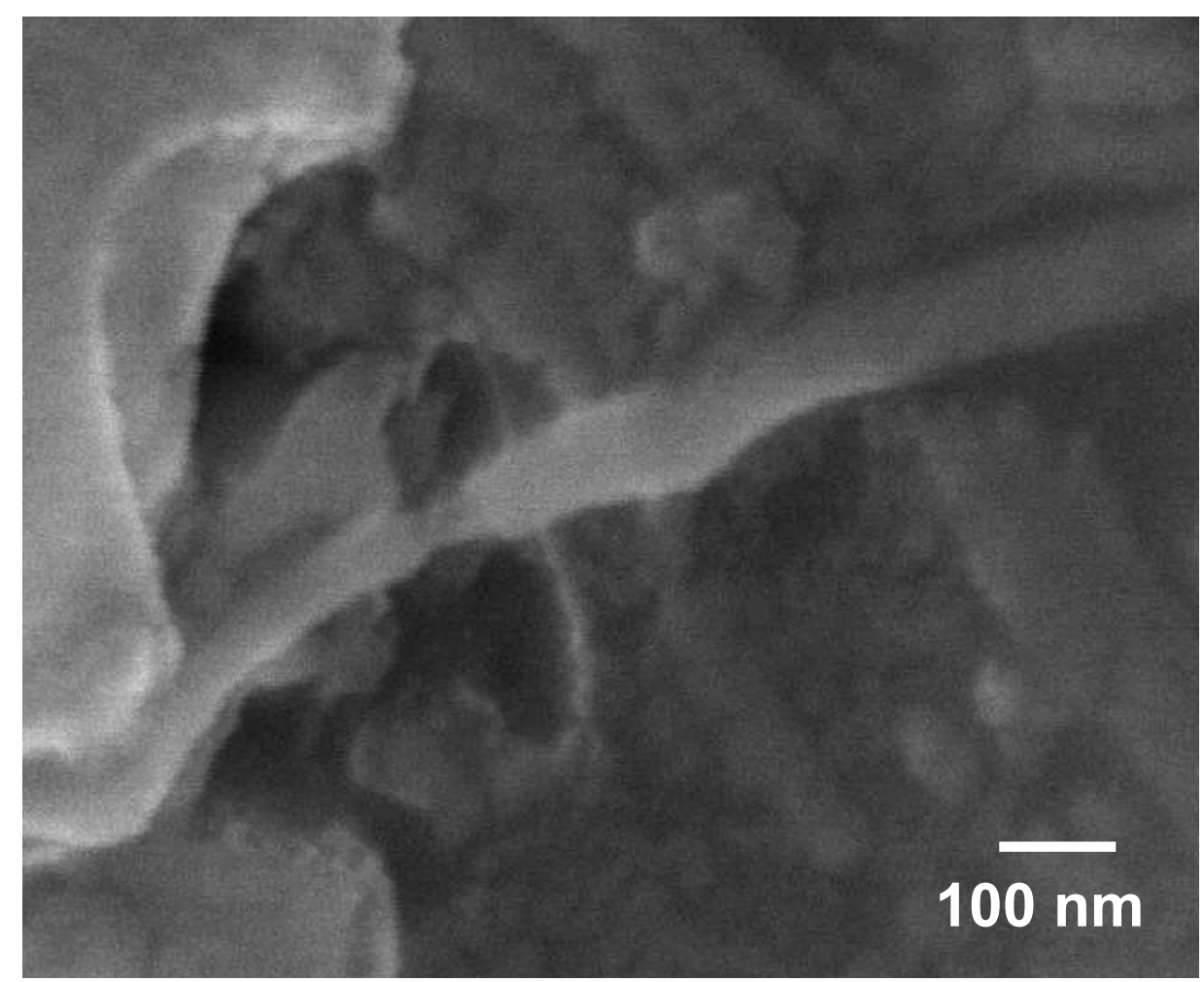

Figure 4.37: 'Sword in sheathe' type structure shown by BNNT upon application of stress. 
'Sword in sheathe' behavior of BNNT is possible at room temperature and is observed in figure 4.37. Sword in sheathe' indicates effective load transfer from the matrix to the outermost wall of BNNT. The transferred load is then carried to inner walls in a stepwise manner upon breakdown of the outer walls, leading to the 'sword in sheathe' structure formation. The gradual sliding of BNNT layers converts the applied force to strain energy. This energy absorption mechanism causes plastic deformation in HA-BNNT composite. As a result, the mass is not totally removed from the track, but gets dislodged towards the periphery, still being held together with BNNT bridges. Figure 4.38 shows a BNNT bridge supporting the dislodged mass on wear track.

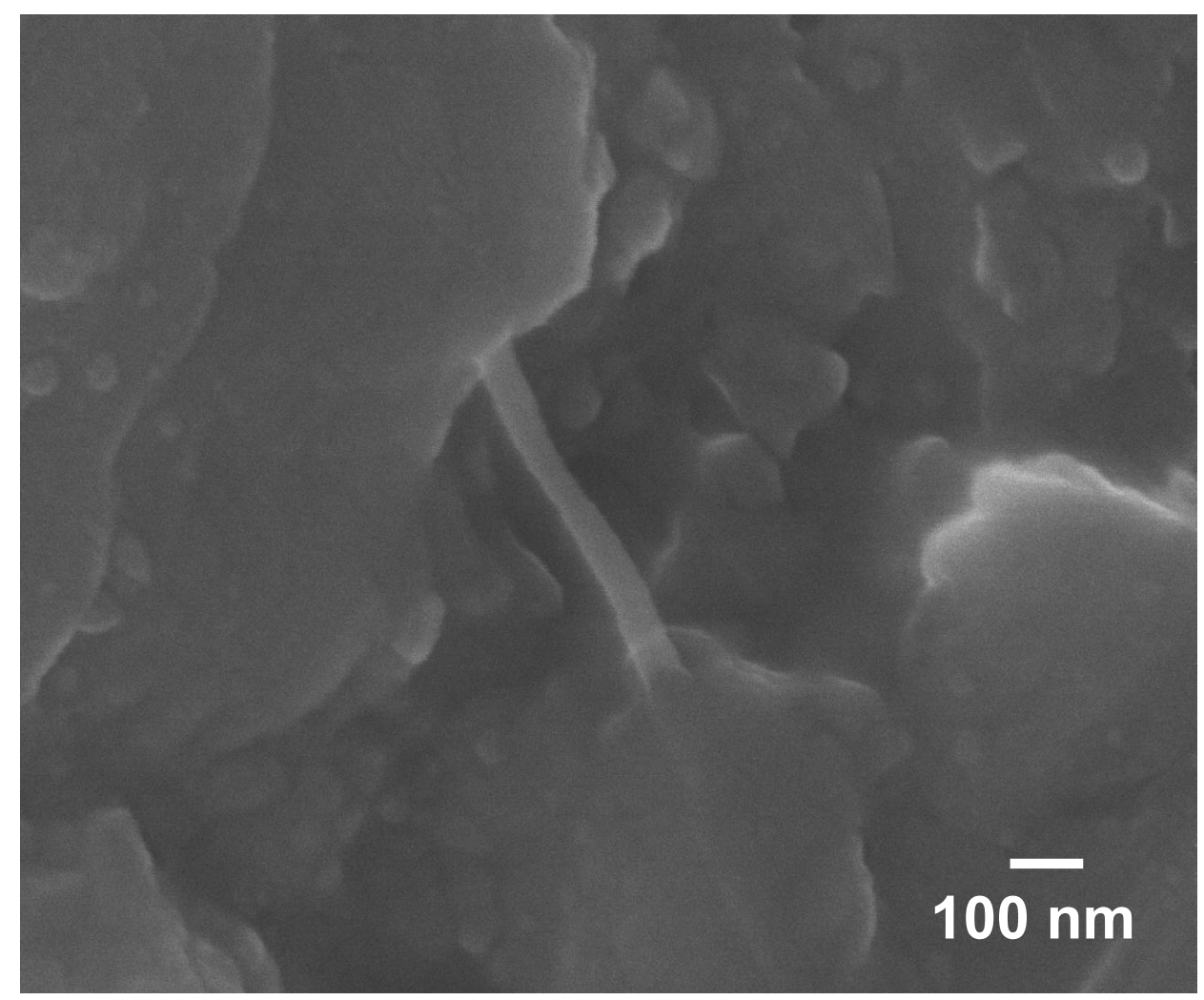

Figure 4.38: BNNT bridging of mass on wear track of HA-BNNT. 
BNNT is found as a potential reinforcement to HA for improving its mechanical and tribological properties. Higher thermal conductivity of BNNT reinforcement results in dense sintered structure due to accelerated diffusion during SPS. BNNT structure was not damaged by high temperature and pressure applied during SPS. A significant $120 \%$ increase in the elastic modulus of HA-BNNT composite is obtained. BNNT also acts as grain refiner by pinning the grain boundary during sintering, which helps in simultaneous increase in hardness (129\%) and fracture toughness (86\%) of the composite structure. High BNNT pullout energy from the matrix helps improving the fracture toughness. A $75 \%$ increase in the wear resistance of HA-BNNT is attributed to the improvement in elastic modulus, hardness and fracture toughness. Wear surface morphology reveals the transformation of brittle abrasive fracture in HA to plastic deformation and pile-up in HA-BNNT. BNNT bridging, effective load transfer at matrix-reinforcement interface and sword in sheathe' phenomenon is responsible for the plastic deformation in HA-BNNT composite.

Microstructural investigations of HA-nanotube composites have proven the potential of $\mathrm{CNT}$ and $\mathrm{BNNT}$ reinforcement in improving mechanical and tribological behavior of HA. The next step towards their orthopedic application is to evaluate their biocompatibility. Following section presents and analyses the results of biocompatibility studies on HA-nanotube composites. 


\subsection{Biocompatibility of HA-Nanotube Composites and Coatings}

This section focuses on biocompatibility of HA-nanotube composites. In-vitro and in-vivo studies have been carried out following ISO-10993 and ASTM - F748, F981 and F1903test guidelines (Appendix-1).

\subsubsection{Biocompatibility of Hydroxyapatite-Carbon Nanotube Composite}

Biocompatibility studies have been carried out on plasma sprayed HA and HACNT coatings. The main objective of this research is to evaluate the effect CNT on the biocompatibility of HA. Considering similar chemical composition (HA-4 wt.\% CNT)for plasma sprayed coating and spark plasma sintered composite, the role of CNT on the biocompatibility of both surfaces should be similar. It is true that density and surface microstructure of coating and sintered composite is different and may lead to differential cell growth behavior. Biocompatibility study of spark plasma sintered HA-4wt.\% CNT composite is suggested as future research.

\subsubsection{In-Vitro Assessment of HA-CNT}

In-vitro assessment of biocompatibility of HA and HA-CNT is carried out by culturing osteoblast on coatings and assessing their viability and proliferation kinetics. The differentiation and mineralization of osteoblast is evaluated by gene expression studies and alkaline phosphatase expression. The cytotoxicity of wear debris is also examined for osteoblasts and macrophages. 


\subsection{Osteoblast Viability}

Osteoblasts attach first to the orthopedic implant surface. Once the osteoblasts cover the implant surface by proliferation and growth, they deposit collagen in the intercellular region, known as osteoids. Further, osteoblasts collect salt ions from the blood to release them on the osteoid matrix for its mineralization and bone formation [7980]. Thus, the growth and proliferation of osteoblast cells on implant surface is extremely important for bone generation and integration. The effect of CNT on the growth and proliferation of osteoblast cells was assessed qualitatively by observing the population of osteoblast cells on the surface after different days of culture. The osteoblast cell viability on HA and HA-CNT surface was also conducted. Viability of osteoblast cells is defined as the quantitative ratio of live to dead cells. FDA stains live cells in green color, whereas, PI stains the nuclei of dead cells in red. The fluorescence images in figure 4.39 show the live cells in green and dead cells in red on HA and HA-CNT surface after 3, 5, and 10 days of growth. The cells in all the images show a typical lens shape characteristic of live osteoblasts suggesting the presence of normal cell growth behavior. After 5 days of culture (figures $4.39 \mathrm{~b}$ and $4.39 \mathrm{e}$ ), cells became more confluent and started forming dense islands. After 10 days of growth, the cell population increased significantly covering the coating surface (figures $4.39 \mathrm{c}$ and $4.39 \mathrm{f}$ ). Increasing numbers of osteoblast cells with time suggests an increase in cell proliferation and/or survival on HA and HACNT surfaces. The osteoblast population was visibly larger on HA-CNT surface than on HA. 
(a)

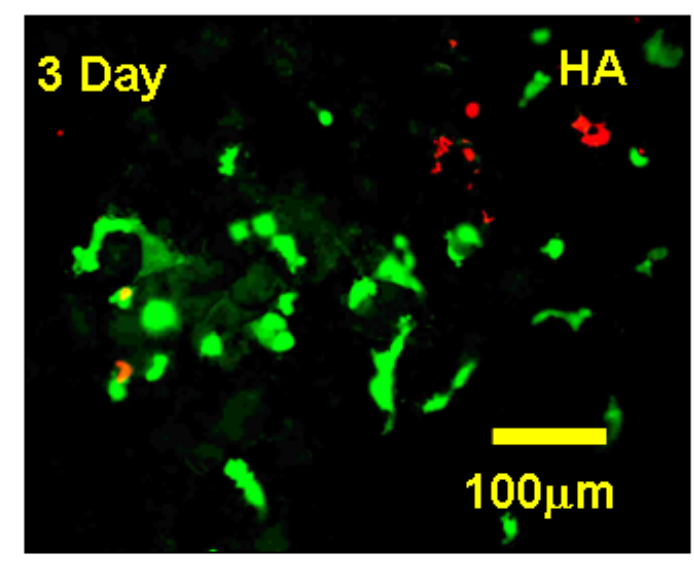

(d)

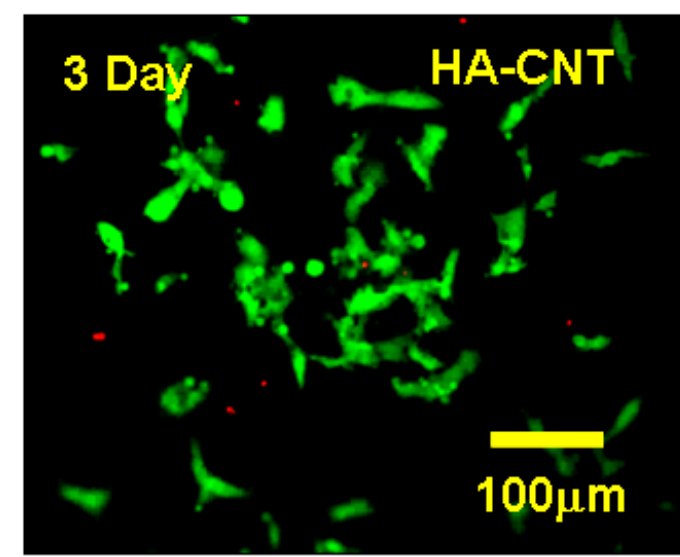

(b)

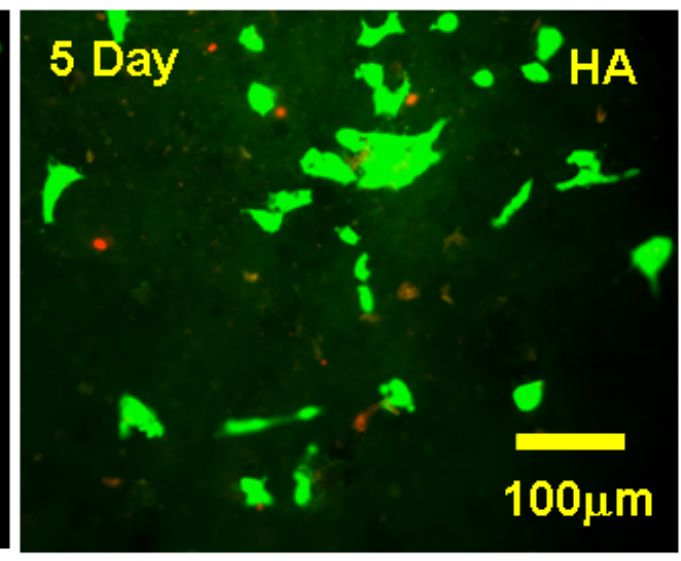

(e) (c)

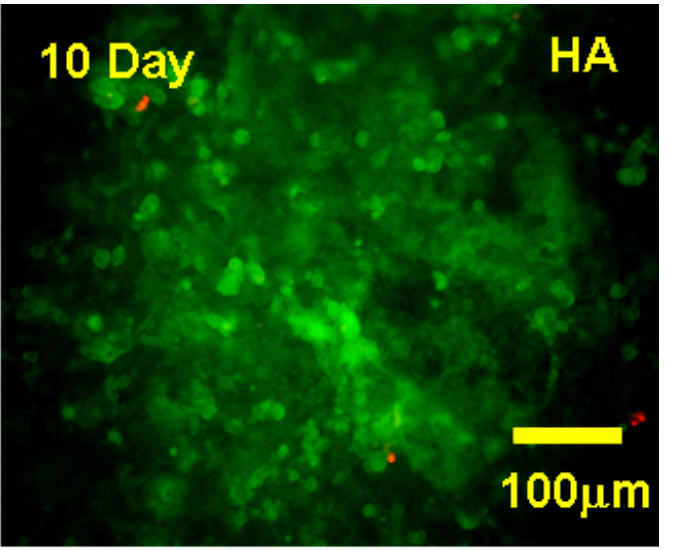

(f)

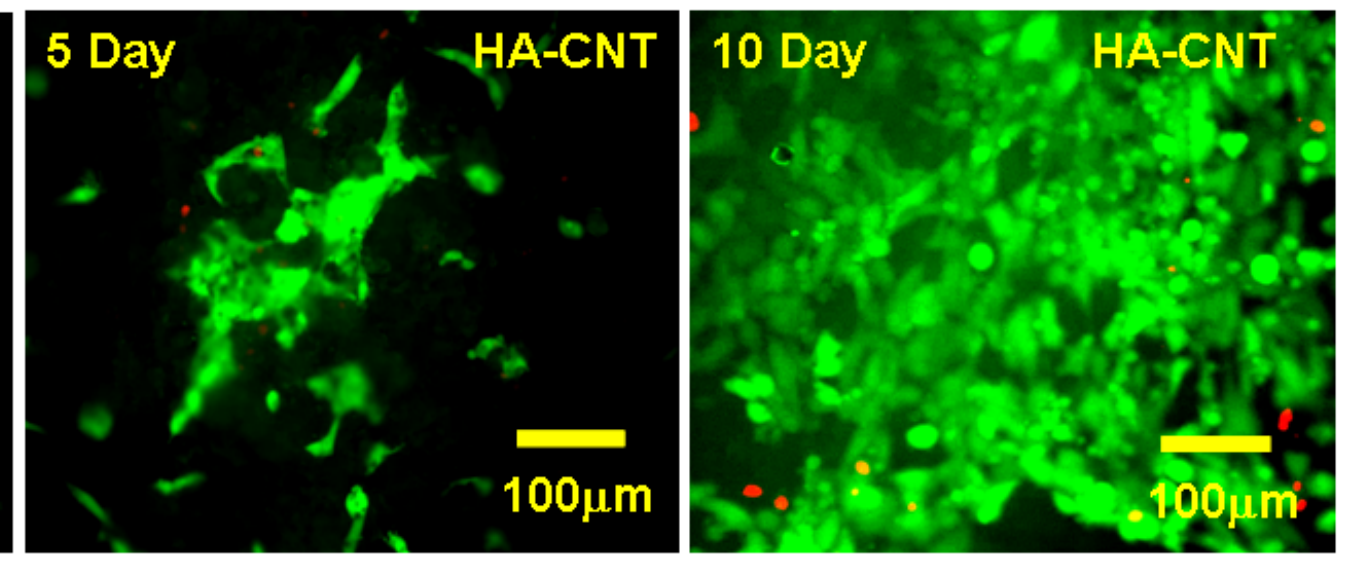

Figure 4.39: Fluorescent images of osteoblast cells grown for 3, 5 and 10 days on (a-c) HA and (d-f) HA-CNT coatings. The live cells are stained in green color with FDA and dead cells in red with PI. 
The ratio of dead and live cells are found comparable for HA and HA-CNT surfaces, indicating their similar behavior towards viability of osteoblast cells. The viability plot (figure 4.40) shows that the \% of live cells on HA-CNT surface is similar or slightly higher than HA surface for a duration of $1-10$ days of culture. Thus, the presence of CNTs does not negatively affect the osteoblast cell viability. On the contrary, the slightly larger osteoblast population and viability on HA-CNT surface with increasing number of days indicates the positive effect of CNTs in osteoblast proliferation.

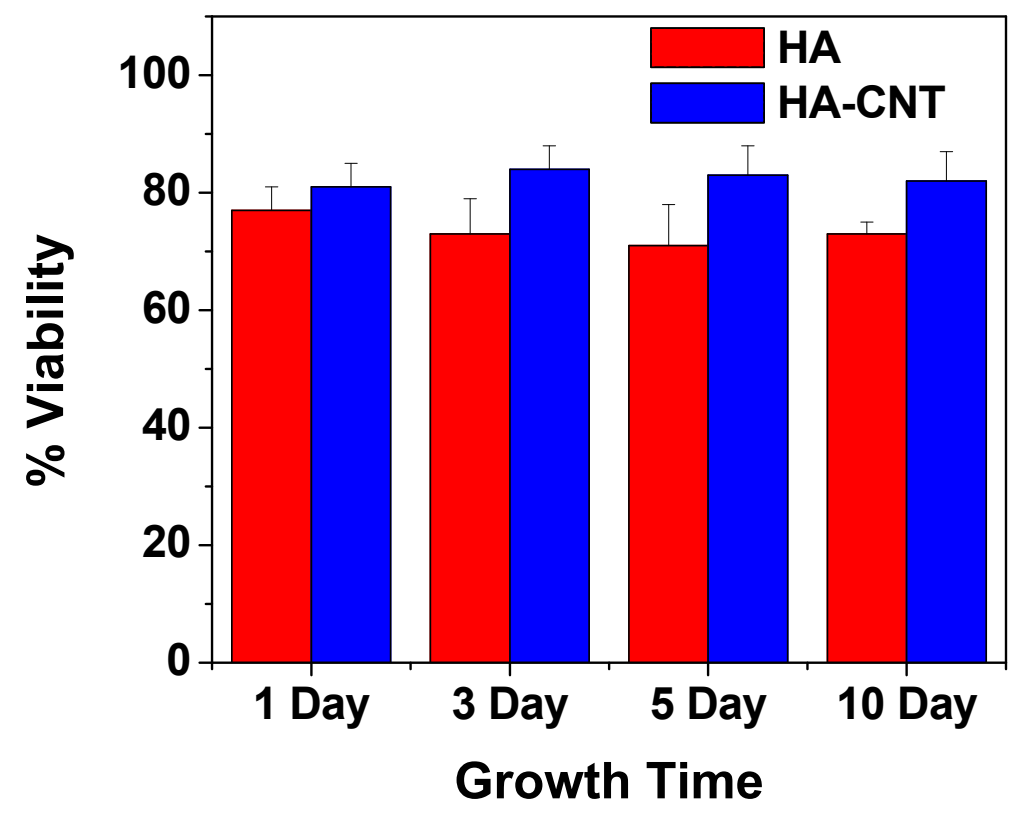

Figure 4.40: Osteoblast cell viability on $H A$ and $H A$-CNT coatings for 1, 3, 5 and 10 days of culture $(p<0.05)$.

The density of both HA (93.7 \%TD) and HA-CNT (94\%TD) coatings being similar in this study, does not pose any differential effect on osteoblast viability and population. Studies on the biocompatibility of HA-CNT composites by other research groups have also suggested that the presence of CNTs in HA promotes osteoblast cell proliferation $[3,33,81-82]$. The protein expression profile of osteoblasts grown on HA- 
CNT surface was shown to be distinct from that of cells grown on HA, and resulted in differences in proliferation [83-84]. These differences observed in cells grown on HACNTs may be explained by recent studies indicating that CNTs can absorb various molecules on their surface [85-86]. Akasaka et al. have shown that both, SWNT and multiwall CNTs absorb proteins from the serum (in cell culture medium) on their surface and positively influence osteoblast cell proliferation [86]. Matsuoka et al. have further observed that the adhesion of osteoblasts on multiwall CNT surface is higher than SWNT, due to the rough and curled surface of the former [85]. More interestingly, both of these studies [85-86] did not use any surface treatment or functionalization for the CNTs.

\subsection{Proliferation Kinetics of Osteoblast}

Proliferation kinetics of osteoblasts on HA and HA-CNT coatings is evaluated by determining the numbers of cells proliferating on a substrate in certain period is evaluated using Bromodeoxyuridine (BrdU) assay. This assay performs quantification of DNA synthesis during cell activation which allows assessment of cell proliferation kinetics.

BrDU is a synthetic nucleoside that is an analogue of the DNA precursor thymidine. During proliferation of a cell, DNA has to be replicated before the division can take place. The cells, which are exposed to BrdU during division, would have BrDU incorporated in their DNA in place of thymidine. The trace of BrdU in the DNA of the proliferated cells can be detected by staining the cell with anti-BrdU fluorescent antibodies and observing under fluorescent microscope. 
Total Cells

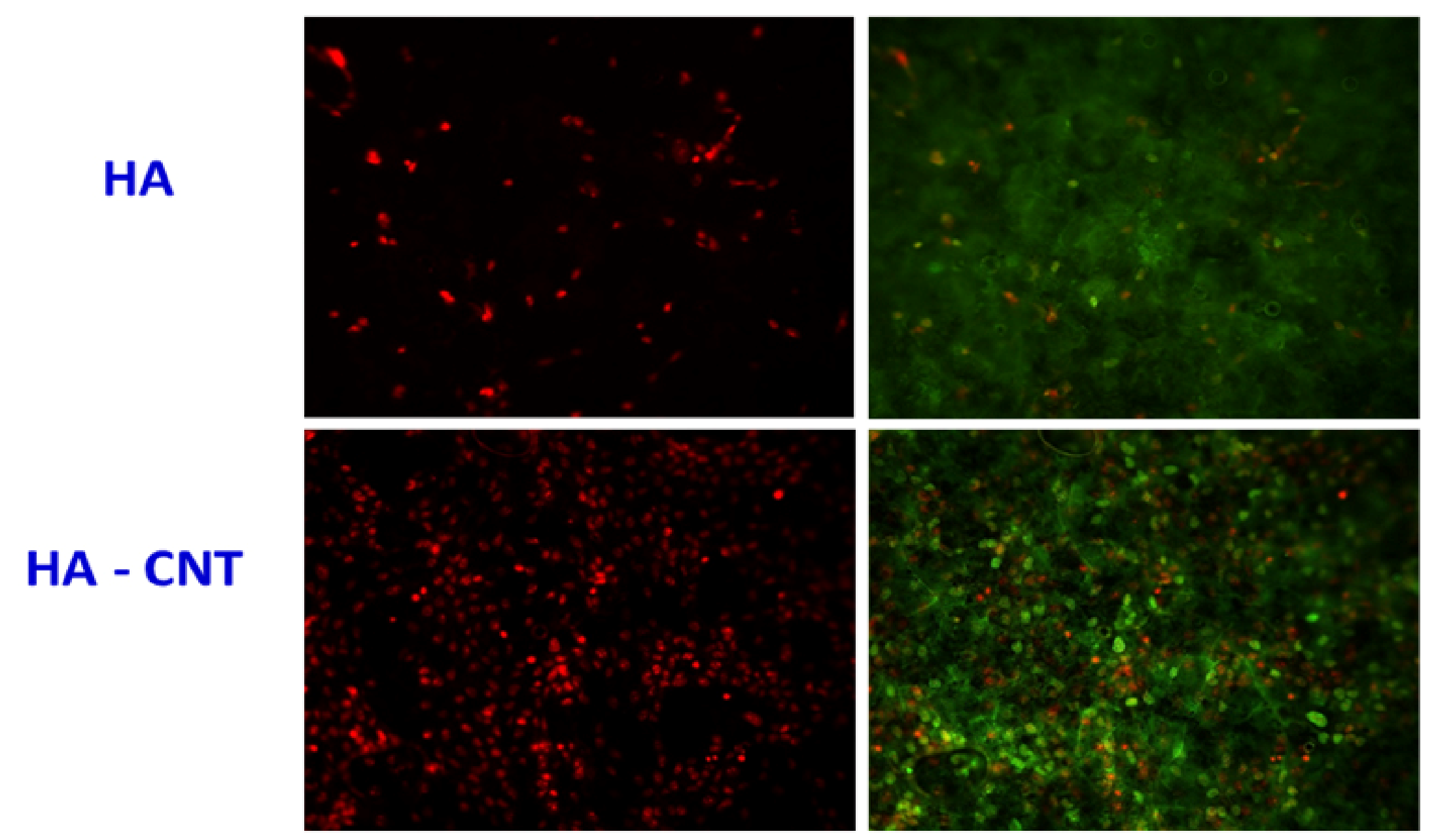

Figure 4.41: Fluorescent images of osteoblasts on HA and HA-CNT coatings, exposed with BrDU after 1 day of incubation. The cells stained in red indicate total number of osteoblasts on substrate, whereas the cells proliferated during $1 \mathrm{hr}$ of BrDU exposure are stained in green.

\section{Proliferated Cells}




\section{Total Cells}

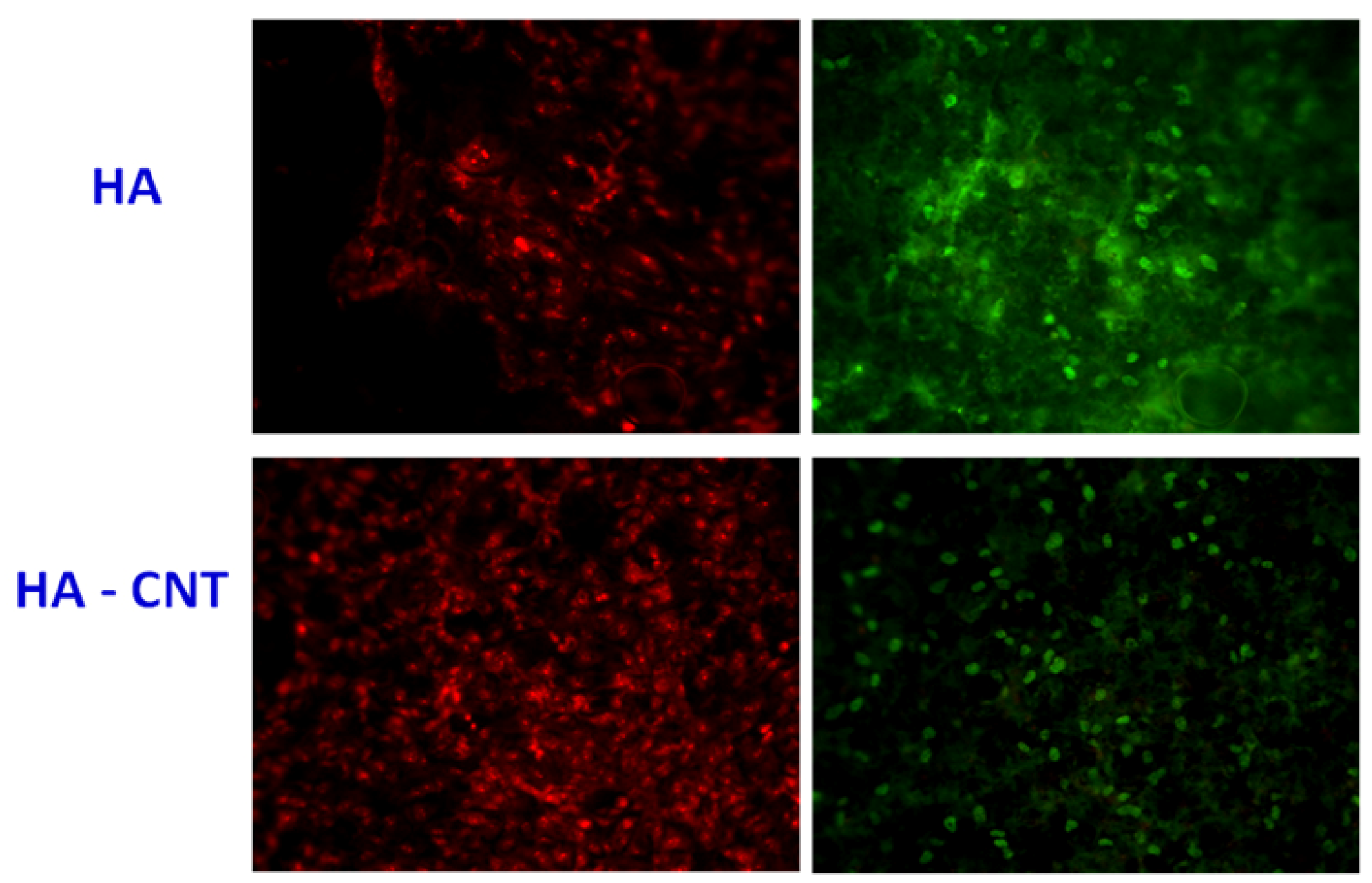

Figure 4.42: Fluorescent images of osteoblasts on HA and HA-CNT coatings, exposed with BrDU after 3 days of incubation.

\section{Proliferated Cells}




\section{Total Cells}

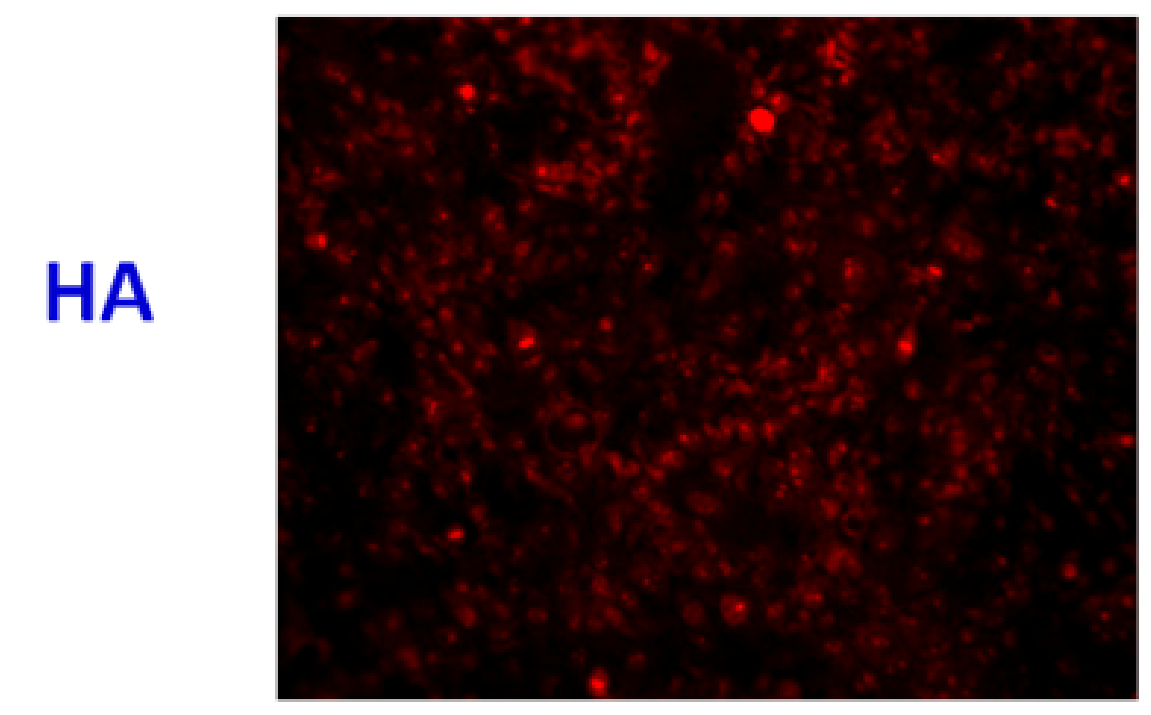

HA - CNT
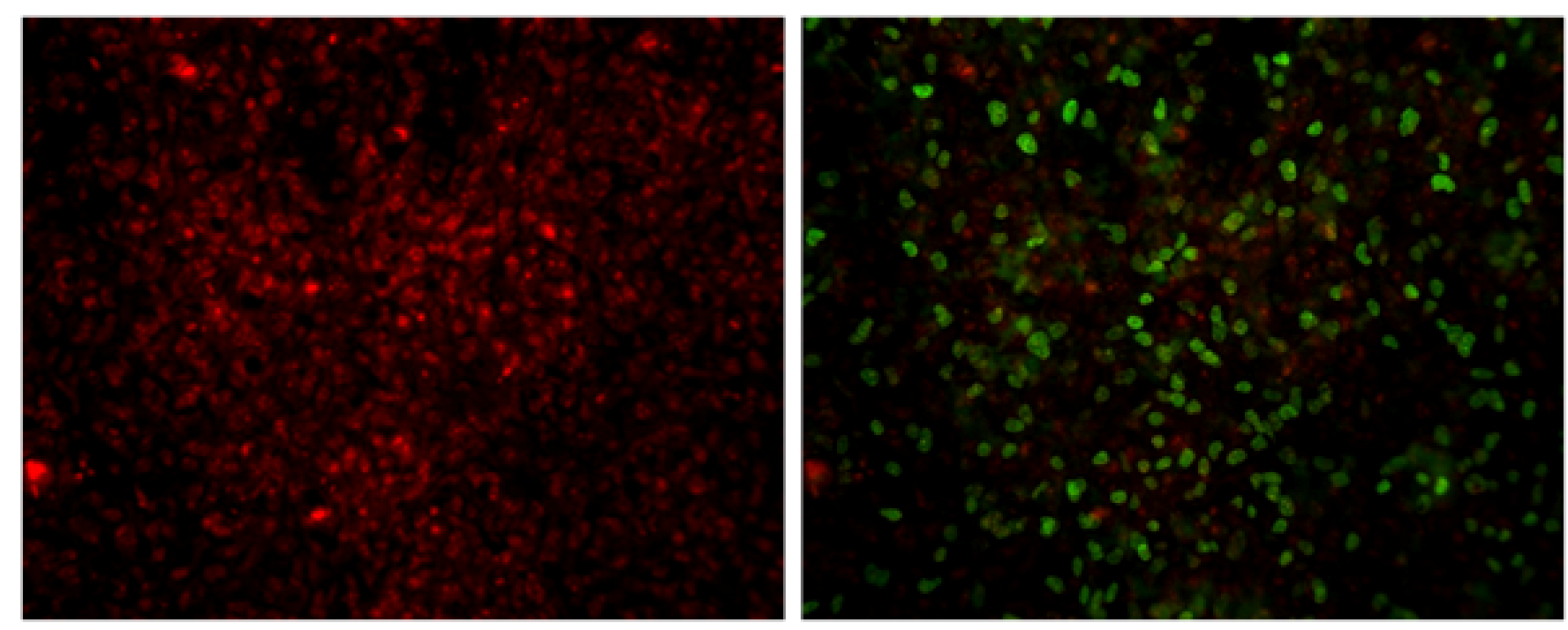

Figure 4.43: Fluorescent images of osteoblasts on HA and HA-CNT coatings, exposed with BrDU after 5 days of incubation. 
In the preset study, the osteoblasts cultured on HA and HA-CNT coatings are exposed to BrDU for $1 \mathrm{hr}$ after the predetermined incubation period of 1,3 and 5 days. The DNA of the cells proliferated during $1 \mathrm{hr}$ is stained with anti-BrDU reagent, while the nucleus of all the cells on the substrate are stained with propidium iodide. The substrates with cells are observed under fluorescence microscope. Figures 4.41. 4.42 and 4.43 present the fluorescence images of osteoblasts on HA and HA-CNT coatings for 1, 3 and 5 days, respectively. All the cells appear stained in red in the left hand images. The right hand images show only the proliferating cells in green, which have incorporated BrDU in their DNA.

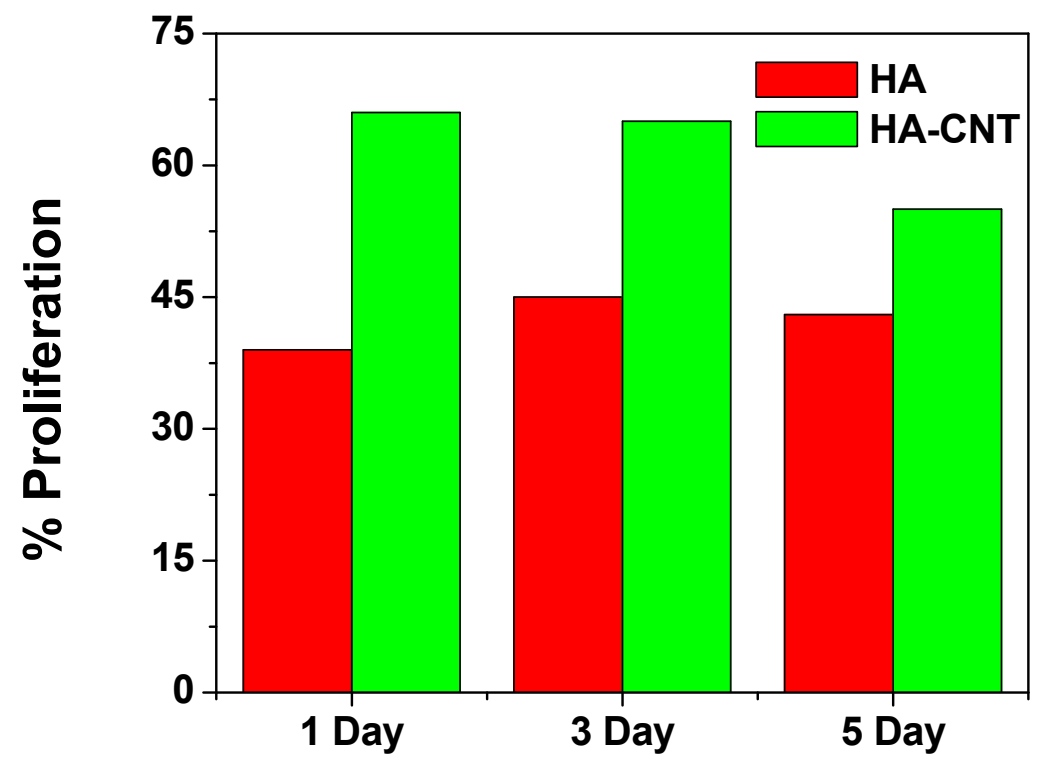

Figure 4.44: Proliferation rate of osteoblasts on HA and HA-CNT coatings after 1, 3 and 5 days of exposure.

A total of 3000 cells were counted in each substrate to calculate the proliferation rate. Figure 4.44 presents a bar chart on the osteoblast proliferation rate for different incubation period on both the coatings. Proliferation rate is always higher in HA-CNT than on HA. This observation indicates positive influence of CNT towards 
osteoblast proliferation. Proliferation rate on HA-CNT seems slightly decreasing at 5 days. But the cell population on HA-CNT after 5 days (figure 4.43) reveals the substrate to be already full of cells. At this condition, the cell will grow on each other. We have performed normal fluorescence microscopy and not the 3D confocal microscopy. Thus, the observation would be limited to the upper surface layer of cells and it would not be possible to the ones that are proliferated at the lower (bottom) layer of cells. A very closer look at osteoblasts on HA-CNT after 5 days of incubation (figure 4.43) reveals faint impressions of BrDU stained cells at lower layers, which are not counted during proliferation rate calculation. This leads to an artificial lowering in the proliferation rate shown in figure 4.44. Another reason could be the higher density of osteoblasts on HACNT causes lowering of proliferation rate after 5 days. Similar observation on lower proliferation rate of osteoblast at higher seeding density is observed by other research groups [87]. But, the same is not true for HA as the surface is yet to be filled up by cells even after 5 days. The higher population of cells on HA-CNT substrate is also indicative of higher cell proliferation rate, as osteoblast needs to proliferate more to achieve higher density. Similar to viability and proliferation (as discussed in section 4.2.1.1.1), the higher osteoblast proliferation rate on HA-CNT could also be explained by attachment of favorable proteins on CNT surface from cell culture medium [85-86].

\subsection{Evaluation of Osteoblast Differentiation through Gene Expression}

Gene expression for osteopontin, osteocalcin and RunX2 are evaluated for osteoblastscultured on HA and HA-CNT coating surfaces for 5 days. Osteopontin is the protein, synthesized by osteoblasts and deposited in the bone matrix where it bonds with 
hydroxyapatite. Osteopontinis involved in cell-to-cell and cell-matrix interactions during cell proliferation and cell migration and helps in attachment of osteoclast to the bone matrix and bone remodeling [88-89]. Osteocalcin is a bone-specific protein synthesized by osteoblasts that represents a good marker for osteogenic maturation. Osteocalcin is secreted by osteoblasts and higher osteocalcin levels are correlated with increases in bone mineral density (BMD) - which means better calcification/mineralization. Choice of osteopontin and osteocalcin as biomarker covers all stages of osteoblast differentiation. In general, osteoblasts express alkaline phosphatase (ALP) and osteopontin at early stage of maturation whereas osteocalcin is expressed later at the onset of matrix mineralization [90]. RunX2 is a master gene that regulates the process of osteoblast differentiation. It controls the expression of other genes such as osteopontin and osteocalcin that are markers of osteoblast differentiation [91]. Thus, expression of all these three genes indicate overall healthy/normal growth, proliferation and differentiation of osteoblast towards maturation and bone formation.

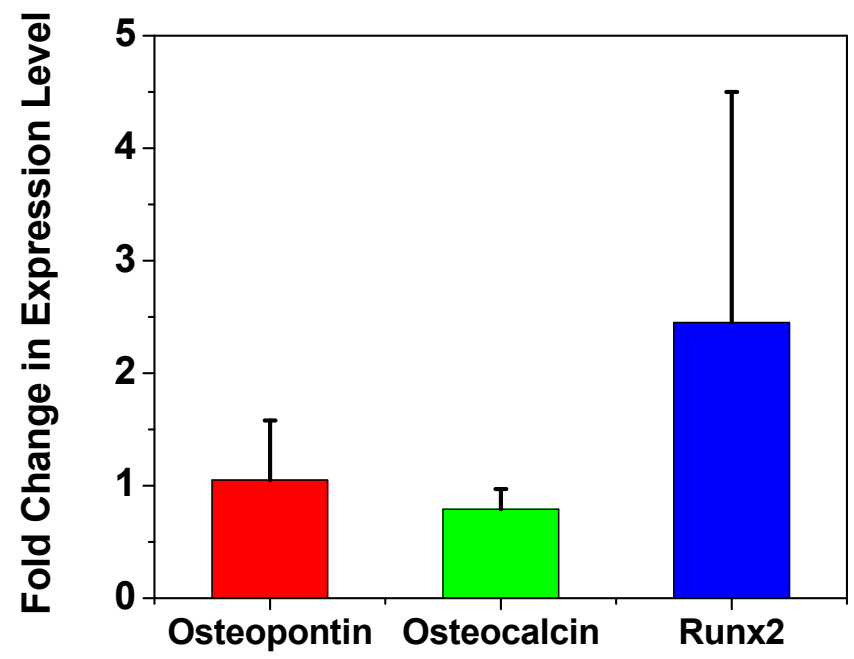

Figure 4.45: Over expression of osteopontin, osteocalcin and RunX2 for osteoblasts cultured on HA-CNT for 5 days as compared to the cells on HA surface. 
Figure 4.45 presents the results on osteopontin, osteocalcin and Runx2 expression for osteoblasts on HA and HA-CNT surfaces. All three genes are expressed more on HA-CNT surface than on HA. The results are presented as over expression on HA-CNT surface as compared to HA surface. The calculation scheme is explained in section 3.6.1.4 of this document. The significant over expression of osteopontin, osteocalcin and RunX2 on HA-CNT surface indicates a positive role played by CNT in faster differentiation and maturation of osteoblast cells leading to accelerated initiation of matrix mineralization and bone formation.

\subsection{Assessment of Mineralization through Alkaline Phosphatase Expression}

Alkaline phosphatase expression is evaluated for osteoblasts grown on HA and HA-CNT surfaces for 5 days, in order to assess the bone mineralization activity. Alkaline phosphatase, a metalloenzyme, is an important component in hard tissue formation and is highly expressed in mineralized tissue. It is secreted by osteoblasts at an early stage of maturation and is biochemical marker for bone formation and skeletal mineralization [90, 92].

Figure 4.46 presents the alkaline phosphatase expression of osteoblasts incubated for 5 days on HA and HA-CNT substrates, determined using colorimetric assay. As the proliferation of osteoblast is different on HA and HA-CNT (figure 4.43), the total number of cells will also differ after 5 days of incubation. Thus the total DNA is quantified for each substrate and alkaline phosphatase activity is normalized by total DNA amount for each sample. This is required to segregate the role of CNT on alkaline phosphatase expression from that on proliferation. Figure 4.46 reveals higher alkaline phosphatase 
expression on HA-CNT, which means more mineralization and faster bone formation in the presence of CNT.

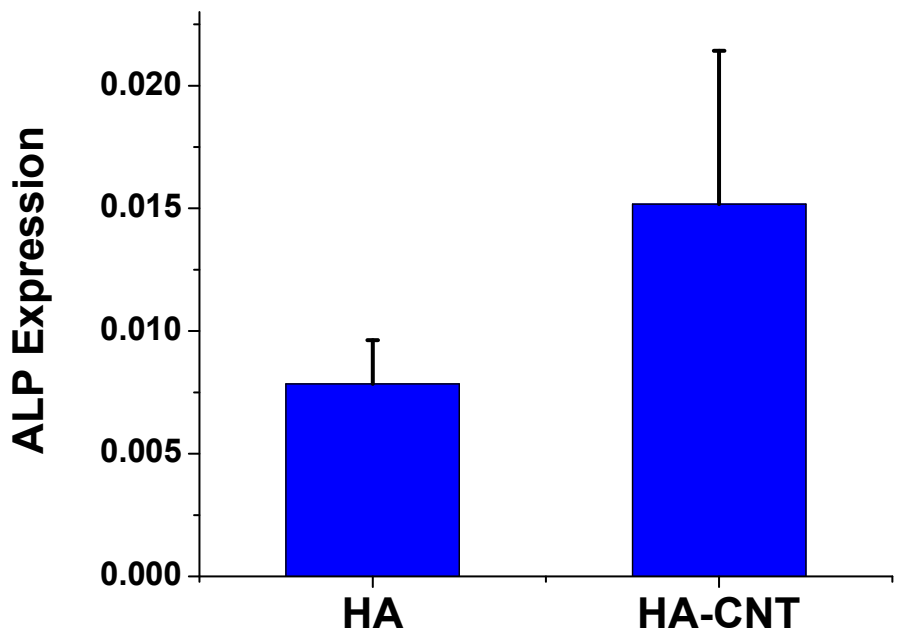

Figure 4.46: Alkaline phosphatase expression for osteoblasts cultured on HA and HACNT surfaces for 5 days.

The results from gene expression studies indicate that presence of CNT positively influence osteopontin, osteocalcin, runx2 and alkaline phosphatase expression for osteoblasts. Similar observations were made by $\mathrm{Li}$ et al. in a study on culturing osteoblasts on CNTs [93]. Osteopontin, osteocalcin and alkaline phosphatase expression increased on CNT surface as compared to the culture plate. Selective absorption of bone morphogenetic protein-2 on CNT surface was responsible for higher osteogenetic expression levels (91). Selective absorption of proteins from cell culture medium could be responsible for accelerated osteoblast differentiation and maturation on HA-CNT surface in our study. Moreover, carbon nanofiber surfaces show better osteoblast adhesion, due to high surface energy, small diameter and aligned structure [94-95]. Similar role played by exposed CNTs on HA-CNT surface could be an additional help towards better functioning of osteoblast leading to faster bone formation. 


\subsection{Cytotoxicity Evaluation for Wear Debris with Osteoblasts and Macrophages}

Cytotoxicity of CNT containing wear debris is checked for osteoblasts and macrophages. Osteoblasts are the bone forming cells and their activity determines the progression of neobone formation at the implant surface and its integration with bone. Thus, it is very important to study the effect of wear debris on osteoblast, because any cytotoxic effect of CNT (in wear debris) can impede the integration of implant with the bone. Further, wear debris could also be introduced into the blood stream in the long run. Wear debris, when generated, is suspended in the body fluid near bone or synovial fluid around the joints. The debris could get introduced in the blood stream from the body fluid at the finer capillary portion of the blood vessels through intercellular space between endothelial cells [96]. In the blood, the debris is first attacked by the macrophages, the security guard of the blood from any foreign element. Macrophages engulf the debris in the blood stream through vesicular internalization process known as phagocytosis, in order to clear them out from the blood. For effective removal of the debris from the blood, the macrophages need to ingest the debris without being harmed. Thus, the evaluation of cytotoxicity of wear debris to macrophages is important.

Wear debris is collected and used for studying in-vitro cytotoxicity to osteoblast

and macrophage cells. The cytotoxicity assay used in this study allows for the rapid and accurate quantification of released lactate dehydrogenase (LDH), a stable cytosolic enzyme released from lysed cells. The amount of LDH released is proportional to the number of dead cells, which is quantified through a colorimetric assay by measuring the absorbance at $490 \mathrm{~nm}$. The percentage absorbance value reported in this study is calculated using the LDH released from the live cells in the medium (after they are 
lysed), with respect to the total LDH released from all the cells (live and dead) in the medium after 3 days of culture with debris.

\section{Cytotoxicity Response of Osteoblasts}

The cytotoxicity of wear debris to osteoblasts is important because these cells stay in direct contact with the implants during the bone growth stage. If osteoblasts die on the implant surface due to the presence of debris then new bone will not be generated. Our results showed that the level of cytotoxity to osteoblasts is smaller when osteoblasts are cultured in the presence of debris from HA-CNT than with debris from HA alone (table4.3). A possible explanation for these results is the fact that presence of CNTs in the debris may have the potential for increasing osteoblast viability as discussed in section 4.2.1.1.1. Another reason could be the size of wear debris. Osteoblasts can phagocytose solid particles available in the culture medium [16]. Bigger particles $(0.3-9.5 \mu \mathrm{m})$ present in HA wear debris, if internalized, can cause more disturbance and harm to osteoblasts. Thus, smaller particle $(0.1-3.1 \mu \mathrm{m})$ size in HA-CNT wear debris will cause lesser disturbance if phagocytosed, resulting in higher population of live osteoblasts in the medium.

\section{Cytotoxicity Response of Macrophages}

The results of the macrophage cytotoxicity assay reveal slightly higher level in the presence of HA-CNT debris than HA (table-4.3). The difference, however, is not statistically significant. This observation indicates that the presence of CNTs in the debris does not alter the cytotoxicity response of macrophages. HA-CNT debris are as 
biocompatible as HA debris to macrophages. In spite of the difference in the debris particle size for HA and HA-CNT, they show similar cytotoxic behavior to macrophages. Olivier et al. have shown that the death of macrophages occur at the same rate for both smaller $(0.45 \mu \mathrm{m})$ and bigger $(3.53 \mu \mathrm{m})$ polystyrene particles, although the underlying mechanisms were different. The smaller particles caused apoptosis, whereas bigger ones induced necrosis of macrophages [97]. Another aspect that needs to be taken into consideration is fiber geometry given its effect on the process of macrophage phagocytosis. Fibers with length $<17 \mu \mathrm{m}$ were found non-cytotoxic to murine alveolar macrophages ( 13 $\mu \mathrm{m}$ diameter) [98]. CNTs used in this study are much smaller (1-3 $\mu \mathrm{m})$ than the critical fiber length $(17 \mu \mathrm{m})$ required shown to cause cytotoxicity. Murine macrophages (J774 Eclone) used in this study are larger ( $\sim 20 \mu \mathrm{m}$ diameter $)$ than alveolar macrophages, which should decrease the chances of cytotoxic effect of loose CNTs in the wear debris. The recent study by Kagan et al. reported that human myeloperoxidase enzymes, contained in macrophages, can biodegrade carbon nanotubes (SWNT) [99]. Thus, it is unlikely that loose CNTs in debris pose a threat to macrophages.

Table 4.3: Percentage LDH absorbance values from live cells obtained through cytotoxicity tests indicating cytotoxicity level in osteoblasts \& macrophages cultured with and without wear debris ( $p$ value $<0.05$ ).

\begin{tabular}{|c|c|c|}
\hline \multirow{2}{*}{ Cell Type } & \multicolumn{2}{|c|}{ \% LDH Absorbance from Live Cells } \\
\cline { 2 - 3 } & With HA Debris & With HA-CNT Debris \\
\hline Osteoblasts & $0.19 \pm 0.04$ & $0.48 \pm 0.03$ \\
\hline Macrophages & $0.50 \pm 0.06$ & $0.46 \pm 0.01$ \\
\hline
\end{tabular}


Wear particles can induce some behavioral changes in macrophages leading to adverse effect on biocompatibility of implant [22]. Metallic wear particles stimulate the macrophages to release hormonal factors like Prostaglandin $\mathrm{E}_{2}$ and Interlukin-1 [17, 100101]. These factors induce osteoclastic differentiation of precursor cells [102-104]. Increase in the osteoclast formation aggravates the bone resorption. The presence of CNTs in the composite coating would be beneficial in such situation as it can reduce bone resorption by inhibiting osteoclast proliferation and growth [105]. Further studies on the effects of wear debris produced in physiological solution, on macrophages and osteoblasts can provide more insight on different aspects of cytocompatibility of HA and HA-CNT coatings for orthopedic implants.

Findings from in-vitro study of HA-CNT suggest no negative influence with biocompatibility related to its orthopedic application. CNTs are, actually, found positively influencing the osteoblastic proliferation, viability, differentiation and bone formation through cytoskeletal mineralization. Non-cytotoxic response of osteoblast and macrophages to CNT containing wear debris during in-vitro exposure is a step towards predicting its safe clinical application. In-vivo studies, explained in next section, would provide further accurate evaluation on this issue.

\subsubsection{In-Vivo Evaluation of HA-CNT Coating}

In-vivo assessment of biocompatibility for HA-CNT and HA coatings are performed using rat model. All the studies mentioned in this section are carried out by our collaborating research group at University of Strasbourg, France. Results from in 
vivo studies are included in this dissertation for the sake of completeness of biocompatibility evaluation of HA-CNT.

\subsection{Implantation using Rat Model}

HA and HA-CNT coated Ti-6Al-4V rods are implanted in the femur of rats. Figure $4.47 \mathrm{a}$ presents the HA-CNT coated $\mathrm{Ti}$ rod used for implantation. A closer observation coating surface reveals network of CNTs in the HA matrix (figure 4.47b).
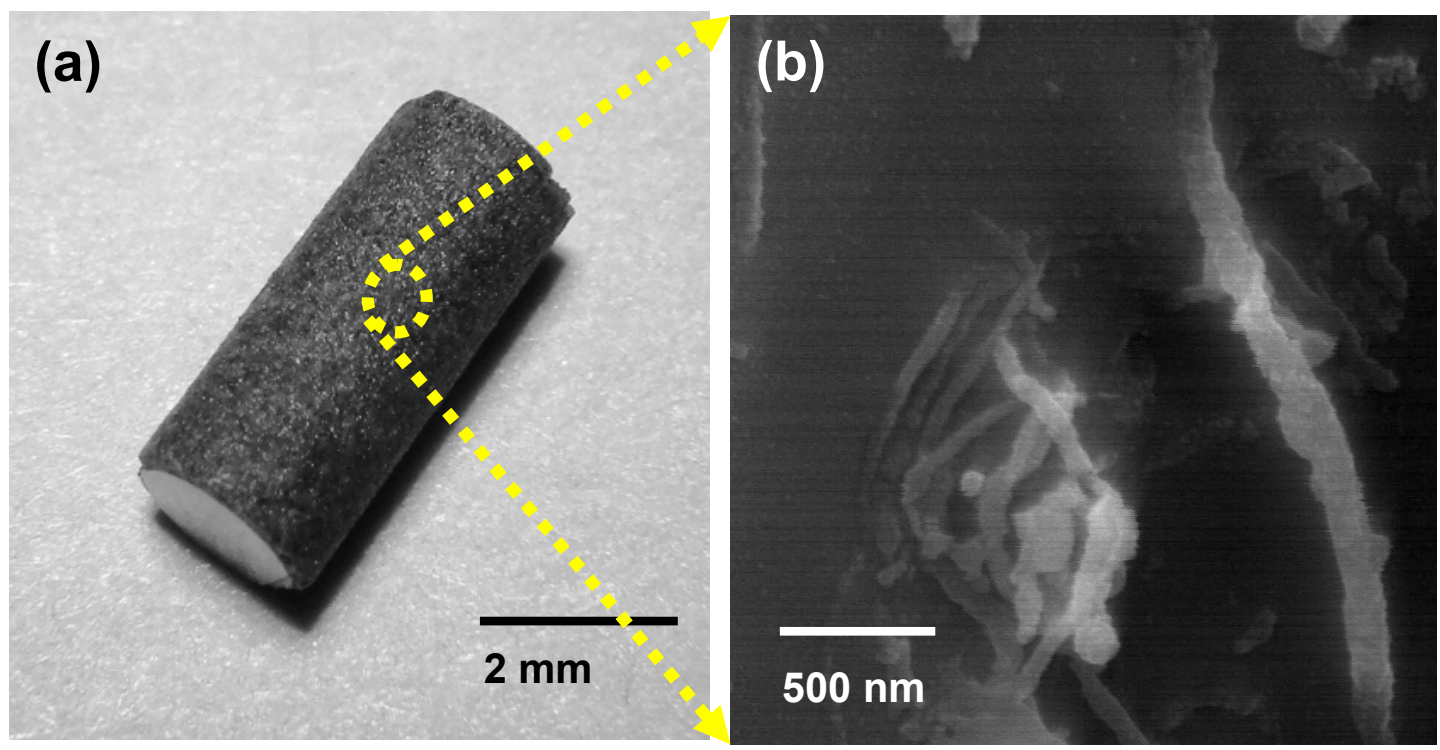

Figure 4.47: (a) Ti-6Al-4V Alloy rod - coated with HA-CNT by plasma spraying; (b) top surface of plasma sprayed HA-CNT coating revealing embedded CNTs in HA matrix.

Clinical results showed that none of the animal died during surgery or after surgery during one month of observation. Rats could walk without any disabilities after the implantation surgery. No infection, no disunion of the scar and a complete range of motions of knee joint are observed. The implanted femur bones are retrieved after one month of implantation. Figure 4.48a presents a picture of the retrieved femur with HA- 
CNT coated implant inside. After dissection and retrieval of all parts of the femoral bone with knee joint, no infected tissues were found.

(a)

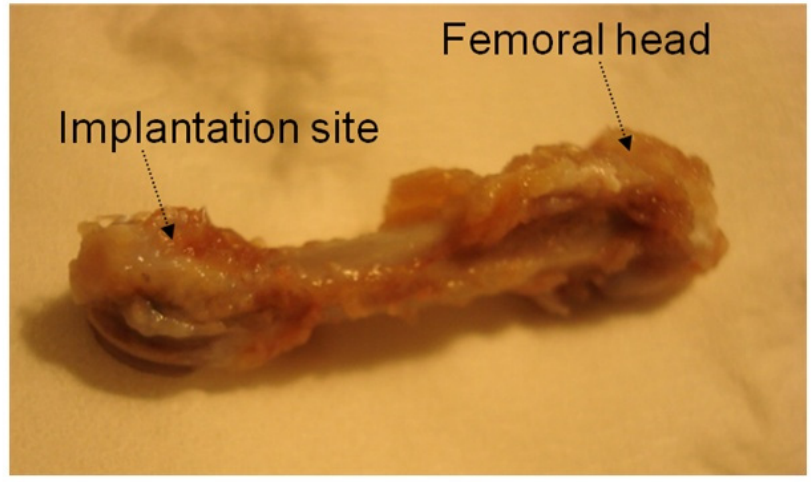

(b)

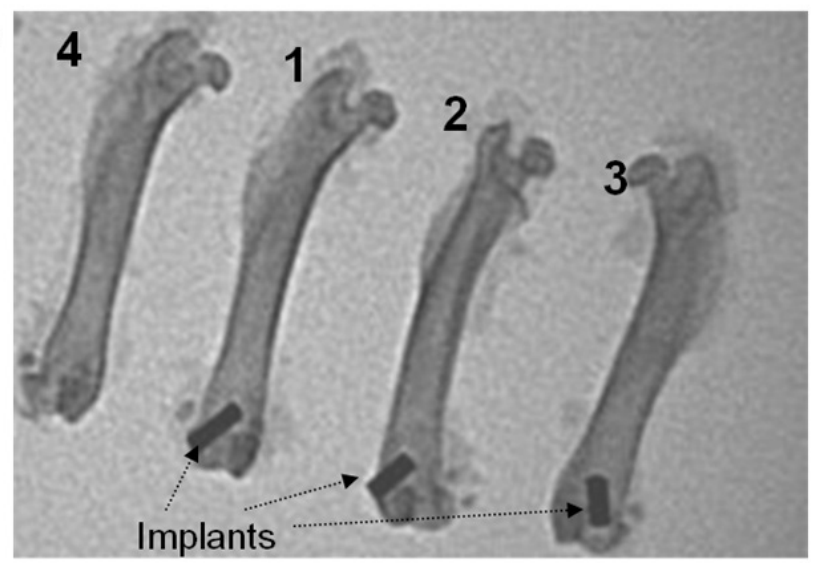

Figure 4.48: (a) Retrieved femoral bone of rat with HA-CNT coated Ti-alloy rod implanted; (b) X-ray images of rat femoral bones after one month implantation. The distal part of femur contains (1) uncoated Ti-alloy implant, (2) HA coated Ti-alloy implant, (3) HA-CNT composite coated Ti-alloy and (4) no implant (normal).

$\mathrm{X}$-ray images of rat femoral bones after one month of implantation are shown for normal, Ti-alloy implanted, HA and HA-CNT coated Ti-alloy implanted bones (figure 4.48b). The implants from groups are found well positioned inside the external femoral condyle. None of the implant is ejected. Cortical bones are restored completely and a good healing is achieved for all the three implants after one month of implantation. 
Periostal tissue was restored in all the bones, without any inflammatory reactions at the perforation location of the cortical bone. Cortical and marrow bones appeared normal around each implant without any specific abnormal tissues. No osteolysis was observed at the periphery of the rod shaped implants embedded inside the external femoral condyle. The implanted bones are compared with normal bone for rat as a control sample for analysis of the results. The approach was to compare ex vivo tissues, cells behavior and bioactivity around implants using histological and TEM studies.

\subsection{Histology of Retrieved Bone with Implant}

For histological observations, all implants are fully removed from the bone using forceps. It was most difficult to detach HA-CNT coated implants as compared to HA coated or bare Ti-alloy implants, which is a qualitative indicator of the strong adherence of CNT containing HA coating with the bone. Semi-thin (1-2 mm) sections are cut from rat's femoral bone with embedded implant for the microscopic observations (figure 4.49).

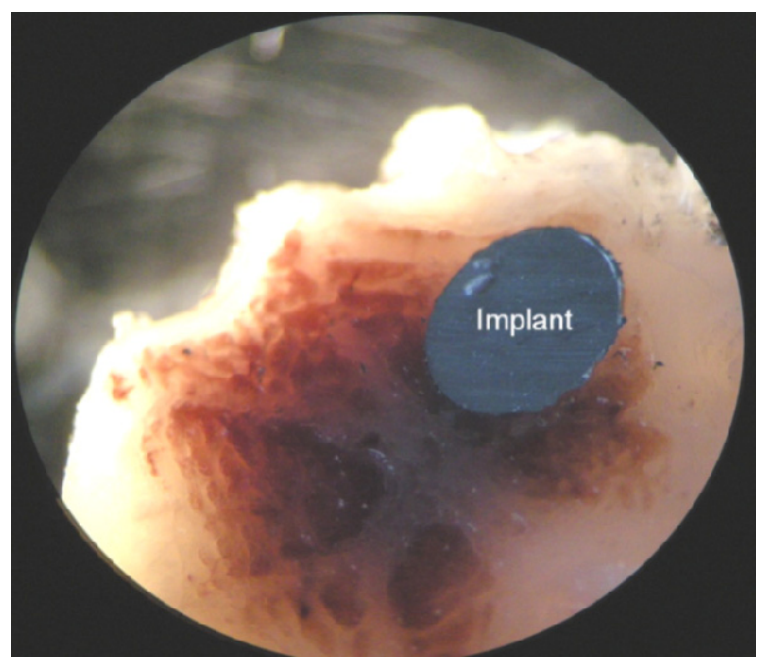

Figure 4.49: Ex-vivo semi-thin (1-2 $\mathrm{mm})$ sections cut from the rat's bone with implant embedded inside (magnification $5 X)$. 
Figure 4.50 shows the histological observations Bare, HA coated and HA-CNT coated implants embedded rat femoral bones. Bone tissues exhibited new grown bone, haematopoietic marrow and trabecular bones which were synthesized with osteocytes into their lacuna. The cortical defect for all bones is also restored due to formation of the neocortical bone. Figure 4.50 also shows a gray layer attached on the bone closed to the cavity (C). The layer is thicker for HA-CNT coated implants as compared to HA coated and uncoated implants.

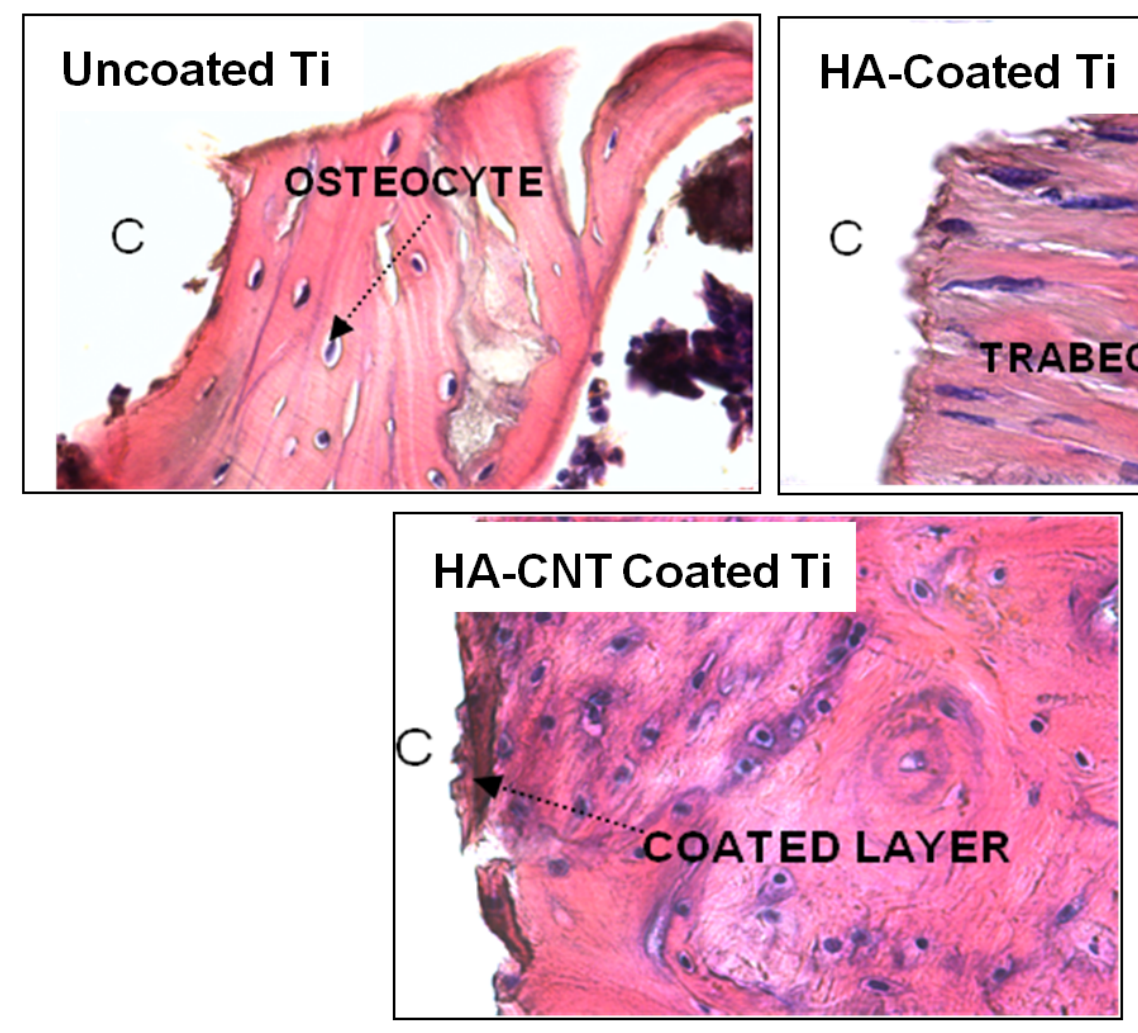

Figure 4.50: Histological results (40X) for rat bones presented through Mallory coloration images for uncoated, HA-coated and HA-CNT coated implants. These images show normal, thick trabecular and hematopoietic marrow bones, without any inflammatory reactions and tissues. A layer is observed attached on the bone closed to the cavity $(C)$ caused by retrieved implant. 
With toluidine blue coloration in figure 4.51, it is clearly observed that HA-CNT coating (gray layer) was strongly attached to the newly grown bone tissues. This explains the difficulty in detaching the HA-CNT coated implant from the bone using forceps. A normal neobone near the HA-CNT coating suggests that bone regeneration is complete.

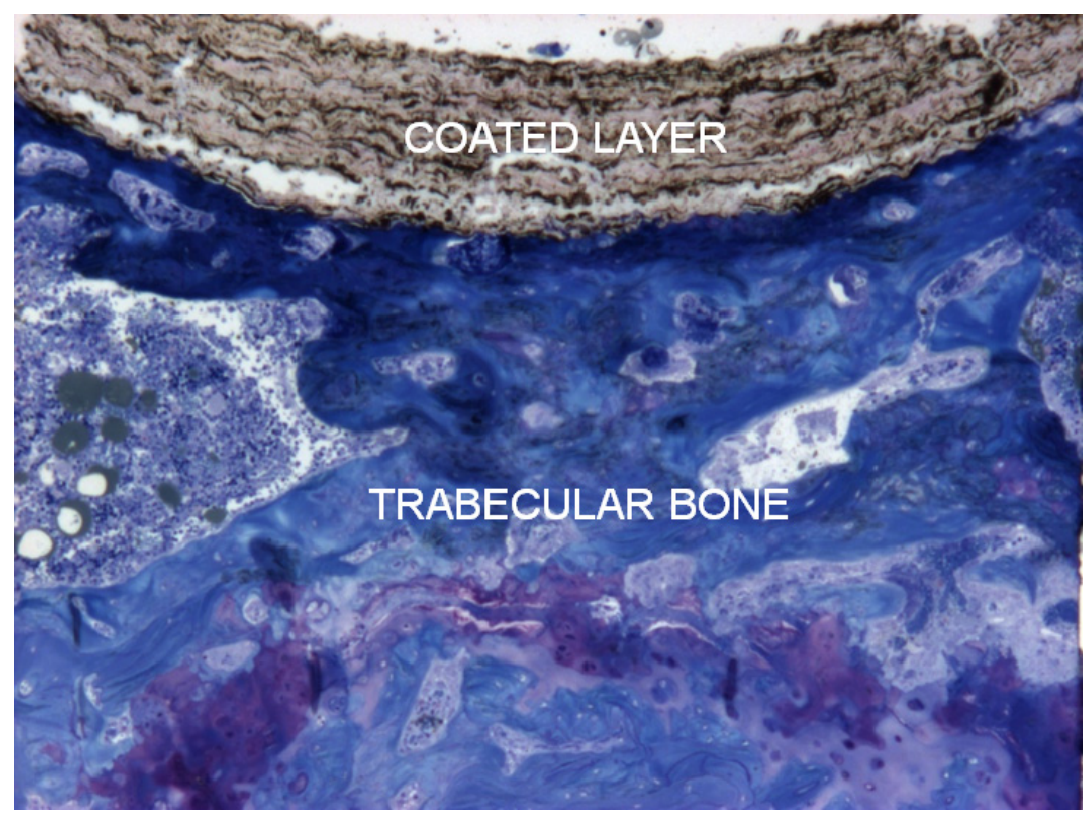

Figure 4.51: Histological image (5X) of HA-CNT coated implant in rat, with toluidine blue coloration, shows HA-CNT coating strongly attached to newly grown bone tissues.

\subsection{TEM Study of Retrieved Bone with Implant}

Transmission electron microscopy (TEM) was employed to study the bone in retrieved implants. Figure 4.52 presents TEM images of bones formed on HA-CNT coated implant surface. Normal bone cells and bony trabeculum were observed without necrosis or inflammatory reaction. There was no tissue degeneration or neutrophil infiltration for HA-CNT coated implants. CNT migration was not observed in either newly formed bone adjacent to the coating or distant bone. Other bones also displayed 
very similar microstructural observations as seen in figure 4.53. In all the bones, cortical or marrow bones and osteoblasts or osteocytes morphologies were normal.
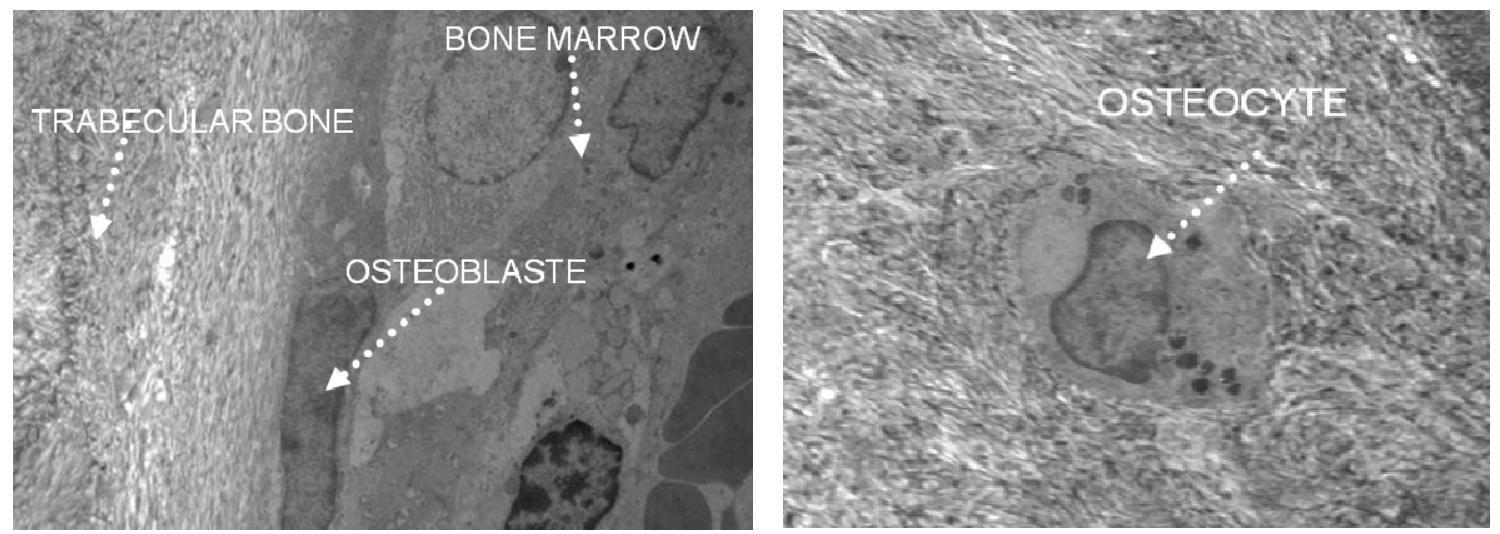

Figure 4.52: TEM images of bone on HA-CNT coated implant at 2200X magnification.
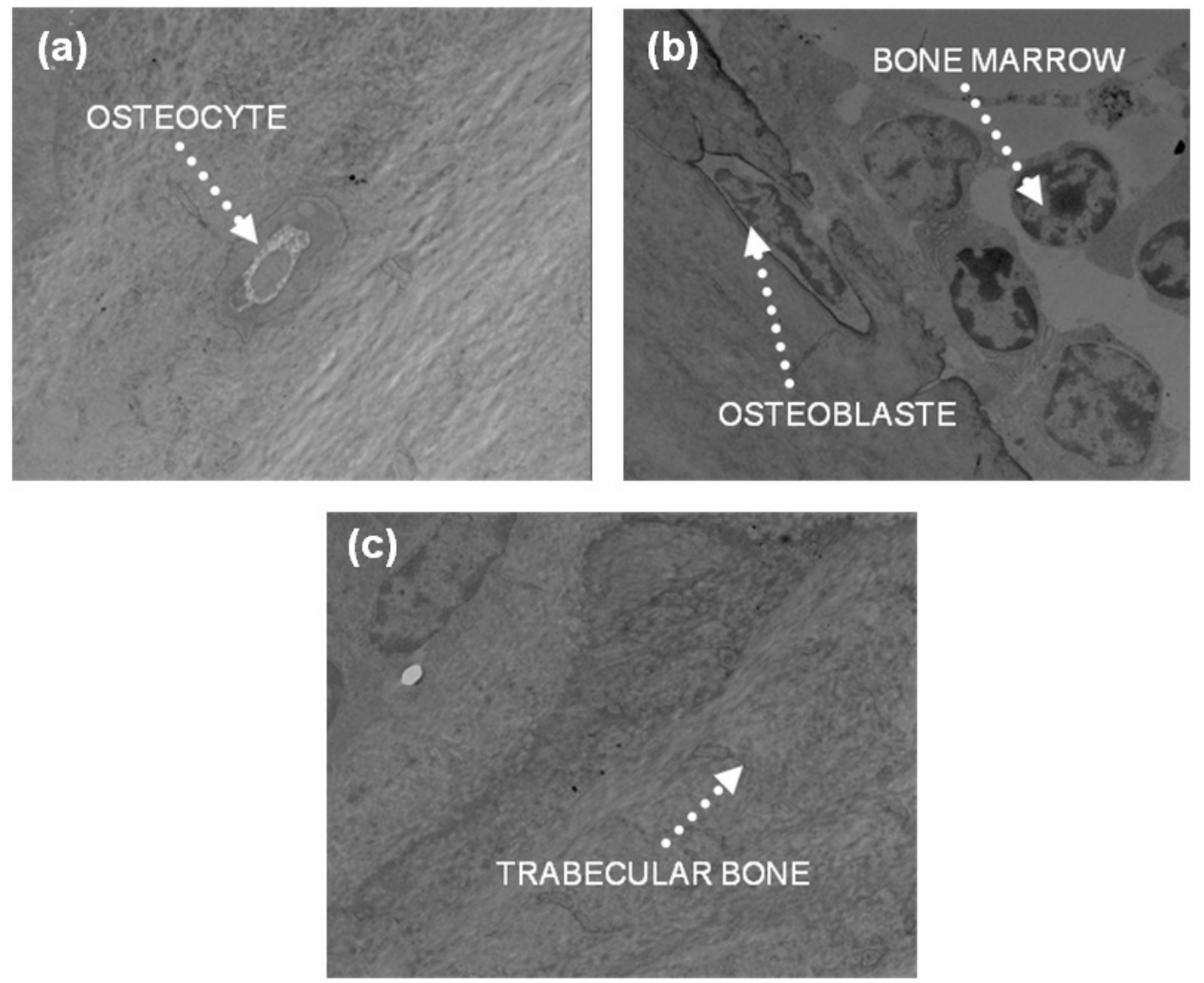

Figure 4.53: TEM images of (a) bone on uncoated Ti implant; (b) bone on HA coated implant; (c) normal bone at $2200 X$ magnification. 
Clinical, histological and TEM observations described above indicate that CNT addition promotes the formation of the new bone without any detrimental effect. Clinical and X-ray results suggest no side effects, no osteolysis and a good osseointegration for CNT-HA coated implant. Biocompatibility with the bone near implant and in distant bone is excellent. Haematopoeitic marrow and trabeculae bones appeared with a good integrity; all different types of cells associated with bone suggested a normal neobone induction. The presence of osteocytes in the lacuna and the observation of bony trabecculum and osteoblatsare are specific for bone formation. Bioactivity and morphology of cells around all implants is completely normal. HA-CNT does not induce necrosis or inflammatory reactions or formation of tissue granulomas. These promising results are largely attributed to the presence of CNTs that accelerate bone growth [106], biomineralization and inhibit osteoclastic bone resorption [105]. The accelerated bone repair occurs because CNTs stimulate osteoblasts proliferation. CNTs can also form an efficient nanomatrix for the growth of HA crystals with a stoichiometric value that complies with natural HA [107-109]. Hence, CNTs act as an effective nucleation surface to induce the formation of a biomimetic apatite coating. A better adhesion is observed for HA-CNT coated implants which could be attributed to the nucleation of apatite on CNT surface that promotes anchorage [3]. The higher surface area of CNTs also promote bonding as observed in figure 4.51, where HA-CNT coating was bonded with newly grown bone. For orthopaedic surgeons, osseointegration of joint replacement continue to be a challenge in terms of quality and duration, which could be improved by CNT addition to HA. 
More interestingly, the phenomena of phagocytosis or necrosis or any strong inflammation reactions are not observed near the implant/coating/bone interface for HACNT coated implant. The same observations are made for new and distant bone cells. The lack of inflammatory response is because enzymatic biodegradation of nanotubes do not induce any inflammatory response [99]. Inflammation could also be explained by the ineffective internalization of non-functionalized nanotubes by phagocytic cells. Nanotubes are actively ingested through phagocytosis in macrophages in significant quantities without cytotoxic effects [110]. Wear debris generated from plasma-sprayed HA-CNT coating also does not alter the cytotoxicity response of macrophages, as seen previously in section 4.2.1.1.5. Some studies even suggested that macrophages induced by nanotubes may mediate bone formation because macrophages can produce osteoinductive factors (TGF- $\beta$, BMP-2) [99].

The major findings of this in vivo study suggest that CNTs can be used as reinforcement to HA composite for orthopedic applications without any negative effect. CNT addition resulted in growth of new bone and improved. Further, the results of this study indicate healing time and osseointegration of biomedical materials for joint replacements could be improved with HA-CNT composite coating.

\subsubsection{In-vitro Biocompatibility of Hydroxyapatite-Boron Nitride Nanotube}

\section{Composite}

The present research pioneers in proposing the application of BNNT in orthopedic. Thus, it is mandatory to check the cytotoxicity of BNNT to osteoblasts and macrophages, the two most important cell lineages for orthopedic. In addition, osteoblast 
proliferation and viability is also checked on HA-BNNT surface, considering the dictating role of osteoblasts in neobone formation on implant surface.

\subsubsection{Cytotoxicity of BNNTs to Osteoblasts and Macrophages}

Cytotoxicity assay for bare BNNTs is performed with osteoblast and macrophage cells to evaluate the suitability of using BNNTs for orthopedic implants. The assay that has been used in this study measures the cell death, by rapid and accurate quantification of the release of lactate dehydrogenase ( $\mathrm{LDH})$, a stable cytosolic enzyme released from lysed cells. The amount of LDH released, which is proportional to the number of dead cells, is quantified through a colorimetric assay by measuring the absorbance at $490 \mathrm{~nm}$. Absorbance value of the culture medium without any cells or BNNT was considered as background and subtracted from the experimental absorbance values obtained for the cells cultured with and without BNNT. Comparative measurement was performed and cytotoxicity values obtained for the cells cultured without BNNT were considered as the reference (value set to 1.00).
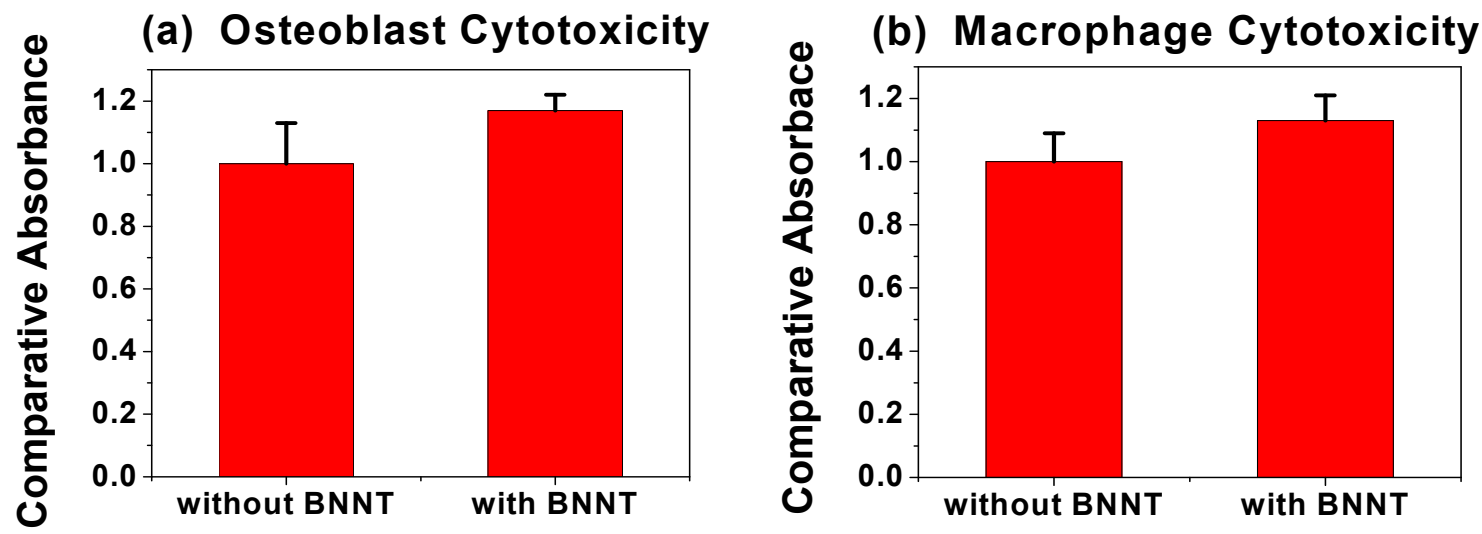

Figure 4.54: Cytotoxicity result of bare BNNTs with (a) osteoblasts and (b) macrophages obtained through LDH assay. 
Results, presented in figure 4.54, show a non-significant cytotoxicity induced by the BNNT for both osteoblasts and macrophages cell lines. The cytotoxicity assay outcomes, in the present study, clearly show that presence of BNNT does not increase the number of dead osteoblast or macrophages cells. Such behavior is attributed to the chemical inertness and structural stability of BNNT [62]. These results suggest safe application of BNNTs in biodegradable orthopedic scaffold, since they are not harmful even if released with wear debris to body fluid or blood stream.

\subsubsection{Osteoblast Proliferation and Viability of SPS HA and HA-BNNT}

Proliferation and viability of osteoblast cells are evaluated on HA and HA-BNNT surface after in-vitro culturing for 1,3 and 5 days. Proliferation of the osteoblast cells is assessed qualitatively by observing the population of FDA stained live cells on HA and HA-BNNT surface by fluorescence microscopy images. Viability of cells on each surface is evaluated by manually measuring number of live and dead cells after different days of culture. Figure 4.55 shows the fluorescent images of live (green) and dead (red) cells after 1 and 3 days of culture on HA and HA-BNNT surfaces. The cells exhibit typical lens shape suggesting the normal cell growth behavior. The population of the osteoblast also increases visibly from 1 to 3 days on both surfaces. This observation indicates that HA and HA-BNNT surfaces are suitable for osteoblast cell proliferation. Population of osteoblast cells is slightly denser on HA-BNNT surface than HA after 3 days of culture.

Figure 4.56 presents the percentage of live osteoblast cells on HA and HA-BNNT surface after 1, 3 and 5 days of culture. The viability of osteoblast cells on HA-BNNT surface is comparable to HA surface. Similar observation of osteoblast proliferation in 
presence of BNNT was reported by the present authors on a biodegradable polymer surface [111]. Gene expression study revealed that presence of BNNT positively influence osteoblast differentiation and proliferation [111]. The reason for such behavior could be attachment of proteins (from the culture medium) on BNNT surface that assists osteoblast proliferation. BNNT also has a natural affinity for protein attachment on its surface [112]. Probability of the protein absorption on the BNNT surface is further supported by the similar behavior established for CNT, the structural analogue for BNNT [85-86].
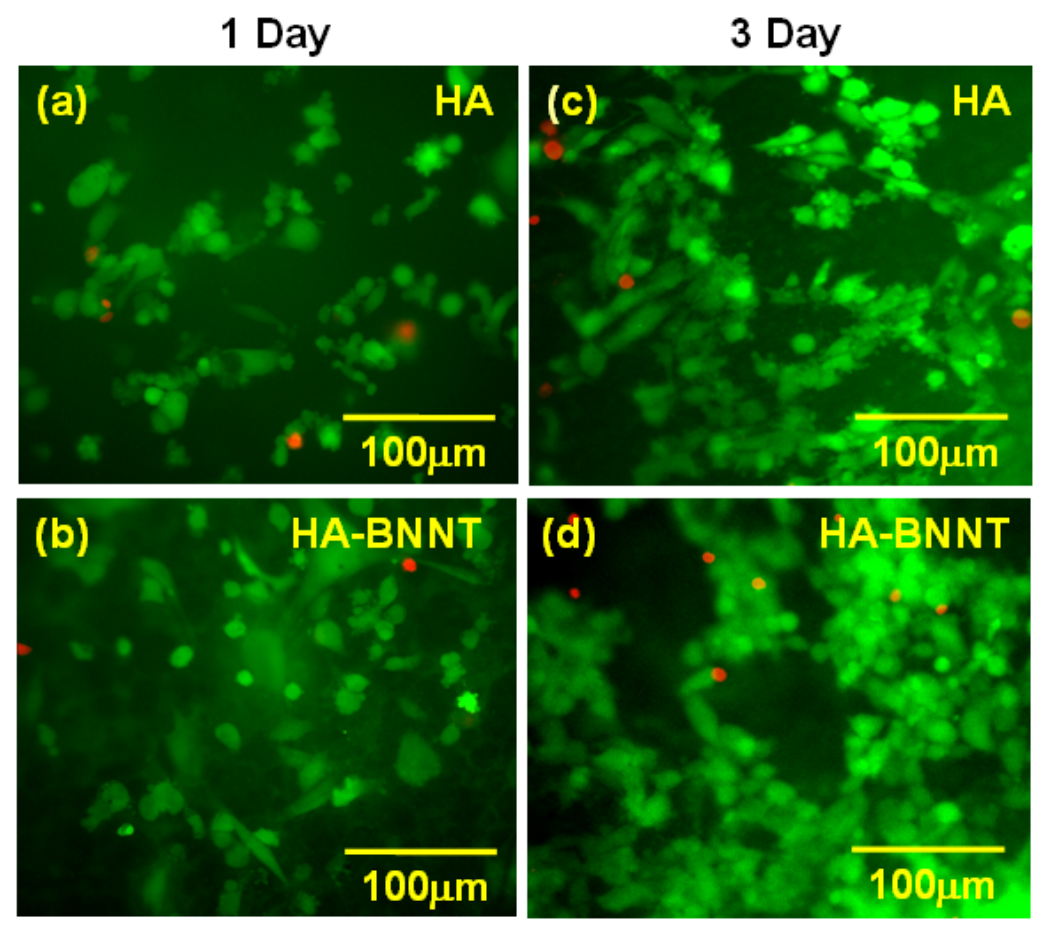

Figure 4.55: Fluorescent images of osteoblasts grown on HA and HA-BNNT surface for (a-b) 1 day and (c-d) 3 days. The live cells are stained in green color with FDA and dead cells in red with PI.

Apart from the bone growth and integration on implant surface, another major concern regarding the orthopedic material could be the cytotoxicity of wear debris. As 
discussed above, BNNTs do not exhibit any cytotoxic response to osteoblasts and macrophages. Thus, the presence of any loose or embedded BNNT in wear debris generated from HA-BNNT implant during service would not impose any negative effect on its biocompatibility.

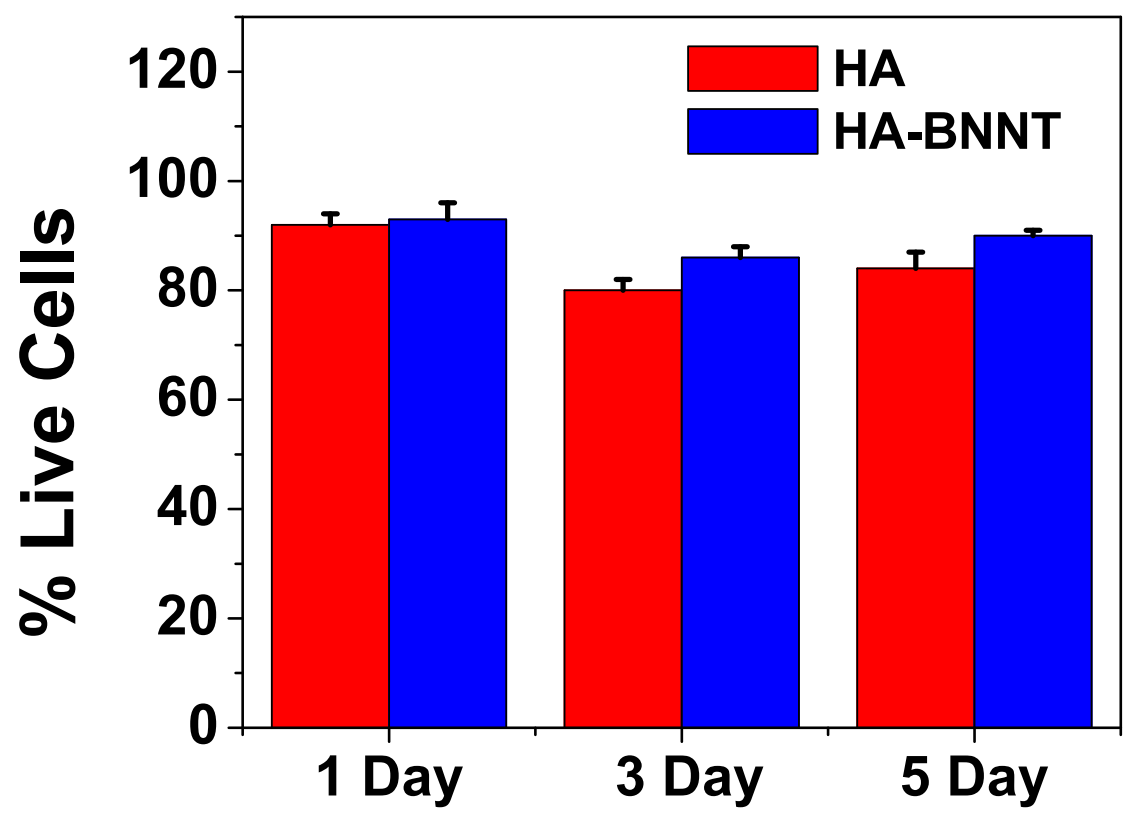

Figure 4.56: Osteoblast viability on HA and HA-BNNT surfaces for 1,3 and 5 days of culture $(p<0.05)$.

\subsection{Osseointegration Ability of Implant}

An important requirement for a successful orthopedic implant is the formation of new bone on its surface for effective osseointegration. During the bone formation process, osteoblast plays active role in laying collagen fiber matrix and then mediate precipitation of hydroxyapatite mineral to form a compact bone. Further, the boneimplant surface should also maintain a mechanical compatibility to inhibit fracture of bone at interface and/or implant failure. Thus, three different processes/phenomena are 
very important for osseointegration on a bioimplant surface, namely (i) adhesion of osteoblast to implant surface; (ii) apatite precipitation ability of implant surface and (iii) mechanical compatibility at bone-implant interface. HA-nanotube composites are checked for these three phenomena, which are accounted in this section.

\subsubsection{Adhesion of Osteoblasts on Implant}

Osseointegration of implants is greatly dependent on the retention of osteogenic cells on the implant surface. Especially, for anchorage-dependent cells, like osteoblasts, chondrocytes etc., initial cell-biomaterial interaction and attachment regulates the subsequent biological cascade of proliferation, differentiation, extracellular matrix production (ECM) and matrix mineralization [113-116]. Unsuccessful attachment of osteogenic cells can even cause dedifferentiation and loss of their phenotype [117-118]. Further, considering the presence of mechanical forces at the implant-bone interface, initial attachment and spread of bone forming cells turns out to be vital for a successful implant [114-115, 119-120]. Proposed materials for orthopedic implant, thus demand a quantitative understanding of the cell adhesion on the surface.

\subsubsection{Adhesion Quantification Techniques for a Single Cell}

Several techniques have been adopted by researchers to evaluate the adhesion of cells with the underlying substrate. Appendix-2 presents a comprehensive compilation of the major techniques with their advantages and limitations in assessing adhesion of the cell. Qualitative techniques are widely used due to their ease in application, though the outcome is always comparative in nature. The quantitative techniques can be broadly 
divided into two categories. The first ones are those in which the adhesion force of a single cell is evaluated using indirect relationships. Examples of such techniques are centrifuging, spinning disc, shearing by hydrodynamic force [121-124]. The second quantitative category consists of the techniques that are capable of measuring the adhesion force of a single cell directly. Atomic force microscopy (AFM), micropipette suction, cytodetacher and shearing by a cantilever are the major techniques in this category. Although AFM is a suitable technique for measuring the adhesion of a single cell [125], the attachment of a cell on the AFM probe or cantilever can pose stress induced damage to the cell due to the long time required for the procedure. Micropipette suction can also measure the force required to detach a cell from the substrate, but inherits a risk of rupturing the cell membrane and damaging the cell due to application of localized tensile force [126]. A very attractive way of quantifying adhesion of a single cell is by the application of shear force to a cell parallel to the substrate and recording the deflection of a cantilever to measure the detachment force [127-129]. This method is suitable as it does not cause cell damage prior to its detachment. Two different groups of researchers have adopted this principle to develop methods for cell adhesion quantification, namely, cytodetacher [129] and shear force measurement [127-128]. In both these techniques, the adhesion force is calculated from the deflection of the cantilever caused by the reaction of the cell. This calculation engages the compliance of the cantilever and incorporates a conversion factor for calculating the force from the deflection, which is often a source of error [130]. Optical technique was used for determination of the cantilever deflection. Highly sensitive piezo-transducer which is capable of recording very low shear forces was unavailable at the time of this study 
[129]. Possible sources of measurement errors in cytodetacher technique include: quantification of small deflection using conjugate system of carbon filament (glued at the back of cantilever), optical microscope and photodiode. Moreover, during pushing the

cell, the cantilever immersed in liquid medium experiences a resistance from the fluid, which also adds to the cell adhesion value, leading to an error in estimation.

The present study proposes nano-scratch technique using a nanoindenter, which uses the principle of shear force measurement to quantify the adhesion of a single cell. However, this technique measures the force directly and does not rely on computation of the force as in the case of cytodetacher. Moreover, scratch made in similar conditions without cells but with culture medium helps to subtract the contribution of the medium and underlying substrate in the lateral force measured for the cell detachment. Thus, this technique overcomes the limitations of existing ones for an accurate measurement of the adhesion force for a single cell. We have demonstrated the success of this technique in our recently published study, where adhesion strength of 1-D structure such as carbon nanotubes (CNTs) and carbon nanocones (CNC) has been quantified for a single nanotube/nanocone [131].

\subsubsection{Quantification of Adhesion Force and Energy of Osteoblast on Composite}

\section{Surface by Nano-scratch Technique}

Adhesion of an osteoblast is quantified in terms of lateral force required for its detachment from different substrates. The substrates used in this study are (i) HA-CNT plasma coated Ti-alloy substrate, (ii) HA-coated substrate and (iii) permanox plastic slide as a control substrate. Figure 3.4 in previous chapter presents a schematic of the nano- 
scratch test with detailed explanation on calculation of adhesion force and energy for a single osteoblast. Figure 4.57 presents the optical image of cells on permanox surface before and after scratch, revealing a distinct movement of the cell by $>9 \mu \mathrm{m}$ due to scratching.

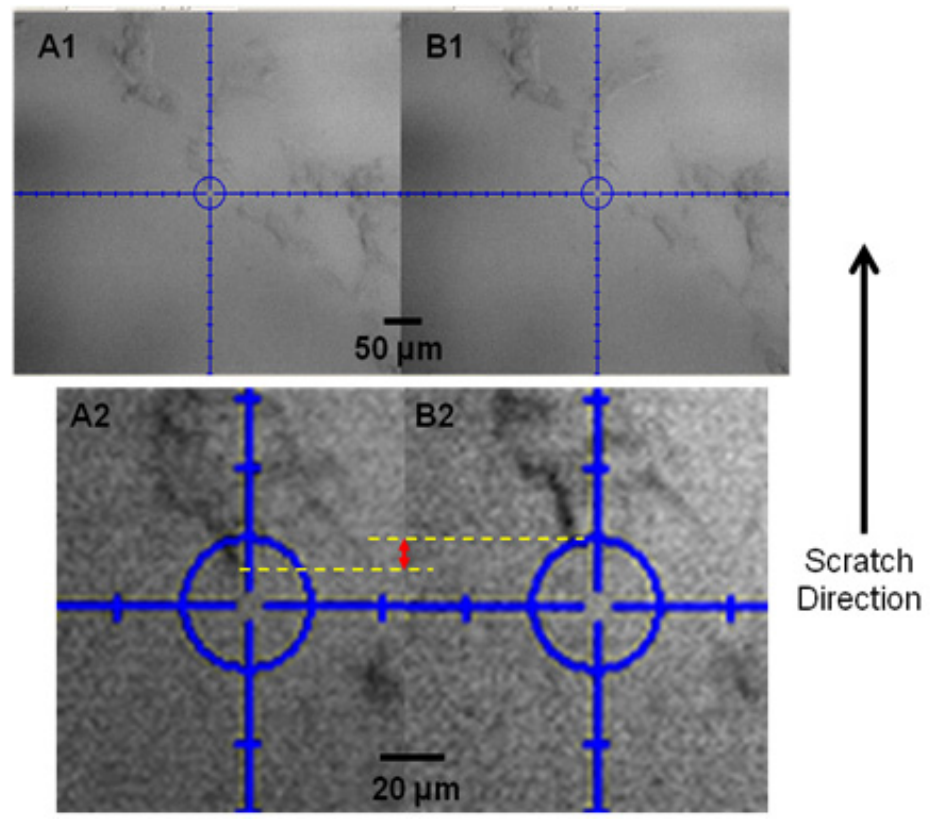

Figure 4.57: Optical Images of osteoblast on permanox surface grown for 3 days (A1) before and (B1) after nano-scratch. A2 and B2 show the magnified part of images A1 and $B 1$, respectively, to clearly visualize the displacement of the cell by nano-scratching.

Figure 4.58a presents the lateral force $v s$. displacement curves for an osteoblast on HA and HA-CNT surfaces after 5 days of incubation. The same figure also includes the lateral force $v s$. displacement curves for scratches made on HA and HA-CNT surfaces in the same conditions but without cells. The hump in the lateral force curves for surfaces with cells is completely absent for the scratches made on the same surfaces without cell. The increase in the lateral force denotes the force required to detach the cell from the underlying substrate. Once the cell is detached, the lateral force shows a drop from its 
peak. A closer observation of this curve reveals that even after detachment of the cell, the lateral force does not drop down to the same level as of the bare substrate. This is because a smaller amount of lateral force is required to push the detached cell along the scratched path/direction. Cell adhesion force is obtained by subtracting the lateral force contribution of the bare scratch from the height of the lateral force hump as explained in Figure 3.3.

(a)

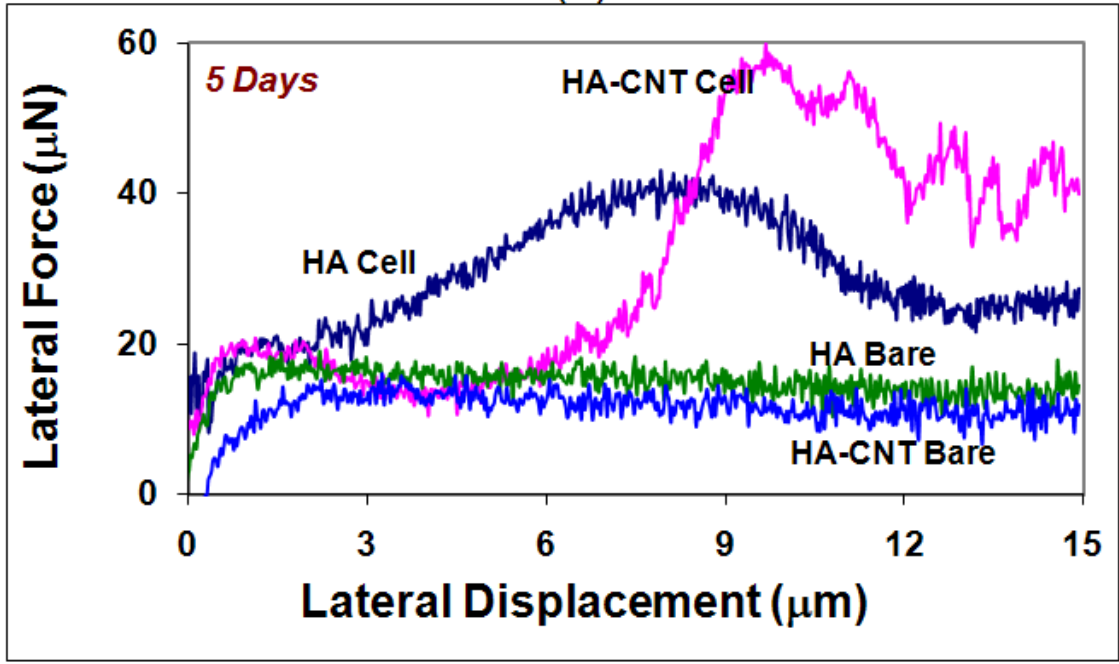

(b)

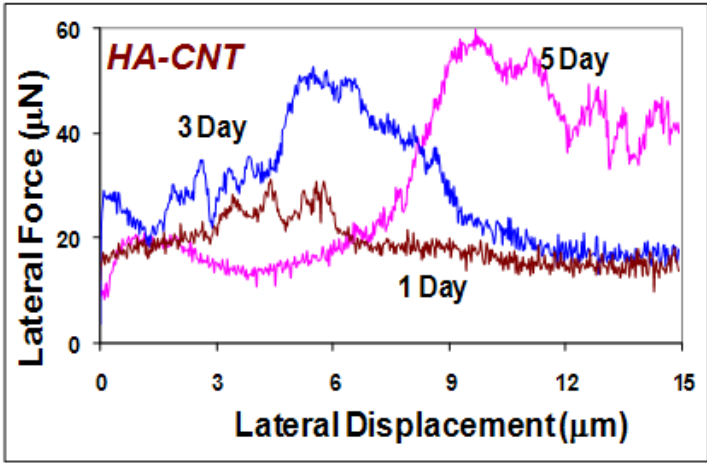

(c)

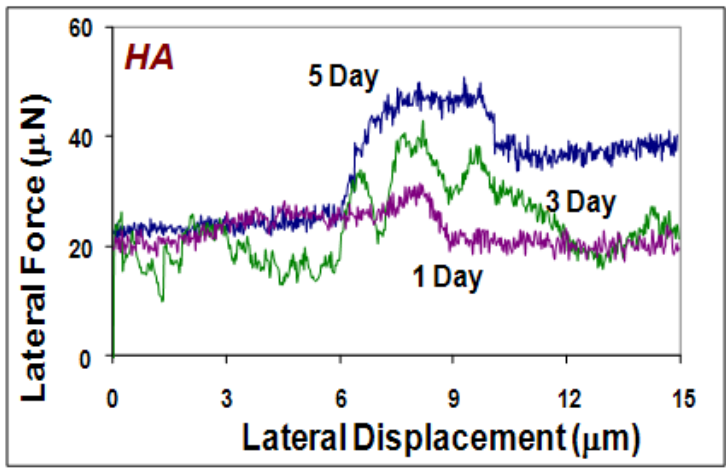

Figure 4.58. Lateral force vs. displacement curves obtained from nano-scratch tests of (a) osteoblasts grown on both HA and HA-CNT substrate for 5 days and the bare substrates in same condition; (b) osteoblasts grown on HA-CNT surface for 1, 3 and 5 days and (c) osteoblasts grown on HA surface for 1, 3 and 5 days. 
Figure 4.58a also provides a comparison of the lateral force required to detach an osteoblast from HA and HA-CNT surfaces. Osteoblast is found to have better adhesion to HA-CNT surface as compared to HA. Figures $4.58 \mathrm{~b}$ and $4.58 \mathrm{c}$ show adhesion force of osteoblasts cultured for 1, 3 and 5 days on HA-CNT and HA surfaces respectively. In both substrates, the adhesion strength increases with the incubation period. Table 4.4 lists the adhesion force of osteoblast on permanox plastic, HA and HA-CNT surfaces for 1, 3 and 5 days of growth period. Adhesion energy of osteoblast computed from the area under the curve is also presented in table 4.4. The change in adhesion energy from one substrate to another and as a function of incubation period can be easily visualized in figure 4.59. Adhesion energy also follows a similar trend as adhesion force. Osteoblast shows the lowest adhesion energy on permanox surface, followed by HA and highest on HA-CNT. The degree of increase in adhesion energy from 1to 5 days is higher for HA and HA-CNT coated surfaces as compared to permanox. At least 8 tests are performed in each condition and the standard deviation is reported based on these results in table 4.4. The consistency of the results makes the difference in adhesion force and energy very significant.

Table 4.4. Adhesion force and energy of osteoblast on permanox plastic, $H A$ and $H A$ CNT for 1, 3 and 5 days of incubation periods

\begin{tabular}{|c|r|r|r|r|r|r|}
\hline \multirow{2}{*}{ Substrate } & \multicolumn{3}{|c|}{ Adhesion Force $(\boldsymbol{\mu}$ N/Cell) } & \multicolumn{3}{c|}{ Adhesion Energy (pJ/Cell) } \\
\cline { 2 - 7 } & 1 Day & $\mathbf{3}$ Days & $\mathbf{5}$ days & 1 Day & 3 Days & 5 days \\
\hline Permanox & $9 \pm 2$ & $12 \pm 2$ & $12 \pm 2$ & $37 \pm 7$ & $65 \pm 5$ & $74 \pm 11$ \\
\hline HA Coating & $11 \pm 2$ & $29 \pm 3$ & $32 \pm 2$ & $45 \pm 10$ & $99 \pm$ & $145 \pm 1$ \\
\hline $\boldsymbol{H A}$ C $\boldsymbol{N T}$ & $17 \pm 2$ & $35 \pm 3$ & $41 \pm 2$ & $78 \pm 14$ & $194 \pm 8$ & $237 \pm 1$ \\
\hline
\end{tabular}




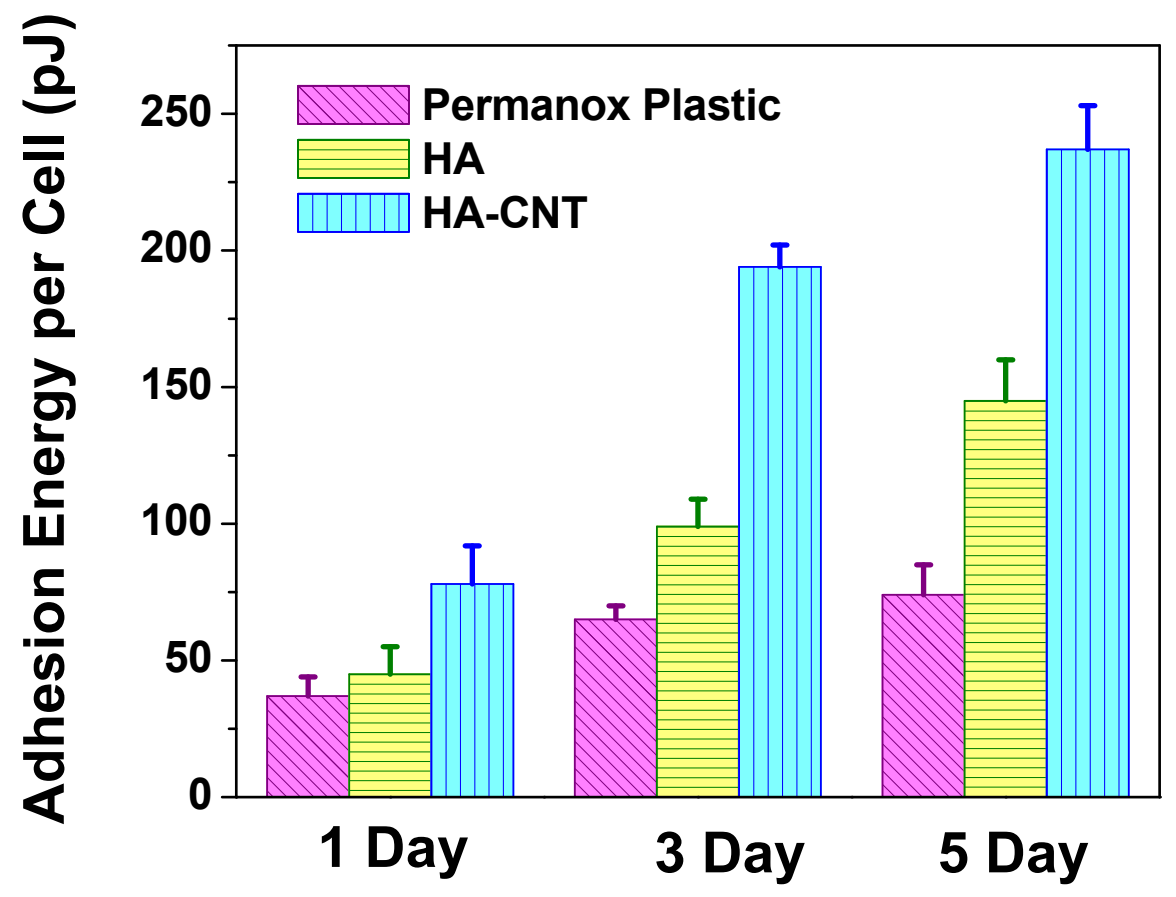

Figure 4.59: Adhesion energy of osteoblast on permanox plastic, $H A$ and HA-CNT substrates for 1, 3 and 5 days of incubation periods.

Osteoblast shows better adhesion on HA and HA-CNT surfaces than on permanox plastic (table-4.4 and figure 4.59). The adhesion increases significantly with the incubation period on HA based surfaces than plastic, which proves those surfaces to be more suitable for osteoblast adhesion. Better adhesion of osteoblast to HA than plastic is also observed by other research groups [132-136], although not quantified. Using gene expression profiling Xie et al. [135] reported enhanced differentiation for osteoblasts cultured on HA when compared to those cultured on plastic with better spreading, which indicates better attachment. The same study also reported better attachment of surface proteins from serum (in cell culture medium) to HA surface, which influences integrin signaling and causes cell attachment [135]. Ozawa and Kasugai showed that osteoblasts 
cultured on HA express higher level of osteopontin than those grown on plastic. Higher levels of osteopontin may allow for better cell attachment at bone remodeling site [132]. Moreover, a single osteoblast was found to synthesize more ECM with type I collagen and osteonectin on HA than on plastic [133-134], which may increase the attachment of the cell with the HA substrate. All these reports, along with the quantitative evaluation of adhesion force and energy presented in the present study, confirms that attachment of a single osteoblast is better on HA based surfaces than plastic. It is also observed that presence of CNTs increases the adhesion energy of an osteoblast on HA based coatings by 73,95 and $63 \%$ for 1,3 and 5 days of cell growth period, respectively (table-4.4). Therefore, to understand the biological changes elicited by CNTs that may promote cell adhesion, actin staining is performed for osteoblasts grown on HA and HA-CNT coated substrates.

\subsubsection{Osteoblast Adhesion Mechanism through Actin Expression}

Actin filaments constitute one of the three major components of the cell's cytoskeleton and are directly involved in defining the cell's shape and rigidity. When osteoblasts adhere to a surface, they form filopodia where parallel actin filaments can be seen organized in bundles. The integrin family of heterodimeric transmembrane receptors connects the actin filaments to the ECM during the process of cell adhesion. Staining of actin filaments reveals the spreading of the cell and its shape on the substrate. Better spreading is an indicator of improved adhesion. Figure 4.60 presents the fluorescence images of the stained actin network of osteoblasts on HA and HA-CNT coatings for 1, 3 and 5 days. 

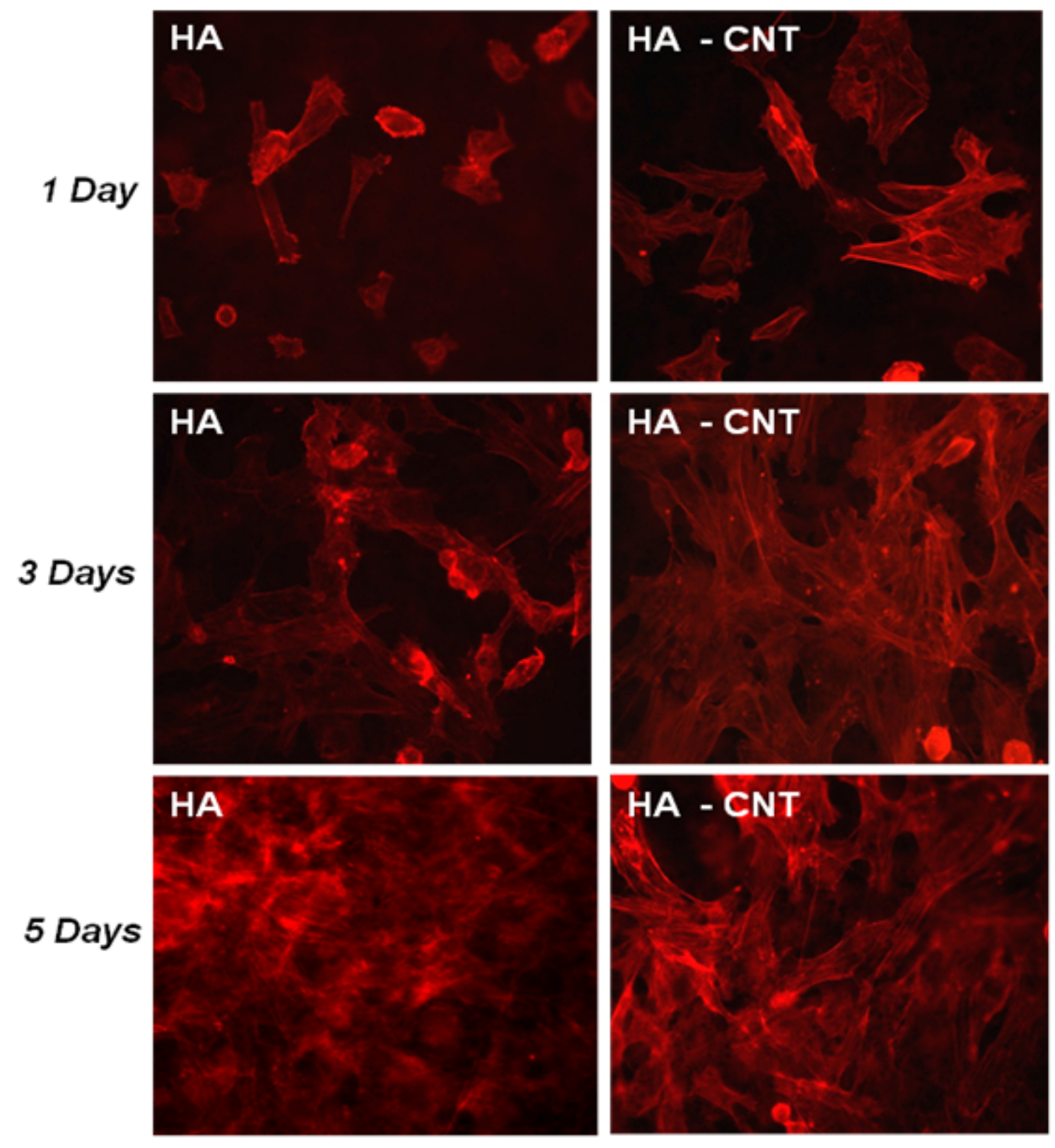

Figure 4.60: Fluorescence images of stained cytoskeleton showing actin network of osteoblasts grown for 1, 3 and 5 days on HA and HA-CNT surfaces. All the images were taken at $40 X$ magnification.

The images of day 1 clearly show more stretched, well oriented and more prominent actin fiber formation in osteoblast cytoskeleton on HA-CNT surface than on HA. When cells are subjected to stress, formation of actin stress fibers is regulated by 
signaling pathways that may involve integrin receptors [137-139]. Bone cells are found to form stress fibers when anchored with collagen, but not within a floating gel [140]. Walcott and Sun have reported faster and greater aggregation of actin filaments with external tensile force applied on the cytoskeleton in addition to the orientation of filaments along force direction [137]. Thus, oriented and prominent actin fiber formation on HA-CNT surface could be due to the better spreading and adhesion of osteoblasts on that surface, which causes tensile force in osteoblast cytoskeleton. This observation is in direct agreement with the higher adhesion energy of osteoblasts on HA-CNT surface measured in the present nano-scratch study. A similar trend continued with the longer incubation periods of 3 and 5 days, when osteoblasts on HA-CNT surface showed increasingly more intense actin staining with nicely developed and oriented actin fibers as compared to that on HA surface (figure 4.60).

Image based analysis is carried out using ImageJ software [141] to obtain a quantitative measurement of osteoblast actin expression in HA and HA-CNT surfaces, by determining the number of stained pixels that represent actin filaments. Figure 4.61a presents the comparative number of stained pixels within a single osteoblast cultured on HA and HA-CNT surfaces for 1 day. Higher numbers of stained pixels found in osteoblasts cultured on HA-CNT surface indicates more actin fiber formation. Figure 4.61b presents the pixel based measurement of the area fraction on HA and HA-CNT surfaces covered by cytoskeletal actin network. All through the incubation period, HACNT surface shows more intense and well spread actin fiber network than HA. Hence figures 4.60 and 4.61 clearly indicate that CNTs enhances the spreading and adhesion of osteoblasts. 
(a)

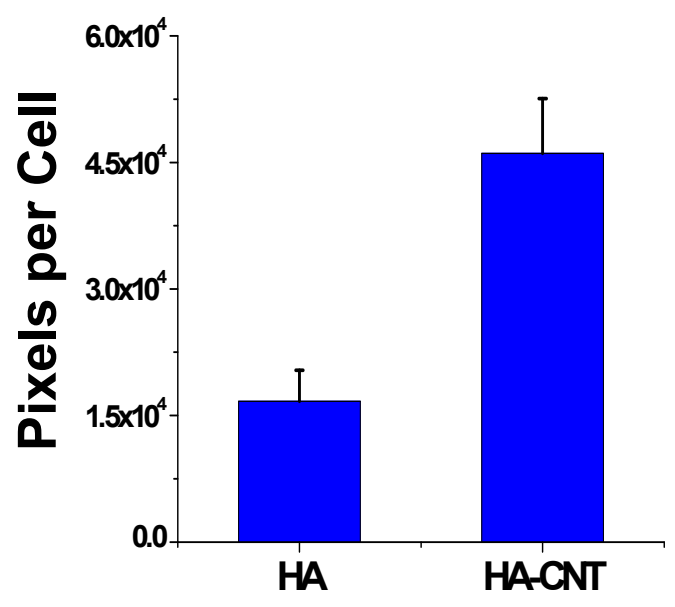

(b)

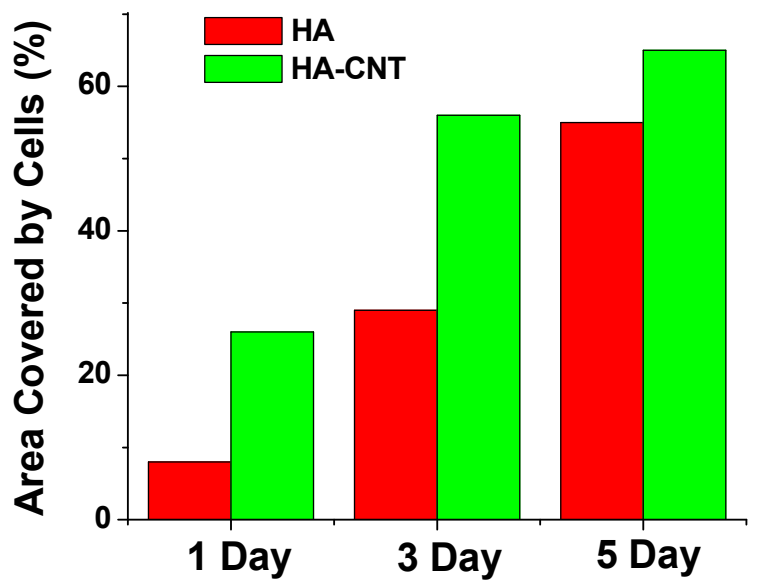

Figure 4.61: (a) Image analysis-based actin expression for substrates incubated with osteoblast for 1 day - showing number of stained pixels/cell for HA and HA-CNT substrates; (b) fraction of area covered by actin network (in \% of total area) at different substrates for different incubation periods - determined by evaluating fraction of stained pixels out of $\sim 8 \times 10^{6}$ pixels in each substrates (images of similar magnification were used for the measurements).

\subsubsection{Role of CNT in Adhesion of Osteoblasts}

It is well established that stable osteoblastic cell adhesion is mediated by integrins that play a major role in survival of primary osteoblasts [142-143]. Integrin receptors comprise of two transmembrane glycoprotein subunits $\alpha$ and $\beta$. The cytosolic domain of integrin associates with adaptor proteins, like $\alpha$-actinin, vinculin, and talin to form a focal adhesion assembly with actin filaments inside the cell. ECM ligands bind with the $\alpha / \beta$ subunit interface at the extracellular domain of integrins. In this manner, the intracellular cytoskeltal actin filaments are linked to the ECM or substrate [144-145]. Attachment of 
osteoblasts with different ECM components or different substrates is mediated by different combinations of integrin subunits [142, 146-147]. For example, $\alpha_{2}, \alpha_{3}, \alpha_{4}, \alpha_{5}, \alpha_{6}$, $\beta_{1}$ and $\beta_{3}$ integrin subunits are expressed on polysterene surface, whereas, $\alpha_{5}, \alpha_{6}$, and $\beta_{1}$ are expressed on metallic surfaces like titanium and cobalt-chrome [142]. $\alpha_{2} \beta_{1}$ was found to mediate osteoblast response on Ti surface [147] and type I collagen [146]. Lai et al. have shown $\alpha_{v} \beta$ integrins to mediate osteoblast response with many bone matrix proteins [146]. CNTs also have specific affinity to integrins [148-151]. Functionalized CNTs were shown to have high affinity to $\alpha_{v} \beta_{3}$ integrin and have been proposed for tumor targeting to deliver radioisotope drugs in cancer therapy [148-151]. Affinity of CNTs to integrin conjugation could also play an active role in the promotion of osteoblast attachment on HA-CNT substrate. CNTs are also reported to attach cell adhesive proteins from serum in cell culture medium [86]. These proteins can further enhance integrin mediated attachment of osteoblasts on CNT surfaces. Figure 4.62 shows a proposed schematic model of the integrin mediated focal adhesion mechanism that may be responsible for linking the osteoblast cytoskeletal actin filaments to CNTs. Figure $4.62 \mathrm{~b}$ reveals the network of exposed CNTs on the surface of HA-CNT coating, on which osteoblasts are cultured. These exposed CNTs are favorable sites for integrin attachment. The additional integrin-CNT attachment over the normal attachment of integrin-ECM has potential to aid in increased adhesion strength on HA-CNT surface. 


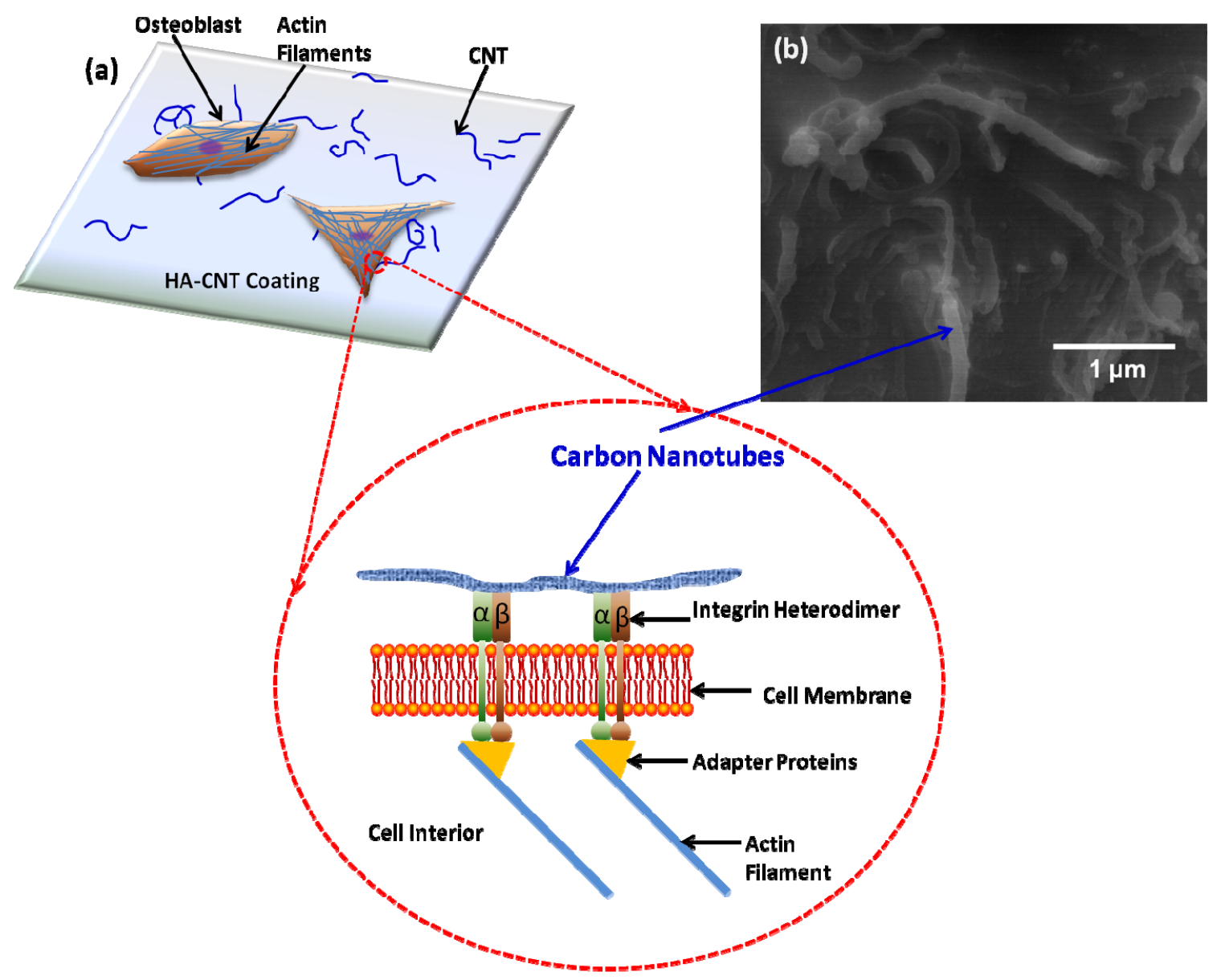

(c)

Figure 4.62: Schematic for proposed model of focal adhesion mechanism for osteoblast showing (a) osteoblast grown on HA-CNT coating; (b) SEM micrograph of HA-CNT coating top surface revealing exposed surfaces of embedded CNTs; (c) osteoblast focal adhesion assembly showing integrin mediated attachment of actin to CNT surface.

A closer look at the adhesion force and energy of osteoblasts on the three surfaces after 1 day (table 4.4) strengthens the hypothesis that CNTs assist osteoblast adhesion. After 1 day of culture, the adhesion force and energy of an osteoblast is almost similar on permanox and HA surfaces but it shows an increase of $55 \%$ and $73 \%$, respectively, on HA-CNT surface. Although ECM production rate per cell is more on HA than on plastic [133-134], the amount generated in 1 day of incubation might not be sufficient to exert a 
significant difference. Thus, the adhesion of an osteoblast on HA shows just a minimal increase when compared to that on plastic. However, in the case of HA-CNT, the surface is already rich with CNT network, providing readily available locations for integrin attachment. Thus, presence of CNTs on surface results in better adhesion of osteoblasts even at an initial period of growth, which should be extremely beneficial for quicker healing and osseointegration.

This study (i) reveals the positive effect of CNTs on adhesion of osteoblasts on orthopedic implant surface and (ii) proposes and establishes nano-scratch technique for an accurate measurement of adhesion force of a single cell with the underlying substrate. The simplistic and universal nature of this technique makes it suitable for measuring adhesion force for a large variety of cells on any kind of flat surface. Adhesion of osteoblasts is quantified on plasma sprayed HA surfaces with and without CNT reinforcement. An osteoblast shows 73, 95 and 63\% higher adhesion energy on HA-CNT surface than HA for 1, 3 and 5 days of incubation, respectively. In agreement with the adhesion force and energy, the staining of osteoblast cytoskeleton also reveals more intense, prominent and well oriented actin filament network on HA-CNT surface than on HA. These results indicate better attachment and spreading of osteoblasts in the presence of CNTs. The suggested reason for such behavior is affinity of CNTs towards integrins and other cell adhesive proteins that mediate attachment of osteoblasts on the substrate.

Thus the initial part of osseointergration by attachment of osteoblast on HAnanotube surface is established. The next part is to assess the role of nanotube in 
mechanical compatibility of implant-bone interface, which is dealt with in the next section.

\subsubsection{Mechanical Compatibility of Implant-Bone Interface}

Mechanical compatibility in terms of elastic modulus is very important at boneimplant interface. Because, dissimilar elastic modulus of bone and implant would result in different strain at interface due to application of force during limb movement. Dissimilar elongation or strain in the adjacent parts at bone/implant interface could cause localized stress generation, followed by fracture and delamination of the implant from the bone. Elastic modulus evaluation at the implant/coating/bone interface becomes very important, considering the huge difference in elastic modulus of bone ( 20-30 GPa) to that of titanium and HA or HA-CNT ( $\geq 100 \mathrm{GPa})$. But, it is more important to evaluate the gradient of elastic modulus at bone-implant interface after in-vivo exposure of the implant. The elastic modulus of implant surface could get modified during in-vivo exposure through osteoblastic activities and bio-chemical cycle of new bone generation process. This is more expected/evitable for HA and HA-CNT coatings, due to their porous structure what allows tissue and bone in-growth. Considering these possibilities, the elastic modulus gradient across bone-implant interface is evaluated using the retrieved implanted rat femurs from in-vivo studies.

\subsubsection{Gradient of Elastic Modulus Across Bone-Implant Interface}

The section of the bones with the embedded implants (figure 4.49) were obtained from the collaborating research group at University of Strasbourg, France and prepared 
for measuring elastic modulus using nano Dynamic Mechanical Analysis (nanoDMA Module, Hysitron Triboindenter) method. A total of four different bones are used for this study. They are (i) uncoated Ti-alloy implanted bone; (ii) HA coated Ti implanted bone; (iii) HA-CNTcoated Ti implanted bone and (iv) normal bone. The elastic modulus of the newly grown bone was measured and compared with the implant to qualitatively compare strain at the interfaces.

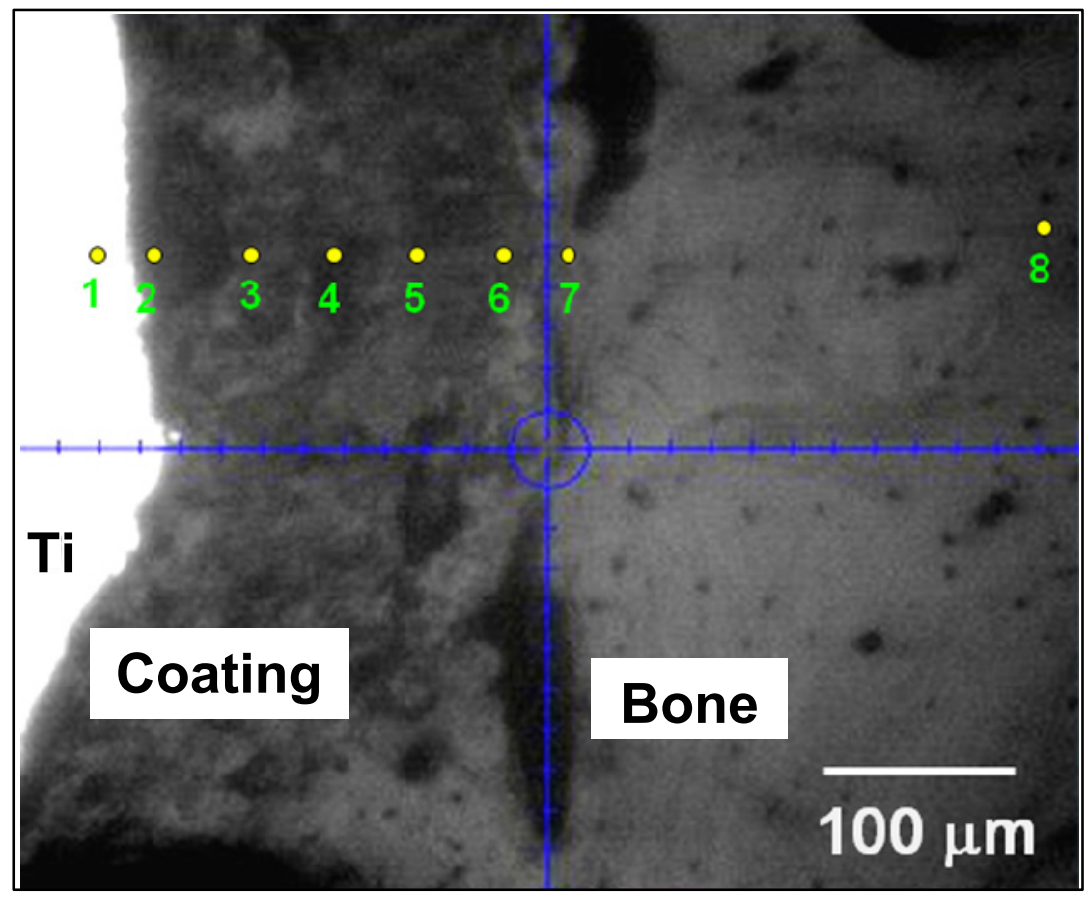

Figure 4.63: Optical cross-sectional micrograph of the rat femur with HA-CNT coated implant, showing the locations of modulus mapping measurements by Arabic numbers. Position 1 denotes the region in titanium part of the implant near coating. Positions $2-6$ are equally placed locations through the thickness of HA/HA-CNT coating starting from titanium side and going towards the bone. Position 7 is in the newly grown bone along the implant surface and position 8 is in a distant region that represents the normal bone.

Figure 4.63 show the locations across the implant/bone interface, where modulus mapping experiments are performed on $\mathrm{HA}$ and HA-CNT coated implants. The 
measurements include one region in the Ti rod near the coating, five equally spaced regions across the coating, one region in the newly generated bone near the coating and one region far from the implant that represents the natural bone. In the case of uncoated Ti implant, measurements are made in three regions viz. in Ti substrate, newly generated bone near $\mathrm{Ti}$ and the natural bone at a distance from the implant. Modulus mapping of cross section of the bone, without any implant is also carried out as the control sample. At least three areas of $5 \mu \mathrm{m}$ X $5 \mu \mathrm{m}$ are used for 2D modulus mapping from each region to get the representative value of the elastic modulus.

Figure 4.64 is a compilation of the modulus maps for all the four samples. The color scale for the elastic modulus has been kept constant between 0-250 GPa for all the images. It enables a clear visualization of the change in elastic modulus across the implant/coating and coating/bone interfaces. Elastic modulus for titanium is similar throughout for all the samples, which indicates no change in Ti properties after in-vivo exposure of one month. But, both HA and HA-CNT coatings show changes in the elastic modulus in the retrieved implant. Elastic modulus of the both HA and HA-CNT coatings is highest near Ti (dark blue in color). Elastic modulus of the coatings reduces gradually through thickness from near Ti to the bone, as observed from the gradual change in the color of modulus in figure 4.64. But uncoated Ti-implant shows a sharp change in modulus (color) at implant/bone interface. The new bone shows lowest modulus for all groups. Similar modulus for normal bones in all four samples indicates no effect of implant material on the mechanical performance of the normal bone. 

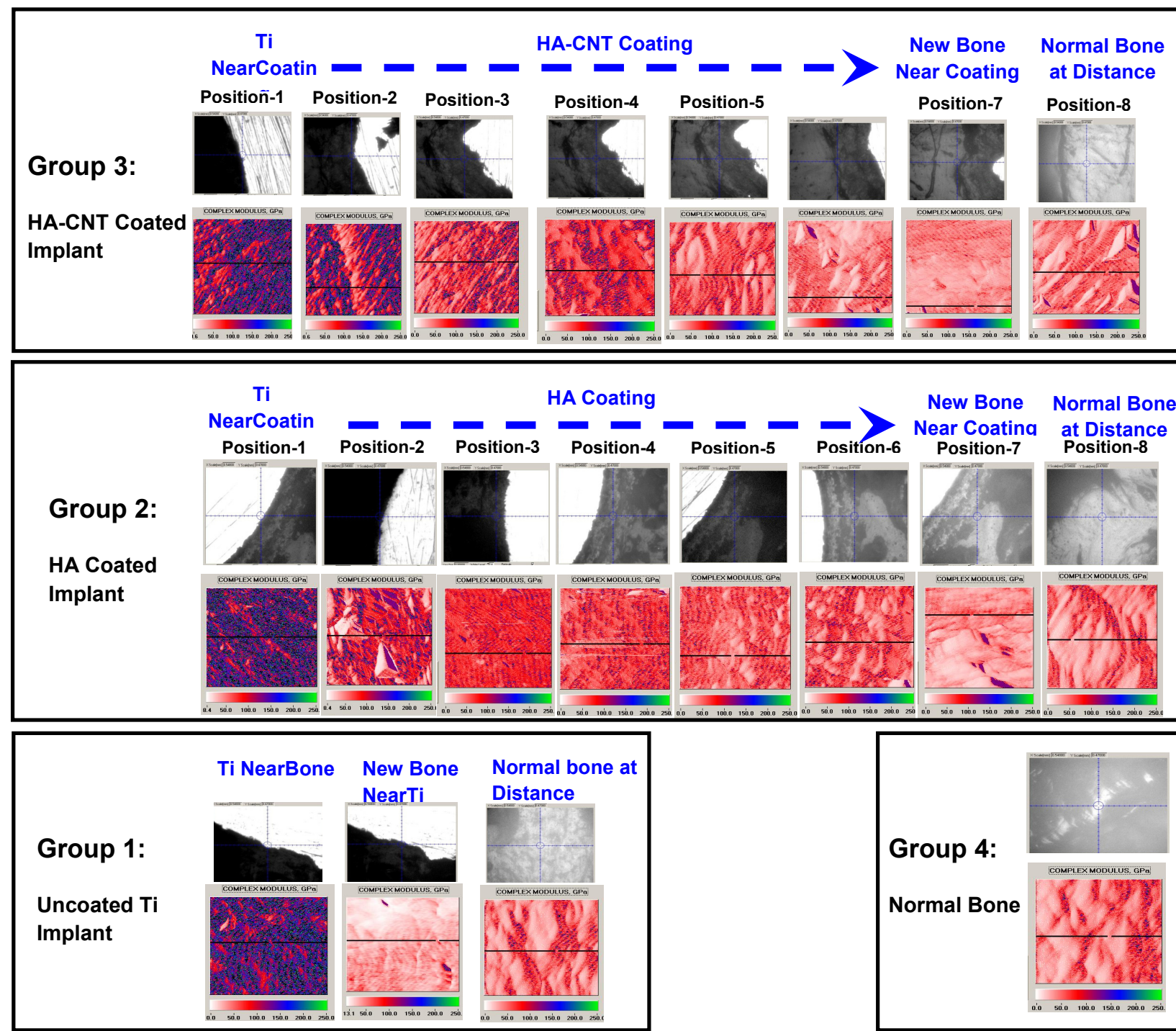

\section{Color Scale in GPa}

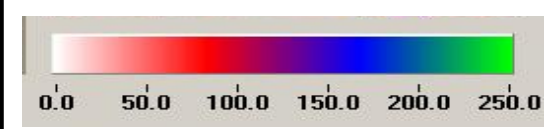

Figure 4.64: Modulus map from different regions in implant/coating/bone interfaces 
(a)

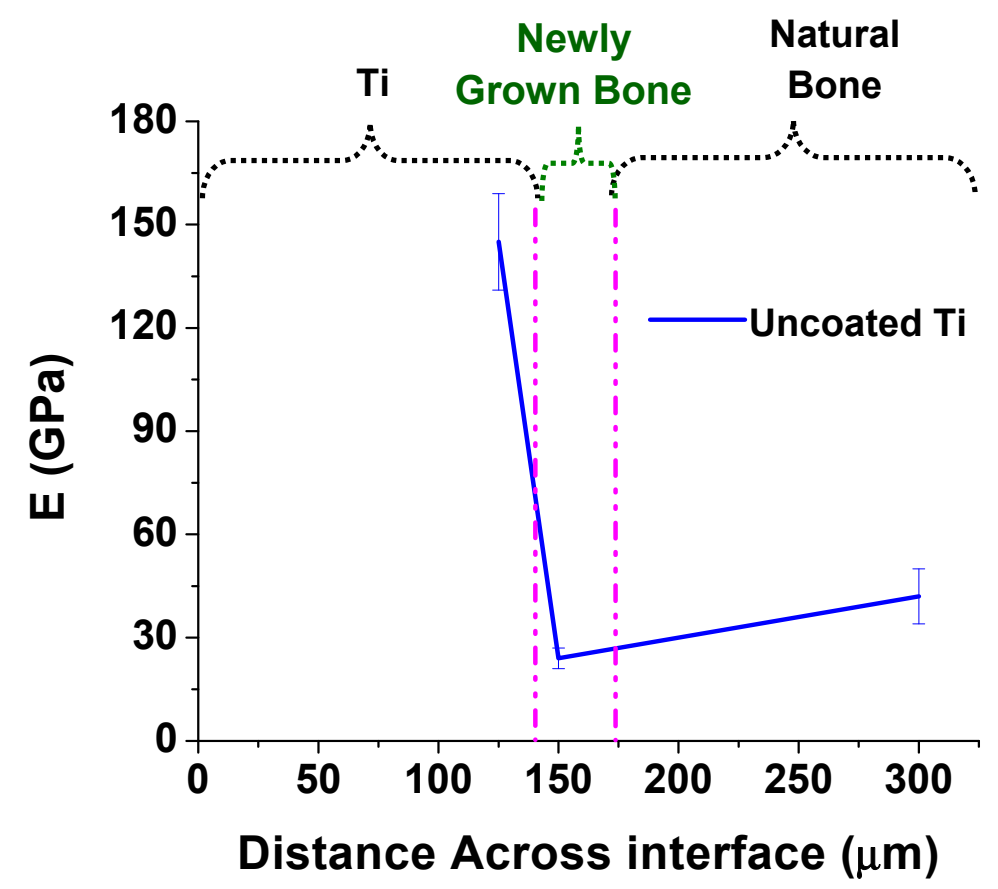

(b)

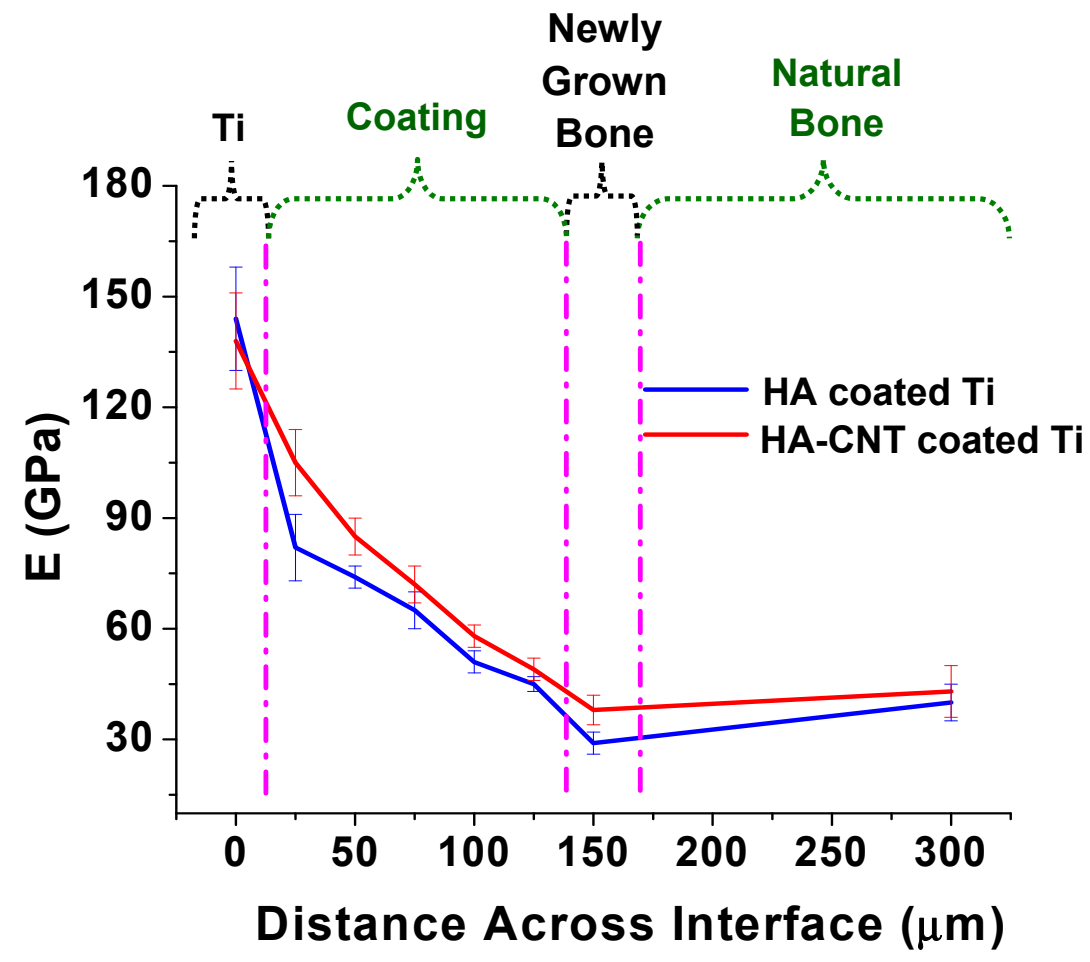

Figure 4.65: Gradient of elastic modulus across bone/implant interface for (a) uncoated Ti implant; (b) HA and HA-CNT coated Ti implants. Elastic modulus values were averaged from the modulus maps presented in figure 4.64. 
Figure 4.65 presents the change in elastic modulus value across the distance over the implant-bone interfaces for all the three types of implants. The elastic modulus values, presented in the plot, are the average of the 3 X 256 X 256 (=199608) modulus values obtained from the three maps performed in each region. The error bar in the plot denotes the standard deviation of elastic modulus measured. Uncoated-Ti implanted bone shows a very sharp change in $\mathrm{E}$ across the bone-implant interface, which possess the risk of, loosening of implant, fracture of bone and delamination from implant surface due to highest strain gradient [152]. Presence of HA-CNT and HA coatings create a smooth gradient of $\mathrm{E}$ across the implant-bone interface, which increases the mechanical compatibility of implant with the bone and reduces the chance of fracture or implant failure. Thus, in addition to bone integration, HA based coatings also play a vital role in maintaining the mechanical health of the implanted bone.

Another novel feature of this study is the elastic modulus measurement of the new bone and its comparison with Ti implant, HA and HA-CNT coatings and distant bone. Such study enables an indirect prediction of the stress and strains at the implant/bone interface which is critical for the mechanical integrity of the implant. Elastic modulus of Ti, HA-coating and HA-CNT coating before implanting was measured to be $109 \pm 3$ $\mathrm{GPa}, 90 \pm 10 \mathrm{GPa}$ and $115 \pm 9 \mathrm{GPa}$, respectively. These coatings were soaked in formalin for 18 days and elastic modulus was measured again in the soaked condition to evaluate if there is any effect of formalin soaking on the modulus of coatings. However, the elastic modulus values of the coatings remain similar before and after formalin soaking, indicating the change in retrieved implants is due to the in-vivo exposure only and not due to their preservation in formalin. 
Figures 4.64 and 4.65 show a decreasing gradient in the elastic modulus of both HA and HA-CNT coatings, which is attributed to the tissue in-growth at coating/bone interface during new bone formation. During the bone formation process, osteoblasts initially play an active role in the collagen matrix formation on the implant surface. HA based coatings have porosity in its plasma sprayed microstructure and also due to possible resorption. Due to the porous nature of the coatings, the collagen fibers get impregnated in the coatings [153], making the integration of bone more effective and at the same time modifying the modulus of the coating. Presence of HA in the coating, that posses similar chemistry of the mineral content of bone, further helps in bone formation and integration on coated implant surface.

\subsubsection{Analyzing Role of CNT in Mechanical Health of Implanted Bone}

Figure 4.65 reveals the effect of CNT addition on the elastic modulus of HA coating. Elastic modulus of the coating remains higher throughout for HA-CNT than HA, which is clearly due to the reinforcing effect of CNT even after in vivo exposure. The newly grown bone near implant surface shows lowest modulus in the case of uncoated Tiimplant. Presence of HA on implant surface helps in better mineralization of the bone and as a result higher modulus than the bone near bare Ti surface. Further, elastic modulus of the bone near HA-CNT surface shows higher value than the bone at HA surface. This observation could be explained by the favorable role of CNT in osteoblast proliferation and differentiation reported in this study earlier and also by other publications $[3,108]$. Osteoblasts are found showing better proliferation and viability in the presence of CNT in the culture surface. Preferential absorption of favorable proteins on CNT surface is found 
responsible for such behavior [85-86]. Implanting raw CNT in mouse skull is also found accelerating in-vivo bone growth [106]. In addition, the presence of CNT is reported inhibiting the osteoclast proliferation, which destructs the bone structure [105]. Thus, presence of CNT also fights out the negative factor of bone growth. As a result, the bone growth near HA-CNT interface becomes more active, causing higher elastic modulus than the bone near HA interface, which is a very impressive and attractive finding to be reported for the first time. Elastic modulus for the normal bone in all the cases, including the bone that was not implanted, shows similar value, indicating no adverse effect of CNT addition on mechanical health of the bone.

This study on mechanical compatibility of HA-CNT coating-bone interface along with the previous findings on in-vivo and in-vitro studies provides a positive picture of its biocompatibility and suitability for orthopedic application. Another important factor of osseointegration is the apatite formation on bioimplant surface. HA possess the same chemical composition as apatite in bone and thus, do not pose any heterogeneity for apatite precipitation and integration. Same is not true for CNT and BNNT, which demands an evaluation of apatite formability on CNT and BNNT to ensure better osseointegration on HA-nanotube composite surface. Apatite formability of CNT surface is well established in the literature $[107,109,154]$ and has been discussed in literature review (Chapter 2) (section 2.1.4.1). Hence, evaluation of apatite formability on CNT surface is not repeated in this dissertation. But, BNNT is proposed for the first time for orthopedic application. Since no study exists on apatite formability of BNNT surface, the same is presented in the following section. 


\subsubsection{Apatite Formation on Boron Nitride Nanotube in Simulated Body Fluid}

An important requirement for a successful orthopedic implant is the formation of new bone on its surface for effective osseointegration. An ideal implant surface should induce apatite formation without interfacial discontinuity. These discontinuities often act as weak points to cause fracture and delamination of the implant from the bone in-vivo. Hence, the apatite formation ability on the BNNT surface is critical for orthopedic application of HA-BNNT composite. The apatite forming ability on BNNT surface is evaluated by immersing it in simulated body fluid (SBF).

\subsubsection{Morphology of Apatite Precipitate on BNNT}

SEM micrographs in figure 4.66(a-d) present the image of as-received BNNTs and apatite precipitated BNNTs after 7,14 and 28 days of soaking in SBF. Figure 4.66a shows a tubular BNNT structure with clean outer surface. Figure 4.66b reveals the onset of HA precipitation on BNNT in forms of few flakes. After 14 days of soaking (figure 4.66c), the precipitates increase in volume and starts becoming thicker, covering the BNNTs. After 28 days, apatite crystals are fully grown with their typical needle shapes in spherical agglomerate that surrounds the BNNTs (figure 4.66d). These visual observations reveal the gradual increase in amount and morphology of apatite precipitate on BNNT with an increasing soaking period. EDS analysis has been carried out for the determination of elemental composition and quantification of volume of precipitated phase. 

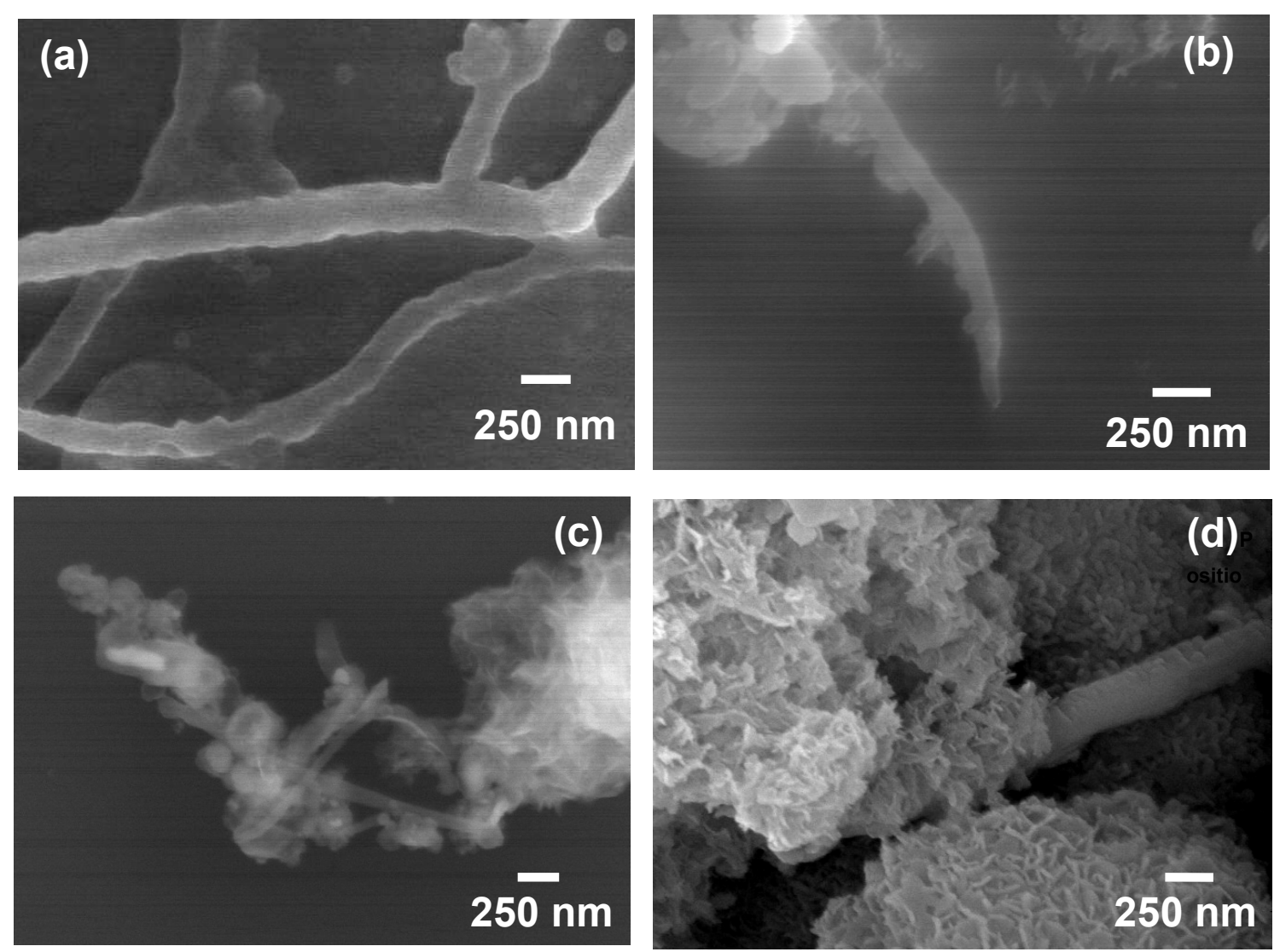

Figure 4.66: SEM images of (a) as-received BNNTs and apatite precipitation on BNNTs after (b) 7; (c) 14; (d) 28 days of soaking in SBF.

\subsubsection{Compositional Analysis and Comparative Volume Determination of Apatite \\ Precipitate}

Figure $4.67(\mathrm{a}-\mathrm{c})$ present representative elemental signature plots for three apatite samples collected after 7, 14 and 28 days of soaking. Boron and nitrogen peaks are observed from BNNTs, whereas the $\mathrm{Ca}$ and $\mathrm{P}$ peaks are generated from HA precipitate. Source of $\mathrm{Si}$ and $\mathrm{Au}$ peaks are from the silicon substrate and the gold coating, respectively, used for EDS. The presence of $\mathrm{Ca}$ and $\mathrm{P}$ peaks in EDS spectrum of the SBF soaked BNNTs reveals the presence of calcium and phosphate containing compound in the precipitate. Absence of any other major elemental peak in the spectrum indicates that 
mainly calcium and phosphorous containing constituents are mineralized from SBF on the BNNT surface. The 28 days precipitate shows a smaller peak of sodium, denoting the precipitation of a small amount of $\mathrm{Na}$ containing salt (figure 4.67c). The 7 days precipitate shows a very strong $\mathrm{B}$ and $\mathrm{N}$ peak, while $\mathrm{Ca}$ and $\mathrm{P}$ peaks are of very low intensity (figure 4.67a). The intensity of $\mathrm{Ca}$ and $\mathrm{P}$ peaks increases as compared to $\mathrm{B}$ and $\mathrm{N}$ peaks after 14 days as observed in figure 4.67b. Precipitates collected after 28 days produce much sharper $\mathrm{Ca}$ and $\mathrm{P}$ peaks of higher intensity than $\mathrm{B}$ and $\mathrm{N}$ peaks (figure 4.67c). The relative height (intensity) of the peaks reveals increasing $\mathrm{Ca}$ and $\mathrm{P}$ content in the precipitate with number of days, as seen in figure 4.67(a-c).
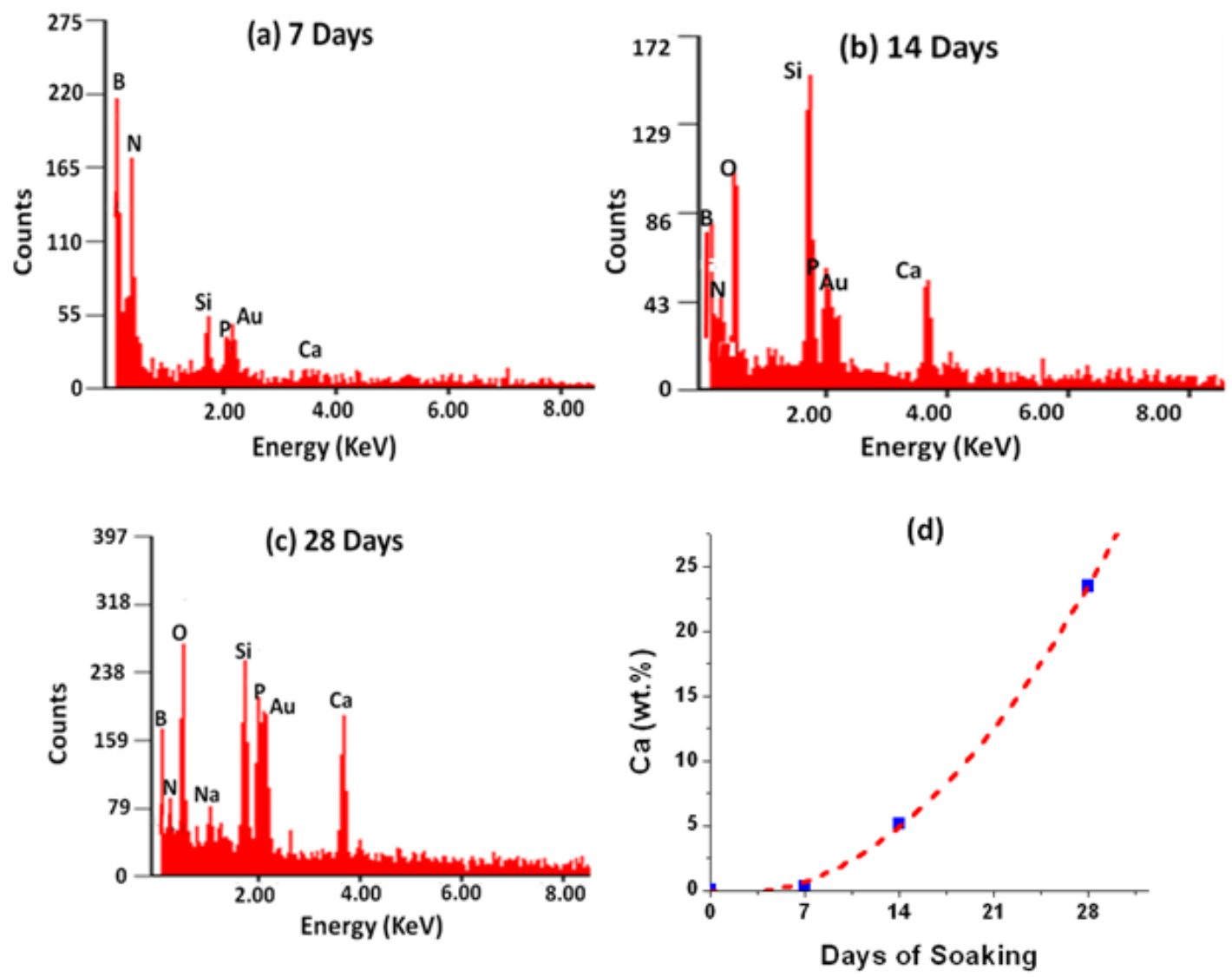

Figure 4.67: EDS spectra of SBF soaked BNNTs for (a) 7; (b) 14 and (c) 28 days. (d) Increasing Ca wt.\% in precipitate as a function of days of soaking. 
The quantification of elemental constituents, in terms of percentage weight, has been carried out for each sample at five different places with a $1 \mu \mathrm{m}$ X $1 \mu \mathrm{m}$ scan area in each measurement. Calcium content, in terms of percentage weight, is measured for all the three samples to estimate the change in amount of HA precipitate on BNNT as a function of soaking period. Figure $4.67 \mathrm{~d}$ presents the wt. $\% \mathrm{Ca}$ in precipitate collected after 7, 14 and 28 days. The error bar on each data is based on five measurements at different areas in each sample. The smaller deviation in the $\mathrm{Ca}$ content denotes the consistency of the data. The value fit a third degree polynomial with the following equation and $R^{2}=0.99952$,

$$
y=0.03483 x^{2}-0.13051 x-0.12082
$$

where, $y$ is $\mathrm{Ca}$ wt.\% and $x$ is the number of soaking days. This semi-quantitative evaluation reveals an increase in HA formation on BNNT between $7-28$ days. Being extrapolated towards lesser soaking period, the fitted curve indicates no HA formation at $\sim 4.6$ days - which might be the incubation period of HA precipitation on BNNT. Akasaka et al. have reported an incubation period of 14 days for HA precipitation on CNT surface when immersed in standard SBF [109]. Nucleation occurs only after the activation energy barrier is exceeded. It takes $\sim 4.6$ days to overcome the energy barrier to nucleate apatite on BNNT surface when immersed in standard SBF. Incubation time for apatite precipitation on CNT is found decreasing with increasing ion concentration in a supersaturated SBF [109]. Functionalized CNT surfaces with carboxylic groups are also reported to accelerate apatite formation [154]. It should be noted that the present study 
have used BNNTs without functionalization. Functionalization of BNNT may accelerate apatite formation due to attraction of favorable ions form SBF.

Micro Raman Spectroscopy analysis is carried out on the as-received BNNTs and BNNTs soaked in SBF for 7, 14 and 28 days. Figure 4.68 presents the Raman spectra for as-received BNNT and three precipitates showing peaks representative of the bonds present in BNNT and HA.

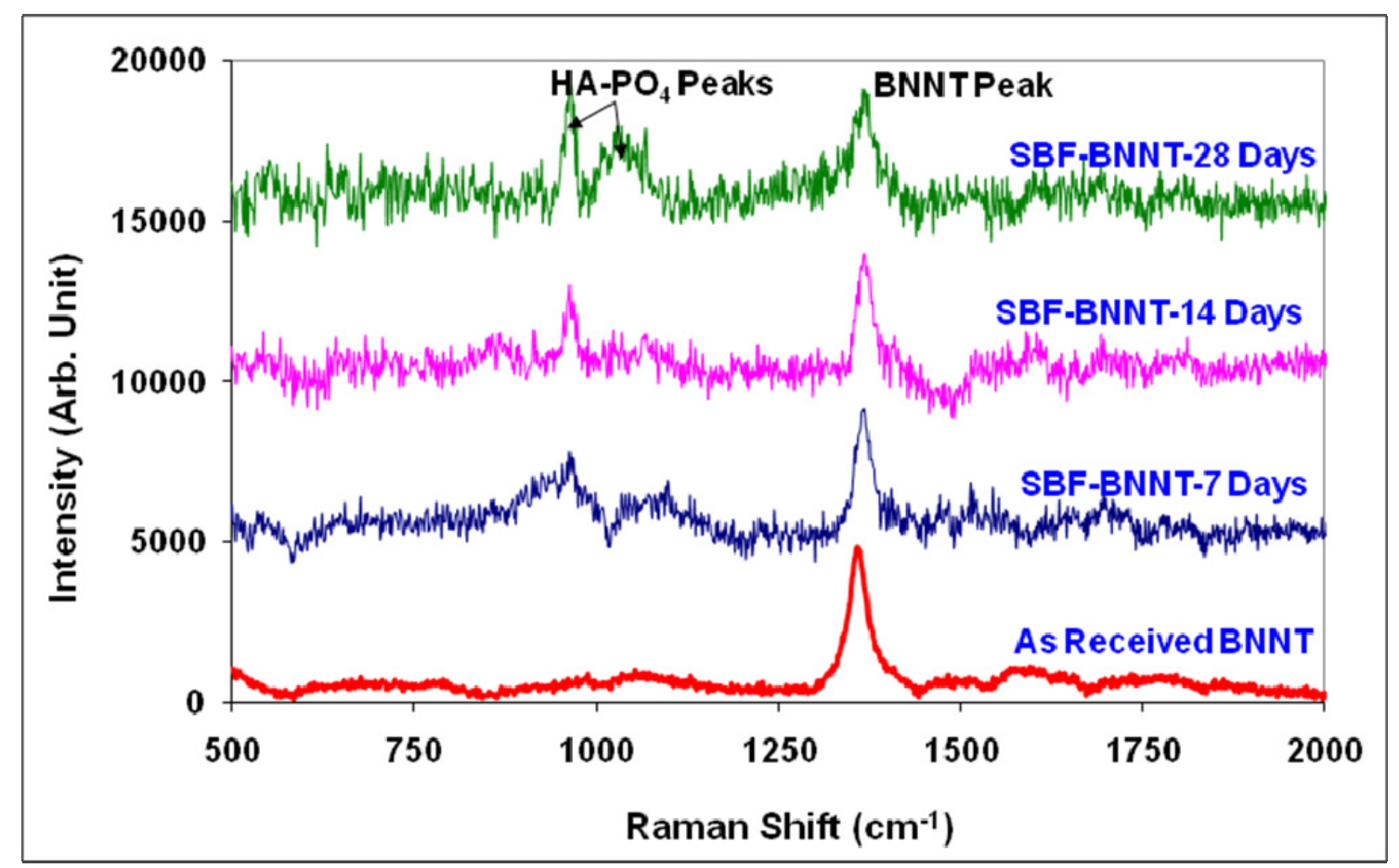

Figure 4.68: Raman spectra of as-received BNNTs and BNNTs soaked in SBF showing h-BN peaks of BNNT and phosphate peaks of $H A$.

The peak at $\sim 1367 \mathrm{~cm}^{-1}$ is an $\mathrm{E}_{2 \mathrm{~g}}$ mode peak from h-BN [63-65]. The presence of HA in precipitates is noted by the twin peaks at lower wave number. The peaks at $\sim 960$ $\mathrm{cm}^{-1}$ is due to $v_{1}$ symmetric stretching vibration of phosphate ion in HA [27]. The relatively broader peak at $\sim 1052-1081 \mathrm{~cm}^{-1}$, which is more prominent in 28 days sample, is $v_{3}$ antisymmetric stretching mode of phosphate ion in HA [27]. The peaks 
corresponding to HA-phosphate bonding are clearly absent in as-received BNNT. Elemental analysis by EDS has proven the presence of $\mathrm{Ca}$ and $\mathrm{P}$ in the precipitates. The signature peaks of bond energy in compound, as observed in Raman spectrum, present the evidence of $\mathrm{Ca}$ and $\mathrm{P}$ forming $\mathrm{HA}$ in the precipitate.

Raman spectra from precipitates also show a relative change in the intensity of $\mathrm{h}$ BN and HA-phosphate peaks in figure 4.68. BNNTs soaked in SBF for 7 days show weak and lesser intensity peak of HA-phosphate group compared to the h-BN peak. But, the relative intensity of the phosphate peak increases after 14 days of soaking. The 28 days sample shows sharp phosphate peaks with intensity similar to h-BN peaks. The intensity of a Raman peak is proportional to the volume concentration of the respective bond, when the spectra are collected at similar spectroscopic conditions [155]. Penel et al. have also reported higher intensity of $v_{1}$ symmetric phosphate peak with increasing apatite content [155]. Raman spectra in the present study are captured under similar settings. Hence, the relative increase of HA-phosphate peak intensity with respect to h$\mathrm{BN}$ peak indicates increasing HA content in the precipitation with number of days. This observation supports the trend of increased HA precipitation on BNNT with soaking period as observed from SEM images and EDS analysis.

The BNNT peak shows a shift from $1357 \mathrm{~cm}^{-1}$ to $1363 \mathrm{~cm}-1$ from as-received to 7 days SBF soaked sample. But, no shift in the same peak is observed for BNNTs soaked for 14 and 28 days. The peak remains in almost constant position $\left(1363-1364 \mathrm{~cm}^{-1}\right)$. The initial shift in BNNT peak could be due to ageing of BNNT in SBF, which do not continue for higher soaking time. Detailed investigation is required to confirm about the initial ageing reaction of BNNT with apatite or SBF. 


\subsubsection{Nucleation and Growth Mechanism of Apatite Precipitate}

HRTEM images of the precipitates provide insight into the nucleation and growth mechanism of HA crystals on BNNT surface. Figure 4.69 shows the TEM images of asreceived BNNT and HA precipitate on nanotube surface after 7, 14 and 28 days of soaking. All BNNTs are bamboo shaped.
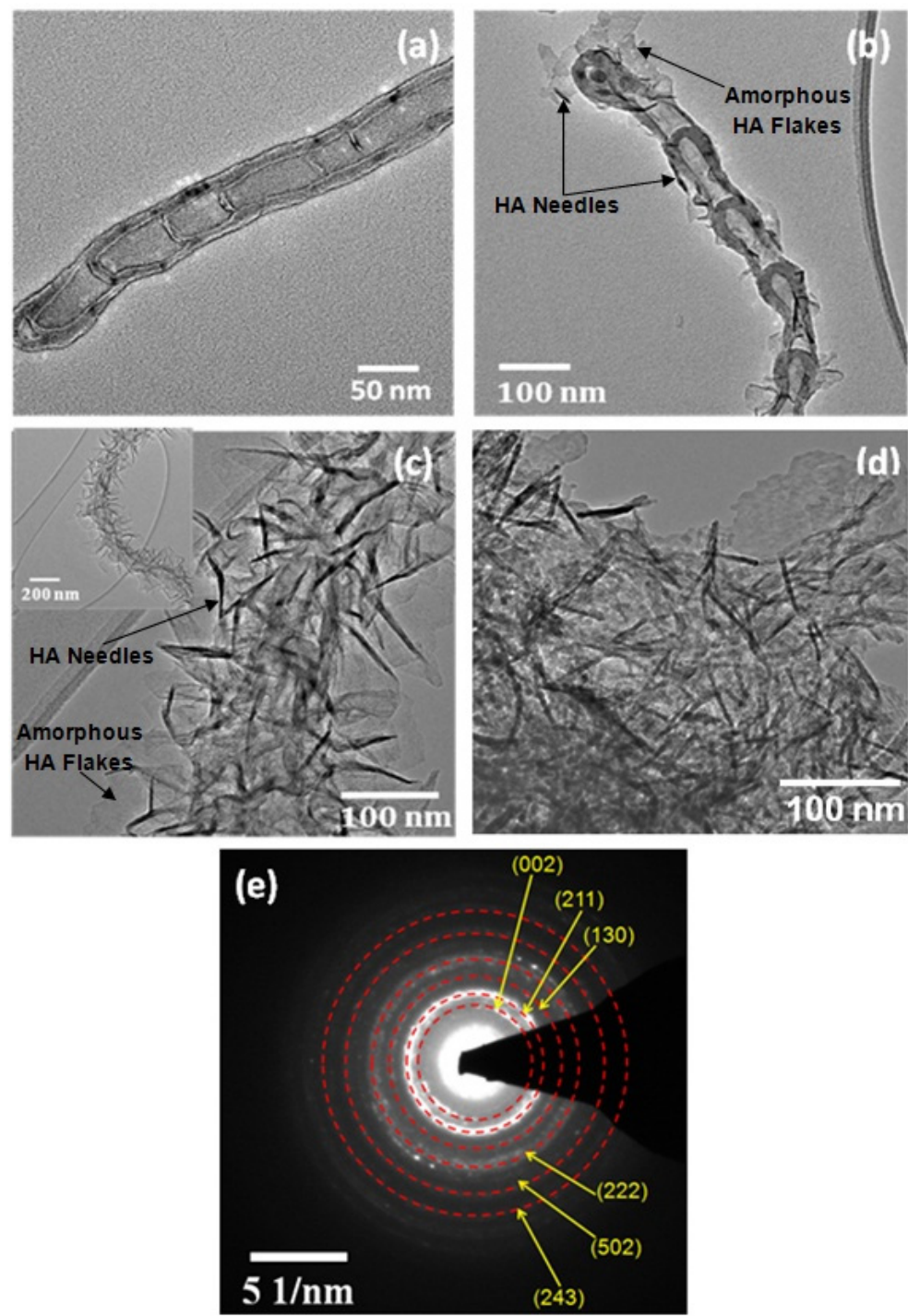

Figure 4.69: TEM image of BNNTs in (a) as-received condition; soaked in SBF for (b) 7;

(c) 14 days and (d) 28 days. (e) Selected Area Diffraction (SAD) pattern of 28 days sample in figure ' $d$ ' showing the presence of crystalline $H A$. 
As-received BNNT, in figure 4.69a, shows a clean outer surface. After 7 days of soaking, amorphous HA flakes precipitate on the BNNT surface along with the nucleation and growth of some tiny HA crystals of needle shape (figure 4.69b). After 14 days of soaking, the HA needles grow significantly in length (figure 4.69c). Amorphous HA flakes are still visible, but a prominent increase in the number of crystalline needles is observed. BNNT surface was fully covered with HA needles after 28 days as observed in figure $4.69 \mathrm{~d}$. Figure $4.69 \mathrm{e}$ presents the selected area diffraction (SAD) pattern of 28 days precipitate corresponding to figure $4.69 \mathrm{~d}$. The bright circular ring in the SAD pattern corresponds to the (211) plane of HA, which produces highest intensity diffraction [JCPDS PDF No. 9-432].

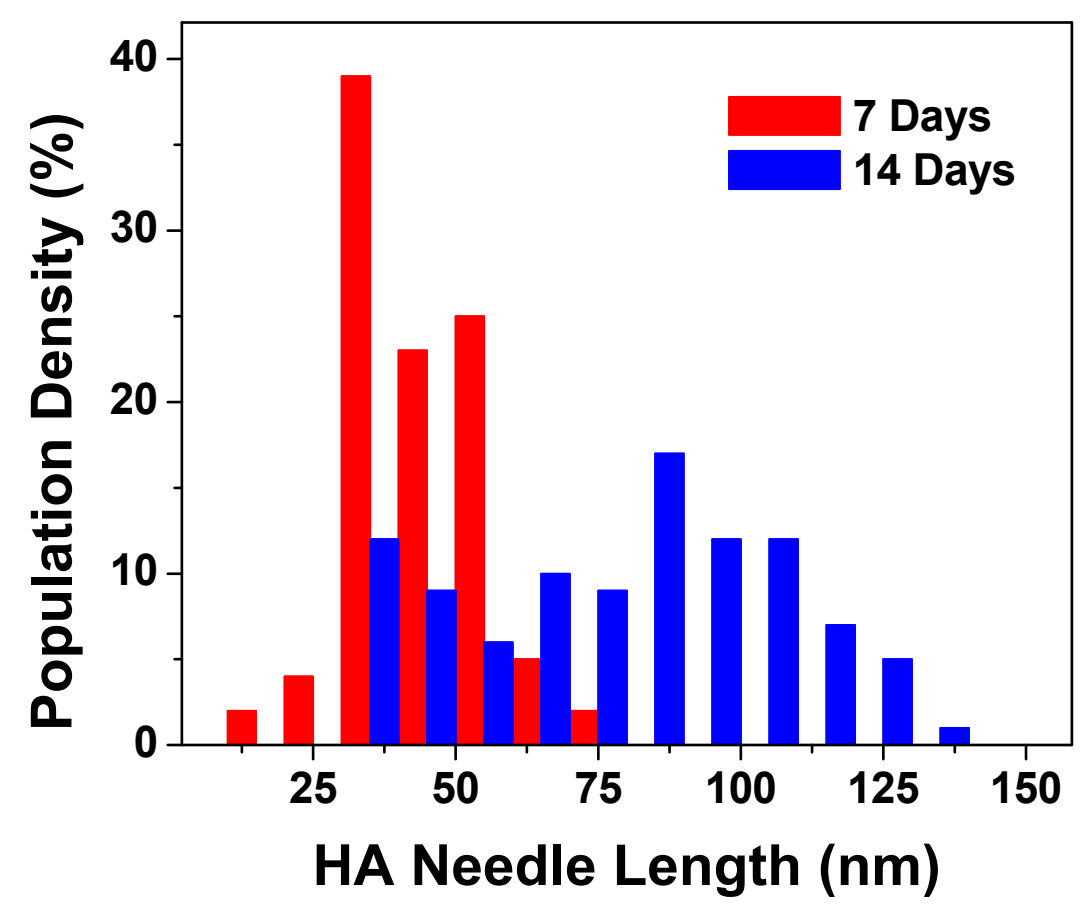

Figure 4.70: Distribution of HA needle length in 7 and 14 days samples.

The bar chart in figure 4.70 represent the needle length distribution in 7 and 14 days precipitates, as measured from several TEM images. A total of 100 needles were 
considered for each case. Due to the dense needle structure of HA precipitate after 28 days, it was not possible to calculate the needle length. Majority of the HA crystalline needles have a length of 30-55 nm after 7 days of soaking. But, the HA needles in 14 days precipitate show a much wider length range of $30-125 \mathrm{~nm}$. This large variation in the length is the result of growth of the needles that have nucleated early and the continuous nucleation of new needles throughout the soaking period. Figure 4.71(a-c) presents higher magnification TEM micrographs of HA precipitates for 7, 14 and 28 days respectively, which also indicate the change in population of HA crystalline needles.
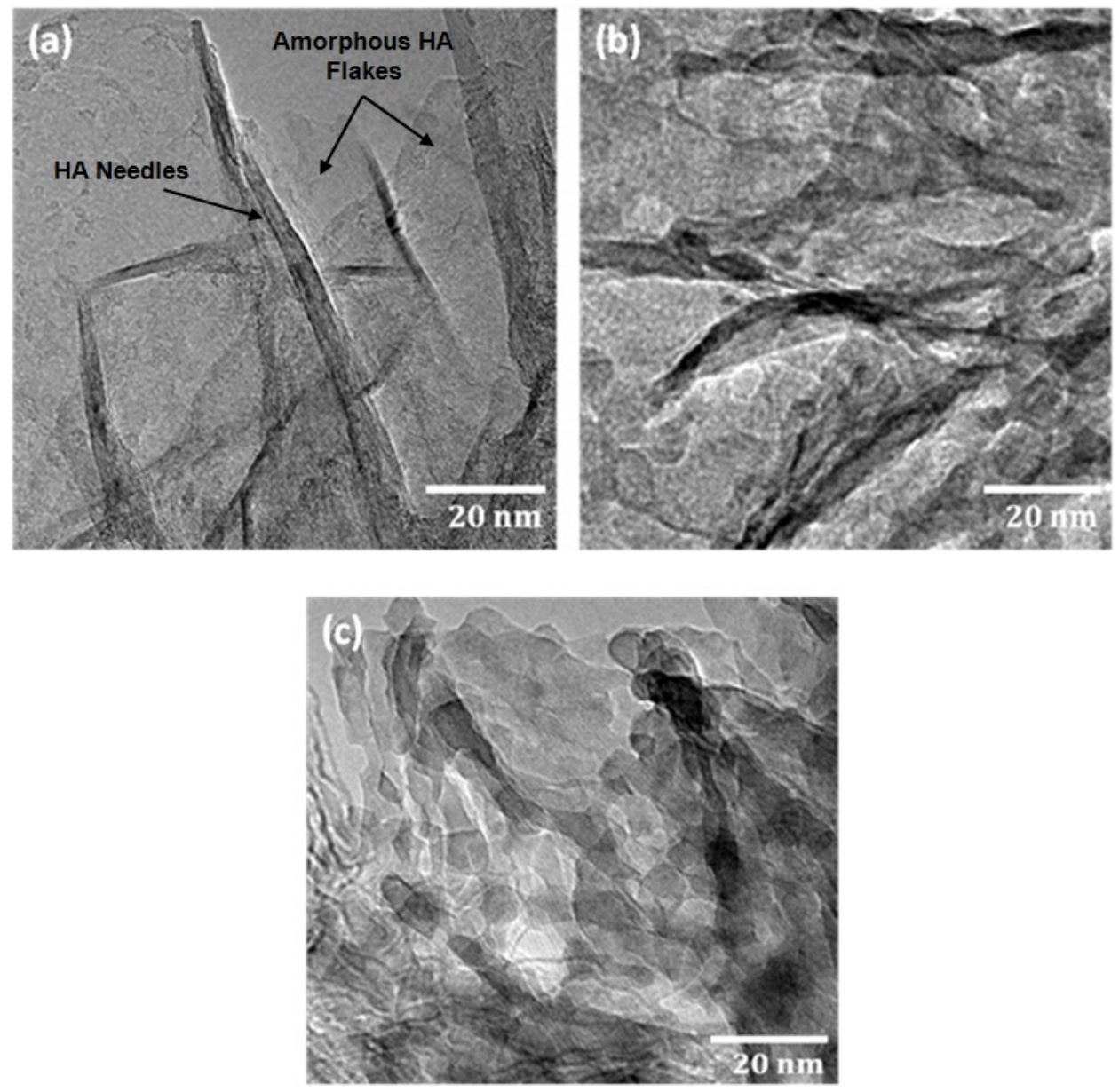

Figure 4.71: TEM images of precipitates showing HA needles and amorphous HA for (a) 7; (b) 14 and (c) 28 days. 
Figure 4.71a shows the 7 days precipitate with amorphous, flaky HA and a few whisker shaped crystalline needles. The number of needles increases after 14 days, though some amount of amorphous phase still exists (figure 4.71b). The precipitates after 28 days, in figure $4.71 \mathrm{c}$, is full of crystalline HA needles. Apart from the needle structure, some equiaxed HA crystal shapes are also observed in 28 days sample. This observation is in agreement with Aryal et al. about formation of diffused HA cubes in CNT matrix after 14 days of reaction with SBF [107].

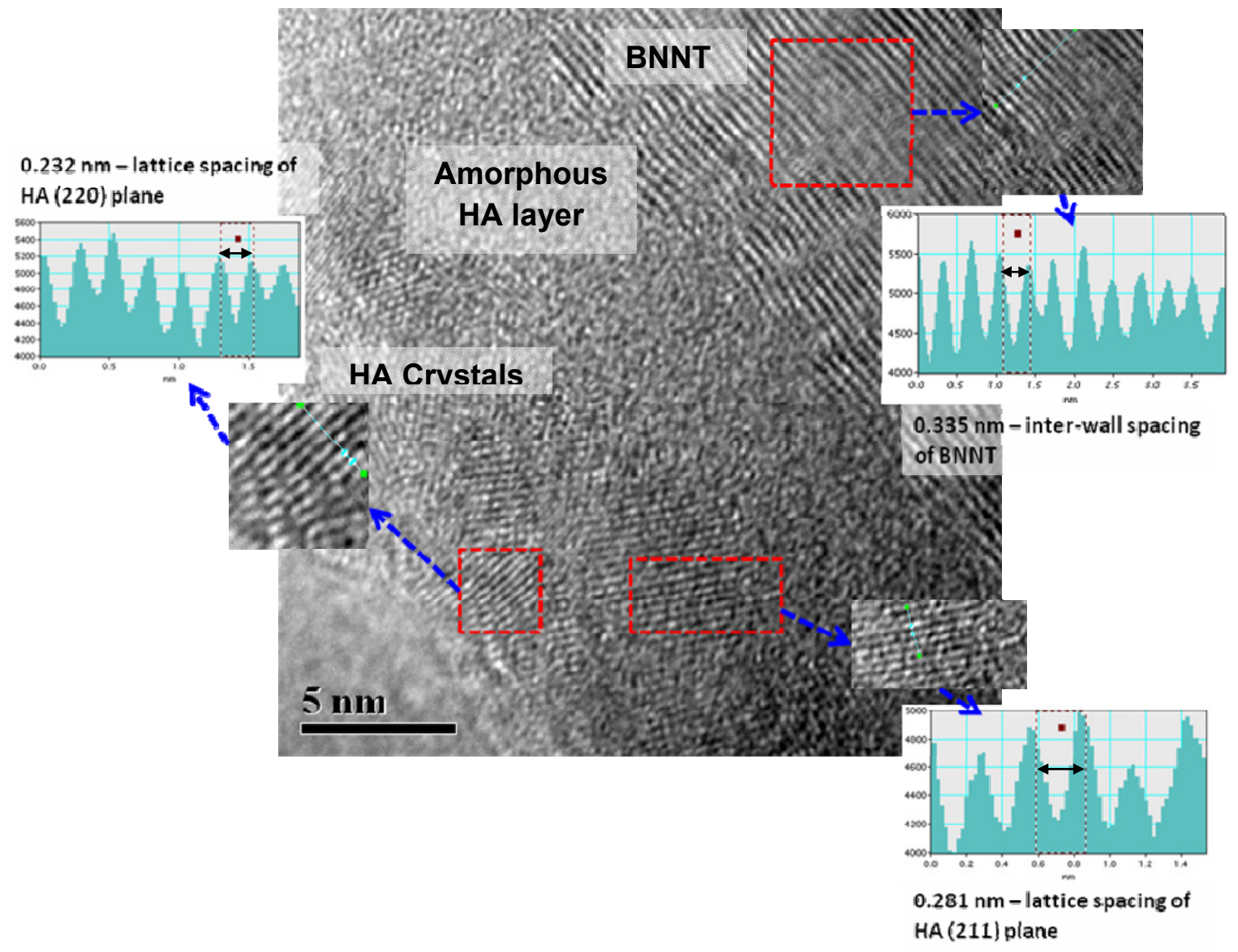

Figure 4.72: HRTEM image of HA precipitate on BNNT surface after 28 days of soaking in $S B F$, revealing both amorphous and crystalline nature of the precipitate.

Figure 4.72 presents high resolution TEM lattice images of a BNNT surface with apatite precipitate on it after 28 days of soaking. Fast Fourier Transformation (FFT) 
analysis is used to accurately determine the interplanar spacing. BNNT is identified by the inter-wall spacing of $0.335 \mathrm{~nm}$. The spacing of h-BN layers in BNNT is $0.33 \mathrm{~nm}$ [66]. HA crystallites of different orientation are found on the BNNT surface. HA crystallites are identified by their lattice spacing of different set of planes (JCPDS PDF No. 9-432). Two such crystallites of HA are marked in figure 4.72, which reveal lattice spacing of (220) and (211) sets of planes. Apart from these crystallites, amorphous HA phase also exists next to the BNNT surface.

Figure 4.73 presents TEM images of few HA needles on the BNNT surface. HA needles are found growing parallel to BNNT surface as well as at different angles. Based on the observations from figures 4.72 and 4.73 , it can be concluded that there is no preferred crystallographic orientation for HA nucleation on BNNT surface. In our investigation on spark plasma sintered HA-BNNT composite, a preferred orientation between HA and BNNT is observed, where (211) planes of HA were arranged at an angle of $65^{\circ}-68^{\circ}$ to the outer wall of BNNT (section 4.1.2.4). This contradictory behavior could be attributed to the mechanism of apatite formation from liquid SBF on BNNT surface as compared to the HA-BNNT integration in solid state through SPS. In the case of SPS, the HA crystallites orient themselves on BNNT wall to generate minimal lattice strain at the interface, thus resulting in a specific orientation relationship. But, in SBF, a thin layer of amorphous HA covers the BNNT surface before the nucleation of HA crystals (figure 4.72). This amorphous layer obstructs the direct contact between BNNT and precipitating HA, and thus disturbs the orientation relationship. The origin of initial amorphous apatite layer precipitation could be related to several possibilities. Crystallinity difference between HA and BNNT could favor amorphous apatite 
precipitation on BNNT surface initially. The kinetics of nucleation could also impact the precipitation. The initial deposition of HA may occur in the amorphous form due to higher concentration of ions in SBF. Once the concentration of ion decreases in SBF, it is possible to get more time for nucleation and growth of crystalline needles. The thin amorphous reaction product layer at the interface could also be due to some physicochemical ageing reaction. A more detailed and thorough investigation focusing on the interface is required in future to fully understand the nucleation mechanism.
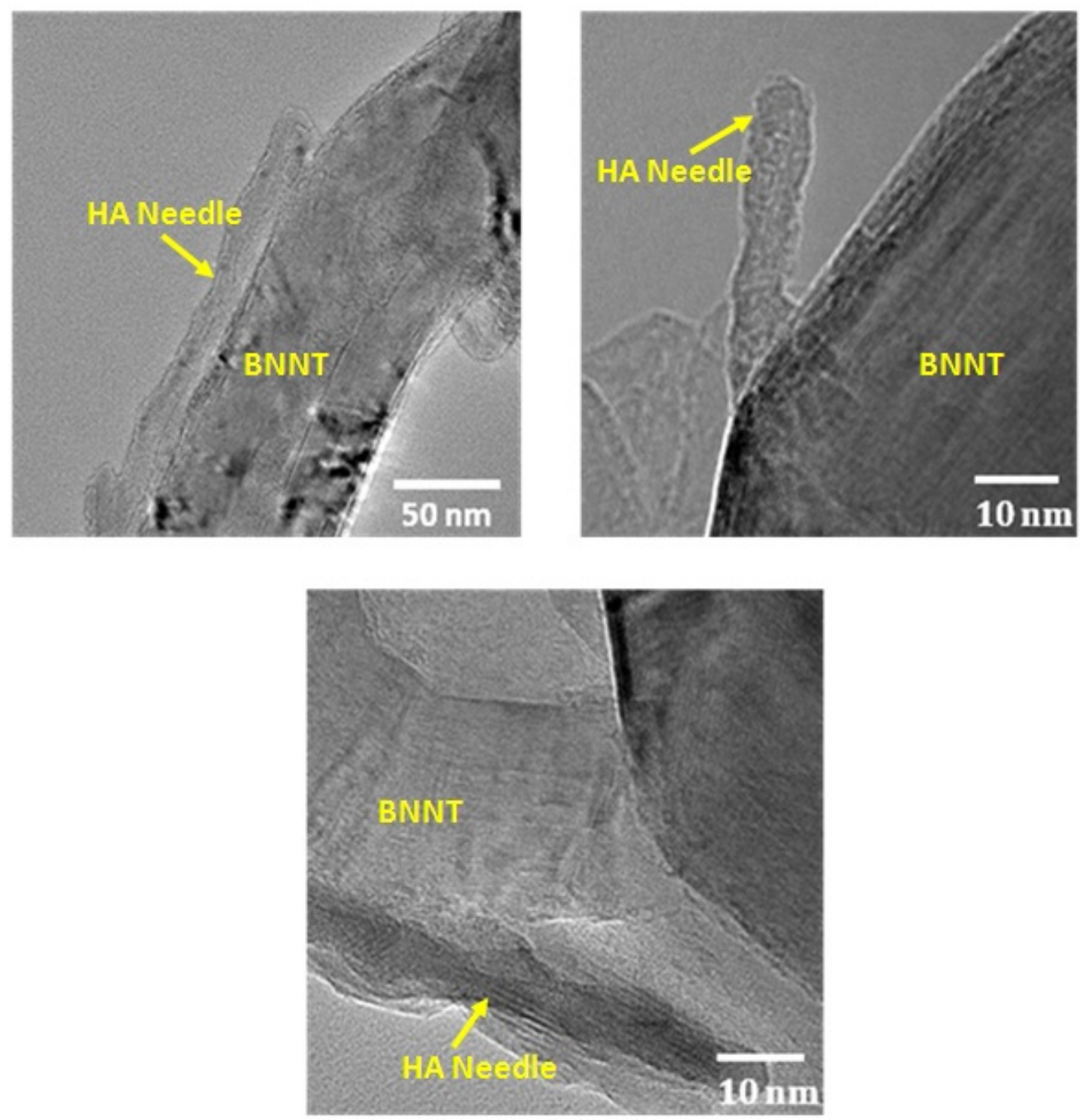

Figure 4.73: HA needles nucleated and grown on BNNT parallel to surface as well as making different angles. 
Investigation on hydroxyapatite formation ability of BNNT surface, soaked in simulated body fluid, reveals that the amount of HA precipitate increases with the soaking period following a polynomial relationship between 7-28 days. HA formation on BNNT requires $\sim 4.6$ days of incubation period. EDS analysis of the precipitate confirms the elemental composition of the precipitate, whereas Raman spectra and HRTEM images proves the presence of HA on the BNNT surface. Amorphous HA flakes with a few crystalline needles are formed on the BNNT surface after 7 days of soaking. Further soaking results in formation of more crystalline HA needles as well as growth of the existing ones. After 28 days of soaking, BNNT surfaces was covered with numerous HA crystallites. HA crystals do not show a definite crystallographic orientation relationship with boron nitride nanotube. In summary, BNNT surfaces induce apatite formation in the physiological solution, which increases their potential towards orthopedic application.

In this dissertation, we have used two different nanotubes as reinforcement to HA, viz. (i) $\mathrm{CNT}$ and (ii) BNNT. Thus it becomes important to compare the outcomes to understand the suitability of each nanotube for the proposed application. The following section presents a comparative analysis on the role played by CNT and BNNT as reinforcement to HA for intended orthopedic application. 


\subsection{Comparison of CNT vs. BNNT: As Reinforcement to HA}

This dissertation has proposed CNT as reinforcement to HA for orthopedic application. Due to the concerns related to cytotoxicity of CNT, BNNT has been proposed as an alternative. The research plan thus includes processing of both HA-CNT and HA-BNNT composites, their characterization for mechanical and tribological behavior and evaluation of biocompatibility. However, due to unavailability of BNNT in large amount, it has not been possible to conduct each type of processing and characterization for HA-BNNT as carried out for HA-CNT. Considering this limitation, the aim of this dissertation has been to present a complete evaluation of HA-BNNT composite in terms of -

(i) feasibility of composite synthesis;

(ii) role of BNNT in toughening of HA;

(iii) tribological behavior of HA-BNNT composite

(iv) cytotoxicity of BNNT to orthopedic specific cell lineages

(v) in-vitro biocompatibility of composite surface to bone forming cells

The outcomes of study on HA-BNNT will become more meaningful when compared with the potential shown by HA-CNT composite in similar aspects. Thus, a comparison between spark plasma sintered HA-CNT and HA-BNNT composites is presented in table 4.5 , highlighting the pros and cons of both in light of their intended orthopedic application. 
Table 4.5: Comparison of spark plasma sintered HA-CNT and HA-BNNT composites

\begin{tabular}{|c|c|c|c|}
\hline Criterion of Comparison & HA- 4 wt. $\%$ CNT & HA- 4 wt. $\%$ BNNT & Remark \\
\hline \multicolumn{4}{|c|}{ Synthesis of Composite } \\
\hline $\begin{array}{l}\text { Retention of nanotube } \\
\text { Structure in SPS }\end{array}$ & $\begin{array}{l}\text { CNT structure gets } \\
\text { disturbed due to high } \\
\text { pressure application in SPS }\end{array}$ & $\begin{array}{l}\text { BNNT structure remains } \\
\text { intact during SPS }\end{array}$ & $\begin{array}{l}\text { BNNT can withstand high strain } \\
\text { without getting permanently } \\
\text { deformed and has higher fracture } \\
\text { strain than CNT }[62,156]\end{array}$ \\
\hline Density of composite & $96.5 \% \mathrm{TD}$ & $97 \% \mathrm{TD}$ & $\begin{array}{l}\text { Both composite show similar } \\
\text { densification }\end{array}$ \\
\hline \multicolumn{4}{|c|}{ Mechanical Property Enhancement } \\
\hline Elastic Modulus & $\begin{array}{l}\frac{25 \% \text { improvement }}{\text { elastic modulus with }} \text { in } \\
\text { wt. } \% \text { CNT addition }\end{array}$ & $\begin{array}{l}\frac{120 \% \text { improvement }}{\text { elastic modulus with }} 4 \\
\text { wt. } \% \text { BNNT addition }\end{array}$ & $\begin{array}{l}\text { CNT gets damaged during SPS - } \\
\text { BNNT remains intact }- \text { thus } \\
\text { enhancement in E for HA-BNNT } \\
\text { becomes more effective }\end{array}$ \\
\hline Fracture Toughness & $\begin{array}{l}\frac{92 \% \text { improvement }}{\text { fracture toughness with } 4} \\
\text { wt.\% CNT addition }\end{array}$ & $\begin{array}{l}\frac{86 \% \text { improvement }}{\text { fracture toughness with } 4} \\
\text { wt. } \% \text { BNNT addition }\end{array}$ & $\begin{array}{l}\text { CNT and BNNT show similar role in } \\
\text { improving fracture toughness - though } \\
\text { HA-BNNT shows higher E and both } \\
\text { CNT and BNNT shows similar strong, } \\
\text { coherent interface and similar density - } \\
\text { this issue needs further investigation }\end{array}$ \\
\hline
\end{tabular}




\begin{tabular}{|c|c|c|c|}
\hline Criterion of Comparison & HA-CNT & HA-BNNT & Remark \\
\hline \multicolumn{4}{|c|}{ Tribological Behavior of Composite } \\
\hline Coefficient of Friction & 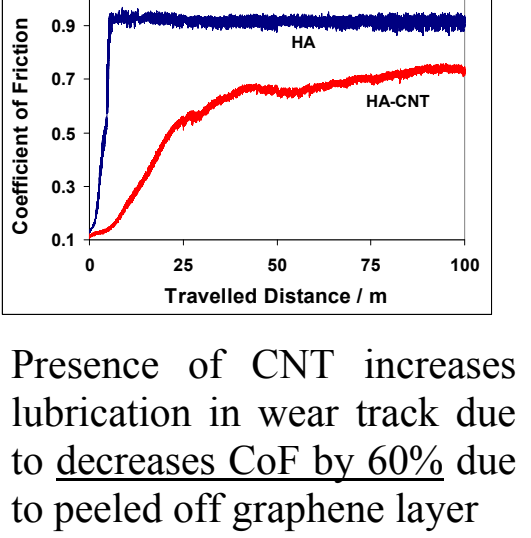 & 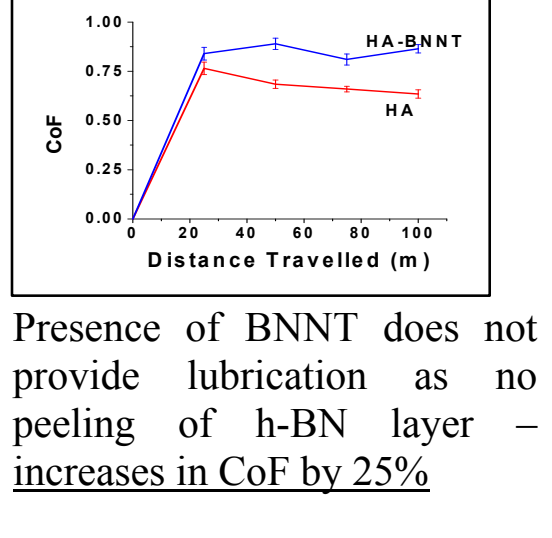 & $\begin{array}{l}\text { Fracture strain for BNNT is } \\
\text { higher than CNT [156], } \\
\text { which restricts peeling of h- } \\
\text { BN walls at similar load at } \\
\text { which graphene layer from } \\
\text { CNT gets peeled off - } \\
\text { lubrication in HA-CNT } \\
\text { helps in increasing wear } \\
\text { resistance }\end{array}$ \\
\hline Wear Volume Loss & $\begin{array}{l}\text { 66\% decrease in wear volume } \\
\text { CNTs get damaged and } \\
\text { broken during wear due to } \\
\text { shear force application - } \\
\text { cannot support or anchor } \\
\text { chipped mass }\end{array}$ & $\begin{array}{l}75 \% \text { decrease in wear volume } \\
\text { BNNTs remain intact - can } \\
\text { anchor chipped and sheared off } \\
\text { mass - decreases wear volume } \\
\text { loss }\end{array}$ & $\begin{array}{l}\text { BNNTs support and anchor } \\
\text { loosened mass on wear track } \\
\text { - increases wear resistance } \\
\text { At the same time CNT offers } \\
\text { lubrication and increases } \\
\text { wear resistance } \\
\text { Finally, HA-BNNT shows } \\
\text { slightly higher wear } \\
\text { resistance improvement } \\
\text { than HA-CNT } \\
66 \% \text { in HA-CNT } \\
75 \% \text { in HA-BNNT }\end{array}$ \\
\hline
\end{tabular}




\begin{tabular}{|c|c|c|c|}
\hline Criterion of Comparison & HA-CNT & HA-BNNT & Remark \\
\hline \multicolumn{4}{|c|}{ Biocompatibility } \\
\hline Osteoblast Viability* & $\begin{array}{l}\text { Slight increase in osteoblast } \\
\text { viability in HA-CNT as } \\
\text { compared to HA surface }\end{array}$ & $\begin{array}{l}\text { Slight increase in osteoblast } \\
\text { viability in HA-BNNT as } \\
\text { compared to HA surface }\end{array}$ & $\begin{array}{l}\text { Both CNT and BNNT does } \\
\text { not negatively influence the } \\
\text { viability of osteoblast on } \mathrm{HA} \\
\text { based surface }\end{array}$ \\
\hline \multicolumn{4}{|c|}{ Osseointegration } \\
\hline Apatite formability & $\begin{array}{l}\text { CNT shows apatite formability } \\
\text { when immersed in SBF - } \\
\text { incubation period for } \\
\text { precipitation is } 14 \text { days [109] }\end{array}$ & $\begin{array}{l}\text { BNNT also shows apatite } \\
\text { formability under } \text { SBF } \\
\text { immersion - incubation period } \\
\text { is } 4.6 \text { days }\end{array}$ & $\begin{array}{l}\text { Lesser apatite precipitation } \\
\text { incubation period on BNNT } \\
\text { would help in faster neobone } \\
\text { formation and post operation } \\
\text { healing period }\end{array}$ \\
\hline
\end{tabular}

*Osteoblast viability for SPS HA-BNT is compared with that of plasma sprayed HA-CNT coating, as no biocompatibility study on SPS HA-CNT is carried out in this dissertation. But, nanotube content is given consideration while evaluating biocompatibility in this research. Considering that, SPS HA-BNNT and plasma sprayed HA-CNT is similar as both of them contain 4 wt.\% nanotube. 
The comparison between HA-CNT and HA-BNNT reveals similar potential of CNT and BNNT towards improvement in fracture toughness and tribological behavior. But, there are some points in favor to BNNT as reinforcement in HA. They are:

- Higher fracture strain, deforming ability and oxidation resistance of BNNT protects it from getting damaged during high temperature and/or pressure exposure required for composite consolidation.

- $\quad$ Though the biocompatibility related results for CNT is positive in this study, but there are some negative reports too in open literature. On the contrary, the present study and all the reports available have always found BNNT to be biocompatible to different cell lineages.

It must be noted that there are very few in-vitro biocompatibility studies on BNNT and no in-vivo study has been conducted on the same. On the other hand, HACNT composites are investigated thoroughly for in-vitro biocompatibility, though in-vivo biocompatibility is evaluated by two research groups, including the study presented in this dissertation. Thus, HA-BNNT composite needs to be more thoroughly evaluated for in-vitro and in-vivo biocompatibility. HA-BNNT coating also needs to be synthesized and characterized for mechanical, tribological and biological behavior to be able to fully compare with HA-CNT coatings. Overall, the outcomes suggest that BNNT has a greater potential as an alternative to CNT for orthopedic application. 


\subsection{References}

1. A.A. White, A.H. Windle, I.A. Kinloch, S.M. Best. Preparation and properties of carbon nanotube reinforced hydroxyapatite. 2008, Key Eng. Mater., Vols. 361363, pp. 419-422.

2. K. Balani, A. Agarwal. Wetting of carbon nanotubes by aluminum oxide. 2008, Nanotechnology, Vol. 19, p. 165701.

3. K. Balani, R. Anderson, T. Laha, M. Andara, J. tercero, E. Crumpler, A. Agarwal. Plasma-sprayed carbon nanotube reinforced hydroxyapatite coatings and their interaction with human osteoblasts in vitro. 2007, Biomaterials, Vol. 28, pp. 618624.

4. K. Balani, S.R. Bakshi, Y. Chen, T. Laha, A. Agarwal. Role of powder treatment and carbon nanotube dispersion in the fracture toughening of plasma sprayed aluminum oxide-carbon nanotube nano-composite. 2007, J. NanoSci. Nanotech., Vol. 7, pp. 1-10.

5. A.K. Keshri, J. Huang, V. Singh, W.B. Choi, S. Seal, A. Agarwal. Synthesis of aluminum oxide coating with carbon nanotube reinforcement produced by chemical vapor deposition for improved fracture and wear resistance. 2010, Carbon, Vol. 48, pp. 431-442.

6. L. Ci, H. Zhu, B. Wei, C. Xu, D. Wu. Annealing amorphous carbon nanotubes for their application in hydrogen storage. 2003, Appl. Surf. Sci., Vol. 205, pp. 39-43.

7. W. Huang, Y. Wang, G. Luo, F. Wei. 99\% Purity multi-walled carbon nanotubes by vacuum high-temperature annealing. 2003, Carbon, Vol. 41, pp. 2528-2590.

8. J. Che, I. Cagin, WA Goddard III. Thermal conductivity of carbon nanotubes. 2000, Nanotechnology, Vol. 11, pp. 65-69.

9. M. Gaona, R.S. Lima, B.R. Marple. Nanostructured titania/hydroxyapatite composite coatings deposited by high velocity oxy-fuel (HVOF) spraying. 2007, Mater. Sci. Eng. A, Vol. 458, pp. 141-149.

10. W. Yi, L. Lu, D.L. Zhang, Z.W. Pan, S.S. Xie. Linear specific heat of carbon nanotubes. 1999, Phys. Rev. B, Vol. 59, pp. 9015-9018.

11. C.M. Roomie, C.D. Craig. Crystallite orientation and anisotropic strains in thermally sprayed hydroxyapatite coatings. 1995, Biomaterials, Vol. 16, pp. 691696.

12. H. Kim, R.P. Camata, S. Chowdhury, Y.K. Vohra. In-vitro dissolution and mechanical behavior of c-axis preferentially oriented hydroxyapatite thin films 
fabricated by pulsed laser deposition. 2010, Acta Biomater., Vol. 6, pp. 32343241.

13. H. Kim, R.P. Camata, S. Lee, G.S. Rohrer, A.D. Rollett, Y.K. Vohra. 2007, Acta Mater., Crystallographic texture in pulsed laser deposited hydroxyapatite bioceramic coatings. Vol. 55, pp. 131-139.

14. A. Agarwal, N.B. Dahotre. Mechanical Properties of Laser Engineered Composite Boride Coating on Steel: A Nanoindentation Approach. 2000, Met. Mater. Trans. A, Vol. 31, pp. 401-408.

15. G.R. Anstis, P. Chantikul, B.R. Lawn, D.B. Marshall. A critical evaluation of indentation technique for measuring fracture toughness: I. Direct crack measurements. 1981, J. Am. Ceram. Soc., Vol. 64 (9), pp. 533-538.

16. D. P. Pioletti, H. Takei, S.Y. Kwon, D. Wood, K.L.P. Sung. The cytotoxic effect of titanium particles phagocytosed by osteoblasts. 1999, J. Biomed. Mater. Res. A, Vol. 46, pp. 399-407.

17. D.R. Haynes, S.J. Hay, S.D. Rogers, S. Ohta, D.W. Howie, S.E. Graves. Regulation of bone cells by particle-activated mononuclear phagocytes. 1997, J Bone Joint Surg. Br., Vol. 79, pp. 988-994.

18. S.D. Neale, D.R. Hayes, D.W. Howie, D.W. Murray, N.A. Athanasou. The effect of particle phagocytosis and metallic wear particles on osteoclast formation and bone resorption in vitro. 2000, J. Arthroplasty, Vol. 15, pp. 654-662.

19. C.H. Lohmann, D.D. Dean, G. Köster, D. Casasola, G.H. Buchhorn, U. Fink, Z. Schwartz, B.D. Boyan. Ceramic and PMMA particles differentially affect osteoblast phenotype. 2002, Biomaterials, Vol. 23, pp. 1855-1863.

20. W. Akar, C.B. Colby, G.J. Atkins, P. Majewski. Biomimetic hydroxyapatite coating on glass coverslips for the assay of osteoclast activity in vitro. 2009, J. Mater. Sci., Vol. 20, pp. 1467-1473.

21. S.B. Goodman, T. Ma. Cellular chemotaxis induced by wear particles from joint replacements. 2010, Biomaterials, Vol. 31, pp. 5045-5050.

22. M. Ipavec, A. Iglic, V.K. Iglic, K. Srakar. Stress distribution on the hip joint articular surface during gait. 1996, Eur. J. Physiol., Vol. 431, pp. R275-276.

23. L. Neumann, K.G. Freund, K.H. Sorenson. Long-term results of Chernley total hip replacement. 1994, J. Bone Joint Surg. Br., Vol. 76, pp. 245-251.

24. M.F. Yu, O. Lourie, M.J. Dyer, K. Moloni, T.F. Kelly, R.S. Rouff. Strength and breaking mechanism of multiwalled carbon nanotube under tensile load. 2000, Science, Vol. 287, pp. 637-640. 
25. A.G. Evans, B. Marshall. Wear mechanisms in ceramics. Editor: D.A. Rigney. Fundametals of Friction and Wear of Materials, 1981, Materials Park, Ohio, pp. 439-452.

26. M.S. Dresselhaus, G. Dresselhaus, R. Saito R, A. Jorio. Raman spectroscopy of carbon nanotubes. 2005, Phys. Report, Vol. 409, pp. 47-99.

27. J. Liu, U.A. Glasmacher, M. Lang, C. Trautman, K.O. Voss, R. Neumann, G.A. Wagner, R. Miletich. Raman spectroscopy of apatite irradiated with swift heavy ions with and without simultaneous exertion of high pressure. 2008, Appl. Phys. A, Vol. 9, pp. 17-22.

28. A. Das, B. Chakraborty, A.K. Sood. Raman spectroscopy of graphene on different substrates and influence of defects. 2009, Bull. Mater. Sci., Vol. 31, pp. 579-584.

29. K. Yang, J. He, Z. Su, J.B. Reppert, M.J. Skove, T.M. Tritt, A.M. Rao. Inter-tube bonding, graphene formation and anisotropic transport properties in spark plasma sintered multi-wall carbon nanotube arrays. 2010, Carbon, Vol. 48, pp. 756-762.

30. S.R. Bakshi, V. Singh, D.G. McCartney, S. Seal, A. Agarwal. Deformation and damage mechanisms of multiwalled carbon nanotubes under high-velocity impact. 2008, Scripta Mater., Vol. 59, pp. 499-502.

31. B. Wei, J. Zhang, J. Liang, D. Wu. The mechanism of phase transformation from carbon nanotube to diamond. 1998, Carbon, Vol. 36, pp. 997-1000.

32. K.S. Kim, S.Y. Moon, S.Y. Bang, B.G. Choi, H. Ham, T. Sekino, K.B. Shim. Fabrication of graphene layers from multiwalled carbon nanotubes using high dc pulse. 2009, Appl. Phys. Lett., Vol. 95, p. 083103.

33. J.L. Xu, K.A. Khor, J.J. Sui, W.N. Chen. 2009, Preparation and characterization of a novel hydroxyapatite/carbon nanotubes composite and its interaction with osteoblast-like cells. Mater. Sci. Eng. C, Vol. 29, pp. 44-49.

34. S.K. Sarkar, M.H. Youn, I.H. Oh, B.T. Lee. Fabrication of CNT reinforced HAp composites by spark plasma sintering. 2007, Mater. Sci. Forum, Vols. 534-536, pp. 893-896.

35. Z. Shen, M. Nygren. Microstructural prototyping of ceramics by kinetic engineering: applications of spark plasma sintering. Chem. Rec., 2005, Vol. 5, pp. 173-184.

36. A.V. Ragulya. Consolidation of ceramic nanopowders. 2008, Adv. Appl. Ceram., Vol. 107, pp. 118-134. 
37. Z.A. Munir, U.A. Tamburini, M. Ohyanagi. The effect of electric field and pressure on the synthesis and consolidation of materials: A review of the spark plasma sintering method. 2006, J. Mater. Sci., Vol. 41, pp. 763-777.

38. E.A. Olevsky, S. Kandukuri, L. Froyen. Consolidation enhancement in spark plasme sintering: Impact of high heating rates. 2007, J. Appl. Phys., Vol. 102, p. 114913.

39. A. Laghzizil, N. El Herch, A. Bouhaouss, G. Lorente, J. Macquete. Comparison of electrical properties between fluoroapatite and hydroxyapatite materials. 2001, J. Solid State Chem., Vol. 156, pp. 57-60.

40. T.W. Ebbesen, H.J. Lezec, H. Hiura, J.W. Bennett, H.F. Ghaemi, T. Thio. Electrical conductivity of individual nanotubes. 1996, Nature, Vol. 382, pp. 5456.

41. M. Miodownik, E.A. Holm, G.N. Hassold. Highly parallel computer simulations of particle pinning: Zener vindicted. 2000, Scripta Mater., Vol. 42, pp. 11731177.

42. N. Sun, B.R. Patterson, J.P. Suni, H. Weiland, L.F. Allard. Characterization of particle pinning potential. 2006, Acta Mater., Vol. 54, pp. 4091-4099.

43. B.C. Kim, J.H. Lee,J.J. Kim, T. Ikegami. Rapid rate sintering of nanocrystalline indium tin oxide ceramics: particle size effect. 2002, Mater. Lett., Vol. 52, pp. 114-119.

44. U. Messerschmidt, M. Bartsch, C. Dietsch, W. Kurtz, Scheu, M. Rühle. HVEM in situ study of fracture of Al2O3/Nb sandwich specimen. Z. Metallkd., 2004, Vol. 95, pp. 785-922004.

45. D.A. Porter, K.E. Easterling. "Phase transformation in metals and alloys. 2nd ed. Cheltenham: CRC Press. 2001, pp. 142-169.

46. Y.T. Cheng, C.M. Cheng. Scaling, dimensional analysis, and indentation measurements. 2004, Mater. Sci. Eng. R, Vol. 44, pp. 91-149.

47. S. Singh, Y. Pei, R. Miller, P.R. Sundararajan. Long-range, entangled carbon nanotube networks in polycarbonate. 2003, Adv. Func. Mater., Vol. 13, pp. 868872.

48. J.D. Eshelby. The determination of elastic field of an ellipsoidal inclusion, and related problems. 1957, Proc. Royal Soc. London, Vol. 241, pp. 376-396.

49. T. Mori, K. Tanaka. Average stress in matrix and average elastic energy of materials with misfitting inclusions. 1973, Acta Mater., Vol. 21, pp. 571-574. 
50. Y. Chen, K. Balani, A. agarwal. Analytical model to evaluate interface characteristics of carbon nanotube reinforced aluminum oxide nanocomposites. 2008, Appl. Phys. Lett., Vol. 92, p. 011916.

51. S. Pramanik, A.K. Agarwal AK, K.N. Rai. Development of High Strength Hydroxyapatite for Hard Tissue Replacement. 2005, Trends Biomater. Artif. Organs, Vol. 19, pp. 46-51.

52. C. Hellimich, F.J. Ulm. Poro-micromechanics of bone: Impact loading and wave propagation. In: Mechanical properties of bioinspired and biological materials. 2005, MRS 2004 Fall Meeting (Boston, USA): Mater. Res. Soc. Symp. Proc., Vol. 844, pp. Y8.6.1-7.

53. A. Nakahira, K. Eguchi. Evaluation of microstructure and some properties of hydroxyapatite/Ti composites. 2001, J. Ceram. Proc. Res., Vol. 2, pp. 108-112.

54. S.B. Cronin, A.K. Swan, M.S. Unlu, B.B. Goldberg, M.S. Dresselhaus, M. Tinkham. Measuring the uniaxial strain of individual single-wall carbon nanotubes: resonance Raman spectra of atomic-force-microscope modified single-wall nanotubes. 2004, Phys. Rev. Lett., Vol. 93, p. 167401.

55. S.R. Bakshi, J.E. Tercero, A. Agarwal. Synthesis and characterization of multiwalled carbon nanotube reinforced ultra high molecular weight polyethylene composite by electrostatic spraying technique. 2007, Composite A, Vol. 38, pp. 2493-2499.

56. M. Mu, S. Osswald, Y. Gogotsi, K.I. Winey. An in situ Raman spectroscopy study of stress transfer between carbon nanotubes and polymer. 2009, Nanotechnology, Vol. 20, p. 335703.

57. J.L. Li, L.J. Wang, T. He, W. Jiang. Surface graphitization and mechanical properties of hot-pressed bulk carbon nanotubes compacted by spark plasma sintering. 2007, Carbon, Vol. 45, pp. 2636-2642.

58. S.R. Bakshi, D. Lahiri, R. Patel, A. Agarwal. Nanoscratch behavior of carbon nanotube reinforced aluminum coatings. 2010, Thin Solid Films, Vol. 518, pp. 1703-1711.

59. C. Zhi, Y. Bando, T. Terao, C. Tang, H. Kuwahara, D. Golberg. Towards thermoconductive, electrically insulating polymeric composites with boron nitride nanotubes as fillers. 2009, Adv. Func. Mater., Vol. 19, pp. 1857-1862.

60. T. Terao, C. Zhi, Y. Bando, M. Mitome, C. Tang, D. Golberg. Alignment of boron nitride nanotubes in polymeric composite films for thermal conductivity improvement. 2010, J. Phys. Chem., Vol. 114, pp. 4340-4344. 
61. Q. Huang, Y. Bando, X. Xu, T. Nishimura, C. Zhi, C. Tang, F. Xu, L. Gao, D. Golberg. Enhancing superplasticity of engineering ceramics by introducing $B N$ nanotubes. 2007, Nanotechnology, Vol. 18, p. 48570.

62. D. Golberg, Y. Bando, C. Tang, C.Zhi. Boron Nitride Nanotubes. 2007, Adv. Mater., Vol. 19, pp. 2413-2432.

63. C. Zhi, Y. Bando, C. Tang, S. Honda, K. Sato, H. Kuwahara, D. Golberg. Purification of boron nitride nanotubes through polymer wrapping. 2006, J. Phys. Chem. B, Vol. 110, pp. 1525-1528.

64. L. Guo, R.N. Singh. Selective growth of boron nitride nanotubes by plasmaenhanced chemical vapor deposition at low substrate temperature. 2008, Nanotechnology, Vol. 19, p. 065601.

65. S.K. Singhal, A.K. Srivastava., R.P. Pant, S.K. Halder,B.P. Singh, A.K. Gupta. Synthesis of boron nitride nanotubes employing mechanothermal process and its characterization. 2008, J. Mater. Sci., Vol. 43, pp. 5243-5250.

66. M. Terrones, J.M. Romo-Herrera, E. Cruz-Silva, F. López-Urías, E. MuñozSandoval, J.J. Velázquez-Salazar, H. Terrones, Y. Bando, D. Golberg. Pure and doped boron nitride nanotubes. 2007, Mater. Today, Vol. 10, pp. 30-38.

67. M.I. Kay, R.A. Young. Crystal structure of hydroxyapatite. 1964, Nature, Vol. 204, pp. 1050-1052.

68. A. Nag, K. Raidongia., K.P.S.S. Hembram, R. Datta, U.V. Waghmare, C.N.R. Rao. Graphene analogues of BN: Novel synthesis and properties. 2010, ACS Nano, Vol. 4, pp. 1539-1544.

69. H.P. Lan, L.H. Ye, S. Zhang, L.M. Peng. Transverse dielectric properties of boron nitride nanotubes by ab initio electric field calculations. 2009, Appl. Phys. Lett., Vol. 94, p. 183110.

70. A.P. Suryavanshi, M.F. Yu, J. Wen, C. Tang, Y. Bando. Elastic modulus and resonance behavior of boron nitride nanotubes. 2004, Appl. Phys. Lett., Vol. 84 (14), pp. 2527-2529.

71. C. Hellimich, F.J. Ulm. Poro-micromechanics of bone: Impact loading and wave propagation. 2005, Mater. Res. Soc. Symp. Proc., Vol. 844, pp. Y8.6.1-Y8.6.7.

72. A. Fritsch, L. Dormieus, C. Hellimich, J. Sanahuja. Mechanical behavior of hydroxyapatite biomaterials: An experimentally validated micromechanical model for elasticity and strength. 2009, J. Biomed. Mater. Res. A, Vol. 88A, pp. 149-161. 
73. A. Nakahira, K. Eguchi. Evaluation of microstructure and some properties of hydroxyapatite/Ti composites. 2001, J. Ceram. Proc. Res., Vol. 2, pp. 108-112.

74. N.G. Chopra, A. Zetll. Measurement of the elastic modulus of a multi walled boron nitride nanotubes. 1998, Solid State Commun., Vol. 105 (5), pp. 297-300.

75. A. Ravaglioli, A. Krajewski. Bioceramics: Materials, properties, application. 1992, London: Chapman \& Hall, p. 44.

76. J. Wang, L.L. Shaw. Nanocrystalline hydroxyapatite with simultaneous enhancements in hardness and toughness. 2009, Biomaterials, Vol. 30, pp. 65656572.

77. Z. Pawlak, T. Kaldonski, R. Pai, E. Bayraktar, A. Oloyede. A comparative study on the tribological behaviour of hexagonal boron nitride (h-BN) as lubricating micro-particles-An additive in porous sliding bearings for a car clutch. 2009, Wear, Vol. 267, pp. 1198-1202.

78. Z. Pawlak, R. Pai, E. Bayraktar, T. Kaldonski, A. Oloyede. Lamellar lubrication in vivo and vitro: Friction testing of hexagonal boron nitride. 2008, BioSystems, Vol. 94, pp. 202-208.

79. F. Bronner, M.C. Farach-Carson. Bone Formation. 2004, London: SpringerVerlag, ISBN: 1-85233-717-6.

80. V. Kumar, A.K. Abbas, F. Nelson, J.C. Aster. Robbins and Cotran Pethologic Basis of Disease. 8th Edition. 2010, Philadelphia: Saunders Elsevier, ISBN: 9781-4160-3121-5.

81. C. Lin, H. Han, F. Zhang, A. Li. Electrophoretic deposition of HA/MWNTs composite coating for biomedical applications. 2008, J. Mater Sci: Mater. Med., Vol. 19, pp. 2569-2574.

82. B.D. Hahn, J.M. Lee, D.S. Park, J.J. Choi, J. Ryu, W.H. Yoon, B.K. Lee, D.S. Shin, H.E. Kim. Mechanical and in vitro biological performances of hydroxyapatite-carbon nanotube composite coatings deposited on Ti by aerosol deposition.2009, Acta Biomater., Vol. 5, pp. 3205-3214.

83. J. Xu, K.A. Khor, J. Sui, J. Zhang, T.L. Tan, W.N. Chen. Comparative petromics profile of osteoblasts cultured on dissimilar hydroxyapatite biomaterials: An iTRAQ-coupled 2-D LC-MS/MS analysis. 2008, Petromics, Vol. 8, pp. 4249-4258.

84. J.L. Xu, K.A. Khor, J. J. Sui, W.N. Chen. 2008, Investigation of multiwalled carbon nanotube modified hydroxyapatite on human osteoblast cell line using iTRAQ petromics technology. Key Eng. Technol., Vols. 361-363, pp. 1047-1050. 
85. M. Matsuoka, T. Akasaka, Y. Totsuka, F. Watari. Strong adhesion of Saos-2 cells to multi-walled carbon nanotubes. 2010, Mater. Sci. Eng. B, Vol. 173, pp. 182186.

86. T. Akasaka, A. Yokoyama, M. Matsuoka, T. Hashimoto, F. Watari. Thin films of single-walled carbon nanotubes promote human osteoblastic cells (Saos-2) proliferation in low serum concentrations. 2010, Mater. Sci. Eng. C, Vol. 30, pp. 391-399.

87. E.A. dos Santos, M. Farina, G.A. Soares. Specific proliferation rates of human osteoblasts on calcium phosphate surfaces with variable concentrations of $\alpha$ TCP. 2007, Mater. Sci. Eng. C, Vol. 27, pp. 61-66.

88. K. merry, R. Dodds, A. Littlewood, M. Gowen. Expression of osteopontin $m R N A$ by osteoclasts and osteoblasts in modelling adult human bone.1993, J. Cell Sci., Vol. 104, pp. 1013-1020.

89. T. Yamate, H. Mocharla, Y. Taguchi, J.U. Igietseme, S.C. Manolagas, E. Abe. Osteopontin expression by osteoclast and osteoblast progenitors in the murine bone marrow: demonstration of its requirement for osteoclastogenesis and its increase after ovariectomy. 1997, Endocrinology, Vol. 138, pp. 3047-3055.

90. T.R. Arnett, B. Henderson. Methods in Bone Biology. 1998, London: Chapman \& Hall, pp. 9-10.

91. P. J. Marie. Transcription factors controlling osteoblastogenesis. 2008, Arch. Biochem. Biophys., Vol. 473, pp. 98-105.

92. E.E. Golub, K. Boesze-Battaglia. The role of alkaline phosphatase in mineralization. 2007, Curr. Opin. Orthopedics, Vol. 18, pp. 444-448.

93. X. Li, H. Gao, M. Uo, Y. Sato, T. Akasaka, S. Abe, Q. Feng, F. Cui, F. Watari. Maturation of osteoblast-like SaoS2 induced by carbon nanotubes. 2009, Biomed. Mater. Vol. 4, p. 015005.

94. R.L. Price, M.C. Waid, K.M. Haberstroh, T.J. Webster. Selective bone cell adhesion on formulations containing carbon nanofibers. 2003, Biomaterials, Vol. 24, pp. 1877-1887.

95. A. Atala, R. Lanza, J. Thomson, R. Nerem. Principles of Regenerative Medicine. 2008, Burlington: Academic Press, Elsevier, pp. 558-561.

96. G.D. Pappas, E.J. Blanchette. Transport of colloidal particles from small blood vessels correlated with cyclic changes in permeability. 1965, Investigative Opthalmology, Vol. 4, pp. 1026-1035. 
97. V. Olivier, J. L. Duva, M. Hindi, P. Pouletaut, M.D.Nagel. Comparative particleinduced cytotoxicity toward macrophages and fibroblasts. 2003, Cell. Bio. Toxicol., Vol. 19, pp. 145-159.

98. T. Blake, V. Castranova, S. Schwegler-Berry, P. Baron, G.J. Deye, L. Ci, W. Jones. Effect of fiber length on glass microfiber cytotoxicity. 1998, Toxicol. Environ. Health, Vol. 54, pp. 243-259.

99. V.E Kagan, N.V. Konduru, W. Feng, B.L. Allen, J. Conroy, Y. Volkov, I.I. Vlasova, N.A. Belikova, N. Yanamala, A. Kapralov, Y.Y. Tyurina, J. Shi, E.R. kisin, A.R. Murray, J. Franks, D. Stolz, P. Gou, J.K. Seetharaman, B. Fadeel, A. Star, A.A. Shvedova. Carbon nanotubes degraded by neutrophil myeloperoxidase induce less pulmonary inflammation. 2010, Nat. Nanotechnol., Vol. 5, pp. 354359.

100. D.R. Haynes, S.D. Rogers, S.Ha, M.J. Pearcy, D.W. Howie. The differences in toxicity and release of bone-resorbing mediators induced by titanium and cobaltchromium-alloy wear particles. 1993, Bone Joint. Surg. Am., Vol. 75, pp. 825834.

101. T.T. Glant, J.J. Jacob, G. Molnar,A.S. Shanbag, M. Valyon, J.O. Galante. Bone resorption activity of particulate-stimulated macrophages. 1993, J. Bone Miner. Res., Vol. 8, pp. 1071-1079.

102. M.R. Wani, K. Fuller, N.S. Kim. Y. Choi. Prostaglandin E2 cooperates with TRANCE in osteoclast induction from hemopoietic precursors: Synergistic activation of differentiation, cell spreading, and fusion. 1999, Endocrinology, Vol. 140, pp. 1927-1935.

103. Y. Kobayashi, T. Mizoguchi, I. Take, S. Kurihara, N. Udagawa, N. Takahashi. Prostaglandin E2 enhances osteoclastic differentiation of precursor cells through protein Kinase A-dependent phosphorylation of TAK1. 57.59. 2005, J. Biol. Chem., Vol. 280, pp. 11395-11403.

104. Z. Yao, L. Xing, C. Qin, E.M. Schwarz, B.F. Boyce. Osteoclast precursor interaction with bone matrix induces osteoclast formation directly by an interleukin-1-mediated autocrine mechanism. 2008, J. Biol. Chem., Vol. 283, pp. 9917-9924.

105. N. Narita, Y. Kobayashi,H. Nakamura, K. Maeda, A. Ishihara, T. Mizoguchi, Y. Usui, K. Aoki, M. Simizu, H. Kato, H. Ozawa, N. Udagawa, M. Endo, N. Takahashi, N. Saito. Multiwalled carbon nanotubes specifically inhibit osteoclast differentiation and function. 2009, Nano Lett., Vol. 9 (4), pp. 1406-1413. 
106. Y. Usui, K. A., N. Narita, N. Murakami, I. Nakamura, K. Nakamura, N. Ishigaki, H. Yamazaki, H. Horiuchi, H. Kato, S. Taruta, Y. A. Kim, M. Endo, N. Saito. Carbon nanotubes with high bone-tissue compatibility and bone-formation acceleration effects. 2008, Small, Vol. 4, pp. 240-246.

107. S. Aryal, S.R. Bhattarai, K.C. R. Bahadur, M.S. Khil, D.R. Lee, H.Y. Kim. Carbon nanotubes assisted biomimetic synthesis of hydroxyapatite from simulated body fluid.2006, Mater. Sci. Eng. A, Vols. 426, pp. 202-207.

108. L.P. Zanello, B. Zhao, H. Hu and R.C. Haddon. Bone cell proliferation on carbon nanotubes. 2006, Nano Lett., Vol. 3, pp. 562-567.

109. T. Akasaka, F. Watari, Y. Sato, K. Tohji. Apatite formation on carbon nanotubes. 2006, Mater. Sci. Eng. C, Vol. 26, pp. 675-678.

110. P. Cherukuri, S.M. Bachilo, S.H. Litovsky, R.B. Weisman. Near-infrared fluorescence microscopy of single-walled carbon nanotubes in phagocytic cells. 2004, J. Am. Chem. Soc., Vol. 126, pp. 15638-15639.

111. D. Lahiri, F. Rouzaud, T. Richard, A.k. Keshri, S.R. Bakshi, L. Kos, A. Agarwal. Boron nitride nanotube reinforced polylactide-polycaprolactone copolymer composite: Mechanical properties and cytocompatibility with osteoblasts and macrophages in vitro. 2010, Acta Biomater., Vol. 6, pp. 3524-3533.

112. C. Zhi, Y. Bando, C. Tang, D. Golberg. Immobilization of proteins on boron nitride nanotubes. 2005, J. Am. Chem. Soc., Vol. 127, pp. 17144-17145.

113. E.A. clark, J.S. Brugge. Integrins and sigmal tranductin pathways: the road taken. 1995, Science, Vol. 268, pp. 233-239.

114. C. Wang, Y. Gong, Y. Zhong, Y. Yao, K. Su, D.A. Wang. The control of anchorage-dependent cell behavior within a hydrogel/microcarrier system in an osteogenic model. 2009, Biomaterials, Vol. 30, pp. 2259-2269.

115. V. Grigoriou, I.M. Shapiro, E.A. Cavalcanti-adam, R.J. Composto, P. Ducheyne, C.S. Adams. Apoptosis and survival of osteoblast-like cells are regulated by surface attachment. 2005, J. Biol. Chem., Vol. 280, pp. 1733-1739.

116. A. Mata, X. Su, A.J. Fleischman, S. Roy, B.A. Banks, S.K. Miller, R.J. Midura. Osteoblast attachment to a textured surface in the absence of exogenous adhesion proteins. 2003, IEEE Trans Nanobiosci., Vol. 2, pp. 287-294.

117. P.D. Benya, J.D. Shaffer. Dedifferentiated chondrocytes reexpress the differentiated collagen phenotype when cultured in agarose gels. 1982, Cell, Vol. 30 , pp. 215-224. 
118. Y. Luo, M.S. Shoichet. A photolabile hydrogel for guided three-dimensional cell growth and migration. 2004, Nature Mater., Vol. 3, pp. 249-253.

119. E. Ruoslathi, J.C. Reed. Anchorage dependence, integrins, and apoptosis. 1994, Cell, Vol. 77, pp. 477-478.

120. F. Iwasa, N. Hori, T. Ueno, H. Minamikawa, M. Yamada, T. Ogawa. Enhancement of osteoblast adhesion to UV-photofunctionalized titanium via an electrostatic mechanism. 2010, Biomaterials, Vol. 31, pp. 2717-2727.

121. A.J. García, P. Ducheyne, D. Boettiger. Effect of surface reaction stage on fibronectin-mediated adhesion of osteoblast-like cells to bioactive glass. 1998, J. Biomed. Mater. Res., Vol. 40, pp. 48-56.

122. M.M. Lotz, C.A.Burdsal, H.P. Erickson,D.R. McClay. Cell Adhesion to Fibronectin and Tenascin: Quantitative Measurements of Initial Binding and Subsequent Strengthening Response. 1989, J. Cell Biol., Vol. 109, pp. 1795-1805.

123. J.W. Piper, R.A.Swerlick, C. Zhu. Determining Force Dependence of TwoDimensional Receptor-Ligand Binding Affinity by Centrifugation. 1998, Biophys. J., Vol. 74, pp. 492-513.

124. M. Kusakabe, Y. Suzuki, A. Nakao, M. Kaibara, M. Iwaki, M. Scholl. Control of endothelial cell adhesion to polymer surface by ion implantation. 2001, Polym. Adv. Technol., Vol. 12, pp. 453-460.

125. G. Weder, O. Guillaume-Gentil, N. Matthey, F. Montagne, H. Heinzelmann. The quantification of single cell adhesion on functionalized surfaces for cell sheet engineering. 2010, Biomaterials, Vol. 31, pp. 6436-6443.

126. V. M. Bowers, L.R. Fisher, G.M. Francis, K.L. Williams. A Micromechanical technique for monitoring cell-substrate adhesiveness: measurements of the strength of a red blood cell adhesion to glass and polymer test surfaces. 1989, J. Biomed. Mater. Res., Vol. 23, pp. 1453-1473.

127. A. Yamamoto, S. Mishima, N. Maruyama, M. Sumita. A new technique for direct measurement of the shear force necessary to detach a cell from a material. 1998, Biomaterials, Vol. 19, pp. 871-879.

128. M. Yamada, T. Miyauchi, A. Yamamoto, F. Iwasa, M. Takeuchi, M. Anpo, K. Sakurai. Enhancement of adhesion strength and cellular stiffness of osteoblasts on mirror-polished titanium surface by UV-photofunctionalization. 2010, Acta Biomater., Vol. 6, pp. 4578-4588.

129. K.A. Athanasiou, B.S. Thoma, D.R. Lanctot, D. Shin, C.M. Agrawa!, R.G. LeBaron. Development of the cytodetachment technique to quantify mechanical adhesiveness of the single cell. 1999, Biomaterials, Vol. 20, pp. 2405-2415. 
130. A.C. Fischer-Cripps. Nanoindentation.2004, New York: Springer-Verlag, ISBN: 0-387-22045-3.

131. I. Lahiri, D. lahiri, S. Jin, A. Agarwal, W. Choi. Carbon nanotubes: how strong is their bond with substrate? 2011, ACSNano, Vol. 5(2), pp. 780-787.

132. S. Ozawa, S. Kasugai. Evaluation of implant materials (hydroxyapatite, glassceramics, Titanium) in rat bone marrow stormal cell culture. 1996, Biomaterials, Vol. 17, pp. 23-29.

133. M. Hott, B. Noel, D. Bernache-Assolant, C. Rey, P.J. Marie. Proliferation and differentiation of human trabecular osteoblastic cells on hydroxyapatite. 1997, J. Biomed. Mater. Res., Vol. 37, pp. 508-516.

134. N. Specchia, A. Pagnotta, M. Cappella, A. Tampieri, F. Greco. Effect of hydroxyapatite porosity on growth and differentiation of human osteoblast-like cells. 2002, J. Mater. Sci., Vol. 37, pp. 577-584.

135. J. Xie, M.J. Boumann, L.R. McCabe. Adsorption of serum fetuin to hydroxylapatite does not contribute to osteoblast phenotype modifications. 2005, J. Biomed. Mater. Res. A, Vol. 73A, pp. 39-47.

136. K. Okamoto, T. Matsuura, R. Hosokawa and Y. Akagawa. RGD peptides regulate the specific adhesion scheme of osteoblasts to hydroxyapatite but not to titanium. 1998, J. Dent. Res., Vol. 77, pp. 481-487.

137. S. Walcott, S.X. Sun. A mechanical model of actin stress fiber formation and substrate elasticity sensing in adherent cells. 2010, Proc. Natl. Acad. Sci. Am., Vol. 107, pp. 7757-7762.

138. M. Amano, K. Chihara, K. Kimura, Y. Fukata, N. Nakamura, Y. Matsuura, K. Kaibuchi. Formation of Actin Stress Fibers and Focal Adhesions Enhanced by Rho-Kinase. 1997, Science, Vol. 275, pp. 1308 - 1311.

139. H. Hirata, H. Tatsumi, M. Sokabe. Dynamics of actin filaments during tensiondependent formation of actin bundles. 2007, Biochimica et Biophysica Acta, Vol. 1770, pp. 1115-1157.

140. J.M.A. Farsi, J.E. Aubin. Microfilament rearrangements during fibroblast induced contraction of three-dimensional hydrated collagen gels. 1986, Cell Motil., Vol. 4, pp. 29-40.

141. M.D. Abramoff, P.J. Magelhaes, S.J. Ram. Image processing with ImageJ. 2004, J Biophotonics Int, Vol. 11, pp. 36-42.

142. S.F. El-Amin, M. Attawia, H.H. lu, A.K. Shah, R. Chang, N.J. Hickok, R.S. Tuan, C.T. Laurencin. Integrin expression by human osteoblasts cultured on degradable 
polymeric materials applicable for tissue engineered bone. 2002, J. Orthopedic Res., Vol. 20, pp. 20-28.

143. C.H. Damsky. Extracellular matrix-integrin interactions in osteoblast function and tissue remodelling. 1999, Bone, Vol. 25, pp. 95-96.

144. B. Alberts, D. Bray, J. Lewis, M. Raff, K. Roberts, J.D. Watson. Molecular Biol. Cells. 3rd Edition, 1994, New York: Garland science, ISBN-10: 0-8153-1619-4.

145. N.D. Gallant, K.E. Michael, A.J. Garcia. Cell adhesion strengthening: contributions of adhesive area, integrin binding and focal adhesion assembly.2005, Mol. Biol. Cell, Vol. 16, pp. 4329-4340.

146. C.F. Lai, S.L. Cheng. Alphavbeta integrins play an essential role in BMP-2 Induction of osteoblast differentiation. 2005, J. Bone Mineral Res., Vol. 20, pp. 330-340.

147. R. Olivares-Navarrete, P. Raz, G. Zhao, J. Chen, M. Wieland, D. L. Cochran, R. A. Chaudhri, A. Ornoy, B.D. Boyan, Z. Schwartz. Integrin $\alpha 2 \beta 1$ plays a critical role in osteoblast response to micron-scale surface structure and surface energy of titanium substrates. 2008, Proc. Natl. Acad. Sci. Am., Vol. 105, pp. 15767 15772.

148. Z. Ou, B. Wu, D. Xing, F. Zhou, H. Wang, Y. Tang. Functional single-walled carbon nanotubes based on an integrin av $\beta 3$ monoclonal antibody for highly efficient cancer cell targetting. 2009, Nanotechnology, Vol. 20, p. 105102.

149. A.D.L. Zerda, C. Zavaleta, S. Keren, S. Vaithilingam, S. Bodapati, Z. liu, J. Levi, B.R. Smith, T.J. Ma, O. oralkan, Z. Cheng, X. Chen, H. Dai, B.T. Khuri-Yakub, S.S. Gambhir. Carbon nanotubes as photoacoustic molecular imaging agents in living mice. 2008, Nature Nanotechnol., Vol. 3, pp. 557-562.

150. Z. Liu, W. Cai, L. He, N. Nakayama, K. Chen, X. Sun, X. Chen, H. Dai. In vivo biodistribution and highly efficient tumour targeting of carbon nanotubes in mice. 2007, Nature Nanotechnol., Vol. 2, pp. 47-52.

151. S.I. Pascu, R.L. Arrowsmith, S.R. Bayly, S. Brayshaw, Z. Hu. Towards nanomedicines: design protocols to assemble, visualize and test carbon nanotube probes for multi-modality biomedical imaging. 2010, Phil. Trans., Vol. 368, pp. 3683-3712.

152. D.C. Mears. Metals in medicine and surgery. 1977, Int. Mater. Rev., Vol. 22, pp. 119-155.

153. X. Liu, G.L. Niebur. Bone ingrowth into a porous coated implant predicted by a mechano-regulatory tissue differentiation algorithm. 2008, Biomech. Model Mechanobiol., Vol. 7, pp. 335-344. 
154. J. Beuvelot, C. Bergeret, R. Mallet, V. Fernandez, J. Cousseau, M.F. Basle, D. Chappard. In vitro calcification of chemically functionalized carbon nanotubes. 2010, Acta Biomater., Vol. 6, pp. 4111-4117.

155. G. Penel, N. Leroy, P. Van Landuyt, B. Flautre, P. Hardouin, J. Lemaitre, C. Leroy. Raman microspectrometry studies of brushite cement: In vivo evolution in a sheep model. 1999, Bone, Vol. 25 (2) Supplement, pp. 81S-84S.

156. H.M. Ghassemi, C.H. Lee, Y.K. Yap, R.S. Yassar. In situ observation of reversible rippling in multi-walled boron nitride nanotubes. 2011, Nanotechnology, Vol. 22, p. 115702. 


\subsection{CONCLUSIONS}

This dissertation presents a complete analysis of HA-nanotube composites, in terms of mechanical, tribological and biological behavior to judge its potential for orthopedic application. Investigations on coating and free standing HA-CNT composite reveal significant improvement in the fracture toughness and wear resistance with CNT reinforcement. In-vitro and in-vivo biocompatibility studies suggest non-cytotoxic response of CNT along with positive influence on the bone cell activity. Presence of CNT also helps in osseointegration on HA based surface. Overall, HA-CNT composites and coatings are established as a very attractive solution to existing problems of HA. Considering the ongoing debate about the cytotoxicity of CNTs in open literature, BNNT is proposed and explored as an alternative reinforcement for HA. HA-BNNT composite also leads to excellent improvement in the fracture toughness and wear resistance, which is comparable to HA-CNT. Biocompatibility study of HA-BNNT shows positive influence of BNNT towards bone cell activity and osseointegration. These findings, along with no negative report on cytocompatibility till date, project BNNT's strong candidature as reinforcement to HA in orthopedic application. The specific conclusions on performance of HA-nanotube composites are listed below:

HA-nanotube composites (coating and free standing) could be successfully synthesized using plasma spraying and spark plasma sintering methods. Both types of nanotube reinforcement (CNT and BNNT) do not show any negative effect on densification. High thermal and electrical conductivity of the nanotubes help in improved and uniform densification of HA structure during spark plasma 
sintering. Nanotubes also help in retaining the fine HA structure by grain boundary pinning.CNT gets damaged during high pressure application in SPS, whereas BNNT withstands the high pressure and remains undamaged.

Both CNT and BNNT show favorable orientation relationship with HA interface in SPS composite, leading to coherent interface with minimal lattice mismatch. As a result of this good interfacial bonding, the nanotube pull-out energy from HA matrix becomes higher than fracture energy of monolithic HA. This leads to fracture toughening of HA with CNT/BNNT reinforcement.

Plasma sprayed HA- 4 wt.\% CNT coating shows $350 \%$ increase in fracture toughness with 4 wt.\% CNT addition. Bridging of cracks and absorption of fracture energy due to good interfacial bonding of CNT with HA matrix are the main toughening mechanisms.

HA- 4 wt. $\%$ CNT coating shows $25 \%$ decrease in coefficient of friction $(\mathrm{CoF})$ and $80 \%$ decrease in the wear volume. Higher wear resistance in HA-CNT is due to higher toughness and lubrication offered by peeled off graphene layers.

SPS processed HA-4 wt.\% CNT and HA-4 wt.\% BNNT composites show similar improvement in fracture toughness ( $92 \%$ and $86 \%$ respectively) with nanotube reinforcement.

SPS HA- 4 wt. $\%$ CNT composite shows $60 \%$ decrease in CoF, due to peeled off graphene layers. SPS HA-4 wt.\% BNNT shows $25 \%$ increase in CoF, as no h-BN 
layer peels off from BNNT surface.SPS HA-4 wt.\% CNT composite resulted in $66 \%$ improvement in wear volume less as compared to HA, whereas SPS HA-4 wt.\% BNNT displayed 75\% improvement in wear volume loss.

In-vitro biocompatibility studies for HA-CNT coating reveal non-cytotoxic and encouraging role played by $\mathrm{CNT}$ on bone forming cell activity. HA-CNT coating shows higher osteoblast proliferation rate and viability than HA surface. The cycle of osteoblast differentiation, maturation and matrix mineralization is accelerated in presence of CNT.

In-vivo exposure of HA-CNT coated implant in animal (rat) indicates active role of CNT towards integration of neobone on coated surface. No sign of CNT induced toxicity is observed.

CNT also plays a very positive role in early stages of osseointegration by offering better adhesion of osteoblasts to implant surface. The adhesion energy of osteoblasts is upto $95 \%$ higher on HA-CNT as compared to HA. Attachment of integrin on exposed CNT network is attributes towards increased osteoblast adhesion strength on HA-CNT surface.

Presence of HA-CNT coating on metallic (Ti) implant provides a smoother elastic modulus gradient at bone-implant interface, when exposed in-vivo. This smoother elastic modulus gradient reduces the stress generated at the interface and inhibits the chance of fracture of bone and failure of implant. Moreover, CNT helps in 
faster mineralization of neobone and increases its elastic modulus, as compared to neobone on HA surface.

$>\quad$ In-vitro studies show BNNT is safe for orthopedic application. Bare BNNT does not produce any cytotoxic effect, while interacting with osteoblast and macrophage.

$>\quad$ HA- 4 wt.\% BNNT surface favors osteoblast proliferation and viability more than HA.

Apatite formability of BNNT surface in physiological solution is the indication of its osseointegration ability. Apatite precipitate incubation is faster on BNNT surface (4.6 days) than on CNT (14 days) when immersed in standard SBF. 


\subsection{RECOMMENDATIONS FOR FUTURE RESEARCH}

The aim of the current research was to explore the potential of HA-nanotube composites and coatings for orthopedic application. The criteria for judgment were the effect of nanotube reinforcement on mechanical, tribological and biological behavior of the composite. The findings of this study establish HA-nanotube composites to be potential alternative for clinically used HA. However, some of the topics need further investigations to progress towards the clinical translation of HA-CNT nanotube. Following is the list of recommendations for advancing the research towards the final accomplishment.

\section{Plasma Sprayed Coating for HA-BNNT}

HA coated metallic implants are already in clinical use. Plasma sprayed HABNNT coating on metallic implants have the potential to be a substitute for HA with improved mechanical and tribological behavior. HA-BNNT coatings could not be made in the current research due to unavailability of BNNT in large amount, which is a key requirement for plasma spraying. Synthesis of BNNTs is still carried out in laboratoryscale. On-going studies by different research groups have the potential to establish a large scale production method for BNNT in the near future. Once BNNT is available in a larger quantity (e.g. 50-100 grams), HA-BNNT coatings should be synthesized by plasma spraying. 


\section{Optimizing SPS Parameters for HA-BNNT}

The present research is the first report on HA-BNNT composite. A parametric study is required to optimize the SPS parameters for consolidation of HA-BNNT composite with varying BNNT concentration, to achieve the optimum fracture toughness and wear resistance.

\section{Optimizing Nanotube Content in Composite}

The present study is carried out with a singular composition of 4 wt.\% nanotube reinforcement in HA. The nanotube content could have a significant effect on strengthening of the composite. The composite synthesized with varying nanotube content needs to be characterized for mechanical and tribological behavior to decide on the reinforcement content. Considering the orthopedic application of the composite, biocompatibility can also be a function of nanotube content in composite. Thus the nanotube content optimization process should take care of both mechanical and biological performance of the composite.

\section{Biocompatibility Evaluation for $\mathrm{HA}-\mathrm{BN} N \mathrm{~T}$}

HA-CNT is evaluated thoroughly for its biocompatibility in the present study, including in-vitro studies for osteoblast proliferation rate, viability, differentiation, mineralization and in-vivo study using rat model. HA-BNNT is also characterized for osteoblast proliferation and viability, along with cytotoxicity of BNNT to osteoblast and macrophage. However, more biocompatibility studies (both in vitro and in vivo) are required to establish HA-BNNTcomposites for orthopedic application. With increasing 
availability of BNNTs, it would be possible to perform extensive biocompatibility evaluation for HA-BNNT in near future.

\section{Effect of Composite Microstructure and Surface on Biocompatibility}

The present research has carried out biocompatibility studies on HA-CNT coating surface and not on SPS synthesized composite. The aim of this study was to determine if CNT reinforcement alters the biocompatibility of composite. From that point of view, both plasma sprayed coating and spark plasma sintered composite was equivalent, owing to same CNT content ( $4 \mathrm{wt. \%})$. But, the microstructure, density and surface properties are very different in both the structures, which might have differential effect on biocompatibility, especially for osteoblast adhesion and neobone formation. It is recommended to perform a comparative evaluation of biocompatibility for plasma sprayed and SPS processed HA-nanotube surfaces.

\section{Longer In-vivo Exposure of Composite}

The present study has carried out one month in-vivo exposure of HA-CNT coated implants, which suggest no cytotoxic effect of CNT. But, in real life, the implants stay inside living body for $>15$ years in most of the cases. It is therefore important to assess the fate of HA-CNT composites, especially CNTs, for longer in-vivo exposure, to assure their safe use. A systematic study of implanting HA-nanotube composite in animal model for longer exposure period ( 3 months, 6 months and 1 year) followed by histocompatibility studies is recommended. 


\section{APPENDICES}

\section{Appendix-1}

The Biocompatibility tests for Hydroxyapatite (HA) -nanotube coatings conducted in this research work are in compliance with ISO and ASTM guidelines referred by FDA [1-8]. The brief is as following:

ISO : 10993 - “Biological evaluation of Medical Devices” - is meant for testing any medical device thoroughly before marketing.

\begin{tabular}{|c|c|c|}
\hline $\begin{array}{c}\text { ISO } \\
\text { Standard ID }\end{array}$ & Purpose & Our Testing Methods \\
\hline $10993-5$ & $\begin{array}{l}\text { Test for cytotoxicity } \\
\text { - In Vitro }\end{array}$ & $\begin{array}{l}\text { Using HA-Nanotube coating/free standing body } \\
\text { as substrate and human osteoblast cell line-- } \\
\text { - viability (FDA/PI staining), } \\
\text { - Actin Fiber staining, } \\
\text { - Genetic expression for osteopontin, } \\
\text { osteocalcin and RunX2, } \\
\text { - Alkaline phosphatase activity } \\
\text { - BrDU Analysis for cell proliferation rate } \\
\text { - Adhesion strength of osteoblasts on } \\
\text { Wear Debris of HA-CNT in medium and } \\
\text { osteoblast and macrophage cell lines } \\
\text { - Cytotoxicity using LDH assay }\end{array}$ \\
\hline $10993-6$ & $\begin{array}{l}\text { Test for local effect } \\
\text { after implantation }\end{array}$ & $\begin{array}{l}\text { Using HA-CNT coated Ti alloy beads and rods } \\
\text { in rodents } \\
\text { - Implanted for one month and then the } \\
\text { implanted bone is retrieved } \\
\text { - Histology and calcium staining to check } \\
\text { bone formation } \\
\text { - TEM to observe nature of new bone and } \\
\text { its comparison with previous one } \\
\text { - Immune response } \\
\text { - To study the mechanical compliance of } \\
\text { implant with bone by mapping elastic } \\
\text { modulus at implant-bone interface }\end{array}$ \\
\hline
\end{tabular}


According to FDA report on "Guidance Document for Testing Orthopedic implants" -

"Material combinations with limited or no history of successful use in orthopedic implants must be determined to exhibit an acceptable biological response equal or better than approved or substantially equivalent devices when tested by ASTM F 748, ASTM F 981, and an animal implant study in which the tissue response to the modified surface ..."

Following is a summary of the tests that complies with the directives in the relevant ASTM Standards

\begin{tabular}{|c|c|c|}
\hline $\begin{array}{c}\text { ASTM } \\
\text { Standard }\end{array}$ & Purpose & Our Testing Methods \\
\hline $\mathrm{F}-748$ & $\begin{array}{l}\text { Standard practice for } \\
\text { selecting generic biological } \\
\text { test methods for materials } \\
\text { and device } \\
\text { - Biocompatibility testing } \\
\text { involves tests either the } \\
\text { material itself or an extract } \\
\text { from it - depending on the } \\
\text { nature of end-use application } \\
\text { - cell culture cytotoxicity }\end{array}$ & $\begin{array}{l}\text { Using HAQ-Nanotube coating/free } \\
\text { standing body as substrate and human } \\
\text { osteoblast cell line- } \\
\text { - viability (FDA/PI staining), } \\
\text { - Actin Fiber staining, } \\
\text { - Genetic expression for osteopontin, } \\
\text { osteocalcin and RunX2, } \\
\text { - Alkaline phosphatase activity } \\
\text { - BrDU Analysis for cell proliferation } \\
\text { rate } \\
\text { - Adhesion strength of osteoblasts on } \\
\text { substrate }\end{array}$ \\
\hline F - 1903 & $\begin{array}{l}\text { Standard practice for testing } \\
\text { for biological responses to } \\
\text { particles in vitro } \\
\text { - to define particles nature, } \\
\text { source, chemistry, size, } \\
\text { shape, method of } \\
\text { sterilization, concentration } \\
\text { (in medium) } \\
\text { - To use established cell } \\
\text { lines, culture conditions, } \\
\text { cell viability assay }\end{array}$ & $\begin{array}{l}\text { Wear Debris of HA-CNT in } \\
\text { recommended medium for osteoblast and } \\
\text { macrophage cell lines } \\
\text { - Debris analysis for composition } \\
\text { - Chemistry known from vendor } \\
\text { - Phases by XRD } \\
\text { - Compound by Raman } \\
\text { - } \quad \text { spectroscopy } \\
\text { and shape } \\
\text { - SEM Image Analysis } \\
\text { Cytotoxicity for osteoblasts and } \\
\text { macrophages using LDH assay }\end{array}$ \\
\hline F - 981 & $\begin{array}{l}\text { Standard practice for } \\
\text { assessment of compatibility } \\
\text { of biomaterials for surgical } \\
\text { implants with respect to }\end{array}$ & $\begin{array}{l}\text { Using HA-CNT coated Ti alloy beads } \\
\text { and rods in rodents } \\
\text { - Implanted for one month and then } \\
\text { the implanted bone is retrieved }\end{array}$ \\
\hline
\end{tabular}




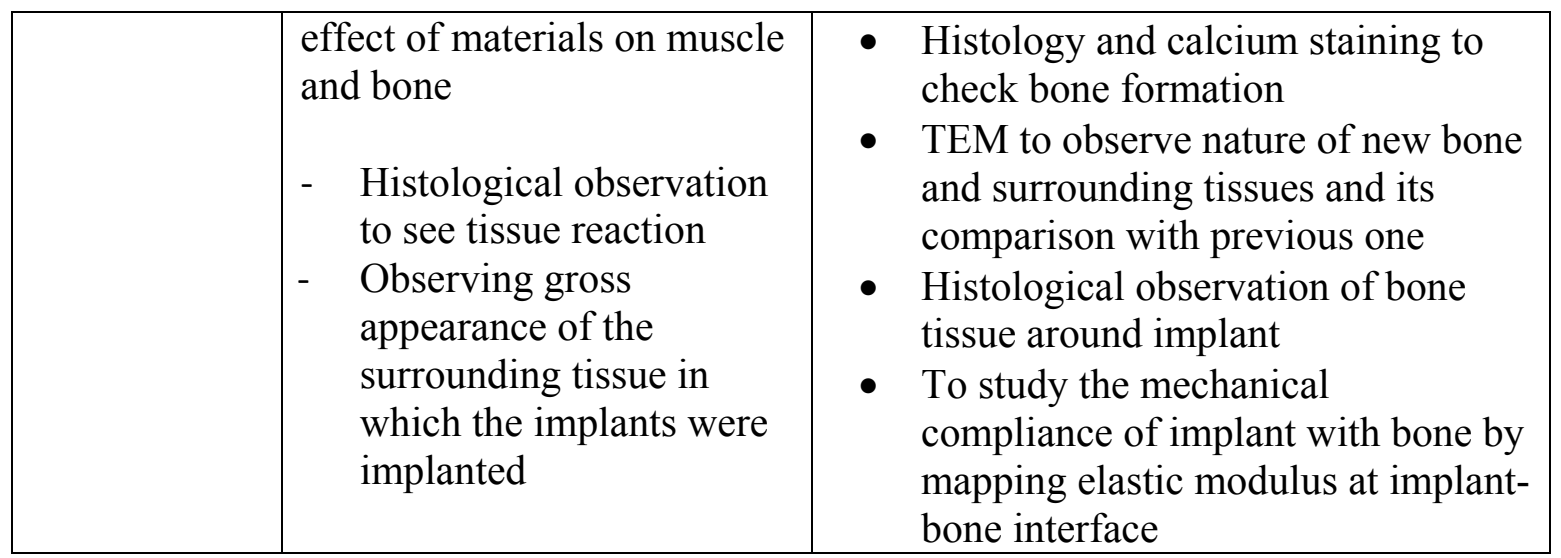

\section{References}

1. G. Kotzar, M. Freas, P. Abel, A. Fleischman, S. Roy, C. Zorman, J.M. Moran, J. Melzak, Evaluation of MEMS materials of construction for implantable medical devices, 2002, Biomaterials, Vol. 23, pp. 2737-2750.

2. http://www.fda.gov/medicaldevices/deviceregulationandguidance/guidancedocum ents/ucm081034.htm

3. http://www.astm.org/Standards/medical-device-and-implant-standards.html

4. http://www.iso.org/iso/iso_catalogue/catalogue_tc/catalogue_detail.htm?csnumber $=36406$

5. http://www.iso.org/iso/iso_catalogue/catalogue_tc/catalogue_detail.htm?csnumb er $=44789$

6. ASTM F748 - 06(2010) Standard Practice for Selecting Generic Biological Test Methods for Materials and Devices, In: ASTM Volume 13.01 Medical and Surgical Materials and Devices, pp. 298-305.

7. ASTM F1903 - 10 Standard Practice for Testing for Biological Responses to Particles In Vitro, In: ASTM Volume 13.01 Medical and Surgical Materials and Devices, pp. 936-939.

8. Standard Practice for Assessment of Compatibility of Biomaterials for Surgical Implants with Respect to Effect of Materials on Muscle and Bone, In: ASTM Volume 13.01 Medical and Surgical Materials and Devices, pp. 375-379. 


\section{Appendix-2}

\section{Major Quantitative Techniques for Cell Adhesion Measurement}

\begin{tabular}{|c|c|c|c|c|}
\hline Technique & Principle & Results (Quantification) & Remarks & References \\
\hline $\begin{array}{l}\text { X-ray } \\
\text { Microcomputed } \\
\text { Tomography }\end{array}$ & $\begin{array}{l}\text { Assessment of 3D } \\
\text { cellular Volume }\end{array}$ & $\begin{array}{l}\text { Adhesion is defined to be } \\
\text { more if cellular volume is } \\
\text { high }\end{array}$ & $\begin{array}{l}\text { - Does not provide the strength/force } \\
\text { for detachment. } \\
\text { - Good for a comparison between } \\
\text { different substrates }\end{array}$ & {$[1]$} \\
\hline Cellular Density & $\begin{array}{l}\text { Measurement of cellular } \\
\text { density in terms of } \\
\text { Number of cells/Area }\end{array}$ & $\begin{array}{l}\text { Adhesion is defined to be } \\
\text { more if the cell density is } \\
\text { high }\end{array}$ & $\begin{array}{l}\text { Does not provide the strength/force } \\
\text { for detachment. } \\
\text { - Good for a comparison between } \\
\text { different substrates }\end{array}$ & {$[2-4]$} \\
\hline Spinning disk & $\begin{array}{l}\text { Cell on the top of } \\
\text { surface is spinned - this } \\
\text { is done basically to } \\
\text { measure the adhesion } \\
\text { with change in shear } \\
\text { force }\end{array}$ & $\begin{array}{l}\text { Number of detached cells } \\
\text { as a function of distance } \\
\text { from centre along radius is } \\
\text { determined - Calculation of } \\
\text { Shear force as a function of } \\
\text { distance from centre gives } \\
\text { force required to detach a } \\
\text { cell }\end{array}$ & $\begin{array}{l}\text { - It is still a comparative one and is not } \\
\text { measured for a single cell }\end{array}$ & {$[5-7]$} \\
\hline $\begin{array}{l}\text { Centrifugal force- } \\
\text { based adhesion } \\
\text { assay }\end{array}$ & $\begin{array}{l}\text { Measure adhesion } \\
\text { strength by centrifuging } \\
\text { attached cells and } \\
\text { finding out the separated } \\
\text { ones }\end{array}$ & Expressed as force unit/cell & $\begin{array}{l}\text { - It is still a comparative one and is } \\
\text { generally expressed as \% of cells } \\
\text { detached or Adhesion Strength / } \\
\text { Unit area of substrate } \\
\text { - The force received by a single cell } \\
\text { across its surface is non-uniform and } \\
\text { its magnitude is unknown }\end{array}$ & {$[8-11]$} \\
\hline $\begin{array}{l}\text { Optical/laser } \\
\text { trapping }\end{array}$ & $\begin{array}{l}\text { Generally for proteins or } \\
\text { molecules attached on } \\
\text { cells. } \\
\text { Filament-bound bead - }\end{array}$ & $\begin{array}{l}\text { The bead being displaced } \\
\text { from tap center - the force } \\
\text { in the trap changes - which } \\
\text { is measurable. }\end{array}$ & $\begin{array}{l}\text { Very low forces measured and very } \\
\text { specific to materials that could be } \\
\text { stained and trapped. } \\
\text { - Mainly used for proteins and other }\end{array}$ & {$[12-14]$} \\
\hline
\end{tabular}




\begin{tabular}{|c|c|c|c|c|}
\hline & $\begin{array}{l}\text { held by the optical trap } \\
\text { and the substrate is } \\
\text { moved in the other } \\
\text { direction. }\end{array}$ & & biomolecules. & \\
\hline Micropipette suction & $\begin{array}{l}\text { Suction of cell by } \\
\text { reducing the pressure of } \\
\text { fluid/environment in the } \\
\text { micropipette. }\end{array}$ & $\begin{array}{l}\text { The adhesion force is } \\
\text { measured in terms of drag } \\
\text { force which is related to the } \\
\text { drop of pressure required } \\
\text { inside micropipette - } \\
\text { measured using pressure } \\
\text { transducer. }\end{array}$ & $\begin{array}{l}\text { - This technique can measure adhesion } \\
\text { strength of a single cell varying in } \\
\text { pN to } \mathrm{nN} \text { range. } \\
\text { - But, posses high chance of rupture or } \\
\text { damage of cells. }\end{array}$ & [15-20] \\
\hline $\begin{array}{l}\text { Flow Shearing - } \\
\text { Hydrodynamic force }\end{array}$ & $\begin{array}{l}\text { Hydrodynamic force is } \\
\text { used through the flow of } \\
\text { liquid to detach cells } \\
\text { from substrate and } \\
\text { assisted by microscopy } \\
\text { to observe when the } \\
\text { cells separate out. }\end{array}$ & $\begin{array}{l}\text { Measured as \% adherent } \\
\text { cells or shear strength/unit } \\
\text { area }\end{array}$ & $\begin{array}{l}\text { - A very popular technique } \\
\text { - Adhesion strength of a single cell can } \\
\text { be assessed by finding the total } \\
\text { adhesion area of a cell from } \\
\text { microscopy. } \\
\text { - Not absolutely/accurately quantifies } \\
\text { the adhesion strength of a single cell. }\end{array}$ & [21-30] \\
\hline AFM & $\begin{array}{l}\text { Cantilever without tip } \\
\text { sits on the cell for } \\
\text { attachment and then } \\
\text { pulls off }\end{array}$ & $\begin{array}{l}\text { The adhesion is measured } \\
\text { from cantilever deflection } \\
\text { (AFM principle) as } \\
\text { detachment force required } \\
\text { for a single cell }\end{array}$ & $\begin{array}{l}\text { - Very suitable technique for } \\
\text { measurement of adhesion strength } \\
\text { for single cell. } \\
\text { - The tip has to sit on cell surface for } \\
\text { attachment - which might take a very } \\
\text { long time in some cases. } \\
\text { - Sitting of cantilever on cell for long } \\
\text { time causes extra stress on cell which } \\
\text { can deviate them from normal } \\
\text { behavior and cause damage also. }\end{array}$ & [31-34] \\
\hline $\begin{array}{l}\text { Confocal reflectance } \\
\text { interference contrast } \\
\text { microscopy }\end{array}$ & $\begin{array}{l}\text { Uses phase contrast } \\
\text { microscopy image } \\
\text { (confocal microscopy) } \\
\text { and C-RIM Images } \\
\text { (laser scanning confocal }\end{array}$ & $\begin{array}{l}\text { Strong adhesion contact of } \\
\text { an adherent cell appears as } \\
\text { a dark or light gray region } \\
\text { in the gray background on } \\
\text { the C-RICM image. }\end{array}$ & $\begin{array}{l}\text { - The adhesion strength measurement } \\
\text { is dependent on the color contrast - } \\
\text { thus calibration is very crucial and } \\
\text { can easily pose large error in } \\
\text { estimation. }\end{array}$ & {$[35-37]$} \\
\hline
\end{tabular}




\begin{tabular}{|c|c|c|c|c|}
\hline & $\begin{array}{l}\text { microscopy). } \\
\text { Color contrast obtained } \\
\text { with change in adhesion } \\
\text { energy, strong contact } \\
\text { zone appears as dark } \\
\text { region on the image }\end{array}$ & $\begin{array}{l}\text { Expresses the adhesion } \\
\text { energy in } \mathrm{J} / \mathrm{m}^{2} \text {. }\end{array}$ & $\begin{array}{l}\text { For calculation of adhesion energy, } \\
\text { many factors like elastic property } \\
\text { and exact thickness of cell membrane } \\
\text { is required. }\end{array}$ & \\
\hline Cyto Detacher & $\begin{array}{l}\text { Applies detaching force } \\
\text { parallel to the base of } \\
\text { single cell through a } \\
\text { cantilever and measures } \\
\text { the resistive force by cell } \\
\text { during movement }\end{array}$ & $\begin{array}{l}\text { The deflection of the } \\
\text { cantilever due to resistance } \\
\text { of cells to detach is } \\
\text { measured by conjugate } \\
\text { optical technique (carbon } \\
\text { filament, optical } \\
\text { microscope and } \\
\text { photodiode) and converted } \\
\text { to adhesion strength }\end{array}$ & $\begin{array}{l}\text { - Suitable technique for measurement } \\
\text { of adhesion strength for single cell. } \\
\text { - Accurate measurement of small } \\
\text { deflection in cantilever, using the } \\
\text { conjugate system of carbon filament } \\
\text { (glued at the back of cantilever), } \\
\text { optical microscope and photodiode, } \\
\text { is challenging and requires custom } \\
\text { made set-up. } \\
\text { - Force is not measured directly - } \\
\text { calibration of cantilever deflection is } \\
\text { very crucial and can be possible } \\
\text { source of error. } \\
\text { - During pushing the cell - cantilever } \\
\text { immersed in liquid medium } \\
\text { experiences resistance force from } \\
\text { fluid - adds an error term in cell } \\
\text { adhesion force value }\end{array}$ & [38-39] \\
\hline $\begin{array}{l}\text { Shear Force by } \\
\text { Cantilever }\end{array}$ & $\begin{array}{l}\text { Application of shear } \\
\text { force parallel to } \\
\text { substrate to detach the } \\
\text { cell }\end{array}$ & $\begin{array}{l}\text { Force is measured by } \\
\text { monitoring cantilever } \\
\text { deflection in opposite } \\
\text { direction of applied force } \\
\text { due to resistance by cell } \\
\text { during detachment }\end{array}$ & $\begin{array}{l}\text { - A suitable technique for } \\
\text { measurement of adhesion strength } \\
\text { for single cell. } \\
\text { - Force is not measured directly - } \\
\text { calibration of cantilever deflection is } \\
\text { very crucial and can be possible } \\
\text { source of error. } \\
\text { - During pushing the cell - cantilever } \\
\text { immersed in liquid medium }\end{array}$ & [40-42] \\
\hline
\end{tabular}




\begin{tabular}{|l|l|l|l|l|}
\hline & & $\begin{array}{l}\text { experiences resistance force from } \\
\text { fluid - adds an error term in cell } \\
\text { adhesion force value }\end{array}$ & \\
\hline
\end{tabular}




\section{References}

1. S.M. Dorsey, S. Lin-Gibson, C.G. Simon, Jr. X-ray microcomputed tomography for the measurement of cell adhesion and proliferation in polymer scaffolds. 2009, Biomaterials, Vol. 30, pp. 2967-2974.

2. P. Krsko, T.E. McCann, T.T. Thach, T.T. Laabs, H.M. Geller, M.R. Libera. Lengthscale mediated adhesion and directed growth of neural cells by surface-patterned poly(ethylene glycol) hydrogels. 2009, Biomaterials, Vol. 30, pp. 721-729.

3. F. Iwasa, N. Hori, T. Uneo, H. Minamikawa, M. Yamada, T. Ogawa. Enhancement of osteoblast adhesion to UV-photofunctionalized titanium via an electrostatic mechanism. 2010, Biomaterials, Vol. 31, pp. 2717-2727.

4. L.K. Randeniya, A. Bendavid, P.J. Martin, M.S. Amin, R. Rohanizadeh, F. Tang, J.M. Cairney. Thin-film nanocomposites of diamond-like carbon and titanium oxide; Osteoblast adhesion and surface properties. 2010, Diamond Related Mater., Vol. 19, pp. 329-335.

5. A.J. García, P.Ducheyne, D. Boettiger. Effect of surface reaction stage on fibronectin-mediated adhesion of osteoblast-like cells to bioactive glass. 1998, J. Biomed. Mater. Res., Vol. 40, pp. 48-56.

6. A.J. García, P.Ducheyne, D. Boettiger. Quantification of cell adhesion using a spinning disc device and application to surface-reactive materials. 1997, Biomaterials, Vol. 18, pp. 1091-1098.

7. N.D. Gallant, K.E. Michael, A.J. Garcia. Cell Adhesion Strengthening: Contributions of Adhesive Area, Integrin Binding, and Focal Adhesion Assembly. 2005, Molecular Biol. Cell, Vol. 16, pp. 4329-4340.

8. M.M. Lotz, C.A.Burdsal, H.P. Erickson,D.R. McClay. Cell Adhesion to Fibronectin and Tenascin: Quantitative Measurements of Initial Binding and Subsequent Strengthening Response. 1989, J. Cell Biol., Vol. 109, pp. 1795-1805.

9. J.W. Piper, R.A.Swerlick, C. Zhu. Determining Force Dependence of TwoDimensional Receptor-Ligand Binding Affinity by Centrifugation. 1998, Biophys. J., Vol. 74, pp. 492-513.

10. S. Qi, C.Yi, S. Ji, C.C. Fong, M. Yang. Cell Adhesion and Spreading Behavior on Vertically Aligned Silicon Nanowire Arrays. 2009, Appl. Mater. Int., Vol. 1, pp. 3034.

11. C.D. Reyes, A.J. Garcia. A centrifugation cell adhesion assay for high-throughput screening of biomaterial surfaces. 2003, J. Biomed. Mater. Res. A, Vol. 67A, pp. 328-333. 
12. M. Hidetake, Y.Ryohei, K. Kinosita Jr. Strength and lifetime of the bond between actin and skeletal muscle alpha-actinin studied with an optical trapping technique. 1996, Biochimica et Biophysica Acta, Vol. 1290, pp. 83-88.

13. L.J. Rinko, M.B.Lawrence, W.H. Guilford. The Molecular Mechanics of P- and LSelectin Lectin Domains Binding to PSGL-1. 2004, Biophys. J., Vol. 86, pp. 544554.

14. P.H. Tsang, G. Li, Y.V. Brun, J.X. Tang. Adhesion of single bacterial cells in the micronewton range. 2006, Proc. Natl. Acad. Sci. Am., Vol. 103, pp. 5764-5768.

15. E. Evans, D. Berk, A. Leung. Detachment of agglutinin-bonded red blood cells 1. Forces to rupture molecular-point attachments. 1991, Biophys. J., Vol. 59, pp. 838848.

16. E. Evans, K.Ritchie, R. Merkel. Sensitive force technique to probe molecular adhesion and structural linkages at biological interfaces. 1995, Biophys. J., Vol. 68 , pp. $2580-2587$.

17. H.J. Ra, C.Picart, H. Feng, H. L. Sweeney, D.E. Discher. Muscle cell peeling from micropatterned collagen: direct probing of focal and molecular properties of matrix adhesion. 1999, J. Cell Sci., Vol. 112, pp. 1425-1436.

18. M.A. Griffin, A.J.Engler, T.A. Barber, K.E. Healy, H.L. Sweeney, D.E. Discher. Patterning, Prestress, and Peeling Dynamics of Myocytes. 2004, Biophys. J., Vol. 86, pp. $1209-1222$.

19. Z. Gao, S.Wang, H. Zhu, C. Su, G. Xu, X. Lian. Using selected uniform cells in round shape with a micropipette to measure cell adhesion strength on silk fibroinbased materials. 2008, Mater. Sci. Eng. C, Vol. 28, pp. 1227-1235.

20. V. M. Bowers, L.R. Fisher, G.M. Francis, K.L. Williams. A Micromechanical technique for monitoring cell-substrate adhesiveness: mesaurements of the strength of a red blood cell adhesion to glass and polymer test surfaces. 1989, J. Biomed. Mater. Res., Vol. 23, pp. 1453-1473.

21. S.P. Tha, J. Shuster, H.L. Goldsmith. Interaction forces between red cells agglutinated by antibody. 1986, Biophys. J., Vol. 50, pp. 1117-1126.

22. R. Alon, D.A. Hammer, T.A. Springer. Lifetime of the P-selectine carbohydrate bond and its response to tensile force in hydrodynamic flow. 1995, Nature, Vol. 374, pp. 539-542.

23. M. Kusakabe, Y. Suzuki, A. Nakao, M. Kaibara, M. Iwaki, M. Scholl. Control of endothelial cell adhesion to plymer surface by ion implantation. 2001, Polym. Adv. Technol., Vol. 12, pp. 453-460. 
24. G. Fu, C.Milburn, S. Mwenifumbo, Y. Cao, G.M. Oparinde, M.O. Adeoye, C. Therialt, A.C. Beye, W.O. Soboyejo. Shear assay measurements of cell adhesion on biomaterials surfaces. 2009, Mater. Sci. Eng. C, Vol. 29, pp. 1293-1301.

25. C. Milburn, J.Chen, Y. Cao, G.M. Oparinde, M.O. Adeoye, A. Beye, W.O. Soboyejo. Investigation of effects of Argenine-Glycine-Aspartate (RGD) and nanoscale titanium coatings on cell spreading and adhesion. 2009, Mater. Sci. Eng. C, Vol. 29, pp. 306-314.

26. A. Rezania, C.H. Thomas, K.E. Healy. A probabilistic approach to measure the strength of bone cell adhesion to chemically modified surfaces. 1997, Annals Biomed. Eng., Vol. 25, pp. 190-203.

27. P. Zortullana, Z. Rong, P. Vadgama, V. Hasirci. Influence of nanopatterns on endothelial cell adhesion: Enhanced cell retention under shear stress. 2009, Acta Biomater., Vol. 5, pp. 2451-2459.

28. A.S. Goldstein, P.A. DiMilla. Effect of adsorbed fibronectin concentration on cell adhesion and deformation under shear on hydrophobic surfaces. 2002, J. Biomed. Mater. Res., Vol. 59, pp. 665-675.

29. E. decave, D. Garrivier, Y. Brechet, B. Fourcade, F. Bruckert. Shear fFlow-induced detachment kinetics of dictyostelium discoideum cells from solid substrate. 2002, Biophys. J., Vol. 82, pp. 2383-2395.

30. C.J. Pendegrass, C.A. Middleton, D. Gordon, J. Jacob, G.W. Blunn. Measuring the strength of dermal fibroblast attachment to functionalized titanium alloys in vitro. 2010, J. Biomed. Mater. Res. A, Vol. 92A, pp. 1028-1037.

31. X. Zhang, V.T. Moy. Cooperative adhesion of ligand-receptor bonds. 2003, Biophys. Chem., Vol. 104, pp. 271-278.

32. P. Hinterdorfer, W.Baumgartner, H.J. Gruber, K. Schilcher, H. Schindle. Detection and localization of individual antibody-antigen recognition events by atomic force microscopy. 1996, Proc. Natl. Acad. Sci., Vol. 93, pp. 3477-3481.

33. F. Li, S.D.Redick, H.P. Erickson. Force Measurements of the a5b1 IntegrinFibronectin Interaction. V.T. Moy. 2003, Biophys. J., Vol. 84, pp. 1252-1262.

34. G. Weder, O. Guillaume-Gentil, N. Matthey, F. Montagne, H. Heinzelmann. The quantification of single cell adhesion on functionalized surfaces for cell sheet engineering. 2010, Biomaterials, Vol. 31, pp. 6436-6443.

35. C. Yin, K.Liao, H.Q. Maoa, K.W. Leong, R.X. Zhuo,Vincent Ch. Adhesion contact dynamics of HepG2 cells on galactose-immobilized substrates. 2003, Biomaterials, Vol. 24, pp. 837-850. 
36. N. Cai, C.C. Wong, Y.X. Gong, S.C.W. Tan, K. Liao. Modulating cell adhesion dynamics on carbon nanotube monolayer engineered with extracellular matrix proteins. 2010, ACS Appl. Mater. Inetrfaces, Vol. 2, pp. 1038-1047.

37. A. Ranella, M. Barberoglou, S. Bakogianni, C. Fotakis, E. Stratakis. Tuning cell adhesion by controlling the roughness and wettability of $3 D$ micro/nano silicon structures. 2010, Acta Biomater., Vol. 6, pp. 2711-2720.

38. K.A. Athanasiou, B.S. Thoma, D.R. Lanctot, D. Shin, C.M. Agrawa!, R.G. LeBaron. Development of the cytodetachment technique to quantify mechanical adhesiveness of the single cell. 1999, Biomaterials, Vol. 20, pp. 2405-2415.

39. W. Huang, B. Anvari, J. H. Torres, R.G. LeBaron, K.A. Athanasiou. Temporal effects of cell adhesion on mechanical characteristics of the single chondrocyte. 2003, J. Orthopedic Res., Vol. 21, pp. 88-95.

40. A. Yamamoto, S. Mishima, N. Maruyama, M. Sumita. A new technique for direct measurement of the shear force necessary to detach a cell from a material. 1998, Biomaterials, Vol. 19, pp. 871-879.

41. M. Yamada, T. Miyauchi, A. Yamamoto, F. Iwasa, M. Takeuchi, M. Anpo, K. Sakurai. Enhancement of adhesion strength and cellular stiffness of osteoblasts on mirror-polished titanium surface by UV-photofunctionalization. 2010, Acta Biomater., Vol. 6, pp. 4578-4588.

42. A. Yamamoto, S. Mishima, N. Maruyama, M. Sumita. Quantitative evaluation of cell attachment to glass, polystyrene, and fibronectin- or collagen-coated polystyrene by measurement of cell adhesive shear force and cell detachment energy. 2000, J. Biomed. Mater. Res., Vol. 50, pp. 114-124. 


\section{Appendix-3}

This section provides a list of publications in peer-reviewed journals during the current

$\mathrm{PhD}$ research.

\section{JOURNAL ARTICLES (Published/Accepted)}

1. D. Lahiri, A.P. Benaduce, L. Kos, A. Agarwal, "Quantification of Carbon Nanotube Induced Adhesion of Osteoblast on Hydroxyapatite using Nano-Scratch Technique”, Accepted for publication in Nanotechnology 2011 (Journal Impact Factor: 3.644).

2. D. Lahiri, V. Singh, A.K. Keshri, S. Seal, A. Agarwal, "Apatite formability of boron nitride nanotube", Nanotechnology, Vol. 22, 2011, pp. 205601 (Journal Impact Factor: 3.644).

3. I. Lahiri", D. Lahiri*, S. Jin, A. Agarwal, W. Choi, "Carbon Nanotubes: How Strong is Their Bond with the Substrate?" ACSNano, Vol. 5(2), 2011, pp. 780-787. (Journal Impact Factor: 9.855)

Highlighted in "Nanowerk" (http://www.nanowerk.com/spotlight/spotid=19707.php).

\section{* Co-first Authors.}

4. S. Facca*, D. Lahiri*, F. Fioretti, N. Messadeq, D. Mainard, N. Jessel, A. Agarwal, "In Vivo Osseointegration of Nano-Designed Composite Coatings on Titanium Implants", ACS Nano, 2011, Vol. 5, pp. 4790-4799 (Journal Impact Factor: 9.855).

* Co-first Authors.

5. D. Lahiri, V. Singh, A.P. Benaduce, S. Seal, L. Kos, A. Agarwal, "Boron nitride nanotube reinforced hydroxyapatite composite: mechanical and tribological performance and in-vitro biocompatibility to osteoblasts", Journal of the Mechanical Behavior of Biomedical Materials, Vol. 4, 2011, pp. $44-56$ (Journal Impact Factor: 3.297).

6. D. Lahiri, A.P. Benaduce, F. Rouzaud, J. Solomon, A.K. Keshri, L. Kos, A. Agarwal, "Wear Behavior and In-vitro Cytotoxicity of Wear Debris Generated from Hydroxyapatite-Carbon Nanotube Composite Coating" Journal of Biomedical Materials Research Part A, Vol. 96A, 2011, pp. 1-12. (This Paper has been cited in the website of 'The International Council on Nanotechnology' and included in 'nanoEHS Virtual Journal'.) (Journal Impact Factor: 3.044) 
7. A.K. keshri, D. Lahiri, A. Agarwal, "Carbon nanotubes improve the adhesion strength of a ceramic splat to the steel substrate", Carbon, 2011, DOI: 10.1016/j.carbon.2011.06.010. (Journal Impact Factor: 4.893)

8. K. Balani, R.R Patel, A.K. Keshri, D. Lahiri, A. Agarwa, "Multi-scale Hierarchy of Chelydra serpentina: Microstructure and Mechanical Properties of Turtle Shell", Journal of the Mechanical Behavior of Biomedical Materials, 2011, DOI: 10.1016/j.jmbbm.2011.05.014. (Journal Impact Factor: 3.297)

9. H. Couvy, D. Lahiri, J. Chen, A. Agarwal, G. Sen, "Nano-hardness and Young's modulus of nano-polycrystalline diamond", Scripta Materialia, 2011, Vol. 64, pp. 1019-1022. (Journal Impact Factor: 2.806)

10. S.R. Bakshi, V. Musharamthota, D.A. Virzi, A.K. Keshri, D. Lahiri, V. Singh, S. Seal, A. Agarwal, "Spark plasma sintered tantalum carbide-carbon nanotube composite: effect of pressure, carbon nanotube length and dispersion technique on microstructure and mechanical properties", Material Science and Engineering A, 2011, Vol. 528, 2538-2547. (Journal Impact Factor: 2.090)

11. S.R. Bakshi, V. Musaramthota, D. Lahiri, V. Singh, S. Seal, A. Agarwal, "Spark Plasma Sintered Tantalum Carbide: Effect of Pressure and nano-Boron Carbide Addition on Microstructure and Mechanical properties", Materials Science and Engineering A, Vol. 528, 2011, pp. 1287-1295. (Journal Impact Factor:2.090)

12. K.K. Jha, N. Suksawang, D. Lahiri, A. Agarwal, "Energy Based Analysis of Nanoindentation Curves for Cementitious Materials", Submitted to ACI Materials Journal on 28 June, 2011. . (Journal Impact Factor: 1.023)

13. D. Lahiri, V. Singh, A. K. Keshri, S. Seal, A. Agarwal, "Carbon Nanotube Toughened Hydroxyapatite by Spark Plasma Sintering: Microstructural Evolution and Multi-Scale Tribological Properties", Carbon, Vol. 48, 2010, pp. 3103-3120. (Journal Impact Factor: 4.893)

14. D. Lahiri, F. Rouzaud, T. Richard, A. K. Keshri, S.R. Bakshi, L. Kos, A. Agarwal, "Boron Nitride Nanotube Reinforced Polylactide-Polycaprolactone Copolymer Composite: Mechanical Properties and Cytocompatibility with Osteoblasts and Macrophages In Vitro", Acta Biomaterialia, Vol. 6, 2010, pp. 3524-3533. (This Paper has been cited in the website of 'The International Council on Nanotechnology' and included in 'nanoEHS Virtual Journal' http://icon.rice.edu/details.cfm?rid=48335). (Journal Impact Factor: 4.822)

15. S.R. Bakshi, D. Lahiri, R.R. Patel, A. Agarwal, "Nanoscratch behavior of carbon nanotube reinforced aluminum coatings", Thin Solid Films, Vol. 518, 2010, pp. 1703-1711. (Journal Impact Factor: 1.909) 
16. S. R. Bakshi, D. Lahiri, A. Agarwal, "Carbon Nanotube Reinforced Metal Matrix Composite - A Review”, International Materials Review, Vol. 55, 2010, pp. 41-64. (Journal Impact Factor: 4.857)

17. S. Goenka, S. Kalmodia, T. Laha, D. Lahiri, B. Basu, K. Balani, "In vitro biocompatibility of spark plasma sintered hydroxyapatite-aluminum oxide-carbon nanotube composite" Material Science and Engineering C, Vol. 30, 2010, pp. 1162-1169. (Journal Impact Factor: 2.178)

18. K. Balani, S.R. Bakshi, D. Lahiri, A. Agarwal, "Grain Growth Behavior of Aluminum Oxide Reinforced with Carbon Nanotubes During Plasma Spraying and Post-Spray", International Journal of Applied Ceramic Technology, Vol. 7, 2010, pp. 846-855. (Journal Impact Factor: 1.627)

19. D. Lahiri, F. Rouzaud, S. Namin, A.K. Keshri, J.J. Valdes, L. Kos, N. Tsoukias, A. Agarwal, "Carbon Nanotube Reinforced Polylactide-Caprolactone Copolymer: Mechanical Strengthening and Interaction with Human Osteoblasts in Vitro", ACS Applied Materials and Interfaces, Vol. 1, 2009, pp. 2470-2476. (Journal Impact Factor: 2.925)

20. D. Lahiri, S.R. Bakshi, A. K. Keshri, Y. Liu, A. Agarwal, "Dual Strengthening Mechanism Induced by Carbon Nanotube in Roll Bonded Aluminum x Composites", Materials Science and Engineering A, Vol. 523, 2009, pp. 263-270. (Journal Impact Factor: 2.090)

21. K. Balani, R. Batista, D. Lahiri, A. Agarwal, "The Hydrophobicity of a Lotus Leaf: A Nanomechanical and Computational Approach", Nanotechnology, Vol. 20, 2009, pp. 305707 (9pp). (Journal Impact Factor: 3.644)

22. T. Laha, Y. Chen, D. Lahiri, A. Agarwal, "Tensile Properties of Carbon Nanotube Reinforced Aluminum Nanocomposite Fabricated by Plasma Spray Forming", Composite Part A, Vol. 40, 2009, pp. 589-594. (Journal Impact Factor: 2.338)

23. J. Tercero, S. Namin, D. Lahiri, K. Balani, N. Tsoukias, A. Agarwal, "Effect of Carbon Nanotube and Aluminum Oxide Addition on Plasma Sprayed Hydroxyapatite Coating's Mechanical Properties and Biocompatibility", Materials Science and Engineering C, Vol. 29, 2009, pp. 2195-2202. (Journal Impact Factor: 2.178)

24. K. Balani, D. Lahiri, A. K. Keshri, S.R. Bakshi, J.E. Tercero, A. Agarwal, "The Nano-scratch Behavior of Biocompatible Hydroxyapatite Reinforced with Aluminum Oxide and Carbon Nanotubes ", Journal of the Minerals, Metals and Materials Society, Vol. 61, 2009, pp. 63-66. (Journal Impact Factor: 1.175) 


\section{JOURNAL ARTICLES (Submitted and Under Review)}

1. A Ferrand, S Facca, C Mendoza-Palomares, D Lahiri, F Fioretti,, G Schlatter, D Mainard, A Agarwal, N Benkirane-Jessel, "Nanomechanical properties of retrieved nanofibers implants for subchondral bone regeneration", Submitted to Biomaterials on 5 July, 2011.

2. K.K. Jha, N. Suksawang, D. Lahiri, A. Agarwal, "On the use of energy based parameter in the analysis of nanoindentation data to compute mechanical properties for cementitious materials”, Submitted to Acta Materialia on 3 June, 2010.

\section{BOOK}

“Carbon Nanotubes Reinforced Metal Matrix Composites", A. Agarwal, S.R. Bakshi, D. Lahiri, Taylor and Francis Publishers, ISBN: 978-1-4398114-9-8.

\section{BOOK CHAPTER}

"Graphene Reinforced Ceramic and Metal Matrix Composites", D. Lahiri, A. Agarwal, in "Graphene: Synthesis and Applications", Editors: W. Choi and J. Lee, Taylor and Francis Publishers, ISBN: 9781439861875 (to be published in August, 2011). 


\title{
Apatite formability of boron nitride nanotubes
}

\author{
Debrupa Lahiri ${ }^{1}$, Virendra Singh ${ }^{2}$, Anup K Keshri ${ }^{1}$, Sudipta Seal ${ }^{2}$ \\ and Arvind Agarwal ${ }^{1,3}$ \\ ${ }^{1}$ Plasma Forming Laboratory, Department of Mechanical and Materials Engineering, \\ Florida International University, Miami, FL 33174, USA \\ ${ }^{2}$ AMPNC and Nanoscience Technology Center, University of Central Florida, \\ 4000 Central FL. Boullvard, AMPAC, Eng 1 Room 381, OL 32816, USA \\ E-mail: agarwala efiucedu
}

Received 14 January 2011, in final form 27 February 2011

Published 28 March 2011

Online at stacks. lop.org/ $\mathrm{Nano} / 22 / 205601$

Abstract

This study investigates the ability of boron nitride nanotubes (BNNTs) to induce apatite formation in a simulated body fluid environment for a period of 7,14 and 28 days. BNNTs, when soaked in the simulated body fluid, are found to induce hydroxyapatite (HA) precipitation on their surface. The precipitation process has an initial incubation period of $\sim 4.6$ days. The amount of HA precipitate increases gradually with the soaking time. High resolution TEM results indicated a hexagonal crystal structure of HA needles. No specific crystallographic orlentation relationship is observed between BNNT and HA, which is due to the presence of a thin amorphous HA layer on the BNNT surface that disturbs a definite orientation relationship.

(Some figures in this article are in colour only in the electronic version)

\section{Introduction}

Boron nitride nanotutes (BNNTs) are tubular and bollow nanostructures comprising two-dimensional hexagonal boron nitride (hBN) sheets. BNNTs possess an excellent elastic modulus (750-1200 $\mathrm{GP}$ [1-3]) and tensile streagth ( $>24 \mathrm{GPa}$ [4]), which makes them a potential reinforcement for strengthening and toughening of composites [5-10]. BNNTs have also shown their potential as a suitable reinforcement for bioceramics and biopolymers for orthopedic implants and scaffolds. In our previous studies, we reported the effect of BNNT reinforcement on biodegradable poly-lactidecaprolactone (PLC) copolymer and hydroxyapatite (HA) $[5,6]$. The addition of BNNT improved the tensile strength of the copolymer by the significant amount of $109 \%$. BNNT addition also resulted in an improvement in osteoblast proliferation and differentiation on PLC copolymer [5]. A similar observation was found for spark plasma sintered HA-4 wt\% BNNT composite, which displayed $86 \%$ improvement in the fracture toughness and $75 \%$ in the wear resistance of HA, while improwing osteoblast viability and proliferation [6].

An important requirement for a successful orthopedic implant is the formation of new bone on its surface for effective

${ }^{3}$ Nuthor to whom any coerespondence should be addressed

$0557-4484 / 11 / 200601+08533300$ osseointegration. During the bone formation process, a collagen fiber matrix is generated followed by the precipitation of hydroxyapatite mineral to form a compact bone [11]. An ideal implant surface should induce apatite formation without interfacial discontinuity. These discontinuities often act as weak points to cause fracture and delamination of the implant from the bone in vivo. Hence, the ability to form apatite on the BNNT surfice is critical for orthopedic application. BNNTs can also be effectively used for bone regeneration in case of a fracture or injury. BNNTs, with their excellent elastic modulus, tensile strength and high deformability [12], are capable of mimicking bone collagen fibers. A 3D scaffold of BNNTs can be used for mineralization of HA and thus regeneration of bone in vitro and in vivo. But in order to achieve this the apatite formation ability of the BNNT surface becomes the deciding factor. Beuvelot et al have proposed a similar application for carbon nanotubes (CNTs) which possess mechanical properties comparable with BNNTs [13]. CNT surfaces are found to be sultable for apatite precipitation [13-15] but their cytotoxicity is still under debate [16]. There are a few studies on the cytotoxicity of BNNTs but none has reported negative effects on different cell types $[5,6,17-19]$. Our previous study has shown that BNNTs are non-cytotoxic to osteoblasts and macrophages, which are the most important 


\title{
Carbon Nanotubes: How Strong Is Their Bond with the Substrate?
}

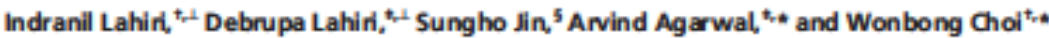

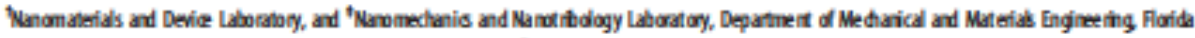

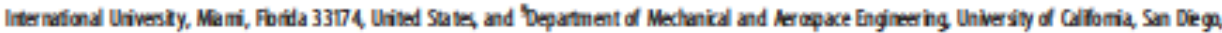

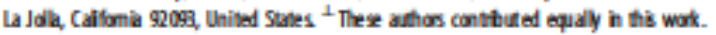

\begin{abstract}
A wide vafety of one-dimensional nanomaterials, such as nanotubes and nanowires are proposed for improved performance of a number of future devices-fromsensos to solar cells. ${ }^{1,2} \mathrm{How}$ ever, desired seamless integration, longtime, reliable pefformance, and extended device lifetime of all these nanomaterialsbased devices necessitates stability of the structure. ${ }^{3-5}$ One of the critical issues in structural stability is the bonding between the nanomaterials and the substrate A poor nanomaterial-substrate adhesion may cause unrel iable behavior and a very short life span of such a device Hence, knowledge about nature and strength of the nanostructure-substrate bond is consideredas one of the most fundamental issues. It is of utmost importance to devise a methodclogy to quantify the nanomaterial-substrate bonding, to apply that technique to understand bonding between different nanostructure-substrate bonding, uttimately leading to development of wellbonded nanostructured devices.

Among all the cne-dimensional (1-D) nanomatefiak, carbon nanotubes (CNT) are probably the most important for a myriad of potential next generabon devices, owing to their fascinating properties, such as unique structures ${ }^{6}$ excellent electronic and electical properties, ${ }^{7 /}$ highspecific strength ${ }^{2}$ good thermal conductivity, ${ }^{10}$ and dhemical inertness." Amost two decades of intense research activibes have resulted in the demonstration of various CNTbased deviaes, for example, nanoelectronics, fet emission displays, ${ }^{12,13}$ biosensors and biomatefals, ${ }^{14,15}$ and hydrogen storge andenergy generstion devices ${ }^{16-110}$ Research efforts (Supporting Information Table 51) to clarfy the nature of bonding between nanomaterials and substrates ane thus mostly restricted to CNTbased systems However, the qualitative methodsufiliad do not offer anystandardiaed
\end{abstract}

ABSTRACT A niable quanffication tech-ique fa interpefog nasonataid-ubstrate bood

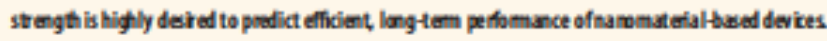
Mdopting a novd nanoseate-bed tedriqu, hare we demonsale quantfication of cabor nanotube (CMT)-sabtrate ad lesion strogth for derse OIT stucture and for patterned cabon nnocone (CNO) strudures Dabonding emeng for a single CMT is illustated to ang between 1 and

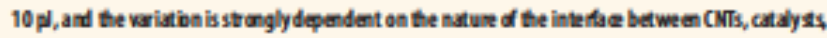

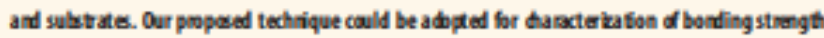
between a wile vaity of namtibes, nanowirs, and other onedineriond ranatrudured materals and their undelyeg stestrate.

KEYWORDSA nanotubes - adhesion - nanosarth - bonding energy

procedure and reproducible results, as the techniques are extremely user sensitive. The quantitative methods experimented so far havesome common ground for compa fison of adhesion strengths, though all of these techniques pose several limitations. First, none of these methods have been calibrated to single out the bonding between only CNT-substrate. Proposed quantitative methods do not show the amount of stress required to pull out the adhesive tape from a blank substate (without having CNTs grown on it.. ${ }^{14,19}$ Thus, the strength values found in these methods are often overestima ted values of exact contribution from the CNT-substrate bond. Second, the adhesion strength was calculated assuming the area of the tape as the contact area The actual contact area, where CNTs are in contact with the tape, is much lower. So, the reported values are likely to be an underestimate of the actual strength required to debond CNTs from the substrate. Third, the number of CNTs in contact with the tape was not known, and hence, none of these methods could predictadhesion strength of a single CNT. Furthermore, it was not known whether the CNTs were breaking at the CNT-substrate interface or at any defective locations along its length or if one or few 


\section{In Vivo Osseointegration of Nano- Designed Composite Coatings on Titanium Implants}

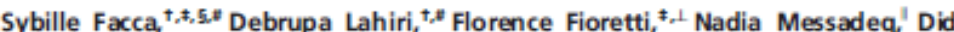
Nadia Benkirane-Jessel, ${ }^{,, 5, *}$ and Arvind Agarwal ${ }^{\dagger, *}$

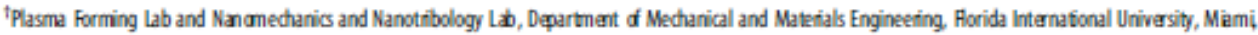

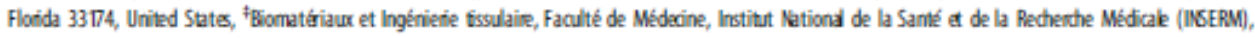
Unite 977, 11 rue Humann, 67085 Straboung Cedex, Fance, "Dqpatment of Hand Sungery, Strasbourg University Hospitds, 10 Avenue Adille Bauman, $6740 B$

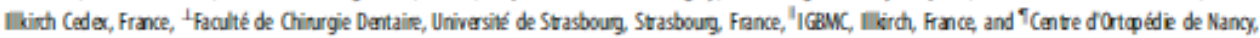
Hòpita Centrd, and UMR 7561, CNRS-Univesité Hent Poincané Nancy 1,29 Avenue du Mréchal de lattre de Taxsigny, 54000 Nancy, France. "These authos contributad equaly to this work

I ydraxyapatite (HA) is used in the biomedical industry as the coating for titanium implants. HA is also used as the structural material for dental, maxillofacial, and orthopedic implants, ${ }^{1,2}$ but HA is known to display rapid wear and failure due to poor fracture toughness. ${ }^{3}$ Therefore, problems such as loosening of arthroplasty prostheses continueto scare surgeons. Hence, there is still a large demand for the accelera. tion of new bone induction around implants for improved osseointegration.

Carbon nanotube (CNT), first reported in $1991,{ }^{4}$ is a potential reinforcement for brittle hydroxyapatite, as it exhibits high Young's modulus ${ }^{5-7}$ and tensile strength. ${ }^{8}$ CNTs are being extensively researched as reinforcement for improving the fracture toughness and wear resistance of brittle ceramics, such as $\mathrm{HA}^{9-12}$ and $\mathrm{Al}_{2} \mathrm{O}_{3} \cdot{ }^{13-15}$ Though biocompatibility of HA is in universal agreement, the same is not true for CNT. The cytotaxicity of CNT is still in debate with contradictory reports by different research groups. ${ }^{16-23}$ However, CNTs have been found to aid in accelerated bone growth and apatite precipitation and induce bonding and reinforcement with the bone matrix. ${ }^{24-27}$ In vitro studies have shown the HA-CNT composites possess improved biocompatibility with osteoblasts ${ }^{2,1028}$ and macrophages. ${ }^{10}$ However, invivo response of $\mathrm{HA}-\mathrm{CNT}$ composite-coated titanium implants in bones has never been explored. There are few in vivo studies performed with bare CNTs, but they are restricted toectopic bone induction, tibial diaphysis, or mice skull. ${ }^{242729,30}$ CNTs help in accelerated new bone growth and guided bone regeneration in the case

FACCA ET AL.
ABSTRACT This is the first in vivo study of plasma-sprayed carbon nanotube (CNT)-reinforced hydroxyapa fite (HA) coating on titanium impla nts embedded in rodents' bone. No advese effect or gtotoxiaty of CNT addition on bone tissues and cells was obsenved. Normal bone growth was observed around HA-CNTccasted implants. CNT addition induces higher osseointegration as compared to HA. Elastic modulus of new bone was compared with the modulus of HA-ONT/bone interface to understand the mechanical integrity of the implant.

KEYWORDS: hydroxyapatite - carbon nanotubes - osseointegration - bone induction in vivo - elastic modulus - titanium implants

of defect recovery. ${ }^{2427}$ There is only one report on in vivo study of the sintered HA-CNT composite, which was performed by implanting in a rat musde tissue but not the bone. ${ }^{31}$ After 14 days of implantation, the muscle tissues look similar to natural undamaged tissue, which indicates recovery and no cytotoxic effect. ${ }^{31}$

This study reports in vivo investigations of HA-CNT-coated Ti-6AL-4V alloy (hereafter referred as titanium) implants of different geometries which were embedded in mice and rats. The effect of CNT addition to HA on bone healing and bone induction is elucidated. The elastic modulus of the newly grown bone was measured and compared with the coating and implant to qualitatively compare strain at the interfaces. A very sharp and large difference in the elastic modulus between the implant and bone can create a large difference in strain at the interface, resulting in failure. This study encompasses all those variables (e.g., in vivo bone implantations, HA coating, CNT reinforcement to HA, real-life titanium alloy implant, and mechanical properties of the new bone and multiple interfaces), which
Address comespondence to sgarwalsefiuedu nadia.jesselgmede dine.us tras bg.fr.

Received for review February 24, 2011 and accepted Mby 19, 2011.

Published online May 19, 2011 $10.1021 / \mathrm{m} 200768 \mathrm{c}$

Q 2011 Aesarian Onemial Sodiaty 


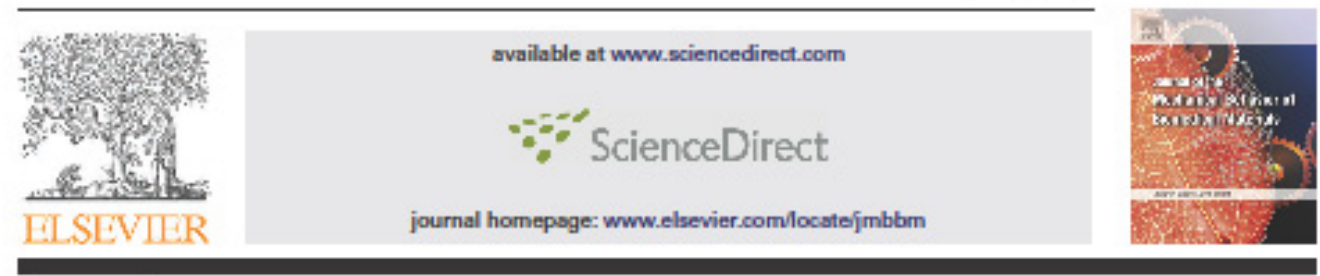

Research paper

\title{
Boron nitride nanotube reinforced hydroxyapatite composite: Mechanical and tribological performance and in-vitro biocompatibility to osteoblasts
}

\author{
Debrupa Lahiri ${ }^{a}$, Virendra Singh ${ }^{b}$, Ana Paula Benaducec, Sudipta Seal ${ }^{b}$, Lidia Kos $^{c}$, \\ Arvind Agarwal ${ }^{a, *}$ \\ a Nanomechanic and Nanotribology Lab and High Temperature Tribology Lab, Department of Mechanioal and Materials Engineering. \\ Florida International University, Miami, FL. 33174, USA \\ b AMPAC and Naroecience Technology Center, 4000 Central FL. Bhod, AMPAC, Eng 1 Room 381, University of Central Florida, \\ Orlando 32816, USA \\ ${ }^{c}$ Department of Biologieal Sciences, Florida International University, Miami, FL. 33174, USA
}

A R TICLE I N F O

\section{Article history:}

Recsived 28 May 2010

Received in revised farm

14 September 2010

Accoptad 17 Saptamber 2010

Published online 21 September 2010

\begin{abstract}
A B STR A C T
This study propcese boron nitride nanotube (BNNT) reinforced hydraxyapatite (HA) as a noval composits material for cothopedic implant applications. The spark plasma sintered (SPS) composite structure shows higher density cocmpared to HA. Minimal lattice mismatch betwoen HA and BNNT leads to coherent bonding and strong intarface. HA-4 wtS BNNT carmpoaite offers excellent mechanicel properties - $120 \mathrm{x}$ incrament in elastic modulus, $129 \%$ higher hardness and $86 \%$ more fracture toughneas, as cormpared to HA. Improwements in the hardneas and fracture toughness are related to grain refinement and crack bridging by BNNTS. HA-BNarT compoaite also shows $75 \%$ improvement in the waar resistance. The wear morpholog sugests localized plastic deformation supported by the sliding of outer walls of BNNI Oateoblast proliferntion and cell viability show no advarse affect of aNNT addition. HA-BNAT cocnposite is, thus, envisioned as a potential material for stronger octhopedic implants.
\end{abstract}

(c) 2010 Elsovier Ltd. All rights reservod.

\section{Introduction}

Hydroxyapatite (HA) possesses chemical compositicn $\left(\mathrm{Ca}_{10}\right.$ $\left.\left(\mathrm{PO}_{4}\right)_{6}(\mathrm{OH})_{2}\right)$, crystal structure and Ca:P ratio $(1.67)$ similar to apatite found in human skeletan (Gu et al., 2002; White et al., 2007; Yu et al., 2003). These features have made HA clinically accepted orthopedic implant material. In spite of its bioactivity, the poor fracture toughness and wear resistance of HA limits its application in load bearing arthopedic implants. Mechanical and tribological perfarmance of HA could be improved by following two different approachesgrain size refinement and secand phase reinforcement.

Researchers have successfully used nanocrystalline HA for the improvernent of mechanical property without having negative effect on its biocompatibility (Li et al., 2007; Guo et al., 2007; Que et al., 2008; Grossin et al., 2009; Wang and Shaw, 2009; Wang et al., 2009). A recent study by Wang and Shaw (2009) has shown that grain size refinement

\footnotetext{
* Correponding author. Tol:- +1 305348 1701; fax +1 3053481932.

E-mail addrasa: agarwaleefiuedu (A. Agarwal).

1751-6161/\$ - see front matter (s) 2010 Elsevier Ltd. All rights reservod.

doi:10.1016/j.jmbbrn.2010.09.005
} 


\title{
Wear behavior and in vitro cytotoxicity of wear debris generated from hydroxyapatite-carbon nanotube composite coating
}

\author{
Debrupa Lahiri,, Ana Paula Benaduce, ${ }^{2}$ Francois Rouzaud, ${ }^{2}$ Jonathan Solomon, ${ }^{1,3}$ \\ Anup Kumar Keshri, ${ }^{1}$ Lidia Kos, ${ }^{2}$ Arvind Agarwal ${ }^{1}$ \\ "Plasma Forming Laboratory and High Temperature Tribology Laboratory, Mechanical and Materials Engineering, Florida \\ International University, Miami, Florida 33174 \\ ²Biological Sciences, Forida International U niversity, Miami, Forida 33174 \\ ${ }^{2}$ Matterials Science and Engineering, University of Florida, Gainesville, Florida 32611
}

Received 25 June 2010; revised 19 July 2010; acoepted 21 July 2010

Published online 13 October 2010 in Wiley Online Library fwileyonlinelibrary.oom). DOt: 10. 1002/bm. a.32952

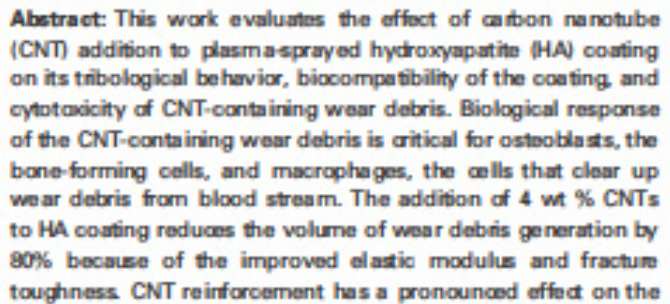

partide size in the was debris and subeequent biological mexponee. There was a slight incroase in the numbers and via: bility of osteoblasts grown on HA-CNT compared with HA alone. The cytctaxic effoct of HA and HA-CNT debris to macro phages and osteoblasts was similar, demonstrating that loose CNT does not pose a problem to these cells. 8 av Wilsy Pstodi. eds, line. J Blomed Maser Res Part A: 96A: 1-12, 2011.

Koy Words: carbon nanotube, hydroxyapatite, wear debris, cytotoxicity, ostoeblast, macrophage

\section{INTRODUCTION}

Hydraxyapatite (HA) is a clinically proven and commercially used coating material for orthopedic implants because of its unique bioactive and osteoconductive properties. The chemical composition of $\mathrm{HA}\left(\mathrm{Ca}_{1}\left(\mathrm{PO}_{4}\right)_{s}(\mathrm{OH})_{2}\right)$, its crystal structure, and calcium to phosphate ratio $(1.67)$ are similar to that of apatite found in the human skeletal system ${ }^{1-3}$ However certain medhanical properties of HA are insufficient for major load-bearing implants The fracture toughness of dense HA (1 $\mathrm{MPam}^{\mathrm{aS}}$ ) is just balf of the minimum reported fracture toughness of artical bone $\left(2-12 \mathrm{MPam}^{0.5}\right)^{2}$ Poor wear resistance of HA is another limiting factor for its successful application in arthopedic implants ${ }^{2-6}$ Low fracture toughness and wear resistanos of HA can be improved by controlling the HA grain size $^{7}$ and/ar adding second-phase reinforoments, such as carbon nanotubes (CNT), that possess high elastic modulus $\left(200-1000 \mathrm{GPa}^{\mathrm{N}}\right)$ and tensile strength $\left(11-63 \mathrm{GPa}^{2}\right)$. Previous studies have shown that CNT is an effective reinforcement to HA for improving the fracture toughness and wear resistance. 5, 3,10

The costed implants stay inside the human body for a long time. For example, $\sim 90 \%$ of the total hip implants stay within the living body for $>20$ years. ${ }^{\text {II }}$ Wear debris is gen- erated from the costed surface during the service period. Henos, it is important to assess the cytotoxicity of the wear debris for the safe use of the coated orthopedic implants. The issue becomes even more critical in case of HA-CNT composite castings, because debris may contain embedded and loose CNTs. Although the cytotoxicity of CNTs is still under debate, ${ }^{12}$ studies have shown ONTs to be biocompatibe to bone celk. ${ }^{\text {D-16 }}$ Usui et al. ${ }^{14}$ have shown that CNTs possess good bone-tissue compatibility without any toxic effect. CNTs were implanted in the bone with the collagen, which resulted in an accelerated bone growth. ${ }^{24}$

Wear debris could be introduced into the blood stream in the lang run. Wear debris when generated, is suspended in the body fluid near the bone or synovial fluid around the jaints The debris could get intruduced into the blood stream from the body fluid at the finer capillary portion of the blood vessels through intercellular space between endor thelial cells. ${ }^{17}$ In the blood, the debris is first attadked by the macrophages the security guard of the blood from any foreign element. Macrophages engulf the debris in the blood stream through a vesicular intermalization process known as phagocytosis to clear them out from the blood. For effective removal of the debris from the blood, the macrophages

Ceerespondenee to: A. Aganval; e-malt agawdapliuede

Contract grant sponsor: NSF CAREER Award; contract grant number NSF.DML0547178 (A.A)

Contract grant sponsor: Otlice of Naval Rescarch; contact grant number: N0001408-1-064 (AA)

Contract grant sponsor: DUPIP program; contnat grant number: N0001406 0675 (A.A)

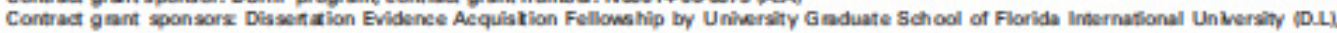

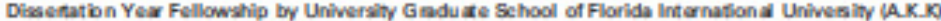




\section{Carbon nanotube toughened hydroxyapatite by spark plasma sintering: Microstructural evolution and multiscale tribological properties}

Debrupa Lahiri ${ }^{a}$, Virendra Singh ${ }^{b}$, Anup K. Keshri ${ }^{a}$, Sudipta Seal ${ }^{b}$, Arvind Agarwal ${ }^{a,}$

- Plasma Forming Laboratory and Nanomechanis and Nanotribdogy Laboratory, Mechanical and Matrials Enginering, 10555 West Flagler Strext, EC 3064, Florida International Univeraity, Miami, FL 33174, USA

- AMPAC and Nanoscience Technology Center, 4000 Cortral FL Bhud, AMPAC, Eng 1 Room 381, Univeraity of Central Florida, Orlando 32816, USA

A RTICLE IN FO

Article history:

Recrived 8 Jenuary 2010

Accepted 23 April 200

Available online $23 \mathrm{May} 2010$

\begin{abstract}
A BST RACT
Carbon nano tube (CNT) reinforosd hydronyapatite (HN) composite synthesixd using spark pleama sintering is imvestigeted in this study. Quantitative microatructural analyala sug. gests that CNTs play a ro le in grain boundary pinning and are responsible for the improwed densification and retention of nanostructure throughout the thideness of the intered pellet. HA crystal forms coherent interface with the CNT, resul ting in a strong interfacial bond The uniform diatribution of $4 \mathrm{wtx}$ CNTs in the HA matrix, good interfadal bonding and fine HA grain size halp to improwe the fracture toughness by $92 \%$ and elastic moduha by $25 \times$ as compared to the HA matrix without CNI. Toughening mechaniama have been explained in terms of interfacial shear strength and pull-out anergy of CNT from the HA matrix. CNT plays a major role in improving the wear resiatance of HA matrix at both macro- and nano-scale. It is conchuded that graphene layer removal from the CNT surface occurs during macro-wear, but not for nano-wear. Thus, the coefficient of friction (CoF) in HA-CNT decreases in macro-wear due to hibrication avail able through delaminated gaphene layers.
\end{abstract}

2010 Elsevier Ltd $\mathrm{All}$ rights reserved.
1.

Introduction

Hydroxyapatits $(\mathrm{HA})$ is an attractive material for bane substi. tution and recanstructian owing to its chemical campositian $\left(\mathrm{Ca}_{10}\left(\mathrm{PO}_{4} / \mathrm{OH}_{2}\right)\right.$, crystal structure and calcium to phosphate ratio (167), which is very sinrilar to that of apatite found in the human skeletal system [1-3]. Because of its unique bios: tive and astrocanductive properties, HA has been clinically tested and accepted for arthopedic applications. However linited stahility of HA causes its dissociation into tricalcrum phosphate (TCP) and tetracalcium phosphate, during prolanged exposure at high temperature processing [1,4]. Fur ther, the mechanical properties of $\mathrm{HA}$ are insufficient for major load bearing implants. The fracture toughness of denas $\mathrm{HA}\left(1 \mathrm{MPa} \mathrm{m}{ }^{0.9}\right)$ is just half of the minimum reparted fracture toughnes of cartical bone (2-12 MPa ma ${ }^{09}$ [3].Poor wear resis tance of $\mathrm{HA}$ is another drawback far its successful application in orthopedic implants $[2,3,5-7]$.

Nhnocrystalline HA has been utilized by zeveral research. ers [3-12] to averome same of these limitations The development of nanoparticles has been accompanied by the need for a suitable procesing technique to retain the fine grain size. Spark plasma sintering (SPS) has proved to be a promis ing technique for the sintering of nanocramics [13-17]. Due to simultaneaus application of pressure, electrical current, and rapid heating nte, SPS offers better densification of

" Comeponding author: Fax: +1 3053481932

E-mail addresa: agarwaladifu edu ( $\Lambda$. Agarwal).

0008-6203/\$ - see front ma ther O 2010 Elsevier Ltd $\mathrm{All}$ rights reserved.

doit-10.1015/jearbon 201004.047 


\title{
Boron nitride nanotube reinforced polylactide-polycaprolactone copolymer composite: Mechanical properties and cytocompatibility with osteoblasts and macrophages in vitro
}

\author{
Debrupa Lahiri ${ }^{2}$, Francois Rouzaud ${ }^{\text {b }}$, Tanisha Richard ${ }^{2}$, Anup K Keshri ${ }^{2}$, Srinivasa R. Bakshi ${ }^{2}$, \\ Lidia Kos ${ }^{\text {b }}$, Arvind Agarwal ${ }^{2 .}$

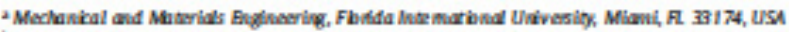

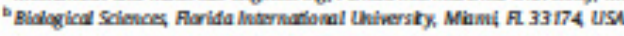

\section{A R TICLE I N F O}

\section{Article hatang:}

Recetved 17 November 2008

Recelved in revilad form 22 February 2010

Accepted 24 Rebruary 2010

Ava Lable onlline 10 March 2010

\section{Kismonts:}

Biodegadable polymer composh:

Borcon nitidie nanombe

Mechanical popertes

Vibillity

Cyowoxidity

\begin{abstract}
A B S T R A C T
Biod grad able polylactide-polycaprolactone copolymer (PLC) has been reinforced with Q, 2 and 5 wt: boron nitride nanotubes (BNNTs) for orthopedic scaffold application. Elastic modulus of the PIC 5 wtr. BNNT mimposite, evaluated through manoindentabon technique, shows a $137 \mathrm{Gr}$ increase. The same amount of BNNI addition to PLC enhances the zensile strength by $109 \%$, without any adverseeffect on the ductility up to $240 \%$ elangation. In zractions of the osteoblasts and macrophages with bare BNNTs prove them to be non-cytotocic. PIC-BNNT composizs displayed increxied osteoblast cell visbility as compared to the PLC matrix. The add ition of BNNTs also resulted in an increase in the expression levels of the Banx 2 gene, the main regulator of astzoblast differentiation. These results indicate that BNNI is a potential reinforement for compocizes for arthopedic applications
\end{abstract}

O 2010 Acta Materialia Inc. Published by Elsevier Itd. All rights reserved.

\section{Introduction}

Biodegradable polymers are classified as the second gener ation bio-implant materials [1] due to their advantages over the firs: generation metallic and ceramic implants because they do not leave undesired foreign elements in the body, even when implant removal is not feasible. One of the main applications of the biodegradable polymers is as temporary scaffolds in bone tissue engineering $\{2-5]$. Amongst the biodegradable polymers, the most commonly used for biomedical applications are poly-e-caprolactone (PCL) and poly lactic acid (PLA), because of their excellent bioresorbability and biocompatibility. There a re some basic differences between PLA and PCL, from both the mechanical properties and the chemistry point of view. PLA is a brittle, crystalline polymer with high strength and low elongation at the fracture point. PLA degrades at a faster rate through enzymatic or alkali hydrolysis [2]. On the other hand, PCL is a semi-crystalline polymer of elastomeric nature. It shows hydrophobic nature and degrades at a slower rate [6]. PCL and PLA can thus be used to form a series of copolymers with tailored combinations of strength, deformability and the degradation rate, suitable for the intended bio-applications [27101.

\footnotetext{
- Comesponding author TAl: +1 305348 1701; 6x +1 3053481932

E-nall aafies: agarwala fiued ( $A$ Marwal)
}

PLC copolymer scaffolds are proposed for the bone and cartilage tissue regeneration in this study. However, the PLC scaffold has elastic modulus and tensile strength that do not match with the properties of the bone tissue or cartilage Subchondral, trabecular and cortical bone tissues show higher elastic modulus [11]. whereas cartilage shows higher tensile strength [12] than P.C. Researchers have used several reinforcements for improving the mechanical properties of PCL, PLA and copolymers. These reinforcements include hydroxyapatite (HA) $[3,4,13-16]$, organic phases like polysaccharides, starch, cellulose, etc. $|17-21|$, silk fibers $[22,23]$ and carbon na notubes (CNT) [24-27]. The biocompatibility of the reinforcement is a major concern. Since reinforcement may remain within the living tissues for a longer time after the biodegradable matrix is absorbed, it is desired that minimum amount of second phase should be added to obtain maximum increase in the mechanical properties. These requirements necessitate that the second phase material must have excellent mechanical properties and biocompatibility. Studies have reported significant improvement in the mechanical properties of PL.A. PCL and PLC copolymer with the addition of carbon nanotubes $[13,24-27]$. However, it must be noted that biocompatibility of CNTs is still being debated and actively researched $[28-32]$. Motivated by this scenaria, we propose in this study the application of a novel material, bor on nitride nanotube (BNNT), as the second phase reinforcemert for the biodegradable PLC copolymer. 


\title{
Carbon Nanotube Reinforced \\ 山 Polylactide-Caprolactone Copolymer: 그 Mechanical Strengthening and Interaction with Human Osteoblasts in Vitro
}

\author{
D. Lahiri, ${ }^{\dagger}$ F. Rouzaud, ${ }^{\dagger}$ S. Namin, ${ }^{5}$ A. K. Keshri, ${ }^{\dagger}$ J. J. Valdẻs, ${ }^{\dagger}$ L. Kos, ${ }^{\dagger}$ N. Tsoukias, ${ }^{5}$ and \\ A. Agarwal**† \\ Mechanical and Materials Engineering, Biological Sciences, and Biomedical Engineering, Florida International \\ University, Mlami, Florida 33174
}

ABSTRACT This study proposes the use of carbon nanotubes (CNTs) as reinforcernent to enhance the mechanical properties of a polylactide-caprolactone copolymer (PLC) matrix. Biological interaction of PLC-CNT composites with human osteoblast cells is also investigated. Addition of $2 \mathrm{wt} *$ CNT shows very uniform dispersion in the copolymer matrix, whereas 5 wt $\%$ CNT shows severe apglomeration and high porosity. PLC -2 wt $\mathrm{Q}$ CNT composite shows an improvement in the mechanical properties with an increase in the elastic modulus by $100 \%$ and tensile strength by $160 \%$, without any adverse effect on the ductility up to $240 \mathrm{X}$ elorigation, An in vitro biocornpatibility study on the composites shows an increase in the viability of human osteoblast cells compared to the PLC matrix, which is attributed to the combined effect of CNT content and surface roughness of the composite films.

KEYWORDS: PLA-PCL. copolymer • carbon nanotube • biodegradable scaffold • strengthening • osteoblast • viability

\section{INTRODUCTION}

he rapid advancement in the field of tissue engineer-
ing and regeneratlve medicine has led to an increased
interest in the use of blodegradable polymers as scaffold materlal, Blodegradable polymers have been used extensively for multiple bone fixation, repalr of osteochondral defects, and ilgarnent and tendon reconstruction (1). Poly-lactic acid (PLA) and poly-E-caprolactone (PCL) are used as scaffold materials for their excellent bioresorbability and blocompatibility. PLA is a crystalline and brittle polymer with high strength and low elongation at break value, it degrades easily at a faster rate through enzymatic or alkall hydrolysis (2). In contrast, PCL. is a semicrystalline polymer of elastomeric nature, which is hydrophobic and degrades at a slower rate (3), PCL. is therefore a sultable comonomer of PLA for the preparation of a series of copolymers with mechanical properties ranging from elastomeric to rigld. PLA-PCL. copolymer (PLC) has good elongatlon characteristics, which makes it interesting for bloapplications where both elasticity and degradability are required (4). Hence, copolymerization of PLA and PCL. provides a controlled way to adjust the degradation rate, sultable for the intended application (2).

It is equally important that the copolymer should possess excellent mechanical properties as the scaffold material, One of the most effective methods of increasing the mechanical

+ Mechanical and Materials Enginerring

+ Biological sdences

Bibmedical Engineering.

Reselved for review fine 19,2009 and aurropted Cotober 4, 2009

DOL: $10.1021 / 2 m 900423 q$

- 2009 American Chemical Sodery properties (elastic modulus and tenslle strength) of a polymer is by relnforcing with a second-phase materlal, Hence, researchers have used different types of second-phase materlals for mechanical strengthening of PLA, PCL, and their copolymers (PLC) (5-16). Among all of these, carbon nanotubes (CNT) seem to be the relnforcement with most potentlal, due to their very high mechanical properties (Young's modulus $0.2-1 \mathrm{TPa}$, tenslle strength $11-63 \mathrm{GPa})(17,18)$ and fiberlike structure. A recent study by usul et al, (19) demonstrated that multiwalled carbon nanotubes (MWCNT) have very good bone-tlssue compatibihty and help in the bone repair by accelerating its growth. Further, CNTs get closely integrated in the grown bone without toxic effect. All these findings have caused carbon nanotubes to be the sutable second-phase reinforcement for blodegradable polymers in orthopedic scaffold applications.

Researchers have studled the effect of CNT addition on the mechanical strengthening for PLA $(20-23)$ and PCL. (24-26). However, there is currently no report avallable on a PLC copolymer-CNT composite, Blocompatibihty of the CNT-reinforced PLC copolymer composite is another very Important issue for scaffold application. Zhang et al, (27) reported that the presence of MWCNTs in PLA inhibits fibroblast cell growth due to less attachment of the cells on the composite surface, although they were not able to establish the cause. Another in vitro study showed that osteoblasts grown on PLA-CNT composite exhibit higher vability and metabolic actlvity, suggesting it to be a favorable environment for the cells (2B). The blocompanbility for PLC-CNT composite is yet to be established, as there are no reports avallable on blostudles of PCL-CNT and PLC-CNT 


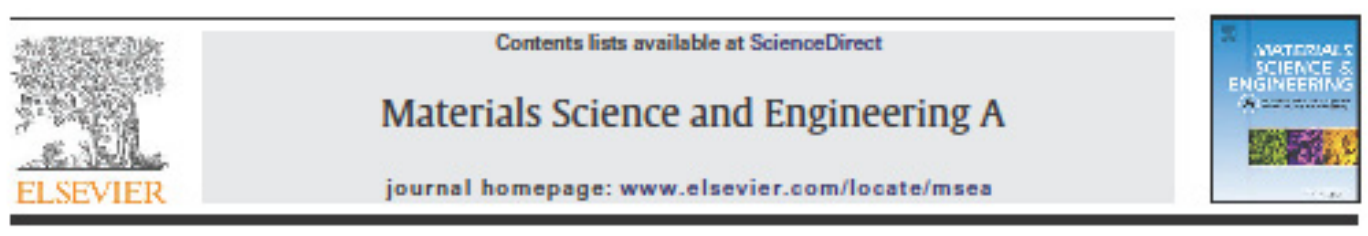

\title{
Dual strengthening mechanisms induced by carbon nanotubes in roll bonded aluminum composites
}

\author{
D. Lahiri ${ }^{2}$, S.R. Bakshi ${ }^{2}$, A.K. Keshri ${ }^{2}$, Y. Liu ${ }^{\text {b }}$, Arvind Agarwal ${ }^{2, *}$

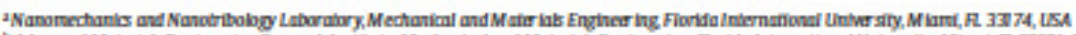

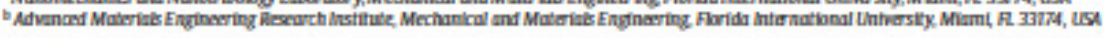

\begin{tabular}{|c|}
\hline $\begin{array}{l}\text { Atick hastor } \\
\text { Received } 14 \text { Mry } 2009 \\
\text { Received in revised furm 28 May } 2000 \\
\text { Accepted 4 Jine } 2009\end{array}$ \\
\hline $\begin{array}{l}\text { Keywort: } \\
\text { N-CNT composite fol } \\
\text { Pol-bonding } \\
\text { Dislocation density } \\
\text { Strain hardening } \\
\text { Dispersion }\end{array}$ \\
\hline
\end{tabular}

\section{Introduction}

Carbon nanotubes (CNTs) have found an increasing interest as reinforcement for aluminum matrix due to their excellent mechanical properties. Elastic modulus of CNT has been reported to vary between 200 and $1250 \mathrm{GPa}[1,2]$, depending on different morphologies. Since the first study by Kuzumaki et al. [3], Al-CNT composite has been successfully processed through different techniques. Most of such investigations have dealt with the powder metallurgy route, often assisted with severe plastic deformation process [4-9]. Researchers have also explored thermal spray methods [10-12], magnetic sputtering [13], liquid innltration [14] and high pressure torsion [ 15] for preparing Al-CNT composites. Dispersion of nanotubes is the most critical step towards enhancernent of mechanical properties of the composite. Improved degree of CNT dispersion in Al matrix has been achieved through molecular level mixing $[9,16]$ and growing CNT on Al powder particles through chemical vapor deposition [5] prior to consolidation. This improvisation has resulted in $350 \%$ increase in the strength [16], proving the role of better dispersion in enhancement of mechanical properties of the composite. Most of the above-listed processing technique involves high ternperature for consolidation, which might result in inducing some damage to CNTs. Hence a room temperature tech-

\footnotetext{
- Carresponding author.

E-mall adter: farwalaanuedu (A. Agrwal).
}

$0021-5003 / 5$ - see front muter 62000 Bxevier B.V. A. rigtits reserved. do: 101016j-mxea2009.06.005 nique, like roll bonding, could be a viable option for processing soft, ductile matrix, e.g. Al or Cu based CNT reinforced composites.

Roll bonding is already an established process for mechanical strengthening of aluminum $[17,18]$. This technique has been successfully used for fabrication of metal (Cu) [19] and ceramic (SiC) [20] reinforced aluminum matrix composites. Choi et al. [8] have shown the positive effect of severe deformation on good dispersion and alignment of CNTs in Al matrix. Li et al. [21] has published the only report on processing of Cu matrix-CNT composite through roll-bonding route. In that study, SWCNTS were acetone sprayed on $10 \mu \mathrm{m}$ thick Cu foils and then 19 such foils, with intermediate layers of CNTs, were roll bonded to form the composite, with 138 increase in strength. But, till date, processing of Al-CNT composite through roll-bonding route has not been reported. Hence, in the present study, Al-CNT composites have been synthesized by roll-bonding technique.

Another aspect of interest is the role of CNTs in the strengthening mechanism of metal matrix. Apart from enhancement in elastic modulus, it has also been concluded that CNTs obstruct dislocation movement, thus creating high dislocation density in the metal matrix composites in case of soft matrix like Al [8], Mg [22-25], Cu $[26]$ and lead free solder (SnAgCu) alloy [ 2$]$ ]. Dislocation pile ups are created due to mismatch in the elastic modulus of CNT and the soft metal matrix [22-24, 7 . This in turn, increases the strain hardening of the matrix metal during plastic deformation. But, till date no report is available on the "quantiflation" of dislocation density and strain hardening of the composite induced by CNTs. 


\section{An analysis of the factors affecting strengthening in carbon} nanotube reinforced aluminum composites

\section{Srinivasa R. Bakshi, Arvind Agarwal * \\ Plasma Forming Laboratory, Nanomechanial and Nanotribology Laboratory, Mechanical and Materials Engineering,} 10555 West Flagler Street, EC 3464, Florida International University, Miami, FL. 33174, USA

A R T I C L E I NFO

Article histary:

Received 25 May 2010

Accepted 28 September 2010

Avalable online 1 November 2010

\begin{abstract}
A B S T R A C T
Tensile strength data of Al/CNT composites from the literat ure is analyzed to understand the effects of CNT dispersion, processing technique, degree of deformation and CNTmatrix interface on the elastic modulus, strength and toughness of composites. Strengthening can be divided in three regimes which show decreasing strengthening effect with an increasing ONT content. The strengthening is highest for CNT content less than 2 vol\% The applicability of the micromechanics models in predicting the strength and elastic modulus of CNT reinforced metal matrix composites is also analyzed. The rule of mixtures is effective in predicting the elastic modulus of the Al/ONT composites for low CNT content $(\kappa 2$ vol.\%) whereas Halpin-Tsai and combined Voigt-Reuss models are bet ter at intermediate QNT content (2-5 vol $\%$. Iffect of degree of deformation such as extrusion ratio during processing on the load transfer to CNT and resulting strengthening is also discussed. Tensile data on $\mathrm{Cu} / \mathrm{CNT}$ and $\mathrm{Mg} / \mathrm{CNT}$ composites is compared with Al/ONT to show that strengthening is not effective when there is no chemical interaction between metal mat rix and $\mathrm{CNT}$. The analysis presented here would be very helpful in the future design of high strength $\mathrm{CNT} /$ metal matrix composites.
\end{abstract}

Q 2010 Elsevier Itd. All rights reserved.

\section{Introduction}

Single and multi-wall carbon nanotubes have created tremendous expectations as strengthening additives for metalic, ceramic and polymer composites due to their high strength and stiffness [1,2]. Recent experiments have shown the elastic modulus of multi-wall carbon nanotube (CNT) to be between 600 and $1100 \mathrm{GPa}$ while the tensile strength ranged from 35 to $110 \mathrm{GPa}$ [3]. The high strength is a result of the near perfect structure and the strong s $\mathrm{s}^{2}$ bonding between the $\mathrm{C}-\mathrm{C}$ bonds. Recently, it has been shown that increasing the inter-wall shear resistance leads to an improvement in tensile strength of the CNTs [4]. A bt of work has been carried out on strengthening of polymer matrices where the enhancement of mechanical properties is clearly visible due to the large ratio of the elastic modulus of the CNTs to the polymer [5].

Research on metal matrix composites has been increasing after the first article appeared in 1998 on $\mathrm{Al} / \mathrm{CNT}$ composite [6]. A comprehensive review of the literature in this area can be found in our previous work [7]. Metal matrix/CNT composites (MM/CNT) have a great potential in load bearing applcations and electronic packaging due to their high specific strength, high themal conductivity and bw coefficient of themal expansion. These properties are advantageous in advanced applica tions like aerospace and automotive structural members where a lower weight leads to savings in energy [8]. There are more than 300 research papers on synthesis and characterization of metalmatrix/CNT composites which have

\footnotetext{
- Corresponding authar: Fux: +1 3053481932.

E-mail address: agarwalaofuedu (A. Agarwal). $0008-6223 / \$$ - see front matter $\$ 2010$ Tleevier Ltd. All rights reserved. doi:10.1016/j.carbon.2010.09.05A
} 


\title{
Nanohardness and Young's modulus of nanopolycrystalline diamond
}

\author{
Hélène Couvy, ${ }^{\mathrm{a}, *}$ Debrupa Lahiri, ${ }^{\mathrm{b}}$ Jiuhua Chen, ${ }^{\mathrm{a}}$ Arvind Agarwal ${ }^{\mathrm{b}}$ and Gautam Sen ${ }^{\mathrm{e}, \mathrm{d}}$ \\ "CeSMEC, Department of Mechanical and Makrials Enginaring Florida Intemational Uning sity, Miami, FL, USA \\ "Nanamechanics and Nanatribabgy Labarabry, Department of Mechanical and Materials Enginaring \\ Farida Intemational Cuinersity, Miami, FL, USA \\ "Department of Ear th \& Environucnt, Fbrida International Uning sity, Miani, FL, USA

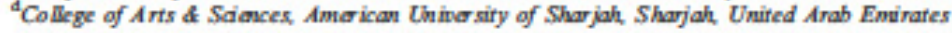 \\ Recelved 23 Jamaary D11; revised 8 Fehruary 2011; acoep ted 9 Febraxy 2011 \\ Avallathle online 12 Fehruary 2011
}

\begin{abstract}
We present the meas urement of the Young's modulus (E) of a nanopolycrystalline diamond (NPD) sample synthesized by the direct conversion of graphite under high pressure and temperature. $E$ has been measured along with the nanohardness ( $H_{w 0}$ ) using the nancindentation techrique $\left(E=707 \mathrm{GPa}\right.$ and $\left.H_{N}=62 \mathrm{GPa}\right)$. Comparing $E$ and $H_{N}$ of NPD with other commerially availa ble superhard anvils and polycrystalline diamonds, we demonstrate its greater hardness and elastic properties.

Published by Elevier Ltd. on behalf of Acta Materialia Inc
\end{abstract}

Kaymond: Nanohandnes; Young's mod ulux; Nanocrystalline dlamond; Nano indentation

Direct of graphite into diamond yields materials that have shown very interesting properties. The result of the total conversion under high static pressure and high-temperature conditions $\left(>15 \mathrm{GPa}\right.$ and $\left.>2300^{\circ} \mathrm{C}\right)$ is a high-purity binderless aggregate of cubic nanodiamond $[1,2]$. It is colorless, optically transparent and stable at high temperature (up to $1200^{\circ} \mathrm{C}$ ) [2]. Hardness tests on nanopolycrystalline diamond (NPD) have found to be isotropic with a Knoop hardness of $120-145 \mathrm{GPa}$, which is equal and even superior to the maximum hardness values of single-crystal diamond (SCD) [3]. Moreover, NPD hardness can be main tained at temperatures up to $1000^{\circ} \mathrm{C}$ while SCD hardness usually decreases at temperatures above $300^{\circ} \mathrm{C}[4,5]$. Finally, the thermal conductivity of the nanodiamond is an order of magnitude lower than that of SCD [6]. The distinctive mechanical properties of NPD have all been explained by its microstructure, purity and the Hall-Petch strengthening effect [7], Indeed, the nanodiamond consists of a mixture of two morphologies: a fine structure of nanometric grains (typical $10-20 \mathrm{~nm}$ ), randomly oriented, and a lamellar structure with elongated grains of up to $200 \mathrm{~nm}[2,8]$. Nanomaterial has the char-

\footnotetext{
* Correz̧onding author. Addrex: Lurie Nanofabrioation Facility. University of Michigan, $\$ 12$ EECS Bullding 1300 Beal Avemae, Ann Atoor, MI 48109, USA. Td $z+1734615$ 966; e-mail: heleneofifumichedu
}

acteristic of a high volume fraction of grain boundaries. Grain boundaries are responsible for blocking the propagation of dislocations from deforming the material, and thus for increasing its hardness. They are also responsible for photon scattering which lowers the thermal conductivity as observed in NPD [6]. The intergranular bonding strength is also believed to be very high due to the absence of impurity or secondary phase in NPD [3]. This strong intergranular bonding and the fact that lamellae block or modify the propagation of cracks improve greatly the fracture toughness of NPD [9], Recently, applications featuring the mechanical properties of NPD have been successfully de veloped. Its high hard. ness even at high temperature makes it an ideal candidate for third-stage anvil in a multi-anvil apparatus as well as in a Knoop indenter $[4,5]$. Its use as an anvil for diamond anvil cells has shown positive results, which have been attributed to its hardness and low thermal conductivity [10]. Even though many physical properties of NPD have been studied, the elastic properties of this material remain unknown, In this study, we have measured the Young's modulus and nanohardness of NPD and compared the data with different superhard polycrystalline materiak.

NPD was synthesized at the Center for the Study of Matter at Extreme Conditions (CeSMEC) using a 500 ton multi-anvil press equipped with a Walker module. A $1.7 \mathrm{~mm} \times 1.1 \mathrm{~mm}$ rod of polycrystalline graphite (99.9995\%; Alfa Aesar) was used as starting material. 
Research paper

\title{
Multi-scale hierarchy of Chelydra serpentina: Microstructure and mechanical properties of turtle shell
}

\author{
Kantesh Balani ${ }^{a, *}$, Riken R. Patel ${ }^{b}$, Anup K. Keshri ${ }^{b}$, Debrupa Lahiri ${ }^{b}$ Arvind Agarwal $^{b}$ \\ ${ }^{2}$ Biomaterials Processing and Characterization Laboratory, Materials Science and Engineering, Indian Institute of Technology Kanpur, \\ Kanpur-208016, India \\ ${ }^{\mathrm{b}}$ Nanomechanics and Nanotribology Laboratory, Mechanical and Materials Engineering, Florida International University, Miami, \\ FL-33174, USA
}

\section{A R T I C L E I N F O}

Article history:

Received 28 December 2010

Received in revised form

6 May 2011

Accepted 7 May 2011

Published online 17 May 2011

\section{Keywords:}

Chelydra serpentina

Turtle's carapace

Nancindentation

Object oriented finite element

modeling

\begin{abstract}
A B S T R A T
Carapace, the protective shell of a freshwater snapping turtle, Chelydra serpentina, shields them from ferocious attacks of their predators while maintaining light-weight and agility for a swim. The microstructure and mechanical properties of the turtle shell are very appealing to materials scientists and engineers for bio-mimicking, to obtain a multifunctional surface. In this study, we have elucidated the complex microstructure of a dry Chelydra serpentina's shell which is very similar to a multi-layered composite structure. The microstructure of a turtle shell's carapace elicits a sandwich structure of waxy top surface with a harder sub-surface layer serving as a shielding structure, followed by a lamellar carbonaceous layer serving as shock absorber, and the inner porous matrix serves as a load-bearing scaffold while acting as reservoir of retaining water and nutrients. The mechanical properties (elastic modulus and hardness) of various layers obtained via nanoindentation corroborate well with the functionality of each layer. Elastic modulus ranged between 0.47 and $22.15 \mathrm{GPa}$ whereas hardness varied between 53.7 and $522.2 \mathrm{MPa}$ depending on the microstructure of the carapace layer. Consequently, the modulus of each layer was represented into object oriented finite element (OOF2) modeling towards extracting the overall effective modulus of elasticity $(-4.75 \mathrm{GPa})$ of a turtle's carapace. Stress distribution of complex layered structure was elicited with an applied strain of $1 \%$ in order to understand the load sharing of various composite layers in the turtle's carapace.
\end{abstract}

Q 2011 Elsevier Ltd. All rights reserved.

\section{Introduction}

Turtles have existed dating 200 million years back. Chelydra serpentina, a freshwater turtle, evolves from the order Testudines and family Chelydridae commonly referred to as a snapping turtle. The upper shell dorsal carapace and the belly portion ventral plastron of a turtle are fused by the bridges (Gilbert et al., 2001). The shell includes portions of ribs and vertebrae, thus the turtle cannot crawl out of its shell. Hence, justifying its name, the turtles snap standing on all four feet when in danger, since it has a short shell not being able to retract the skull completely.

\footnotetext{
+Corresponding author. Tel.: +91512 2596194.

$\mathrm{E}$-mail address: kbalaniaiitk. ac.in ( $\mathrm{K}$. Balani).

1751-6161/\$ - see front matter $@ 2011$ Elsevier Ltd. All rights reserved. doi-10.1016/j.jmbbm.2011.05.014
} 


\title{
Spark plasma sintered tantalum carbide-carbon nanotube composite: Effect of pressure, carbon nanotube length and dispersion technique on microstructure and mechanical properties
}

\author{
Srinivasa R. Bakshi ${ }^{2}$, Vishal Musaramthota ${ }^{2}$, David A. Virzi ${ }^{2}$, Anup K. Keshri ${ }^{2}$, Debrupa Lahiri ${ }^{2}$, \\ Virendra Singh ${ }^{\mathrm{b}}$, Sudipta Seal ${ }^{\mathrm{b}}$, Arvind Agarwal ${ }^{\mathrm{a}, 4}$

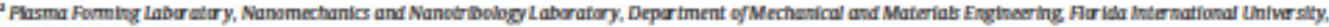 \\ Mieni, FL.33174, LAM

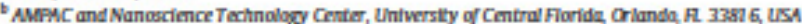

A RTICLE I N F O

Articie htstory:

Received 11 Octaber 2010

Riecelved in revised form

23 November 2010

Accepted 3 Decermber 2010

Avaluble online 10 December 2010

Keyword:

Tantalum carbide

Carbon nanotube:

Spark plasmu sintering

Grain gruwth

Dersincation

\begin{abstract}
A B S T R A C T
$\mathrm{TaC}-4$ wt. 9 CNT composites were synthesized using spark plasma sintering. Two kinds of CNTs, having long $(10-20 \mu \mathrm{m})$ and short $(1-3 \mu \mathrm{m})$ length, were dispersed by wet chemistry and spray drying techniques respectively. Spark plasma sintering was carried out at $1850^{\circ} \mathrm{C}$ at pressures of 100,255 and $363 \mathrm{MP} 2$ Addition of CNTs leads to an increase in the density of $100 \mathrm{MPa}$ sample from $89 \%$ to $95 \%$. Short CNTs are more effective in increasing the density of the composites whereas long $\mathrm{CNTs}$ are more effective grain growth inhibitors. The longer QNTs are more effective in increasing the fracture toughness and an increase up to $60 \%$ was observed for $363 \mathrm{MPa}$ sample. Hardness and elastic modulus are found to increase by $22 \%$ and $18 \%$ respectively for $100 \mathrm{MPa}$ samples by addition of long CNTs. Raman spectroscopy, SEM and TEM images indicated that the CNTs were getting transformed into flaky graphitic structures at pressure higher than $100 \mathrm{MPz}$
\end{abstract}

C 2010 Elsevier B.V. All rights reserved.

\section{Introduction}

The borides and carbides of group IV-V metals such as zirconium, hafnium, and tantalum have high hardness, high melting points and good corrosion resistance. These compounds and their composites are particularly suitable for ultra-high temperature applications such as rocket nozzle throat liners, nose and edge components of re-entry vehicles, jet components and protection of carbon-carbon composites [1-6]. Properties such as high temperature stability, high temperature oxidation resistance, thermal shock resistance and high thermal conductivity are desirable for such applications. Pressure-less sintering [7-10], hot pressing [211-18] reactive hot pressing [19-21] and plasma spraying $[22,23]$ have been utilized for the fabrication of monolithic or composite structures and coatings of these ultra high temperature ceramics (UHTCs). Spark plasma sintering is a relatively novel consolidation method that has the advantage of short sintering duration and has shown better densification and properties than hot pressing [24]. SPS has been extensively used for synthesis of UHTCs and their composites $[11,14,17,25-29]$.

\footnotetext{
* Corresponding author. Tel: + 1305348 1701: fax: +1 3053481932

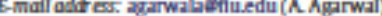

0921-5093/5 - see frunt matter o 2010 Elsevier RV. All rights reserved dol:10.1016]|msea2010.12017
Tantalum carbide has high melting point of $3880^{\circ} \mathrm{C}$ and is stable for a large range of $\mathrm{C} / \mathrm{Ta}$ ratio of $0.76-1$ [13]. The strong covalent-ionic bond results in high hardness of > $20 \mathrm{GPa}$ and elastic modulus of up to 550GPa [30] which makes it extremely suitable for rocket components involving combustion temperatures up to $3500 \mathrm{~K}$ TaC has been consolidated using techniques such as pressureless sintering $[7,31$ L hot pressing $[13,15,16,18,32$ L high frequency induction heating $[33,34]$, vacuum plasma spraying [22] and spark plasma sintering $[27,35,36]$. Due to the low self diffusion coefficient, additives such as $\mathrm{B}_{4} \mathrm{C}[15,18,36]$, carbon [15,31] $\mathrm{TaB}_{2}[16,31]$ and $\mathrm{TaSi}_{2}[34]$ have also been utilized to aid the sintering.

In the present study, we have synthesized TaC composites reinforced with carbon nanotubes (CNTs) using spark plasma sintering. CNTs have proved as effective reinforcement in increasing the fracture toughness of ceramic materials through mechanisms such as crack bridging and crack deflection [37-40]. Improwement in the CNT dispersion leads to significant improvement in fracture and wear properties of the composites and coatings $[38,41,42]$. Carbon fiber reinforced TaC composites have been prepared by infiltration of carbon fiber weave Ta and C containing solutions followed by vacuum sublimation treatments [43]. Recently, Khaleghi et al. have synthesized a TaC- 0.77 wt. $x$ CNT composite using spark plasma sintering. The relative density of the composite synthe- 


\title{
Spark plasma sintered tantalum carbide: Effect of pressure and nano-boron carbide addition on microstructure and mechanical properties
}

\author{
Srinivasa R. Bakshi ${ }^{a}$, Vishal Musaramthota ${ }^{a}$, Debrupa Lahiri ${ }^{a}$, Virendra Singh ${ }^{\text {b }}$, Sudipta Seal ${ }^{\text {b }}$, \\ Arvind Agarwal ${ }^{2, *}$
}

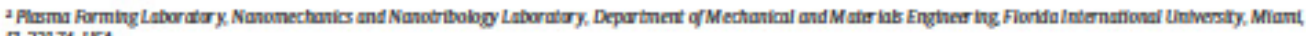
R. 33074 , USA

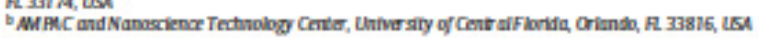

ARTICLE INE D

\section{Arick hastory}

lieceived 18) jaly 2010

Deceived in revised form 6 October 2010

\section{Krywords:}

Tantalum cartide

Boron cartide

Spark plasma sintering

Crain growth

\begin{abstract}
A B STRACT
TaC and TaC-1 wt $X \mathrm{~B}_{4} \mathrm{C}$ powders were consolidated using spark plasma sintering (SPS) at 1850-C and varying pressure of 100, 255 and 363 MP2. The effect of pressure on the densification and grain size is evaluated. The role of nano-stzed $\mathrm{B}_{\mathrm{q}} \mathrm{C}$ as sintering aid and grain growth inhibitor is studied by means of XKD, SEM and high resolution TEM. Fully dense TaC samples were produced at a pressure of $255 \mathrm{MPa}$ and higher at $1850-\mathrm{C}$. The increasing pressure also resulted in an increase in TaC grain size. Addition of $\mathrm{B}_{4} \mathrm{C}$ leads to an increase in the density of $100 \mathrm{MPa}$ sample from $89 x$ to 972 . $\mathrm{B}_{4} \mathrm{C}$ nano-powder resists grain growth even at high pressure of 363 MP2. The formation of $\mathrm{T} 2 \mathrm{~B}_{2} / \mathrm{C}$ arbon at $\mathrm{TaC}$ grain boundaries helps in pinning the grain boundary and inhibiting grain growth. The effect of $\mathrm{B}_{4} \mathrm{C}$ addition on hardness and elastic modulus measured by nanoindentation and the indentation fracture toughness has been studied. Relative fracture toughness increased by up to $93 \pi$ on $\mathrm{B}_{4} \mathrm{C}$ addition.
\end{abstract}

C 2010 Elsevier HV. All rights reserved.

\section{Introduction}

Applications involving temperatures more than $2000 \cdot C$ such as rocket nozzles, scramjets and supersonic re-entry vehicles require materials with ultra high melting point, high temperature strength and high temperature erosion resistance. Tantalum carbide (TaC) is an interesting compound due to its remarkable high temperature properties. The high hardness $(>20 \mathrm{GPa})$, high melting point $(>3800-C)$ and resistance to chemical attack and oxidation can be attributed to the presence of strong covalent-metallic bond [1-3] $\mathrm{TaC}$ also possesses good catalytic properties along with good thermal and electrical conductivity [3]. Tantalum carbide (TaC) has the NaCl type structure (B1, space group $F \mathrm{~m} \overline{3} \mathrm{~m}$ ) and can accommodate large vacancies in the carbon lattice, without changing the crystal structure [4]. Tantalum also forms a hexagonal carbide $\mathrm{Ta}_{2} \mathrm{C}$. The $\zeta$-phase having a composition of $\mathrm{Ta}_{4} \mathrm{C}_{3}$ have been reported in twoand three-phase samples in the $\mathrm{Ta}-\mathrm{C}$ system between hexagonal $\mathrm{TaC}_{0.52}$ and cubic $\mathrm{TaC}_{0.73}$ compositional range [5]. The mechaniCal properties of TaC depend on the C/Ta atomic ratio. The highest fracture toughness of $12.7 \pm 0.7 \mathrm{MPam}^{1 / 2}$ is reported for $\mathrm{TaC}_{0.5}$ and highest hardness value of $20 \pm 0.5 \mathrm{GPa}$ for TaC0.8 carbides [6].

\footnotetext{
* Correpoeding author. Tel: +1 3053481701.

E-mall adters a garwalashundu (A Aparwal)
}

Tantalum carbide has been difflcult to sinter due to its covalent bonding, low self-diffusion coefncient and high activation energy for viscous flow $[7,8]$. It was reported that even hot pressing at a temperature of $3050-\mathrm{C}$ at $15.7 \mathrm{MPa}$ produced a density of only 90.5\% [7]. Employing smaller particle size for $\mathrm{TaC}(<0.6 \mu \mathrm{m})$ led to a densification of up to $975 \%$ by pressureless sintering at $2300^{\circ} \mathrm{C}[9]$. Zhang et al. have used $\mathrm{C}$ and $\mathrm{B}_{4} \mathrm{C}$ as the sintering aid for improving the densiflcation [8]. A relative density of $97 \%$ was achieved in hot pressed $\mathrm{TaC}$ containing 0.78 wt. $\mathrm{C} \mathrm{C}$ at $2300^{\circ} \mathrm{C}$ and $30 \mathrm{MPa}$ pressure with a $\mathbf{4 5}$ min hold time in helium atmosphere. Addition of 0.36 wt: $x \mathrm{~B}_{4} \mathrm{C}$ produced a $98 \%$ dense $\mathrm{TaC}$ sample at a lower temperature of $2200^{\circ} \mathrm{C}$, which shows the emcacy of boron carbide over $\mathrm{C}$, as the sintering aid $[8,10]$. A recent study has shown that addition of larger amounts of $\mathrm{B}_{4} \mathrm{C}\left(1-8\right.$ molar ratio $\left.-3.5 \mathrm{wt} . \mathrm{x} \mathrm{B}_{4} \mathrm{C}\right)$ to nanosized $\mathrm{TaC}$ powder leads to densiffcation at $1800^{\circ} \mathrm{C}$ without any application of pressure [11]. Kim et al. have used a high trequency induction heating sintering (HFHS) apparatus for the fast sintering of nine $\mathrm{TaC}$ powders. Sintering of $33 \mathrm{~nm}$ grain size $\mathrm{TaC}$ powder at $80 \mathrm{MPa}$ pressure took only $3 \mathrm{~min}$ to reach $96 \%$ theoretical density with termperatures reaching up to $1400^{\circ} \mathrm{C}[12]$. Similar technique has been used by Sciti et al. for the consolidation of $\mathrm{TaC}-15$ vols MoSiz and $\mathrm{TaC}-15$ vol. $\%$ TaSiz composites to achieve $96 \%$ density within 20 min for temperatures below $1850 \circ \mathrm{C}[13]$. Free standing $\mathrm{TaC}$ components have also been synthesized using Vacuum Plasma Spray technique [14]. However, the high temperature and low pressure conditions resulted in the partial decomposition of $\mathrm{TaC}_{\mathrm{C}} \mathrm{T} \mathrm{Ta}_{2} \mathrm{C}$ 


\section{Author's personal copy}

Tain Solid Films $518(2010) 1203-171$

\section{Nanoscratch behavior of carbon nanotube reinforced aluminum coatings}

Srinivasa R. Bakshi, Debrupa Lahiri, Riken R. Patel, Arvind Agarwal *

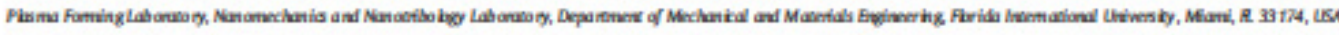

A RTICLE I N F O

\section{Artilehádary}

Beretived 2 May $200:$

Received ia revied fom 1 Sepoem in 2009

Acceped 24 Nove ater 200

Nudiste mine 1 Derenter 200

\section{Kismorik:}

Mlama payak

Cutin micoute

Nenosiciach tex

Med maxictamparte

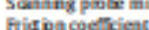

A BST RACT

Nanorath experiments hwe been caried out on plasma sprayed alum inum alloy coatings reinforced with Q, 5 and 10 wtz carbon nanotubes (CVIT). Scrathes hrve been performed II lasis of 1000, 2000 and $3000 \mu \mathrm{N}$ load using Berkontch indenter. The contact and true wear volumes of the scratches hrve been calculated. The nanoscale wear resistance is shown to increase by 4 times by additbn of 10 wtx CNTs. calculated. The nanoscale wear resistance is shown to increase by 4 times by addition of lowtz CVIs. recovery by addition $d$ CNRE. Direct evidence of increxsed recowery and small decrexse in the coeffident of friction with CN contrnt is provided using the true and instantaneas depth plots and the comresponding scanning probe microx ce and scanning dectron micoscope images $d$ the scrarther. Fitction coeffident was found to te load independent and was found to vary sllighty with the CNT content

Q 2009 Elovier B.V. All rights resewed.

\section{Introduction}

Nanascratch testing using a nanoindenter is a unique method for characterizing the wear properties of asurfixe at the nano-scale. This kind of testing is significant for predicting the wear behavior in case of micro- and nano-electro mechanical devices [1] emplaying moving parts like gears and applications imvolving thin watings like magnetic storagedevices $|2|$ Also it helps in understanding processes in which nano-scale patterning is carried out by removal of material Nanoscratch experiments have been carried out to study the wear behaviar of different kinds of materials like palymer/polymer camposites [3-6], hard oramics [7-10] and diamond-like carbon caatings [11-13] self lubricating transition metal dichakngenide [14] metals [15-17] and human hir [18]. The focus of these studie has been to understand the near-surface wear mecharisms, deformation and damage in the wake of the indenter, critical load for delamination and adhesion strength of thin films, effect of reinfarce. ment an the wear resistanceor understanding the frictian behavior of the coatings Dasari at al [4] have used nanoscratch studies on nylan 66-SEBS-arganoday nanocomposites to understand the effect of dispersion of exfoliated day between the hard and soft regions on the ranoscratch properties. Yuan et al [5] have observed the formation of ripple type tracks during nanosaratching of polypropylene-day compos ites which were analogous to period ic ripples former during micro-scratching at larger lasds. This suggested that the mechunism of microscale deformation could be applied at the nano-

\footnotetext{
- Cereponding arthe

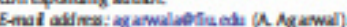

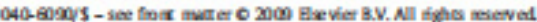
doieterot 6f or anon 1107 scale. An in-depth stres analysis around the indenter was presented by Chosh et al [10] $b$ show that a maximum shear stress occurred in front of the indenter and maximum principal tensile stress in the wake of the indenter. These stress distributions were respons ible for the ohserved slip hands and microcracks an the wear track Lafiye and Troyon [16] have analyzel the variation of friction farce with the applied normal farce and provided a model based on the datic rearvery. Wei and Bhushan have used the ranascratch technique to study the properties of human hair treated with different canditioners and shampoos [18]. It was mentioned that these studies may help in developing or testing hair care produds. Thus, nanoscratch tedhrique is very important technique and the interest and understandingofthis technique is rapidly growing.

Carbon nanotubes (CNIs) have been propased as reinfarcement for polymer, ceramic and metalic matrices due to their excellent mechanical properties [19-23]. Balani et al have shown tremendous improverment of wear resistance of alumina cantings rein forced with ONTs both at nano ( 18 times) and macroscale ( 49 times) [ 8 ]. Excellent improvement in the mechanical properties has been reparted far $\mathrm{Al}$ [23-29], Cu [30,31] and Ni [32,33] matrix composites Zhou et 1 . [29] have shown a $30 \%$ decrease in the wear rate and friction coefficient of AJ-ONT al lays by aldi bion of 20 vol\% of ONTs. Mar e than 2006 increase in the yield strength $h \mathrm{~s}$ been observed in 10 voLICNT rein for $\mathrm{ced} C \mathrm{Cu}$ composites prepared by a molecular level mixing method which shows the significanceof dispersion in effective strengthening [31].A decrease in the macroscale wear lass by $80 \%$ and mefficient of friction by $40 \%$ by aldition of ONTs was observed in Ni-P-ONT cuating prepared by electraless deposition tedurique [33]. These results indicate that CNT addition could result in improvement in mechanical and wear properties 


\title{
Carbon nanotube reinforced metal matrix composites - a review
}

\author{
S. R. Bakshi, D. Lahiri and A. Agarwal*
}

This review summarises the research work carried out in the field of carbon nanotube (CNT) metal matrix composites (MMCs). Much research has been undertaken in utilising CNTs as reinforcement for composite material. However, CNT-reinforced MMCs have received the least attention. These composites are being projected for use in structural applications for their high specific strength as well as functional materials for their exciting themal and electrical characteristics. The present review focuses on the critical issues of CNT-reinforced MMCs that include processing techniques, nanctube dispersion, interface, strengthening mechanisms and mechanical properties. Processing techniques used for synthesis of the composites have been critically reviewed with an objective to achieve homogeneous distribution of carbon nanotubes in the matrix. The mechanical property improvements achieved by addition of CNTs in various metal matrix systems are summarised. The factors determining strengthening achieved by CNT reinforcement are elucidated as are the structural and chemical stability of CNTs in different metal matrixes and the importance of the CNT/metal interface has been reviewed. The importance of CNT dispersion and its quantification is highlighted. Carbon nanotube reinforced MMCs as functional materials are summarised. Future work that needs attention is addressed.

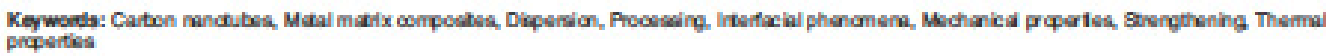

\section{Introduction}

The need for hightweight, high strength materiak has been recogrised since the invention of the sirplane. As the strength and stiffness of a matrial increases, the dimensions, and consequently, the mass, of the material requiral for a certain load bearing application is reduced. This kads to several advantages in the case of aircraft and automobiles such as increase in payload and improvement of the fuel efficiency. With global oil resources on a decline, increase in the fuel efficiency of engines has become highly desirable. The inadequacy of metals and alloys in providing both strength and stiffness $b$ a structure has led to the development of metal matrix composites (MMCs), whereupon the strength and ductility is providal by the metal matrix and the strength and/or stiffness is provided by the reinforcement that is either a ceramic or high stiffness metal basal particubte or fibre. Metal matrix composites can be designed to possess qualities such as low coefficient of thermal apansion and high thermal conductivity which make them suitable for use in electronic packaging applications. Metal matrix composites today

Plasma Forming Laboracry. Nanomechanics and Nanobibology

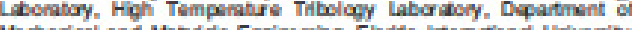
Macharioal and Masilda Enginesing Flenda linsmationd Uniwerity.

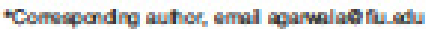

ane extensively usal in automobile and aerospace apphications. ${ }^{1-4}$

In 1960, Roger Bacon ${ }^{5}$ demonstrated the formation of graphite whiskers (diameter ranging between fractions of a micrometre to a couple of micrometres) that were flexible and had a tensile strength of up to $20 \mathrm{GPa}$ Subsequent research led to development of proasses for arge scale production of these fibres by carbonisation of Rayon, poly-acrilonitrile (PAN), or pitch. Manufacture of carbon fibres of high strangth in the $1960 \mathrm{~s}$ and $1970 \mathrm{~s}$ made than the first choice for the manufacture of advanced composites for use in rocket norzle ait cones, missile nose tips, roentry heat shiekls, padkaging and thermal management. Since 1970, carbon fibre reinforced composites have been extensively used in a wide array of apphications such as aircraft brakes, space structures, military and commercial planes, lithium batteries, sporting goods and structursl reinforcement in construction. Research in the fiekd of carbon was revolutionisal by the discovery of carbon nanotubea (CNTs) by bjima $^{6}$ in 1991. Although CNTs might have been synthesised in 1960 by Bacon, ${ }^{5}$ it took the ganius of bijina to realise that they are tubes made by rolling a graphene shed onto itself. A multiwalled carbon manotube (MWCNT) is made up of many single walled carbon nanotubes (SWCNT) arranged in a concentric manner. Unkess otherwise specified, CNT in this work refars to MWCNTs. Experiments and simulations showed that CNTs have extraondinary mechanical 
Microstructure, mechanical properties, and in vitro biocompatibility of spark plasma sintered hydroxyapatite-aluminum oxide-carbon nanotube composite

\author{
Sushma Kalmodia ${ }^{2}$, Shilpi Goenka ${ }^{\text {h.c }}$, Tapas Laha ${ }^{\text {de, }}$, Debrupa Lahiri ${ }^{f}$, Bikramjit Basu ${ }^{2}$, Kantesh Balani ${ }^{2, *}$

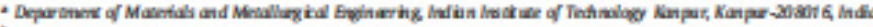

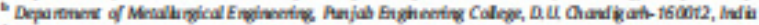

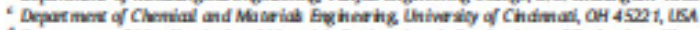

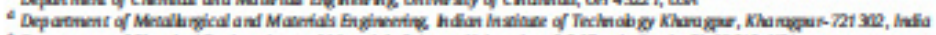

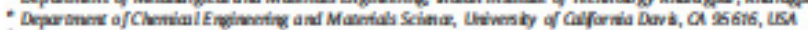

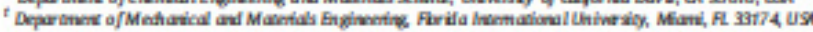

\section{A RTICLE I N F O}

\section{Araticit hisary:}

Rereived 20 Derenter 2009

Recrived in meved form 10 May 2010

Nocoped 11 Jux 2010

Anelitie odix 29 Jax 2010

\section{Kicomordis}

Hydraxyanke (HW)

Numia $(N<0 x)$

Cution matios (CMIs)

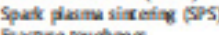

hachere wagho:

secompaliticy

\begin{abstract}
A B STRACT
In the present work HA reinforced with $\mathrm{N}_{2} \mathrm{O}_{2}$ and multiwa lled carbon nanotubes (CNIs) is procesced uaing spark plasmasintering (SPS). Vickes micro indentation and nanoindenta bon of the samples revesled contrany)

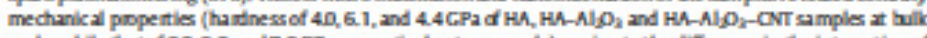
scale, while that $d 80,9.9$ and $7.0 \mathrm{CP} a \mathrm{~m}$ sper twdy at nanoscale), own $\mathrm{g}$ to the difference in the interaction of the indenter with the m atrilal at two different length scales. The addibon of $\mathrm{Al}, \mathrm{O}, \mathrm{s}$ reinforcement $\mathrm{h} x \mathrm{~s}$ been shown to enhance the indentation fracture toughness of $\mathrm{HA}$ matrix from $1.18 \mathrm{MPa} \mathrm{m}^{1 / 2}$ to $207 \mathrm{MPa} \mathrm{m} \mathrm{m}^{1 / 2}$. Purther GNT reh forcement has increxed the facture taughness to 23 times $\left(272 \mathrm{MPa} \mathrm{m}^{1 / 2}\right)$, th vto blocompatiality of CNT reinforced $\mathrm{HA}-\mathrm{N}_{2} \mathrm{O}_{2}$ compocitr has been evaluated using $\mathrm{MT}$ aseay an mouse fibrothast 1929 cell line. Cell adhesion and proliferation hwe been chancterined using scanning dectron microscopy (SEM), and have been quantified using UV spectrophotometzr. The combination of cell vilablility data as well as microsocpic obrenatoms of cultured surtaces sugzest tha SPS sintered $\mathrm{HA}-\mathrm{N}_{2} \mathrm{O}_{2}-\mathrm{CNT}$ composiles exhilat the ability to promote cell adhesion and poliferzation on their surtace and prove to be
\end{abstract} promising new blocompatble materiak.

Q 2010 Hoevier R.V. All itghts reserved.

\section{Introduction}

Hydraxyapatite ( $\left.\mathrm{HA}, \mathrm{Ca}_{10}(\mathrm{PO})_{4}(\mathrm{OH})_{2}\right)$ is ane of the mast promising bioceramic materiak, used for human tissue implantation due t $b$ its simil arity with apatite in human skeletal system and bone in terms of the chemical composition $(\mathrm{C} / \mathrm{P}$ ntio of 167$)$ and cystal structure (hexagral) [1,2]. HA findsimmense use in bone suls titution and bone. reconstruction as well $\mathbf{s}$ other arthopedic applications due $\mathbf{b}$ its good biasctivity ie bane band ing ability and os teacanductive properties [3-5] However, medhanical properties ofHA ( fradure toughess of $0.28-108 \mathrm{MPa} \mathrm{m} \mathrm{m}^{2}$ ) need to be improved far majar lasd bearing biomedical applications $\mid 6$. One of the most commonlyused methods $\mathrm{t}$ improve the medunical properties has been the production of HA based camposites with reinfarcements like $\mathrm{YSZ}, \mathrm{Al}_{2} \mathrm{O}_{2}$. $\mathrm{ZrO}_{2} \mathrm{TSO}_{2}$ ONTs, etr. [1,7-11]. To date, several stuclies exist an composites reinforced with cabon nanotubes (CNT). ONT impant strength and toughness (up th $56 \%$ ) to brittle HA bioceramics, by $x$ ting as a strong reinforement [1]. Exceptianal strengthening via CNT reinforcement is attributed to their high stiffness (Young's modulus of $-1 \mathrm{TPa}$ ) and unifarm dispersion [12].

\footnotetext{
- Cormponding entor. Tel: +915122926194
}

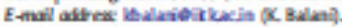

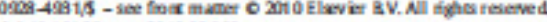
da: $10.1016 /$ masec.20100600
ONIs have demanstrated th assist apatite mineralization, encouragal cell ingrow th, and shown good cell morphology of cultured asteoblasts [1,13-15] Chlopek et.al have studied cell proliferation of fithroblas ts an multi walled carbon nanotu bes [16].

Spark plasma sintering (SPS) is a relatively new processing edhrique and there exist anly a few reparts on its use to synthesize HA based composites for biomedical applications [17-19] SPS allows the sintering of compucted powder under uniform hesting to high density at a relativ dy lower temperature and in a much shorter durabian (generally few minutes), as campared to the canventianal sintering methods [20-22]. Reaently. Xu etal have prepared multiwalled ONT reinforced HA compasites by SPS method and stucied it interadion with hPOB 1.19 asteoblast cells [23]. A wide variation of the elstic modulus (31-131 GPa) was observed for various sintering temperatures, which was attributed to a combined effect of dersification, grain growth and ONT reinforcernent [23].

Reinforcement of ceramics with harder nanopartides (such as SiC) has shown to enhance the mechanical properties of $\mathrm{Al}_{2} \mathrm{O}_{3}$ matrix [24) In addition, $\mathrm{Al}_{2} \mathrm{O}_{3}$ reinforcement enhunaed the medharical properties of HA (fracture bughness enhuncement by 15 times) while retaining cytacampatibility due to its biainert nature [25] Therefore, the supplemented reinforament of $\mathrm{HA}$ with $\mathrm{Ab}_{2} \mathrm{O}_{3}$ manopartides and ONTs is expected to impart toughening of HA 


\title{
$\mid$

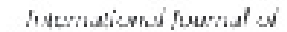 \\ Applied Ceramic \\ TECHNOLOGY
}

Int J. Appl Cenam. Technol, 7 (6) 846-855 (2010)

DON:10.11116.17447402.2009.02385x

Ceramic Product Development and Commeräalizarion

\section{Grain Growth Behavior of Aluminum Oxide Reinforced with Carbon Nanotube During Plasma Spraying and PostSpray Consolidation}

\author{
Kantesh Balani, Srinivasa R. Bakshi, Debrupa Lahiri, and Arvind Agarwal \\ Mechaniatland Material's Engineaing, Flarida Intanational University, Marmi, Fbrida 33174 \\ Kantesh Balani

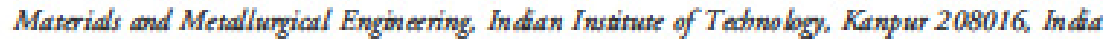

\begin{abstract}
Grain boundary mobility of the plasma sprayed ahuminum oxide $\left(\mathrm{Al}_{2} \mathrm{O}_{3}\right)$-arbon nanotube $(\mathrm{CNI})$ am posizs is anahared in thecurrent work. Grain mobility is evaluazd from the grain growth within the spraydried partides and thermal history experienced during high-temperature plasma processing CN'Ts form an interfacial grain boundary layer during thermal exposure, limiting the grain growth of plasmasprayed anatings. Consequent hot isostatic pressing (HIPing) of $\mathrm{CNT}$-reinforced $\mathrm{Al}_{2} \mathrm{O}_{3}$ at $1773 \mathrm{~K}$ shows differences in gain growth kinetics, degree of densification, and pore strinlag. Densification of HIPed anarings is oberved to be dictated by CNIs phase tanformation, initial grain sine, and time of themal processing. $\mathrm{CNI}$ 's have shown to impede the $\mathrm{Al}_{2} \mathrm{O}_{3}$ grin growth by serving as grain pinning obsades. Impediment of grain-boundary mobility with variabion of CNT conzent, and time and zmperature of the heat treatment of ahuminum aride $\left(\mathrm{Al}_{2} \mathrm{O}_{3}\right)-\mathrm{CNT}$ nanocomposite is addressed in deail.
\end{abstract}

\section{Introduction}

High-temperature strength, wear resistance, and chemical stability of $\mathrm{Al}_{2} \mathrm{O}_{3}$ inevitably finds application as ceramic lines, heaters, crucibles, and so forth. ${ }^{1-9}$ Low

agmalefinadu

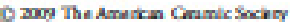

fracture toughness of brittle $\mathrm{Al}_{2} \mathrm{O}_{3}$ ceramic can be improved by utilizing nano or submicrometer $\mathrm{Al}_{2} \mathrm{O}_{3}$ powder particles, followed by sintering at high temperatures. ${ }^{2,-6}$ However, high sintering temperature $\left(>1450^{\circ} \mathrm{C}\right)$ needed for densification also promotes abnormal grain growth. ${ }^{25-7}$ Addition of sintering additives and secondary reinforcements have shown to restrict grain growth by acting as barriers. ${ }^{5}$ High-tem- 


\title{
The hydrophobicity of a lotus leaf: a nanomechanical and computational approach
}

\author{
Kantesh Balani ${ }^{1,2,3}$, Ruben Galiano Batista ${ }^{2}$, Debrupa Lahiri ${ }^{2}$ and \\ Arvind Agarwal ${ }^{2}$ \\ ${ }^{1}$ Materials and Metallurgical Engineering, Faculty Building, Rocm 409, Indian Institute of \\ Technology Kanpur, Kanpur-208 016, India \\ ${ }^{2}$ Mechanical and Materials Engineering, Forida International University, EC 3464, \\ 10555 W Flagler Street, Miami, FL-33174, USA \\ E-mail: kbalaniตiitk ac.in
}

Recelved 25 February 2009, in final form 20 May 2009

Published 8 July 2009

Online at stacks.lop.org/Nano/20/305707

\begin{abstract}
The multi-scale microstructure of a lotus leaf is rendered non-wetting by micro-protrusions and nano-hairs present on its surface. The mechanical properties of the surface become important since the waler droplet has to be supported on the micro-protrusions without wetting the surface. Current work correlates the non-wetting behavior of the lotus leaf with its mechanical properties (Young's modulus and critical flexing stress) and areal spread of micro-protrusions on the leaf surface. Quasistatic nanoindentation of nano-hairs on the lotus leaf surface has shown a variation of elastic modulus between 359 and $870 \mathrm{MP2}$, which in turn dictates the critical flexing strength and consequent non-wetting. Computational fluid dynamics modeling is utilized to correlate wetting phenomena with the areal spread of micro-protrusions. A qualitative model is proposed for the way nature has chosen to render the lotus leaf surface non-wetting.
\end{abstract}

(Some figures in this article are in colour only in the electronic version)

\section{Introduction}

Nature is simple, but its science is not. The lotus leaf is known to grow at the bottom of ponds, but emerges above the water surface as if untouched by the contamination of the dirty water that it grows in [1]. The non-wetting lotus leaf is often related to extreme purity, which restricts bacteria and pathogens growing on its surface [2]. Water droplets roll over the leaf's surface taking away all the dirt and leaving a clean surface behind. A high contact angle $\left(>160^{\circ}\right)$ of the water droplet is attributed to two levels of microstructure of the lotus leaf $[1,3-6]$. It is well documented in the literature that a material with the lowest surface energy renders a contact angle of $120^{\circ}$ [5]. It is reasoned that only an increased surface roughness (apart from surface chemistry) can further enhance the contact angle of water with the surface [5].

${ }^{3}$ Author to whom any coerespondence should be addressed

$0857-4484090 / 305707+0953000$
A variety of surfaces have been pattemed for mimicking lotus leaf structure and rendering hydrophobic surfaces with high contact angles for potential applications such as waterrepellent glass in the automobile industry, surgical antiboofouling instruments, self-cleaning clothes and paints, molecular motors, and low-drag marine vessels [7-9]. Polycarbonate wis roughened by a hydrophobic coating of POSS (polyhedral oligomentc silsesquioxane) resulting in a contact angle of greater than $165^{\circ}$ for the structure [3]. PET fabric modified with silver/polystyrene (PS) displayed a superhydrophobic contact angle of $157^{\circ}$ because of the enhanced roughness effect of hydrophobic PS [10]. Similarly, carbon nanotubes were deposited on cotton fabrics using ultrasonic imadiation to roughened the surface and achieve a superhydrophobic surface ( $>150^{\circ}$ contact angle) [11]. Further, nanocasting has been performed on polyvinyl chlonde (PVC) in two steps: (i) achieving a negative surface of a lotus leaf using polydimethylsiloxane (PDMS), and (ii) casting
Q 2005 woP Puenshing L.Ad Printed in the UK 


\title{
Tensile properties of carbon nanotube reinforced aluminum nanocomposite
} fabricated by plasma spray forming

\author{
T. Laha ${ }^{2+}$, Y. Chen ${ }^{2}$, D. Lahiri ${ }^{2}$, A Agarwal ${ }^{2 *}$

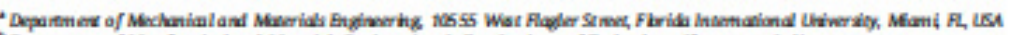

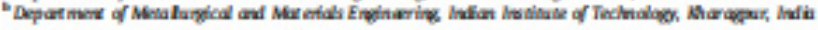

\section{A RTICLE IN FO}

\section{Aralde Hitary}

heceivel to Sepoember 200 .

keceived in revied form 31 jecary 200

Aceped 5 Fitodicy 2000

\section{Kigmont:}

A. Cutan manco

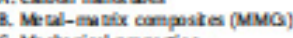

C. Medarical popríle:

D. Plaim sayng

\section{A BSTRACT}

Uniarial tensile tests were performed on plasma spray formed (PSF) AL-Si alloy reinforced with mult:walled carbon nanotubes (MWCNTs). The add iton of CNTs leads to $78 x$ increase in the elastic modulus of the composit $t$. There was a marginal increase in the tend le strength of CNT reinforced composite with degradation in strain to fallure by 4 ax. The computed cribcal pullout length of CNTs ranges from 21 to

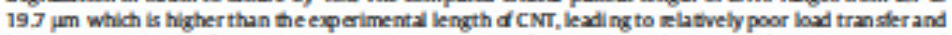
law tenalle stren gh of PSF nanocomposites. Fracture surtace validates that tensile fracture is gowerned strongly by the constitutive hierarchical microstructure of the plasma spayed AL-CNT n anocomposite The facture path in N-CNT nanocomposite ocous in AL-S matrix adjacent to SC layer on CNI surbe e 2009 Elevier Lat All rights reserved.

\section{Introduction}

Carbon nanotubes (ONTs) exhibit relatively low density ranging from $\sim 1.2 \mathrm{~g} / \mathrm{cm}^{3}$ for single walled CNT to $\sim 1.8 \mathrm{~g} / \mathrm{cm}^{3}$ for multiwalled ONTs $[1,2]$ and subsequently have high specific strength of $\sim 55.5 \mathrm{GPa} /\left(\mathrm{mg} / \mathrm{m}^{3}\right)[2]$. These unique mechanical properties of CNTs make them promising reinforcements for synthesizing light weight, high streng th metal matrix structural composites Reoent efforts have been devoted to synthesize $\mathrm{Al}$ and $\mathrm{Mg}$ nanocomposites reinfor aed with carbon nanotubes (CNT) for the purpose of lightweight high-strength structural materials $[2-27]$. However, the unique mechanical properties of individual CNTs have never been translated to bulk composites with nanotube reinfor cements. The question of realizing nanoscale properties at bulk scale is an existing challenge for the scientific community which is yet $t$ find a convincing answer. Though there is a fair deg ree of agreement that problems are largely associated with consolidation or processing of nanocomposites. Fabrication of bulk namocmposite structur ewith CNT reinforcement has always become difficult primarily due to (i) problem of in homogeneous ONT distribution and retention, and (ii) insufficient adhesion at the matrix-reinforcement interface for effective lasd transfer. Consequently, measuring the mechanical properties of CNT reinforaed nanocamposites has been extremely difficult and often ambiguous, attributed to the difficulties of preparing mechanical testing samples Most of the mechanical property data reported on CNT reinforod nanocomposites has been

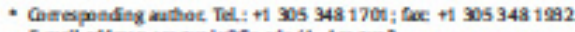

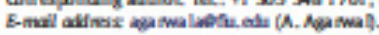

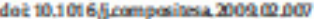

hased on microhardness nanoindentation, miniaturized sample or computational theories. Table 1 lists the available information on the tensile properties of $\mathrm{CNT}$ reinforced aluminum composites Few research groups have conducted tersile tests on relatively large (15 mm gauge length) AJ-CNT composite but obtained very different yield strength values $[3,22$, Kurumaki et al [3] obtained the yield strength of $80 \mathrm{MPa}$ for 5-10 voll ONT whereas Deng et al [22] reported yield strength of $336 \mathrm{MPa}$ for mere $1 \mathrm{wt} \mathrm{CNT}$ addjtion to Al matrix.It is evident from Table 1 that the tensile properties of CNT reinforced aluminum composites synthesized by different processes display a wide scatter in the medhanical properties attributed to the variance in the microstructural features, defects, poros ity level caused by processing and lack of consistency in mechanical testing techriques and samples [28].

Our research group has demonstrated successful fabrication of multiwalled carbon nanotube (MWONT) reinfarced hypereutectic Al-Si bulk nanocomposite structures by plasma spray forming (PSF) $[7,10,12,27]$ The nanocmposite exhibits retention and homogenous distribution of MWONTs in $\mathrm{Al}-\mathrm{Si}$ matrix [27]. In the present effort, relativdy large sumples ( $50 \mathrm{~mm}$ overall length) for tersile tests have been machined out of the cylindrical Al-Si nanocmposite structure reinforced with MWCNTs. Uniaxial tensile testing has been carried out to evaluate stress-strain behavior. The experimental values of ultimate tensile strength were compared with theoretically computed values based on fiber pull out theory [29]. Additionally, the aim of this imestigation is to evaluate the mechanical properties of "large" ONT reinforced aluminum nanocomposites from the viewpoint of canstitutive hierarchical microstructure, to bridge therelationship among mecharical prop- 


\section{Effect of carbon nanotube and aluminum oxide addition on plasma-sprayed hydroxyapatite coating's mechanical properties and biocompatibility}

Jorge E. Tercero ${ }^{2}$, Shabnam Namin ${ }^{\text {b }}$, Debrupa Lahiri ${ }^{2}$, Kantesh Balani ${ }^{2, c}$, Nikolaos Tsoukias ", Arvind Agarwal ${ }^{2, *}$

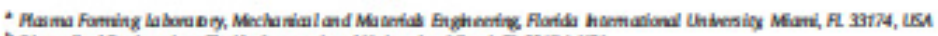

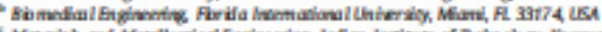

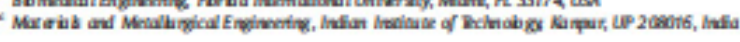

\section{A RTICLE I N F O}

Areilehatory

heceived 6 Apd 200

Necepead 1 May zon

Avilulie adix a May ano

Kegmontis

Hyd expyacike

Pleama sowing

Freare waygras.

Biocompatily

Cutm anoute:

\section{A BST R ACT}

This study repoits $\mathrm{an}$ the synthesis $d$ n avel bloceramic compositr coating of hydracyapatit (HA) reinforced with carbon nanotubes (CNIs) and aluminum oride $\left(\mathrm{Al}_{2} \mathrm{O}_{\mathrm{A}}\right)$ wesing plasma spray technique fracture

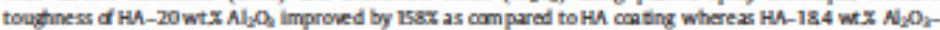
15 wtx CNT showed an impiowement of $300 \%$ Carbon nanotules provided reh forcement via rebar mechanism. Human filer ortechlast cellgrowth studies showed thrit bixampatbility of the coaring

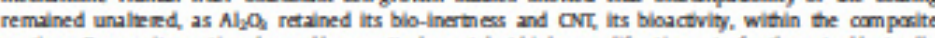

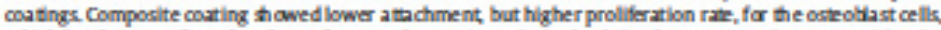
which has been attributed to the surtace roughness. An optim ined rehtson between couxing composition, it: blocompatiblity and mexhanical propertes was extablshed to predict the most sulted coaring material for orthopedic implanta. $\mathrm{HA}-\mathrm{Al}_{2} \mathrm{O}_{2}$ - ONT composity coating displyyed mos improved mechanical poopertes while retaining its tocompatbility.

D 2009 Elisevier R.V. All rights resenved.

\section{Introduction}

Hydraxyapatite (HA) is a cakium phos phate based ceramic ( $\mathrm{C} 2 / \mathrm{P}$ ratio of 166$)$ with a chemical composition $\left(\mathrm{Ca}_{10}\left(\mathrm{PO}_{4}\right) \mathrm{s}(\mathrm{OH})_{2}\right)$ similar to that of bone HA has been widdy acoepted in the biamedical industry for orthopedics, maxillof $x$ ial surgery and dental implants because of its biocmpatibility and asseointegration [1]. One of the main applications of HA is that as matings on metallic medical implants. Despite the advantages that HA presents, the brittle nature, and low fracture toughness of HA caating often result in rapid werr, and premature fracture of the conted layer. Hence, there is a need to improve the mecharical properties of the HA castings without compromising the biocompatibility.

As a response to such ned, many effarts have attempted to enhance the mechanical properties of HA via the addition of secondary ceramic reirforcement materials such $2 \mathrm{Al}_{2} \mathrm{O}_{2}, \mathrm{ZrO}_{2}, \mathrm{TO}_{2}$ [1-10. Gautier and $\mathrm{m}$-warkers have reported four times increase in the fracture toughness with 20 voli of $\mathrm{Al}_{2} \mathrm{O}_{3}$ addition in the form of platdets $\left[\mathrm{B}\right.$. Li et al also agreed to the fact that $\mathrm{AL} \mathrm{O}_{3}$ addition helps increasing the bending strength of $\mathrm{HA}$ composite [4]. Evis and Daremus [7] studied $\mathrm{HA}-\mathrm{ZrO}_{2}$ cmposites and reported the increase in hardness and fracture toughness of the camposite with $\mathrm{ZrO}_{2}$ addition. Findings of Fu et al also follow the same trend, and in

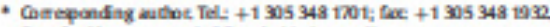

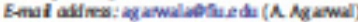

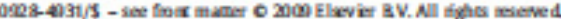

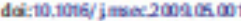

addition they reparted the significant reduction in decrmpasition of HA during high temperature plasma processing [2]. Wang et al [1] has studied the wer property as an effect of partially stabilined $\mathrm{ZrO}_{2}$ additian to $\mathrm{HA}$ against ultra high molecular weight polyethylene in humar plasma lubrication (asimulated bio implant ervironment) and has reparted improvement in the wear resistance due to the secand phase particle addition.

A few researchers have attempted carbon nanotubes (ONTs) as the reinfarcement in HA-based composites to improve its mechanical strength [11- 15] Chen and co-workers [13] were first to report such an effort where they have shown improvement in wear resistunce with addition of up to 20 wtI ONB in HA-composites Meng et al reported an increzse in flexural strength of HA-CNT composite by -10 val $q$ CNT addition [11] Kaya ha observed the increase in bond strength of mating with Th-6A]-4Vimplant substrite with addition of $2 x$ ONT to HA [12]. Theses matings also demons trated increase in the elastic modulus, hardness and fradure toughnes [12,13]. Balani et al. from our research group reported $56 x$ increase in the fracture toughness [14] and $60 \%$ decrease in the wer volume [15] of the plasme-sprayed composite casting of HA with 4 wtI OVI. The biocompatibility of the ONT reinforced HA coating was established by ell growth and proliferation [14]

In general addition of ceramics or second phase partides hops in increasing the mechanical properties of HA-based composite caating for biamedical implants. Recently an attempt was madeby Kalem et il. [16] to overcomeceramic brittleness by dual strengthening via add ibion 


\section{The Nano-scratch Behavior of Biocompatible Hydroxyapatite Reinforced with Aluminum Oxide and Carbon Nanotubes}

Kantesh Balani, Debrupa Lahiri, Anup K. Keshri, S.R. Bakshi, Jorge E. Tercero, and Arvind Agarwal

Hydroxyapatite (HA) reinforced with sub-micrometer $\mathrm{Al}_{2} \mathrm{O}_{3}$ and carbon nanotubes (CNTs) has been synthesized as a coating on the $T i-6 \mathrm{Al}-4 \mathrm{~V}$ substrate via plasma spraying. The addition of $\mathrm{Al}_{2} \mathrm{O}_{3}$ and $\mathrm{CNTS}$ to $\mathrm{HA}$ has shown improvement in the hardness and elastic modulus by $65 \%$ and $50 \%$, respectively, when compared to HA. Consequently, $\mathrm{HA}-\mathrm{Al}_{2} \mathrm{O}_{3}-\mathrm{CNT}$ coatings have been nano-scratched to understand their wear performance. Reinforcement of $\mathrm{HA}$ by $\mathrm{Al}_{2} \mathrm{O}$, shows a decrease in the wear volume by more than 13 times, whereas $\mathrm{HA}-\mathrm{Al}_{2} \mathrm{O}_{3}-\mathrm{CNT}$ coating demonstrated further wear volume reduction of five times compared to that of $\mathrm{HA}_{-} \mathrm{Al}_{2} \mathrm{O}_{3}$ coating.

\section{INTRODUCTION}

Biocompatibillity of hydroxyapatite (HA) has been established by various researchers because of its chemical structure similar to that of bone and teeth $(\mathrm{Ca} / \mathrm{P} \text { ratio of } 1.66)^{1,2}$ Application of $\mathrm{HA}$ as a structural material is limited because of its brittleness and low fracture toughness $\left(-0.4 \mathrm{MPa} \mathrm{m}^{12}\right)$. Hence arises the need to reinforce the HA without hampering its biocompatibility. Introduction of ceramics (e.g., $\mathrm{ZrO}_{2}, \mathrm{Al}_{2} \mathrm{O}_{3}$, mullite, $\mathrm{Ni}, \mathrm{Al}$, and $\mathrm{SiC}$, ) with better mechanical properties has been applied to enhance the energy absorption by HA matrix. ${ }^{2-6}$ It becomes a special concern to retain the biocompatibility of the composite while reinforcing secondary materials to improve mechanical properties of HA matrix. ${ }^{24}$ Extensive interfacial reaction between $\mathrm{HA}$ and $\mathrm{ZrO}_{2}$ to form tricalcium phosphate (TCP) limits its utilization.4 Toxicity of $\mathrm{SiC}_{\mathbf{n}}$ restricts its reinforcement in HA matrix. ${ }^{7}$ Alumina $\left(\mathrm{Al}_{2} \mathrm{O}_{3}\right)$, being a bioinert material, has shown much promise. ${ }^{2 x}$ Reinforcement with carbon nanotubes (CNTs) has shown frac-

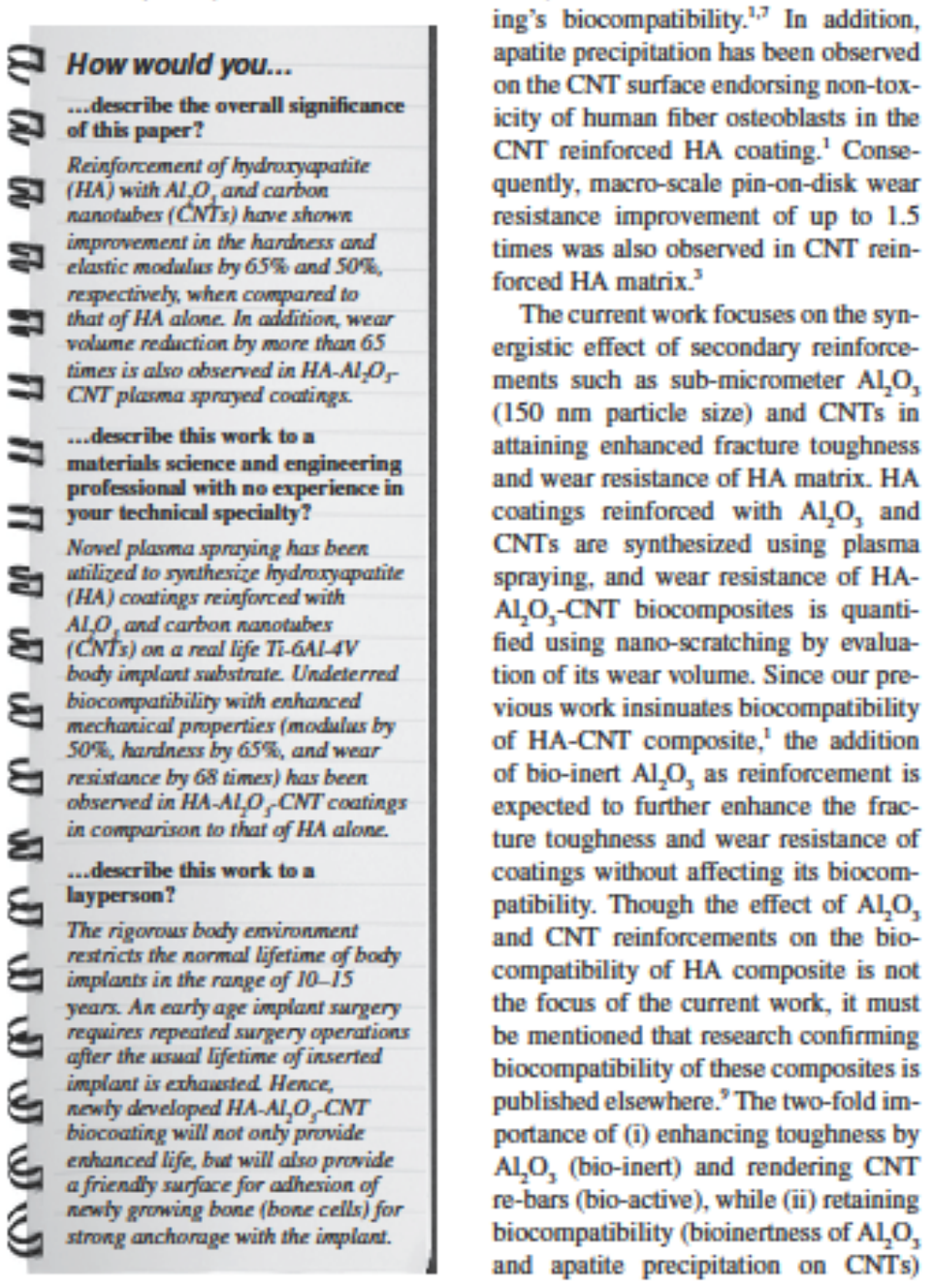

ture toughness enhancement by up to three times, HA crystallinity increase by $27 \%$, bending strength of up to 180 $\mathrm{MPa}$, without deterioration of the coaton the CNT surface endorsing non-toxicity of human fiber osteoblasts in the resistance improvement of up to 1.5 The current work focuses on the synreinforce(150 nm particle size) and CNTs in attaining enhanced fracture toughness CNTs are synthesized using plasma spraying, and wear resistance of HA-CNT biocomposites is quantition of its wear volume. Since our previous work insinuates biocompatibility -CNT composite, ${ }^{1}$ the addition ure toughness and wear resistance of its biocomand $\mathrm{CNT}$ reinforcements on the biocompatibility of HA composite is not biocompatibility of these composites is published elsewhere. 'The two-fold imand apatite precipitation on CNTs 
The back cover description that will be placed here will be routed separately from the copywriting department. You will have a chance to review and make corrections at that time.

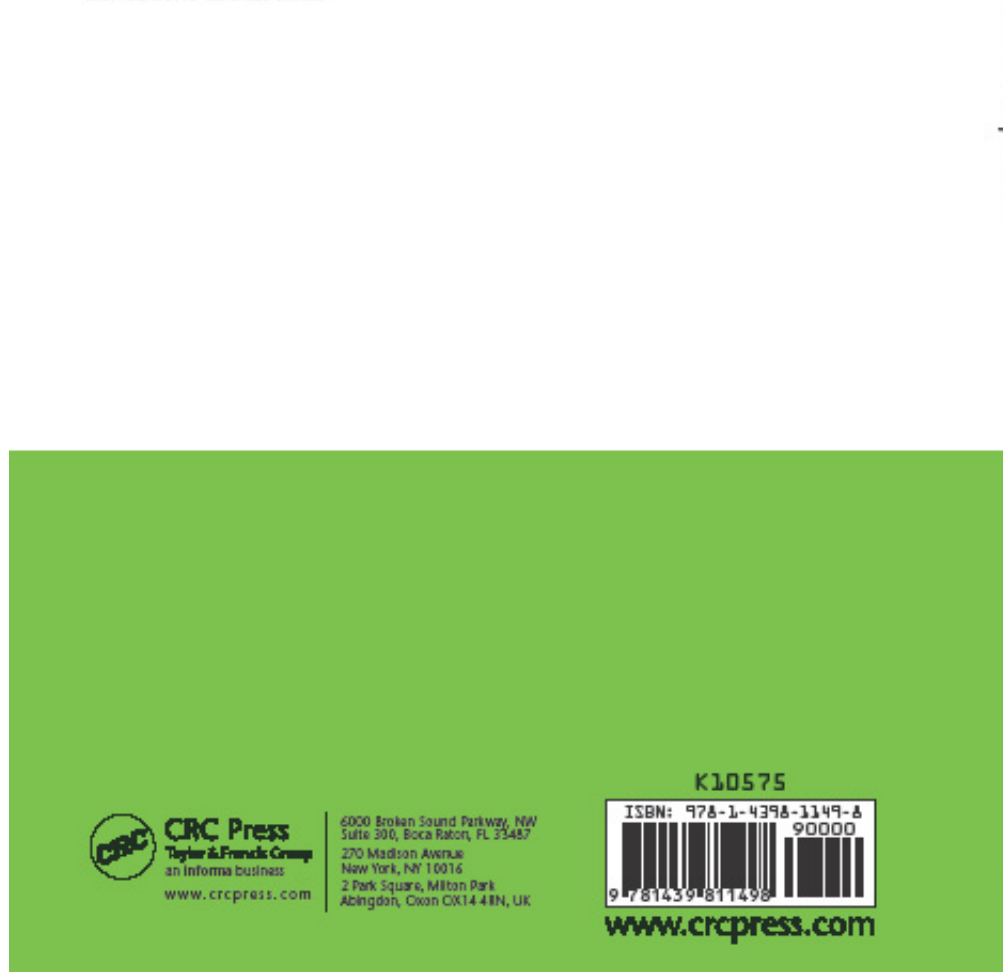

Carbon Nanotubes

Reinforced

Metal Matrix Composites

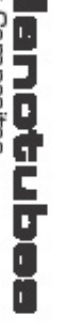

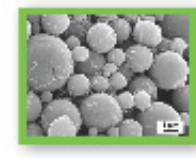

\section{.}

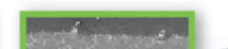

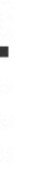

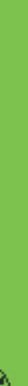

Arvind Agarwal

Srinivasa Rao Bakshi cac press

Debrupa Lahiri 
VITA

\section{DEBRUPA LAHIRI}

EDUCATION

Aug. 1994 - Jun. 1998

Aug. 1998 - Jun. 2000

Aug. 2007 - May. 2011

PROFESSIONAL EXPERIENCE

May. 1999 - Jan. 2001

Jul. 2001 - Jul. 2003

Aug. 2003 - Jul. 2007
: Bachelor of Engineering (B.E) in Metallurgical Engineering, Bengal Engineering College, Shibpur, India (Third position in class of 1998)

: Master of Technology (M. Tech) in Metallurgical and Materials Engineering, Indian Institute of Technology, Kanpur, India (First position in class of 2000)

: Doctoral Candidate (Ph.D) in Florida International University, Miami, USA (GPA - 3.93/4.0)

: Metallurgist, R\&D in Indian Aluminium Co. Ltd. (INDAL), ALCAN group, Belur, India

: Visiting Scientist, Nuclear Fuel Complex, Dept. of Atomic Energy, Govt. of India, Hyderabad, India : Scientific Officer D, Nuclear Fuel Complex, Dept. of Atomic Energy, Govt. of India, Hyderabad, India

\section{ACADEMIC AWARDS AND HONORS}

1. Recipient of Dissertation Year Fellowship (DYF) by University Graduate School, FIU for Spring-Summer, 2011.

2. Second best oral presentation award for symposium on "Surfaces and Heterostructures at Nano- or Micro-Scale and Their Characterization, Properties, and Applications" in TMS Annual meeting, San Diego, CA, 27Feb - 3 Mar, 2011.

3. First place in student poster competition, MS\&T, Houston, TX, 17-21 Oct. 2010.

4. Recipient of Dissertation Evidence Acquisition (DEA) Fellowship by University Graduate School, FIU for 2009-2010.

5. First place in student poster competition - Materials Processing and Manufacturing Division, TMS, San Francisco, CA, 14 - 18 Feb, 2009.

6. Selected as student delegate to PCSA-AcerS President's Council of Student Advisors for 2009-2010.

7. Outstanding Member Award for Materials Advantage, Council of Student Organizations, FIU, 2008-2009 (among more than 175 student clubs).

8. Nominated for membership of Sigma Xi Honors Society 2008-2009 by Department of Mechanical and Materials Engineering, FIU. 


\section{PUBLICATIONS}

1.D. Lahiri, A.P. Benaduce, L. Kos, A. Agarwal, "Quantification of Carbon Nanotube Induced Adhesion of Osteoblast on Hydroxyapatite using Nano-Scratch Technique”, Nanotechnology 2011 (in press).

2.D. Lahiri, V. Singh, A.K. Keshri, S. Seal, A. Agarwal, "Apatite Formability of Boron Nitride Nanotube”, Nanotechnology, Vol. 22, 2011, pp. 205601.

3.I. Lahiri", D. Lahiri*, S. Jin, A. Agarwal, W. Choi, "Carbon Nanotubes: How Strong is Their Bond with the Substrate?”ACS Nano, Vol. 5(2), 2011, pp. 780-787. Highlighted in "Nanowerk". * Co-first Authors.

4.S. Facca*, D. Lahiri*, F. Fioretti, N. Messadeq, D. Mainard, N. Jessel, A. Agarwal, "In Vivo Osseointegration of Nano-Designed Composite Coatings on Titanium Implants", ACS Nano, 2011, Vol. 5, pp. 4790-4799* Co-first Authors.

5.D. Lahiri, V. Singh, A.P. Benaduce, S. Seal, L. Kos, A. Agarwal, "Boron Nitride Nanotube Reinforced Hydroxyapatite Composite: Mechanical and Tribological Performance and In-Vitro Biocompatibility to Osteoblasts", Journal of the Mechanical Behavior of Biomedical Materials, Vol. 4, 2011, pp. 44-56.

6.D. Lahiri, A.P. Benaduce, F. Rouzaud, J. Solomon, A.K. Keshri, L. Kos, A. Agarwal, "Wear Behavior and In-vitro Cytotoxicity of Wear Debris Generated from Hydroxyapatite-Carbon Nanotube Composite Coating" Journal of Biomedical Materials Research Part A, Vol. 96A, 2011, pp. 1-12.

7.D. Lahiri, V. Singh, A. K. Keshri, S. Seal, A. Agarwal, "Carbon Nanotube Toughened Hydroxyapatite by Spark Plasma Sintering: Microstructural Evolution and Multi-Scale Tribological Properties”, Carbon, Vol. 48, 2010, pp. 3103-3120.

8.D. Lahiri, F. Rouzaud, T. Richard, A. K. Keshri, S.R. Bakshi, L. Kos, A. Agarwal, "Boron Nitride Nanotube Reinforced Polylactide-Polycaprolactone Copolymer Composite: Mechanical Properties and Cytocompatibility with Osteoblasts and Macrophages In Vitro", Acta Biomaterialia, Vol. 6, 2010, pp. 3524-3533.

9.D. Lahiri, F. Rouzaud, S. Namin, A.K. Keshri, J.J. Valdes, L. Kos, N. Tsoukias, A. Agarwal, "Carbon Nanotube Reinforced Polylactide-Caprolactone Copolymer: Mechanical Strengthening and Interaction with Human Osteoblasts in Vitro", ACS Applied Materials and Interfaces, Vol. 1, 2009, pp. 2470-2476.

(Listed 9 out of 31 published journal articles, 3 more papers are under review in peer reviewed journals)

\section{BOOK}

"Carbon Nanotubes Reinforced Metal Matrix Composites", A. Agarwal, S.R. Bakshi, D. Lahiri, Taylor and Francis Publishers, ISBN: 978-1-4398114-9-8.

\section{BOOK CHAPTER}

"Graphene Reinforced Ceramic and Metal Matrix Composites", D. Lahiri, A. Agarwal, in "Graphene: Synthesis and Applications", Editors: W. Choi and J. Lee, Taylor and Francis Publishers, ISBN: 9781439861875 (to be published in October, 2011). 